\title{
$\mathrm{f}_{7}^{\mathrm{y}}$ \\ UNIVERSITAT \\ JAUME• |
}

Ph.D. Thesis

\section{Molecular insights into the promiscuity of serine hydrolases. Towards a computationally guided protocol for the redesign of enzymes}

Author

Miquel Àngel Galmés Ordinas

Supervisors

Dr. Vicent Moliner Ibáñez Dr. Katarzyna Patrycja Świderek 



\title{
P⿻ㄱำ \\ UNIVERSITAT \\ JAUME•I
}

Doctoral Program in Theoretical Chemistry and Computational Modelling

\author{
Universitat Jaume I Doctoral School
}

\section{Molecular insights into the promiscuity of serine hydrolases. Towards a computationally guided protocol for the redesign of enzymes}

Report submitted by Miquel Àngel Galmés Ordinas in order to be eligible for a doctoral degree awarded by the Universitat Jaume I

\begin{tabular}{|c|c|c|}
\hline $\begin{array}{l}\text { Miquel Àngel Galmés } \\
\text { Ordinas } \\
\text { (Ph.D. candidate) }\end{array}$ & $\begin{array}{l}\text { Dr. Vicent Moliner } \\
\text { Ibáñez } \\
\text { (Supervisor) }\end{array}$ & $\begin{array}{c}\text { Dr. Katarzyna } \\
\text { Patrycja Świderek } \\
\text { (Supervisor) }\end{array}$ \\
\hline $\begin{array}{l}\text { rmado por MIQUEL ANGEL } \\
\text { ALMES ORDINAS - } \\
\text { IF:41523286Y el día } \\
\text { b:11/2021 con un certificado } \\
\text { nitido por ACCVCA-120 }\end{array}$ & $\begin{array}{ll}\text { VICENTE } & \begin{array}{l}\text { Firmado digitalmente } \\
\text { por VIIENTEMOLINER }\end{array} \\
\text { MOLINER IBANNEZ IBANEZ- NIF:25388050Y } \\
\text { - NIF:25388050Y Fecha: 2021.11.130 } \\
11: 56: 54+011^{\prime} 00^{\prime}\end{array}$ & 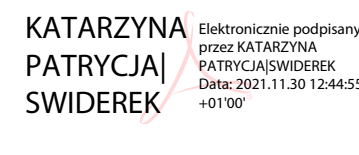 \\
\hline
\end{tabular}

Castelló de la Plana (Spain), November 2021 



\section{Funding:}

- Universitat Jaume I through the predoctoral grant PREDOC 2017/23.

- Universitat Jaume I through the project UJI B2017- 31 and UJIA2019-04.

- Spanish Ministerio de Ciencia, Innovación y Universidades through the grant PGC2018-094852-B-C21

- Spanish Ministerio de Economía y Competitividad through the grant MAT2015-65184-C2-2-R.

- Generalitat Valenciana through the grant AICO/2019/195 and SEJI/2020/007.

- USA National Institute of Health through the project Ref No. NIH R01 GM065368. 



\section{Thesis by compendium of publications:}

(1) Galmés, M. A.; García-Junceda, E.; Świderek, K.; Moliner, V. Exploring the Origin of Amidase Substrate Promiscuity in CALB by a Computational Approach. ACS Catal. 2020, 10, 1938-1946. DOI: 10.1021/acscatal.9b04002. Impact Factor: 13.084.

Contribution: MAG did all the calculations and the experimental kinetics and contributed to the writing of the manuscript.

(2) Galmés, M. À.; Swiderek, K.; Moliner, V. Computational Studies Suggest Promiscuous Candida Antarctica Lipase B as an Environmentally Friendly Alternative for the Production of Epoxides. 7. Chem. Inf. Model. 2021. DOI: 10.1021 /acs.jcim.1c00425. Impact Factor: 4.956.

Contribution: MAG did all the calculations and contributed to the writing of the manuscript.

(3) Galmés, M. À.; Nödling, A. R.; Luk, L.; Świderek, K.; Moliner, V. Combined Theoretical and Experimental Study to Unravel the Differences in Promiscuous Amidase Activity of Two Nonhomologous Enzymes. ACS Catal. 2021, 8635-8644. DOI: 10.1021/acscatal.1c02150. Impact Factor: 13.084.

Contribution: MAG did all the calculations and contributed to the writing of the manuscript.

(4) Galmés, M. À.; Nödling, A. R.; He, K.; Luk, L.; Świderek, K.; Moliner, V. Computational Design of an Amidase by Combining the Best Electrostatic Features of Two Promiscuous Hydrolases. 2021. Manuscript submitted for publication.

Contribution: MAG did all the calculations and contributed to the writing of the manuscript.

\section{"This thesis has been accepted by the co-authors of the publications listed above that have waved the right to present them as a part of another PhD thesis"}



Per a tu mumare, i per ets teus ulls plorosos dets que tantes vegades m'he sentit responsable 

Overhead the albatross

Hangs motionless upon the air And deep beneath the rolling waves

In labyrinths of coral caves

The echo of a distant time

Comes willowing across the sand And everything is green and submarine And no one showed us to the land And no one knows the where's or why's But something stirs and something tries

And starts to climb toward the light

Strangers passing in the street

By chance, two separate glances meet

And I am you and what I see is me

And do I take you by the hand

And lead you through the land

And help me understand the best I can?

And no one calls us to move on And no one forces down our eyes

No one speaks and no one tries

No one flies around the sun Cloudless everyday

You fall upon my waking eyes

Inviting and inciting me to rise

And through the window in the wall

Come streaming in on sunlight wings A million bright ambassadors of morning

And no one sings me lullabies And no one makes me close my eyes So, I throw the windows wide And call to you across the sky

Echoes. Pink Floyd 



\section{Acknowledgments}

Some years have passed since I started this adventure and now that is finishing, I can say that it has been an unforgettable experience. Lots of things happened during these years, good and bad, but this overwhelming feeling is part of the predoctoral journey. I can gladly say that I met many wonderful people from whom I learned a lot, not only from the scientific perspective but the human angle. It would be tedious for a Ph.D. thesis to write the names of all the people who, in some way or another, have made an impact on the final outcome of this document. For this reason, I might first apologize if I forget somebody below these lines.

This thesis would not been possible without the advice and the encouragement of my supervisors Vicent Moliner and Katarzyna Świderek. They always helped to make some sense of the confusion. I would like to thank my tutor Sergio Martí, without whom I would be still lost and who always tried to teach me something. Immense gratitude to Raquel Castillo for her patience and help along this tough time writing this thesis. I would like to give my gratitude to Eduardo García-Junceda for the time I spent with in Madrid, and for all the knowledge he transmitted to me.

I can't put into words the wonderful friendship I found in Natalia, Daria and Eduardo. It will be too long to tell all the good (and bad) moments we had together for that reason I only want you to know that this journey would have been incredibly disappointing without you. I would like to thank the rest of the members of the group for their always useful advice and help.

During this period, I had many moments of happiness and annoyance, but I have been a lucky person, who could share all these feelings with many friends that always supported me. I would like to give to Victoria, Xesca, Silvia, Joana, Marta, Sabina, Carol, Rocío, Gabri, Cati, Diana, Guiem, 
Bruno, Juanma, Andreu, Dury, Pomar, Balles, Boni, Pablo and Dani, my sincere gratitude to welcome me every time I went to Mallorca, even for a short and without notice visit. To Jessica, Elena and Juanca, that although this pandemic has prevented us to meet often you have been always there for a quick virtual chat. My most affectionate thanks to Ander and Nerea for all the time we spent rambling about the problems of life and for how all that helped me. To Rauli, for the many upcoming conversations that will come in the future. To Ana and Nadia, whose happiness is so contagious that it is impossible to be sad by their side. Finally, to all my friends around Spain, Laura P., Laura S., Helia, Ana, Cris, Zoraima, Lara, Sussy, Elena, Rafets, Raulito, Dani, Àngel, Pablo, Kike and Jorge. Many weddings (and kids) happened along these years, and it was always a pleasure to share those moments with you.

I left the most important people in my life for the end. There is nothing I can say to them that they don't actually know. To my parents, Cati and Nofre, simply thank you for everything. You always made my life easier and full of love and happiness. To my sister Marina, thank you for your vision of life, it was always useful, and thank you to try to make me smile whenever you could. I tried to do my best as the older brother. Finally, to my companion in life, Cristi. You know that I will always love you no matter what happens. Your kindness and love saved my life. Thank you for being by my side in this long journey. 


\section{Abstract}

The aim of the present thesis is to give insights into the molecular mechanisms of enzyme promiscuity from a computational and an experimental perspective. The obtained knowledge encourages further research focused on the exploration of new computational schemes valuable for enzyme (re)design procedures.

Enzyme promiscuity is understood as the ability to catalyze secondary reactions apart from the ones that have evolved to be dominant. In this sense, these promiscuous or moonlighting functions might serve as a scaffold for evolution either in vivo or in vitro. A better understanding of the molecular mechanisms that regulate this behavior is crucial for the development of new tools and techniques to be used in industry and in academia.

Two serine hydrolases, Candida antarctica Lipase B and para-nitrobenzyl esterase from Bacillus subtilis, were used as a model to study enzyme promiscuity through quantum mechanics / molecular mechanics methods and experimental enzymes kinetics in the present Ph.D. thesis. Therefore, both the catalytic and the substrate promiscuity were studied. Firstly, the hydrolysis of amides catalyzed by Candida antarctica Lipase B was explored, proving that this enzyme catalyzed the amidase reaction, although some specific features of the substrate are required. Secondly, the epoxidation of short alkenes catalyzed by the same enzyme was proposed as an ecofriendly alternative to the traditional chemical synthesis and for the epoxide production in the industry.

Based on these previous studies, a computational scheme for the redesign of the para-nitrobenzyl esterase from Bacillus subtilis was also proposed. We found that this serine hydrolase catalyzed the hydrolysis of amides in a similar mechanistic manner to Candida antarctica Lipase B. From the computational and experimental studies, we proved that they had similar 


\section{Abstract}

catalytic performance. This finding encouraged us to carry out a deep mechanistic and structural comparison between both enzymes. The electrostatic environment around the active site was analyzed and a map of structural determinants in the vicinity of the active site pocket was done using 3D Convolutional Neural Networks. Once the most appropriate protein scaffold was selected, the proposed computationally guided protocol for the mutagenesis of enzymes based on the detailed analysis of the electrostatic environment of two structurally aligned trajectories using rotation quaternions was applied. A new mutant variant, the F398D, of the para-nitrobenzyl esterase from Bacillus subtilis was suggested as an improved catalytic variant by combining the best electrostatic features of both enzymes. Although, little improvement was achieved in terms of catalytic activity, this protocol might serve as starting point to propose new catalytically improved variants.

To conclude, the work presented in this thesis has contributed to the knowledge in the field of molecular mechanisms of enzyme promiscuity from a broad perspective. Moreover, the proposed protocol for the rational redesign of enzymes could contribute to future general strategies to be used in the computational enzyme design toolbox. 


\section{Index}

Acknowledgments .......................................................................................... XIII

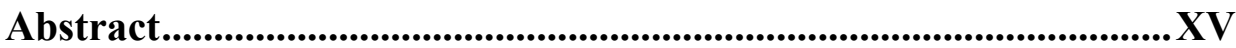

Chapter 1. Introduction .......................................................................1

1.1. Enzyme Promiscuity ...............................................................................3

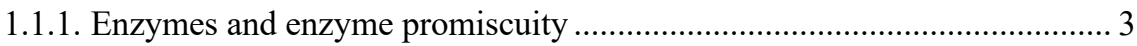

1.1.2. Evolutive perspective of enzyme promiscuity........................................ 7

1.1.3. Applications of enzyme promiscuity to enzyme engineering .................. 11

1.2. Carboxylic Ester Hydrolases ............................................................. 15

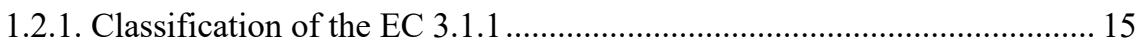

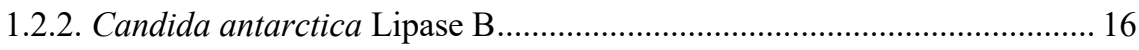

1.2.3. para-nitrobenzyl esterase from Bacillus subtilis .................................... 19

1.3. Protein engineering........................................................................................ 22

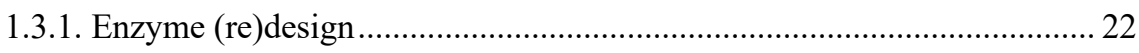

1.3.2. Experimental strategies in enzyme (re)design....................................... 25

1.3.3. Computational strategies in enzyme (re)design....................................... 26

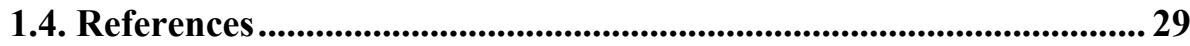

Chapter 2. Objectives ...........................................................................41

Chapter 3. Computational and Experimental Methods ......................45

3.1. Structure and energy ...................................................................................... 47

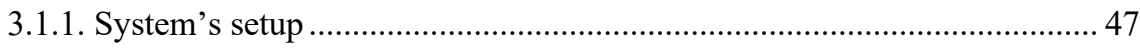

3.1.2. Molecular Mechanics potentials ........................................................... 50

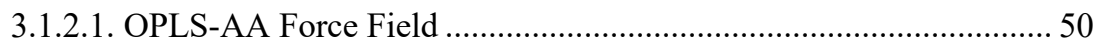

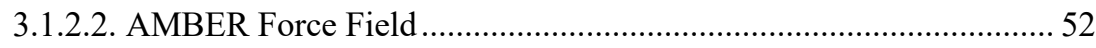

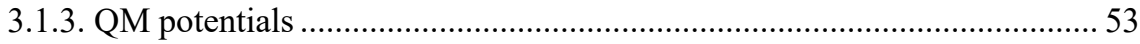

3.1.3.1. Semiempirical approximations ………........................................ 53 
3.1.3.2. Density Functional Theory approximations ................................. 54

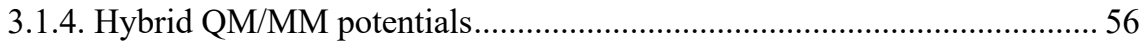

3.2. Exploring reactivity ...............................................................6 60

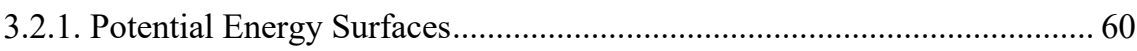

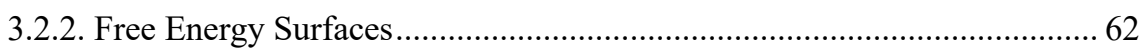

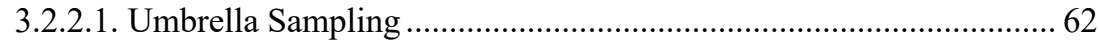

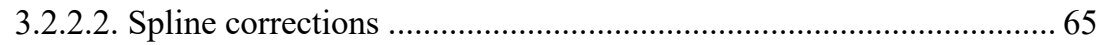

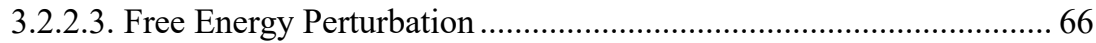

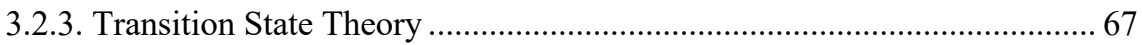

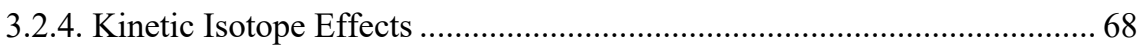

3.3. Exploring protein-ligand binding step .......................................70

3.4. Convolutional Neural Networks ..................................................... 72

3.4.1. A brief introduction to Convolutional Neural Networks.......................... 72

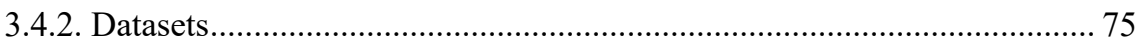

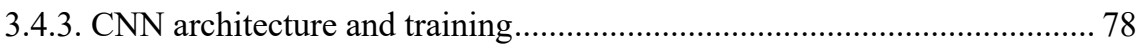

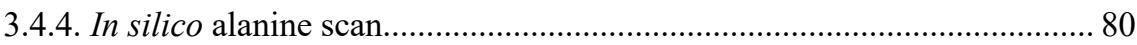

3.5. Rational enzyme redesign methods ................................................. 82

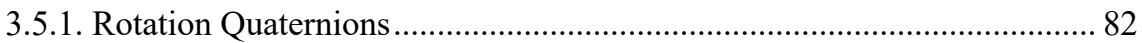

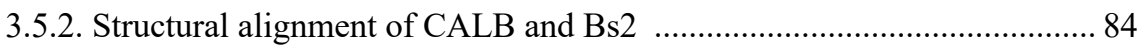

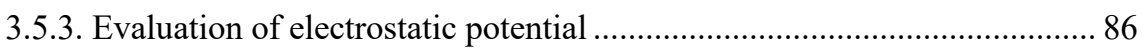

3.6. Experimental determination of kinetic parameters..........................87

3.6.1. Synthesis of the benzylbutyramide........................................................ 87

3.6.2. Synthesis of the $N$-(4-nitrophenyl)-butyramide....................................... 88

3.6.3. Kinetic assays of the hydrolysis of benzylbutyramide ............................... 88

3.6.4. Kinetic assays of the hydrolysis of $N$-(4-nitrophenyl)-butyramide .......... 89

3.7. References...................................................................................................... 91

Chapter 4. Results and Discussion .........................................................97

4.1. Promiscuity studies in CALB............................................................... 100

4.1.1. Hydrolysis of the amide bond catalyzed by CALB (Article 1) ............... 100

4.1.2. Direct epoxidation of short alkenes catalyzed by CALB (Article 2) ...... 113

4.2. A computer-assisted rational redesign................................................... 129 
4.2.1. Structural-mechanistic comparison of Bs2 and CALB (Article 3).... 129

4.2.2. Redesign of Bs2 based on electrostatic features of CALB (Article 4).... 143

4.3. References.................................................................................................. 173

Chapter 5. Conclusions and future perspectives..................................175

Appendix. List of abbreviations ...............................................................181

Annex A. Supporting Information of Article 1 .....................................183

Annex B. Supporting Information of Article 2 .................................213

Annex C. Supporting Information of Article 3 ....................................231

Annex D. Supporting Information of Article 4 ...................................269

Annex E. Co-author's consent ......................................................313 

Chapter 1

Introduction 



\subsection{Enzyme Promiscuity}

\subsubsection{Enzymes and enzyme promiscuity}

Enzymes are the driven force of life. They are involved in all the processes that maintain the cells alive. ${ }^{1}$ Enzymes not only catalyze almost every single reaction in the cells ${ }^{2}$ but help to sustain the structural integrity of its components such as $\mathrm{DNA}^{3,4}$ and $\mathrm{RNA},{ }^{5}$ participate in the transport tasks of the cell, ${ }^{6}$ and in the metabolism of lipids ${ }^{7}$ and sugars. ${ }^{8}$ They are also involved in the energy production that living organisms need for survival. ${ }^{9}$ Enzymes participate in the regulation of vital functions, forming very complex networks in the organism. ${ }^{10}$ But despite their crucial role, a lot of mystery still surrounds enzyme biochemistry. For instance, although we have powerful theories to predict how molecules behave, having tools to predict the tertiary structure of enzymes is not a trivial task. ${ }^{11}$ In fact, Levinthal's paradox highlights how complex the prediction of the tertiary structure of proteins can be. In 1969 Cyrus Levinthal pointed out, in his very famous paper entitled How to Fold Graciously, that an average protein of 150 residues of amino acid has $10^{300}$ possible conformations. ${ }^{12}$ This is an astronomic number, so, it is evident that the protein does not explore all possible conformers when folding, since only the most thermodynamically favorable ones are observed. Even though, which are the laws that govern protein folding are still unknown. This fact introduces significant difficulties from the biochemical perspective, especially for the rational design of new biocatalysts. Therefore, the use of computational-based methods in combination with experimental approaches is the gold-standard in protein engineering. ${ }^{13}$ From this scenario, two main trends can be followed, the rational (re)design, such as the de novo design, ${ }^{14,15}$ the design of catalytic antibodies ${ }^{16,17}$ or the redesign of promiscuous enzymes, and the directed evolution approaches (see section 1.3 for a detailed explanation). Rational 


\subsection{Enzyme Promiscuity}

redesign is usually based on the reshaping of the active site pocket, or the close vicinity, of a promiscuous or non-specific enzyme. The presence of secondary activities gives an excellent opportunity to redesign the enzyme towards other latent activities. Additionally, the de novo design is a completely different strategy. In this case, the methodology intends to design from scratch a novel biocatalyst. This ambitious strategy is usually based on information related to the transition state, and has been applied with limited success. ${ }^{13}$ On the other hand, directed evolution strategies exploit enzyme promiscuity for the evolution in vitro, to render improved biocatalysts with impressive performance. ${ }^{18}$

When a protein is mutated, it is almost impossible to predict what will be the final effect on it. Most of the time the mutations are deleterious affecting its stability and promoting the misfolding, hence its inactivation. ${ }^{19}$ But what seems at first glance a disastrous disadvantage, nature has been able to transform it into its major force for evolution. Evolution is the consequence of the progressive adaptation of living organisms by the accumulation of mutations at DNA level, and it is undoubtably that this is the reason of the wonderful complexity of organisms we observe on the Earth. As Richard Dawkins said in his book The blind watchmaker, "the theory of evolution by cumulative natural selection is the only theory we know of that is in principle capable of explaining the existence of organized complexity". ${ }^{20}$ Life has developed routes of adaptation to the continuous changing environment. This has allowed the spread of life in a wonderful way even in the most hostile environment.

Mutations happen at the DNA level and, consequently, they are translated to proteins, and thus enzymes. The main outcome of these modifications in the primary sequence of the proteins is the alteration of their tertiary structure that can have a positive, a negative or a neutral effect on their function. In some cases, the effects on enzymatic activities can be notorious. Since the catalytic performance of enzymes is directly related to their structure every mutation might alter in an undefined manner the 
function that a specific enzyme performs. In this sense, due to the progressive accumulation of mutations and the correct positive selection, an enzyme can reshape its function towards another that provides an advantage under certain environmental conditions. ${ }^{21}$ For this reason, enzyme promiscuity might serve as a reservoir for the development of new catalytic activities.

The concept of enzyme promiscuity was first discussed by Jensen ${ }^{22}$ in 1976 and afterwards extended by O'Brien and Herschlag23 in 1999. Enzyme promiscuity is understood more as a widespread characteristic in enzymes rather than a rare event. ${ }^{24}$ Although its definition is often vague it can be recognized as the ability that many enzymes possess to catalyze other reactions than the native, or the reaction that has become to be dominant or key for the survival of the host. 25,26

Enzymes have evolved through evolution to be optimized for a specific chemical transformation on a specific type of substrate. ${ }^{27}$ In this sense, the optimization of specific features of the enzymes through mutations and selection confers the ability to be more adapted to a specific environment, hence giving more changes for its survival. Then, these secondary activities, or promiscuous activities, can be the starting point for the evolution of enzymes in vivo. ${ }^{28}$

Enzyme promiscuous activities are usually latent functions that are not under selective pressure, therefore they are normally very low compared to the primary reaction. ${ }^{18} \mathrm{It}$ is estimated that most enzymes vary between $10^{5}$ and $10^{8} \mathrm{~mol}^{-1} \mathrm{~s}^{-1}$ in terms of $k_{\text {cat }} / K_{\mathrm{M}}$ for its native activity. ${ }^{24}$ But in terms of promiscuous activity, it varies in orders of magnitude when compared to the primary reaction. However, weak promiscuous activities can serve as scaffold for the arise of new functions which can even become dominant in the propitious selective environment.

Classification of enzyme promiscuity can sometimes be imprecise due to the wide variety of functions that enzymes perform. But, in general, it can be classified into three main classes: ${ }^{27}$ 


\subsection{Enzyme Promiscuity}

- Catalytic promiscuity. This type of promiscuity is often referred to the enzymes that catalyze two reactions that follow a different reaction mechanism. This fact means that the bond that takes part in the reaction must be different and should go through a different Transition State (TS) than the native reaction.

- Substrate promiscuity. Some enzymes can bind different substrates and consequently catalyze the same reaction but on a different molecule, thus displaying a broad substrate specificity.

- Condition promiscuity. This promiscuous behavior is related to the enzymes that can catalyze a reaction in different conditions than the natural ones, such as $\mathrm{pH}$, temperature, or solvent in which the reaction takes place.

Besides the former classification, some hints can be highlighted. For instance, catalytic promiscuity can be a consequence of either accidental catalytic promiscuity or induced catalytic promiscuity. ${ }^{29}$ In the first one the wildtype enzyme displays promiscuous behavior on substrates that are not the natural ones, meanwhile, in the latter, the catalytic promiscuity is induced by a different co-factor or through protein engineering, such as directed evolution.

How enzymes can display natural or accidental promiscuous behavior within the same active site is a complex question to be addressed. Many factors are involved in the different possible scenarios. For example, alternate co-factor binding can lead to a promiscuous behavior in some enzymes. For example, the dihydroxyacetone kinase from Citrobacter

freundii, which catalyzes either the phosphorylation of the dihydroxyacetone or the cyclization of the flavin adenine dinucleotide, is dependent on the divalent cation that forms the complex with the substrate. ${ }^{30}$ In some cases, the promiscuous behavior can be explained by a different protonation state of key residues. This is the case of the tautomerase family, where the activity of the catalytic Pro is modulated by its $\mathrm{pK}_{\mathrm{a}}$, thus it can act as a general acid or general base, exhibiting, 
promiscuous behavior depending on its protonation state. ${ }^{31,32}$ The conformational diversity and plasticity of enzymes also play a fundamental role in enzyme promiscuity. Enzymes are very flexible structures, and they are subjected to changes in conformational stability due to mutations. For instance, a single mutation in the $\alpha$-lytic protease produces a shift towards the promiscuous activity which can increase by a factor of $10^{5}$, with only a slight decrease in the native activity. Another example of conformational plasticity can be found in the endo-glycoceramidase II from Rhodococcus sp. where a single point mutation alters the transglycosylation/hydrolysis partition. ${ }^{33}$ Similarly, in has been seen that the $\mathcal{N}$-deacetylation pattern in the chitin de- $\mathcal{N}$-acetylase family is dependent on the loops decorating the active site. ${ }^{34}$ Finally, in some cases, promiscuity is a consequence of the activity of different subsites from the same active site. For example, Candida antarctica Lipase B (CALB), which native activity is the hydrolysis of triacylglycerides, is also capable to catalyze a plethora of reactions, ${ }^{25,35-38}$ among which the carbon-carbon bond formation has special interest. ${ }^{39,40}$ The native activity is thanks to a triad formed by Ser-His-Asp, but in the case of the carbon-carbon bond formation, the catalysis goes without the intervention on the catalytic Ser. In fact, the mutation of this Ser to Ala shifts the catalytic performance towards the promiscuous activity.

A hot topic in recent years is the use of non-conventional solvents, like organic and ionic liquids, in enzyme catalysis. The ability to work under different environmental conditions is described as condition promiscuity, and it is observed in the case of many lipases ${ }^{41}$ and laccases ${ }^{42}$. In fact, it is extensively used in industrial processes and organic synthesis.

\subsubsection{Evolutive perspective of enzyme promiscuity}

Many questions arise from the biochemical and evolutionary perspective when dealing with enzyme promiscuity. Undoubtedly, natural evolution has played a crucial role in the wide variety of functions that can be found among families of enzymes. It is obvious that enzyme organization and 


\subsection{Enzyme Promiscuity}

efficiency are imperative for life. ${ }^{43}$ The existence of enzymes that permit the use of valuable chemical resources by the microorganisms in their environmental niches is determinant for their survival and adaptation and it has served as starting point for the evolution of new chemical functions. ${ }^{44}$ In this context, enzyme promiscuity has been crucial. This idea is supported by Voordeckers et. al. who found that when reconstructing ancestral enzymes, they displayed a wider range of substrate promiscuity although its activity was lower. ${ }^{45}$ This fact suggests that evolution in biological systems is driven by evolutionary pressure. The presence of new valuable molecules in the environment guides the enzymes towards the optimization of their promiscuous activities and leads to higher and more efficient biocatalysts. These promiscuous reactions are often less efficient, with their catalytic activity being limited to the selective pressure in the environment. ${ }^{18}$ There is some evidence suggesting that the evolution of new kinetics activities goes through the recruitment and optimization of pre-existing functions in enzymes. ${ }^{46}$ However, which is the origin of this widespread behavior is still under debate.

Several models have been proposed to explain such promiscuous and moonlighting functions in proteins. In the model proposed by $\mathrm{Ohno}^{47}$ and later expanded with the contributions of Kimura and Ohta, ${ }^{48}$ it was suggested that novel functions in proteins may arise from gene duplication events. In this sense, gene duplication and amplification provide an opportunity to search in the sequence space accumulating mutations that can at any point provide a new function. At the same time, the duplication of the genes also allows that those deleterious mutations which might be accumulated on one allele can be buffered by the other copies of the gene. ${ }^{21}$ Under positive selective pressure these duplicated genes can go through a series of neo-functionalization events whose outcome would be two proteins with a specialized different activity.

The Innovation-Amplification-Divergence ${ }^{49}$ (IAD) model goes further and gives an extended vision of Ohno's model (Figure 1.1). This model 
assumes that a series of mutagenic events occur before the duplication, so either a promiscuous enzyme or a protein with moonlighting function is need before the amplification events. After the gene duplication, these secondary activities are free to follow different trajectories. Two possible scenarios can be originated from this fact. Firstly, the rapid accumulation of mutations can provide a bifunctional enzyme that displays promiscuous behavior. This gene at the same time can suffer a duplication event, providing another copy that could go under an independent path. ${ }^{49,50}$ Secondly, the promiscuous functions can go under selection obtaining in the end enzymes with specialized function. From the optimization of these latent secondary activities, new functions can arise.

Assuming that smooth transitions occur in the evolutionary path, enzyme promiscuity might serve as a starting point for enzyme divergence. ${ }^{24}$ Moreover, a promiscuous activity could potentially go under selection and can become the native function in an alternative scenario. This divergent trajectory is common among families of enzymes. For example, in the enolase superfamily, the process of duplication and divergence has led to the existence of families that, despite conserving a similar tertiary structure, they catalyze more than 20 types of reactions. ${ }^{51}$

The outcome of this evolutionary path certainly depends on various factors, such as the frequency of gene duplication, their mutation rate, the size and content of the amplified region, and the number of copies. ${ }^{21}$ The fitness that a mutation provides to the protein in the specific environment will be crucial for preserving the copy of the gene. This optimization seems to occur gradually and smoothly in the sequence space ${ }^{52}$ which might explain why mutations are crucial in the evolution of enzymes. These mutations are constrained by chemical aspects of the new molecules that the enzyme has to adapt to. ${ }^{53}$ Consequently, the adaptation of enzymes will depend on the electrostatic properties and geometrical complementarities with their substrates which will restrict the eruption of new chemical functions in the evolving enzymes. ${ }^{54}$ 
1.1. Enzyme Promiscuity

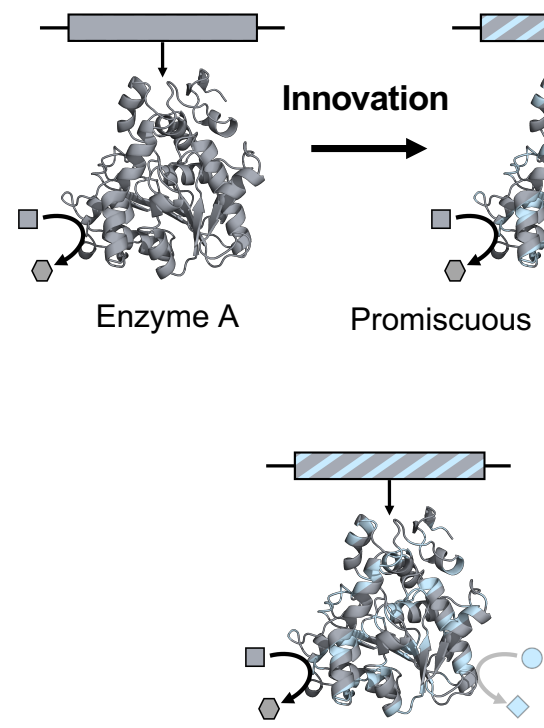

Promiscuous

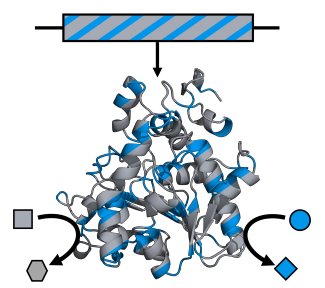

Bifunctional

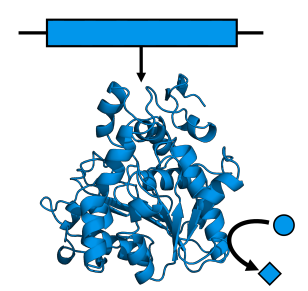

Enzyme B
Amplification

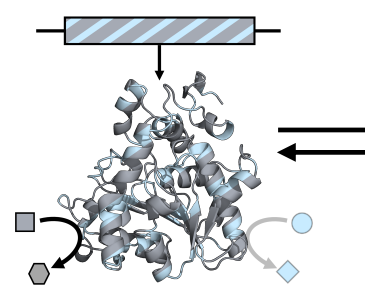

Promiscuous

Divergence

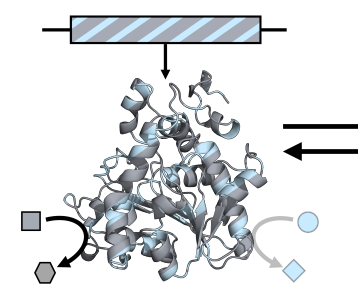

Promiscuous

\section{Specialization}

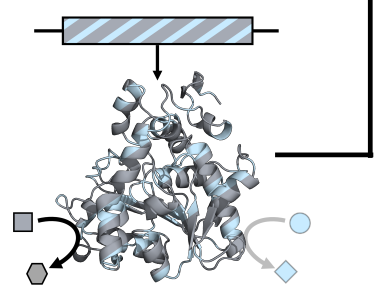

Promiscuous

Figure 1.1. Arise of new functions from an evolutionary perspective. A promiscuous enzyme goes under an amplification process where multiple copies of the same gene diverge due to selective pressure. The result of the diverge renders a bifunctional enzyme that can go under a specialization path to lose function A and become a B specialist. 
Since enzymes are also involved in the metabolic pathways of the cell, their promiscuous properties could be evolutionarily useful in what is called underground metabolism. ${ }^{55}$ Metabolic networks are inherently complex and interconnected. Some products of a pathway are the substrates of another, some metabolites are shared within pathways and some by-products of promiscuous enzymes are set unused and consequently degraded. ${ }^{56}$ Enzyme promiscuity could play a role in this underground metabolism, contributing to the evolution of the metabolic pathways in the organisms by replacing the pathways that are unused or inefficient with alternative pathways and recruiting promiscuous enzymes to expand or re-route the actual network. Not only that but they can also integrate the by-products of these enzymes into a complex network. ${ }^{57}$

Until now, a divergent perspective of enzyme promiscuity has been exposed. To conclude this section it is worth mentioning that even though many functions of enzymes have appeared due to divergent events, other enzymes have been evolved to catalyze the very same reaction even though they are non-homologous enzymes. ${ }^{58}$ This fact can be explained because they share a similar or common active site. The first evidence of convergent evolution was found in the serine protease family. ${ }^{59,60}$ Since then, other convergent enzymes have been reported. ${ }^{58,61,62}$ Moreover, the recruitment of ancestral enzymes that undergo change in specificity can lead to mechanistic analogs. ${ }^{63}$ This is the case for example of the Serine Hydrolase (SH) family which has a conserved catalytic Ser-His-Aps/Glu triad geometry. ${ }^{64}$

\subsubsection{Applications of enzyme promiscuity to enzyme engineering}

The interest in the study of the mechanisms of promiscuous enzymes has recently increased in the field of biomolecular transformations. ${ }^{24-26,28,65,66}$ Deep knowledge on how these mechanisms work at a molecular level can 


\subsection{Enzyme Promiscuity}

lead to the obtention of highly desirable chemical compounds, with improved yields, ecological procedures, and in a wide variety of conditions. In a world that demands greener sources of products and where ecological consciousness is an urgent topic, enzymatic approaches are preferred over chemical synthesis. ${ }^{67}$

Thus, understanding the molecular basis of enzyme promiscuity is crucial. Huge efforts are invested in the research on this field in the last years ${ }^{68-70}$ in order to increase the knowledge on the chemistry that gives this promiscuous character to the enzymes. For this reason, this characteristic has been widely studied as a starting point to design new biocatalysts not present in nature by directed evolution and other molecular biology techniques ${ }^{71}$. So, enzyme promiscuity has gained a lot of interest in academia and industry due to its potential application as a tool to design new and optimized enzymes, and to be used as scaffold for directed evolution to enhance the non-native reactions ${ }^{72,73}$. In this regard, many applications of enzyme promiscuity together with protein engineering techniques have been described for a wide spectrum of fields. Some examples are described below focusing on the enhancement of latent promiscuous activities.

Lipases are well-known to be highly promiscuous. Their broad substrate specificity and the fact that they are highly active in a variety of organic solvents, make them potential candidates to be used in the obtention of high-value chemicals in the biotechnological and pharmaceutical industry. ${ }^{25,26}$ In recent years, many studies reported the use of lipases for carbon-carbon bond formation. ${ }^{74}$ These reactions are important in organic synthesis and in the pharmaceutical industry. ${ }^{74}$ For example, as mentioned, CALB is capable to catalyze $\mathrm{C}-\mathrm{C}$ bond formation through aldol addition. ${ }^{39}$ The mutation of the catalytic Ser to Ala completely shifts the native and promiscuous activities. The native reaction, which involves the hydrolysis of the ester bond in triacylglycerides, is dependent on the catalytic triad formed by Ser-His-Asp. But the aldol addition goes through a mechanism 
where the catalytic His acts as an acid/base and the Ser plays no role. So, the variant where the Ser is mutated to Ala suppresses the primary hydrolytic activity, enhancing the secondary aldol addition. This very same enzyme has been seen to catalyze the Michael addition of 1,3-dicarbonyl compound and an $\alpha / \beta$-unsaturated aldehyde. ${ }^{75}$ In this case, the Ser/Ala variant shifted the activity towards the secondary reaction. Lipases are also involved in oxidative processes, such as the formation of peroxycarboxylic acids $^{76}$ from a carboxylic acid and hydrogen peroxide. This reaction at the same time can be coupled to the formation of epoxides from alkenes. ${ }^{77}$ The direct epoxidation of alkenes is also an interesting reaction catalyzed by lipases from the perspective of its applicability in the industry. Moreover, it is remarkable the proposal of using lipases in the degradation of polyethylene terephthalate derivatives ${ }^{78}$ which opens a promising future direction in the recycling and waste industry.

Although serine proteases are very specific enzymes, similarly to lipases, they display secondary activity towards some substrates, probably because they sometimes share a similar catalytic triad formed by Ser-His-Asp/Glu. For instance, porcine trypsin, a protease catalyzing the cleavage of peptide bonds after charged Arg/Lys residues, ${ }^{79}$ has been demonstrated to be involved in the formation of Si-O-Si bonds in the condensation of silanols, increasing the catalysis by 10-fold with respect to the spontaneous condensation. ${ }^{80}$ Recently a variant of the protease Caspase- 2 was proposed as a universal tool for N-terminal fusion-tag cleavage, due to the shift in substrate specificity. ${ }^{81}$ This implies a major advance in the molecular biology toolbox. Other serine proteases have therapeutic interest, for example, the earthworm serine protease, which possesses fibrinolytic, antitumor, anti-viral, and anti-inflammatory properties. ${ }^{82}$ The broad substrate promiscuity showed by these proteases make them also ideal candidates for a wide variety of applications, such as clot dissolution, ${ }^{83,84}$ potential antitumor agents ${ }^{85}$ to be used in oncological treatments or in the treatment of some viral diseases in plants like the tobacco or the cucumber mosaic virus. ${ }^{86}$ 


\subsection{Enzyme Promiscuity}

Apart from lipases and proteases, there's a potential niche of interesting enzymes in the archaea domain. Extremophile microorganisms can be found within the archaea domain and comprise those organisms that have been evolved to live in extreme environments. Therefore, their enzymes have been adapted to such extreme conditions. Enzyme properties that allow operating under hostile conditions are fascinating from the biochemical and biotechnological perspective. Few promiscuous enzymes in archaea are reported when compared to those found in bacteria and eukaryotes, but it may be due to the fact that the latter have been extensively studied. ${ }^{87}$ For example the inositol monophosphatase from Methanococcus jannaschii and Archaeoglobus fulgidus exhibit both inositol monophosphatase and fructose-1,6-bisphosphatase activity. ${ }^{88,89}$ Another interesting example is the DNA primase of Pyrococcus abyssi ${ }^{90}$ which can catalyze the priming synthesis in the single-stranded DNA and, at the same time, the elongation of DNA and RNA acting as a polymerase. 


\subsection{Carboxylic Ester Hydrolases}

\subsubsection{Classification of the EC 3.1.1}

The Enzyme Commission (EC) number ${ }^{91}$ is a system that tries to put some order in the classification of the enzymes based on the reaction they catalyze. Formed by four digits, the first one refers to one of six classes based on the general type of reaction, and they are progressively subclassified based on the reaction properties and the substrate they catalyze the reaction on. Regarding the enzymes that exhibit substrate promiscuity or substrate ambiguity, EC numbers may only differ in the last $4^{\text {th }}$ digit. In the case of catalytic promiscuity, EC of the same enzyme catalyzing the same reaction on various substrates can differ in the $3^{\text {rd }}$ or even in the $2^{\text {nd }}$ digit, which refers to a different chemistry of the reaction. ${ }^{24}$

The enzymes studied in this thesis belong to the EC 3.1.1 which classifies the Carboxylic Ester Hydrolases ( $\mathrm{CEH})$ into a big functional domain, which englobes the hydrolases that act on ester bonds. Their main catalytic activity is the hydrolysis of ester bonds into the respective carboxylic acid and alcohol. CEHs, which include SHs, are a huge family of enzymes that are ubiquitous and that have been identified in all domains of life and viruses. ${ }^{92}$ At the moment of writing this document (August of 2021) there are 4130 reviewed entries in the Uniprot ${ }^{93}$ database and a total of 269 proteins with available crystallographic data in the Protein Data Bank $(\mathrm{PDB})^{94}$ database. The huge amount of information available reflects the inherent interest in the scientific community for this type of enzymes.

Depending on the hydrolyzed substrate, the EC 3.1.1 group can be divided into 111 subgroups from 3.1.1.1 to 3.1.1.118, with 7 deleted entries. Other aims to classify CEHs have been proposed. For example, the CASTLE (CArboxylic eSTer hydroLasE) ${ }^{95}$ database classifies CEHs into 5 clans (from A to E) depending on their general fold. Other more specialized 


\subsection{Carboxylic Ester Hydrolases}

databases cover some particular CEHs, for instance, the ESTHER (ESTerases and alpha/beta-Hydrolase Enzymes and Relatives) ${ }^{96}$ database which classifies the superfamily of $\alpha / \beta$ fold CEHs. The CAZy (Carbohydrate-Active enZYmes) ${ }^{97}$ database is an initiative which aim is the classification of carbohydrate-active hydrolases. Finally, LED (Lipase Engineering Database ${ }^{98}$ integrates the information of lipases and other proteins that share the common $\alpha / \beta$ hydrolase fold.

The most common mechanism of hydrolysis of the ester bond involves a characteristic triad formed by Ser-His-Asp/Glu, but other nonconventional active sites have been also identified. ${ }^{99}$ Based on sequence alignment and analysis of tertiary structure different active site conformations can be highlighted. ${ }^{100}$ Some other identifiable triads involve, for example, Ser-His-His, His-His-Asp, or Ser-Ser-Lys as catalytic residues. Some of them contain Cys or Thr as a nucleophile like Cys-HisAsn/Asp. Aspartyl dyads containing a signature formed by Asp-Asp are also common in the CEH family. Other common dyads can be formed by Cys-His, His-Asp, or Ser-Lys. ${ }^{101}$

In the CEHs family, there are two well-known groups, the lipases (EC 3.1.1.3) and the carboxylesterases (EC 3.1.1.1). Lipases have a preference for substrates with long-chain acyl esters, while esterases act preferably on short-chain substrates. ${ }^{102}$ In this thesis we have focused on the study of a member of each group and their main characteristics will be described in the following sections.

\subsubsection{Candida antarctica Lipase $B$}

One of the most studied and well-known promiscuous enzymes is CALB. It belongs to EC 3.1.1.3 group which includes the triacylglycerol lipases. CALB is a SH whose active form consists of 317 residues of amino acid with a total molecular weight of $33 \mathrm{kDa}$. Its native activity comprises the hydrolysis of the ester bond in triacylglycerides. The inactive pro-protein 
is activated after the cleavage of a short pro-peptide, positions 19-25, in the N-terminus.

According to $\mathrm{CATH}^{103}$ database, CALB is classified as an $\alpha / \beta$ protein belonging to the CATH functional family 3.40.50.182. It exhibits a characteristic Rossman fold core formed by six $\beta$-strands surrounded by alpha-helices forming a three-layered sandwich (Figure 1.2a). It also has a single mono-glycosylation point in Asn74, far from the active site, which is not important neither for the activity nor for the structural stabilization. ${ }^{104}$
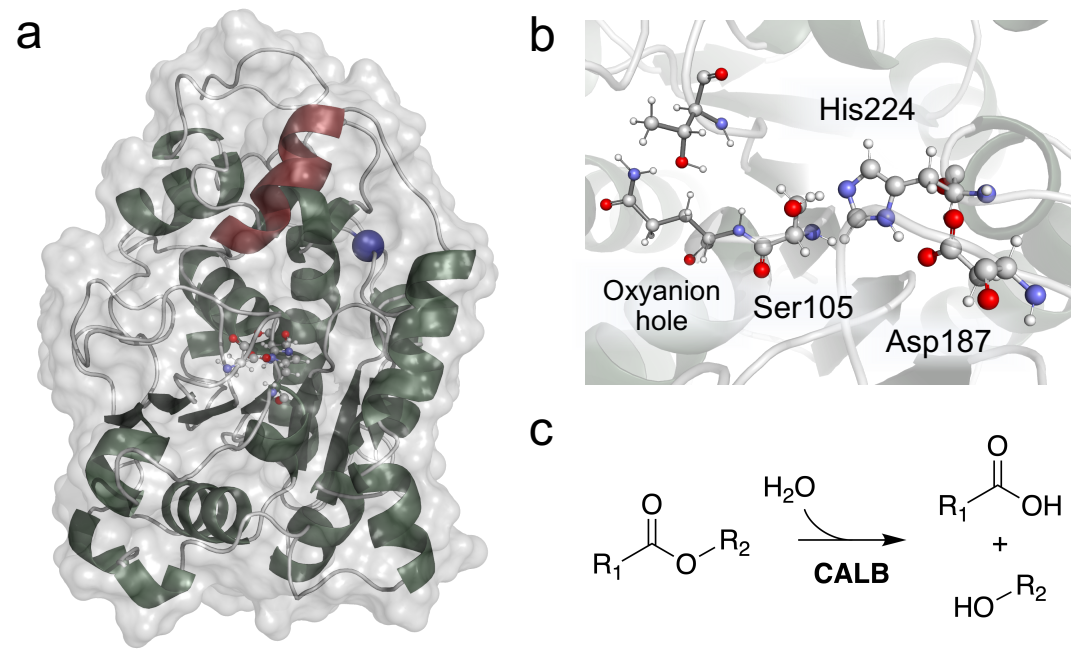

Figure 1.2. Structural and functional highlights of CALB. Panel a: overall tertiary structure of CALB, with the residues belonging to the catalytic triad displayed as balls and sticks. The helix $\alpha 5$ is shown in red and the glycosylation site Asn74 is displayed as a blue sphere. Panel $\mathbf{b}$ : detailed view of the active site, formed by the catalytic triad Ser105-His224-Asp187 and the oxyanion hole formed by the residues Thr40 and Gln106. Panel c: general ester hydrolysis catalyzed by CALB.

Like many other CEHs, CALB performs its activity by a catalytic triad composed by Ser105-His224-Asp187 (Figure 1.2b), where the histidine has a relevant role in the mechanism by acting as an acid/base residue. This acid/base character of His224 is governed by the aspartate residue which modulates its $\mathrm{pK}_{\mathrm{a}}$. In the lipase family, it is very common that the enzyme 


\subsection{Carboxylic Ester Hydrolases}

undergoes a conformational change when the substrate binds into the cavity. Basically, they have a lid that closes when the substrate binds, positioning the catalytic residues in a proper active conformation and catalyzing the reaction. ${ }^{105}$ In CALB the catalytic triad is located in a very open active side pocket which does not contain a lid domain, ${ }^{106}$ but instead of that, it has a lid-forming alpha-helix, helix $\alpha 5$. This alpha-helix is delimited by Trp 104 which has a potential role in the stereospecificity of lipases. ${ }^{107}$

Additionally, an oxyanion hole formed by the residues Thr40 and Gln 106 is also present in the active site pocket. This oxyanion hole has a double role. Firstly, it allows the substrate to adopt the correct pose into the active site pocket, avoiding its diffusion to the bulk and ensuring the chemical transformation. Secondly, it stabilizes the negative charge accumulated on the substrate during the hydrolysis. The proposed mechanism of the hydrolysis of ester natural substrates catalyzed by CALB is proposed to be a four steps reaction ${ }^{108}$ that involves the attack of the catalytic Ser105 to the carbonyl carbon of the ester (see Figure 1.3). In the second step, the release of the leaving group occurs as an effect of the acid-base character of His224. The third and the last chemical steps involve the hydrolysis of the acyl-enzyme complex yielding the products, composed of the carboxylic acid and the alcohol, and regenerating the active site for the subsequent catalytic iteration.

Lipases are known to display substrate promiscuous behaviour, ${ }^{25}$ accepting a broad range of substrates. In CALB, the characteristic open structure of the active site and the lack of a structured lid confers to it a highly promiscuous behavior. For this reason, CALB has been reported to catalyze a plethora of reactions. ${ }^{25,26}$ It was shown to be active in reactions of synthesis of esters and amides, transacylation of alcohols, ${ }^{35}$ epoxidation reactions, ${ }^{36}$ aldol additions and Michael type additions, ${ }^{25,37}$ and hydrolysis of plastic derivatives ${ }^{78}$ among many others. ${ }^{38}$ CALB has also been reported to be very active in organic solvents ${ }^{109,110}$ and ionic liquids, ${ }^{111}$ which is 
interesting from the industrial perspective. Moreover, it is highly resistant to immobilization on a wide variety of surfaces and materials, ${ }^{112-114}$ which is useful in the biotechnological industry for the recovery and reutilization of the protein in aqueous and organic media.

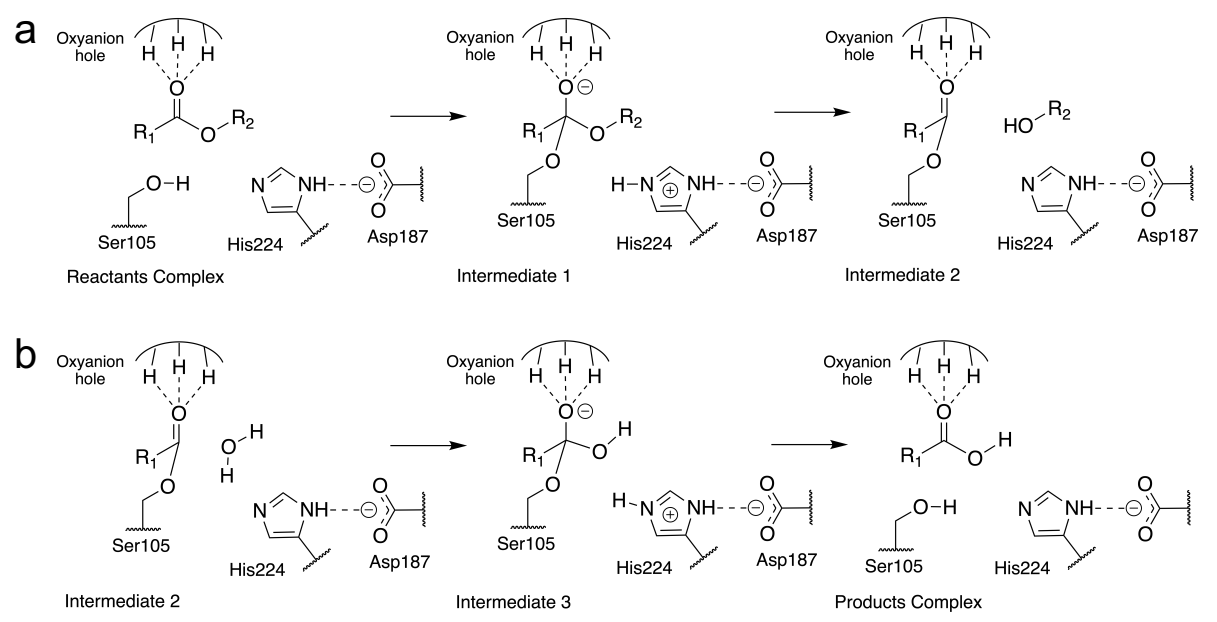

Figure 1.3. Proposed general mechanism for the native ester hydrolysis catalyzed by CALB. Panel a: acylation step, where in the first step the nucleophilic attack of the Ser 105 renders the tetrahedral intermediate 1. In the second step, the His224 promotes the braking of the $\mathrm{C}-\mathrm{O}$ bond, leading to the formation of the first product, the alcohol, and the formation of the acyl-enzyme complex. Panel b: hydrolysis step, where a water molecule enters the active site and the hydrolysis of the acyl-enzyme complex occurs in two steps, rendering the formation of the last product, the acid, and the regeneration of the active site.

Apart from its high promiscuity, over the years CALB has been widely studied, ${ }^{25,26,115-117}$ including mutagenic studies, and consequently extensive information is available in the literature. ${ }^{118-121}$ Therefore, CALB can serve as a very good candidate for understanding the origin of enzymatic promiscuity.

\subsection{3. para-nitrobenzyl esterase from Bacillus subtilis}




\subsection{Carboxylic Ester Hydrolases}

As described before, esterases belonging to EC 3.1.1.3 are widely studied. This is the case of para-nitrobenzyl esterase form Bacillus subtilis (Bs2). This intracellular serine hydrolase performs the hydrolysis of various $\beta$-lactam antibiotics esters, ${ }^{122}$ through a triad formed by Ser189-His399-Glu310. In a similar manner as CALB, the catalytic activity of His399 is modulated by Glu310, which influences its $\mathrm{pK}_{\mathrm{a}}$. According to BRENDA database ${ }^{123}$ it is classified in EC 3.1.1.1 group, which comprises the carboxylesterases. It has a total of 489 residues of amino acid and a molecular weight of $54 \mathrm{kDa}$. According to CATH database it belongs, as well as CALB, to the functional family 3.40.50.182, adopting an $\alpha / \beta$ fold. It is composed of 13strands of $\beta$ sheets which are surrounded by $15 \alpha$-helices. The active site is located inside a cavity that is decorated with four loops. Similar as in CALB, an oxyanion hole is present in the active site pocket formed by residues Ala107 and Ala190 (Figure 1.4). Although the crystal structure of this specific variant is not available a similar structure, PDB 1QE3, ${ }^{124}$ is available, differing in only 11 residues.

Bs2, displays promiscuous behavior, similarly as CALB. Even though its primary reaction is the hydrolysis of ester bonds, ${ }^{122,125} \mathrm{Bs} 2$ has been proved to be capable to catalyze the hydrolysis of amides. ${ }^{69}$ Moreover, it can also bind other non-native substrates such as PET derivatives. ${ }^{126}$
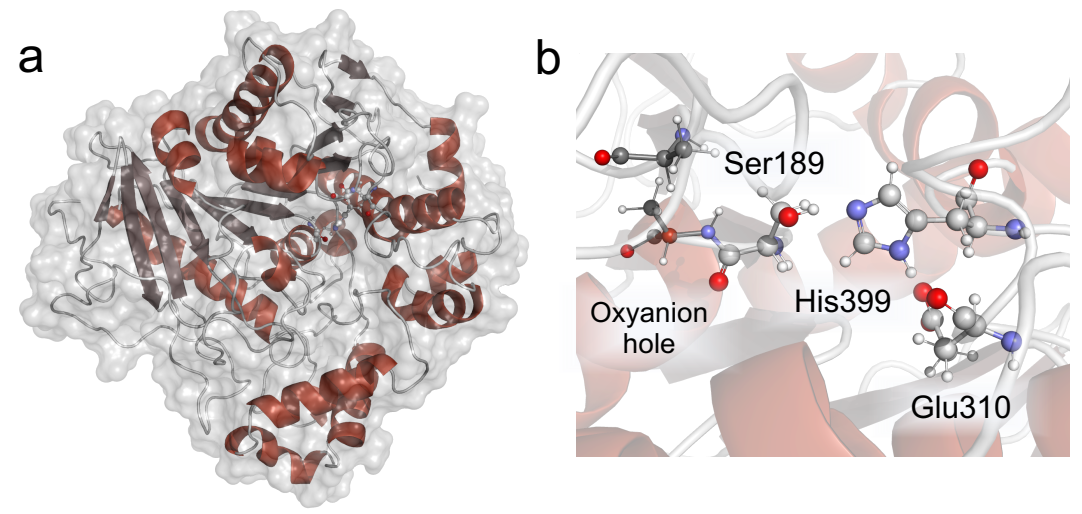

Figure 1.4. Structure and active site of Bs2. In panel a: general view of Bs2, where the backbone is represented as cartoon and the catalytic triad as balls and 


\subsection{Carboxylic Ester Hydrolases}

sticks. In panel $\mathbf{b}$ : detailed view of the active site pocket with the catalytic triad formed by Ser189-His399-Glu310 and the oxyanion hole formed by the residues Ala107 and Ala190.

Various mutagenic studies have been also conducted on Bs2, providing information not only about thermostability ${ }^{124}$ but also on the origin of its catalytic promiscuity. ${ }^{127}$

Among all previously exposed information, Bs2, as well as CALB, is a promising model for the exploration of promiscuous molecular mechanisms in the CEHs family. In this thesis, both enzymes have been extensively studied by means of theoretical and experimental techniques. 


\subsection{Protein engineering}

\subsubsection{Enzyme (re)design}

There is a growing interest in the scientific community to explore the use of biocatalysts as a tool for obtaining valuable products. There is expanding evidence about the use of enzymes for specific chemical transformations which evolved into the creation of novel and functional designed structures. ${ }^{128-132}$ This fact is, in part, due to the development of efficient biomolecular techniques to explore the conformational space and fitness of enzymes, such as directed evolution techniques. ${ }^{133}$ With these advances, protein technologies have become a mainstream technique in the chemistry and pharmaceutical industries. ${ }^{81}$

Many examples of engineered proteins are available in the recent literature covering a wide variety of fields, such as the pharmaceutical industry for drugs manufacture, ${ }^{134}$ therapeutic proteins, ${ }^{135,136}$ and biofuel production. ${ }^{137}$

As mentioned in section 1.1.2, the natural evolution of enzymes is the outcome of many billions of years of continuous adaptation of living organisms to the changing environment. Nature has been successful on optimizing enzymes from ancestral promiscuous genes to specialized enzymes. ${ }^{132}$ Some of these promiscuous behaviors have been retained in modern enzymes, therefore can be used experimentally to improve these biocatalysts in the laboratory. Hence, having tools to enhance these secondary reactions that to be used to obtain high-value chemicals is of vital importance.

Many questions arise when dealing with effective strategies to modify or mutate enzymes in order to increase their catalytic efficiency. A proper preorganization of the active site is crucial for the kinetics of the protein. 
In this sense, the chemistry of the protein can be explained by the electrostatic and steric effects in the surroundings of the active site. Finding the proper characteristics that guide the protein towards a specific reaction is not trivial and can be a challenging task. In this regard, two main approaches can be followed, rational (re)design and directed evolution. ${ }^{138}$ In the same way, rational approaches can be specified into rational redesign, semi-rational redesign and de novo design. New computational techniques, such as Machine Learning (ML) approaches, could serve as complementary techniques that can give additional insights to either rational (re)design or directed evolution approaches (Figure 1.5).

Rational (re)design of protein catalysts can be defined as the strategy that uses previous mechanistic or structural knowledge to propose mutations with the aim to obtain highly efficient enzymes. ${ }^{13}$ In the rational redesign some hot-spots are targeted based in previous available information. ${ }^{139}$ The complexity of enzyme structure and function limits the applicability of these approaches. On the other hand, an interesting strategy within the rational (re)design framework is the de novo design of proteins, ${ }^{140}$ where a protein scaffold is built from scratch. Semi-rational redesign refers to the strategy based on applying some 'smart' libraries on specific hot-spots selected by rational means, such as iterative saturation mutagenesis. ${ }^{141-143}$ This strategy allows the exploration of a vast sequence space with few resources and overcomes the limitations of some rational approaches.

Directed evolution of enzymes is a completely different strategy. The main advantage is that minimal previous structural or mechanistic information is needed to obtain improved enzymes. Directed evolution of enzymes tries to mimic the natural evolution but in a much shorter timescale. ${ }^{132}$

Next, a summary of the main and most successful strategies in the field of protein engineering, specifically applied to enzymes, will be briefly discussed, not only from the experimental perspective but also giving insights from the computational approaches to enzyme (re)design. 


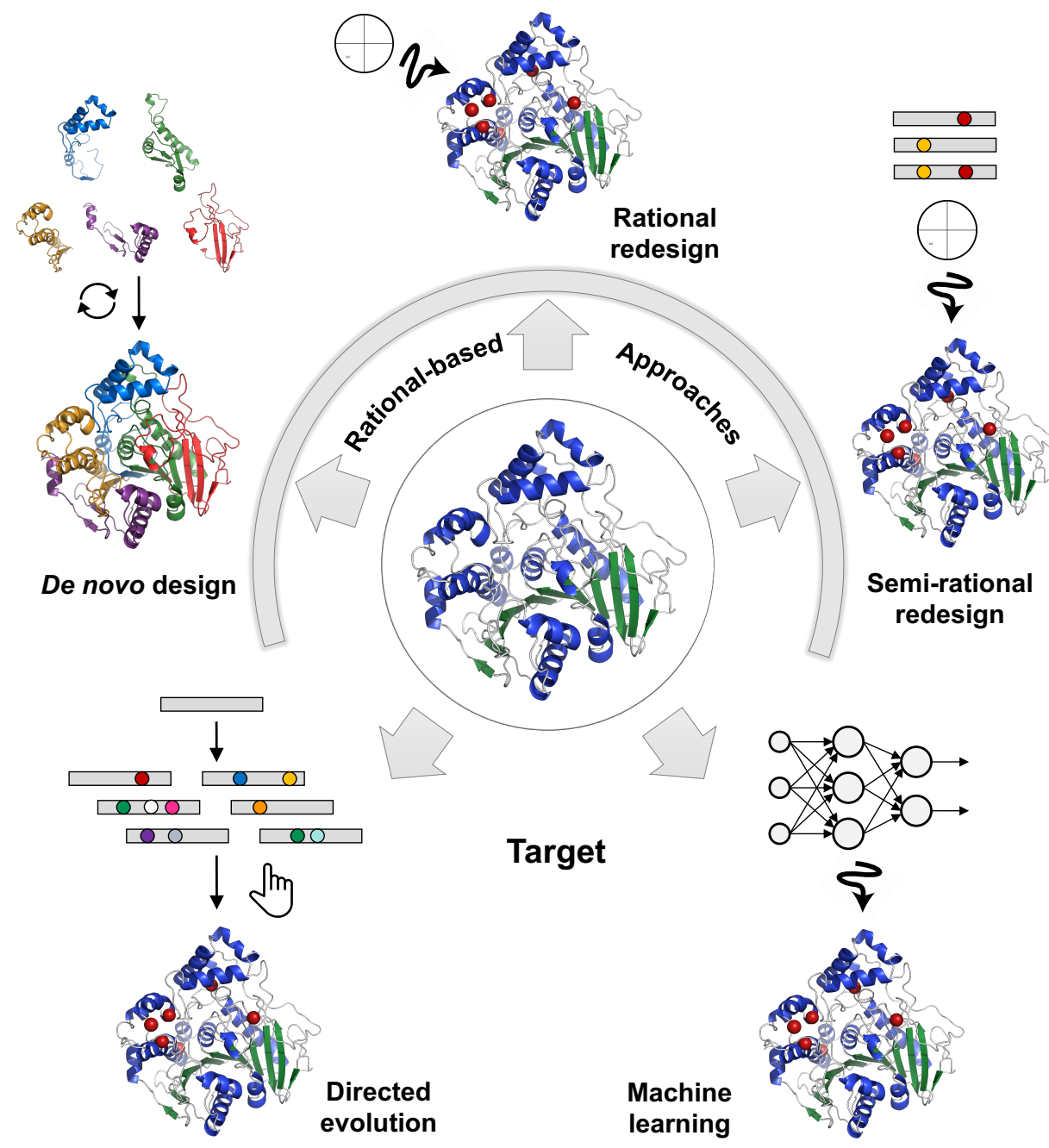

Figure 1.5. Strategies in enzyme (re)design. Different approaches exist in the protein engineering toolbox towards enzyme (re)design, from rational and semirational strategies based on deep structural and mechanistic knowledge to directed evolution and ML approaches, or de novo design which intends to build an enzyme from only a guess of the transition state. 


\subsubsection{Experimental strategies in enzyme (re)design}

Several strategies can be followed in enzyme (re)design depending on the availability of useful sequence, structural or mechanistic information of the target enzyme.

Rational design is limited to those cases where valuable structural or mechanistic information can be used to propose a specific set of target mutations. From the accurate analysis of the structure and mechanism of the reaction, a combination of direct mutations is proposed to shift the desired property. This is a challenging task since the availability of efficient tools to predict the impact of these mutations on the protein is limited.

Recent advances in molecular biology engineering tools, such as the directed evolution of enzymes, are aimed to overcome these limitations. This strategy has become mainstream in the protein engineering field. ${ }^{133,144-149}$ Considering the principle of mimicking the process of natural evolution, proteins can be optimized experimentally in a short timescale by introducing random mutations iteratively and selecting the active or improved variants. One of the main advantages is that minimal or no structural information is needed for this strategy. Not only that but distant regions from the active site that can influence the performance of the protein are also identifiable. In some cases, the distant mutations from the active site can have a huge impact on the given enzyme properties. ${ }^{150}$ Nevertheless, directed evolution is limited by the fact that, even when the most efficient high-throughput system is used, only a fraction can be sampled within a set timeframe. ${ }^{151}$

Even though a random examination of the protein sequence space might seem an inefficient strategy, it has been the standard technique in the last years. Moreover, this technology can be too costly and other semi-rational approaches are preferred. In semi-rational approaches, structural or sequence information is used to propose a series of random mutations to be done in a specific target location of the enzyme. ${ }^{142}$ This fact can have a 


\subsection{Protein engineering}

tremendous impact on the screening of new variants since it uses a reduced library size. Additionally, in semi-rational redesign, computational techniques are useful tools for the analysis of the structural impact of amino acid substitutions on the catalyzed chemical reaction and the prediction of target sites. ${ }^{142}$ These computational approaches are discussed in the following section.

\subsubsection{Computational strategies in enzyme (re)design}

Recent advances in computational sciences and an exponential increase in computational power have led to the rise of a computational paradigm in enzyme (re)design. The challenge in enzyme (re)design, specially in the de novo design, has always been limited by the understanding of which is the molecular basis of enzyme catalysis. Based on the pioneering ideas of Pauling, ${ }^{152}$ the hypothesis proposed by Warshel and co-workers that the enzyme is a preorganized structure that stabilizes the charge distribution of the substrate in the TS, thus decreasing the activation barrier, has been adopted as the new paradigm of enzyme catalysis in the recent decades. ${ }^{153,154}$ Nevertheless, only in the last years the reorganization of charges and the electric field optimization have been considered targets of interest for enzyme redesign. ${ }^{155-160}$ Recently, the idea of a set of dynamically organized enzyme motions ${ }^{161}$ together with an electrostatic preorganization of the enzyme has been contemplated as an important factor in enzyme performance.

Many different computational strategies to obtain a universal protocol for enzyme (re)design have been explored in the last years. The de novo design is a well-known strategy in enzyme design which starts with the elucidation of a theozyme computed at a high quantum mechanical level, where the TS is resolved in gas phase and residues assisting the reaction are added. ${ }^{162}$ Then a suitable protein scaffold is explored by placing the theozyme in a library of predefined active sites. Finally, the scaffold is adapted by introducing the correspondent modifications. ${ }^{159}$ This strategy is usually 
combined with iterative cycles of directed evolution to obtain enzymes with the desired characteristics. ${ }^{138}$

Other strategies can be followed such as the redesigning of existing proteins. ${ }^{163}$ In a similar manner as rational redesign is done experimentally, structural information of the enzyme can help to suggest new variants whose performance can be analyzed by computational methods such as Molecular Dynamics (MD) simulations using classical Molecular Mechanics (MM) Force Fields (FF) or hybrid Quantum Mechanics / Molecular Mechanics (QM/MM) potentials. ${ }^{164}$ The major limitation of this strategy is the high computational cost associated with the analysis of a large number of variants in terms of Quantum Mechanics (QM) or QM/MM methodologies. ${ }^{164}$

Recently, in silico directed evolution frameworks have also been proposed. In this sense, CADEE (Computer-Aided Directed Evolution of Enzymes ${ }^{165}$ is a semi-automated approach for computer-assisted directed evolution of enzymes, based on the Empirical Valence Bond ${ }^{166}$ (EVB) method.

Recent ML methods have been proposed to expedite evolution and expand the number of properties that can be optimized. ${ }^{167,168}$ Some of the ML frameworks for protein (re)design rely on the analysis of huge amounts of sequence data. ${ }^{169}$ These strategies can be used together with rational (re)design and directed evolution to speed up the obtention of improved biocatalysts. In this sense, Convolutional Neural Networks (CNN) have been proved to be very useful in classification tasks in a wide range of fields, such as image, speech, and movement recognition, together with text analysis. ${ }^{170}$ There are expanding applications of CNNs in the analysis of protein structure. ${ }^{171-174}$ Although some 3D CNNs have been $\operatorname{proposed}^{171,173}$ for the analysis of protein structures, the reduced amount of non-redundant crystal structures limits the use of these methodologies.

Overall, the number of successful designs of new enzymes is limited and their improved activity is modest compared with the wild-type enzymes. In 


\subsection{Protein engineering}

fact, as argued by Warshel and co-workers, the high activities of the best artificial enzymes are largely due to directed evolution, therefore the contribution of computation was comparatively modest. ${ }^{175}$ However, there is still room for the improvement in the computational assisted methods used in the (re)design of new enzymes. 


\subsection{References}

(1) Almonacid, D. E.; Babbitt, P. C. Toward Mechanistic Classification of Enzyme Functions. Curr. Opin. Chem. Biol. 2011, 15, 435-442.

(2) Cooper, G. M. The Central Role of Enzymes as Biological Catalysts - The Cell: A Molecular Approach. The Cell: A Molecular Approach. 2000.

(3) Champoux, J. J. DNA Topoisomerases: Structure, Function, and Mechanism. Annu. Rev. Biochem. 2001, 70, 369-413.

(4) Nevinsky, G. A. Main Factors Providing Specificity of Repair Enzymes. Biochem. 2011, 76, 94-117.

(5) Ishitani, R.; Yokoyama, S.; Nureki, O. Structure, Dynamics, and Function of RNA Modification Enzymes. Curr. Opin. Struct. Biol. 2008, 18, 330-339.

(6) Nishimura, T.; Stefan, C. J. Specialized ER Membrane Domains for Lipid Metabolism and Transport. Biochim. Biophys. Acta - Mol. Cell Biol. Lipids 2020, 1865,158492 .

(7) Hannun, Y. A.; Luberto, C.; Argraves, K. M. Enzymes of Sphingolipid Metabolism: From Modular to Integrative Signaling. Biochemistry 2001, 40, 4893-4903.

(8) Ruan, Y. L. Sucrose Metabolism: Gateway to Diverse Carbon Use and Sugar Signaling. Annu. Rev. Plant Biol. 2014, 65, 33-67.

(9) Yoshida, M.; Muneyuki, E.; Hisabori, T. ATP Synthase - A Marvellous Rotary Engine of the Cell. Nat. Rev. Mol. Cell Biol. 2001, 2.

(10) Hatzimanikatis, V.; Li, C.; Ionita, J. A.; Broadbelt, L. J. Metabolic Networks: Enzyme Function and Metabolite Structure. Curr. Opin. Struct. Biol. 2004, 14, 300-306.

(11) Gao, M.; Skolnick, J. A General Framework to Learn Tertiary Structure for Protein Sequence Characterization. Front. Bioinforma. 2021, 1.

(12) Levinthal, C. How to Fold Graciously. Mössbauer Spectrosc. Biol. Syst. Proc. 1969, 24, 22-24.

(13) Świderek, K.; Tuñon, I.; Moliner, V. Predicting Enzymatic Reactivity: From Theory to Design. Wiley Interdiscip. Rev. Comput. Mol. Sci. 2014, 4, 407-421.

(14) Kries, H.; Blomberg, R.; Hilvert, D. De Novo Enzymes by Computational Design. Curr. Opin. Chem. Biol. 2013, 17.

(15) Kiss, G.; Çelebi-Ölçüm, N.; Moretti, R.; Baker, D.; Houk, K. N. Computational Enzyme Design. Angew. Chemie Int. Ed. 2013, 52, 5700-5725.

(16) Hilvert, D. Critical Analysis of Antibody Catalysis. Annu. Rev. Biochem. 2000, 


\subsection{References}

69, 751-793.

(17) De Raffele, D.; Martí, S.; Moliner, V. A QM/MM Study on the Origin of RetroAldolase Activity of a Catalytic Antibody. Chem. Commun. 2021, 57.

(18) Copley, S. D. Shining a Light on Enzyme Promiscuity. Curr. Opin. Struct. Biol. 2017, 47, 167-175.

(19) Camps, M.; Herman, A.; Loh, E.; Loeb, L. A. Genetic Constraints on Protein Evolution. Crit. Rev. Biochem. Mol. Biol. 2007, 42, 313-326.

(20) Dawkins, R. The Blind Watchmaker - Evidence of Evolution Reveals a Universe without Design; 1986.

(21) Copley, S. D. An Evolutionary Perspective on Protein Moonlighting. Biochem. Soc. Trans. 2014, 42, 1684-1691.

(22) Jensen, R. A. Enzyme Recruitment in Evolution of New Function. Annu. Rev. Microbiol. 1976, 30, 409-425.

(23) O'Brien, P. J.; Herschlag, D. Catalytic Promiscuity and the Evolution of New Enzymatic Activities. Chem. Biol. 1999, 6, R91-R105.

(24) Tawfik, D. S.; Khersonsky, O. Enzyme Promiscuity: A Mechanistic and Evolutionary Perspective. Annu. Rev. Biochem. 2010, 79, 471-505.

(25) Busto, E.; Gotor-Fernández, V.; Gotor, V. Hydrolases: Catalytically Promiscuous Enzymes for Non-Conventional Reactions in Organic Synthesis. Chem. Soc. Rev. 2010, 39, 4504-4523.

(26) Gotor-Fernández, V.; Brieva, R.; Gotor, V. Lipases: Useful Biocatalysts for the Preparation of Pharmaceuticals. J. Mol. Catal. B Enzym. 2006, 40, 111-120.

(27) Humble, M. S.; Berglund, P. Biocatalytic Promiscuity. European J. Org. Chem. 2011, 2011, 3391-3401.

(28) Sheldon, R. A.; Brady, D. The Limits to Biocatalysis: Pushing the Envelope. Chem. Commun. 2018, 54, 6088-6104.

(29) Hult, K.; Berglund, P. Enzyme Promiscuity: Mechanism and Applications. Trends Biotechnol. 2007, 25, 231-238.

(30) Sánchez-Moreno, I.; Iturrate, L.; Martín-Hoyos, R.; Jimeno, M. L.; Mena, M.; Bastida, A.; García-Junceda, E. From Kinase to Cyclase: An Unusual Example of Catalytic Promiscuity Modulated by Metal Switching. ChemBioChem 2009, $10,225-229$.

(31) Susan C. Wang; William H. Johnson, J. . and; Whitman*, C. P. The 4Oxalocrotonate Tautomerase- and YwhB-Catalyzed Hydration of 3EHaloacrylates: Implications for the Evolution of New Enzymatic Activities. $J$. Am. Chem. Soc. 2003, 125, 14282-14283.

(32) Poelarends, G. J.; Veetil, V. P.; Whitman, C. P. The Chemical Versatility of the $\beta-\alpha-\beta$ Fold: Catalytic Promiscuity and Divergent Evolution in the Tautomerase Superfamily. Cell. Mol. Life Sci. 2008, 65, 3606-3618. 
(33) Durand, J.; Biarnés, X.; Watterlot, L.; Bonzom, C.; Borsenberger, V.; Planas, A.; Bozonnet, S.; O’Donohue, M. J.; Fauré, R. A Single Point Mutation Alters the Transglycosylation/Hydrolysis Partition, Significantly Enhancing the Synthetic Capability of an Endo-Glycoceramidase. ACS Catal. 2016, 6, 8264-8275.

(34) Andrés, E.; Albesa-Jové, D.; Biarnés, X.; Moerschbacher, B. M.; Guerin, M. E.; Planas, A. Structural Basis of Chitin Oligosaccharide Deacetylation. Angew. Chemie Int. Ed. 2014, 53, 6882-6887.

(35) Wikmark, Y.; Humble, M. S.; Bäckvall, J. E. Combinatorial Library Based Engineering of Candida Antarctica Lipase a for Enantioselective Transacylation of Sec-Alcohols in Organic Solvent. Angew. Chemie - Int. Ed. 2015, 54, 42844288 .

(36) Humble, M. S.; Carlqvist, P.; Branneby, C.; Allnér, O.; Frise, A.; Hult, K.; Berglund, P.; Brinck, T. Direct Epoxidation in Candida Antarctica Lipase B Studied by Experiment and Theory. ChemBioChem 2008, 9, 2443-2451.

(37) Chen, X. Y.; Chen, G. J.; Wang, J. L.; Wu, Q.; Lin, X. F. Lipase/AcetamideCatalyzed Carbon-Carbon Bond Formations: A Mechanistic View. Adv. Synth. Catal. 2013, 355, 864-868.

(38) Sarmah, N.; Revathi, D.; Sheelu, G.; Rani, K. Y.; Sridhar, S.; Mehtab, V.; Sumana, C. Recent Advances on Sources and Industrial Applications of Lipases. Biotechnol. Prog. 2018, 34, 5-28.

(39) Branneby, C.; Carlqvist, P.; Magnusson, A.; Hult, K.; Brinck, T.; Berglund, P. Carbon-Carbon Bonds by Hydrolytic Enzymes. J. Am. Chem. Soc. 2003, 125, 874-875.

(40) Carlqvist, P.; Svedendahl, M.; Branneby, C.; Hult, K.; Brinck, T.; Berglund, P. Exploring the Active-Site of a Rationally Redesigned Lipase for Catalysis of Michael-Type Additions. ChemBioChem 2005, 6, 331-336.

(41) Zhao, H.; Jones, C. L.; Cowins, J. V. Lipase Dissolution and Stabilization in Ether-Functionalized Ionic Liquids. Green Chem. 2009, 11, 1128.

(42) Itoh, T. Ionic Liquids as Tool to Improve Enzymatic Organic Synthesis. Chem. Rev. 2017, 117, 10567-10607.

(43) Ulusu, N. N. Evolution of Enzyme Kinetic Mechanisms. J. Mol. Evol. 2015, 80, 251-257.

(44) Copley, S. D. Toward a Systems Biology Perspective on Enzyme Evolution. J. Biol. Chem. 2012, 287, 3-10.

(45) Voordeckers, K.; Brown, C. A.; Vanneste, K.; van der Zande, E.; Voet, A.; Maere, S.; Verstrepen, K. J. Reconstruction of Ancestral Metabolic Enzymes Reveals Molecular Mechanisms Underlying Evolutionary Innovation through Gene Duplication. PLoS Biol. 2012, 10, e1001446-e1001446.

(46) Yang, G.; Miton, C. M.; Tokuriki, N. A Mechanistic View of Enzyme Evolution. Protein Sci. 2020, 29, 1724-1747.

(47) Ohno, S. Evolution by Gene Duplication; Springer Berlin Heidelberg, 1970. 


\subsection{References}

(48) Kimura, M.; Ohta, T. On Some Principles Governing Molecular Evolution. Proc. Natl. Acad. Sci. U. S. A. 1974, 71, 2848.

(49) Bergthorsson, U.; Andersson, D. I.; Roth, J. R. Ohno's Dilemma: Evolution of New Genes under Continuous Selection. Proc. Natl. Acad. Sci. 2007, 104, 17004 17009.

(50) Hughes, A. L. The Evolution of Functionally Novel Proteins after Gene Duplication. Proc. R. Soc. London. Ser. B Biol. Sci. 1994, 256, 119-124.

(51) Gerlt, J.; Babbitt, P.; Jacobson, M.; Almo, S. Divergent Evolution in Enolase Superfamily: Strategies for Assigning Functions. J. Biol. Chem. 2012, 287, 2934.

(52) Kaltenbach, M.; Tokuriki, N. Dynamics and Constraints of Enzyme Evolution. $J$. Exp. Zool. Part B Mol. Dev. Evol. 2014, 322, 468-487.

(53) Glasner, M. E.; Gerlt, J. A.; Babbitt, P. C. Evolution of Enzyme Superfamilies. Curr. Opin. Chem. Biol. 2006, 10, 492-497.

(54) Mitchell, J. B. Enzyme Function and Its Evolution. Curr. Opin. Struct. Biol. 2017, 47, 151-156.

(55) D’Ari, R.; Casadesús, J. Underground Metabolism. BioEssays 1998, 20, 181-186.

(56) Glasner, M. E.; Truong, D. P.; Morse, B. C. How Enzyme Promiscuity and Horizontal Gene Transfer Contribute to Metabolic Innovation. FEBS J. 2020, 287, 1323-1342.

(57) Noda-Garcia, L.; Liebermeister, W.; Tawfik, D. S. Metabolite-Enzyme Coevolution: From Single Enzymes to Metabolic Pathways and Networks. Annu. Rev. Biochem. 2018, 87, 187-216.

(58) Gherardini, P. F.; Wass, M. N.; Helmer-Citterich, M.; Sternberg, M. J. E. Convergent Evolution of Enzyme Active Sites Is Not a Rare Phenomenon. J. Mol. Biol. 2007, 372, 817-845.

(59) Drenth, J.; Hol, W. G.; Jansonius, J. N.; Koekoek, R. A Comparison of the ThreeDimensional Structures of Subtilisin BPN' and Subtilisin Novo. Cold Spring Harb. Symp. Quant. Biol. 1972, 36, 107-116.

(60) Kraut, J. Serine Proteases: Structure and Mechanism of Catalysis. Annu. Rev. Biochem. 1977, 46, 331-358.

(61) Zhang, M.; Van Etten, R. L.; Stauffacher, C. V. Crystal Structure of Bovine Heart Phosphotyrosyl Phosphatase at 2.2-A Resolution. Biochemistry 1994, 33, 11097 11105.

(62) Penning, T. M.; Bennett, M. J.; Smith-Hoog, S.; Schlegel, B. P.; Jez, J. M.; Lewis, M. Structure and Function of 3 Alpha-Hydroxysteroid Dehydrogenase. Steroids 1997, 62, 101-111.

(63) Hegyi, H.; Gerstein, M. The Relationship between Protein Structure and Function: A Comprehensive Survey with Application to the Yeast Genome. $J$. Mol. Biol. 1999, 288, 147-164. 
(64) Doolittle, R. F. Convergent Evolution: The Need to Be Explicit. Trends Biochem. Sci. 1994, 19, 15-18.

(65) Leveson-Gower, R. B.; Mayer, C.; Roelfes, G. The Importance of Catalytic Promiscuity for Enzyme Design and Evolution. Nat. Rev. Chem. 2019, 3, 687705.

(66) Rix, G.; Watkins-Dulaney, E. J.; Almhjell, P. J.; Boville, C. E.; Arnold, F. H.; Liu, C. C. Scalable Continuous Evolution for the Generation of Diverse Enzyme Variants Encompassing Promiscuous Activities. Nat. Commun. 2020, 11, 5644.

(67) Sheldon, R. A.; Brady, D. Broadening the Scope of Biocatalysis in Sustainable Organic Synthesis. ChemSusChem 2019, 12, 2859-2881.

(68) Freund, G. S.; O’Brien, T. E.; Vinson, L.; Carlin, D. A.; Yao, A.; Mak, W. S.; Tagkopoulos, I.; Facciotti, M. T.; Tantillo, D. J.; Siegel, J. B. Elucidating Substrate Promiscuity within the FabI Enzyme Family. ACS Chem. Biol. 2017, 12, 2465-2473.

(69) Kourist, R.; Bartsch, S.; Fransson, L.; Hult, K.; Bornscheuer, U. T. Understanding Promiscuous Amidase Activity of an Esterase from Bacillus Subtilis. ChemBioChem 2008, 9, 67-69.

(70) Lan, D.; Popowicz, G. M.; Pavlidis, I. V.; Zhou, P.; Bornscheuer, U. T.; Wang, Y. Conversion of a Mono- and Diacylglycerol Lipase into a Triacylglycerol Lipase by Protein Engineering. ChemBioChem 2015, 16, 1431-1434.

(71) Renata, H.; Wang, Z. J.; Arnold, F. H. Expanding the Enzyme Universe: Accessing Non-Natural Reactions by Mechanism-Guided Directed Evolution. Angew. Chemie - Int. Ed. 2015, 54, 3351-3367.

(72) Molina-Espeja, P.; Viña-Gonzalez, J.; Gomez-Fernandez, B. J.; Martin-Diaz, J.; Garcia-Ruiz, E.; Alcalde, M. Beyond the Outer Limits of Nature by Directed Evolution. Biotechnol. Adv. 2016, 34, 754-767.

(73) Aharoni, A.; Gaidukov, L.; Khersonsky, O.; Gould, S. M.; Roodveldt, C.; Tawfik, D. S. The "evolvability" of Promiscuous Protein Functions. Nat. Genet. 2005, 37, 73-76.

(74) Kapoor, M.; Gupta, M. N. Lipase Promiscuity and Its Biochemical Applications. Process Biochem. 2012, 47, 555-569.

(75) Maria Svedendahl; Karl Hult, and; Berglund*, P. Fast Carbon-Carbon Bond Formation by a Promiscuous Lipase. J. Am. Chem. Soc. 2005, 127, 17988-17989.

(76) Björkling, F.; Godtfredsen, S. E.; Kirk, O. Lipase-Mediated Formation of Peroxycarboxylic Acids Used in Catalytic Epoxidation of Alkenes. J. Chem. Soc. Chem. Commun. 1990, No. 19, 1301-1303.

(77) Chávez, G.; Hatti-Kaul, R.; Sheldon, R. A.; Mamo, G. Baeyer-Villiger Oxidation with Peracid Generated in Situ by CaLB-CLEA Catalyzed Perhydrolysis. J. Mol. Catal. B Enzym. 2013, 89, 67-72.

(78) Carniel, A.; Valoni, É.; Nicomedes, J.; Gomes, A. da C.; Castro, A. M. de. Lipase from Candida Antarctica (CALB) and Cutinase from Humicola Insolens Act 


\subsection{References}

Synergistically for PET Hydrolysis to Terephthalic Acid. Process Biochem. 2017, $59,84-90$.

(79) Johnson, A.; Krishnaswamy, S.; Sundaram, P.; Pattabhi, V. The First Structure at 1.8 A Resolution of an Active Autolysate Form of Porcine Alpha-Trysoin. Acta Crystallogr. D. Biol. Crystallogr. 1997, 53, 311-315.

(80) Bassindale, A. R.; Brandstadt, K. F.; Lane, T. H.; Taylor, P. G. Enzyme-Catalysed Siloxane Bond Formation. J. Inorg. Biochem. 2003, 96, 401-406.

(81) Öhlknecht, C.; Sprenger, B.; Oostenbrink, C.; Petrov, D.; Engele, P.; Kröß, C.; Fischer, A.; Lingg, N.; Schneider, R. Enhancing the Promiscuity of a Member of the Caspase Protease Family by Rational Design. Proteins 2020, 88, 1303-1318.

(82) Verma, M. K.; Pulicherla, K. K. Enzyme Promiscuity in Earthworm Serine Protease: Substrate Versatility and Therapeutic Potential. Amino Acids 2016, 48, 941-948.

(83) H, M.; H, S.; T, Y.; H, M.; R, I.; M, S.; M, M. A Novel Fibrinolytic Enzyme Extracted from the Earthworm, Lumbricus Rubellus. Jpn. J. Physiol. 1991, 41, 461-472.

(84) Mihara, H.; Maruyama, M.; Sumi, H. Novel Thrombolytic Therapy Discovered from Traditional Oriental Medicine Using the Earthworm. Southeast Asian J. Trop. Med. Public Health 1992, 23 Suppl 2, 131-140.

(85) Zhao, J.; Li, L.; Wu, C.; He, R. Q. Hydrolysis of Fibrinogen and Plasminogen by Immobilized Earthworm Fibrinolytic Enzyme II from Eisenia Fetida. Int. J. Biol. Macromol. 2003, 32, 165-171.

(86) Ueda, M.; Noda, K.; Nakazawa, M.; Miyatake, K.; Ohki, S.; Sakaguchi, M.; Inouye, K. A Novel Anti-Plant Viral Protein from Coelomic Fluid of the Earthworm Eisenia Foetida: Purification, Characterization and Its Identification as a Serine Protease. Comp. Biochem. Physiol. Part B Biochem. Mol. Biol. 2008, $151,381-385$.

(87) Jia, B.; Shihong, G. C. Multifunctional Enzymes in Archaea: Promiscuity and Moonlight. Extremophiles 2013, 17, 193-203.

(88) Stec, B.; Yang, H.; Johnson, K. A.; Chen, L.; Roberts, M. F. MJ0109 Is an Enzyme That Is Both an Inositol Monophosphatase and the "missing" Archaeal Fructose-1,6-Bisphosphatase. Nat. Struct. Biol. 2000, 7, 1046-1050.

(89) Stieglitz, K. A.; Johnson, K. A.; Yang, H.; Roberts, M. F.; Seaton, B. A.; Head, J. F.; Stec, B. Crystal Structure of a Dual Activity IMPase/FBPase (AF2372) from Archaeoglobus Fulgidus. The Story of a Mobile Loop. J. Biol. Chem. 2002, 277, 22863-22874.

(90) Le Breton, M.; Henneke, G.; Norais, C.; Flament, D.; Myllykallio, H.; Querellou, J.; Raffin, J. P. The Heterodimeric Primase from the Euryarchaeon Pyrococcus Abyssi: A Multifunctional Enzyme for Initiation and Repair? J. Mol. Biol. 2007, 374, 1172-1185.

(91) Tipton, K. F. Enzyme Nomenclature 1992. Recommendations of the 
Nomenclature Committee of the International Union of Biochemistry and Molecular Biology on the Nomenclature and Classification of Enzymes. Acad. Press 1992, 6.

(92) Levisson, M.; Oost, J. van der; Kengen, S. W. M. Carboxylic Ester Hydrolases from Hyperthermophiles. Extremophiles 2009, 13, 567-581.

(93) UniProt: The Universal Protein Knowledgebase in 2021. Nucleic Acids Res. 2021, 49, D480-D489.

(94) Berman, H. M.; Westbrook, J.; Feng, Z.; Gilliland, G.; Bhat, T. N.; Weissig, H.; Shindyalov, I. N.; Bourne, P. E. The Protein Data Bank. Nucleic Acids Res. 2000, $28,235-242$.

(95) Chen, Y.; Black, D. S.; Reilly, P. J. Carboxylic Ester Hydrolases : Classification and Database Derived from Their Primary, Secondary, and Tertiary Structures. Protein Sci. 2016, 00, 1-12.

(96) Lenfant, N.; Hotelier, T.; Velluet, E.; Bourne, Y.; Marchot, P.; Chatonnet, A. ESTHER, the Database of the $\alpha / \beta$-Hydrolase Fold Superfamily of Proteins: Tools to Explore Diversity of Functions. Nucleic Acids Res. 2013, 41, D423-D429.

(97) Lombard, V.; Golaconda Ramulu, H.; Drula, E.; Coutinho, P. M.; Henrissat, B. The Carbohydrate-Active Enzymes Database (CAZy) in 2013. Nucleic Acids Res. 2014, 42, D490-D495.

(98) Bauer, T. L.; Buchholz, P. C. F.; Pleiss, J. The Modular Structure of $\alpha / \beta$ Hydrolases. FEBS J. 2020, 287, 1035-1053.

(99) Dodson, G.; Wlodawer, A. Catalytic Triads and Their Relatives. TIBS 1998, 23, 347-352.

(100) Oh, C.; Kim, T. D.; Kim, K. K. Carboxylic Ester Hydrolases in Bacteria : Active Site, Structure, Function and Application. Crystals 2019, 9, 597.

(101) Furnham, N.; Holliday, G. L.; de Beer, T. A. P.; Jacobsen, J. O. B.; Pearson, W. R.; Thornton, J. M. The Catalytic Site Atlas 2.0: Cataloging Catalytic Sites and Residues Identified in Enzymes. Nucleic Acids Res. 2014, 42, D485-D489.

(102) Chahinian, H.; Nini, L.; Boitard, E.; Dubès, J. P.; Comeau, L. C.; Sarda, L. Distinction between Esterases and Lipases: A Kinetic Study with Vinyl Esters and TAG. Lipids 2002, 37, 653-662.

(103) Dawson, N. L.; Lewis, T. E.; Das, S.; Lees, J. G.; Lee, D.; Ashford, P.; Orengo, C. A.; Sillitoe, I. CATH: An Expanded Resource to Predict Protein Function through Structure and Sequence. Nucleic Acids Res. 2017, 45, D289-D295.

(104) Hong, S. Y.; Jung, E. J.; Joo, J. C.; Yoo, Y. J. Soluble Expression of Candida Antarctica Lipase B in Escherichia Coli by Fusion with Skp Chaperone. Biotechnol. Bioprocess Eng. 2012, 17, 687-692.

(105) Khan, F. I.; Lan, D.; Durrani, R.; Huan, W.; Zhao, Z.; Wang, Y. The Lid Domain in Lipases: Structural and Functional Determinant of Enzymatic Properties. Front. Bioeng. Biotechnol. 2017, 5, 16. 


\subsection{References}

(106) Martinelle, M.; Holmqvist, M.; Hult, K. On the Interfacial Activation of Candida Antarctica Lipase-A and Lipase-B as Compared with Humicola-Lanuginosa Lipase. Biochim. Biophys. Acta 1995, 1258, 272-276.

(107) Uppenberg, J.; Ohrner, N.; Norin, M.; Hult, K.; Kleywegt, G. J.; Patkar, S.; Waagen, V.; Anthonsen, T.; Jones, T. a. Crystallographic and MolecularModeling Studies of Lipase B from Candida Antarctica Reveal a Stereospecificity Pocket for Secondary Alcohols. Biochemistry 1995, 34, 16838-16851.

(108) Świderek, K.; Martí, S.; Moliner, V. Theoretical Study of Primary Reaction of Pseudozyma Antarctica Lipase B as the Starting Point to Understand Its Promiscuity. ACS Catal. 2014, 4, 426-434.

(109) Li, C.; Tan, T.; Zhang, H.; Feng, W. Analysis of the Conformational Stability and Activity of Candida Antarctica Lipase B in Organic Solvents: Insight from Molecular Dynamics and Quantum Mechanics/Simulations. J. Biol. Chem. 2010, 285, 28434-28441.

(110) Trodler, P.; Pleiss, J. Modeling Structure and Flexibility of Candida Antarctica Lipase B in Organic Solvents. BMC Struct. Biol. 2008, 8, 9.

(111) Ventura, S. P. M.; Santos, L. D. F.; Saraiva, J. A.; Coutinho, J. A. P. Ionic Liquids Microemulsions: The Key to Candida Antarctica Lipase B Superactivity. Green Chem. 2012, 14, 1620-1625.

(112) Nicolas, P.; Lassalle, V. V.; Ferreira, M. L. M. L.; Nicolás, P.; Lassalle, V. V.; Ferreira, M. L. M. L. Immobilization of CALB on Lysine-Modified Magnetic Nanoparticles: Influence of the Immobilization Protocol. Bioprocess Biosyst. Eng. 2018, 41, 171-184.

(113) Zhong, N.; Li, Y.; Cai, C.; Gao, Y.; Liu, N.; Liu, G.; Tan, W.; Zeng, Y. Enhancing the Catalytic Performance of Candida Antarctica Lipase B by Immobilization onto the Ionic Liquids Modified SBA-15. Eur. J. LIPID Sci. Technol. 2018, 120.

(114) Rodrigues, A. S.; Cavalcante, G. P.; Ferrei, A. L. O.; Goncalves, L. R. B. Immobilization of Candida Antarctica Lipase Type B by Adsorption on Activated Carbon. Chem. Biochem. Eng. Q. 2008, 22, 125-133.

(115) Escorcia, A. M.; Molina, D.; Daza, M. C.; Doerr, M. Acetylation of (R,S)Propranolol Catalyzed by Candida Antarctica Lipase B: An Experimental and Computational Study. J. Mol. Catal. B Enzym. 2013, 98, 21-29.

(116) Raza, S.; Fransson, L.; Hult, K. Enantioselectivity in Candida Antarctica Lipase B: A Molecular Dynamics Study. Protein Sci. 2001, 10, 329-338.

(117) Engström, K.; Vallin, M.; Syrén, P. O.; Hult, K.; Bäckvall, J. E. Mutated Variant of Candida Antarctica Lipase B in (S)-Selective Dynamic Kinetic Resolution of Secondary Alcohols. Org. Biomol. Chem. 2011, 9, 81-82.

(118) Hamber, A.; Maurer, S.; Hult, K. Rational Engineering of Candida Antarctica Lipase B for Selective Monoacylation of Diols. Chem. Commun. 2012, 1001310015.

(119) Montanier, C. Y.; Chabot, N.; Emond, S.; Guieysse, D.; Remaud-Siméon, M.; 
Peruch, F.; André, I. Engineering of Candida Antarctica Lipase B for Poly( $\varepsilon-$ Caprolactone) Synthesis. Eur. Polym. J. 2017, 95, 809-819.

(120) Santos, Y. L. de los; Chew-Fajardo, Y. L.; Brault, G.; Doucet, N. Dissecting the Evolvability Landscape of the CalB Active Site toward Aromatic Substrates. Sci. Rep. 2019, 9, 1-14.

(121) Xu, J.; Cen, Y.; Singh, W.; Fan, J.; Wu, L.; Lin, X.; Zhou, J.; Huang, M.; Reetz, M. T.; Wu, Q. Stereodivergent Protein Engineering of a Lipase to Access All Possible Stereoisomers of Chiral Esters with Two Stereocenters. J. Am. Chem. Soc. 2019, 141, 7934-7945.

(122) Zock, J.; Cantwell, C.; Swartling, J.; Hodges, R.; Pohl, T.; Sutton, K.; Rosteck, P.; McGilvray, D.; Queener, S. The Bacillus Subtilis PnbA Gene Encoding PNitrobenzyl Esterase: Cloning, Sequence and High-Level Expression in Escherichia Coli. Gene 1994, 151.

(123) Chang, A.; Jeske, L.; Ulbrich, S.; Hofmann, J.; Koblitz, J.; Schomburg, I.; Neumann-Schaal, M.; Jahn, D.; Schomburg, D. BRENDA, the ELIXIR Core Data Resource in 2021: New Developments and Updates. Nucleic Acids Res. 2021, 49, D498-D508.

(124) Spiller, B.; Gershenson, A.; Arnold, F. H.; Stevens, R. C. A Structural View of Evolutionary Divergence. Proc. Natl. Acad. Sci. 1999, 96, 12305-12310.

(125) Schmidt, M.; Henke, E.; Heinze, B.; Kourist, R.; Hidalgo, A.; Bornscheuer, U. T. A Versatile Esterase from Bacillus Subtilis: Cloning, Expression Characterization, and Its Application in Biocatalysis. Biotechnol. J. 2007, 2, 249253.

(126) Ribitsch, D.; Heumann, S.; Trotscha, E.; Herrero Acero, E.; Greimel, K.; Leber, R.; Birner-Gruenberger, R.; Deller, S.; Eiteljoerg, I.; Remler, P.; Weber, T.; Siegert, P.; Maurer, K. H.; Donelli, I.; Freddi, G.; Schwab, H.; Guebitz, G. M. Hydrolysis of Polyethyleneterephthalate by P-Nitrobenzylesterase from Bacillus Subtilis. Biotechnol. Prog. 2011, 27, 951-960.

(127) Hackenschmidt, S.; Moldenhauer, E. J.; Behrens, G. A.; Gand, M.; Pavlidis, I. V.; Bornscheuer, U. T. Enhancement of Promiscuous Amidase Activity of a Bacillus Subtilis Esterase by Formation of a $\pi-\pi$ Network. ChemCatChem 2014, $6,1015-$ 1020 .

(128) Chen, K.; Arnold, F. H. Tuning the Activity of an Enzyme for Unusual Environments: Sequential Random Mutagenesis of Subtilisin E for Catalysis in Dimethylformamide. Proc. Natl. Acad. Sci. U. S. A. 1993, 90, 5618-5622.

(129) Arnold, F. H. Combinatorial and Computational Challenges for Biocatalyst Design. Nature 2001, 409, 253-257.

(130) Blomberg, R.; Kries, H.; Pinkas, D. M.; Mittl, P. R. E.; Grütter, M. G.; Privett, H. K.; Mayo, S. L.; Hilvert, D. Precision Is Essential for Efficient Catalysis in an Evolved Kemp Eliminase. Nature 2013, 503, 418-421.

(131) Huang, P.-S.; Boyken, S. E.; Baker, D. The Coming of Age of de Novo Protein Design. Nature 2016, 537, 320-327. 


\subsection{References}

(132) Zeymer, C.; Hilvert, D. Directed Evolution of Protein Catalysts. Annu. Rev. Biochem. 2018, 87, 131-157.

(133) Arnold, F. H. Directed Evolution: Bringing New Chemistry to Life. Angew. Chemie Int. Ed. 2018, 57, 4143-4148.

(134) Savile, C. K.; Janey, J. M.; Mundorff, E. C.; Moore, J. C.; Tam, S.; Jarvis, W. R.; Colbeck, J. C.; Krebber, A.; Fleitz, F. J.; Brands, J.; Devine, P. N.; Huisman, G. W.; Hughes, G. J. Biocatalytic Asymmetric Synthesis of Chiral Amines from Ketones Applied to Sitagliptin Manufacture. Science (80-. ). 2010, 329, 305-309.

(135) Kamionka, M. Engineering of Therapeutic Proteins Production in Escherichia Coli. Curr. Pharm. Biotechnol. 2011, 12, 268-274.

(136) Mandeep; Sinha, R.; Shukla, P. Protein Engineering for Improved Health : Technological Perspectives. Curr. Protein Pept. Sci. 2019, 20, 856-860.

(137) Foo, J. L.; Ching, C. B.; Chang, M. W.; Su, S.; Leong, J. The Imminent Role of Protein Engineering in Synthetic Biology. Biotechnol. Adv. 2012, 30, 541-549.

(138) Świderek, K.; Tuñon, I.; Moliner, V.; Bertran, J. Computational Strategies for the Design of New Enzymatic Functions. Arch. Biochem. Biophys. 2015, 582, 68-79.

(139) Mate, D. M.; Alcalde, M. Laccase Engineering: From Rational Design to Directed Evolution. Biotechnology Advances. 2015.

(140) Jiang, L.; Althoff, E. A.; Clemente, F. R.; Doyle, L.; Röthlisberger, D.; Zanghellini, A.; Gallaher, J. L.; Betker, J. L.; Tanaka, F.; Barbas, C. F.; Hilvert, D.; Houk, K. N.; Stoddard, B. L.; Baker, D. De Novo Computational Design of Retro-Aldol Enzymes. Science (80-. ). 2008, 319, 1387-1391.

(141) Reetz, M. T.; Carballeira, J. D. Iterative Saturation Mutagenesis (ISM) for Rapid Directed Evolution of Functional Enzymes. Nat. Protoc. 2007, 2, 891-903.

(142) Lutz, S. Beyond Directed Evolution - Semi-Rational Protein Engineering and Design. Curr. Opin. Biotechnol. 2011, 21, 734-743.

(143) Sebestova, E.; Bendl, J.; Brezovsky, J.; Damborsky, J. Computational Tools for Designing Smart Libraries. Methods Mol. Biol. 2014, 1179, 291-314.

(144) Stemmer, W. P. C. Searching Sequence Space. Bio/Technology 1995, 13, 549553.

(145) Kuchner, O.; Arnold, F. H. Directed Evolution of Enzyme Catalysts. Trends Biotechnol. 1997, 15, 523-530.

(146) Bornscheuer, U. T. Directed Evolution of Enzymes. Angew. Chemie Int. Ed. 1998, 37, 3105-3108.

(147) MacBeath, G.; Kast, P.; Hilvert, D. Redesigning Enzyme Topology by Directed Evolution. Science (80-. ). 1998, 279, 1958-1961.

(148) Yoshikuni, Y.; Ferrin, T. E.; Keasling, J. D. Designed Divergent Evolution of Enzyme Function. Nature 2006, 440, 1078-1082.

(149) Otten, R.; Pádua, R. A. P.; Bunzel, H. A.; Nguyen, V.; Pitsawong, W.; Patterson, 
M.; Sui, S.; Perry, S. L.; Cohen, A. E.; Hilvert, D.; Kern, D. How Directed Evolution Reshapes the Energy Landscape in an Enzyme to Boost Catalysis. Science (80-. ). 2020, 370, 1442 LP - 1446.

(150) Wilding, M.; Hong, N.; Spence, M.; Buckle, A. M.; Jackson, C. J. Protein Engineering: The Potential of Remote Mutations. Biochem. Soc. Trans. 2019, No. March, 1-11.

(151) Bunzel, H. A.; Garrabou, X.; Pott, M.; Hilvert, D. Speeding up Enzyme Discovery and Engineering with Ultrahigh-Throughput Methods. Curr. Opin. Struct. Biol. 2018, 48, 149-156.

(152) Pauling, L. Molecular Architecture and Biological Reactions. Chem. Eng. News 1946, 24, 1375-1377.

(153) Warshel, A. Electrostatic Origin of the Catalytic Power of Enzymes and the Role of Preorganized Active Sites. J. Biol. Chem. 1998, 273, 27035-27038.

(154) Warshel, A.; Sharma, P. K.; Kato, M.; Xiang, Y.; Liu, H.; Olsson, M. H. M. Electrostatic Basis for Enzyme Catalysis. Chem. Rev. 2006, 106, 3210-3235.

(155) Roca, M.; Martí, S.; Andrés, J.; Moliner, V.; Tuñón, I.; Bertrán, J.; Williams, I. H. Theoretical Modeling of Enzyme Catalytic Power: Analysis of "Cratic" and Electrostatic Factors in Catechol O-Methyltransferase. J. Am. Chem. Soc. 2003, $125,7726-7737$.

(156) García-Meseguer, R.; Martí, S.; Ruiz-Pernía, J. J.; Moliner, V.; Tunóón, I. Studying the Role of Protein Dynamics in an SN2 Enzyme Reaction Using FreeEnergy Surfaces and Solvent Coordinates. Nat. Chem. 2013, 5, 566-571.

(157) Krzemińska, A.; Moliner, V.; Świderek, K. Dynamic and Electrostatic Effects on the Reaction Catalyzed by HIV-1 Protease. J. Am. Chem. Soc. 2016, 138, 1628316298.

(158) Świderek, K.; Tunón, I.; Williams, I. H.; Moliner, V. Insights on the Origin of Catalysis on Glycine N-Methyltransferase from Computational Modeling. J. Am. Chem. Soc. 2018, 140, 4327-4334.

(159) Welborn, V. V.; Head-gordon, T. Computational Design of Synthetic Enzymes. Chem. Rev. 2018, 119, 6613-6630.

(160) Serrano-Aparicio, N.; Moliner, V.; Świderek, K. Nature of Irreversible Inhibition of Human 20S Proteasome by Salinosporamide A. The Critical Role of Lys-Asp Dyad Revealed from Electrostatic Effects Analysis. ACS Catal. 2021, 11, 35753589.

(161) Nagel, Z. D.; Klinman, J. P. A 21st Century Revisionist's View at a Turning Point in Enzymology. Nat. Chem. Biol. 2009, 5, 543-550.

(162) Tantillo, D. J.; Chen, J.; Houk, K. N. Theozymes and Compuzymes: Theoretical Models for Biological Catalysis. Curr. Opin. Chem. Biol. 1998, 2, 743-750.

(163) Samish, I.; MacDermaid, C. M.; Perez-Aguilar, J. M.; Saven, J. G. Theoretical and Computational Protein Design. Annu. Rev. Phys. Chem. 2011, 62, 129-149. 


\subsection{References}

(164) Barrozo, A.; Borstnar, R.; Marloie, G.; Kamerlin, S. C. L. Computational Protein Engineering: Bridging the Gap between Rational Design and Laboratory Evolution. Int. J. Mol. Sci. 2012, 13, 12428-12460.

(165) Amrein, B. A.; Steffen-Munsberg, F.; Szeler, I.; Purg, M.; Kulkarni, Y.; Kamerlin, S. C. L. CADEE: Computer-Aided Directed Evolution of Enzymes. IUCrJ 2017, 4, 50-64.

(166) Warshel, A.; Weiss, R. M. An Empirical Valence Bond Approach for Comparing Reactions in Solutions and in Enzymes. J. Am. Chem. Soc. 1980, 102, 6218-6226.

(167) Fox, R. J.; Davis, S. C.; Mundorff, E. C.; Newman, L. M.; Gavrilovic, V.; Ma, S. K.; Chung, L. M.; Ching, C.; Tam, S.; Muley, S.; Grate, J.; Gruber, J.; Whitman, J. C.; Sheldon, R. A.; Huisman, G. W. Improving Catalytic Function by ProSARDriven Enzyme Evolution. Nat. Biotechnol. 2007, 25, 338-344.

(168) Yang, K. K.; Wu, Z.; Arnold, F. H. Machine-Learning-Guided Directed Evolution for Protein Engineering. Nat. Methods 2019, 16, 687-694.

(169) Wu, Z.; Kan, S. B. J.; Lewis, R. D.; Wittmann, B. J.; Arnold, F. H. Machine Learning-Assisted Directed Protein Evolution with Combinatorial Libraries. Proc. Natl. Acad. Sci. 2019, 116, 8852 LP - 8858.

(170) LeCun, Y.; Bengio, Y.; Hinton, G. Deep Learning. Nature 2015, 521, 436-444.

(171) Jiménez, J.; Škalič, M.; Martínez-Rosell, G.; De Fabritiis, G. KDEEP: ProteinLigand Absolute Binding Affinity Prediction via 3D-Convolutional Neural Networks. J. Chem. Inf. Model. 2018, 58, 287-296.

(172) Alley, E. C.; Khimulya, G.; Biswas, S.; AlQuraishi, M.; Church, G. M. Unified Rational Protein Engineering with Sequence-Based Deep Representation Learning. Nat. Methods 2019, 16, 1315-1322.

(173) Cao, H.; Wang, J.; He, L.; Qi, Y.; Zhang, J. Z. DeepDDG: Predicting the Stability Change of Protein Point Mutations Using Neural Networks. J. Chem. Inf. Model. 2019, 59, 1508-1514.

(174) Senior, A. W.; Evans, R.; Jumper, J.; Kirkpatrick, J.; Sifre, L.; Green, T.; Qin, C.; Žídek, A.; Nelson, A. W. R.; Bridgland, A.; Penedones, H.; Petersen, S.; Simonyan, K.; Crossan, S.; Kohli, P.; Jones, D. T.; Silver, D.; Kavukcuoglu, K.; Hassabis, D. Improved Protein Structure Prediction Using Potentials from Deep Learning. Nature 2020, 577, 706-710.

(175) Jindal, G.; Slanska, K.; Kolev, V.; Damborsky, J.; Prokop, Z.; Warshel, A. Exploring the Challenges of Computational Enzyme Design by Rebuilding the Active Site of a Dehalogenase. Proc. Natl. Acad. Sci. 2019, 116, 389 LP - 394. 
Chapter 2

Objectives 
- 42 - 
Enzyme promiscuity has been of remarkable interest in the scientific community for its intrinsic implications in enzyme evolution and activity, as stated in the previous chapter. Nevertheless, how these promiscuous mechanisms work at a molecular level is still under debate. Moreover, enzyme promiscuity is often used as starting point for enzyme evolution in the laboratory, which is key for the obtention of powerful tools and biocatalysts to be used in the molecular biology field and industry. This Ph.D. thesis is centered on the study of reaction mechanisms of promiscuous enzymes and the subsequent application in the generation of improved biocatalysts. The specific objectives that are addressed in the present study, based on the use of computational and experimental techniques, are detailed below.

- To study the promiscuous amidase activity of CALB. The catalytic and substrate promiscuity are studied from an experimental and theoretical perspective.

- To give insights on the promiscuous epoxidation reaction that CALB displays on short alkenes.

- To analyze the similarities and differences that can be found on the amidase activity of CALB and the Bs2 esterase.

- To propose a new rational QM/MM MD protein engineering strategy based on the combination of the best electrostatic properties of enzymes with activity on a common reaction, in this case Bs2 and CALB 

Chapter 3

Computational and Experimental

Methods 
- 58 - 


\subsection{Structure and energy}

To study the nature of promiscuous mechanisms at the molecular level from a theoretical perspective, a series of computational simulations have been carried out. In this thesis, the reactivity was studied in terms of multiscale QM/MM approaches, where the part of the system where the chemical transformation takes place is treated with QM theory, and the rest is described within the classical MM framework. Hence, the large region of the system that was not directly involved in the reaction, the MM part, was described by classical FF, while the QM part, which comprises the region involved in the reaction, was described using Semiempirical (SE) methods or Density Functional Theory (DFT) approximations.

\subsubsection{System's setup}

The initial preparation of the biological system was done in each case intending to simulate the system in the closest conditions to the experimental ones. Then, a proper setup was needed to accommodate the crystal geometry of the protein into a condensed phase media. The setup was in general the same for all the systems presented in this thesis with some peculiarities which depend on the proper nature of the protein, for instance the protonation state of titratable residues and the overall charge of the system, the substrate, and the studied conditions. Consequently, a general scheme is provided, while the specific peculiarities of each system are summarized in Table 1.

The initial geometries of the proteins were obtained from the respective PDB structures, either CALB with PDB ID $1 \mathrm{TCA}^{1}$ or Bs2 with PDB ID 1QE3. ${ }^{2}$ The protonation state of titratable residues were determined at $\mathrm{pH}$ equivalent to 7 using the PropKa ver. 3.0.3 program to calculate the possible $\mathrm{pK}_{\mathrm{a}}$ shifts, based on the $3 \mathrm{D}$ structures. ${ }^{3,4}$ Protonated residues for 


\subsection{Structure and energy}

each protein are summarized in Table 1. Moreover, in the case of CALB three disulfide bridges between residues Cys22 and Cys64, Cys216 and Cys258, and Cys293 and Cys311 were identified. No disulfide bridges were set in the case of Bs2.

Hydrogen atoms were added to the protein structures and the systems were solvated by placing them in a $100 \times 80 \times 80 \AA^{3}$ pre-equilibrated box of TIP3 $\mathrm{P}^{5}$ water molecules. Any water with an oxygen atom lying in a radius of $2.8 \AA$ from a heavy atom of the protein was deleted. In order to neutralize the systems, the appropriate number of counterions $\left(\mathrm{Na}^{+}\right)$were added in the most electrostatically favorable positions using LEaP program as implemented in Ambertools. ${ }^{6}$

The corresponding substrates were manually built into the active site pocket of the preequilibrated system, considering that the electrophilic oxygen of the substrate should be placed in the correct position defined by location of the oxyanion hole.

With the purpose to equilibrate the total system, classical MD simulations were done in each system. The substrates were parametrized with Antechamber. ${ }^{7}$ The missing $\mathrm{FF}$ parameters of the substrates were generated using the General Amber Force Field (GAFF), ${ }^{8}$ and the SE Austin Model 1 (AM1 $)^{9}$ Hamiltonian was used to compute the partial charges. All parameters can be found in the Supporting Information of the respective research articles in the annexes.

After initial energy minimizations, the systems were heated with $0.1 \mathrm{~K}$ temperature increment up to the reference temperature given in Table 1, and equilibrated during short 100 ps NPT MD simulations followed by non-accelerated classical NVT MD simulations with AMBER ff03 FF, ${ }^{10}$ as implemented in NAMD software. ${ }^{11}$ During the NVT MD simulations all atoms were free to move within periodic boundary conditions and cut-offs for nonbonding interactions with an internal cut-off of $14.5 \AA$ and an external of $16 \AA$. To maintain the temperature constant the Langevin thermostat ${ }^{12}$ was used. 
Table 1. Summary of the systems studied in this thesis. Enzyme information, as well as the substrate used, is given for each system. The number of atoms stands for protein/substrate/counterions/water. The number of QM atoms is presented as QM atoms/link atoms. The corresponding articles where each system is studied are presented in Chapter 4.

\begin{tabular}{|c|c|c|c|c|c|}
\hline ID & Enzyme & Variant & PDB ID & \multicolumn{2}{|c|}{ Substrate } \\
\hline 1 & CALB & wild-type & 1TCA & \multicolumn{2}{|c|}{ benzylbutyramide } \\
\hline 2 & CALB & wild-type & $1 \mathrm{TCA}$ & \multicolumn{2}{|c|}{ N-(4-nitrophenyl)-butyramide } \\
\hline 3 & CALB & $\mathrm{S} 105 \mathrm{~A}$ & $1 \mathrm{TCA}$ & \multicolumn{2}{|c|}{ 2-butenal + hydrogen peroxide } \\
\hline 4 & Bs2 & wild-type & 1QE3 & \multicolumn{2}{|c|}{ N-(4-nitrophenyl)-butyramide } \\
\hline 5 & Bs2 & F398D & 1QE3 & \multicolumn{2}{|c|}{ N-(4-nitrophenyl)-butyramide } \\
\hline 6 & Bs 2 & F398D-H+ & 1QE3 & \multicolumn{2}{|c|}{ N-(4-nitrophenyl)-butyramide } \\
\hline 7 & Bs 2 & D66I/L335K & 1QE3 & \multicolumn{2}{|c|}{ N-(4-nitrophenyl)-butyramide } \\
\hline ID & \multicolumn{2}{|c|}{ Protonated residues } & Charge (a.u.) & Ions Added & \#QM atoms \\
\hline 1 & \multicolumn{2}{|c|}{ D134 } & 0 & - & $53 / 3$ \\
\hline 2 & \multicolumn{2}{|c|}{ D134 } & 0 & - & $52 / 3$ \\
\hline 3 & \multicolumn{2}{|c|}{ D134 } & 0 & - & $32 / 2$ \\
\hline 4 & \multicolumn{2}{|c|}{ E188,E214,E402 } & -20 & $\mathrm{Na}+$ & $55 / 3$ \\
\hline 5 & \multicolumn{2}{|c|}{ E188,E214,E402 } & -21 & $\mathrm{Na}+$ & $55 / 3$ \\
\hline 6 & \multicolumn{2}{|c|}{ E188,E214,D398,E402 } & -20 & $\mathrm{Na}+$ & $55 / 3$ \\
\hline 7 & \multicolumn{2}{|c|}{ E188,E214,E402 } & -18 & $\mathrm{Na}+$ & $55 / 3$ \\
\hline ID & \multicolumn{2}{|c|}{ \#Atoms } & length (ns) & Ref. Temp. (K) & Ref. Article \\
\hline 1 & \multicolumn{2}{|c|}{$4625 / 28 / 0 / 60015$} & 5 & 303 & Article 1 \\
\hline 2 & \multicolumn{2}{|c|}{$4625 / 27 / 0 / 60015$} & 5 & 303 & Article $1,3,4$ \\
\hline 3 & \multicolumn{2}{|c|}{$4624 / 15 / 0 / 59406$} & 200 & 293 & Article 2 \\
\hline 4 & \multicolumn{2}{|c|}{$7568 / 27 / 20 / 65544$} & 100 & 303 & Article 3,4 \\
\hline 5 & \multicolumn{2}{|c|}{$7533 / 27 / 21 / 65544$} & 100 & 303 & Article 4 \\
\hline 6 & \multicolumn{2}{|c|}{$7534 / 27 / 20 / 65544$} & 100 & 303 & Article 4 \\
\hline 7 & \multicolumn{2}{|c|}{$7551 / 27 / 18 / 65544$} & 100 & 303 & Article 4 \\
\hline
\end{tabular}

In some cases, the MD simulations were carried out on an intermediate of the reaction to avoid unbinding and diffusion of the substrates from the active site. In the cases where the MD was done on the Michaelis complex, a restraint was applied with a force constant of $2000 \mathrm{~kJ} \cdot \mathrm{mol}^{-1} \cdot \AA^{-2}$. 


\subsection{Structure and energy}

The final structures to be used as starting geometries for the reactivity studies were chosen from MD simulations after careful analysis of the distances of the reaction coordinate. A series of QM/MM minimizations were applied to the representative geometry of the system, ${ }^{13}$ consisting on a series of steepest descent conjugated gradient minimizations with a gradient tolerance of $5 \mathrm{~kJ} \cdot \mathrm{mol}^{-1} \cdot \AA^{-1}$, followed by a L-BFGS-B minimization with a final gradient tolerance of $0.1 \mathrm{~kJ} \cdot \mathrm{mol}^{-1} \cdot \AA^{-1}$.

\subsubsection{Molecular Mechanics potentials}

As already explained, the part of the system not directly involved in the chemical reaction is defined in terms of MM potentials. In this sense, two different FFs, that are briefly detailed below, were used to describe the forces and potential energy of the atoms belonging to the protein (except the residues participating in the reaction) and the ions. To describe the energy and behavior of water molecules, the TIP3 $\mathrm{P}^{5} \mathrm{FF}$ was used.

\subsubsection{OPLS-AA Force Field}

The Optimized Potentials for Liquid Simulations FF (OPLS-AA) was initially developed in 1996 by Jorgensen and co-workers. ${ }^{14}$ This FF has been demonstrated to be consistent in the simulations of proteins in condensed phase. Non-bonded parameters are optimized to reproduce liquid phase properties. Additionally, torsional parameters are parametrized to fit experimental or quantum data. The OPLS-AA force field was used to describe the atoms belonging to the protein in the QM/MM calculations. In its functional form, the total energy of the system is computed as the sum of the components in eqs. 3.1 and 3.2.

$$
\begin{gathered}
E(r)=E_{\text {bonded }}+E_{\text {non-bonded }} \\
E(r)=E_{\text {bond }}+E_{\text {angle }}+E_{\text {dihedral }}+E_{\text {improper }}+E_{\mathrm{vdW}}+E_{\text {elec }}
\end{gathered}
$$


The energetics for bond stretching and bond bending are computed as harmonic potentials, as shown in eqs. 3.3 and 3.4,

$$
\begin{gathered}
E_{\text {bond }}=\sum_{\text {bonds }} K_{r}\left(r-r_{\mathrm{eq}}\right)^{2} \\
E_{\text {angle }}=\sum_{\text {angles }} K_{\theta}\left(\theta-\theta_{\mathrm{eq}}\right)^{2}
\end{gathered}
$$

where $K_{r}$ and $K_{\theta}$ are the force constants for bonds and angles respectively, and $r_{\mathrm{eq}}$ and $\theta_{\mathrm{eq}}$ are the equilibrium bond length and bond angle.

The dihedral and improper energy is computed as a Fourier expansion as described in eq. 3.5,

$$
\begin{aligned}
E_{\mathrm{dihe} / \mathrm{impr}}= & \sum_{\text {dihe } / \text { impr }} \frac{V_{1}^{i}}{2}\left[1+\cos \left(\phi_{i}\right)\right]+\frac{V_{2}^{i}}{2}\left[1-\cos \left(2 \phi_{i}\right)\right] \\
& +\frac{V_{3}^{i}}{2}\left[1-\cos \left(3 \phi_{i}\right)\right]+\frac{V_{4}^{i}}{2}\left[1+\cos \left(4 \phi_{i}\right)\right]
\end{aligned}
$$

where $V_{1}, V_{2}, V_{3}$ and $V_{4}$ are the fitted Fourier coefficients for a given dihedral and $\phi_{i}$ the dihedral or improper angle.

In the case of the non-bonding interactions, the energy is computed as the contributions of the electrostatic and vdW interactions expressed as Coulombic potential energy and Lennard-Jones potential, as represented in eqs. 3.6 and 3.7 .

$$
\begin{gathered}
E_{\text {elec }}=\sum_{i=1}^{N-1} \sum_{j=i+1}^{N} \frac{q_{i} q_{j}}{4 \pi \epsilon_{0} r_{i j}} f_{i j} \\
E_{\mathrm{vdW}}=\sum_{i=1}^{N-1} \sum_{j=i+1}^{N} 4 \epsilon_{i j}\left[\left(\frac{\sigma_{i j}}{r_{i j}}\right)^{12}-\left(\frac{\sigma_{i j}}{r_{i j}}\right)^{6}\right] f_{i j}
\end{gathered}
$$




\subsection{Structure and energy}

Here, $q_{i}$ and $q_{j}$ correspond to the partial charges of atoms $i$ and $j$, and $r_{i j}$ stands for the distance between them. The values of $\epsilon$ and $\sigma$ correspond to depth of the potential and the distance where the potential in zero, respectively. Combination rules for the $\epsilon_{i j}$ and $\sigma_{i j}$ between two different atom types are computed as $\epsilon_{i j}=\sqrt{\epsilon_{i i} \epsilon_{j j}}$ and $\sigma_{i j}=\sqrt{\sigma_{i i} \sigma_{j j}}$, respectively. Intramolecular non-bonding interactions were computed with the same expression where $f_{i j}=1.0$ except in the $1-4$ bonds where $f_{i j}=0.5$.

\subsubsection{AMBER Force Field}

The Assisted Model Building with Energy Refinement FF (AMBER) ${ }^{15}$ is a set of FFs that are designed to work on different types of biomolecules. Initially developed and parametrized by Kollman and co-workers, ${ }^{16}$ has gained a reputation for working effectively in molecular modeling of proteins in the condensed phase. In this work, the AMBER ff03 FF has been used to describe the parameters of the proteins for long MD simulations, as well as the $\mathrm{GAFF}^{8}$ force field used to generate the missing parameters for non-standard molecules in this case substrates. The AMBER force field employs the same energetic scheme as shown in eqs. 3.1 and 3.2, and the original parametrization was done using fixed partial charges centered on atoms. Bonds and bond angles are described as harmonic potentials as shown in eqs. 3.3 and 3.4, respectively. Electrostatic interactions are described in terms of eq. 3.6 with a scaling factor of $1 / 1.2$ for 1-4 bond interactions. Lennard-Jones potentials are described following the addition showed in eq. 3.8,

$$
E_{\mathrm{vdW}}=\sum_{i=1}^{N-1} \sum_{j=i+1}^{N} 4 \epsilon_{i j}\left[\left(\frac{r_{i j}^{0}}{r_{i j}}\right)^{12}-2\left(\frac{r_{i j}^{0}}{r_{i j}}\right)^{6}\right] f_{i j}
$$


being the equilibrium distance $r_{i j}^{0}=2^{1 / 6} \cdot \sigma_{i j}$. Combination rules for $\epsilon_{i j}$ and $\sigma_{i j}$ are computed as $\epsilon_{i j}=\sqrt{\epsilon_{i i} \epsilon_{j j}}$ and $\sigma_{i j}=\frac{1}{2}\left(\sigma_{i i}+\sigma_{j j}\right)$, respectively. The scaling factor $f_{i j}$ was 0.5 for $1-4$ bonded atoms, and 1.0 for the rest.

The torsion term is described as a Fourier series in the form of eq. 3.9,

$$
E_{\text {dihe } / \mathrm{impr}}=\sum_{\text {dihe/impr }} \sum_{n} \frac{V_{i}^{n}}{2}\left[1+\cos \left(n \phi_{i}-\gamma\right)\right]
$$

where $\phi$ is the dihedral or improper angle; $V^{n}$ is the corresponding $n^{\text {th }}$ force constant of the set of parameters describing the dihedral or improper; and $\gamma$ denotes the phase which takes values of either $0^{\circ}$ or $180^{\circ}$.

\subsubsection{QM potentials}

The phenomena where electronic rearrangement occurs must be described by QM methodologies. But when describing big biological systems, the computational cost is a limiting factor. Hence, the use of SE or DFT based Hamiltonians is common within the QM/MM framework. In this thesis, two main Hamiltonians have been used to study reactivity, the semiempirical $\mathrm{AM} 1^{9}$ as a low-level Hamiltonian, and the Minnesota M06$2 \mathrm{X}^{17}$ or the B3LYP (Becke 3-parameter Lee-Yang-Parr) ${ }^{18,19}$ functionals as high-level DFT potentials. In the following subsections a brief introduction on the SE and DFT approximations used will be exposed.

\subsubsection{Semiempirical approximations}

SE methods have been developed to overcome the limitations of computational cost in QM calculations. In electronic structure theory, Molecular Orbitals (MO) are approximated as linear combination of a basis set functions represented by atomic orbitals. Usually, in the 


\subsection{Structure and energy}

conventional Hartree-Fock (HF) approximations all electrons are expanded in the atomic orbital sets. This fact scales the computational cost rapidly and makes it unfeasible for large systems. On the other site, SE methods typically only treat the valence electrons, which are assumed in the field of the nuclei and the inner-shell electrons. ${ }^{20}$ This is typically done by neglecting certain integrals, which overcomes the bottleneck in a conventional HF approximation.

In this thesis, the $\mathrm{AM} 1^{9}$ method has been used, which is based in the Neglect of Differential Diatomic Overlap (NDDO) ${ }^{21}$ approximation. In fact, it is a further reparameterization of the Modified Neglect of Diatomic Overlap (MNDO) ${ }^{22}$ developed by Dewar and Thiel. The MNDO improves NDDO by replacing the two-center two-electron integrals by approximate integrals which were derived from multipole interactions, and the core-core interactions terms in the one-electron operator were improved. In the case of AM1 up to four Gaussian functions were added to the core-core term of MNDO by means of eq. 3.10, to improve the short-range and the hydrogen bond interactions.

$$
V_{N}(\mathrm{~A}, \mathrm{~B})=V_{\mathrm{AB}}^{\mathrm{MNDO}}+\frac{Z_{\mathrm{A}} Z_{\mathrm{B}}}{r_{\mathrm{AB}}} \sum_{i=1}^{4}\left\lfloor a_{\mathrm{A}, i} e^{-b_{\mathrm{A}, i}\left(r_{\mathrm{AB}}-c_{\mathrm{A}, i}\right)^{2}}+a_{\mathrm{B}, i} e^{-b_{\mathrm{B}, i}\left(r_{\mathrm{AB}}-c_{\mathrm{B}, i}\right)^{2}}\right\rfloor
$$

Where $V_{\mathrm{AB}}^{\mathrm{MNDO}}$ is the MNDO core-core term, $Z_{\mathrm{A}}$ and $Z_{\mathrm{B}}$ are the core charges, $r_{\mathrm{AB}}$ stands for the core-core distance, while the $a, b$ and $c$ terms correspond to the fitted parameters of atoms $\mathrm{A}$ and $\mathrm{B}$.

\subsubsection{Density Functional Theory approximations}

DFT is one of the most successful and widely used approximation in quantum chemistry. It is based on the work carried out by Hohenberg and Kohn who proofed that the ground state electronic energy can be determined by the electron density. ${ }^{23}$ The main goal of DFT methods is to produce functionals that correctly connect the energy of the system with 
the electron density. The first attempts in DFT theory tried to express all the energy components as a functional of the electron density. However, due to their poor performance, wave-function based methods were preferred. However, the paradigm changed with the work of Kohn and Sham who suggest that the kinetic electron energy should be calculated from an auxiliary set of orbitals used to represent the electron density. ${ }^{24}$ Hence, the exchange-correlation energy is the only unknown, and even the crudest approximations provide quite accurate results.

The key to Kohn-Sham theory is to calculate the kinetic energy assuming non-interacting electrons, whose difference with the exact kinetic energy is small, and it is in part absorbed into an exchange-correlation term. A general DFT energy expression can be described as shown in eq. 3.11.

$$
E_{\mathrm{DFT}}[\rho]=T_{\mathrm{s}}[\rho]+E_{\mathrm{ne}}[\rho]+J[\rho]+E_{\mathrm{xc}}[\rho]
$$

In this general expression $T_{\mathrm{s}}[\rho]$ defines the kinetic energy, $E_{\mathrm{ne}}[\rho]$ is the interaction with the external potential including the electron-nuclei interaction, $J[\rho]$ is the Coulomb functional, and the last $E_{\mathrm{xc}}[\rho]$ is the exchange-correlation term.

By equating $E_{\mathrm{DFT}}$ to the exact energy the $E_{\mathrm{xc}}$ can be defined as,

$$
E_{\mathrm{xc}}[\rho]=\left(T[\rho]-T_{\mathrm{s}}[\rho]\right)+\left(E_{\mathrm{ee}}[\rho]-J[\rho]\right)
$$

where the first parenthesis can be considered as the kinetic correlation energy, and the last contains the potential correlation and exchange energy. In Kohn-Sham theory the main goal is to develop approximations to the exchange-correlation energy functional.

The main difference between DFT methods is the choice of the functional for the exchange-correlation energy. In this thesis, two hybrid functionals have been used, the B3LYP ${ }^{18,19}$ functional, and the M06-2X ${ }^{17}$ functional from the Minnesota M06 suite.

B3LYP is a wide extended functional defined as, 


$$
E_{\mathrm{xc}}^{\mathrm{B} 3 \mathrm{LYP}}=(1-a) E_{\mathrm{x}}^{\mathrm{LSDA}}+a E_{\mathrm{x}}^{\mathrm{HF}}+b \Delta E_{\mathrm{x}}^{\mathrm{B} 88}+(1-c) E_{\mathrm{c}}^{\mathrm{LSDA}}+c E_{\mathrm{c}}^{\mathrm{LYP}}
$$

where the $a, b$ and $c$ parameters are determined by fitting to experimental data with typical values being $a=0.20, b=0.72$ and $c=0.81$.

The M06-2X functional, developed by Truhlar and co-workers, is based in the meta-generalized gradient approximation. A general definition of the M06 family of functionals can be defined as in eq. 3.14.

$$
E_{\mathrm{xc}}^{\mathrm{M} 06}=\frac{X}{100} E_{\mathrm{x}}^{\mathrm{HF}}+\left(1-\frac{X}{100}\right) E_{\mathrm{x}}^{\mathrm{DFT}}+E_{\mathrm{c}}^{\mathrm{DFT}}
$$

The M06-2X functional has the 54\% of the HF exchange and introduces 32 empirically optimized parameters into the exchange-correlation functional.

\subsubsection{Hybrid QM/MM potentials}

Multiscale or hybrid QM/MM methods were firstly proposed by Warshel, Levitt and Karplus in the 1970s and their essential role in modern computational biochemistry was later recognized and awarded with the Nobel Prize. ${ }^{25-27}$ It is based on the combination of QM and MM methods allowing the inclusion of environmental effects described by MM approximations on the modelling of reactivity which is described by QM methods. The QM/MM scheme employed in the present thesis is the additive scheme, by contrast to the substrative scheme employed in, for instance, the ONIOM method of Morokuma and co-worwers. ${ }^{28}$ The hybrid effective Hamiltonian in the additive scheme is presented in eq. 3.15 .

$$
\widehat{H}_{\mathrm{eff}}=\widehat{H}_{\mathrm{QM}}+\widehat{H}_{\mathrm{QM}-\mathrm{MM}}+\widehat{H}_{\mathrm{MM}}
$$


Here, $H_{\mathrm{QM}}$ and $H_{\mathrm{MM}}$ describe the quantum and classical Hamiltonians, respectively, whereas $\widehat{H}_{\mathrm{QM}-\mathrm{MM}}$ represents the interaction between the quantum and the classical partitions.

One of the advantages of this additive scheme is that the QM region is calculated considering the environmental effects, which are described by cheap classical FFs. On the other hand, the system suffers from an abrupt change in the boundary of the QM and the MM regions where the system goes from an electronic description to a very simplified classical description where electrons are not considered.

In this scheme, the QM region is often described by either SE or DFT approaches due to the low computational cost by comparison with more expensive post-HF ab initio methods. More precisely, aforementione in this thesis, AM1 has been used as SE method for low-level calculations, and M06-2X or B3LYP functionals as high-level QM calculations.

The $\widehat{H}_{\mathrm{MM}}$ term is described by classical force fields. The OPLS-AA and TIP3P force fields have been used herein as described in section 3.1.2.

The coupling $\widehat{H}_{\mathrm{QM}-\mathrm{MM}}$ term represents the interaction energy between the QM and the MM regions and can be decomposed in the terms of eq. 3.16.

$$
\widehat{H}_{\mathrm{QM}-\mathrm{MM}}=\widehat{H}_{\mathrm{QM}-\mathrm{MM}}^{\mathrm{bond}}+\widehat{H}_{\mathrm{QM}-\mathrm{MM}}^{\mathrm{elec}}+\widehat{H}_{\mathrm{QM}-\mathrm{MM}}^{\mathrm{vdW}}
$$

In general, the electrostatic interaction $E_{\mathrm{QM}-\mathrm{MM}}^{\mathrm{elec}}$ can be treated in different schemes such as mechanical, electrostatic, and polarization embeddings. In this thesis, electrostatic embedding was used, where the point charges of the MM part are introduced into the QM Hamiltonian as external charge distribution. The electrostatic coupling is calculated as,

$$
\widehat{H}_{\mathrm{QM}-\mathrm{MM}}^{\mathrm{elec}}=-\sum_{i} \sum_{J} \frac{q_{J}}{r_{i J}}+\sum_{\alpha} \sum_{J} \frac{q_{J} Q_{\alpha}}{R_{\alpha J}}
$$




\subsection{Structure and energy}

where, $q_{J}$ are the MM point charges, and $Q_{\alpha}$ are the nuclear charges of the QM region. Here $i$ and $\alpha$ run for the electrons and nuclei of the QM part, respectively, and $J$ accounts for the MM atoms.

The van der Waals interaction is described through force field terms as in eq. 3.18,

$$
\widehat{H}_{\mathrm{QM}-\mathrm{MM}}^{\mathrm{vdW}}=\sum_{i} \sum_{J} 4 \epsilon_{i J}\left[\left(\frac{\sigma_{i J}}{r_{i J}}\right)^{12}-\left(\frac{\sigma_{i J}}{r_{i J}}\right)^{6}\right]
$$

where $i$ runs for the MM atoms and $J$ stands for the QM atoms. The bonded coupling term $\widehat{H}_{\mathrm{QM}-\mathrm{MM}}^{\mathrm{bond}}$ is calculated as the same time through force field parameters as described in eq. 3.19.

$$
\begin{aligned}
\widehat{H}_{\mathrm{QM}-\mathrm{MM}}^{\mathrm{bond}}= & \sum_{\text {bonds }} K_{r}\left(r_{I J}-r_{e q}\right)^{2} \\
& +\sum_{\text {angles }} K_{\theta}\left(\theta_{I J K}-\theta_{e q}\right)^{2} \\
& +\sum_{\text {torsions }} \sum_{n} \frac{V_{I J K L}^{n}}{2}\left[1+\cos \left(n \phi_{I J K L}-\gamma\right)\right]
\end{aligned}
$$

Note that here the torsion term is described as general Fourier series. In this case at least one of the atoms $I, J, K$, and $L$ belongs to the QM region. Nevertheless, this term is calculated classically, so it is often included into the $\widehat{H}_{\mathrm{MM}}$ term of the effective Hamiltonian.

The total energy of the system is determined by the sum of the contributions as showed in eq. 3.20. The expectation value of the effective Hamiltonian over the wave function gives the energy of the hybrid QM/MM system, as shown in eq. 3.21, whose ground state is determined by its lowest eigenvalue.

$$
E=E_{\mathrm{QM}}+E_{\mathrm{QM}-\mathrm{MM}}+E_{\mathrm{MM}}
$$




$$
E=\frac{\left\langle\Psi\left|\widehat{H}_{\mathrm{eff}}\right| \Psi\right\rangle}{\langle\Psi \mid \Psi\rangle}
$$

In MO-based approaches, the total energy of the system can be determined as presented in eq. 3.22.

$$
E=\frac{\left\langle\Psi\left|\widehat{H}_{\mathrm{QM}}+\widehat{H}_{\mathrm{QM} / \mathrm{MM}}^{\mathrm{elec}}\right| \Psi\right\rangle}{\langle\Psi \mid \Psi\rangle}+E_{\mathrm{QM}-\mathrm{MM}}^{\mathrm{vdW}}+E_{\mathrm{QM}-\mathrm{MM}}^{\mathrm{bond}}+E_{\mathrm{MM}}
$$

In order to saturate the valence of the QM region where a bond is truncated, a link atom, hydrogen in this case, was included in the QM part. ${ }^{27,29}$ Although the use of link atoms can have some effects on the overpolarization of the QM region, they are widely used and the effects tend to be small if a small atom-centered basis set is used, as in the case of semiempirical methods with a minimal basis set. ${ }^{30}$

Finally, to speed up the calculations without losing precision, a series of cut-offs were applied to the non-bonding interactions. More precisely, a switch function was used with an internal cut-off of $14.5 \AA$ and an external of $16.0 \AA$. The advantage of using this type of function is that it vanishes smoothly, avoiding abrupt disruptions in the non-bonding calculations between pairs of atoms. 


\subsection{Exploring reactivity}

\subsubsection{Potential Energy Surfaces}

The Potential Energy Surface (PES) arises from the Born-Oppenheimer approximation, ${ }^{31}$ and is a representation of the energetics of the system with respect to the coordinates of all the nuclei. A deep analysis of the PES allows the energetic description of molecular phenomena.

In the PES, stationary points are defined as those where the gradient, or first derivative of the potential energy with respect to the position of the nuclei, eq. 3.23 , is equal to zero, $\vec{g}=0$.. From the chemical point of view, two interesting types of stationary points can be located on the PES, the minima, and the first-order saddle points. The nature of the stationary points is analyzed by computing the matrix of second energy derivatives with respect to the nuclei, Hessian matrix, which is calculated as in eq. 3.24 .

$$
\begin{gathered}
\vec{g}=\left(\begin{array}{c}
\frac{\partial V}{\partial x_{1}} \\
\frac{\partial V}{\partial y_{1}} \\
\vdots \\
\frac{\partial V}{\partial z_{N}}
\end{array}\right) \\
\mathrm{H}=\left(\begin{array}{cccc}
\frac{\partial^{2} V}{\partial x_{1}^{2}} & \frac{\partial^{2} V}{\partial x_{1} \partial y_{1}} & \cdots & \frac{\partial^{2} V}{\partial x_{1} \partial z_{N}} \\
\frac{\partial^{2} V}{\partial y_{1} \partial x_{1}} & \frac{\partial^{2} V}{\partial y_{1}^{2}} & \cdots & \frac{\partial^{2} V}{\partial y_{1} \partial z_{N}} \\
\vdots & \vdots & \ddots & \vdots \\
\frac{\partial^{2} V}{\partial z_{N} \partial x_{1}} & \frac{\partial^{2} V}{\partial z_{N} \partial y_{1}} & \cdots & \frac{\partial^{2} V}{\partial z_{N}^{2}}
\end{array}\right)
\end{gathered}
$$


The diagonalization of the Hessian gives information about the nature of the stationary point. Thus, a minimum is defined when all the eigenvalues of the Hessian are positive values. This is the case of reactants $(\mathrm{R})$, possible intermediates $(\mathrm{I})$, and products $(\mathrm{P})$. On the other hand, first-order saddle points are defined when all the eigenvalues of the Hessian are positive except one that has a negative value. This means that this point is a minimum in all directions except one in which is a maximum. This is the case of TSs that will exhibit an imaginary frequency. The corresponding normal mode of vibration corresponds to the motion of the atoms along the reaction coordinate.

In this thesis, the exploration of the PESs was carried out by choosing the appropriate combination of internal coordinates $\left(\xi_{i}\right)$ in every single step of the reaction. A harmonic constraint was used to maintain the proper interatomic distances along the reaction coordinate, and a series of conjugate gradient optimizations ${ }^{32}$ and L-BFGS-B ${ }^{33}$ optimization algorithms were applied to obtain the final potential energy of the minimized constrained geometry. For the exploration of the PES, the QM sub-set of atoms was described by the AM1 semiempirical Hamiltonian.

After the exploration of stationary points on the PES, the structures corresponding to R, P, Is, and TSs were localized applying Baker's algorithm. ${ }^{34}$ It is a quasi-Newton-like algorithm that uses the first and second-order derivatives to follow the eigenvectors of the Hessian to refine the initial guess iteratively until convergency.

Minimum Energy Path (MEP), referred to the curved line along the reaction coordinate that connects $\mathrm{R}$ to $\mathrm{P}$ through the TS, was traced down following the Intrinsic Reaction Coordinate ${ }^{35}$ (IRC) method from every localized TS structure. The IRC is a mass-weighted steepest descent path on the PES from the first-order saddle point, i.e., the TS. ${ }^{36}$ It is the solution of the differential equation, 


\subsection{Exploring reactivity}

$$
\frac{d q(s)}{d s}=v(s)
$$

where, $q$ is the mass-weighted cartesian coordinates, $s$ is the coordinate along the IRC, and $v$ is the tangent vector which corresponds to the unit vector parallel to the mass-weighted gradient $\vec{g}$, eq. 3.26.

$$
v= \begin{cases}\frac{-\vec{g}}{|\vec{g}|} & \text { for } s>0 \\ \frac{\vec{g}}{|\vec{g}|} & \text { for } s<0\end{cases}
$$

\subsubsection{Free Energy Surfaces}

\subsubsection{Umbrella Sampling}

Estimating free energy barriers is of key importance for the understanding of enzyme reactivity because it is the magnitude that will allow predicting values of rate constants directly comparable with the experimental kinetics measurements, as described in section 3.6, by applying the Transition State Theory (TST), which is described in section 3.4. The description of the Free Energy Surface (FES) dictates the final estimation of the activation energies and limits the technique in terms of computational cost.

In this thesis, FESs were most of the times obtained in terms of Potential Mean Force (PMF), ${ }^{37}$ for every step of the reaction using the Umbrella Sampling (US) approach ${ }^{37,38}$ combined with the Weighted Histogram Analysis Method (WHAM). ${ }^{39}$

The PMF along a reaction coordinate is defined from the average distribution function $\langle\rho(\xi)\rangle$ as depicted in eq. 3.27, 


$$
W(\xi)=W\left(\xi^{*}\right)-k_{\mathrm{B}} T \ln \left[\frac{\langle\rho(\xi)\rangle}{\langle\rho(\xi *)\rangle}\right]
$$

where $\xi^{*}$ and $W\left(\xi^{*}\right)$ are arbitrary constants and $k_{\mathrm{B}}$ is the Boltzmann constant. At the same time, the average distribution function along the reaction coordinate is obtained from eq. 3.28.

$$
\langle\rho(\xi)\rangle=\frac{\int d R \delta\left(\xi^{\prime}[R]-\xi\right) e^{-U(R) / k_{\mathrm{B}} T}}{\int d R e^{-U(R) / k_{B} T}}
$$

Here, $U(R)$ is the total energy of the system as a function of the coordinates $R$, and $\xi^{\prime}[R]$ represents a function depending on several degrees of freedom of the dynamical system.

The procedure for the PMF calculation is straightforward and requires a series of molecular dynamics simulations in which the distinguished reaction coordinate variable, $\xi$, is constrained around particular values. In the US approach, the system is simulated introducing a biasing window potential. This biasing potential helps to enhance the sampling around particular values of the $\xi$. To obtain the PMF for the whole range of $\xi$ it is necessary to perform the simulations along the $\xi$ to sample region of interest. Finally, the values of the variables sampled during the simulations are pieced together to construct the distribution function from which the $\mathrm{PMF}$ is obtained as a function of the distinguished reaction coordinate $(W(\xi))$. This is done using the WHAM approach which allows the reconstruction of the average distribution function from the biased distribution,

$$
\langle\rho(\xi)\rangle=\frac{\sum_{i=1}^{N} n_{i}\langle\rho(\xi)\rangle_{i}}{\sum_{j=1}^{N} n_{j} e^{\left[-w(\xi)-F_{j}\right] / k_{\mathrm{B}} T}}
$$

where $\langle\rho(\xi)\rangle_{i}$ is the biased distribution function, $n$ represents the number of windows used in the estimations of averaged distribution, $w(\xi)$ is the 


\subsection{Exploring reactivity}

biasing potential and $F_{j}$ are the free energy constants which are determined by eq. 3.30 .

$$
e^{-F_{i} / k_{B} T}=\int d \xi e^{-w(\xi) / k_{\mathrm{B}} T}\langle\rho(\xi)\rangle
$$

The distribution function depends on the free energy constants $F_{i}$, so it must be solved self-consistently.

In the end, the activation free energy can be then expressed as,

$$
\Delta G^{\ddagger}(\xi)=W\left(\xi^{\ddagger}\right)-\left[W\left(\xi^{\mathrm{R}}\right)+G_{\xi}\left(\xi^{\mathrm{R}}\right)\right]
$$

where the superscripts indicate the value of the reaction coordinate at the $\mathrm{R}$, and at the TS $(\ddagger)$, and $G_{\xi}\left(\xi^{\mathrm{R}}\right)$ is the free energy associated with setting the reaction coordinate to a specific value at the reactant state. Normally this last term makes a small contribution, and the activation free energy is directly estimated from the PMF change between the maximum of the profile and the reactant's minimum, by means of eq. 3.32.

$$
\Delta G^{\ddagger}(\xi) \approx W\left(\xi^{\ddagger}\right)-W\left(\xi^{R}\right)=\Delta W^{\ddagger}(\xi)
$$

The selection of the reaction coordinate is usually trivial when the mechanism can be driven by a single internal coordinate or a simple combination (as the antisymmetric combination of two interatomic distances). However, this is not the case when more coordinates are participating. Instead, two coordinates: $\xi_{1}$ and $\xi_{2}$ are needed to describe the two-dimensional (2D) PMF, which is related to the probability of finding the system at particular values of these two distinguished coordinates and can be expressed in terms of eq. 3.33.

$$
W(\xi)=W\left(\xi^{*}\right)-k_{\mathrm{B}} T \ln \int \rho\left(r^{N}\right) \delta\left(\xi_{1}\left(r^{N}\right)-\xi_{1}\right) \delta\left(\xi_{2}\left(r^{N}\right)-\xi_{2}\right) d r^{N-2}
$$


To estimate the activation free energy from this quantity, we recovered one-dimensional PMF changes tracing a maximum probability reaction path on the 2D-PMF surface and integrating over the perpendicular coordinate.

In this thesis, the procedure to obtain the PMFs was the following, despite the nature of the chemical step (i.e., hydride or proton transfer reactions). A series of MD simulations were performed adding a constraint for the selected reaction coordinates with an umbrella force constant of 2500 $\mathrm{kJ} \cdot \mathrm{mol}^{-1} \cdot \AA^{-2}$. In every window, QM/MM MD simulations, with the semiempirical AM1 Hamiltonian, were performed with a total of 5 ps of equilibration and $20 \mathrm{ps}$ of production at the given temperature using the Langevin-Verlet algorithm ${ }^{40}$ with a time step of 1 fs. Structures obtained in previously computed PESs were used as starting points for the MD simulations in every window. Finally, the FES was obtained using the WHAM approach, as explained above, using the data collected during the biased MD simulations.

\subsubsection{Spline corrections}

In order to improve the lower quality results associated with the low-level semiempirical calculations, high-level corrections were applied using DFT. This approach, initially proposed by Truhlar and co-workers for reactions in solution ${ }^{41}$ and later applied to biological systems, ${ }^{42,43}$ is based on a spline correction term, $S\left[\Delta E_{\mathrm{LL}}^{\mathrm{HL}}\left(\xi_{1}, \xi_{2}\right)\right]$, which is interpolated to any value along the reaction coordinates in the FES. A continuous energy function is used to obtain the corrected PMFs,

$$
E\left(\xi_{1}, \xi_{2}\right)=E_{\mathrm{LL} / \mathrm{MM}}+S\left[\Delta E_{\mathrm{LL}}^{\mathrm{HL}}\left(\xi_{1}, \xi_{2}\right)\right]
$$

where $S$ is the two-dimensional spline function and $\Delta E_{\mathrm{LL}}^{\mathrm{HL}}$ is the difference between the energies obtained at low-level (LL) and high-level (HL) of the theory of the QM part. The AM1 semiempirical Hamiltonian was used as 


\subsection{Exploring reactivity}

the LL method, while the DFT method was selected for the HL energy calculation. In particular, HL energy calculations were performed by means of the hybrid M06-2X or B3LYP functionals using the standard 6$31+\mathrm{G}(\mathrm{d}, \mathrm{p})$ basis set.

\subsubsection{Free Energy Perturbation}

Free Energy Perturbation ${ }^{44,45}$ (FEP) is a method used to obtain free energy differences between two states, in this case between species along the reaction path. Two major applications of FEP methods have been explored in this thesis. Firstly, FEP was used to obtain the free energy landscape of a chemical reaction. Secondly, it was used to estimate the binding free energy of the substrates in the Michaelis complex. A brief description of FEP methods to study chemical reactivity is provided below, and an explanation on FEP approaches for estimating binding energies is provided in section 3.3.

To obtain the free energy landscape of a chemical reaction, FEP requires the sampling of the environment over a previously traced IRC down to $\mathrm{R}$ and $\mathrm{P}$ from the TS. In this thesis, when using the FEP method, the QM region was treated at the DFT level with the B3LYP functional with the 6$31+\mathrm{G}(\mathrm{d}, \mathrm{p})$ basis set. The MM region was treated with the OPLS-AA force field and the waters with the TIP3P model. The fact that the sampling is obtained over a set of structures along the IRC, implies that the free energy profile is obtained over a realistic reaction coordinate. In this case, the MM charges of the environment polarize the wave function of the QM part and allow the exploration of the reaction path at a high level of theory.

The structures used in FEP calculations were extracted from the traced IRC, and are characterized by a single $s$ coordinate,

$$
s=s_{i-1}+\sqrt{\sum_{j \in \mathrm{QM}} m_{i}\left(\left(x_{j, i}-x_{j, i-1}\right)^{2}-\left(y_{j, i}-y_{j, i-1}\right)^{2}-\left(z_{j, i}-z_{j, i-1}\right)^{2}\right)}
$$


where $x_{j, i}, y_{j, i}$ and $z_{j, i}$ are the coordinates of the $i^{\text {th }}$ structure and $j^{\text {th }} \mathrm{QM}$ atom of the structure, and $m_{i}$ are the corresponding masses of the QM atoms.

So, the free energy relative to reactants can be expressed as a function of $s$ as presented in eq. 3.36,

$$
\begin{aligned}
\Delta G_{\mathrm{FEP}}\left(s^{\mathrm{R}}-s^{j}\right)= & \left(E_{\mathrm{QM}}^{0}\left(s^{j}\right)-E_{\mathrm{QM}}^{0}\left(s^{\mathrm{R}}\right)\right)+\left(Z P E\left(s^{j}\right)-Z P E\left(s^{\mathrm{R}}\right)\right) \\
& -k_{\mathrm{B}} T \sum_{i=R}^{j} \ln \left(\left.\exp \left(-\frac{E_{\mathrm{QM} / \mathrm{MM}}\left(s^{i+1}\right)-E_{\mathrm{QM} / \mathrm{MM}}\left(s^{i}\right)}{k_{\mathrm{B}} T}\right)\right|_{\mathrm{MM}, i}\right.
\end{aligned}
$$

where $E_{\mathrm{QM}}^{0}$ is the gas-phase energy of the QM sub-set computed at DFT level, ZPE is the zero-point energy, $k_{\mathrm{B}}$ is the Boltzmann constant and $T$ is the temperature. QM/MM contribution to the free energy between two values of $s$ is calculated by averaging the QM/MM interaction energy over all the MM atoms obtained during the MD simulation.

In the particular case where FEP methods were used in this thesis, 20 ps of QM/MM MD simulation were done for each window along the IRC path at the given temperate (see Table 1) using the NVT ensemble, maintaining the position of atoms from the QM subset fixed during the simulation.

\subsubsection{Transition State Theory}

The free energy barriers obtained using the approaches used in this thesis can be directly compared with the experimental kinetic parameters in the framework of the TST. ${ }^{46}$

TST, proposed by Eyring, Evans, and Polanyi in the 1930s, ${ }^{47,48}$ is based on the following assumptions: ${ }^{49}$

1. When passing from an initial to a final state over the PES, the reacting system must cross a region called the transition state, whose potential energy is the highest on the path. 


\subsection{Exploring reactivity}

2. The chemical species in the TS are in equilibrium with the reactant state.

3. The rate of the reaction is equal to the product of the concentration of the TS species which are formed from the reactant state, and the frequency with which this species passes on to the product state.

In the end, the Eyring equation (eq. 3.37) derived from the TST allows the estimation of the kinetic constants when the free activation energy of the reaction, $\Delta G^{\ddagger}$, is known.

$$
k(\xi, T)=\frac{k_{\mathrm{B}} T}{h} e^{\left(\frac{-\Delta G^{\ddagger}(\xi, T)}{R T}\right)}
$$

In eq. $3.27, h$ stands for the Planck's constant, $k_{\mathrm{B}}$ is the Boltzmann constant, $R$ is the universal gas constant and $T$ is the temperature. In this regard, the direct comparison of the activation free energy barriers, obtained from the analysis of the FES, with the experimental $k_{\text {cat }}$ was done in this thesis in the framework of the TST.

\subsubsection{Kinetic Isotope Effects.}

Averaged Kinetic Isotope Effects (KIE) were calculated from the isotopic substitution of key atoms from localized R and TS structures. From the definition of the free energy of a state and using the TST, the ratio between the rate constants corresponding to the heavy and the light atoms can be computed by means of eq. 3.38 .

$$
K I E=\frac{\left(\frac{Q_{\mathrm{TS}}}{Q_{\mathrm{R}}}\right)_{\mathrm{L}}}{\left(\frac{Q_{\mathrm{TS}}}{Q_{\mathrm{R}}}\right)_{\mathrm{H}}} e^{(-1 /(R T))\left(\Delta Z P E_{\mathrm{L}}-\Delta Z P E_{\mathrm{H}}\right)}
$$


Here, $Q$ refers to the total partition function which was computed as the product of the translational, rotational, and vibrational partition functions of isotopologs in $\mathrm{R}$ and TS structures, and $\triangle Z P E$ refers to the difference of the zero-point energy between $\mathrm{R}$ and TS in the light $(\mathrm{L})$ and heavy $(\mathrm{H})$ isotopologs. ${ }^{50,51} \mathrm{KIEs}$ were computed at both M06-2X/MM with the standard $6-31+\mathrm{G}(\mathrm{d}, \mathrm{p})$ basis set and AM1/MM levels of theory. Structures representing each of the TS and the $\mathrm{R}$ were extracted from a QM/MM MD simulation constraining the key distances to maintain their characteristic geometries. These structures were later refined using Baker's algorithm. The Hessian was computed for the QM subset of atoms. 


\subsection{Exploring protein-ligand binding step}

To evaluate the interaction energy between the substrates and the protein in the Michaelis complex, alchemical FEP method can be applied. ${ }^{52-54}$ In this approach the free energy difference will be computed between the Michaelis complex and the substrate in solution. To do that, a series of QM/MM MD were carried out in the enzyme-substrate complex in the reactants state, and in the substrate solvated in a box of water molecules. Only the substrates were treated at QM level using the AM1 semiempirical Hamiltonian. The energy of the system was computed introducing two parameters, $\lambda$ and $\gamma$, in the electrostatic and van der Waals interaction terms respectively, as explained in ref. 53 and showed in eq. 3.39.

$$
\begin{gathered}
E(\lambda)=\left\langle\Psi\left|\widehat{H}_{0}\right| \Psi\right\rangle+\lambda\left(\sum_{J}\left\langle\Psi\left|\frac{q_{\mathrm{J}}}{r_{i J}}\right| \Psi\right\rangle+\sum_{\alpha} \sum_{J} \frac{q_{J} Q_{\alpha}}{R_{\alpha J}}\right) \\
+\gamma E_{\mathrm{QM} / \mathrm{MM}}^{\mathrm{vdW}}+E_{\mathrm{QM} / \mathrm{MM}}^{\mathrm{bond}}+E_{\mathrm{MM}}
\end{gathered}
$$

Then, changes were smoothly introduced in $\lambda$ and $\gamma$ parameters from 1.0 to 0.0 to annihilate the electrostatic and van der Waals interaction contributions. The calculation of the free energy difference between two consecutive steps is obtained, and then the total free energy change is computed as the sum of all the contributions between the steps covering the whole transformation from the initial to the final states.

In the case explored in this thesis the binding free energy between 2butneal and CALB was computed (Section 4.1.2). To do this, a total of 100 windows were done to evaluate the electrostatic and van der Waals interaction terms, with a step for $\lambda$ and $\gamma$ of 0.01 (from 1.0 to 0.0 ). In each window, a total of $20 \mathrm{ps}$ of AM1/MM MD at the given temperature (see Table 1) were performed using the NVT ensemble. Finally, the binding 
3.3. Exploring protein-ligand binding step

free energy can be computed as the difference between $\Delta G_{\mathrm{W}}$ in the water solvent and $\Delta G_{\mathrm{E}}$ in the Michaelis complex, as shown in eq. 3.40.

$$
\Delta G=\Delta G_{\mathrm{W}}-\Delta G_{\mathrm{E}}
$$




\subsection{Convolutional Neural Networks}

\subsubsection{A brief introduction to Gonvolutional Neural Networks}

In the ML paradigm, GNNs have been proved to be very useful in classification tasks in a wide range of fields, such as image, speech, and movement recognition, together with text analysis. ${ }^{55}$ Although there are expanding applications in science, little research has been done in the analysis of protein structure. ${ }^{56-59}$

CNNs emerged from the study of the brain's visual cortex, and although they gained popularity in recent years, due to the increase in computational power, they have been used in image recognition tasks since the 1980s.

The building blocks of the CNNs are the convolutional layers. A convolution is a mathematical operation that slides one function over another and evaluates the integral of the pointwise product. A CNN takes a set of values, for example the pixel values of an image, and applies convolution operations in every layer of the network. The networks are usually formed by the connection of multiple layers where the output of the preceding is the input of the subsequent layer. One of the main advantages of the CNN architecture is that each neuron of one layer is not connected to all the neurons of the preceding but only to those in its perceptive field. This type of architecture allows the network to concentrate on small lowlevel features, in the first hidden layer and assemble them into larger higher-level features in the next hidden layer.

A series of filters are applied in each hidden layer. A filter is simply a mathematical operation that is applied to the perceptive field that results in activation of the neuron. The application of the same filter within a layer 
outputs the feature map, which highlights the areas of the input that activate the filter the most.

The activation of the neurons is dictated by an activation function. It defines the output of the neuron given a set of inputs. There are several types of activation functions. One of the most widely used is the Rectified Linear Unit function (ReLU), which is used in this thesis. The ReLU can be defined as,

$$
\operatorname{ReLU}(z)=\max (0, z)
$$

where $z$ is the input of the neuron. The main advantage is that it is computationally cheap, but it is not differentiable at $z=0$, and its derivative is 0 for $z<0$ which is inconvenient when using Gradient Descent (GD) methods.

In the end, the network is composed of a series of layers with stacked feature maps, which detect the interesting features of the given input. As a summary, each neuron computes the weighted sum of all inputs plus the bias term.

In this thesis, 3D convolutional operations were applied, due to the threedimensional nature of protein geometry. The mathematics behind each neuron can be described in terms of eq. 3.42.

$$
\begin{aligned}
& z_{i, j, k, m}=b_{m}+\sum_{u=0}^{f_{\mathrm{h}}-1} \sum_{v=0}^{f_{\mathrm{W}}-1} \sum_{t=0}^{f_{\mathrm{d}}-1} \sum_{m^{\prime}=0}^{f_{n^{\prime}-1}} x_{i^{\prime}, j^{\prime}, k^{\prime}, m^{\prime}} \\
& \cdot w_{u^{\prime}, v^{\prime}, t^{\prime}, m^{\prime}, m} \quad \text { with }\left\{\begin{array}{l}
i^{\prime}=i \times s_{\mathrm{h}}+u \\
j^{\prime}=i \times s_{\mathrm{w}}+v \\
k^{\prime}=i \times s_{\mathrm{d}}+t
\end{array}\right.
\end{aligned}
$$

In this equation $z_{i, j, k, m}$ is the output of the neuron located in the position $i, j, k$ of the feature map $m$ of the convolutional layer $l ; s_{\mathrm{h}}, s_{\mathrm{w}}$ and $s_{\mathrm{d}}$ are the strides while $f_{\mathrm{h}}, f_{\mathrm{w}}$ and $f_{\mathrm{d}}$ are the dimensions of the perceptive field in 


\subsection{Convolutional Neural Networks}

each dimension (height, width, and depth), and $f_{n^{\prime}}$ is the number of feature maps in the previous layer, $x_{i^{\prime}, j^{\prime}, k^{\prime}, m^{\prime}}$ is the output located in the layer $l-$ 1 , at positions $i^{\prime}, j^{\prime}, k^{\prime}$ of the feature map $m^{\prime} ; b_{m}$ defines the bias term of the feature map $m$ and $w_{u^{\prime}, v^{\prime}, t^{\prime}, m^{\prime}, m}$ is the connection weight between any neuron of the feature map $m$ of the layer $l$ located at position $u, v, t$, relative to the perceptive field and the feature map $m^{\prime}$.

The bias term $b_{m}$ and the weights $w_{u^{\prime}, v^{\prime}, t^{\prime}, m^{\prime}, m}$ are the parameters that are optimized during the training of the CNN. The optimization of these parameters is done through a process called backpropagation. The backpropagation training algorithm was introduced in 1986 by Rumelhart, Hilton, and Williams. ${ }^{60}$ It is a GD method that computes the gradients of each parameter of the network, the biases and the weights, based on the actual error of the network. In summary, the algorithm works as follows:

1. The training inputs pass through the network in a batch, and the network predicts the outputs in the forward pass.

2. The error of the network is measured by a loss function, where the actual output is compared with the ground truth.

3. Then it computes how much each parameter contributed to the error, through the chain rule.

4. Finally, it computes the gradient of each parameter and performs the GD step where the biases and the weights are updated. The step towards the gradient is called the learning rate.

In this way, the network tries to minimize the error towards making better predictions. In modern implementations, several optimizers are implemented besides the GD. For example, in this thesis, the Adam ${ }^{61}$ optimizer, combined with the categorical cross-entropy as loss function to compute the error, was used.

Besides the convolution operations, several types of layers and operations are used within the GNN framework whose aim is to increase performance. 
Although they are not going to be explained here, a short description of the most common layers and operations used in the CNN framework are listed below.

- Pooling layer. It is used to subsample the input to reduce the computational cost.

- Dense layer. Also known as the fully connected layer, it is used to connect all the neurons between two layers. Normally used on the top of the CNN architecture just before the output layer.

- Output layer. It is the top layer of the $\mathrm{CNN}$ architecture and represents the predictions. In the case of CNNs used in classification tasks, a Softmax activation function is used to obtain the probabilities for a certain input to belong to a specific class.

- Batch normalization. Usually used to normalize the data within the batch used for training.

- Dropout. It is used to avoid the activation of certain neurons. It is useful to avoid overfitting.

\subsubsection{Datasets}

As mentioned above the neural networks must be trained to optimize the weights within the neurons. This is done by feeding the network with a set of labeled data. The network will use this dataset to learn the features and make accurate predictions after the training process. The validation of the performance of the network is important, so usually, the original dataset is divided into a training, testing, and validation dataset. The network will use the training dataset to update the weights, while the testing dataset will be used during training to monitor the real performance of the network if it is capable to generalize and avoid overfitting. Finally, the validation dataset will be used as an independent dataset to validate the performance of the GNN. In the applications of CNN carried out in the present thesis 


\subsection{Convolutional Neural Networks}

(section 4.2.1), crystallographic data of three functional families of enzymes was used as a dataset.

Crystallographic data of enzymes belonging to EC 3.1.1.1, EC 3.1.1.3, and EC 3.1.1.4 was taken from the $\mathrm{PDB}^{62}$ database. These proteins were selected based on their classification in Uniprot ${ }^{63}$ database. To avoid the overrepresentation of some structures, redundancy was cleaned based on sequence alignment between all the sets of proteins. All proteins that shared more than $85 \%$ of sequence identity were removed. The alignment was done using the Needleman-Wunch ${ }^{64}$ algorithm. A final list of the codes used to build the dataset is showed in Table 2 .

The main aim of this thesis was to study of the vicinity of the active site. To do that, the catalytic site of each protein was labeled based on the curated information available in Uniprot. Then, a cube of $20 \times 20 \times 20 \AA^{3}$ was centered on the geometrical center of the catalytic site. Placing the cube in the catalytic site allows the analysis of only the near vicinity of the active site. The cube was then divided into voxels of $1 \AA$ to ensure that only one atom lay inside each voxel (Figure 3.1a).

To properly mimic the chemistry inside the cube, the atoms that lay inside a voxel were selected and codified into a vector of 13 features. Each position of the vector represented a specific characteristic of the codified residue or atom, i.e., atom type $(\mathrm{C}, \mathrm{N}, \mathrm{O}$ or $\mathrm{S})$, atom belonging to the backbone, methyl group, negative or positive charge, polar or non-polar, hydrogen bond donor or acceptor, or aromatic residue. If the characteristic was present in the atom the corresponding position of the vector was set to 1.0, and a 0.0 otherwise. A final cube of dimension $20 \times 20 \times 20 \AA^{3}$ and 13 channels was obtained for each protein of the initial dataset. To mimic the electron density in a real protein, a series of Gaussian filters were applied in each channel. These filters were calibrated to resemble the de van der Waals radius of each atom type. 
Table 2. PDB IDs of the proteins used for the GNN training.

\begin{tabular}{ll}
\hline EC number & PDB ID \\
\hline EC 3.1.1.1 & 2O7V, 5FV4, 1K4Y, 1AUR, 3P2M, 5THM, 3KVN, 1TQH, \\
& 4JGG, 2R11, 4CCY \\
EC 3.1.1.3 & 5CUR, 3RAR, 1THG, 1GZ7, 1THG, 1LLF, 3GUU, 1K8Q, \\
& 1HLG, 1HPL, 1N8S, 1ETH, 1GPL, 2PVS, 1BU8, 4FKB, 5LIP, \\
& 2ES4, 1EX9, 6QPR, 1LGY, 6XS3 \\
& 1QD6, 4BP2, 5IZR, 1TC8, 1OZ6, 1POB, 1GP7, 1M8T, 1P7O, 3.1 .1 .4 \\
& 4RFP, 1PP2, 1IJL, 1VAP, 1BUN, 1OO1, 6AL3, 5TFV, 2NOT, \\
& 5VET, 3G8G, 4HG9, 1PO8, 1VIP, 1RGB, 2GHN, \\
\hline
\end{tabular}

One of the major drawbacks of CNN training is the huge amount of information needed in the training process. Only a few proteins of each family were chosen after removing the redundancy (Table 2). This fact limits the power of the network. But some strategies can be applied to overcome this limitation and up-sample the dataset. In this case, a series of random rotations were applied to the cubes in the $\mathrm{x}, \mathrm{y}$ and $\mathrm{z}$-axis.

Another important factor to consider is that a balanced dataset is preferred during training, i.e., a dataset with a similar number of instances in each class. Due to the different number of proteins in each of the EC classes, the dataset was balanced up-sampling each class independently. The network was trained with a ratio train:test:validation of 0.7:0.15:0.15. In this case, we are not talking about a specific number of samples in each dataset because the cubes and the random rotations were done on-the-fly while the network was trained so each mini batch was in this sense different. For this reason, the CNN was trained until the convergence of the loss. 


\subsection{Convolutional Neural Networks}

\subsubsection{GNN architecture and training}

Several GNN architectures are widely available in modern ML implementations. One of the most well-known and successful architectures in the Residual Network (ResNet). Proposed by Kaiming He et. al. ${ }^{65}$ in 2015, it rapidly increased in popularity achieving astonishing accuracies in short training runs.

It is based on what is called residual learning. When initializing a network, the weights are close to zero, so the output is also close to zero. But if a skip connection is added, the output is close to the input, so the network models the identity. This allows the network to speed up the learning process and progress rapidly. So, the ResNet architecture is composed of several residual units interconnected through skip connections. Each block is composed by two convolutional layers followed by a normalization layer. These blocks are concatenated forming the core of the network. On the top, a dense layer connects the last convolutional layer with the final output layer, which gives the predictions of the model.

Several architectures in the ResNet framework can be found but they only differ in the depth of the network, i.e., the number of residual units stacked together. In this thesis, a ResNet5065 architecture was chosen to build the CNN. This is a network with 50 hidden layers in depth. In this case, a set of $3 \mathrm{D}$ convolutional layers was applied to maintain the original architecture of the network. A final output layer, a vector with three components (one for each class), was set with a Softmax activation function, eq. 3.43. Thus, the outputs can be treated as real probabilities of belonging to each class.

$$
\sigma(s(x))_{k}=\frac{e^{s_{k}(x)}}{\sum_{i=1}^{K} e^{s_{j}(x)}}
$$

In eq. $3.43, K$ is the number of classes in the classifier, $s(x)$ is a vector containing the scores of each class, for instance, $x$, and $\sigma(s(x))_{k}$ is the 
estimated probability that the instance $x$ belongs to class $k$ given the scores of each class for that instance (Figure 3.1b).
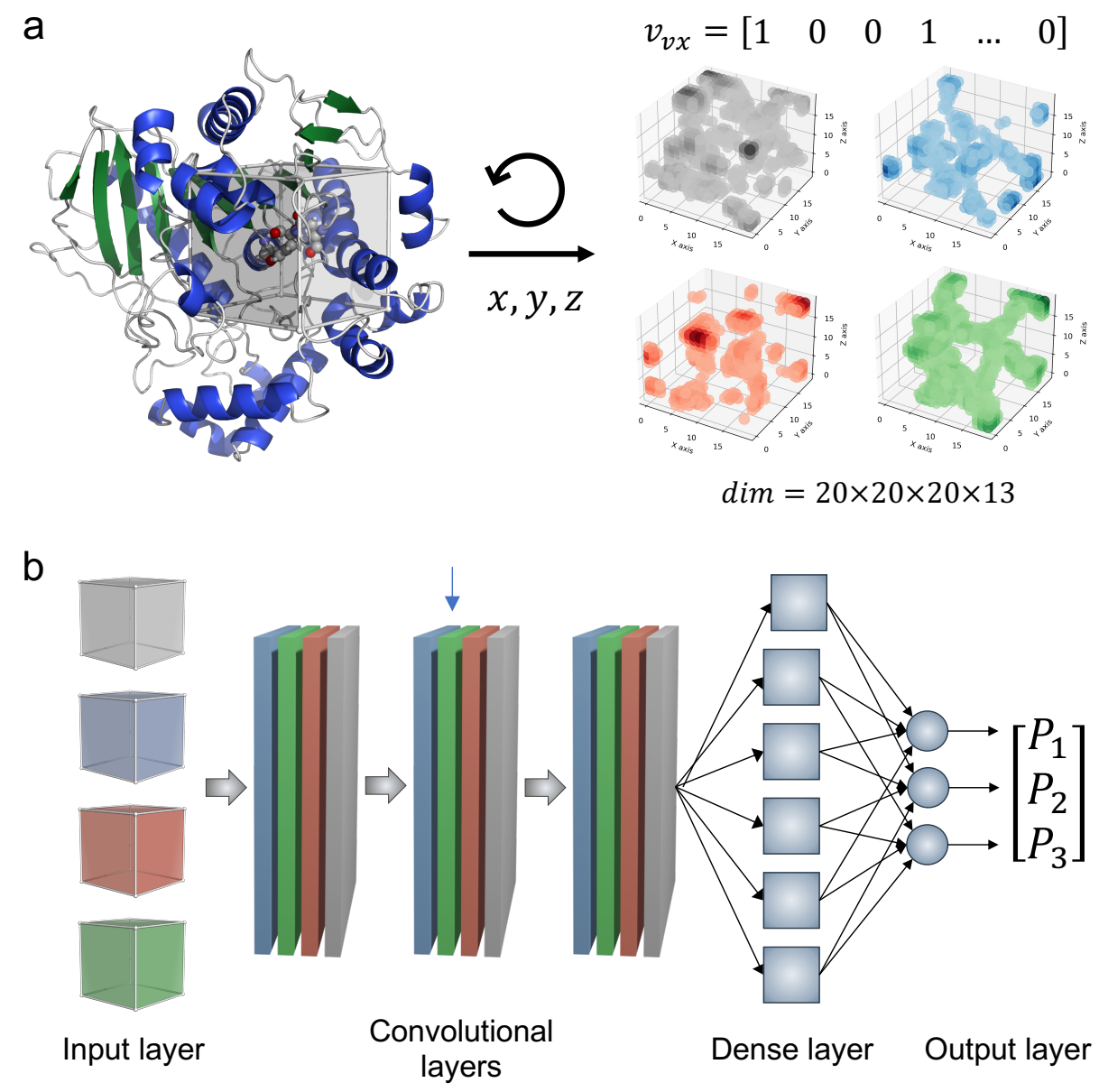

Figure 3.1. Workflow of the GNN datasets and architecture. Panel a: cube construction. The cube is centered on the triad and divided into voxels of 1 A 3 . Each cube is randomly rotated, and each voxel is defined by a vector of 13 chemical features. Panel $\mathbf{b}$ : schematic representation of the CNN architecture. The inputs, the cubes, go through a series of convolutional layers. A final dense layer and the output layer withdraw the probabilities of each class.

For the training of the GNN, the Adam ${ }^{61}$ optimizer was used and the categorical cross-entropy was chosen as a loss function, as described in eq. 


\subsection{Convolutional Neural Networks}

3.44. A mini-batch regime was set for each iteration, and an exponential decay learning rate was scheduled, which can be defined in terms of eq. 3.45 .

$$
\begin{gathered}
C E=-\frac{1}{m} \sum_{i=1}^{m} \sum_{k=1}^{K} y_{k}^{i} \log \left(p_{k}^{i}\right) \\
l r=l r_{0} \cdot\left(1-\frac{t}{100}\right)^{\text {epoch }}
\end{gathered}
$$

In eq. 3.44, $K$ is the number of classes, $m$ is the number of instances, while $y_{k}^{i}$ and $p_{k}^{i}$ are the target and actual probabilities that the instance $i$ belongs to class $k$. In eq. $3.45, l r$ is the actual learning rate, $l r_{0}$ is the initial learning rate, $t$ represents the decay rate and the epoch is the number of complete iterations done in the complete train dataset.

\subsubsection{In silico alanine scan}

In most cases it is difficult to know what the $\mathrm{CNN}$ has learned to be common among a specific class which make it capable to classify the inputs into their correct classes. To understand what the classifier has learned, an alanine scan approach was done.

Similarly as it is done experimentally, mutating the residues of the protein in the vicinity of the active site to Ala, can give an idea if the mutated residue, or the chemistry of the mutated residue, is important in the class. Once the mutation is done, if the $\mathrm{GNN}$ is not capable anymore to correctly classify the protein it means that the residue was key in the classification, hence it is structurally important. So, all the residues that had an atom inside the cube were mutated to alanine consecutively, obtaining a library of mutants. Then, 1000 predictions were done by applying random rotations. A classification score was computed by the difference between 
the classification ratio of the actual class and the ratio of the most probable of the remaining classes as described in eq. 3.46.

$$
s=\frac{c_{i 1}}{t_{i 1}}-\frac{c_{\max (i>1)}}{t_{\max (i>i)}}
$$

In the above equation $s$ stands for the score, $c_{i 1}$ is the number of correctly classified instances of the most probable class, $t_{i 1}$ is the total number of instances in the most probable class, while $c_{\max (i>1)}$ and $t_{\max (i>i)}$ represent the correctly classified instances and the total number of instances of the second most probable class. In this sense, a score of 1.0 corresponds to a perfectly classified mutant and a score of -1.0 is the one completely misclassified. Finally, after each iteration, the mutation that dropped in greater extend the score was accumulated for the next iteration (Figure 3.2).

This approach allowed highlighting which were the important residues in a given protein and helped to map the near vicinity of the active site, which can be useful to suggest mutations.

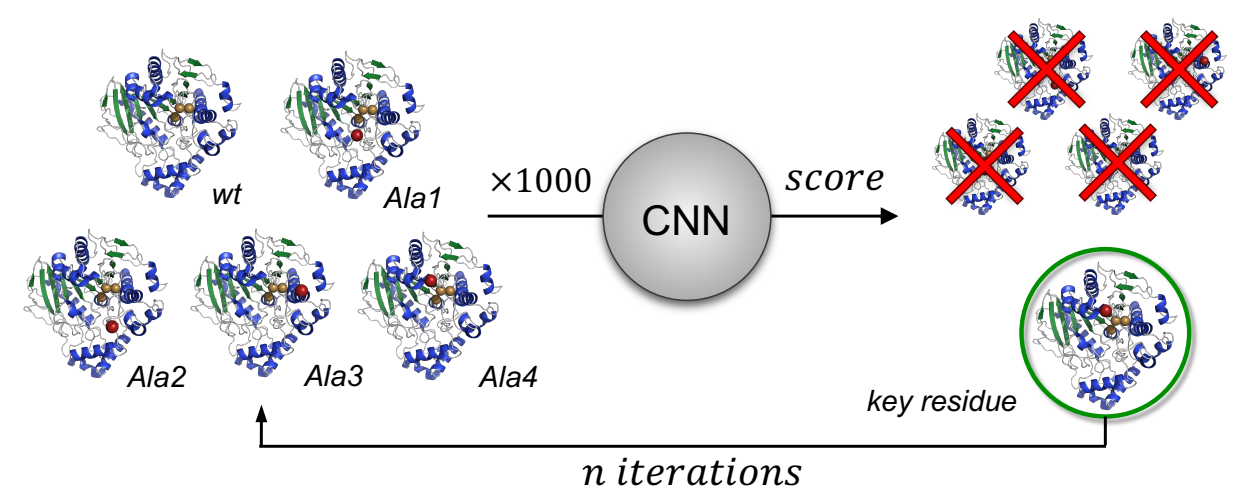

Figure 3.2. In silico alanine scan. Ala-mutants are evaluated by the trained CNN. The classification score helps to identify key residues and the mutations are accumulated through successive iterations. 


\subsection{Rational enzyme redesign methods}

\subsubsection{Rotation Quaternions}

Structural alignment of molecules, especially in the case of proteins, is in most cases limited to the ones that share the same number of atoms disposed in a similar manner. For example, the structural alignment of two proteins is often limited by the percentage of identity. Of course, a strategy is to use only the common parts, so, the regions that can be aligned are those which are common along the primary sequence. However, vast regions of the protein are needed to properly align two structures, and the errors associated with common rotation matrices can be limiting in the distal regions of the protein. Rotation Quaternions (RQ) are in this sense more efficient and numerically stable compared to simple rotation matrices. Hence, this strategy allows the comparison of the active site of both proteins, although they are structurally completely different.

RQ applied to the rotation of molecules were proposed by Kneller in $1990 .{ }^{66}$ It can be considered as an algorithm to find the optimal orientation of a rigid molecule with respect to a template structure by minimizing the weighted sum of squared deviations of the rotated structure with respect to the template. The rotation is then parameterized by a quaternion whose components are the eigenvectors of the matrix $M$ described in eq. 3.47, which is defined in terms of the coordinates that are superimposed.

$$
M=\sum_{\alpha} w_{\alpha} M_{\alpha}
$$

$M_{\alpha}$ is a symmetric square $4 \times 4$ matrix whose terms can be computed as defined below in eq. 3.48 . 
3.5. Rational enzyme redesign methods

$$
\begin{aligned}
& M_{\alpha, 1,1}=x_{\alpha}^{2}+y_{\alpha}^{2}+z_{\alpha}^{2}+x_{0 \alpha}^{2}+y_{0 \alpha}^{2}+z_{0 \alpha}^{2}-2 x_{\alpha} x_{0 \alpha}-2 y_{\alpha} y_{0 \alpha}-2 z_{\alpha} z_{0 \alpha} \\
& M_{\alpha, 1,2}=2\left(y_{\alpha} z_{0 \alpha}-z_{\alpha} y_{0 \alpha}\right) \\
& M_{\alpha, 1,3}=2\left(-x_{\alpha} z_{0 \alpha}+z_{\alpha} x_{0 \alpha}\right) \\
& M_{\alpha, 1,4}=2\left(x_{\alpha} y_{0 \alpha}-y_{\alpha} x_{0 \alpha}\right) \\
& M_{\alpha, 2,2}=x_{\alpha}^{2}+y_{\alpha}^{2}+z_{\alpha}^{2}+x_{0 \alpha}^{2}+y_{0 \alpha}^{2}+z_{0 \alpha}^{2}-2 x_{\alpha} x_{0 \alpha}+2 y_{\alpha} y_{0 \alpha}+2 z_{\alpha} z_{0 \alpha} \\
& M_{\alpha, 2,3}=-2\left(x_{\alpha} y_{0 \alpha}+y_{\alpha} x_{0 \alpha}\right) \\
& M_{\alpha, 2,4}=-2\left(x_{\alpha} z_{0 \alpha}+z_{\alpha} x_{0 \alpha}\right) \\
& M_{\alpha, 3,3}=x_{\alpha}^{2}+y_{\alpha}^{2}+z_{\alpha}^{2}+x_{0 \alpha}^{2}+y_{0 \alpha}^{2}+z_{0 \alpha}^{2}+2 x_{\alpha} x_{0 \alpha}-2 y_{\alpha} y_{0 \alpha}+2 z_{\alpha} z_{0 \alpha} \\
& M_{\alpha, 3,4}=-2\left(y_{\alpha} z_{0 \alpha}+z_{\alpha} y_{0 \alpha}\right) \\
& M_{\alpha, 4,4}=x_{\alpha}^{2}+y_{\alpha}^{2}+z_{\alpha}^{2}+x_{0 \alpha}^{2}+y_{0 \alpha}^{2}+z_{0 \alpha}^{2}+2 x_{\alpha} x_{0 \alpha}+2 y_{\alpha} y_{0 \alpha}-2 z_{\alpha} z_{0 \alpha}
\end{aligned}
$$

Here, $x_{\alpha}, y_{\alpha}$ and $z_{\alpha}$ are the coordinates of the atom $\alpha$ to be rotated, and $x_{0 \alpha}, y_{0 \alpha}$ and $z_{0 \alpha}$ are the coordinates of the atom $\alpha$ belonging to the template.

The eigenvector belonging to the smallest eigenvalue of $M$, defines the optimal rotation of the structure towards the template. The complete $M$ matrix is obtained as the weighted sum of $M_{\alpha}$ over all the sites of the alignment region. 


\subsubsection{Structural alignment of GALB and Bs2}

Bs2 and CALB, despite being mechanistically related, they are two nonhomologous enzymes, and structurally very different in terms of both primary and tertiary structure. In order to compare the vicinity of the active site of the rate-determining TS, a structural alignment between two trajectories of QM/MM MD on the TS1 of both Bs2 and CALB was done using RQs (Figure 3.3a). Due to the poor percentage of sequence identity between enzymes, a structural alignment based on sequence similarity was unachievable. For that reason, the geometrical overlapping of the positions of atoms of the amide bond and the neighboring carbon atoms was used to build the $M$ matrix shown in eq. 3.47.

The trajectories of each Bs2 and CALB protein derived from constrained QM/MM MD simulations were aligned, and structures were compared based on the position of the centers of mass of every residue of the protein. The centers of mass along the trajectory were computed and averaged to reduce the error. The alignment was then done by comparing the distance between the centers of mass of each residue of both proteins (Figure 3.3b). Residues of Bs2 and CALB were paired based on the proximity of their centers of mass, i.e., the pair of a residue from Bs2 is the residue from CALB with the closest center of mass.

This approach allows the comparison of distant related proteins with low sequence identity and gives information about the differences in the spatial domain. It is a realistic way of comparing the chemistry surrounding the active site. Although this method can be applied universally, the real utility is restrained to enzymes sharing major features, such as the catalytic residues, or key features for their activity. 


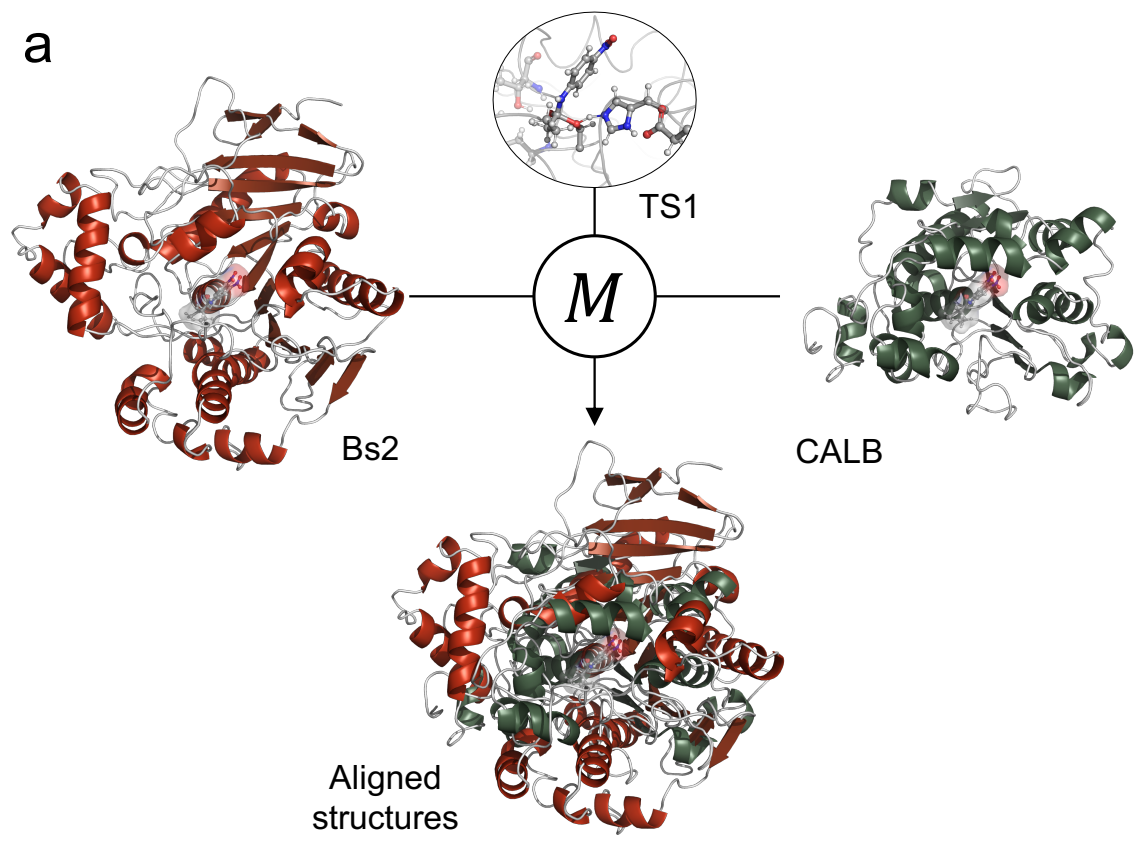

b

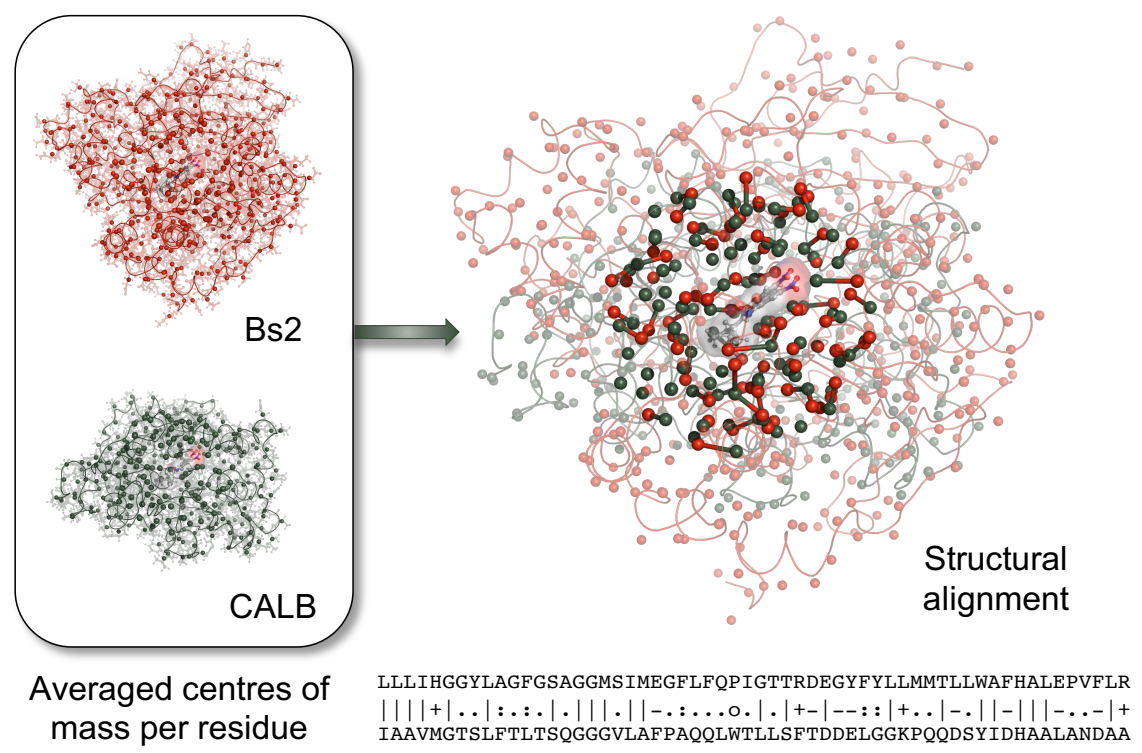

Figure 3.3. Structural alignment of Bs2 and CALB. Panel a: matrix $M$ is built using quaternions and the structural information of the TS. Panel b: structural alignment is based on the computation of the centers of mass per residue. Which are paired based on spatial criteria. 


\subsubsection{Evaluation of electrostatic potential}

The comparison of aligned structures can be done in terms of the electrostatic potential. It is believed that the electrostatics generated by the enzyme in the vicinity of the active site dictates somehow the catalysis of the reaction. ${ }^{67-75}$ In this regard, the electrostatic potential generated on key atoms can be, as previously done in our laboratory (i.e. see ref 69 or 70). Electrostatic potential per residue $\left(V_{\text {res }}\right)$ was computed based on eq. 3.49,

$$
V_{\mathrm{res}}=\sum_{i=1}^{n} \frac{q_{i}}{4 \pi \varepsilon r_{i}}
$$

where $n$ corresponds to the number of atoms in the residue, $q_{i}$ is the charge of the atom taken from the OPLS-AA force field, and $r_{i}$ is the distance between atom $i$ and the key selected atom. A switch function was applied with cut-offs of 14.5 and $16 \AA$. Finally, the electrostatic potential per residue was averaged per residue along the trajectories. 


\subsection{Experimental determination of kinetic}

parameters

Rate constants of the hydrolysis of the amide bond in CALB were carried experimentally, in a research stay in the Bio-Organic Chemistry Group leaded by Dr. Eduardo García-Junceda, at the Institute of General Organic Chemistry, which belongs to the CSIC (Consejo Superior de Investigaciones Cientificas). The determination of the hydrolysis of two substrates, benzylbutyramide and $\mathcal{N}$-(4-nitrophenyl)-butyramide, was carried out using the commercial free form of CALB. A brief description of the synthesis of both substrates and the kinetic measurements is detailed in the following sections.

\subsubsection{Synthesis of the benzylbutyramide}

CALB is also capable to synthetize the amide molecule from its respective precursors. Hence, an enzymatic approach was used for the synthesis of the benzylbutyramide. The commercial immobilized CALB (Novozyme 435) was used in this case. The use of immobilized enzymes has some advantages from the purification of the products once the reaction has finished and accepts the use of organic solvents. The substrate was synthetized from benzylamine and butyric acid. The synthesis was done using equimolar solution of $50 \mathrm{mM}$ of butyric acid and benzylamine in 2methyl-2-butanol, which was incubated during 120 hours in sealed vessels with argon atmosphere and molecular sieves to minimize the available soluble water that is formed in the reaction. The use of molecular sieves serves at this point to avoid the hydrolysis of the synthetized amide. The reaction was followed by Thin-Layer Chromatography (TLC), with a mobile phase of hexane:ethyl acetate (2:3), until complete conversion. Then, the product was purified by a Silica column with hexane:ethyl 
acetate (1:5) as mobile phase. Finally, the solvent was evaporated in a rotatory evaporator, obtaining pure product, which was characterized by mass spectrometry $(\mathrm{m} / \mathrm{z}=178.2)$ and Nuclear Magnetic Resonance (NMR).

\subsubsection{Synthesis of the $N$-(4-nitrophenyl)-butyramide}

The synthesis of $\mathcal{N}$-(4-nitrophenyl)-butyramide was done using a different approach. Chemical synthesis was preferred in this case, due to the availability of the chemical precursors. So, butyryl chloride was dissolved in $5 \mathrm{~mL}$ anhydrous dichloromethane $(4.32 \mathrm{mmols})$ and was added to 10 $\mathrm{mL}$ of a solution of 4-nitroaniline $(3.60 \mathrm{mmols})$. Pyridine $(7.20 \mathrm{mmols})$ in anhydrous dichloromethane was also added to act as a base to catalyze the reaction. The molar ratio of butyryl chloride:4-nitroaniline:pyridine was set to 1.2:1:2. The reaction was carried out in sealed vessels with argon atmosphere. The reaction was let at room temperature over-night and followed by TLC. Once completed, further washes with $10 \% \mathrm{HCl}$ were done. Finally, $\mathcal{N}$-(4-nitrophenyl)-butyramide was purified, as in the previous case, by a Silica column with hexane:ethyl acetate (4:1) as mobile phase. The solvent was evaporated in a rotatory evaporator obtaining yellow crystals. The final product was characterized by NMR.

\subsubsection{Kinetic assays of the hydrolysis of benzylbutyramide}

Experimental kinetics parameters of hydrolysis of benzylbutyramide were obtained in order to be compared with the computational studies. For this, the commercial free form of wild type CALB was purchased from SigmaAldrich. The use of the free form of CALB was preferred over the Novozyme 435 because the computational studies were done with the free form of the enzyme in solution. The kinetic assays were carried out in 20 
$\mathrm{mM}$ phosphate buffer at $\mathrm{pH} 7$, with $10 \%$ of acetonitrile. The poor solubility of benzylbutyramide in aqueous solution makes necessary the addition of a certain concentration of organic solvent. Different organic solvents were discarded due to interferences with the signal during HighPerformance Liquid Chromatography (HPLC) acquisitions, which was used to follow the reaction. Real concentration of enzyme in the commercial powder was determined by Bradford assay. The final concentration of enzyme in the assay was adjusted to $105 \mu \mathrm{g} \mathrm{mL} \mathrm{m}^{-1}$ and concentrations of benzylbutyramide were set to $0 \mathrm{mM}, 0.2 \mathrm{mM}, 0.5 \mathrm{mM}$, $1 \mathrm{mM}, 5 \mathrm{mM}$ in a final volume of $500 \mu \mathrm{L}$. Finally, reactions were done at $37^{\circ} \mathrm{C}$ and $100 \mathrm{rpm}$. Aliquots of $50 \mu \mathrm{L}$ were taken at $0 \mathrm{~h}, 3 \mathrm{~h}, 5 \mathrm{~h}, 7 \mathrm{~h}, 24 \mathrm{~h}$ and $48 \mathrm{~h}$, and were frozen at $-20^{\circ} \mathrm{C}$ to stop the progression of the reaction. Formation of the benzylamine was monitored by HPLG (JASCO UK Limited). A diluted sample of each aliquot was injected into a C18 column and a mixture of water:acetonitrile 50:50 with $0.5 \%$ of trifluoroacetic acid was used as a mobile phase with a $1 \mathrm{~mL} \mathrm{~min}^{-1}$ flow. The elution of products (benzylamine) was followed by absorbance at $206 \mathrm{~nm}$ (retention time of benzylamine $=3.1 \mathrm{~min}$; retention time of benzylbutyramide $=4.5 \mathrm{~min}$ ).

\subsubsection{Kinetic assays of the hydrolysis of $N$-(4- nitrophenyl)-butyramide}

The hydrolysis of $\mathcal{N}$-(4-nitrophenyl)-butyramide by CALB was done in a similar way as explained in section 3.6.3. The commercial free form CALB (Sigma-Aldrich) was also used. As explained before the poor solubility of the substrate requires the addition of organic solvent, Dimethyl Sulfoxide (DMSO) in this case. Reactions were done in $20 \mathrm{mM}$ phosphate buffer at $\mathrm{pH} 7$, with $10 \%$ of DMSO. The assay concentrations of $\mathcal{N}$-(4-nitrophenyl)butyramide were set to $0 \mathrm{mM}, 0.5 \mathrm{mM}, 1.0 \mathrm{mM}, 1.5 \mathrm{mM}, 2.0 \mathrm{mM}, 2.5$ $\mathrm{mM}$ and $3 \mathrm{mM}$. The final concentration of protein was set to $35 \mu \mathrm{g} \mathrm{mL}^{-1}$. The reactions were carried out in a final volume of $150 \mu \mathrm{L}$ in microtiter 
plates at $37{ }^{\circ} \mathrm{C}$ and $100 \mathrm{rpm}$. The progression of the reaction was monitored during 24 hours by the increment of absorbance at $405 \mathrm{~nm}$ using a spectrophotometer (Spectra max Plus 384 from Molecular Devices). The use of this spectrophotometric technique allows the use of DMSO instead of acetonitrile, which was used in the case of benzylbutyramide. Since similar peaks of absorbance of substrates and products are obtained in the case of benzylbutyramide (maximum absorbance peak of benzylbutyramide, substrate: $250 \mathrm{~nm}$; and benzylamine, product: $245 \mathrm{~nm}$ ) spectrophotometric technics can not be used.

The kinetic acquisitions were done in triplicates. Finally, the kinetic parameters were obtained by means of Michalis-Menten model, to get the $k_{\text {cat }}$ and the $K_{M}$, magnitudes that can be directly compared with the computational predictions within the framework of the TST, as explained in section 3.2.3. The model was obtained using the GraphPad Prism v.9.1.0 for MacOS (www.graphpad.com). 


\subsection{References}

(1) Uppenberg, J.; Morgens, H.; Shamkant, P.; Alwyn, J. T. The Sequence, Crystal Structure Determination and Refinement of Two Crystal Forms of Lipase B from Candida Antarctica. Structure 1994, 2, 293-308.

(2) Spiller, B.; Gershenson, A.; Arnold, F. H.; Stevens, R. C. A Structural View of Evolutionary Divergence. Proc. Natl. Acad. Sci. 1999, 96, 12305-12310.

(3) Olsson, M. H. M.; SØndergaard, C. R.; Rostkowski, M.; Jensen, J. H. PROPKA3: Consistent Treatment of Internal and Surface Residues in Empirical p Kapredictions. F. Chem. Theory Comput. 2011, 7, 525-537.

(4) Søndergaard, C. R.; Olsson, M. H. M.; Rostkowski, M.; Jensen, J. H. Improved Treatment of Ligands and Coupling Effects in Empirical Calculation and Rationalization of PKa Values. F Chem Theory Comput 2011, 7, 2284-2295.

(5) Jorgensen, W. L.; Chandrasekhar, J.; Madura, J. D.; Impey, R. W.; Klein, M. L. Comparison of Simple Potential Functions for Simulating Liquid Water. F. Chem. Phys. 1983, 79, 926-935.

(6) Case, D. A.; Aktulga, H. M.; Belfon, K.; Ben-Shalom, I. Y.; Brozell, S. R.; Cerutti, D. S.; Cheatham, T. E. I. I. I.; Cruzeiro, V. W. D.; Darden, T. A.; Duke, R. E.; et al. Amber 2021. University of California: San Francisco 2021.

(7) Wang, J.; Wang, W.; Kollman, P. A.; Case, D. A. Automatic Atom Type and Bond Type Perception in Molecular Mechanical Calculations. F. Mol. Graph. Model. 2006, 25, 247-260.

(8) Wang, J.; Wolf, R. M.; Caldwell, J. W.; Kollman, P. A.; Case, D. A. Development and Testing of a General Amber Force Field. F. Comput. Chem. 2004, 25, $1157-$ 1174 .

(9) Dewar, M. J. S.; Zoebisch, E. G.; Healy, E. F.; Stewart, J. J. P. Development and Use of Quantum Mechanical Molecular Models. 76. AM1: A New General Purpose Quantum Mechanical Molecular Model. f. Am. Chem. Soc. 1985, 107, 3902-3909.

(10) Zhang, W. E. I.; Yang, R.; Cieplak, P.; Luo, R. A. Y.; Lee, T.; Caldwell, J.; Wang, J.; Kollman, P. A Point-Charge Force Field for Molecular Mechanics Simulations of Proteins Based on Condensed-Phase. F. Comput. Chem. 2003, 24, 1999-2012.

(11) Phillips, J. C.; Braun, R.; Wang, W. E. I.; Gumbart, J.; Tajkhorshid, E.; Villa, E.; Chipot, G.; Skeel, R. D.; Poincare, H. Scalable Molecular Dynamics with NAMD. f. Comput. Chem. 2005, 26, 1781-1802.

(12) Grest, G. S.; Kremer, K. Molecular Dynamics Simulation for Polymers in the Presence of a Heat Bath. Phys. Rev. A 1986, 33, 3628-3631.

(13) Świderek, K.; Martí, S.; Moliner, V. Theoretical Study of Primary Reaction of 


\subsection{References}

Pseudozyma Antarctica Lipase B as the Starting Point to Understand Its Promiscuity. ACS Catal. 2014, 4, 426-434.

(14) Jorgensen, W. L.; Maxwell, D. S.; Tirado-Rives, J. Development and Testing of the OPLS All-Atom Force Field on Conformational Energetics and Properties of Organic Liquids. F. Am. Chem. Soc. 1996, 118, 11225-11236.

(15) Duan, Y.; Wu, C.; Chowdhury, S.; Lee, M. C.; Xiong, G.; Zhang, W. E. I.; Yang, R.; Cieplak, P.; Luo, R. A. Y.; Lee, T.; et al. A Point-Charge Force Field for Molecular Mechanics Simulations of Proteins Based on Condensed-Phase. 7 . Comput. Chem. 2003, 24, 1999-2012.

(16) Bayly, C. I.; Merz, K. M.; Ferguson, D. M.; Cornell, W. D.; Fox, T.; Caldwell, J. W.; Kollman, P. A.; Cieplak, P.; Gould, I. R.; Spellmeyer, D. C. A Second Generation Force Field for the Simulation of Proteins, Nucleic Acids, and Organic Molecules. F. Am. Chem. Soc. 1995, 117, 5179-5197.

(17) Zhao, Y.; Truhlar, D. G. The M06 Suite of Density Functionals for Main Group Thermochemistry, Thermochemical Kinetics, Noncovalent Interactions, Excited States, and Transition Elements: Two New Functionals and Systematic Testing of Four M06-Class Functionals and 12 Other Function. Theor. Chem. Acc. 2008, 120, 215-241.

(18) Lee, C.; Yang, W.; Parr, R. G. Development of the Colle-Salvetti CorrelationEnergy Formula into a Functional of the Electron Density. Phys. Rev. B 1988, 37, 785-789.

(19) Becke, A. D. Density-functional Thermochemistry. III. The Role of Exact Exchange. f. Chem. Phys. 1993, 98, 5648-5652.

(20) Christensen, A. S.; Kubař, T.; Cui, Q.; Elstner, M. Semiempirical Quantum Mechanical Methods for Noncovalent Interactions for Chemical and Biochemical Applications. Chem. Rev. 2016, 116, 5301-5337.

(21) Pople,J. A.; Schleyer, P. V.; Hehre, W. J.; Radom, L. AB INITIO Molecular Orbital Theory; 1986.

(22) Dewar, M.J. S.; Thiel, W. Ground States of Molecules. 38. The MNDO Method. Approximations and Parameters. F. Am. Chem. Soc. 1977, 99, 4899-4907.

(23) Hohenberg, P.; Kohn, W. Inhomogeneous Electron Gas. Phys. Rev. 1964, 136, B864-B871.

(24) Kohn, W.; Sham, L. J. Self-Consistent Equations Including Exchange and Correlation Effects. Phys. Rev. 1965, 140, A1133-A1138.

(25) Warshel, A.; Karplus, M. Calculation of $\Pi \pi^{*}$ Excited State Conformations and Vibronic Structure of Retinal and Related Molecules. F. Am. Chem. Soc. 1974, 96, 5677-5689.

(26) Warshel, A.; Levitt, M. Theoretical Studies of Enzymatic Reactions - Dielectric, Electrostatic and Steric Stabilization of Carbonium-Ion in Reaction of Lysozyme. f. Mol. Biol. 1976, 103, 227-249.

(27) Field, M. J.; Bash, P. A.; Karplus, M. A Combined Quantum Mechanical and 
Molecular Mechanical Potential for Molecular Dynamics Simulations. F. Comput. Chem. 1990, 11, 700-733.

(28) Chung, L. W.; Sameera, W. M. C.; Ramozzi, R.; Page, A. J.; Hatanaka, M.; Petrova, G. P.; Harris, T. V.; Li, X.; Ke, Z.; Liu, F.; et al. The ONIOM Method and Its Applications. Chem. Rev. 2015, 115.

(29) Reuter, N.; Dejaegere, A.; Maigret, B.; Karplus, M. Frontier Bonds in QM/MM Methods: A Comparison of Different Approaches. F. Phys. Chem. A 2000, 104, 1720-1735.

(30) Senn, H. M.; Thiel, W. QM/MM Methods for Biomolecular Systems. Angew. Chemie - Int. Ed. 2009, 48, 1198-1229.

(31) Born, M.; Oppenheimer, R. Zur Quantentheorie Der Molekeln. Ann. Phys. 1927, $389,457-484$.

(32) Hestenes, M. R.; Stiefel, E. Methods of Conjugate Gradients for Solving Linear Systems. F. Res. Natl. Bur. Stand. (1934). 1952, 49, 409.

(33) Byrd, R. H.; Lu, P.; Nocedal, J.; Zhu, C. A Limited Memory Algorithm for Bound Constrained Optimization. SLAM J. Sci. Comput. 1995, 16, 1190-1208.

(34) Baker, J. An Algorithm for the Location of Transition States. F. Comput. Chem. 1986, 7, 385-395.

(35) Fukui, K. The Path of Chemical Reactions - The IRC Approach. Acc. Chem. Res. 1981, 14, 363-368.

(36) Maeda, S.; Harabuchi, Y.; Ono, Y.; Taketsugu, T.; Morokuma, K. Intrinsic Reaction Coordinate: Calculation, Bifurcation, and Automated Search. Int. F. Quantum Chem. 2015, 115, 258-269.

(37) Roux, B. The Calculation of the Potential of Mean Force Using ComputerSimulation. Comput. Phys. Commun. 1995, 91, 275-282.

(38) Torrie, G. M.; Valleau, J. P. Non-Physical Sampling Distibutions in Monte-Carlo Free-Energy Estimation - Umbrella Sampling. f. Comput. Phys. 1977, 23, 187199.

(39) Kumar, S.; Rosenberg, J. M.; Bouzida, D.; Swendsen, R. H.; Kollman, P. A. The Weighted Histogram Analysis Method for Free-energy Calculations on Biomolecules. I. The Method. F. Comput. Chem. 1992, 13, 1011-1021.

(40) Verlet, L. Computer "Experiments" on Classical Fluids. I. Thermodynamical Properties of Lennard-Jones Molecules. Phys. Rev. 1967, 159, 98-103.

(41) Chuang, Y. Y.; Corchado, J. C.; Truhlar, D. G. Mapped Interpolation Scheme for Single-Point Energy Corrections in Reaction Rate Calculations and a Critical Evaluation of Dual-Level Reaction Path Dynamics Methods. F. Phys. Chem. A 1999, 103, 1140-1149.

(42) Ruiz-Pernía, J. J.; Silla, E.; Tuñón, I.; Martí, S.; Moliner, V. Hybrid QM/MM Potentials of Mean Force with Interpolated Corrections. 7. Phys. Chem. B 2004, 108, 8427-8433. 


\subsection{References}

(43) Ruiz-Pernía, J. J.; Silla, E.; Tuñón, I.; Martí, S. Hybrid Quantum Mechanics/Molecular Mechanics Simulations with Two-Dimensional Interpolated Corrections: Application to Enzymatic Processes. 7. Phys. Chem. B 2006, 110, 17663-17670.

(44) Bash, P. A.; Field, M. J.; Karplus, M. Free Energy Perturbation Method for Chemical Reactions in the Condensed Phase: A Dynamic Approach Based on a Combined Quantum and Molecular Mechanics Potential. F. Am. Chem. Soc. 1987, 109, 8092-8094.

(45) Świderek, K.; Tuñón, I.; Martí, S.; Moliner, V.; Bertrán, J. Role of Solvent on Nonenzymatic Peptide Bond Formation Mechanisms and Kinetic Isotope Effects. 7. Am. Chem. Soc. 2013, 135, 8708-8719.

(46) Truhlar, D. G.; Garrett, B. C.; Klippenstein, S. J. Current Status of TransitionState Theory. F. Phys. Chem. 1996, 100, 12771-12800.

(47) Eyring, H. The Activated Complex in Chemical Reactions. F. Chem. Phys. 1935, 3, 107-115.

(48) Evans, M. G.; Polanyi, M. Some Applications of the Transition State Method to the Calculation of Reaction Velocities, Especially in Solution. Trans. Faraday Soc. 1935, 31, 875-894.

(49) Connors, A. K. Chemical Kinetics: The Study of Reaction Rates in Solution; 1990; Vol. 180.

(50) Martí, S.; Moliner, V.; Tuñón, I.; Williams, I. H. QM / MM Calculations of Kinetic Isotope Effects in the Chorismate Mutase Active Site. Org. Biomol. Chem. 2003, 1, 483-487.

(51) Martí, S.; Moliner, V.; Tuñón, I. Improving the QM/MM Description of Chemical Processes: A Dual Level Strategy to Explore the Potential Energy Surface in Very Large Systems. 7. Chem. Theory Comput. 2005, 1, 1008-1016.

(52) Świderek, K.; Paneth, P. Binding Ligands and Cofactor to L-Lactate Dehydrogenase from Human Skeletal and Heart Muscles. F. Phys. Chem. B 2011, 115, 6366-6376.

(53) Świderek, K.; Martí, S.; Moliner, V. Theoretical Studies of HIV-1 Reverse Transcriptase Inhibition. Phys. Chem. Chem. Phys. 2012, 14, 12614-12624.

(54) Krzemińska, A.; Paneth, P.; Moliner, V.; Świderek, K. Binding Isotope Effects as a Tool for Distinguishing Hydrophobic and Hydrophilic Binding Sites of HIV-1 RT. F. Phys. Chem. B 2015, 119, 917-927.

(55) LeCun, Y.; Bengio, Y.; Hinton, G. Deep Learning. Nature 2015, 521, 436-444.

(56) Jiménez, J.; Škalič, M.; Martínez-Rosell, G.; De Fabritiis, G. KDEEP: ProteinLigand Absolute Binding Affinity Prediction via 3D-Convolutional Neural Networks. F. Chem. Inf. Model. 2018, 58, 287-296.

(57) Alley, E. C.; Khimulya, G.; Biswas, S.; AlQuraishi, M.; Church, G. M. Unified Rational Protein Engineering with Sequence-Based Deep Representation Learning. Nat. Methods 2019, 16, 1315-1322. 
(58) Cao, H.; Wang,J.; He, L.; Qi, Y.; Zhang, J. Z. DeepDDG: Predicting the Stability Change of Protein Point Mutations Using Neural Networks. 7. Chem. Inf. Model. 2019, 59, 1508-1514.

(59) Senior, A. W.; Evans, R.; Jumper, J.; Kirkpatrick, J.; Sifre, L.; Green, T.; Qin, C.; Žídek, A.; Nelson, A. W. R.; Bridgland, A.; et al. Improved Protein Structure Prediction Using Potentials from Deep Learning. Nature 2020, 577, 706-710.

(60) Rumelhart, D. E.; Hinton, G. E.; Williams, R. J. Learning Representations by Back-Propagating Errors. Nature 1986, 323, 533-536.

(61) Kingma, D. P.; Ba, J. Adam: A Method for Stochastic Optimization. CoRR 2015, abs $/ 1412.6$.

(62) Berman, H. M.; Westbrook, J.; Feng, Z.; Gilliland, G.; Bhat, T. N.; Weissig, H.; Shindyalov, I. N.; Bourne, P. E. The Protein Data Bank. Nucleic Acids Res. 2000, 28, 235-242.

(63) UniProt: The Universal Protein Knowledgebase in 2021. Nucleic Acids Res. 2021, 49, D480-D489.

(64) Needleman, S. B.; Wunsch, C. D. A General Method Applicable to the Search for Similarities in the Amino Acid Sequence of Two Proteins. F. Mol. Biol. 1970, 48, 443-453.

(65) He, K.; Zhang, X.; Ren, S.; Sun, J. Deep Residual Learning for Image Recognition. In 2016 IEEE Conference on Computer Vision and Pattern Recognition (CVPR); 2016; pp 770-778.

(66) Kneller, G. R. Superposition of Molecular Structures Using Quaternions. Mol. Simul. 1991, 7, 113-119.

(67) Świderek, K.; Tuñon, I.; Moliner, V.; Bertran, J. Protein Flexibility and Preorganization in the Design of Enzymes. The Kemp Elimination Catalyzed by HG3.17. ACS Catal. 2015, 5, 2587-2595.

(68) Krzemińska, A.; Moliner, V.; Świderek, K. Dynamic and Electrostatic Effects on the Reaction Catalyzed by HIV-1 Protease. F. Am. Chem. Soc. 2016, 138, 1628316298.

(69) Świderek, K.; Tuñón, I.; Williams, I. H.; Moliner, V. Insights on the Origin of Catalysis on Glycine N-Methyltransferase from Computational Modeling. f. Am. Chem. Soc. 2018, 140, 4327-4334.

(70) Roca, M.; Martí, S.; Andrés, J.; Moliner, V.; Tuñón, I.; Bertrán, J.; Williams, I. H. Theoretical Modeling of Enzyme Catalytic Power: Analysis of "Cratic" and Electrostatic Factors in Catechol O-Methyltransferase. 7. Am. Chem. Soc. 2003, 125, 7726-7737.

(71) García-Meseguer, R.; Martí, S.; Ruiz-Pernía, J. J.; Moliner, V.; Tunón, I. Studying the Role of Protein Dynamics in an SN2 Enzyme Reaction Using FreeEnergy Surfaces and Solvent Coordinates. Nat. Chem. 2013, 5, 566-571.

(72) Welborn, V. V.; Head-gordon, T. Computational Design of Synthetic Enzymes. Chem. Rev. 2018, 119, 6613-6630. 


\subsection{References}

(73) Serrano-Aparicio, N.; Moliner, V.; Świderek, K. Nature of Irreversible Inhibition of Human 20S Proteasome by Salinosporamide A. The Critical Role of Lys-Asp Dyad Revealed from Electrostatic Effects Analysis. ACS Catal. 2021, 11, 35753589 .

(74) Warshel, A. Electrostatic Origin of the Catalytic Power of Enzymes and the Role of Preorganized Active Sites. F. Biol. Chem. 1998, 273, 27035-27038.

(75) Warshel, A.; Sharma, P. K.; Kato, M.; Xiang, Y.; Liu, H.; Olsson, M. H. M. Electrostatic Basis for Enzyme Catalysis. Chem. Rev. 2006, 106, 3210-3235. 
Chapter 4

Results and Discussion 
- 98 - 
In this chapter, the main results of this thesis are highlighted and divided into two main sections. In the first section, two promiscuous reactions of CALB were explored in two separate articles. The first article (Article 1) of this section studies the hydrolysis of the amide bond as a secondary reaction of CALB which was explored from a theoretical-experimental perspective. It appeared to be clearly dependent on specific features of the substrate. In the second article (Article 2) the enzymatic epoxidation of short alkenes was studied and the use of CALB was proposed as a green alternative to the production of epoxides. In the second section, a computer-assisted rational design was suggested. Two articles compose this section. The first one (Article 3) is focused on the comparison of the amidase activity of CALB and Bs2 from a structural and mechanistic level and using QM/MM and ML approaches. The final article (Article 4) of this thesis is centered on rational redesign of $\mathrm{Bs} 2$, which came from the analysis of the electrostatic environment of aligned CALB and Bs2 structures using RQs.

Each article is preceded by a brief summary where the main findings are highlighted. A QR code linked to the published original research article is also provided in each section. 


\subsection{Promiscuity studies in GALB}

\subsubsection{Hydrolysis of the amide bond catalyzed by GALB (Article 1)}

As mentioned in section 1.1.3, lipases are useful biocatalysts to be used in the industry and academia, for their versatility and high promiscuity. ${ }^{1,2}$ In this sense, CALB is the perfect enzyme to study the molecular mechanisms that govern this promiscuous behavior. With the hydrolysis of esters as its primary reaction, CALB displays high catalytic, substrate, and condition promiscuity. ${ }^{1,3-7}$ Among these reactions, the hydrolysis of the amide bond has a special interest. ${ }^{8}$ In this work we demonstrated experimentally and theoretically that CALB is capable to hydrolyze the amide bond of certain substrates. However, this ability is strongly dependent on specific molecular features of the substrates (Figure 4.1).
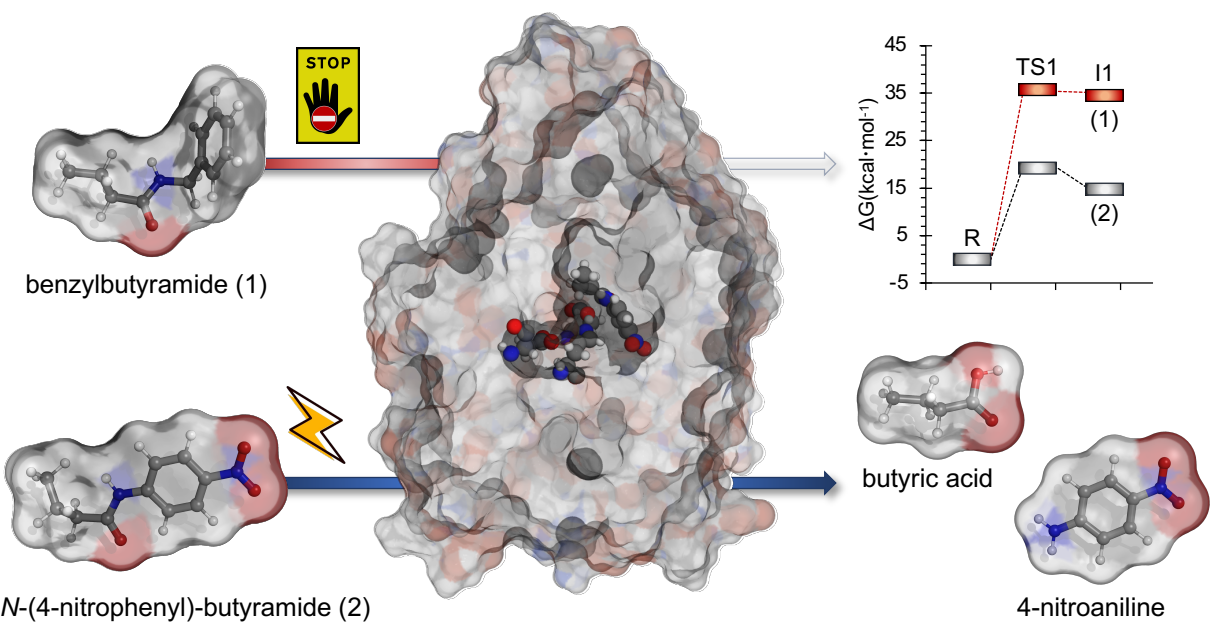

Figure 4.1. Schematic summary of the main findings of the Article. CALB is not capable to hydrolyze $\mathbf{1}$ due to the high energy barrier observed in the first step of the reaction. On the other hand, CALB hydrolyses 2 to butyric acid and 4nitroaniline. The nitro group facilitates the attack of the catalytic Ser 105. 
We studied the hydrolysis of two different benzyl-acyl amides. The first one was the benzylbutyramide (Figure 4.1), with a short 4-carbon acyl chain. We proved experimentally that CALB was not capable to hydrolyze this substrate. This fact was due to the very high activation energy barrier for the first step of the reaction, which was derived from the QM/MM free energy calculations. This step involves the nucleophilic attack of the Ser 105 to the carbonyl carbon, leading to the formation of a tetrahedral intermediate, I1 (see Figure 4.2a).
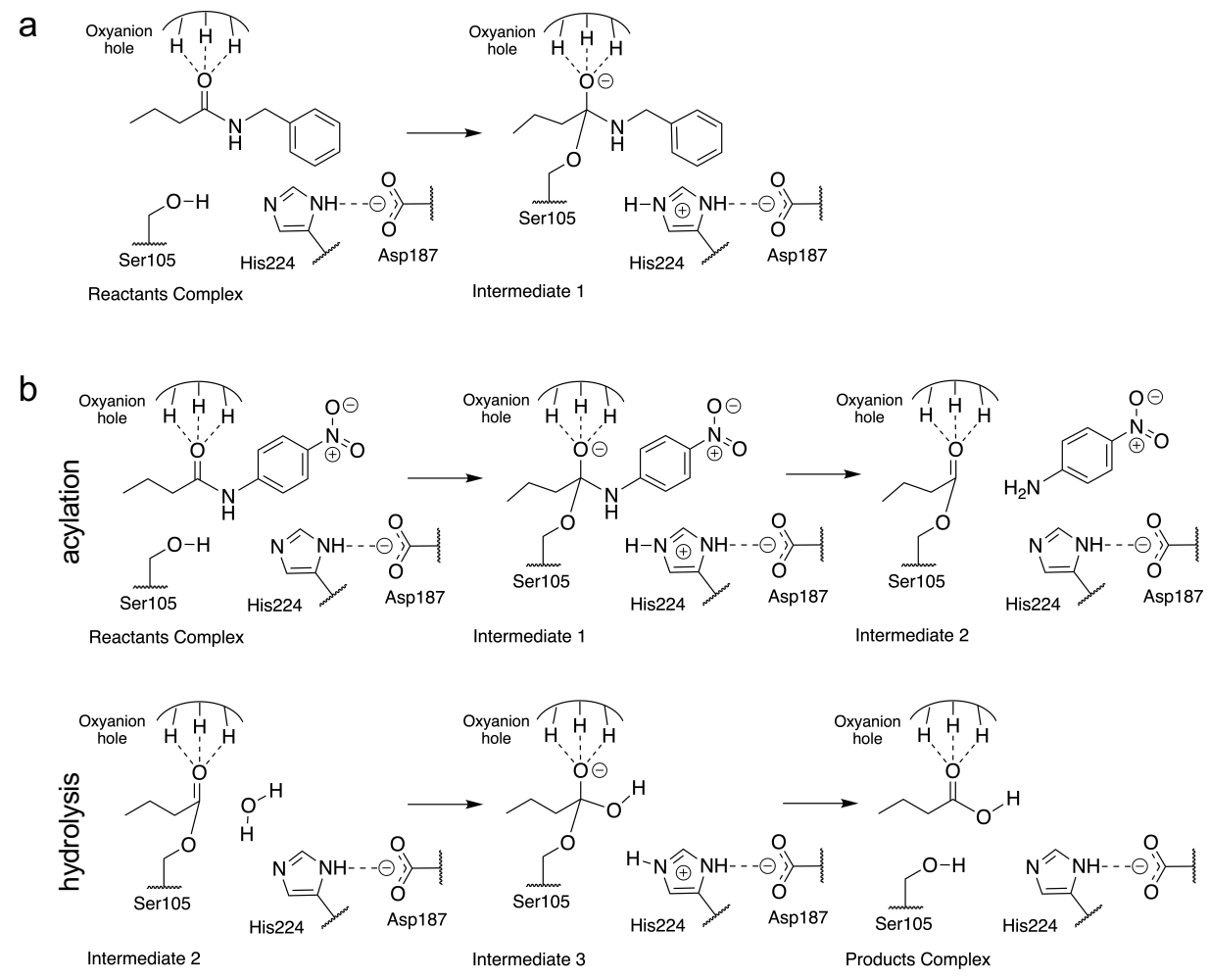

Figure 4.2. Explored mechanisms for the amidase reaction in CALB. Panel a shows the first step of the reaction in benzylbutiramide, while in panel $\mathbf{b}$ the complete mechanism of hydrolysis of $\mathcal{N}$-(4-nitrophenyl)-butyramide is depicted.

To overcome this limitation, we decided to introduce a para-nitro group on the ring of the substrate. From the theoretical calculations with this new substrate, which was the $\mathcal{N}$-(4-nitrophenyl)-butyramide (Figure 4.1 ), we 


\subsection{Promiscuity studies in CALB}

found that this nitro group promotes the displacement of the electrons towards the ring with a subsequent change in the charge of the carbonyl carbon, increasing its electrophilic character. This fact facilitates the attack of the catalytic Ser105 decreasing the barrier of the first step of the reaction. We solved the reaction mechanism for the hydrolysis of $\mathcal{N}$-(4nitrophenyl)-butyramide by CALB (Figure 4.2b). The kinetic constants were also experimentally determined using the commercial free form of CALB and the synthetized substrate. The obtained experimental parameters were in very good agreement with the theoretical energy barriers (Figure 4.3).

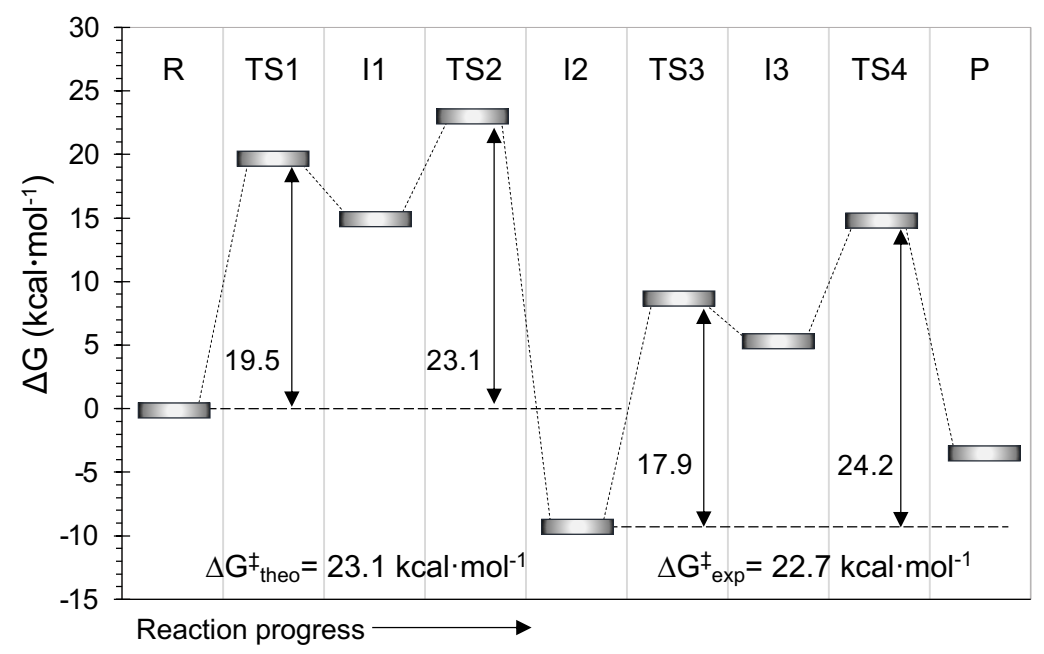

Figure 4.3. Free energy profile of the hydrolysis of $\mathcal{N}$-(4-nitrophenyl)-butyramide by CALB. The first two steps correspond to the acylation step while the last two are those related with the hydrolysis of the acyl-enzyme complex.

To conclude, although CALB is capable to hydrolyze the amide bond, its activity strongly depends on the electrophilic character of the carbonyl group, which can be modulated by specific features of the substrate. This finding invites to explore new promiscuous reactions that can be tuned by either substrate's features or the electrostatic preorganization of the enzyme by introducing adequate mutations.

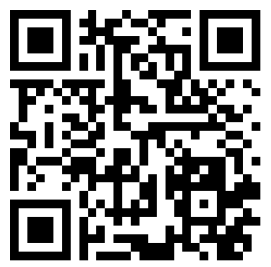




\title{
Exploring the Origin of Amidase Substrate Promiscuity in CALB by a Computational Approach
}

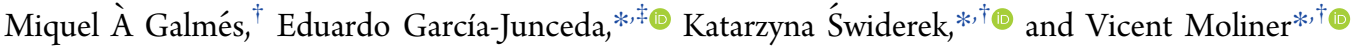 \\ †Departament de Química Física i Analítica, Universitat Jaume I, 12071 Castellón, Spain \\ ‡Departamento de Química Orgánica Biológica, Instituto de Química Orgánica General, CSIC Juan de la Cierva 3, 28006 Madrid, \\ Spain
}

\section{Supporting Information}

ABSTRACT: Enzyme promiscuity attracts the interest of the industrial and academic sectors because of its application in the design of biocatalysts. The amidase activity of Candida antarctica lipase B (CALB) on two different substrates has been studied by theoretical quantum mechanics/molecular mechanics methods, supported by experimental kinetic measurements. The aim of the study is to understand the substrate promiscuity of CALB in this secondary reaction and the origin of its promiscuous catalytic activity. The computational results predict activation free energies in very good agreement with the kinetic data and confirm that the activity of CALB as an amidase, despite

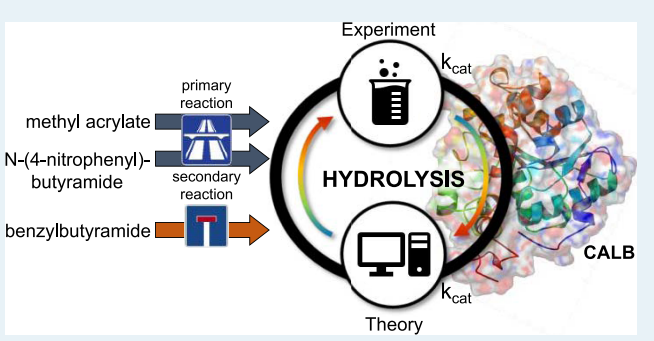
depending on the features of the amide substrate, is dictated by the electrostatic effects of the protein. The protein polarizes and activates the substrate as well as stabilizes the transition state, thus enhancing the rate constant. Our results can provide guides for future designs of biocatalysts based on electrostatic arguments.

KEYWORDS: computational chemistry, enzyme catalysis, enzyme promiscuity, QM/MM, molecular dynamics, free energy surfaces

\section{INTRODUCTION}

Enzymes have evolved along millions of years to catalyze specific chemical transformations on specific type of substrates. ${ }^{1}$ However, some enzymes conserve secondary activities other than the primary one, although their catalytic efficiency is almost always very poor compared to the primary reaction. This fact is due to the lack of the selective pressure over the evolution period. ${ }^{2}$ The presence of these secondary reactions, known as catalytic promiscuity, can be the starting point for the evolution of enzymes in vivo, ${ }^{3}$ which has become a very interesting field from the industrial and the academic points of view. ${ }^{4-10}$ In addition, many enzymes show substrate promiscuity, which involves the ability to accept a range of different substrates. Having tools to enhance the rate constant of a secondary reaction on a particular substrate can be used to obtain high-value chemicals, which is nowadays of vital importance. ${ }^{11,12}$ In addition, understanding the molecular basis of enzyme promiscuity is crucial for the rational design of new environmentally friendly catalysts.

It is reported that many lipases show promiscuous behavior, ${ }^{4}$ and Candida antarctica lipase B (CALB) is one of the best wellknown highly promiscuous enzyme. CALB's primary activity comprises the hydrolysis of the ester bond in triacylglycerides (Figure 1A), but it is also capable of catalyzing a plethora of different reactions such as the synthesis of esters and amides, transacylation of alcohols, ${ }^{13}$ epoxidation reactions, ${ }^{14}$ aldol additions, Michael-type additions, ${ }^{4,15}$ among many others. ${ }^{16}$
As a carboxylic ester hydrolase, CALB performs its activity by a characteristic catalytic triad composed of Ser-His-Asp (Figure 1B). Here, the His224 residue has a very relevant role in the activity by acting as an acid/base residue during the full catalytic process. This acid/base character of histidine is governed by the aspartate residue that modulates its $\mathrm{p} K_{\mathrm{a}}$. Additionally, an oxyanion hole formed by the residues Thr 40 and Gln106 is also present in the active site pocket. As confirmed by computational studies, this oxyanion hole has a double role: first, it is responsible for orienting the substrate in the active site, and second, it stabilizes the negative charge accumulated on the substrate during the hydrolysis. ${ }^{18}$ The hydrolysis of ester natural substrates is proposed to be a fourstep reaction ${ }^{18}$ that involves, in the first step, the attack of the catalytic serine to the carbonyl carbon of the amide. In the second step, the release of the leaving group occurs as an effect of the acid-base character of His224. The products are released due to the hydrolysis of the acyl-enzyme complex in the third and the last chemical steps.

Among all of the secondary reactions that CALB is capable to catalyze, the hydrolysis of the amide bond has special interest (Figure 1A). This reaction is present ubiquitously in nature, from the proteolysis to the inhibition of some

Received: September 18, 2019

Revised: October 22, 2019

Published: November 1, 2019 
A<smiles>[R]OC([R])=O</smiles><smiles>C[Ge](O)C(C)(C)O</smiles>

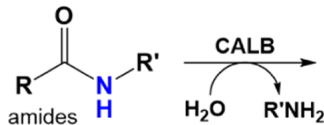<smiles>[R]C(=O)O</smiles>

\section{Primary reaction}

\section{Secondary reaction}

B

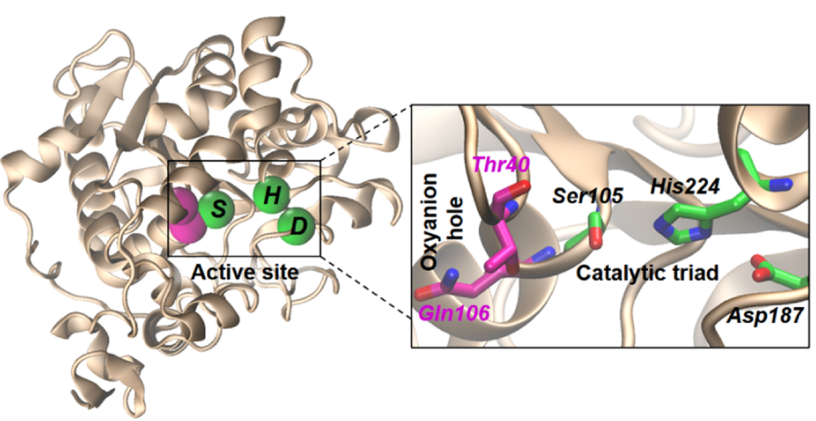

Figure 1. (A) General scheme of the primary and secondary reactions, ester and amide hydrolysis, catalyzed by CALB. (B) Crystal structure of CALB (PDB ID 1TCA) ${ }^{17}$ together with the detail of the active site indicating the key residues.

antibiotics by the molecular machinery of pathogens. ${ }^{19}$ CALB has been widely studied, rendering a significant amount of experimental data that is available in the literature. ${ }^{4,5,20-22}$ In particular, the hydrolysis of the amide bond catalyzed by CALB was experimentally studied by López-Munguia and coworkers, ${ }^{23}$ providing evidence that the enzyme is capable to hydrolyze the amides of several vanillyl derivatives. Obviously, the catalytic efficiency of the amidase reaction on these type of substrates is considerably lower than that of the native ester hydrolysis. Thus, CALB is an appropriate candidate that can serve as a good example to understand the origin of promiscuity in enzymes and to propose mutations that can enhance the rate constant of secondary reactions. The present work is focused on the study of the reaction mechanism of the hydrolysis of the amide bond carried out by CALB on two different substrates, combining experimental kinetic studies and theoretical quantum mechanics/molecular mechanics (QM/MM) methods. The description of the full reaction mechanism at atomistic level derived from the simulations sheds light on the substrate promiscuity of CALB in this secondary reaction, and on the origin of its catalytic activity. We believed that the obtained results will not only allow understanding its promiscuity but also exploring the possibility of using this protein as a scaffold to design new improved and more efficient biocatalysts.

\section{COMPUTATIONAL METHODS}

An initial equilibrated system was taken from a previous study on the primary reaction catalyzed by CALB, ${ }^{18}$ prepared from the crystal structure available in the Protein Data bank under PDB ID 1TCA. ${ }^{17}$ Further details of the setting up of the system are reported in the Supporting Information.

The amidase reaction was studied with two different substrates, benzylbutyramide (1) and $\mathrm{N}$-(4-nitrophenyl)butyramide (2). Compound 1 is a derivative of vanillyl with a short acyl chain of four carbons, which was proposed by
López-Munguia and co-workers. ${ }^{23}$ In 2, the lone pair of the aniline nitrogen can be delocalized into the aromatic ring, making it a far weaker base and better leaving group compared to benzylamine where the nitrogen lone pair cannot delocalize due to the presence of an additional $\mathrm{sp}^{3}$ carbon that, a priori, can increase the positive charge character on the anilide $\mathrm{C} 1$ compared to benzylamide. After equilibration of the systems by classical molecular dynamics (MD) simulations, the full system was partitioned into a $\mathrm{QM}$ and a MM region (see Figure 2). $\mathrm{AM}^{24}$ and $\mathrm{M} 06-2 \mathrm{X}^{25}$ Hamiltonians were selected to describe the QM region, while the OPLS-AA force field ${ }^{26,27}$ was used to describe the residues of the protein ( 4625 atoms) and TIP $3 \mathrm{P}^{28}$ was the force field selected to describe the water molecules

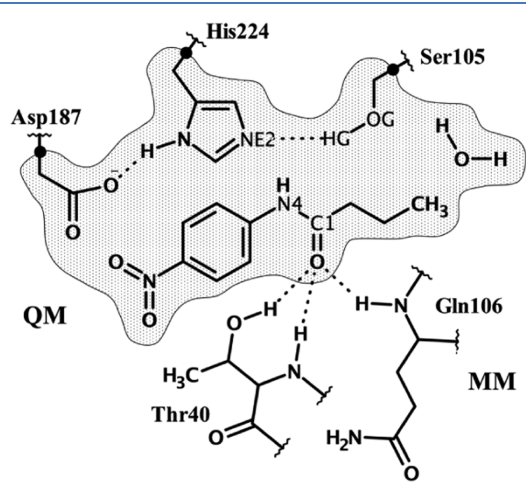

Figure 2. Schematic representation of the active site of CALB with substrate 2. The QM region is represented as a shaded region. Black dots represent link atoms between $\mathrm{QM}$ and $\mathrm{MM}$ regions. The interaction between 2 and the oxyanion hole (Thr40 and Gln106) is shown as dashed lines. 

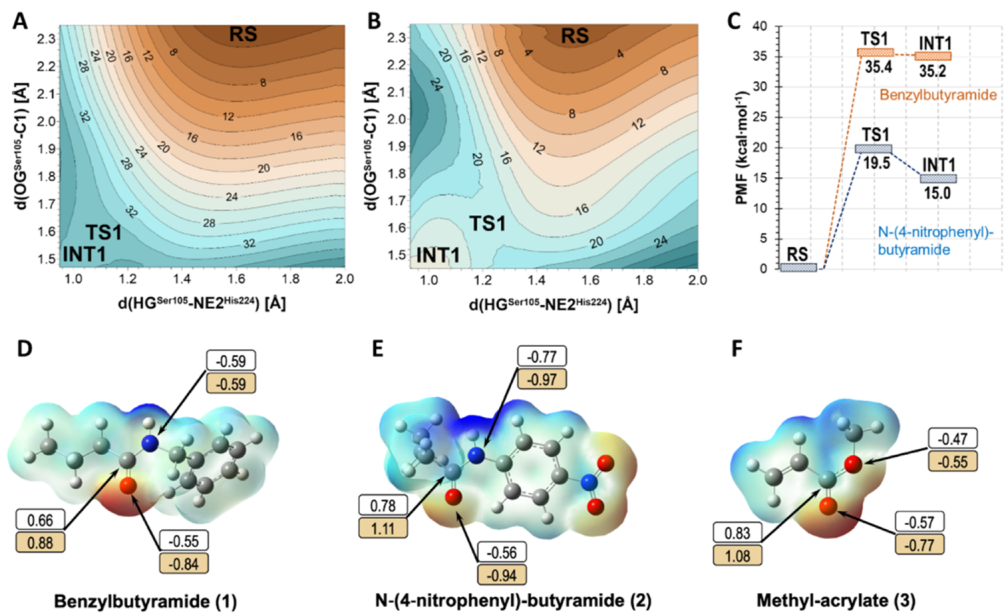

F

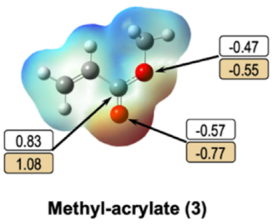

Figure 3. 2D-PMF of the first chemical step of the hydrolysis of 1 (A) and 2 (B) computed at the M06-2X:AM1/MM level. (C) Free energy profiles derived from the 2D-PMFs. Distances are in angstroms, and energies of isoenergetic lines are in kcal mol ${ }^{-1}$. Maps of electrostatic potential computed at the M06-2X/6-31+G** level in the gas phase for the two substrates of the CALB secondary reaction, 1 (D) and 2 (E), and for the substrate of the CALB primary reaction methyl acrylate (3) (F). Charge values in panels (D) - (F) (in au) were computed in the gas phase (white squares) and in the active site of the protein (brown squares).

(60014 atoms). The standard $6-31+\mathrm{G}(\mathrm{d}, \mathrm{p})$ basis set was employed when the density functional theory (DFT) Hamiltonian was used to describe the QM subset of atoms. Hydrogen link atoms were placed in the QM-MM frontier bonds, as depicted in Figure 2.

$\mathrm{QM} / \mathrm{MM}$ free energy surfaces (FESs) were obtained, in terms of two-dimensional potentials of mean force $(2 \mathrm{D}$ PMF), ${ }^{29-31}$ for every step of the reaction using the umbrella sampling approach ${ }^{29,32}$ combined with the weighted histogram analysis method (WHAM) ${ }^{33}$ with the fDYNAMO library. ${ }^{34}$ To correct the possible source of error derived from the low level of theory used to describe the $\mathrm{QM}$ region during the $\mathrm{MD}$ simulations, in this case, the AM1 semiempirical Hamiltonian, spline under tension corrections were used, based on the original work of Truhlar and co-workers. ${ }^{35-37}$ As described in previous papers, ${ }^{38,39}$ a correction term is interpolated to any value along the reaction coordinates in the FES based on multiple structures derived from the AM1/MM simulations. A continuous energy function is used to obtain the corrected PMFs using the hybrid M06-2X functional with the standard 6$31+G(d, p)$ basis set (see the Supporting Information for details). These calculations were carried out using the Gaussian09 program. ${ }^{40}$

Kinetic isotope effects (KIEs) were calculated, at M06-2X/ $\mathrm{MM}$ and $\mathrm{AM1} / \mathrm{MM}$ levels, from isotopic substitution of the key atoms in the reactants and the different transition state structures, as described in previous papers. ${ }^{18,41,42}$ Details and further discussions are provided in the Supporting Information.

\section{EXPERIMENTAL METHODS}

Experimental kinetic studies in CALB were performed to evaluate the hydrolysis of the amide bond of both substrates. For that, compound $\mathbf{1}$ was synthetized using an enzymatic approach (see the Supporting Information for details). Briefly, equimolar solution of benzylamine and butyric acid was incubated in 2-methyl-2-butanol and the reaction was followed by thin-layer chromatography (TLC). The final product was then purified by a silica column and characterized by mass spectrometry $(m / z=178.2)$ and NMR. A final overall yield of $48 \%$ was obtained. 2 was chemically synthesized from a solution of butyryl chloride, 4-nitroaniline, and pyridine (with a molar ratio of 1.2:1:2) in anhydrous dichloromethane. The product was purified by the silica column, and it was finally characterized by NMR. The overall yield was $74 \%$.

Kinetic parameters of amidase reaction were determined for both substrates (see the Supporting Information for details). Only summarizing here, kinetic assays were performed with the commercial free form of CALB. Reactions were carried out at increasing concentrations of substrates in $20 \mathrm{mM}$ phosphate buffer at $\mathrm{pH} 7$ and $37{ }^{\circ} \mathrm{C}$ with $10 \%$ organic solvent due to the poor solubility of substrates. The progression of the reaction was followed by high-performance liquid chromatography (HPLC) in the case of substrate $\mathbf{1}$ and by spectrophotometric techniques (increment of the absorbance at $405 \mathrm{~nm}$ ) in the case of substrate 2 .

\section{RESULTS AND DISCUSSION}

Hydrolysis of the Amide Bond of 1 Catalyzed by CALB. As mentioned, the first goal of this work is to study the substrate promiscuity in the hydrolysis of the amide bond catalyzed by CALB. The exploration of the differences in the kinetics over two substrates can lead to find the key features that control the reaction rate that will serve as a starting point to obtain more efficient biocatalysts. Thus, first, the hydrolysis of the amide bond of substrate $\mathbf{1}$ was studied by kinetic experiments and by computational methods. This compound is a vanillyl derivative that was previously proposed by LópezMunguia $^{23}$ and co-workers but no kinetic constants were reported, so no experimental data was available to be directly compared with theoretical results.

$Q M / M M$ Simulations. Following the molecular mechanism suggested for the primary reaction of CALB, ${ }^{18}$ the first step of 

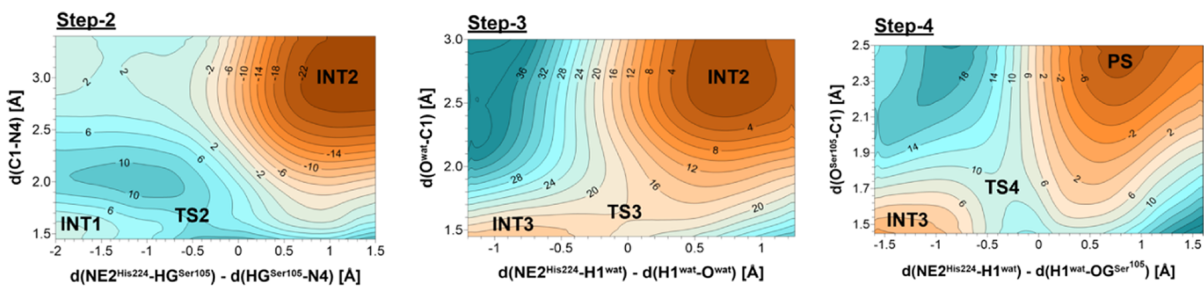

Figure 4. 2D-PMFs surfaces for the hydrolysis of 2 obtained at the M06-2X:AM1/MM level of theory. The surfaces represent the last three steps of the reaction, as depicted in Figure 5. Distances are in ångstroms, and energies of isoenergetic lines are in $\mathrm{kcal} \mathrm{mol}^{-1}$.

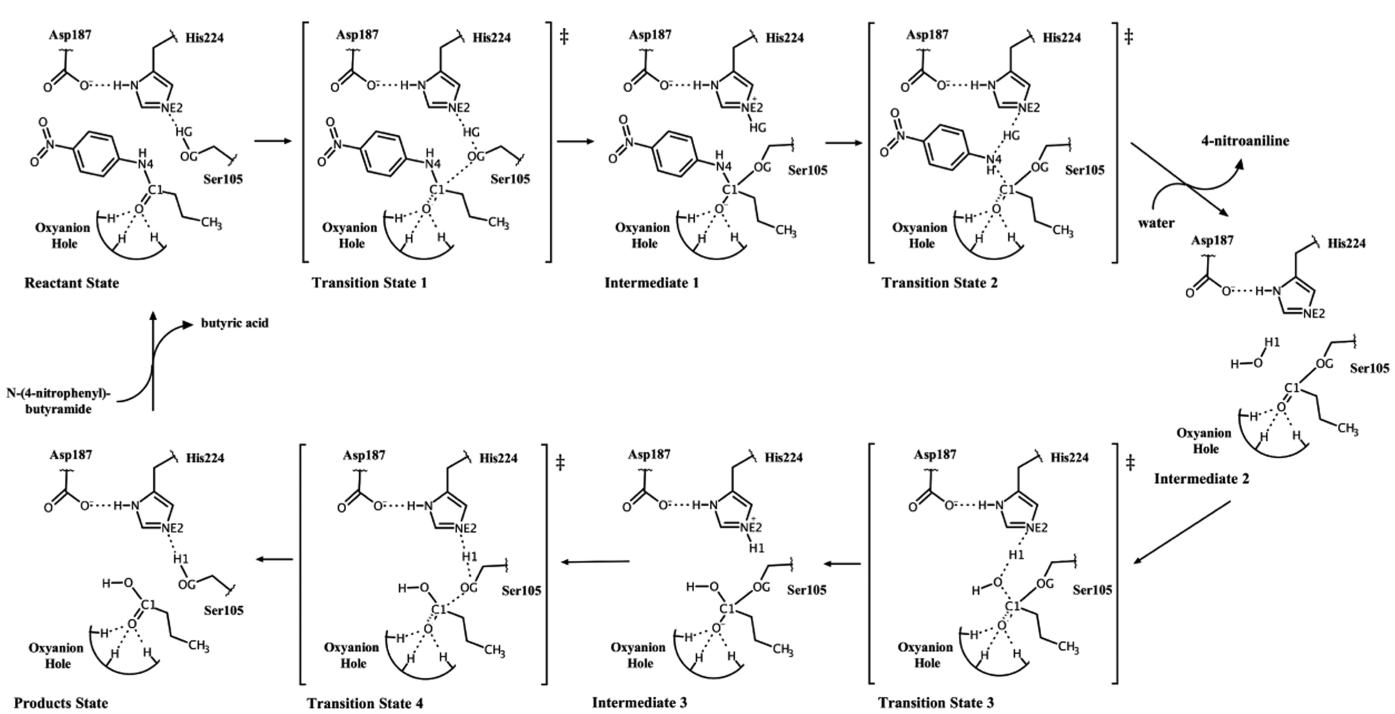

Figure 5. Schematic representation of the hydrolysis of the 2 catalyzed by CALB. The reaction yields a molecule of 4-nitroaniline (second step, INT2) and butyric acid (last step, product state).

the enzymatic hydrolysis of $\mathbf{1}$ is proposed to involve the attack of the catalytic Ser105 to the carbonyl carbon of the amide. The FES of this chemical step, computed in terms of 2D-PMF at the M06-2X:AM1/MM level, is depicted in Figure 3A, and its corresponding free energy profile in Figure 3C. In this step, the His 224 residue acts as a base, allowing the activation of Ser105 and permitting consequently the formation of a tetrahedral intermediate, INT1. The negative charge of the oxygen of the amide is stabilized by strong hydrogen bond interactions in the oxyanion hole, formed by the $\mathrm{HN}$ of the backbone of Gln106 and Thr40, and the hydroxyl group of the lateral chain of Thr40. The FES shown in Figure 3A reveals a concerted mechanism where the transition state, TS1, is displaced close to the region of the slightly stable INT1. The free energy barrier computed for this first step is equal to 35.4 $\mathrm{kcal} \mathrm{\textrm {mol } ^ { - 1 }}$ (Figure 3C). According to the transition state theory, ${ }^{43}$ this barrier would correspond to a $k_{\text {cat }}=1.9 \times 10^{-13}$ $\mathrm{s}^{-1}$ at $303 \mathrm{~K}$. This rate constant is definitely too low for a reaction occurring in mild conditions similar to those of biological systems. Consequently, this means that the hydrolysis of the amide bond on $\mathbf{1}$ cannot take place in a reasonable time scale. Despite this computational prediction, experimental evaluation of the amidase reaction on this substrate was carried out to confirm that CALB is not capable to hydrolyze this short-acyl chain amide.

Experimental Evaluation. To assess the enzymatic hydrolysis of the amide bond, commercial wild-type CALB in its free form was used. As mentioned before, as a result of the poor solubility of $1,10 \%$ acetonitrile was used in the reactions. A tributyrin assay in microtiter plates was performed to confirm that CALB was active in this conditions. The reactions at increasing concentrations of substrate $\mathbf{1}$ (from 0 to $5 \mathrm{mM}$ ) were followed by HPLC during $48 \mathrm{~h}$. The kinetic measurements reveal no hydrolysis of this substrate in any of the reactions under these conditions. Thus, this result is in agreement with the high energy barrier predicted by our QM/ MM calculations.

Hydrolysis of the Amide Bond of 2 Catalyzed by CALB. To explore the amidase activity of CALB with a different substrate, the specific features of the initial substrate 1 were modified. Assuming that the efficiency of CALB can be substrate-dependent, modifications on originally used compound 1 were explored to try to find a more reactive substrate. In particular, and taking into account the character of the first step of the hydrolysis deduced from our QM/MM simulations, the inclusion of chemical groups that could increase the positive charge on the carbonyl carbon of the substrate was 
proposed. Thus, the idea was to generate a better electrophilic center on $\mathrm{C} 1$ position to facilitate the attack of Ser105. After analysis of possible substitutions on the benzyl ring, a nitro group was introduced in the para position, together with a shortening of the acyl chain. Prior to the complete analysis of this new substrate by experimental and QM/MM methods, the change of the charge generated by the introduced substitution on the atoms of the amide bond was computed in the gas phase, and inside the active site of the enzyme, using the $\mathrm{CHelpG}^{44}$ method at the M06-2X level with the standard 6$31+\mathrm{G}^{* *}$ basis set. The resulting maps of electrostatic potentials are shown in Figure 3D,E. These preliminary results confirmed the more positive character of the carbonyl carbon of the amide in $\mathbf{2}$, compared to that in $\mathbf{1}$. An increase in the negative charge on the nitrogen atom of the peptide bond is additionally observed. It is important to point out the additional electrostatic effects of the protein on the charge distribution of the substrate, as can be deduced from the comparison between the charges computed in the gas phase and in the active site of the enzyme. Thus, we proceed to study the hydrolysis of 2 catalyzed by CALB by following the same strategy as the one carried out with substrate 1 .

QM/MM Simulations. The FES of the first step of the hydrolysis of 2, shown in Figure 3B, renders a free energy barrier of $19.5 \mathrm{kcal} \mathrm{mol}^{-1}$, which represents a dramatic decrease of $15.9 \mathrm{kcal} \mathrm{mol}^{-1}$ with respect to 1 . As expected, this meaningful decrease can be associated with the change in the charge of the carbonyl carbon of the amide that increases the electrophilic character of the carbon atom and facilitates the attack of the Ser105. Additionally, it was also observed that INT1 is more stable than in the case of 1 . This is due to the specific interaction between the substrate and the oxyanion hole, which stabilizes the formation of the tetrahedral intermediate and the negative charge developed on the oxygen atom.

These results convert $\mathbf{2}$ into an interesting substrate to study the full mechanism of hydrolysis catalyzed by CALB. Thus, on the basis of these encouraging results, the subsequent steps of the reaction were explored. The corresponding FESs are shown in Figure 4, and a scheme of the full reaction is displayed in Figure 5. Key distances on all different states, structures of the different TSs localized at M06-2X/MM level, averaged values of key interatomic distances obtained at AM1/MM level, and a list of charges computed at DFT/MM level on the key atoms for all structures appearing along the reaction are presented in the Supporting Information.

The second step of the reaction involves the proton transfer from His 224 to the nitrogen atom of the amide. This leads to the breaking of the $\mathrm{C}-\mathrm{N}$ bond and the formation of the 4nitroaniline leaving group (INT2). Interestingly, the amphoteric character of His224 is modulated by the interaction with Asp 187, revealed by the analysis of the interatomic distances reported in Table S4 of the Supporting Information. Thus, the $\mathrm{HD} 1_{\mathrm{His} 224}-\mathrm{OD} 2_{\mathrm{Asp} 187}$ distance was significantly shorter during the first step (including reactants state, $2.02 \AA$ ) than in the second step. This result suggests that the protein adopts a conformation favoring the basic character of His224 that has to accept the proton from Ser105, originally protonated in reactants. In the second step, where His224 is releasing the proton to $\mathrm{N} 4$ and thus acting as an acid, this distance reaches the largest value $(2.19 \AA)$. The His224-Asp187 distance will be reduced in the third step when the former residue acts as base to abstract a proton from an active site water molecule.
Finally, and perhaps unexpectedly, the protein adopts a conformation in products where, despite His224 is neutral, the distance between this two residues of the dyad is really close $(2.00 \AA)$. This suggests that the enzyme is regenerated and ready to accept again the proton from Ser105. Turning to the analysis of second step, the position of TS2 on the FES (Figure 4) reveals that the $\mathrm{C}-\mathrm{N}$ bond breaking takes place almost spontaneously after the proton transfer, thus evolving to a very stable INT2. The remainder of the substrate is maintained anchored to the protein through a covalent interaction with Ser105 and a very strong hydrogen bonding network established between the $\mathrm{O} 2$ oxygen atom and the Thr40 and Gln106 residues.

The third and fourth steps involve the regeneration of the enzyme through the hydrolysis of the acyl-enzyme complex. The third step is initiated by the attack of a water molecule to the activated $\mathrm{C} 1$ atom. In fact, the charge on $\mathrm{C} 1$ carbon atom in INT2 is $0.82 \pm 0.03 \mathrm{au}$, which is noticeably higher than its value in the gas phase, 0.78 au. As occurred in the reactant state, where the $\mathrm{C} 1$ atom reached a high positive value favoring the nucleophilic attack of Ser105 (Figure 3E), the protein has an active electrostatic effect in this step, making $\mathrm{C} 1$ a better electrophile for the attack of the water molecule in INT2. The increase of the positive charge on $\mathrm{C} 1$ is assisted by the polarization of the $\mathrm{C} 1-\mathrm{O} 2$ bond through the interactions between $\mathrm{O} 2$ and the oxyanion hole of the active site. As shown in Tables S4 and S5, the negative charge accumulated on $\mathrm{O} 2$ in INT1 $(-1.104 \pm 0.017 \mathrm{au})$ is stabilized by strong interactions with Gln106 and Thr40, and especially with the $\mathrm{OH}$ of the later, whose interatomic distance reaches one of the shortest value $(1.66 \AA)$ in INT2. The distance between $\mathrm{C} 1$ and $\mathrm{N} 4$ of the substrate in INT2 is $2.84 \AA$, but it is elongated up to $3.81 \AA$ to leave space enough for the water to be correctly posed. Interestingly, the polarization of the substrate is achieved not only by the oxyanion hole (Thr40 and Gln106) but also by the contribution of the catalytic triad (Ser105, His224, and Asp187) that appears to be essential to reach the final values obtained when the full enzyme is considered into the calculations (see Table S6 of the Supporting Information). The water is anchored in the active site by a hydrogen bond interaction with His224 that, in turn, activates it for the attack to the $\mathrm{C} 1$ carbonyl carbon. During this process, a new tetrahedral intermediate, INT3, is formed (see Figure 5). The release of the butyric acid and the regeneration of the active site take place in the last step. Interestingly, the breaking of the bond between the acyl chain of the substrate and Ser105 (C1OG), thus generating a molecule of butyric acid, is assisted by the transfer of the proton from His 224 to Ser105.

From the free energy landscape of the whole reaction, as shown in Figure 6, we can conclude that the rate-limiting step is the regeneration of the enzyme, determined by the TS4 in the case of the amidase activity. Nevertheless, the breaking of the amide bond rendering the 4-nitroaniline (reactant state to INT2), which is the step we are experimentally monitoring since initial velocities are measured, is kinetically controlled by the second step of the reaction, in which the $\mathrm{C}-\mathrm{N}$ bond of the amide is broken. This step gives a total activation free energy $\left(\Delta G^{\ddagger}\right)$ of $23.1 \mathrm{kcal} \mathrm{mol}^{-1}$, which corresponds to a $k_{\mathrm{cat}}=1.4 \times$ $10^{-4} \mathrm{~s}^{-1}$. By comparison with the free energy profile of the primary reaction of CALB, ${ }^{18}$ both the amidase and the esterase reaction mechanisms proceed by a four-step mechanism but, as expected, the free energy barrier of the $\mathrm{C}-\mathrm{N}$ bond breaking of the substrate in the secondary reaction is noticeably higher 


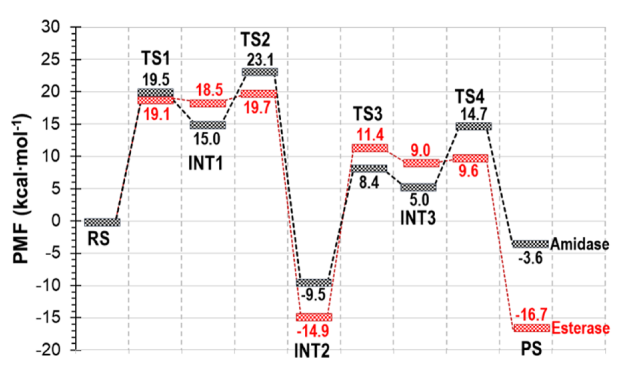

Figure 6. Free energy profile of the hydrolysis of 2 catalyzed by CALB obtained at the M06-2X:AM1/MM level (black lines). Energies are derived from the FESs displayed in Figures 3 and 4. The free energy profile of the primary reaction of CALB with 3 (Figure $3 F$ ), from ref 18 , is shown by red lines.

than that of the $\mathrm{C}-\mathrm{O}$ bond breaking in the primary reaction (see the red profile in Figure 6).

Experimental Evaluation. Experimental kinetics using commercial CALB was studies to confirm the predictions derived from the theoretical simulations with compound $\mathbf{2}$ as a substrate. Specific activities as a function of the concentration of substrate were fitted in a Michaelis-Menten curve. The derived kinetic constants (Table 1 ) were determined to be $k_{\text {cat }}$

Table 1. Substrate Dependency on Kinetic Values of the Hydrolysis of the Amide Bond by CALB Derived from Experimental Studies ${ }^{1}$

$\begin{array}{lcc}\text { substrate } & \begin{array}{c}\text { benzylbutyramide } \\ (\mathbf{1})\end{array} & \begin{array}{c}\text { N-(4-nitrophenyl)- } \\ \text { butyramide (2) }\end{array} \\ V_{\max } \times 10^{-4}\left(\mathrm{U} \mathrm{mg}^{-1}\right) & \mathrm{n} / \mathrm{a} & 5.24 \pm 1.07 \\ k_{\mathrm{cat}} \times 10^{-4}\left(\mathrm{~s}^{-1}\right) & \mathrm{n} / \mathrm{a} & 2.9 \pm 0.6 \\ K_{\mathrm{m}} \times 10^{-3}(\mathrm{M}) & \mathrm{n} / \mathrm{a} & 4.2 \pm 1.3 \\ k_{\mathrm{cat}} / K_{\mathrm{m}} \times 10^{-2}\left(\mathrm{M}^{-1} \mathrm{~s}^{-1}\right) & \mathrm{n} / \mathrm{a} & 6.90 \pm 2.60 \\ { }^{1} \text { No activity could be determined on substrate } \mathbf{1 .}\end{array}$

$=(2.9 \pm 0.6) \times 10^{-4} \mathrm{~s}^{-1}$ and $K_{\mathrm{m}}=(4.2 \pm 1.3) \times 10^{-3} \mathrm{M}$. A total activation free energy of $\Delta G^{\ddagger}=22.7 \mathrm{kcal} \mathrm{mol}^{-1}$ can be derived from the measured $k_{\text {cat }}$ within the framework of the transition state theory, which is in extraordinary good agreement with the theoretical predicted value derived from the exploration of the FES $\left(\Delta G^{\ddagger}=23.1 \mathrm{kcal} \mathrm{mol}^{-1}\right)$. Either theory or experiment suggests that CALB is capable to break the amide bond, but it is very dependent on specific features of the substrate. This result can be considered in agreement with kinetic studies of Bornscheuer and co-workers, who measured esterase and amidase activities of an esterase from Bacillus subtilis (BS2, E.C. 3.1.1.3.) on p-nitro-phenyl butyrate and the corresponding anilide. ${ }^{45}$ We herein show that the impact of introducing a nitro group in the phenyl group and the shortening of the acyl chain of the substrate is dramatic on the first step of the reaction that leads to an improvement of the promiscuous amidase reaction carried out by CALB. The free energy profile, as deduced from our $\mathrm{QM} / \mathrm{MM}$ calculations, shows that despite the first step is not determining the rate of the hydrolysis of the substrate the stabilization of this TS1 and the INT1 has an effect on the energy of the TS2 relative to the reactant state. In addition, the protein appears to have significant electrostatic effects on different states of the reaction as deduced from the comparison of the atomic charges in the gas phase and in the protein active site.
Kinetic Isotope Effects. To support the proposed reaction mechanism, as summarized in Figures 5 and 6, the localized TS structures at M06-2X/MM were used to compute KIEs after isotopic substitution of key heavy atoms involved in the reaction, i.e., ${ }^{12 / 14} \mathrm{C},{ }^{14 / 15} \mathrm{~N}$, and ${ }^{16 / 18} \mathrm{O}$ of the amide bond. The use of different TSs appearing along the reaction path allows computing KIEs by assuming different possible rate-determining steps. It is important to point out that the inherent broad distribution of enzyme configurations dictates that values such as geometrical coordinates, tunneling and recrossing transmission coefficients, KIEs, etc. should be reported as ensemble averages at a certain temperature. ${ }^{46}$ In this case, while the KIE values at a high level were obtained from a combination of a single couple of structure of TS and reactants, due to computational limitations, the use of 10 different structures of each TSs and reactants state at AM1/MM level allows obtaining an arithmetic mean and reporting the corresponding standard deviations (deposited in the Supporting Information). These suggest that the uncertainty on the M06-2X/MM values, as reported in Table 2, can be on the third decimal place.

Table 2. KIEs Computed at the M06-2X/MM Level for the Reaction of Hydrolysis of 2 Using the Different TS Structures Located along the Reaction Path

\begin{tabular}{ccccc} 
& TS1 1 & TS2 & TS3 & TS4 \\
{$\left[1-{ }^{14} \mathrm{C}\right]$} & 1.050 & 1.039 & 1.056 & 1.052 \\
{$\left[4-{ }^{-15} \mathrm{~N}\right]$} & 1.009 & 1.017 & & \\
{$\left[2{ }^{18} \mathrm{O}\right]$} & 1.008 & 1.010 & 1.002 & 1.015 \\
\hline
\end{tabular}

From the values of the KIEs computed at the M06-2X/MM level, the ${ }^{12 / 14} \mathrm{C}$ isotopic substitution of $\mathrm{C} 1$ presents a normal KIE $(>1)$ when using any of the TSs. As deduced from the scheme of the reaction depicted in Figure 5, a change of $\mathrm{sp}^{2}$ hybridization to $\mathrm{sp}^{3}$ on C1 occurs in TS1 and TS3, while an inverse rehybridization, $\mathrm{sp}^{3}$ to $\mathrm{sp}^{2}$, takes place in TS2 and TS4. Thus, an inverse ${ }^{14} \mathrm{C}$-KIE could be expected if TS1 or TS 3 was the rate-limiting step, and a normal KIE in TS2 and TS4. Nevertheless, multiple changes occur on $\mathrm{C} 1$ in every step. For instance, in the first step, formation of the C1-OG bond is associated with the elongation of the $\mathrm{C} 1-\mathrm{O} 2$ distance (from 1.26 to $1.29 \AA$ ) and an elongation of the $\mathrm{C} 1-\mathrm{N} 4$ bond (from 1.36 to $1.44 \AA$ ). All of these changes render a normal KIE of $5.0 \%$. In TS3, similar changes occur and thus again a normal $5.6 \% \mathrm{KIE}$ is observed. In TS2, the $\mathrm{C} 1-\mathrm{N} 4$ bond is broken and consequently a normal, and expected, effect of $3.9 \%$ is observed, the same as in the last step where the breaking of the bond between $\mathrm{C} 1$ and Ser105-OG governs the KIE, leading a normal 5.6\% KIE. In the case of the ${ }^{14 / 15} \mathrm{~N}$ substitution, the force constants associated with the $\mathrm{N} 4$ atom are almost invariant in the first step, where the $\mathrm{C} 1-\mathrm{N} 4$ bond is just slightly elongated (from $1.36 \AA$ in reactants state to $1.44 \AA$ in TS1). Consequently, a very small $0.9 \%$ normal ${ }^{15} \mathrm{~N}$-KIE is obtained by considering the first step as the rate-limiting step. In the second step, the $\mathrm{C} 1-\mathrm{N} 4$ bond is broken but a hydrogen transfer takes place from His224. In all, a slightly higher normal ${ }^{15} \mathrm{~N}$-KIE of $1.7 \%$ is obtained for the second step. Since N4 belongs to the leaving group and does not participate in steps 3 and 4, no KIEs for last two steps were computed. Finally, the ${ }^{16 / 18} \mathrm{O}$ substitution renders normal KIEs for any of the four TS structures selected as possible rate-determining steps. As observed in Table 2, and despite the $\mathrm{O} 2-\mathrm{C} 1$ bond is changing 
from double to single, or vice versa, in the different chemical steps of the proposed mechanism, a normal KIE is obtained in all cases. This can be rationalized based on the strong hydrogen bond interactions established between the $\mathrm{O} 2$ atom and the oxyanion hole formed by Gln106 and Thr40. In those steps where the $\mathrm{O} 2-\mathrm{C} 1$ bond is becoming a single bond (TS1 and TS3), the negative charge accumulated in O2 favors the establishment of stronger interactions with the oxyanion hole and, consequently, stiffer force constants that compensate the loss of the $\mathrm{C} 1-\mathrm{O} 2$ force constant. These results suggest that measurements of KIEs would not provide conclusive values that allow determining the rate-determining step.

Amidase vs Esterase Activity of CALB. Our computational results, summarized in Figures 5 and 6, combined with our previous study on the CALB primary reaction, ${ }^{18}$ show how the promiscuous esterase CALB employs the same molecular mechanism to catalyze the hydrolysis of $\mathbf{2}$ as the natural ester substrate. Thus, in both cases, the reaction is initiated by the attack of the hydroxyl oxygen atom of Ser105 on the carbonyl carbon atom of the substrate, followed by the $\mathrm{C}-\mathrm{N}$ or $\mathrm{C}-\mathrm{O}$ bond breaking, for the amidase or esterase activity, respectively. The comparison of the free energy profiles for both reactions (Figure 6) shows how these two first steps determine the decomposition of the substrate. In the case of methyl acrylate, the relative free energy of the TS associated with the $\mathrm{C}-\mathrm{O}$ bond breaking with respect to reactants was $19.7 \mathrm{kcal} \mathrm{mol}^{-1}$, $^{18}$ while in the present study, the TS of the C$\mathrm{N}$ bond breaking is $23.1 \mathrm{kcal} \mathrm{mol}^{-1}$, higher than the energy of reactants state. This result is in agreement with the experimentally evidence of a higher initial velocities of CALB for the primary reaction (as esterase) than for the secondary reaction (as amidase). The population analysis of both substrates computed in gas phase, as reported in Figure $3 \mathrm{E}, \mathrm{F}$, shows how the positive charge on $\mathrm{Cl}$ carbonyl carbon is significantly higher in compound $3(0.83 \mathrm{au})$ than in $2(0.78$ $\mathrm{au})$, which is in agreement with a more favorable electrophilic attack of the hydroxyl oxygen atom of Ser105 in the first step of the reaction for the primary reaction. However, the understanding of catalysis by CALB is not limited to the discussion of the electrostatic nature of the substrates in the gas phase. Once the substrates are posed in the active site of CALB, the charge of $\mathrm{C} 1$ atom is increased in the amide and in the ester, to 1.11 and 1.08 au, respectively. Thus, this promiscuous enzyme is able to activate both substrates, which explains its catalytic activities as esterase and amidase (despite for certain amides, as proved above). Nevertheless, the larger positive charge of $\mathrm{C} 1$ in the amide substrate than in the ester reveals that the higher esterase activity cannot be rationalized based on just the polarization of the substrate. The protein is also polarizing the breaking bond in the first intermediate, stabilizing the TS2 and thus facilitating the second step by an adequate charge distribution of the active site residues and a synchronized reorganization. In both cases, the positive charge on $\mathrm{Cl}$ atom is increased, as well as the negative charge of the departing $\mathrm{N}$ atom (see the Supporting Information and charges reported in ref 18). Interestingly, the electrostatic effects of the protein decomposed by residues reveal how the total effect of the enzyme is basically due to short-distance electrostatic interactions with the residues of the oxyanion hole and the catalytic triad of the active site.

\section{CONCLUSIONS}

In this work, the promiscuous amidase activity of CALB has been studied with two different short-acyl chain substrates. The results derived from experimental kinetic studies and $\mathrm{QM} / \mathrm{MM}$ molecular dynamics (MD) simulations are in excellent agreement and show how the secondary activity of CALB is very dependent on specific features of the substrate. In particular, CALB can present amidase activity when introducing a nitro group into the para position of the substrate phenyl group, together with a reduction of the acyl chain. This conclusion is in agreement with the studies of Bornscheuer and co-workers ${ }^{45}$ and Hult and co-workers, ${ }^{47}$ who proposed compound $\mathbf{2}$ as a substrate for an esterase of Bacillus subtilis and for CALB, respectively. This is due to the electrophilic character of the nitro group that causes the displacement of the electrons toward the direction of the ring and the proximity of the lone pair of the aniline nitrogen to the ring that facilitates the electron delocalized into the aromatic ring. Nevertheless, our $\mathrm{QM} / \mathrm{MM}$ simulations reveal the remarkable role of the protein in the catalysis by generating an electric field in the active site that polarizes and activates the substrate, as well as stabilizes the charge distribution on the rate-determining TS of the decomposition of the substrate. Our results show how arguments based on just the features of the substrate in the gas phase cannot explain the substrate dependence of the activity of CALB as amidase. ${ }^{48}$

CALB uses the same molecular mechanism to catalyze the secondary reaction and the primary reaction previously studied in our laboratory. ${ }^{18}$ Our $\mathrm{QM} / \mathrm{MM}$ simulations indicate that the breaking of the $\mathrm{C}-\mathrm{N}$ bond of substrate 2 and the regeneration of the protein take place by a four steps mechanism, with an overall activation free energy for the decomposition of the amide molecule of $23.1 \mathrm{kcal} \mathrm{mol}^{-1}$. This barrier is very close to the one derived from our rate constant corresponding to the measurements of the initial velocities that, within the frame of the transition state theory, gives a free energy of activation of $22.7 \mathrm{kcal} \mathrm{mol}^{-1}$. CALB activates both kind of substrates by increasing the electrophile character of the carbonyl carbon atom, thus facilitating the attack of the Ser105 and stabilizing the negative charge developed in the oxygen atom of the original carbonyl group. In addition, the protein polarizes the scissile bond, thus reducing the free energy barrier of the following and decisive breaking bond step. Interestingly, while this is an almost a barrierless process in the primary reaction, the $\mathrm{C}-\mathrm{N}$ bond breaking costs ca. 8 $\mathrm{kcal} \mathrm{mol}^{-1}$, thus explaining the different activities of CALB at short times.

$\mathrm{QM} / \mathrm{MM}$ simulations show that measurements of heavy atoms KIEs would render small normal effects, which could confirm our predicted description of the reaction at the atomic level. However, they are not able to differentiate the ratelimiting step 2 from the other steps described in this mechanism.

The analysis of geometries, energies, and electronic features of the stationary states appearing along the reaction path of the hydrolysis of the natural substrate (primary reaction) or the promiscuous amide substrate (secondary reaction) explains the way CALB is catalyzing both reactions. Our computational results suggest that the barrier that determines the rate constant of the formation of the first product (the 4nitroaniline) could be reduced by specific mutations on the protein. In particular, as deduced from the analysis of the 
corresponding FESs, mutations should be oriented in producing electrostatic effects that favor the rate-determining proton transfer from His 225 to the $\mathrm{N} 4$ atom of the substrate that precedes the $\mathrm{C} 1-\mathrm{N} 4$ bond breaking. Further studies should be also focused in trying to stabilize the TSs of the second stage of the reaction (i.e., the regeneration of the enzyme) that, in both primary and secondary reaction consist in the attack of a water molecule and the departure of the acid (acrylic or butyric acid in the case of the primary or secondary reactions, respectively). In all, the present study illustrates how merging experimental and computational techniques can speed up our understanding of these complex biological catalysts and how to modify them to generate new biotechnological tools.

\section{ASSOCIATED CONTENT}

\section{S Supporting Information}

The Supporting Information is available free of charge at https://pubs.acs.org/doi/10.1021/acscatal.9b04002.

Details of computational and experimental methods; time dependence of RMSD of backbone atoms in the equilibration $\mathrm{MD}$ simulations; transition state structures localized at M06-2X/MM; average KIEs and geometries of key states obtained at the AM1/MM level; charges on key atoms along the reaction at the M06-2X/MM level; NMR spectra; and kinetics of hydrolysis of $\mathrm{N}$-(4nitrophenyl)-butyramide (PDF)

\section{AUTHOR INFORMATION}

\section{Corresponding Authors}

*E-mail: eduardo.junceda@csic.es.

*E-mail: swiderek@uji.es.

*E-mail: moliner@uji.es.

\section{ORCID}

Eduardo García-Junceda: 0000-0002-2344-8743

Katarzyna Świderek: 0000-0002-7528-1551

Vicent Moliner: 0000-0002-3665-3391

\section{Author Contributions}

M.À.G. carried out all of the calculations and the kinetic experiments and E.G.-J., K.S., and V.M. designed the project and analyzed the results. All authors contributed to writing the manuscript.

\section{Notes}

The authors declare no competing financial interest.

\section{ACKNOWLEDGMENTS}

This work was supported by the Spanish Ministerio de Ciencia, Innovación y Universidades (Grant PGC2018-094852-B-C21), the Spanish Ministerio de Economía y Competitividad (Grant MAT2015-65184-C2-2-R), Universitat Jaume I (project UJIB2017- 31), and the National Institutes of Health (Ref no. NIH R01 GM065368). K.S. thanks the MINECO for a Juan de la Cierva-Incorporación (ref IJCI-2016-27503) contract. M.À.G. thanks Universitat Jaume I for a doctoral FPI grant (PREDOC/2017/23). The authors acknowledge computational resources from the Servei d'Informàtica of Universitat Jaume I. The authors also thank Dr. I. Sánchez and Dr. R. Benito for technical assistance in the kinetic studies and chemical syntheses.

\section{REFERENCES}

(1) Humble, M. S.; Berglund, P. Biocatalytic Promiscuity. Eur. J. Org. Chem. 2011, 2011, 3391-3401.

(2) Copley, S. D. Shining a Light on Enzyme Promiscuity. Curr. Opin. Struct. Biol. 2017, 47, 167-175.

(3) Sheldon, R. A.; Brady, D. The Limits to Biocatalysis: Pushing the Envelope. Chem. Commun. 2018, 54, 6088-6104.

(4) Busto, E.; Gotor-Fernández, V.; Gotor, V. Hydrolases: Catalytically Promiscuous Enzymes for Non-Conventional Reactions in Organic Synthesis. Chem. Soc. Rev. 2010, 39, 4504-4523.

(5) Gotor-Fernández, V.; Brieva, R.; Gotor, V. Lipases: Useful Biocatalysts for the Preparation of Pharmaceuticals. J. Mol. Catal. B: Enzym. 2006, 40, 111-120.

(6) Ramirez-Escudero, M.; Molina-Espeja, P.; de Santos, G.; Hofrichter, M.; Sanz-Aparicio, J.; Alcalde, M. Structural Insights into the Substrate Promiscuity of a Laboratory-Evolved Peroxygenase. ACS Chem. Biol. 2018, 13, 3259-3268.

(7) Renata, H.; Wang, Z. J.; Arnold, F. H. Expanding the Enzyme Universe: Accessing Non-Natural Reactions by Mechanism-Guided Directed Evolution. Angew. Chem., Int. Ed. 2015, 54, 3351-3367.

(8) Bloom, J. D.; Arnold, F. H. In the Light of Directed Evolution: Pathways of Adaptive Protein Evolution. Proc. Natl. Acad. Sci. U.S.A. 2009, 106, 9995-10000.

(9) Nobeli, I.; Favia, A. D.; Thornton, J. M. Protein Promiscuity and Its Implications for Biotechnology. Nat. Biotechnol. 2009, 27, 157167.

(10) Sánchez-Moreno, I.; Iturrate, L.; Martín-Hoyos, R.; Jimeno, M. L.; Mena, M.; Bastida, A.; García-Junceda, E. From Kinase to Cyclase: An Unusual Example of Catalytic Promiscuity Modulated by Metal Switching. ChemBioChem 2009, 10, 225-229.

(11) Molina-Espeja, P.; Viña-Gonzalez, J.; Gomez-Fernandez, B. J.; Martin-Diaz, J.; Garcia-Ruiz, E.; Alcalde, M. Beyond the Outer Limits of Nature by Directed Evolution. Biotechnol. Adv. 2016, 34, 754-767.

(12) Aharoni, A.; Gaidukov, L.; Khersonsky, O.; Gould, S. M.; Roodveldt, C.; Tawfik, D. S. The "evolvability" of Promiscuous Protein Functions. Nat. Genet. 2005, 37, 73-76.

(13) Wikmark, Y.; Humble, M. S.; Bäckvall, J. E. Combinatorial Library Based Engineering of Candida Antarctica Lipase a for Enantioselective Transacylation of Sec-Alcohols in Organic Solvent. Angew. Chem., Int. Ed. 2015, 54, 4284-4288.

(14) Maria, S.; Carlqvist, P.; Branneby, C.; Allnér, O.; Frise, A.; Hult, K.; Berglund, P.; Brinck, T. Direct Epoxidation in Candida Antarctica Lipase B Studied by Experiment and Theory. ChemBioChem 2008, 9, 2443-2451.

(15) Chen, X. Y.; Chen, G. J.; Wang, J. L.; Wu, Q.; Lin, X. F. Lipase/ Acetamide-Catalyzed Carbon-Carbon Bond Formations: A Mechanistic View. Adv. Synth. Catal. 2013, 355, 864-868.

(16) Sarmah, N.; Revathi, D.; Sheelu, G.; Rani, K. Y.; Sridhar, S.; Mehtab, V.; Sumana, C. Recent Advances on Sources and Industrial Applications of Lipases. Biotechnol. Prog. 2018, 34, 5-28.

(17) Uppenberg, J.; Morgens, H.; Shamkant, P.; Alwyn, J. T. The Sequence, Crystal Structure Determination and Refinement of Two Crystal Forms of Lipase B from Candida Antarctica. Structure 1994, 2, 293-308.

(18) Świderek, K.; Martí, S.; Moliner, V. Theoretical Study of Primary Reaction of Pseudozyma Antarctica Lipase B as the Starting Point to Understand Its Promiscuity. ACS Catal. 2014, 4, 426-434.

(19) Buchholz, K. A Breakthrough in Enzyme Technology to Fight Penicillin Resistance-Industrial Application of Penicillin Amidase. Appl. Microbiol. Biotechnol. 2016, 100, 3825-3839.

(20) Escorcia, A. M.; Molina, D.; Daza, M. C.; Doerr, M. Acetylation of (R,S)-Propranolol Catalyzed by Candida Antarctica Lipase B: An Experimental and Computational Study. J. Mol. Catal. B: Enzym. 2013, 98, 21-29.

(21) Raza, S.; Fransson, L.; Hult, K. Enantioselectivity in Candida Antarctica Lipase B: A Molecular Dynamics Study. Protein Sci. 2001, 10, 329-338.

(22) Engström, K.; Vallin, M.; Syrén, P. O.; Hult, K.; Bäckvall, J. E. Mutated Variant of Candida Antarctica Lipase B in (S)-Selective 
Dynamic Kinetic Resolution of Secondary Alcohols. Org. Biomol. Chem. 2011, 9, 81-82.

(23) Torres-Gavilán, A.; Castillo, E.; López-Munguía, A. The Amidase Activity of Candida Antarctica Lipase B Is Dependent on Specific Structural Features of the Substrates. J. Mol. Catal. B: Enzym. 2006, 41, 136-140.

(24) Dewar, M. J. S.; Zoebisch, E. G.; Healy, E. F.; Stewart, J. J. P. Development and Use of Quantum Mechanical Molecular Models. 76. AM1: A New General Purpose Quantum Mechanical Molecular Model. J. Am. Chem. Soc. 1985, 107, 3902-3909.

(25) Zhao, Y.; Truhlar, D. G. The M06 Suite of Density Functionals for Main Group Thermochemistry, Thermochemical Kinetics, Noncovalent Interactions, Excited States, and Transition Elements: Two New Functionals and Systematic Testing of Four M06-Class Functionals and 12 Other Function. Theor. Chem. Acc. 2008, 120, 215-241.

(26) Jorgensen, W. L.; Tirado-rives, J. The OPLS Potential Functions for Proteins. Energy Minimizations for Crystals of Cyclic Peptides and Crambin. J. Am. Chem. Soc. 1988, 110, 1657-1666.

(27) Jorgensen, W. L.; Maxwell, D. S.; Tirado-Rives, J. Development and Testing of the OPLS All-Atom Force Field on Conformational Energetics and Properties of Organic Liquids. J. Am. Chem. Soc. 1996, 118, 11225-11236.

(28) Jorgensen, W. L.; Chandrasekhar, J.; Madura, J. D.; Impey, R. W.; Klein, M. L. Comparison of Simple Potential Functions for Simulating Liquid Water. J. Chem. Phys. 1983, 79, 926-935.

(29) Roux, B. The Calculation of the Potential of Mean Force Using Computer-Simulation. Comput. Phys. Commun. 1995, 91, 275-282.

(30) Thomas, A.; Jourand, D.; Bret, C.; Amara, P.; Field, M. J. Is There a Covalent Intermediate in the Viral Neuraminidase Reaction? A Hybrid Potential Free-Energy Study. J. Am. Chem. Soc. 1999, 121, 9693-9702.

(31) Poulsen, T. D.; Garcia-Viloca, M.; Gao, J.; Truhlar, D. G. Free Energy Surface, Reaction Paths, and Kinetic Isotope Effect of ShortChain Acyl-CoA Dehydrogenase. J. Phys. Chem. B 2003, 107, 95679578.

(32) Torrie, G. M.; Valleau, J. P. Non-Physical Sampling Distibutions in Monte-Carlo Free-Energy Estimation - Umbrella Sampling. J. Comput. Phys. 1977, 23, 187-199.

(33) Kumar, S.; Rosenberg, J. M.; Bouzida, D.; Swendsen, R. H.; Kollman, P. A. The Weighted Histogram Analysis Method for Freeenergy Calculations on Biomolecules. I. The Method. J. Comput. Chem. 1992, 13, 1011-1021.

(34) Field, M. J.; Albe, M.; Bret, C.; Proust-De Martin, F.; Thomas, A. The Dynamo Library for Molecular Simulations Using Hybrid Quantum Mechanical and Molecular Mechanical Potentials. J. Comput. Chem. 2000, 21, 1088-1100.

(35) Corchado, J. C.; Coitiño, E. L.; Chuang, Y. Y.; Fast, P. L.; Truhlar, D. G. Interpolated Variational Transition-State Theory by Mapping. J. Phys. Chem. A 1998, 102, 2424-2438.

(36) Nguyen, K. A.; Rossi, I.; Truhlar, D. G. A Dual-Level Shepard Interpolation Method for Generating Potential Energy Surfaces for Dynamics Calculations. J. Chem. Phys. 1995, 103, 5522-5530.

(37) Chuang, Y. Y.; Corchado, J. C.; Truhlar, D. G. Mapped Interpolation Scheme for Single-Point Energy Corrections in Reaction Rate Calculations and a Critical Evaluation of Dual-Level Reaction Path Dynamics Methods. J. Phys. Chem. A 1999, 103, 1140-1149.

(38) Javier Ruiz-Pernía, J. J.; Silla, E.; Tuñón, I.; Martí, S.; Moliner, V. Hybrid QM/MM Potentials of Mean Force with Interpolated Corrections. J. Phys. Chem. B 2004, 108, 8427-8433.

(39) Javier Ruiz-Pernía, J. J.; Silla, E.; Tuñón, I.; Martí, S. Hybrid Quantum Mechanics/Molecular Mechanics Simulations with TwoDimensional Interpolated Corrections: Application to Enzymatic Processes. J. Phys. Chem. B 2006, 110, 17663-17670.

(40) Frisch, M. J.; Trucks, G. W.; Schlegel, H. B.; Scuseria, G. E.; Robb, M. A.; Cheeseman, J. R.; Scalmani, G.; Barone, V.; Mennucci, B.; Petersson, G. A.; Nakatsuji, H.; Caricato, M.; Li, X.; Hratchian, H. P.; Izmaylov, A. F.; Bloino, J.; Zheng, G.; Sonnenberg, J. L.; Hada, M.; Ehara, M.; Toyota, K.; Fukuda, R.; Hasegawa, J.; Ishida, M.;
Nakajima, T.; Honda, Y.; Kitao, O.; Nakai, H.; Vreven, T. Montgomery, J. A., Jr.; Peralta, J. E.; Ogliaro, F.; Bearpark, M.; Heyd, J. J.; Brothers, E.; Kudin, K. N.; Staroverov, V. N.; Kobayashi, R.; Normand, J.; Raghavachari, K.; Rendell, A.; Burant, J. C.; Iyengar, S. S.; Tomasi, J.; Cossi, M.; Rega, N.; Millam, J. M.; Klene, M.; Knox, J. E.; Cross, J. B.; Bakken, V.; Adamo, C.; Jaramillo, J.; Gomperts, R.; Stratmann, R. E.; Yazyev, O.; Austin, A. J.; Cammi, R.; Pomelli, C.; Ochterski, J. W.; Martin, R. L.; Morokuma, K.; Zakrzewski, V. G.; Voth, G. A.; Salvador, P.; Dannenberg, J. J.; Dapprich, S.; Daniels, A. D.; Farkas, O.; Foresman, J. B.; Ortiz, J. V.; Cioslowski, J.; Fox, D. J. Gaussian 09, revision E.01; Gaussian, Inc.: Wallingford, CT, 2009.

(41) Martí, S.; Moliner, V.; Tuñón, I.; Williams, I. H. QM/MM Calculations of Kinetic Isotope Effects in the Chorismate Mutase Active Site. Org. Biomol. Chem. 2003, 1, 483-487.

(42) Swiderek, K.; Moliner, V. Computational Studies of Candida Antarctica Lipase B to Test Its Capability as a Starting Point to Redesign New Diels-Alderases. J. Phys. Chem. B 2016, 120, 20532070.

(43) Eyring, H. The Activated Complex in Chemical Reactions. J. Chem. Phys. 1935, 3, 107-115.

(44) Breneman, C. M.; Wiberg, K. B. Determining Atom-centered Monopoles from Molecular Electrostatic Potentials. The Need for High Sampling Density in Formamide Conformational Analysis. J. Comput. Chem. 1990, 11, 361-373.

(45) Kourist, R.; Bartsch, S.; Fransson, L.; Hult, K.; Bornscheuer, U. T. Understanding Promiscuous Amidase Activity of an Esterase from Bacillus Subtilis. ChemBioChem 2008, 9, 67-69.

(46) Pu, J.; Gao, J.; Truhlar, D. G. Multidimensional Tunneling, Recrossing, and the Transmission Coefficient for Enzymatic Reactions. Chem. Rev. 2006, 106, 3140-3169.

(47) Syrén, P. O.; Hendil-Forssell, P.; Aumailley, L.; Besenmatter, W.; Gounine, F.; Svendsen, A.; Martinelle, M.; Hult, K. Esterases with an Introduced Amidase-Like Hydrogen Bond in the Transition State Have Increased Amidase Specificity. ChemBioChem 2012, 13, 645648.

(48) Świderek, K.; Pabis, A.; Moliner, V. A Theoretical Study of Carbon-Carbon Bond Formation by a Michael-Type Addition. Org. Biomol. Chem. 2012, 10, 5598-5605. 



\subsubsection{Direct epoxidation of short alkenes catalyzed by CALB (Article 2)}

Enzyme catalysis is often preferred over traditional chemical synthesis, because of its environmentally friendly character, and high efficiency and selectivity under mild conditions of temperature. ${ }^{9}$ Thus, the use of enzymes to produce epoxides is an attractive approach.

In this article, we studied the direct epoxidation of a short alkene, 2butenal, by CALB, more precisely the Ser105Ala variant, using hydrogen peroxide. We compared this approach with the synthesis of the same epoxide through the Prilezhaev reaction ${ }^{10}$ occurring in an organic solvent, which uses peroxy-acids to render the epoxide from the alkene. From the QM/MM calculations, we concluded that the reaction mechanism of epoxidation of alkenes in CALB with hydrogen peroxide takes place through a two-step mechanism (Figure 4.4a), as proposed in other studies. ${ }^{4}$

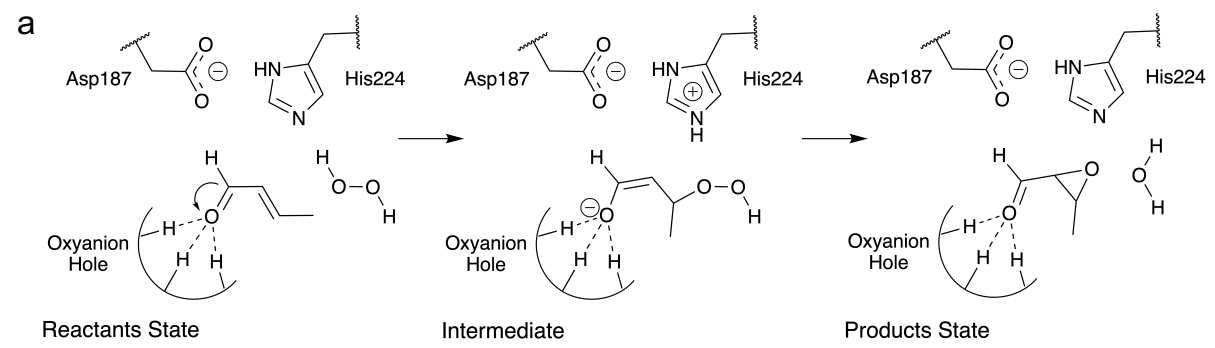

b

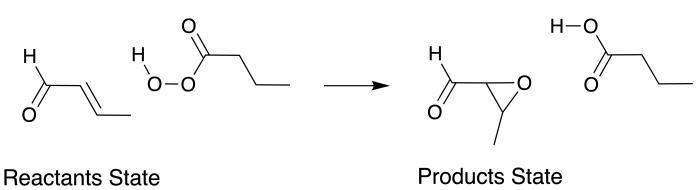

Figure 4.4. Mechanism of the epoxidation of 2-butenal by CALB, depicted in panel $\mathbf{a}$, and the epoxidation in solution using peroxyacids in $\mathbf{b}$. 


\subsection{Promiscuity studies in CALB}

The asymmetric carbon of the product defines its final enantiomeric nature, $(\mathrm{R})$ - and $(\mathrm{S})$ - enantiomers (Figure 4.5), which is decided in the first step of the reaction according to our calculations. Interestingly, although the first step of both $(\mathrm{R})$ - and $(\mathrm{S})$ - enantiomers is energetically equivalent, in the second step the formation of (S)- is energetically favored (Figure 4.6). Nevertheless, the lack of enantioselectivity showed experimentally ${ }^{4}$ could be explained by the population analysis of unbiased MD simulations and the free energies of binding obtained by employing alchemical FEP methods which showed a preference towards the $(\mathrm{R})$ - enantiomer, canceling out the catalytic effect that favors the (S)- product.

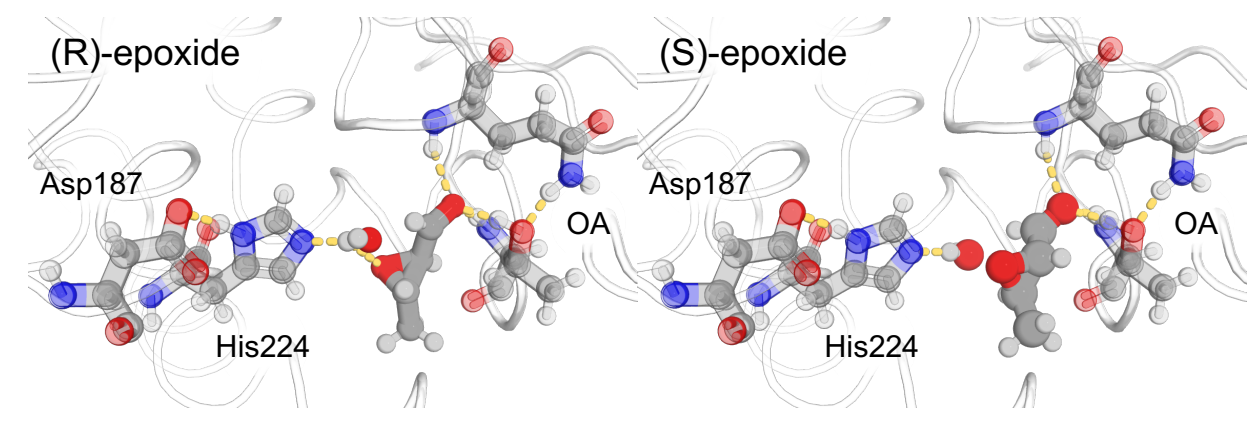

Figure 4.5. Detailed representation of the final enantiomeric products inside the active site of CALB.

We compared the enzymatic epoxidation with the formation of the same epoxide using peroxy-acids in chloroform (Figure 4.3b). We found that, from the energetical perspective, the enzymatic reaction is favored over the chemical synthesis. With this fact, it can be concluded that the use of CALB to produce epoxides from short alkenes is an efficient and environmentally friendly alternative (Figure 4.7). Moreover, the enantioselective character of CALB could be potentially modulated in future studies to produce specific enantiomers.

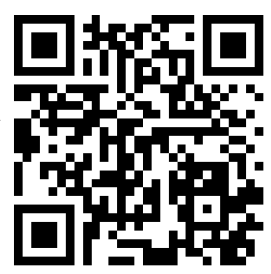



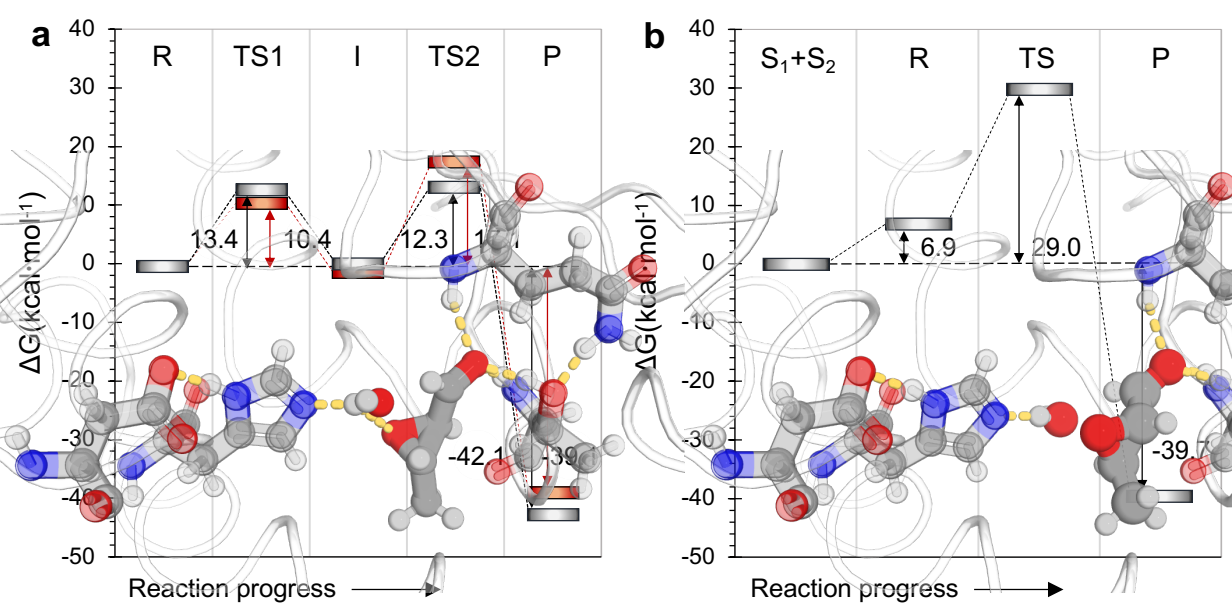

Figure 4.6. Free energy profile obtained at B3LYP/OPLS-AA level. In panel a the epoxidation of 2-butenal catalyzed by CALB is showed. In red the (R)enantiomer while in black the (S)-epoxide is depicted. In panel $\mathbf{b}$ the epoxidation in chloroform of the 2-butenal by peroxyacids is showed.

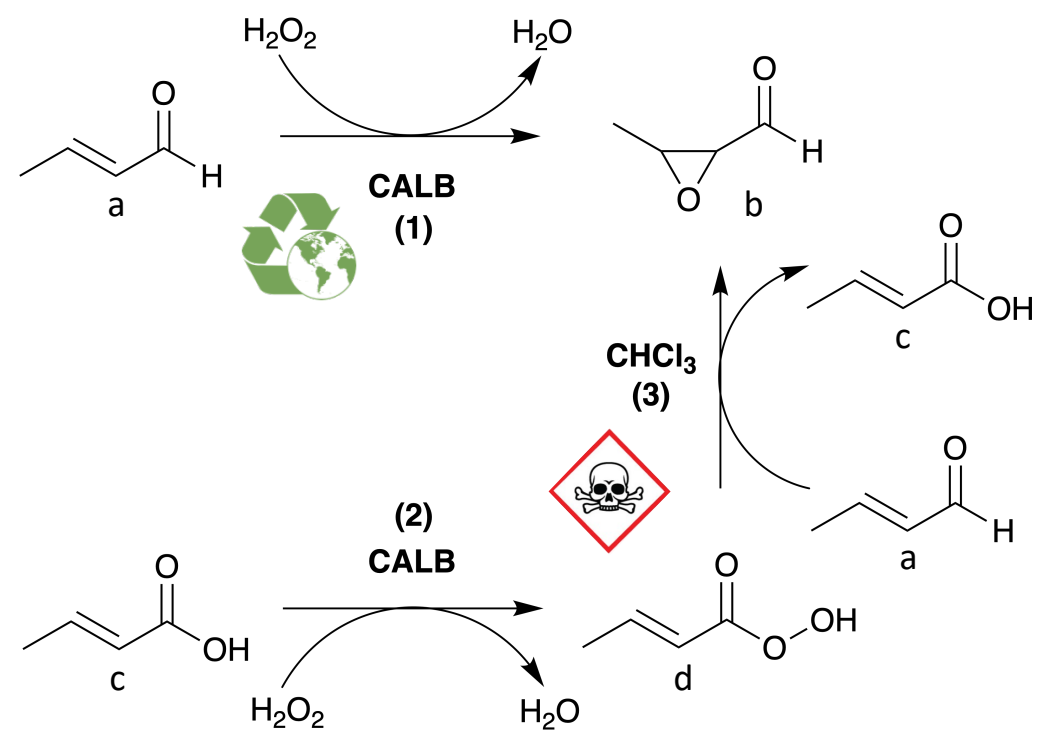

Figure 4.7. Schematic representation of the different routes for the epoxidation of alkenes. In the scheme: (1) is the direct epoxidation (1) of alkenes in CALB as a green alternative to the epoxidation using peracids (2) and (3). The production of peracids by CALB (2) is coupled to the epoxidation in solution with peracids (3). 



\title{
Computational Studies Suggest Promiscuous Candida antarctica Lipase B as an Environmentally Friendly Alternative for the Production of Epoxides
}

\author{
Miquel A. Galmés, Katarzyna Świderek,* and Vicent Moliner* \\ Cite This: J. Chem. Inf. Model. 2021, 61, 3604-3614 \\ Read Online
}

ACCESS | W Metrics \& More | 国 Article Recommendations | S1 Supporting Information

ABSTRACT: Environmentally friendly processes are nowadays a trending topic to get highly desired chemical compounds and, in this sense, the use of enzyme-catalyzed routes is becoming a promising alternative to traditional synthetic methods. In the present paper, a hybrid quantum mechanics/molecular mechanics (QM/MM) computational study on the epoxidation of alkenes catalyzed by the Ser105Ala variant of the promiscuous Candida antarctica lipase B (CALB) is presented in an attempt to search for alternative paths to get useful intermediates in industries. The catalyzed reaction, described at the atomistic level with a model of the full solvated in a box of water molecules, is compared with the alternative epoxidation of alkenes by peroxy acids in chloroform. Freeenergy profiles obtained at the density functional theory (DFT)/MM level show how Ser105Ala CALB is capable of epoxide short alkenes in a two-

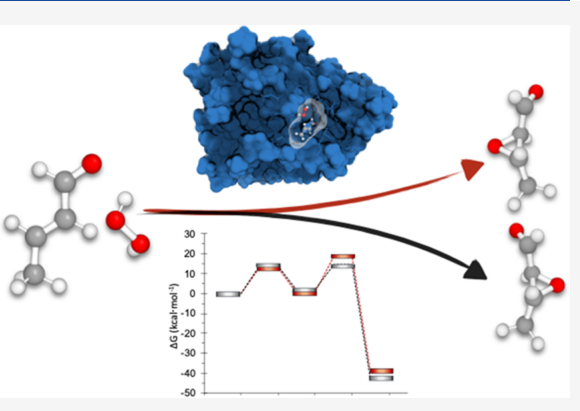
step process with free-energy barriers, in agreement with available experimental data, that are significantly lower than those of the single-step reaction in solution. The possible $(R)$-enantioselectivity dictated by the binding step, explored by means of alchemical $\mathrm{QM} / \mathrm{MM}$ free-energy perturbation (FEP) methods, and the preference for the $(S$ )-enantiomer derived from the free-energy landscape of the chemical steps would cancel out, thus predicting the lack of enantioselectivity experimentally observed. In general, our results provide general information on the molecular mechanism employed by a highly promiscuous enzyme, with potential applications in biotechnology.

\section{INTRODUCTION}

The interest in the study of mechanisms of promiscuous enzymes has recently increased in the field of biomolecular transformations. ${ }^{1-6}$ In-depth knowledge on how these mechanisms work at a molecular level can lead to new tools that permit obtaining highly desirable chemical compounds, with improved yields, ecological procedures, and in a wide variety of conditions. In a world that demands greener sources of products and where ecological consciousness is an urgent topic, enzymatic approaches are preferred over chemical synthesis.

Enzymes are known for their ability to catalyze chemical reactions in aqueous solution with high efficiency and selectivity under mild conditions of temperature or pressure, resulting in few unwanted side products. This converts the enzymatic synthesis into an extremely useful tool to obtain chemical compounds in a more ecologically responsible way than the traditional methods that are usually potentially harmful to the environment. An example of these classical chemical methods can be found in the epoxidation of alkenes employing peroxy acids, known as the Prilezhaev reaction. ${ }^{8}$ First described at the beginning of the 20th century, epoxidation of alkenes using peroxy acids proceeds through a single concerted step where it is believed that the peroxide is positioned perpendicular to the plane of the alkene in the transition state (TS). This mechanism is also known as the butterfly mechanism as coined in the middle of the 20th century by Bartlett, ${ }^{9}$ later confirmed by Azman and coworkers, ${ }^{10}$ and by Houk and co-workers ${ }^{11}$ based on computational studies and kinetic isotope effect (KIE) determinations.

Epoxides are very useful and important chemicals in industries. They are used in the fabrication of surfactants and detergents, insecticides, resins, and aerosols, or as stabilizers in coatings and the plastic industry. ${ }^{12,13}$ The main drawback in the peroxidation of alkenes is the non-environmentally green character of their synthesis. For instance, the use of stoichiometric amounts of peroxy acids for epoxidation of alkenes, ${ }^{14}$ metal-based catalyzed reactions, ${ }^{15}$ epoxidations using hydrogen peroxide in ionic liquids or deep eutectic

Received: April 14, 2021

Published: July 12, 2021 
solvents, ${ }^{16}$ and manganese salts ${ }^{17}$ are some of the established methods for the chemical synthesis of peroxides. For this reason, chemoenzymatic synthesis of epoxides is gaining attention in industries for its greener character. The use of enzymes would permit to reduce the number of oxidants needed for the epoxidations of alkenes due to their highly efficient and selective behavior. In this regard, haloperoxidases and monooxygenases are enzymes that catalyze the epoxidation of alkenes but their use in industries is very limited due to their low versatility in cheap and efficient conditions. ${ }^{18,19}$

Over the years, many lipases have been reported to be capable of performing secondary reactions, ${ }^{1,2}$ but the understanding of the molecular mechanism that governs this promiscuity is still poor. Lipases are very robust enzymes that can work in a wide range of conditions. Moreover, their resistance to organic solvents and oxidants makes this family of enzymes an attractive biocatalyst for industries. Additionally, it has been described that many lipases can use hydrogen peroxide as an oxidant, which is preferred due to its low environmental impact. ${ }^{16,20,21}$

One of the best-characterized enzymes in this field is Candida antarctica lipase B (CALB). CALB is widely used in industries and has served as a model to study enzyme promiscuity, either by experimental or theoretical approaches. ${ }^{20,22-25}$ CALB has been demonstrated to be able to perform a wide variety of promiscuous reactions, such as synthesis of esters and amides, transacylation of alcohols, ${ }^{26}$ epoxidation reactions, ${ }^{20}$ aldol additions, and Michael-type additions, $^{2,27}$ among many others. ${ }^{2,28}$ It has been proposed that this serine hydrolase performs its activity due to the presence of a catalytic triad Ser/His/Asp in the active site, as well as an oxyanion hole formed by Thr and Gln, which stabilizes the negative charge accumulating on the carbonyl oxygen atom of the substrate in different states along the reaction. The hydrolytic character of CALB is achieved by the catalytic Ser105. CALB has been previously reported to display epoxidation activity on short alkenes, ${ }^{20}$ but its hydrolytic activity also permits the ring opening of epoxides. ${ }^{23}$ For this reason, the mutation of Ser105 to Ala105 has been demonstrated to maximize its catalytic activity in the epoxidation reaction. $^{20,21}$

In addition to the direct epoxidation of alkenes, CALB has also been used to produce epoxides from the generation of peroxy acids. ${ }^{29}$ This strategy is also interesting from the chemical point of view and a good alternative to the direct epoxidation and/or the classical chemical epoxidation methods. Both epoxidation pathways catalyzed by CALB are schematically illustrated in Figure 1.

In this work, we have focused on the study of the direct epoxidation reaction catalyzed by CALB on short alkenes by means of multiscale quantum mechanics/molecular mechanics (QM/MM) methods, using the Ser105Ala CALB variant to avoid possible undesired hydrolytic activity. 2-Butenal served as a substrate for the direct epoxidation reaction, and hydrogen peroxide was chosen as an oxidant due to its mild environmental character, availability, and solubility, which permit the use of very low concentrations. The selection of this particular oxidant molecule was additionally dictated by the fact that CALB has been reported to tolerate moderate concentrations of hydrogen peroxide. ${ }^{20,21}$

As mentioned above, apart from this enzymatic approach, epoxidation of alkenes can be also achieved through the epoxidation of peroxy acids in solution. Here, the main

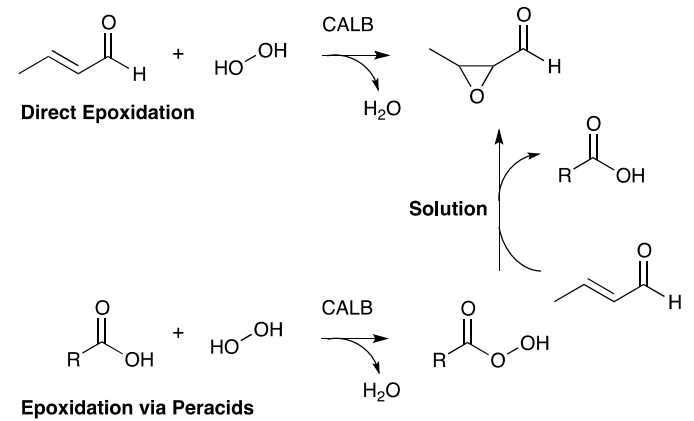

Figure 1. Epoxidation of short alkenes by CALB using two different pathways: the direct epoxidation of alkenes using hydrogen peroxide (top) and the epoxidation of alkenes in solution from peroxy acids generated by CALB (bottom). In this work, $R$ - is defined as $\left(\mathrm{CH}_{2}\right)_{2} \mathrm{CH}_{3}$.

question arises: which is the most efficient way to produce epoxides in terms of catalytic performance, the enzymatic approach via direct epoxidation or the chemical synthesis using peroxy acids? In this work, we have also studied the Prilezhaev reaction in an organic solvent using a short peroxy acid of four carbons as a model (perbutyric acid). Comparison of the freeenergy landscape for the two reactions can indicate which of the studied epoxidation pathways can be an environmentally friendly alternative for the production of epoxides. The results of this work also provide general information about the molecular mechanism employed by promiscuous enzymes.

\section{COMPUTATIONAL METHODS}

System Setup. Initial coordinates of the protein were obtained from the PDB structure of $C$. antarctica lipase B (PDB ID: 1TCA).$^{30}$ Mutation of the catalytic Ser105 to Ala was done using Mutator Plugin in Visual Molecular Dynamics (VMD). ${ }^{31}$ The corresponding substrates, 2-butenal and hydrogen peroxide, were built using MOLDEN software ${ }^{32}$ into the active-site pocket of the pre-equilibrated CALB. ${ }^{22} 2$ Butenal was built manually using as an anchor atom carbonyl oxygen of the substrate studied in previous works. ${ }^{22}$ Hydrogen peroxide was produced using a water molecule as an anchor that lied in a suitable position in the vicinity of the catalytic diad, especially focusing on its proper orientation toward His 224 whose role is its activation and the electrophilic center that will be attacked. The protonation state of titratable residues was determined at $\mathrm{pH} 7$ using the PropKa ver. 3.0.3 program. ${ }^{33,34}$ Asp134 with $\mathrm{pK}_{\mathrm{a}}$ of 7.6 was protonated at the OD2 position, and only one histidine present in the protein, catalytic His224, was treated as neutral with a hydrogen atom added at the $\mathrm{N} \delta$ position. Three disulfide bridges were identified between residues Cys22 and Cys64, Cys216 and Cys258, and Cys293 and Cys31. Afterward, all missing hydrogen atoms were added to the structure and the system was solvated into a $100 \times 80 \times 80 \AA^{3}$ pre-equilibrated box of TIP3P ${ }^{35}$ water molecules. Water molecules with an oxygen atom within $2.8 \AA$ of any heavy atom were removed. The total charge of the system was zero; therefore, no counterions were added. The full system was minimized by a series of optimization algorithms, as described elsewhere. ${ }^{22}$ Such a prepared model was composed of 64045 atoms, of which 4624 
correspond to the protein residues, 59406 to water molecules, and 15 to the substrates.

After initial energy minimizations, the system was heated to $303 \mathrm{~K}$ with a $0.1 \mathrm{~K}$ temperature increment and equilibrated during short (100 ps) NPT MD simulations, followed by nonaccelerated classical 200 ns NVT MD simulations with the AMBER force field, ${ }^{36}$ as implemented in NAMD software. ${ }^{37}$ According to the time evolution of the root-mean-square deviation (RMSD), the system can be considered as equilibrated (Supporting Information Figure S1). The missing force-field parameters for the substrates were generated using the GAFF $^{38}$ and the Antechamber ${ }^{39}$ tool (Supporting Information Tables S1 and S2). Partial charges were computed using the Austin Model 1 (AM1) ${ }^{40}$ semiempirical Hamiltonian as implemented in Antechamber. During 200 ns nonbiased NVT MD simulations with a time step of $1 \mathrm{fs}$, all atoms were free to move within periodic boundary conditions and cutoffs for nonbonding interactions with an internal cutoff of $14.5 \AA$ and an external of $16 \AA$. To avoid diffusion of the substrates, a restrain in the position between the oxygen of the aldehyde and the oxyanion hole, and hydrogen of hydrogen peroxide and the NE2 of the catalytic His224, was set up. The Langevin thermostat $^{41}$ was used. The relative orientation of the 2butenal along the dynamics was analyzed by measuring the angle formed by the His 224 ring, the asymmetric carbon of the substrate, and the hydrogen atom bonded to it. The values were normalized and clustered using $K$-means ${ }^{42}$ clustering to divide the sample into the geometries that could lead to $(R)$ and $(S)$-enantiomers. Two snapshots of the equilibrated system were extracted from the $\mathrm{MD}$ trajectory, taking into account that the initial geometry of the substrates could lead to two possible enantiomers, $(R)$ - and $(S)$-, i.e., a structure whose relative orientation was close to 0 and another close to 1 for $(R)$ - and $(S)$-, respectively. The selected structures were then used for QM/MM calculations after minimization of the full system using a combination of the conjugate gradient ${ }^{43}$ and LBFGS- $^{44}$ algorithms, as implemented in the $\mathrm{fDynamo}^{45}$ library.

To carry out the QM/MM simulations, the system was divided into two subsets of atoms. The $\mathrm{QM}$ region was described at the density functional theory (DFT) level using the B3LYP ${ }^{46,47}$ functional and the standard $6-31+\mathrm{G}(\mathrm{d}, \mathrm{p})$ basis set, as implemented in Gaussian09. ${ }^{48}$ It was composed of the lateral chains of the catalytic His224 and Asp187 residues, as well as the full substrates, i.e., 2-butenal and hydrogen peroxide (34 atoms), as shown in Figure 2. To saturate the valence between QM and MM boundary, two link atoms ${ }^{49}$ were placed

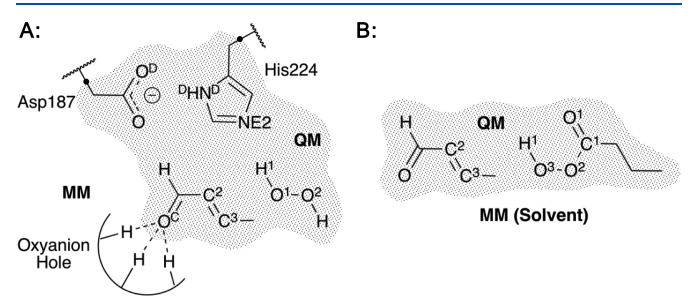

Figure 2. Schematic representation of QM partition. (A) CALB active site and its QM subset represented as the shaded region. Black dots represent link atoms introduced between $\mathrm{QM}$ and $\mathrm{MM}$ regions. (B) $\mathrm{QM}$ region for the epoxidation reaction using peroxy acids in chloroform. The solvent was treated as MM. between $\mathrm{C} \alpha-\mathrm{C} \beta$ of side chains of His224 and Asp187. The remaining atoms of the system were treated by the OPLS-AA ${ }^{50}$ and TIP $3 \mathrm{P}^{35}$ force fields for the protein and water molecules, respectively. A truncation function (shift function) was used to treat the nonbonded interactions, with an internal cutoff at $14.5 \AA$ and an external cutoff at $16 \AA$.

For the study of the Prilezhaev reaction in chloroform, both substrates, perbutyric acid and 2-butenal, were solvated with an $80 \times 80 \times 80 \AA^{3}$ solvation box of chloroform molecules. A short classical MD simulation of $5 \mathrm{~ns}$ using the NVT ensemble was done to equilibrate the box of chloroform using the same approach as described above at $303 \mathrm{~K}$. The substrates were treated at the DFT level, as in the case of the reaction in the enzyme. Parameters to describe the chloroform molecules were taken from the Amber parameter database. ${ }^{51}$ Parameters to describe perbutyric acid were computed following the same procedure as described above using GAFF and Antechamber (Supporting Information, Table S3). The system consists of 10001 atoms (26 atoms for the substrates and 9975 for chloroform molecules).

Localization of Structures at the B3LYP/MM Level. Transition state structures for each system were located at the B3LYP/MM level using Baker's algorithm ${ }^{52}$ using Gaussian09 combined with the fDynamo library. Convergence tolerance for localization of structures was set to $1.2 \mathrm{~kJ} \cdot \mathrm{mol}^{-1} \cdot \AA^{-1}$. Subsequently, Hessian was calculated for the optimized structures to confirm the nature of the TS structure, yielding only one negative eigenvalue. Then, minimum energy paths from the localized TS structures were traced using the IRC method. A total of 200 iterative steps in both directions were done with a step of $0.1 \AA$. The geometry of reactants, intermediates, and products from the IRC was optimized using the same Baker's algorithm with a $1.2 \mathrm{~kJ} \cdot \mathrm{mol}^{-1} \cdot \AA^{-1}$ convergence.

Free-Energy Calculations. To obtain the free-energy landscape of the reactions, the free-energy perturbation (FEP) method was used. ${ }^{53,54}$ FEP requires the sampling of the environment over a previously traced IRC down to reactant state (RS) and product state (PS) from the TS, in our case at the B3LYP/MM level of theory. For this reason, the freeenergy profile is obtained over a realistic reaction coordinate. In this case, the MM charges of the environment polarize the wave function of the QM part and permit the exploration of the reaction path at a high level of theory.

The structures used in FEP calculations were extracted from the traced IRC and are characterized by a single $s$ coordinate

$$
\begin{aligned}
s_{i}=s_{i-1}+ & \left\{\sum _ { j \in \mathrm { QM } } m _ { i } \left[\left(x_{j, i}-x_{j, i-1}\right)^{2}+\left(y_{j, i}-y_{j, i-1}\right)^{2}\right.\right. \\
& \left.\left.+\left(z_{j, i}-z_{j, i-1}\right)^{2}\right]\right\}
\end{aligned}
$$

where $x_{j, i}, y_{j, j}$, and $z_{j, i}$ are the coordinates of the $i$ th structure and $j$ th $\mathrm{QM}$ atom of the structure, and $m_{i}$ is the corresponding mass of the QM atoms.

So, the free energy relative to reactants can be expressed as a function of $s$, as presented in eq 2 


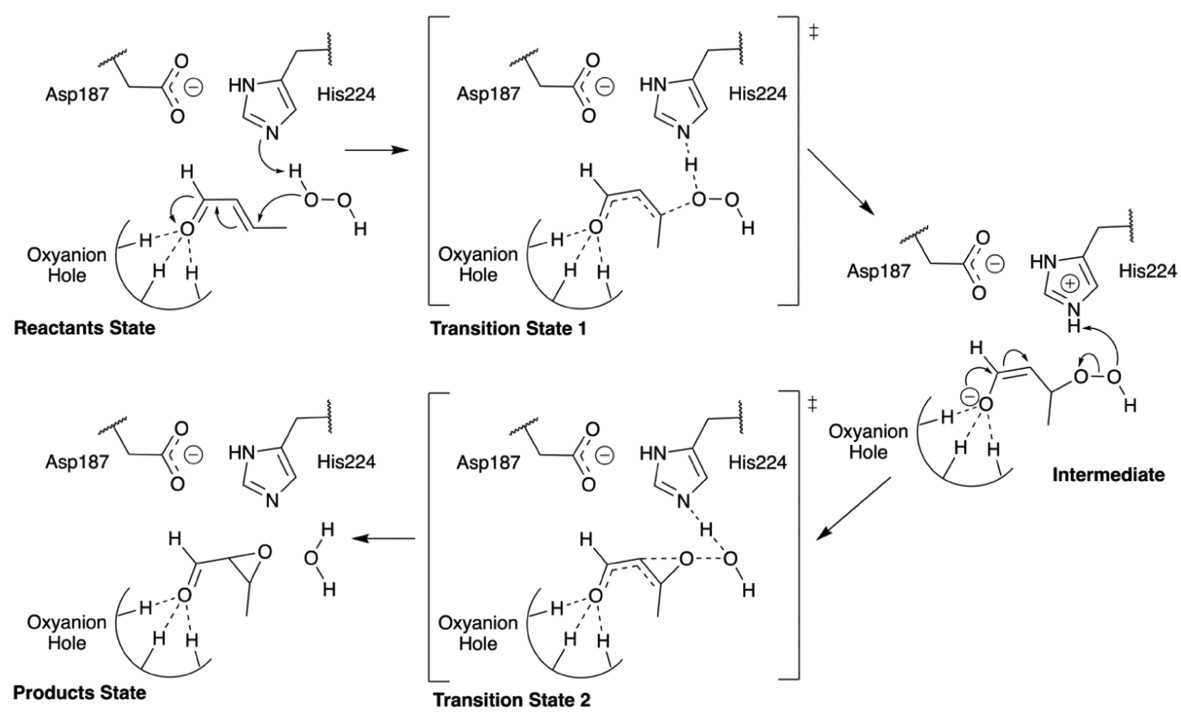

Figure 3. Schematic representation of the reaction mechanism of the direct epoxidation of 2-butenal with hydrogen peroxide catalyzed by CALB.

$$
\begin{aligned}
& \Delta G_{\mathrm{FEP}}\left(s^{R}-s^{j}\right)=\left[E_{\mathrm{QM}}^{0}\left(s^{j}\right)-E_{\mathrm{QM}}^{0}\left(s^{R}\right)\right] \\
& +\left[\mathrm{ZPE}\left(s^{j}\right)-\mathrm{ZPE}\left(s^{R}\right)\right]-k_{\mathrm{B}} T \\
& \quad \sum_{i=R}^{j} \ln \left\langle\exp \left[-\frac{E_{\mathrm{QM} / \mathrm{MM}}\left(s^{i+1}\right)-E_{\mathrm{QM} / \mathrm{MM}}\left(s^{i}\right)}{k_{\mathrm{B}} T}\right]\right\rangle_{\mathrm{MM}, i}
\end{aligned}
$$

where $E_{\mathrm{QM}}^{0}$ is the gas-phase energy of the QM subset computed at the DFT level, ZPE is the zero-point energy, $k_{\mathrm{B}}$ is the Boltzmann constant, and $T$ is the temperature. QM/MM contribution to the free energy between two values of $s$ is calculated by averaging the QM/MM interaction energy over all of the MM atoms obtained during the molecular dynamics (MD) simulation. In particular, $20 \mathrm{ps}$ of $\mathrm{QM} / \mathrm{MM} \mathrm{MD}$ simulation were done for each window along the IRC path at $293 \mathrm{~K}$ using the NVT ensemble, maintaining the QM subset frozen during the simulation. The total number of windows employed to generate the full energy path was 49 and 108 for first and second steps taking place in the active site of CALB in the case of the $(R)$-enantiomer, 51 and 113 for the $(S)$ enantiomer, and 88 windows to study the chemical transformation of the peroxy acid in chloroform, respectively. Free energy between the solvated separated substrates in solution and the reactant complex (RC) was estimated using the $\mathrm{SMD}^{55}$ continuum solvation model. Finally, to take into account dispersion effects produced by the oxyanion hole, empirical dispersion was considered by computing single-point calculations along the IRC using the empirical DFT$\mathrm{D} 3(\mathrm{BJ})^{56,57}$ as implemented in Gaussian09. Statistical errors to the FEP calculations were computed by means of first-order expansion, as proposed by Chipot ${ }^{58}$ (Supporting Information, Tables S4 and S8).

Binding Free Energy. To evaluate the interaction energy between the substrates and the protein, the alchemical free perturbation method was used, as described in previous works. ${ }^{59-61}$ A series of QM/MM MD simulations were carried out in the enzyme-substrate complex in the Michaelis complex state and in the substrate solvated in a box of water molecules. The QM subset of atoms, consisting, in this case, of just the substrates, was treated by the Austin Model 1 (AM1 $)^{40}$ semiempirical Hamiltonian with the standard $6-31+\mathrm{G}(\mathrm{d}, \mathrm{p})$ basis set. Two parameters $\lambda$ and $\gamma$ were introduced in the electrostatic and van der Waals interaction terms, respectively, as shown in eq 3

$$
\begin{aligned}
E_{\mathrm{QM} / \mathrm{MM}}(\lambda)= & \left\langle\Psi\left|\hat{H}_{0}\right| \Psi\right\rangle+\lambda \mid \sum\left\langle\Psi\left|\frac{q_{\mathrm{MM}}}{r_{\mathrm{e}, \mathrm{MM}}}\right| \Psi\right\rangle \\
& \left.+\sum \sum \frac{Z_{\mathrm{QM}} q_{\mathrm{MM}}}{r_{\mathrm{QM}, \mathrm{MM}}}\right)+\gamma E_{\mathrm{QM} / \mathrm{MM}}^{\mathrm{vdW}}+E_{\mathrm{MM}}
\end{aligned}
$$

Then, changes were smoothly introduced in $\lambda$ and $\gamma$ parameters from 1 to 0 to annihilate the electrostatic and van der Waals interaction contributions. The calculation of the free-energy difference between two consecutive steps is obtained, and then the total free-energy change is obtained by the sum of all of the contribution between all of the steps covering the whole transformation from the initial to the final states. Here, we used a total of 100 windows to evaluate the electrostatic and van der Waals interaction term, with a step for $\lambda$ and $\gamma$ of 0.01 (from 1 to 0 ). In each window, a total of $20 \mathrm{ps}$ of AM1/MM MD at $293 \mathrm{~K}$ was performed using the NVT ensemble. Finally, the binding free energy can be computed as the difference between $\Delta G_{\mathrm{W}}$ in the water solvent and $\Delta G_{\mathrm{E}}$ in the Michaelis complex.

$$
\Delta G=\Delta G_{\mathrm{W}}-\Delta G_{\mathrm{E}}
$$

Kinetic Isotope Effects. KIEs were calculated from the isotopic substitution of the key atoms from localized RS and TS structures. From the definition of the free energy of a state and using the transition state theory, ${ }^{62}$ the ratio between the 


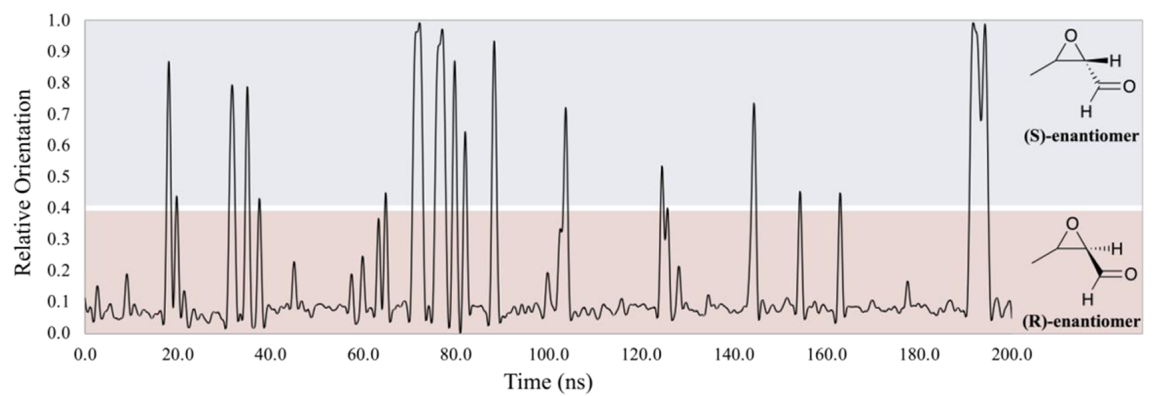

Figure 4. Relative orientation of the substrate 2-butenal along $200 \mathrm{~ns}$ of classical MD. Baseline 0.0 corresponds to the $(R)$-enantiomer, and 1.0 is the corresponding $(S)$-geometry. Cutoff between $R$ - and $S$-geometry was computed applying $K$-means clustering. ${ }^{42}$
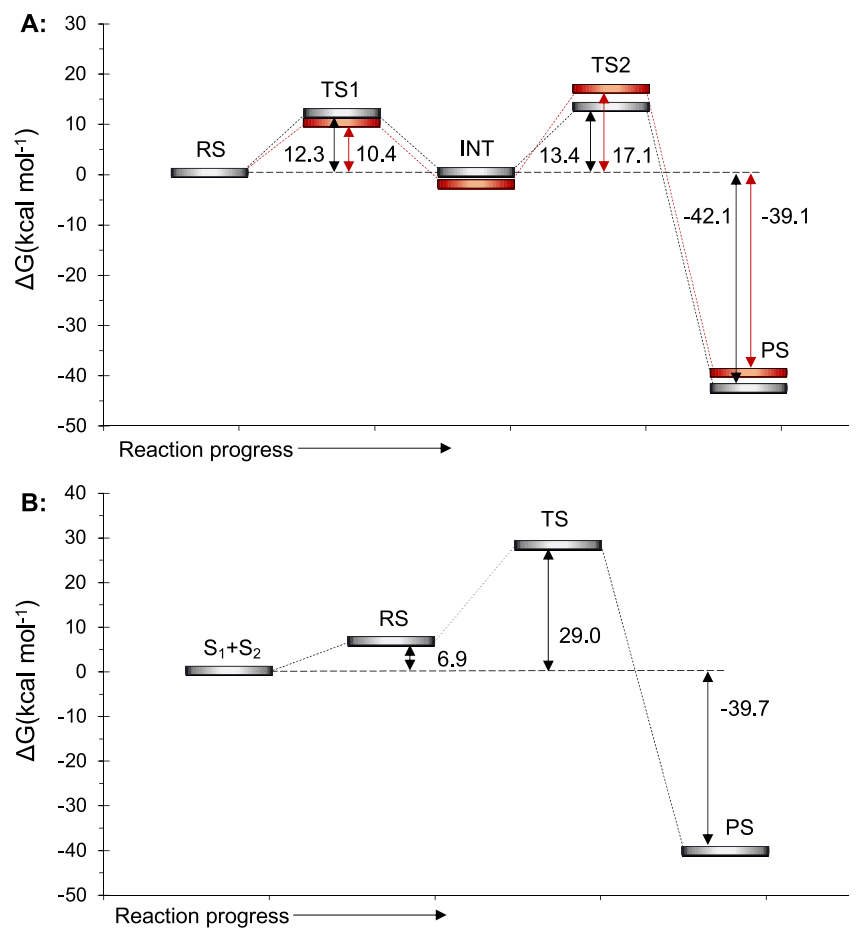

Figure 5. B3LYP-D3(BJ)/MM free-energy profile of the epoxidation of 2-butenal. (A) Reaction catalyzed by CALB to render the (R)- (red line) and (S)- (gray line)epoxide enantiomers. (B) Epoxidation of 2-butenal by perbutyric acid in chloroform.

rate constants corresponding to the heavy and the light atoms can be computed by means of

$$
\mathrm{KIE}=\frac{\left(\frac{Q_{\mathrm{TS}}}{Q_{\mathrm{RS}}}\right)_{\mathrm{L}}}{\left(\frac{\mathrm{Q}_{\mathrm{TS}}}{\mathrm{Q}_{\mathrm{RS}}}\right)_{\mathrm{H}}} \mathrm{e}^{-\left(\Delta \mathrm{ZPE}_{\mathrm{L}}-\Delta \mathrm{ZPE}_{\mathrm{H}}\right) / R T}
$$

where $Q$ is the total partition function, which was computed as the product of the translational, rotational, and vibrational partition functions of isotopologues in RS and TS structures, and $\triangle \mathrm{ZPE}$ is the difference of the zero-point energy between $\mathrm{RS}$ and TS in the light $(\mathrm{L})$ and heavy $(\mathrm{H})$ isotopologues. ${ }^{6,64}$
KIEs were computed at the B3LYP/MM level of theory, and one RS structure and one TS structure for each step of the reaction were used to compute the KIE. The Hessian matrices were computed for the QM subset of atoms.

\section{RESULTS AND DISCUSSION}

Direct Epoxidation Catalyzed by CALB. As mentioned in previous sections, a short alkene 2-butenal has been used as a model of the substrate in this study. A previous study for the direct epoxidation by hydrogen peroxide, derived from a computational study based on a reduced cluster model to mimic the active site of CALB, ${ }^{20}$ suggested that the reaction takes place in two steps, as shown in Figure 3. According to 


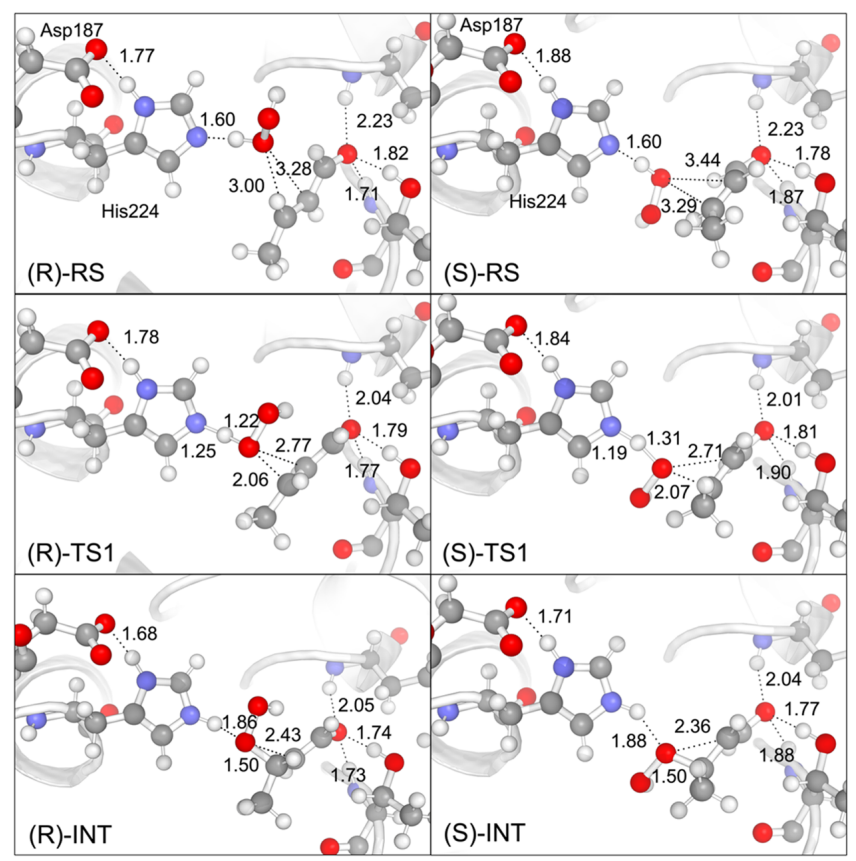

Figure 6. Representative structures of the state localized at the B3LYP/MM level of the first step of the direct epoxidation of 2-butenal with hydrogen peroxide catalyzed by CALB. The left panel corresponds to the $(R)$-enantiomer, while the right panel corresponds to the $(S)$-enantiomer. Key residues and substrates are shown in a ball-and-stick representation. All distances are in $\AA$.

this proposal, we explored the two chemical steps by means of QM/MM FEP methods with the full enzyme solvated in a box of water molecules. The first step involves the attack of the oxygen $\left(\mathrm{O}^{1}\right)$ of the hydrogen peroxide to the carbon $\left(\mathrm{C}^{3}\right)$ of the alkene, concomitant with the proton transfer from the oxygen $\left(\mathrm{O}^{1}\right)$ to His224. In this first step, a negative charge is developed in the carbonyl oxygen atom of the substrate, which is stabilized by the oxyanion hole formed by Thr40 and Gln106. The role of this oxyanion hole is well known and has been stated in other reactions catalyzed by CALB. ${ }^{22-24,65}$ The formation of the epoxide ring by the bond forming between $\mathrm{C}^{2}$ and $\mathrm{O}^{1}$ takes place in the second step. This second step is assisted by the acid/base character of the His224 that permits the transfer of the proton, $\mathrm{H}^{1}$, to oxygen, $\mathrm{O}^{2}$, thus forming a water molecule.

Due to the nature of this short alkene and the presence of an asymmetric carbon atom in the products of the reaction, $\mathrm{C}^{2}$, two different enantiomers can be formed, as shown in Figure 4, which is dictated by the relative orientation of both substrates, 2-butenal and hydrogen peroxide, in the reactant state. This relative orientation is determined by the 2-butenal pose established in the active site. Thus, possible orientations of this substrate were explored during a $200 \mathrm{~ns}$ classical MD. The orientation of the substrate that determines the final $(R)$ epoxide is represented as values close to 0 , while relative orientation values around 1 lead to $(S)$-epoxide. As shown in Figure 4, although both orientations were identified along the MD trajectory, $(R)$-like geometry seems to be the dominant one (90.9\% of overall explored structures). Similar results were obtained when starting the MD simulation from the $(S)$ - enantiomer (Supporting Information, Figure S2). Interestingly, analysis of the interaction energies between the two possible conformations of the substrate and the protein reveals that there is no single evident interaction that can explain the results but a global effect of the protein (Supporting Information, Figure S3). Nevertheless, this result can not be considered as a final conclusion about the propensity of obtaining one or the other enantiomer and exploration of the subsequent chemical reaction is required. Once the substrate binds to the active site, the rotation around the $\mathrm{C}^{1}-\mathrm{C}^{2}$ bond is restricted, and this fact leads to the formation of one or other enantiomers because of the defined relative orientation of alkene and hydrogen peroxide. Since this fact is a priori very attractive from the biocatalytic point of view, both possible mechanisms were studied. The resulting free-energy profiles are shown in Figure 5; average structures of the key states appearing along the reaction are presented in Figures 6 and 7, while key distances and charges of localized structures of the reactant state (RS), TSs, intermediate (INT), and product state (PS) are given in the Supporting Information.

The analysis of the free-energy profiles of the chemical steps of the process catalyzed by CALB, as depicted in Figure 5, and the geometrical analysis of the structures obtained along the reaction pathway, as illustrated in Figures 6 and 7, confirm the proposed two-step mechanism and show that the formation of the $(S)$-enantiomer is kinetically and thermodynamically more favorable than formation of the $(R)$-enantiomer. The experimentally measured rate constant $k_{\mathrm{cat}}$ of $0.56 \mathrm{~s}^{-120}$ that, within the framework of the transition state theory, ${ }^{62}$ corresponds to a free energy barrier of $17.5 \mathrm{kcal} \cdot \mathrm{mol}^{-1}$ at 


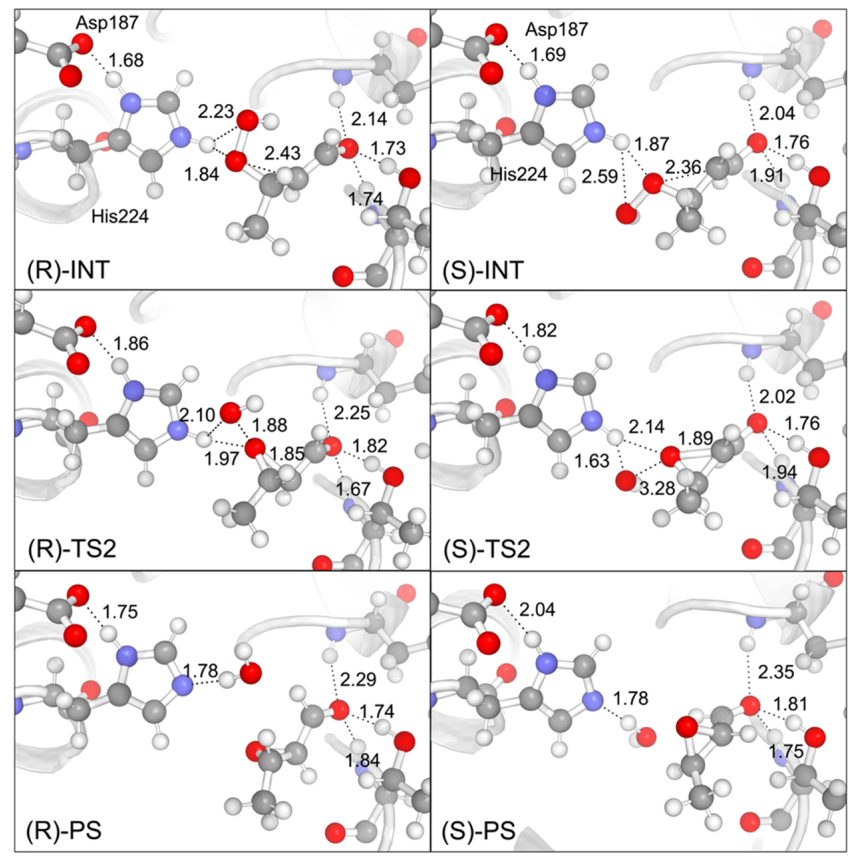

Figure 7. Representative structures of the state localized at the B3LYP/MM level of the second step of the direct epoxidation of 2-butenal with hydrogen peroxide catalyzed by CALB. The left panel corresponds to the $(R)$-enantiomer and the right panel corresponds to the $(S)$-enantiomer. Key residues and substrates are shown in a ball-and-stick representation. Distances are in Å.

$293 \mathrm{~K}$ is in between the values computed for the $(R)$ - and $(S)$ enantiomers at $298 \mathrm{~K}$. Experiments performed by Brinck and co-workers suggested that this reaction leads to a racemic mixture without a preference toward any enantiomer, showing no enantioselectivity. ${ }^{20}$ Considering the differences in the activation and reaction energies for the formation of both products deduced from our simulations $(3.7$ and $3.0 \mathrm{kcal}$. $\mathrm{mol}^{-1}$, respectively), the experimental results would not match with our reactivity results if the reaction was carried out either under kinetic or thermodynamic control.

In general, although no significant geometrical differences are detected between the geometries of the different states of both paths, the different reactivities between $(R)$ - and $(S)$ enantiomers can be rationalized based on the different relative orientations of the substrate in INT and TS2. Thus, the C2O1 distance in the $(S)$-INT is slightly shorter than in the $(R)$ INT enantiomer ( 2.36 vs $2.43 \AA)$. This, together with a longer distance in the $(S)$-TS2 than in the $(R)$-TS2 (1.89 vs $1.85 \AA$ ) implies a larger variation of this reaction coordinate from INT to the TS2 in the $(R)$ - than in the $(S)$-conformer $(0.58$ vs 0.47 $\AA$ ), which is in agreement with a larger activation free energy. According to these results, TS2 is closer to the INT in $(S)$ than in $(R)$-, which is in agreement with the Hammond postulate that predicts that TSs are closer to reactants for reactions with low activation energies and large reaction energies, as is the case when comparing the energy profile for the $(R)$ - and the $(S)$-conformers. Regarding the H1 transfer from NE2 to O2, a different scenario is obtained. Thus, the $\mathrm{H} 1-\mathrm{O} 2$ distance is 1.84 and $1.88 \AA$ in the $(R)$ - and $(S)$-INT, respectively. This would suggest a slightly better conformation for the proton transfer in the $(R)$-conformer, taking into account that $\mathrm{H} 1-\mathrm{O} 2$ is the bond to be formed in this step. Nevertheless, the stronger interaction established between the transferring $\mathrm{H} 1$ atom and $\mathrm{O} 1$ in $(R)-\mathrm{TS} 2$, in comparison with the $(S)$-TS2 (1.97 and $2.14 \AA$, respectively), provokes a delay in the $\mathrm{H} 1$ transfer from $\mathrm{NE} 2$ to $\mathrm{O} 2$ in $(R)-(\mathrm{H} 1-\mathrm{O} 2$ distance equal to $2.10 \AA$ in TS2), in comparison with the (S)-conformer ( $\mathrm{H} 1-\mathrm{O} 2$ distance equal to $1.63 \AA$ in TS2). In fact, the larger accumulation of negative charge on the $\mathrm{O} 2$ atom in the $(R)$ TS2 than in the $(S)$-TS2 $(-0.794$ and $-0.692 \mathrm{au}$, respectively, as shown in the Supporting Information) is in agreement with these detected geometrical differences that explain the different computed energy barriers.

The geometrical analysis of the interatomic distance between the substrate and the active site also shows the role of the Asp187-His224 diad or the oxyanion hole formed by residues Thr40 and Gln106. Thus, in general, the shortest distances between $\mathrm{O}^{\mathrm{c}}$ and $\mathrm{H}^{\mathrm{Thr} 40}$ and $\mathrm{H}^{\mathrm{Gln} 106}$ are detected in the INT, where the charge on $\mathrm{O}^{\mathrm{c}}$ reaches the most negative value (see Tables S5-S7 in the Supporting Information).

Kinetic Isotope Effects. To provide other magnitudes that could be directly compared with experiments to confirm the proposed results, kinetic isotope effects (KIEs) were computed for the CALB-catalyzed reactions rendering the $R$ and $S$ enantiomers. KIEs were computed with TS1 and TS2 in an attempt to identify any difference in either the first or second steps, assuming that intrinsic KIEs can be measured for this reaction. According to the values reported in Table 1, and taking into account the uncertainty associated with the 
method, the KIEs obtained for the $R$ and $S$ enantiomers can be considered equivalent.

Table 1. KIEs Computed at the B3LYP/MM Level for the Direct Epoxidation of 2-Butenal Catalyzed by CALB

\begin{tabular}{cccccc} 
& \multicolumn{2}{c}{ enantiomer $R$} & & \multicolumn{2}{c}{ enantiomer $S$} \\
\cline { 2 - 3 } \cline { 5 - 6 } \cline { 5 - 6 } & TS1 & TS2 & & TS1 & TS2 \\
{$\left[2-{ }^{14} \mathrm{C}\right]$} & 1.011 & 0.972 & & 1.017 & 0.980 \\
{$\left[3-{ }^{14} \mathrm{C}\right]$} & 1.017 & 1.058 & & 1.001 & 1.034 \\
{$\left[1-{ }^{18} \mathrm{O}\right]$} & 0.990 & 1.046 & & 1.031 & 1.046 \\
{$\left[2-{ }^{18} \mathrm{O}\right]$} & 0.994 & 1.010 & & 0.994 & 1.030 \\
\hline
\end{tabular}

Substrate-Enzyme Binding Step. Once the chemical reaction steps are analyzed, it must be taken into account that the overall catalytic efficiency is dictated not only by $k_{\text {cat }}$ but also by the ability of the substrate to bind to the enzyme cavity, $K_{\mathrm{M}}$. To get information about this crucial step that defines the pose of the substrate that will lead to one or another enantiomer, binding free energies $\Delta G_{\text {bind }}$ were calculated by means of alchemical FEP methods. Binding free energies were computed as the difference of electrostatics and van der Waals contributions between the substrate in the Michaelis complex (MC) in the active site of the protein and the solvated reactants in aqueous solution. As shown in Table 2, a difference of $\Delta G_{\mathrm{bind}(R)}-\Delta G_{\mathrm{bind}(S)}$ of $2.1 \mathrm{kcal} \cdot \mathrm{mol}^{-1}$ was found between the geometries that will lead to the two enantiomers. This value suggests that the $R$ orientation would bind the active site with slightly higher probability than the $S$ orientation, which is in agreement with the selectivity that can be deduced from the population analysis of the MC complexes based on classical MD simulations (see Figure 4), but in contrast to the predicted trend that emerged from the difference in the chemical catalytic performance of the two systems, $R$ and $S$, as studied above.

Epoxidation Reaction in Solution. To answer the question of whether the most efficient way to produce epoxides, in terms of catalytic performance, is the enzymatic approach via direct epoxidation or the chemical synthesis using peroxy acids, the Prilezhaev reaction was studied in chloroform as proposed in previous studies. ${ }^{66} \mathrm{~A}$ short peroxy acid, perbutyric acid, was used as a model, as suggested in previous works. $^{67,68}$ The reaction goes through what is called a butterfly mechanism, as depicted in Figure 8. The study of the reaction at the B3LYP/MM level shows that it occurs in a single step where the transfer of the proton $\mathrm{H}^{1}$ toward carbonyl oxygen $\mathrm{O}^{1}$ of the peroxy acid takes place concomitant with the peroxy acid oxygen $\mathrm{O}^{3}$ attack on carbon of the alkene, the closing of the epoxide ring between $\mathrm{C}^{2}-\mathrm{C}^{3}$ of 2-butenal and the formation of butyric acid. Key interatomic distances and atomic charges for localized states for the reaction are shown in Tables S9 and S10 in the Supporting Information.
From the energy point of view, both enantiomers are equally probable when the reaction takes place in solution since the specific solvent-substrate interaction in both enantiomers is indistinguishable. The free-energy profiles of the reaction, also studied using FEP methods, are shown in Figure 5. As observed, the obtained energy barrier, $29.0 \mathrm{kcal} \cdot \mathrm{mol}^{-1}$, is significantly higher than the values obtained when the reaction is catalyzed by the enzyme. The barrier is too high for this reaction to take place in a reasonable timescale and, consequently, the use of this reaction in solution must be discarded, in comparison with the alternative enzymatic approach, as a green strategy to get epoxides.

\section{CONCLUSIONS}

In this paper, we present a $\mathrm{QM} / \mathrm{MM}$ computational study of the epoxidation of alkenes catalyzed by the Ser105Ala CALB enzyme and compare it with the alternative epoxidation of alkenes by peroxy acids in chloroform. Free-energy profiles obtained with FEP methods at the B3LYP/MM level show how the epoxidation of 2-butenal-catalyzed Ser105Ala CALB takes place in a two-step manner. The first step involves the attack of one of the oxygens of hydrogen peroxide $\left(\mathrm{O}^{1}\right)$ on one of the carbon atoms of the double bond of the alkene $\left(\mathrm{C}^{3}\right)$, concomitant with the proton transfer from the same oxygen atom $\left(\mathrm{O}^{2}\right)$ to His224. The closing of the epoxide ring between takes place in the second step, which is assisted by the acid/ base character of His 224 that permits the transfer of proton $\mathrm{H}^{1}$ to oxygen $\mathrm{O}^{2}$, thus forming a water molecule. The calculations show how the negative charge developed in the carbonyl oxygen atom of the substrate is stabilized by the oxyanion hole formed by Thr40 and Gln106. The presence of an asymmetric atom on the substrate opens the possibility of two different enantiomeric products. An in-depth analysis of the evolution of the geometries and charges in the active site of the enzyme along the reaction reveals how the relative orientation of the substrate, when producing the $(S)$-enantiomer, facilitates the presence of intermolecular interactions that increase the reactivity of the substrate to form a peroxide ring in the second step. The active-site His224 residue, posed closer to the substrate when generating the $(R)$-enantiomer, interacts by means of a strong hydrogen bond with the attacking oxygen $\left(\mathrm{O}^{1}\right)$ of the hydrogen peroxide oxygen atom, thus reducing its negative charge and provoking an increase in the energy barrier. The exploration of the free-energy landscape of the chemical steps of the full process catalyzed by Ser105Ala CALB suggests that the enzyme should show enantioselectivity favoring the $S$ enantiomer. Nevertheless, the population analysis of the two possible orientations of the substrate in the active site, based on unbiased MD simulations, and the study of the protein-substrate binding step, explored by means of alchemical FEP methods, suggest a possible $(R)$ enantioselectivity. Thus, the $R$-enantioselectivity that would be

Table 2. Binding Free Energy Computed at AM1/MM of 2-Butenal in the Active Site of CALB to Generate, after the Chemical Reaction, the Two $(R)$ - and $(S)$-Enantiomers of the Epoxide ${ }^{a}$

\begin{tabular}{|c|c|c|c|c|c|}
\hline & \multirow[b]{2}{*}{ solution } & \multicolumn{2}{|c|}{ geometry for $(R)$-enantiomer } & \multicolumn{2}{|c|}{ geometry for $(R)$-enantiomer } \\
\hline & & $\mathrm{RC}$ & $\Delta G_{\mathrm{E}}-\Delta G_{\mathrm{W}}$ & $\mathrm{RC}$ & $\Delta G_{\mathrm{E}}-\Delta G_{\mathrm{W}}$ \\
\hline elec. & $-6.99 \pm 0.13$ & $-9.22 \pm 0.09$ & $-2.23 \pm 0.15$ & $-8.1 \pm 0.11$ & $-1.11 \pm 0.17$ \\
\hline $\mathrm{vdW}$ & $-9.86 \pm 0.03$ & $-16.26 \pm 0.03$ & $-6.4 \pm 0.05$ & $-15.27 \pm 0.02$ & $-5.41 \pm 0.04$ \\
\hline total & $-16.9 \pm 0.13$ & $-25.5 \pm 0.09$ & $-8.6 \pm 0.16$ & $-23.4 \pm 0.11$ & $-6.5 \pm 0.17$ \\
\hline
\end{tabular}

${ }^{a}$ Electrostatic and van der Waals contributions are given separately. Energies are given in $\mathrm{kcal} \cdot \mathrm{mol}^{-1}$. 
A:

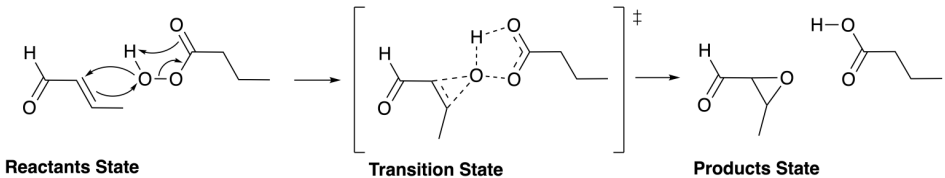

B:

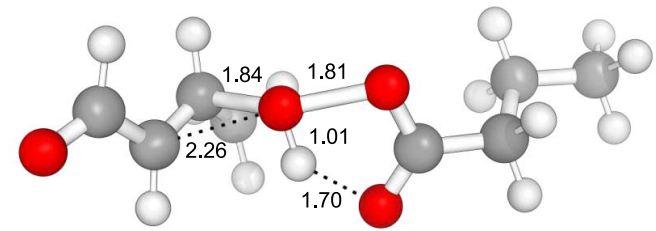

Figure 8. (A) Schematic representation of the epoxidation of 2-butenal by perbutyric acid in solution. (B) Representation of the TS structure of the reaction in chloroform, as obtained at the B3LYP/MM level.

dictated by the binding step and the preference for the $(S)$ enantiomer derived from the chemical step of the full catalyzed process could cancel out, thus rendering the lack of enantioselectivity experimentally observed.

According to our results, the alternative route to get the epoxides in chloroform, the Prilezhaev reaction, would take place in a single step but with a significantly higher free-energy barrier. Thus, our results support the use of a promiscuous modified Ser105Ala CALB enzyme as an environmentally friendly alternative for the production of epoxides in industries. Further redesigns of the enzyme could be studied in the future to increase the efficiency and selectivity of the biocatalyzed process.

\section{ASSOCIATED CONTENT}

\section{SI Supporting Information}

The Supporting Information is available free of charge at https://pubs.acs.org/doi/10.1021/acs.jcim.1c00425.

Free-energy surfaces of all mechanisms, key distances and charge tables, evolution of the RMSD during MD, substrate-protein interaction energies for the $(R)$ - and $(S)$-2-butenal isomers, and parameters of the substrates (PDF)

\section{AUTHOR INFORMATION}

\section{Corresponding Authors}

Katarzyna Świderek - BioComp group, Institute of Advanced Materials (INAM), Universitat Jaume I, 12071 Castellón, Spain; @ orcid.org/0000-0002-7528-1551; Email: swiderek@uji.es

Vicent Moliner - BioComp group, Institute of Advanced Materials (INAM), Universitat Jaume I, 12071 Castellón, Spain; ๑ orcid.org/0000-0002-3665-3391;

Email: moliner@uji.es

\section{Author}

Miquel A. Galmés - BioComp group, Institute of Advanced Materials (INAM), Universitat Jaume I, 12071 Castellón, Spain

Complete contact information is available at: https://pubs.acs.org/10.1021/acs.jcim.1c00425

\section{Author Contributions}

M.A.G. carried out all of the calculations. K.S. and V.M. designed the project and analyzed the results. All authors contributed to writing the manuscript.

\section{Notes}

The authors declare no competing financial interest.

1TCA PDB structure can be downloaded from www.rcsb.org/ structure/1TCA. VMD v1.9.2 can be downloaded free of charge from www.ks.uiuc.edu/Research/vmd. NAMD v2.14 can be downloaded free of charge from www.ks.uiuc.edu/ Research/namd. PROPKA3 v3.2 can be freely downloaded from github.com/jensengroup/propka. MOLDEN v5.8.1 can be downloaded under academic license from www3.cmbi. umcn.nl/molden. Antechamber is part of AmberTools20 that can be obtained from ambermd.org/AmberTools.php. Gaussian09 D01 can be purchased from gaussian.com. fDYNAMO v2.2 can be freely downloaded from www.pdynamo.org/ downloads.

\section{ACKNOWLEDGMENTS}

The authors would like to thank the Spanish Ministerio de Ciencia e Innovación (grant nos. PGC2018-094852-B-C21 and PID2019-107098RJ-I00), the Generalitat Valenciana (grant nos. AICO/2019/195 and SEJI/2020/007), and the Universitat Jaume I (grant nos. UJI-B2017-31 and UJI-A201904). M.A.G. thanks the Universitat Jaume I for FPI-UJI grant (PREDOC/2017/23). The authors acknowledge computational resources from the Servei d'Informàtica of Universitat Jaume I.

\section{REFERENCES}

(1) Gotor-Fernández, V.; Brieva, R.; Gotor, V. Lipases: Useful Biocatalysts for the Preparation of Pharmaceuticals. J. Mol. Catal. B: Enzym. 2006, 40, 111-120.

(2) Busto, E.; Gotor-Fernández, V.; Gotor, V. Hydrolases: Catalytically Promiscuous Enzymes for Non-Conventional Reactions in Organic Synthesis. Chem. Soc. Rev. 2010, 39, 4504-4523.

(3) Tawfik, D. S.; Khersonsky, O. Enzyme Promiscuity: A Mechanistic and Evolutionary Perspective. Annu. Rev. Biochem. 2010, 79, 471-505.

(4) Sheldon, R. A.; Brady, D. The Limits to Biocatalysis: Pushing the Envelope. Chem. Commun. 2018, 54, 6088-6104.

(5) Leveson-Gower, R. B.; Mayer, C.; Roelfes, G. The Importance of Catalytic Promiscuity for Enzyme Design and Evolution. Nat. Rev. Chem. 2019, 3, 687-705. 
(6) Rix, G.; Watkins-Dulaney, E. J.; Almhjell, P. J.; Boville, C. E.; Arnold, F. H.; Liu, C. C. Scalable Continuous Evolution for the Generation of Diverse Enzyme Variants Encompassing Promiscuous Activities. Nat. Commun. 2020, 11, No. 5644.

(7) Sheldon, R. A.; Brady, D. Broadening the Scope of Biocatalysis in Sustainable Organic Synthesis. ChemSusChem 2019, 12, 2859-2881.

(8) Prileschajew, N. Oxydation Ungesättigter Verbindungen Mittels Organischer Superoxyde. Ber. Dtsch. Chem. Ges. 1909, 42, 48114815.

(9) Bartlett, P. D. Recent Work on the Mechanisms of Peroxide Reactions. Rec. Chem. Prog. 1905, 11, 47-51.

(10) Plesnicar, B.; Tasevski, M.; Azman, A. The Transition State for the Epoxidation of Ethylene with Peroxyformic Acid. An Ab Initio Molecular Orbital Study. J. Am. Chem. Soc. 1978, 100, 743-746.

(11) Singleton, D. A.; Merrigan, S. R.; Liu, J.; Houk, K. N Experimental Geometry of the Epoxidation Transition State. J. Am. Chem. Soc. 1997, 119, 3385-3386.

(12) Azeez, A. A.; Rhee, K. Y.; Park, S. J.; Hui, D. Epoxy Clay Nanocomposites - Processing, Properties and Applications: A Review. Composites, Part B 2013, 45, 308-320.

(13) Jin, F. L.; Li, X.; Park, S. J. Synthesis and Application of Epoxy Resins: A Review. J. Ind. Eng. Chem. 2015, 29, 1-11.

(14) Orellana-Coca, C.; Camocho, S.; Adlercreutz, D.; Mattiasson, B.; Hatti-Kaul, R. Chemo-Enzymatic Epoxidation of Linoleic Acid: Parameters Influencing the Reaction. Eur. J. Lipid Sci. Technol. 2005, $107,864-870$

(15) Scotti, N.; Ravasio, N.; Psaro, R.; Evangelisti, C.; Dworakowska, S.; Bogdal, D.; Zaccheria, F. Copper Mediated Epoxidation of High Oleic Natural Oils with a Cumene-O-2 System. Catal. Commun. 2015, 64, 80-85.

(16) Zhou, P.; Wang, X.; Yang, B.; Hollmann, F.; Wang, Y. Chemoenzymatic Epoxidation of Alkenes with Candida antarctica Lipase B and Hydrogen Peroxide in Deep Eutectic Solvents. RSC Adv. 2017, 7, 12518-12523.

(17) Tong, K. H.; Wong, K. Y.; Chan, T. H. A Chemoenzymic Approach to the Epoxidation of Alkenes in Aqueous Media. Tetrahedron 2005, 61, 6009-6014.

(18) Zaks, A.; Dodds, D. R. Chloroperoxidase-Catalyzed Asymmetric Oxidations - Substrate-Specificity and Mechanistic Study. J. Am. Chem. Soc. 1995, 117, 10419-10424.

(19) de Visser, S. P.; Ogliaro, F.; Sharma, P. K.; Shaik, S. What Factors Affect the Regioselectivity of Oxidation by Cytochrome P450? A DFT Study of Allylic Hydroxylation and Double Bond Epoxidation in a Model Reaction. J. Am. Chem. Soc. 2002, 124, 11809-11826.

(20) Svedendahl, M.; Carlqvist, P.; Branneby, C.; Allnér, O.; Frise, A.; Hult, K.; Berglund, P.; Brinck, T. Direct Epoxidation in Candida antarctica Lipase B Studied by Experiment and Theory. ChemBioChem 2008, 9, 2443-2451.

(21) Wang, X. P.; Zhou, P. F.; Li, Z. G.; Yang, B.; Hollmann, F.; Wang, Y. H. Engineering a Lipase B from Candida Antactica with Efficient Perhydrolysis Performance by Eliminating Its Hydrolase Activity. Sci. Rep. 2017, 7, No. 44599.

(22) Swiderek, K.; Martí, S.; Moliner, V. Theoretical Study of Primary Reaction of Pseudozyma Antarctica Lipase B as the Starting Point to Understand Its Promiscuity. ACS Catal. 2014, 4, 426-434.

(23) Bordes, I.; Recatalá, J.; Świderek, K.; Moliner, V. Is Promiscuous CALB a Good Scaffold for Designing New Epoxidases? Molecules 2015, 20, 17789-17806.

(24) Swiderek, K.; Moliner, V. Computational Studies of Candida antarctica Lipase B to Test Its Capability as a Starting Point to Redesign New Diels-Alderases. J. Phys. Chem. B 2016, 120, 20532070.

(25) Galmés, M. A.; García-Junceda, E.; Świderek, K.; Moliner, V. Exploring the Origin of Amidase Substrate Promiscuity in CALB by a Computational Approach. ACS Catal. 2020, 10, 1938-1946.

(26) Wikmark, Y.; Humble, M. S.; Bäckvall, J. E. Combinatorial Library Based Engineering of Candida antarctica Lipase a for
Enantioselective Transacylation of Sec-Alcohols in Organic Solvent. Angew. Chem., Int. Ed. 2015, 54, 4284-4288.

(27) Chen, X. Y.; Chen, G. J.; Wang, J. L.; Wu, Q.; Lin, X. F. Lipase/ Acetamide-Catalyzed Carbon-Carbon Bond Formations: A Mechanistic View. Adv. Synth. Catal. 2013, 355, 864-868.

(28) Sarmah, N.; Revathi, D.; Sheelu, G.; Rani, K. Y.; Sridhar, S.; Mehtab, V.; Sumana, C. Recent Advances on Sources and Industrial Applications of Lipases. Biotechnol. Prog. 2018, 34, 5-28.

(29) Chávez, G.; Hatti-Kaul, R.; Sheldon, R. A.; Mamo, G. BaeyerVilliger Oxidation with Peracid Generated in Situ by CaLB-CLEA Catalyzed Perhydrolysis. J. Mol. Catal. B: Enzym. 2013, 89, 67-72.

(30) Uppenberg, J.; Morgens, H.; Shamkant, P.; Alwyn, J. T. The Sequence, Crystal Structure Determination and Refinement of Two Crystal Forms of Lipase B from Candida antarctica. Structure 1994, 2, 293-308.

(31) Humphrey, W.; Dalke, A.; Schulten, K. VMD - Visual Molecular Dynamics. J. Mol. Graphics 1996, 14, 33-38.

(32) Schaftenaar, G.; Noordik, J. H. Molden: A Pre-and PostProcessing Program for Molecular and Electronic Structures. $J$ Comput.-Aided Mol. Des. 2000, 14, 123-134.

(33) Olsson, M. H. M.; SØndergaard, C. R.; Rostkowski, M.; Jensen, J. H. PROPKA3: Consistent Treatment of Internal and Surface Residues in Empirical p Kapredictions. J. Chem. Theory Comput. 2011, 7, 525-537.

(34) Søndergaard, C. R.; Olsson, M. H. M.; Rostkowski, M.; Jensen, J. H. Improved Treatment of Ligands and Coupling Effects in Empirical Calculation and Rationalization of PKa Values. J. Chem. Theory Comput. 2011, 7, 2284-2295.

(35) Jorgensen, W. L.; Chandrasekhar, J.; Madura, J. D.; Impey, R. W.; Klein, M. L. Comparison of Simple Potential Functions for Simulating Liquid Water. J. Chem. Phys. 1983, 79, 926-935.

(36) Duan, Y.; Wu, C.; Chowdhury, S.; Lee, M. C.; Xiong, G.; Zhang, W.; Yang, R.; Cieplak, P.; Luo, R.; Lee, T.; et al. A PointCharge Force Field for Molecular Mechanics Simulations of Proteins Based on Condensed-Phase Quantum Mechanical Calculations. J. Comput. Chem. 2003, 24, 1999-2012.

(37) Phillips, J. C.; Braun, R.; Wang, W. E. I.; Gumbart, J.; Tajkhorshid, E.; Villa, E.; Chipot, C.; Skeel, R. D.; Poincare, H.; et al. Scalable Molecular Dynamics with NAMD. J. Comput. Chem. 2005, 26, 1781-1802.

(38) Wang, J.; Wolf, R. M.; Caldwell, J. W.; Kollman, P. A.; Case, D. A. Development and Testing of a General Amber Force Field. J. Comput. Chem. 2004, 25, 1157-1174.

(39) Wang, J.; Wang, W.; Kollman, P. A.; Case, D. A. Automatic Atom Type and Bond Type Perception in Molecular Mechanical Calculations. J. Mol. Graphics Modell. 2006, 25, 247-260.

(40) Dewar, M. J. S.; Zoebisch, E. G.; Healy, E. F.; Stewart, J. J. P. Development and Use of Quantum Mechanical Molecular Models. 76. AM1: A New General Purpose Quantum Mechanical Molecular Model. J. Am. Chem. Soc. 1985, 107, 3902-3909.

(41) Grest, G. S.; Kremer, K. Molecular Dynamics Simulation for Polymers in the Presence of a Heat Bath. Phys. Rev. A 1986, 33, 3628-3631.

(42) MacQueen, J. In Some Methods for Classification and Analysis of Multivariate Observations, Proceedings of the Fifth Berkeley Symposium on Mathematical Statistics and Probability, 1967.

(43) Hestenes, M. R.; Stiefel, E. Methods of Conjugate Gradients for Solving Linear Systems. J. Res. Natl. Bur. Stand. 1952, 49, 409.

(44) Byrd, R. H.; Lu, P.; Nocedal, J.; Zhu, C. A Limited Memory Algorithm for Bound Constrained Optimization. SIAM J. Sci. Comput. 1995, 16, 1190-1208.

(45) Field, M. J.; Albe, M.; Bret, C.; Proust-De Martin, F.; Thomas, A. The Dynamo Library for Molecular Simulations Using Hybrid Quantum Mechanical and Molecular Mechanical Potentials. J. Comput. Chem. 2000, 21, 1088-1100.

(46) Lee, C.; Yang, W.; Parr, R. G. Development of the ColleSalvetti Correlation-Energy Formula into a Functional of the Electron Density. Phys. Rev. B 1988, 37, 785-789. 
(47) Becke, A. D. Density-functional Thermochemistry. III. The Role of Exact Exchange. J. Chem. Phys. 1993, 98, 5648-5652.

(48) Frisch, M. J.; Trucks, G. W.; Schlegel, H. B.; Scuseria, G. E.; Robb, M. A.; Cheeseman, J. R.; Scalmani, G.; Barone, V.; Petersson, G. A.; Nakatsuji, H. et al. Gaussian 09, Revision E.01; Gaussian, Inc. Wallingford, CT, 2009

(49) Field, M. J.; Bash, P. A.; Karplus, M. A Combined Quantum Mechanical and Molecular Mechanical Potential for Molecular Dynamics Simulations. J. Comput. Chem. 1990, 11, 700-733.

(50) Jorgensen, W. L.; Maxwell, D. S.; Tirado-Rives, J. Development and Testing of the OPLS All-Atom Force Field on Conformational Energetics and Properties of Organic Liquids. J. Am. Chem. Soc. 1996, $118,11225-11236$.

(51) Cieplak, P.; Caldwell, J.; Kollman, P. Molecular Mechanical Models for Organic and Biological Systems Going beyond the Atom Centered Two Body Additive Approximation: Aqueous Solution Free Energies of Methanol and N-Methyl Acetamide, Nucleic Acid Base, and Amide Hydrogen Bonding and Chloroform/. J. Comput. Chem. 2001, 22, 1048-1057.

(52) Baker, J. An Algorithm for the Location of Transition States. J. Comput. Chem. 1986, 7, 385-395.

(53) Bash, P. A.; Field, M. J.; Karplus, M. Free Energy Perturbation Method for Chemical Reactions in the Condensed Phase: A Dynamic Approach Based on a Combined Quantum and Molecular Mechanics Potential. J. Am. Chem. Soc. 1987, 109, 8092-8094.

(54) Świderek, K.; Tuñón, I.; Martí, S.; Moliner, V.; Bertrán, J. Role of Solvent on Nonenzymatic Peptide Bond Formation Mechanisms and Kinetic Isotope Effects. J. Am. Chem. Soc. 2013, 135, 8708-8719.

(55) Marenich, A. V.; Cramer, C. J.; Truhlar, D. G. Universal Solvation Model Based on Solute Electron Density and on a Continuum Model of the Solvent Defined by the Bulk Dielectric Constant and Atomic Surface Tensions. J. Phys. Chem. B 2009, 113, 6378-6396.

(56) Grimme, S.; Antony, J.; Ehrlich, S.; Krieg, H. A Consistent and Accurate Ab Initio Parametrization of Density Functional Dispersion Correction (DFT-D) for the 94 Elements H-Pu. J. Chem. Phys. 2010 132, No. 154104.

(57) Grimme, S.; Ehrlich, S.; Goerigk, L. Effect of the Damping Function in Dispersion Corrected Density Functional Theory. J. Comput. Chem. 2011, 32, 1456-1465.

(58) Chipot, C. Free Energy Calculations in Biological Systems. How Useful Are They in Practice?, In New Algorithms for Macromolecular Simulation; Leimkuhler, B., Chipot, C., Elber, R. Laaksonen, A., Mark, A., Schlick, T., Schütte, C., Skeel, R., Eds.; Springer, 2006; pp 185-211.

(59) Swiderek, K.; Paneth, P. Binding Ligands and Cofactor to LLactate Dehydrogenase from Human Skeletal and Heart Muscles. J. Phys. Chem. B 2011, 115, 6366-6376.

(60) Świderek, K.; Martí, S.; Moliner, V. Theoretical Studies of HIV1 Reverse Transcriptase Inhibition. Phys. Chem. Chem. Phys. 2012, 14, $12614-12624$.

(61) Krzemińska, A.; Paneth, P.; Moliner, V.; Świderek, K. Binding Isotope Effects as a Tool for Distinguishing Hydrophobic and Hydrophilic Binding Sites of HIV-1 RT. J. Phys. Chem. B 2015, 119, 917-927.

(62) Truhlar, D. G.; Garrett, B. C.; Klippenstein, S. J. Current Status of Transition-State Theory. J. Phys. Chem. A 1996, 100, 1277112800.

(63) Martí, S.; Moliner, V.; Tuñón, I.; Williams, I. H. QM / MM Calculations of Kinetic Isotope Effects in the Chorismate Mutase Active Site. Org. Biomol. Chem. 2003, 1, 483-487.

(64) Martí, S.; Moliner, V.; Tuñón, I. Improving the QM/MM Description of Chemical Processes: A Dual Level Strategy to Explore the Potential Energy Surface in Very Large Systems. J. Chem. Theory Comput. 2005, 1, 1008-1016.

(65) Galmés, M. A.; García-Junceda, E.; Świderek, K.; Moliner, V. Exploring the Origin of Amidase Substrate Promiscuity in CALB by a Computational Approach. ACS Catal. 2019, 1938-1946.
(66) Abdulmalek, E.; Arumugam, M.; Mizan, H. N.; Abdul Rahman, M. B.; Basri, M.; Salleh, A. B. Chemoenzymatic Epoxidation of Alkenes and Reusability Study of the Phenylacetic Acid. Sci. World J. 2014, 2014, No. 756418.

(67) Liu, W.; Chen, J.; Liu, R.; Bi, Y. Revisiting the Enzymatic Epoxidation of Vegetable Oils by Perfatty Acid: Perbutyric Acid Effect on the Oil with Low Acid Value. J. Am. Oil Chem. Soc. 2016, 93, 1479-1486.

(68) Chen, J.; Zhou, J.; Liu, W.; Bi, Y.; Peng, D. Enzymatic Epoxidation of Soybean Oil in the Presence of Perbutyric Acid. Chem. Pap. 2017, 71, 2139-2144. 



\subsection{A computer-assisted rational redesign}

\subsubsection{Structural-mechanistic comparison of Bs2 and CALB (Article 3)}

As already mentioned in Chapter 1 (see section 1.2.2), Bs2 is a SH that has been reported to show catalytic and substrate promiscuity, similarly to CALB. ${ }^{1-15}$ This fact is not surprising considering that they share a similar catalytic triad. However, they are non-homologous enzymes with only a scarce $11 \%$ of sequence identity (Figure 4.8). Many questions arise from the molecular perspective about how two non-homologous enzymes have converged into a similar promiscuous behavior. Thus, we pretended to do a deep structural and mechanistic comparison of these two enzymes.

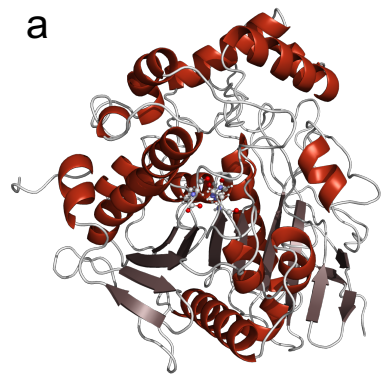

Bs2

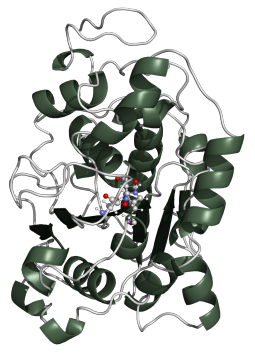

CALB
C<smiles>CCCC(=O)Nc1ccc([N+](=O)[O-])cc1</smiles><smiles>Nc1ccc(O[18O]c2ccc([18OH])cc2)cc1</smiles>

b

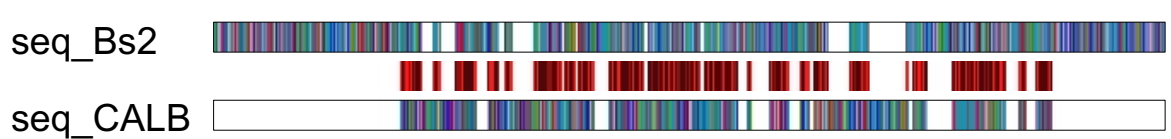

Figure 4.8 Structural comparison of Bs2 and CALB. Panel a shows a cartoon representation of Bs2 (red) and CALB (green) of the tertiary structure. In panel $\mathbf{b}$, a representation of a pairwise sequence alignment of both proteins is presented. Each type of residue of amino acid is represented by a different color, while in the middle the red lines represent the similarity along the alignment. The general reaction catalyzed by both Bs2 and CALB enzymes is shown in panel $\mathbf{c}$. 


\subsection{A computer-assisted rational redesign}

We studied the mechanism of reaction of the promiscuous amidase activity in Bs2. We experimentally proved that Bs2, as CALB, was able to catalyze the hydrolysis of $\mathcal{N}$-(4-nitrophenyl)-butyramide. Moreover, from the QM/MM calculations, we saw that Bs2 followed a similar reaction mechanism as CALB (see Figure 4.2). Not only that but they also rendered similar energy barriers, which at the same time were in strong good agreement with our experimental results (Figure 4.9). Although both enzymes are structurally very different, they catalyze the hydrolysis of $\mathcal{N}$ (4-nitrophenyl)-butyramide in a mechanistically similar way. With these results, we were encouraged to perform a deeper comparison of both proteins, at the mechanistic and structural levels.

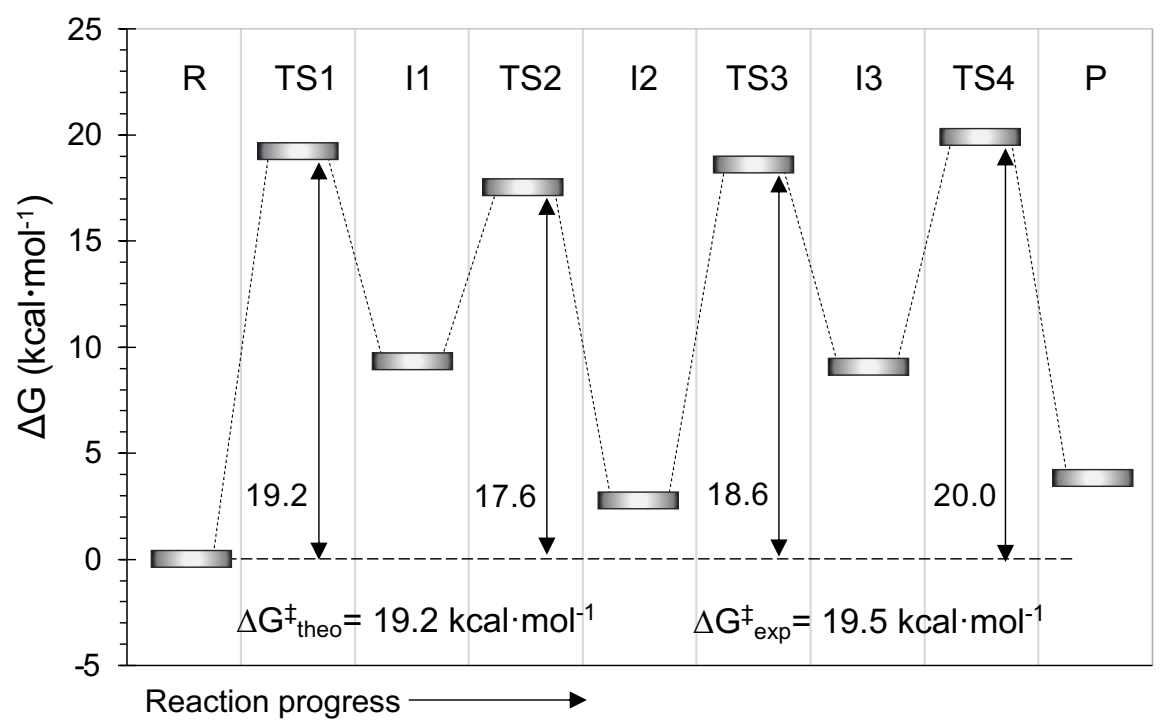

Figure 4.9. Free energy profile of the hydrolysis of $\mathcal{N}$-(4-nitrophenyl)-butyramide catalyzed by Bs2 at M06-2X/OPLS-AA level.

From the mechanistic perspective, we found that the second step of the reaction, which involves the breaking of the $\mathrm{G}-\mathrm{N}$ bond, was different between Bs2 and CALB. In Bs2 the breaking of the amide bond was followed by the transfer of the proton from the catalytic His399 to the nitrogen of the leaving group, while in CALB the transfer of the proton 
preceded the amide bond breaking. This fact could be explained by the difference in the electrostatic forces generated by the enzyme on the $\mathrm{C}-\mathrm{N}$ bond (Figure 4.10). At the same time, the peculiarities in the electrostatic potential generated on each of the active sites could be explained by the structural nature of both enzymes.

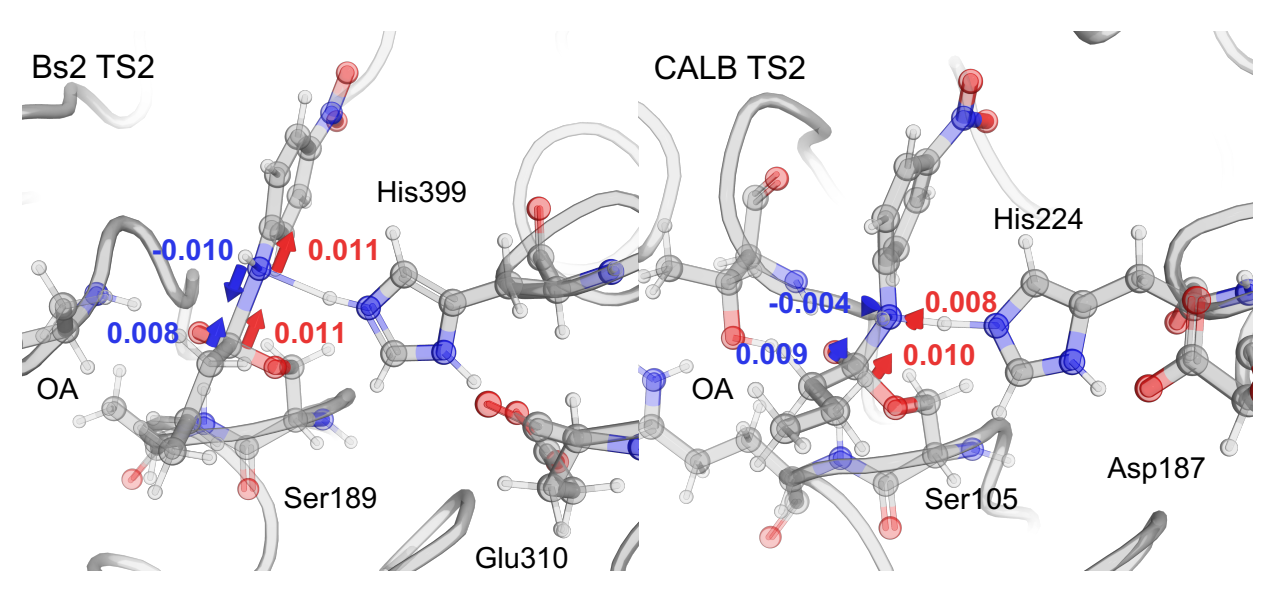

Figure 4.10. TS2 structures of both Bs2 and CALB are showed. The projection of the electric field in red arrows is represented in the direction of the $\mathrm{C}-\mathrm{N}$ bond of the substrate, while blue arrows represent the electrostatic forces (given in a.u. $\left.\times 10^{3}\right)$.

To explain these facts, we decided to map the structural features of the proteins using CNNs. To summarize, a 3D-CNN was built to classify enzymes from the same functional EC number. Then, an in silico alanine scan was done to determine which were the structural key residues of Bs2 and CALB that classify them in the correct family. Finally, a map of structural determinants, in both Bs2 and CALB, was built (Figure 4.11). Despite they are structurally very different in terms of primary sequence, they share some common chemistry within the vicinity of the active site. Moreover, based on the alanine scan, we conclude that Bs2 might be a more robust scaffold for future mutagenesis studies, which were explored in the consecutive article (Article 4).

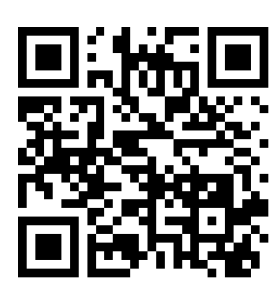




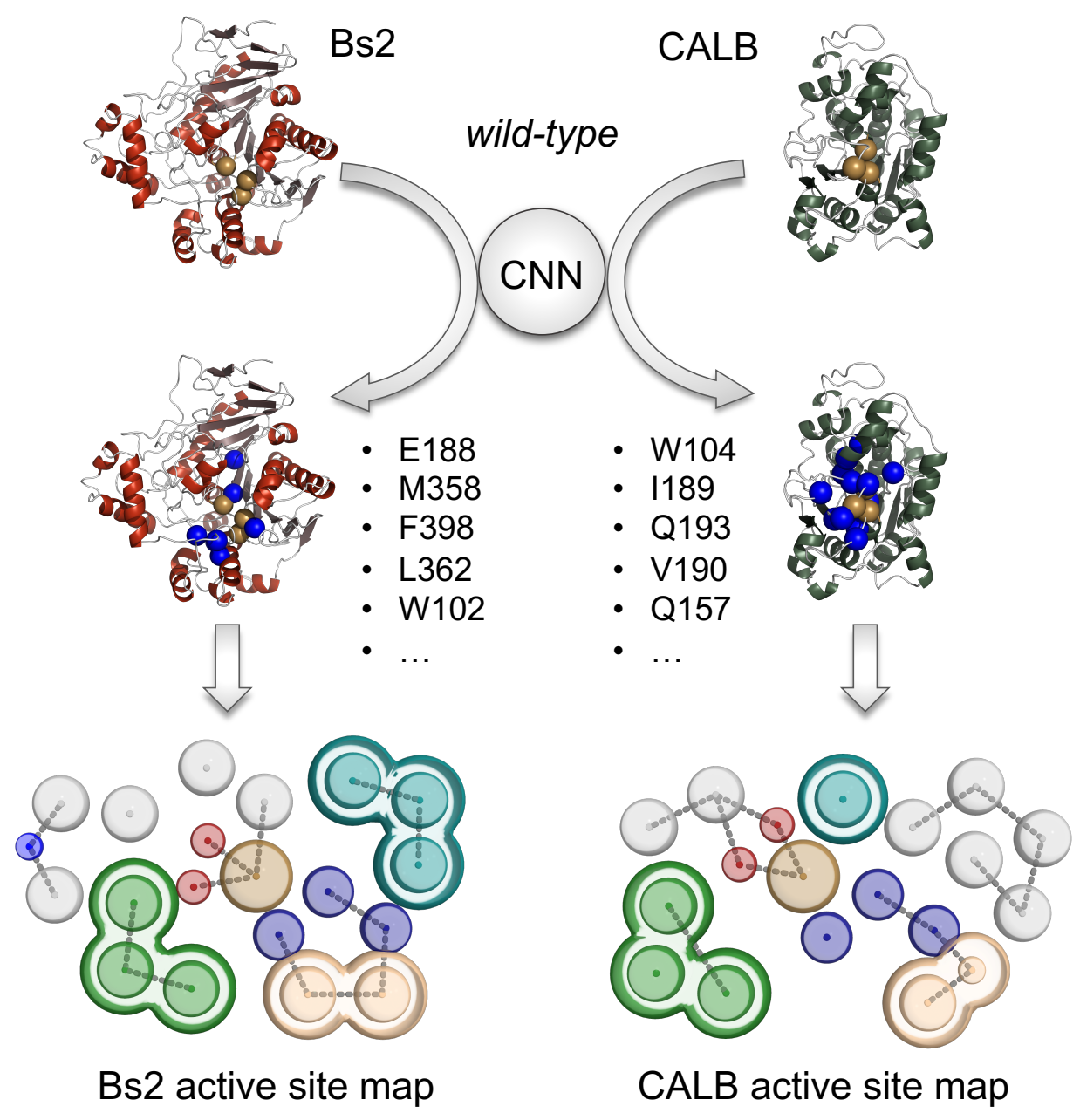

Figure 4.11. Representation of the in-silico alanine scan to map the active site of Bs2 and CALB. Iterative cycles of mutations are done in both proteins and analyzed by the CNN to map the structural key residues in the vicinity of the active site. Finally, a reconstruction of the active site is done based on the residues detected by the GNN. In the active site map, the brown sphere in the center represents the substrate, the dark blue spheres represent the catalytic triad and in red the oxyanion hole. Moreover, the region marked in green highlights the hydrophobic pocket, in turquoise a pi-pi network, and in orange a region responsible of the stabilization of the catalytic triad. 


\title{
Combined Theoretical and Experimental Study to Unravel the Differences in Promiscuous Amidase Activity of Two Nonhomologous Enzymes
}

\author{
Miquel À. Galmés, Alexander R. Nödling, Louis Luk,* Katarzyna Świderek,* and Vicent Moliner*
}

Cite This: ACS Catal. 2021, 11, 8635-8644

Read Online

ACCESS | Lل|ll Metrics \& More | 稩 Article Recommendations | sl Supporting Information

ABSTRACT: Convergent evolution has resulted in nonhomologous enzymes that contain similar active sites that catalyze the same primary and secondary reactions. Comparing how these enzymes achieve their reaction promiscuity can yield valuable insights to develop functions from the optimization of latent activities. In this work, we have focused on the promiscuous amidase activity in the esterase from Bacillus subtilis (Bs2) and compared with the same activity in the promiscuous lipase B from Candida antarctica (CALB). The study, combining multiscale quantum mechanics/molecular mechanics ( $Q M / M M)$ simulations, deep machine learning approaches, and experimental character-

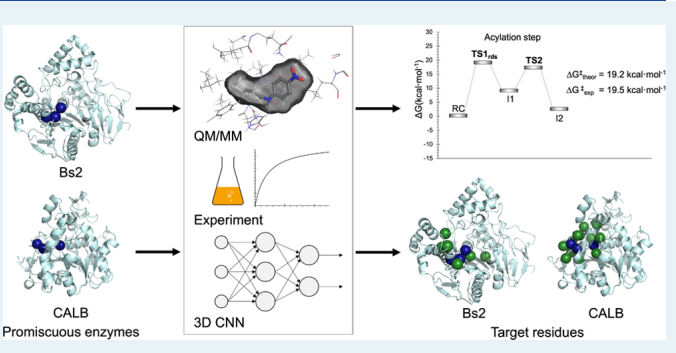
ization of Bs2 kinetics, confirms the amidase activity of Bs2 and

CALB. The computational results indicate that both enzymes offer a slightly different reaction environment reflected by electrostatic effects within the active site, thus resulting in a different reaction mechanism during the acylation step. A convolutional neural network ( $\mathrm{CNN}$ ) has been used to understand the conserved amino acids among the evolved protein family and suggest that Bs2 provides a more robust protein scaffold to perform future mutagenesis studies. Results derived from this work will help reveal the origin of enzyme promiscuity, which will find applications in enzyme (re)design, particularly in creating a highly active amidase.

KEYWORDS: enzyme promiscuity, QM/MM, free energy surfaces, convolutional neural network, $C A L B, B s 2$, amidase activity

\section{INTRODUCTION}

Enzyme promiscuity serves as a reservoir for new catalytic activities, playing a vital role in survival and adaptation during the course of evolution. ${ }^{1,2}$ Recent work by Voordeckers et al. has illustrated ancestral enzymes displayed a much wider range of substrate promiscuity at lower activity, ${ }^{3}$ whereas many enzymes identified to date catalyze secondary reactions whose activities are often less efficient but can be improved upon (laboratory) selection. ${ }^{4}$ Hence, it has been proposed that, under selective pressure (e.g., presence of new valuable chemical resources), reaction promiscuity enables the creation of new enzymes with a modified reaction profile and/or substrate specificity.

From a molecular perspective, enzyme evolution takes place by modifying the electrostatic properties and geometrical complementarities of the active site such that the chemical aspects of the new molecules can be accommodated. ${ }^{6-8}$ This optimization process occurs gradually and smoothly in the sequence space, ${ }^{9}$ and closely related enzymes can act on different substrates as a consequence of divergent evolution. ${ }^{10}$ On the other hand, many enzymes have evolved to catalyze the same reaction whilst having no sequence homology. ${ }^{11}$ Such a phenomenon can be partially explained by the fact that they share similar or same active sites. Indeed, the first evidence of this convergent evolution event has been reported in the study of the serine protease family (amidase) ${ }^{11-15}$ Furthermore, the recruitment of ancestral enzymes that change specificity might lead to mechanistic analogues. ${ }^{16}$ Hence, many serine hydrolases (SHs) also contain the conserved catalytic Ser-His-Aps/Glu triad. ${ }^{17}$ Nevertheless, finding the proper characteristics that guide a protein or a family of proteins toward a specific activity or their capabilities to catalyze other than a primary reaction can be a challenging task. In this sense, convolutional neural networks (CNN) have been proved to be very useful in classification tasks in a wide range of fields, such as image, speech, and movement recognition, together with text analysis. ${ }^{18}$ Although there are expanding applications of CNN, little research has been conducted in the analysis of the protein structure. $^{19-22}$

para-Nitrobenzyl (PNB) esterase from Bacillus subtilis (Bs2) and lipase B from Candida antarctica (CALB) are non-

Received: May 12, 2021

Revised: June 17, 2021

Published: June 30, 2021 


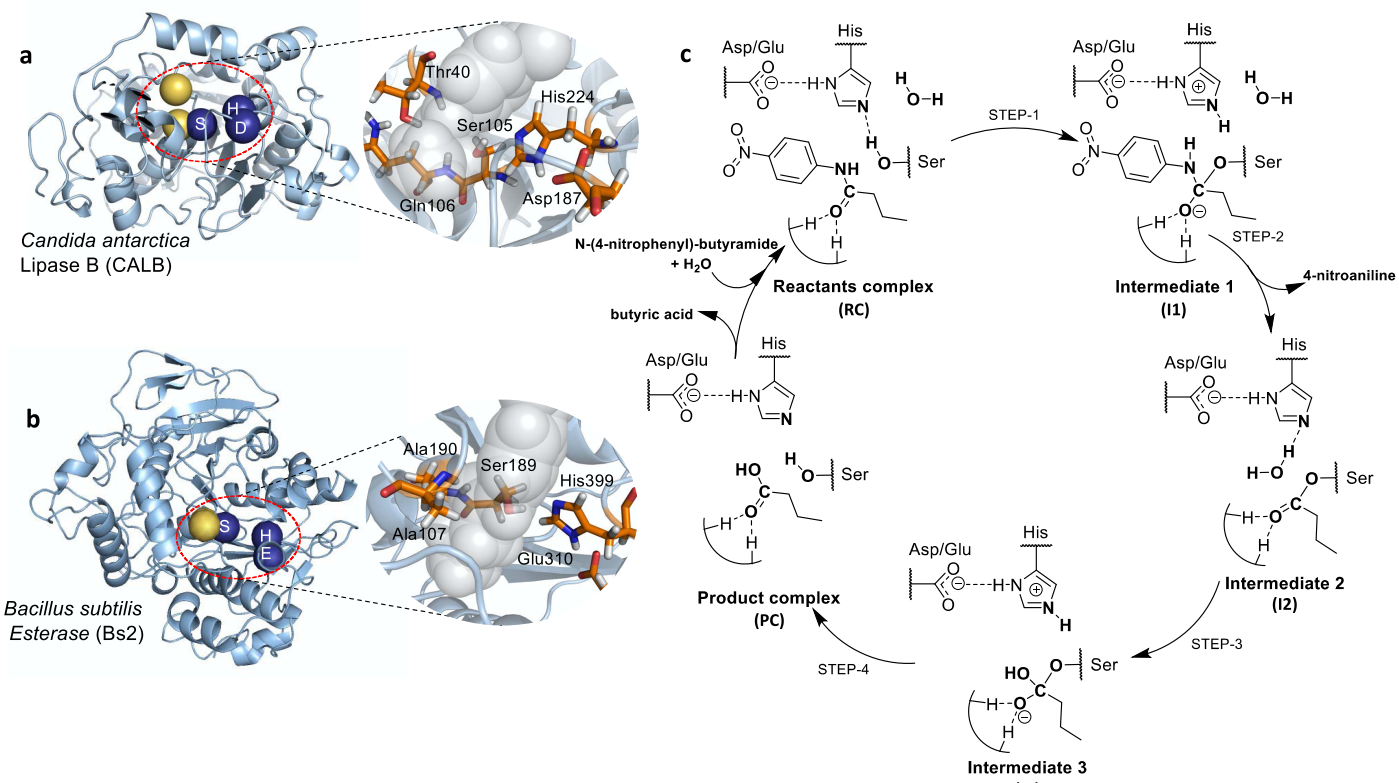

(13)

Figure 1. Structure and mechanism of CALB and Bs2. (a) Structure of CALB (Protein Data Bank (PDB) ID 1TCA) with details of its active site. (b) Structure of Bs2 (PDB ID 1QE3) with details of its active site. Blue spheres in panels (a) and (b) represent the catalytic triad, while yellow spheres indicate the oxyanion hole residues. (c) Schematic representation of the hydrolysis of $N$-(4-nitrophenyl)-butyramide catalyzed by CALB/Bs2. The reaction yields a molecule of 4-nitroaniline (second step, intermediate 2 (I2)) and butyric acid (last step, product complex).

homologous enzymes belonging to the functional family EC 3.1.1, which accounts for carboxylic ester hydrolases. Bs2 can be further subclassified as a carboxylesterase (EC 3.1.1.1) according to the Brenda database, ${ }^{23}$ whereas CALB belongs to the lipase family (EC 3.1.1.3). Bs2 and CALB share only $11 \%$ of identity (see the Supporting Information) and originate in organisms that belong to a different taxonomical rank, with the former from a prokaryote whereas the latter from a eukaryote. Despite the distant relation, they have been found to catalyze similar promiscuous reactions including hydrolysis of paranitrophenyl-butyrate ${ }^{24-26}$ and poly(ethylene terephthalate) ${ }^{27,28}$ Furthermore, both enzymes contain a characteristic triad whereby Ser serves as a nucleophile, His as an acid/base residue, and Asp (in Bs2)/Glu (in CALB) as a base to modulate the $\mathrm{p} K_{\mathrm{a}}$ of the histidine residue (Figure 1). Moreover, the presence of an oxyanion hole in proximity to the catalytic triad permits the proper placement of the carbonyl oxygen of the tetrahedral intermediate in the active site. Interestingly, both enzymes have a promiscuous reaction behavior; we have recently confirmed that CALB can catalyze the hydrolysis of $\mathrm{N}$-(4-nitrophenyl)-butyramide, ${ }^{26}$ while previous works showed that Bs2 is capable to catalyze identical processes. ${ }^{24,25}$ This has encouraged us to study the amidase activity of Bs2 in an aim to find similarities in these nonhomologous enzymes.

We envisioned that the concept of convergent evolution of enzyme promiscuity can be applied in enzyme (re)design. This must take into account that mutations in both the active site and distal location are crucial to create an active enzyme variant. ${ }^{29}$ To put this concept forward into an application, mechanistic studies are essential. In particular, it remains unclear if there is any parallelism in the preorganization of the active site that drives their catalytic activity in an analogous manner. Hence, hydrolysis of $\mathrm{N}$-(4-nitrophenyl)-butyramide catalyzed by Bs2 (see Figure 1) was analyzed, employing theoretical methods based on multiscale quantum mechanics/molecular mechanics $(\mathrm{QM} / \mathrm{MM})$ and kinetic measurements. The results were analyzed and compared with those derived from our previous study on CALB, ${ }^{26}$ including the energetics, geometries, and electrostatic properties obtained from the full $Q M / M M$ free energy landscape of the most favored reaction paths. Deep CNN approach was performed complementarily to analyze protein residues that are conserved during the evolution of the two convergently related enzymes, generating a map of structural determinants in the vicinity of the active site.

\section{METHODS}

Computational Methods. Wild-type $p$-nitrobenzyl (PNB) esterase sequence from B. subtilis (with ID P37967) was initially taken from UniProt. ${ }^{30}$ Because the crystal structure for this specific variant is lacking, a model was prepared based on the reported structure of PNB esterase isolated from a different organism (Bs2; PDB ID: 1QE3), ${ }^{31}$ and the missing residues and required mutations were introduced using Modeller $^{32}$ (see Figure S1). A N-(4-nitrophenyl)-butyramide substrate was placed inside the active site pocket covalently bound to the catalytic Ser189 in the form of INT1 to avoid the possible substrate dissociation to the solvent during the equilibration molecular dynamics (MD). The protonation state of titratable residues was determined at $\mathrm{pH} 7$ using the empirical program PropKa v.3.0.3..$^{33,34}$ along with detailed inspection of the surroundings of each histidine residue. Twenty counterions $\left(\mathrm{Na}^{+}\right)$were placed in optimal electrostatic positions (those 
where the potential reaches maximum negative values) around the enzyme (further than $10.5 \AA$ from any atom of the system and $5 \AA$ from any other counterion, using a regular grid of $0.5 \AA$ ) to obtain the electroneutrality of the system. Finally, the protein, counterions, and the substrate $\mathrm{N}$-(4-nitrophenyl)-butyramide were placed in a $100 \times 80 \times 80 \AA^{3}$ pre-equilibrated orthorhombic box of TIP3P ${ }^{35}$ water molecules.

After initial energy minimizations, the system was heated to $303 \mathrm{~K}$ with $0.1 \mathrm{~K}$ temperature increment and equilibrated during short NPT MD simulations, followed by nonaccelerated classical NVT MD simulations with AMBER force field, ${ }^{36}$ as implemented in NAMD software. ${ }^{37}$ The substrate was described with the same force field parameters as determined in our previous work. ${ }^{26}$ During MD simulations, all atoms were free to move with periodic boundary conditions and cut-offs for nonbonding interactions. Time-dependent evolution of the root mean square deviation (RMSD) together with the $B$-factor calculation confirmed that the model was equilibrated (Figure S2).

The reaction was studied using a $\mathrm{QM} / \mathrm{MM}$ approach from the equilibrated structures. The most populated reactive enzyme (based on the distances of the reaction coordinates) was extracted to be used as initial geometry for subsequent calculations. The semiempirical AM1 ${ }^{38}$ method and the M06$2 \mathrm{X}^{39}$ density functional were used to describe the QM subset of atoms, corresponding to the substrate and the catalytic residues of the active site (as illustrated in Figure 2). A water molecule

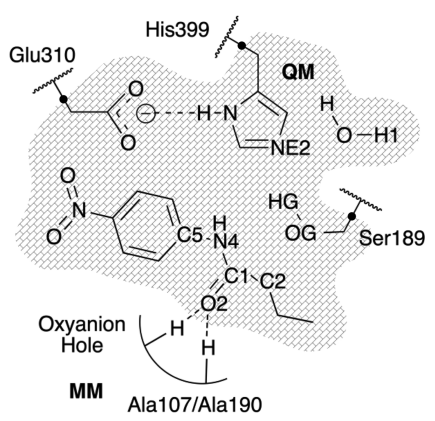

Figure 2. Schematic representation of the $\mathrm{QM}$ subset region (shadowed region). Black dots represent link atoms between QM and $\mathrm{MM}$ regions. The $\mathrm{H}$-bond interactions between the $\mathrm{N}$-(4nitrophenyl)-butyramide substrate and the oxyanion hole are shown as dashed lines.

was also included in the QM regions for the deacylation step. The optimized potentials for liquid simulations all-atom (OPLS$\mathrm{AA})^{40}$ and TIP3P $\mathrm{P}^{35}$ classical force fields were used to treat the protein and the solvent water molecules, respectively, as implemented in fDynamo library. ${ }^{41}$ After potential energy surfaces (PESs) were computed, the appropriate distinguished reaction coordinates were explored, and free energy surfaces (FESs) for each of the chemical steps were calculated in terms of potentials of mean forces (PMFs) at AM1/MM level and subsequently improved at M06-2X/MM level by means of spline corrections. Structures corresponding to the stationary points were finally optimized at M06-2X/MM level and the nature of these structures as minima or saddle point of order one was verified by computing the frequency analysis. Detailed description of the computational methods can be found in the Supporting Information.

A three-dimensional (3D) CNN was built to analyze the near vicinity of the active sites in Bs2 and CALB. In particular, 11, 22, and 25 nonredundant structures from the PDB database classified as EC 3.1.1.1, EC 3.1.1.3, and EC 3.1.1.4 were extracted, respectively. A box of $20 \AA$ was placed in the geometrical center of the catalytic residues. The box was divided into voxels of 1 A side, so only one atom lay in a voxel. Each atom was codified using a vector with 13 features (see details in the Supporting Information), making a cube of $20 \times 20 \times 20 \AA^{3}$ with 13 channels per voxel. This strategy ensured that each amino acid has a particular combination of characteristics making it unique. This also permits analogous residues, such as Asp and Glu, to be treated nonequally though they share similar chemical properties dictated by the nature of their lateral chains. Hence, to ensure that only these specific properties were seen by the network and to avoid a learning based in backbone geometry, the atoms belonging to the backbone were not taken into account. Finally, Gaussian filters were applied to the cube to resemble each atom class. One of the main limitations is the very small initial sample of structures in each family making it poorly diverse. To overcome this limitation, random rotations toward the center of the cube were applied, upsampling the dataset in this way. The initial dataset was divided into train, test, and validation datasets with a ratio of $0.7: 0.15: 0.15$, respectively. We chose a ResNet $50^{42}$ architecture for the training of the network, and it was build using Keras v.2.4.3 ${ }^{43}$ and Tensorflow v.2.4.1 ${ }^{44}$ as a backend. The alanine scan was performed mutating each residue that lies inside the box to Ala using Modeller v.9.25. ${ }^{32}$ Then, 1000 predictions on each mutant protein were done applying random rotations. The final classification score was measured as the difference between the ratio of correct classifications and the ratio of the most probable remaining class.

Experimental Methods. The synthesis of $\mathrm{N}$-(4-nitrophenyl)-butyramide was performed according to a known procedure. ${ }^{26}$ The production of Bs 2 was based on a previously reported procedure. ${ }^{24}$ For the 96 well-plate kinetic assays, stock solutions of $\mathrm{Bs} 2$ (in $50 \mathrm{mM} \mathrm{NaP}, \mathrm{pH} 7.0$ ) and $\mathrm{N}$-(4nitrophenyl)-butyramide (in dimethyl sulfoxide (DMSO)) were prepared. The protein stock solution was kept on ice until use and was freshly prepared before each usage. DMSO and substrate stock solution were added to wells of a 96 transparent well-plate to a total of $15 \mu \mathrm{L}$. Buffer $\left(50 \mathrm{mM} \mathrm{NaP}_{\mathrm{i}}, \mathrm{pH} 7.0\right)$ was added to a total volume of $135 \mu \mathrm{L}(150 \mu \mathrm{L}$ in the case of controls to monitor the substrate stability). The plate was transferred into a plate reader, double orbitally shaken for $5 \mathrm{~s}$ and the absorption at $\lambda_{\mathrm{Ex}}=405 \mathrm{~nm}$ measured to check the correct substrate distribution. Then, $15 \mu \mathrm{L}$ of protein stock solution was added to each well except the enzyme-free controls within $5 \mathrm{~min}$. The final assay conditions were $150 \mu \mathrm{L}$ volume, $10 \%$ DMSO, $N$-(4nitrophenyl)-butyramide (10, 50, 100, $250500,1000,2000$, $3000 \mu \mathrm{M})$, and $20 \mu \mathrm{g} \cdot \mathrm{mL}^{-1}$ protein. The plate was sealed with an airtight and UV-vis transparent self-adhesive plastic cover sheet. After sealing, the plate was placed into the plate reader and the assay was monitored for $14 \mathrm{~h}$.

\section{RESULTS AND DISCUSSION}

Bs2 vs CALB Sequence Comparison. Bs2 and CALB are remarkably different from a structural point of view, although they present some common features such as, as commented above, a common characteristic catalytic triad formed by a serine serving as a nucleophile, a histidine that acts as an acid/base 
residue, and Asp/Glu as a base to modulate the $\mathrm{p} K_{\mathrm{a}}$ of the histidine residue. Moreover, according to the CATH database, ${ }^{45}$ which provides a hierarchical classification of protein domains based on their folding patterns, they both belong to the $\alpha / \beta$ hydrolase superfamily. Based on the phylogenetic tree of the 450 manually selected protein sequences of EC 3.1.1.1, EC 3.1.13, and EC 3.1.1.4 deposited in Uniprot ${ }^{46}$ (Figure 3), it can be

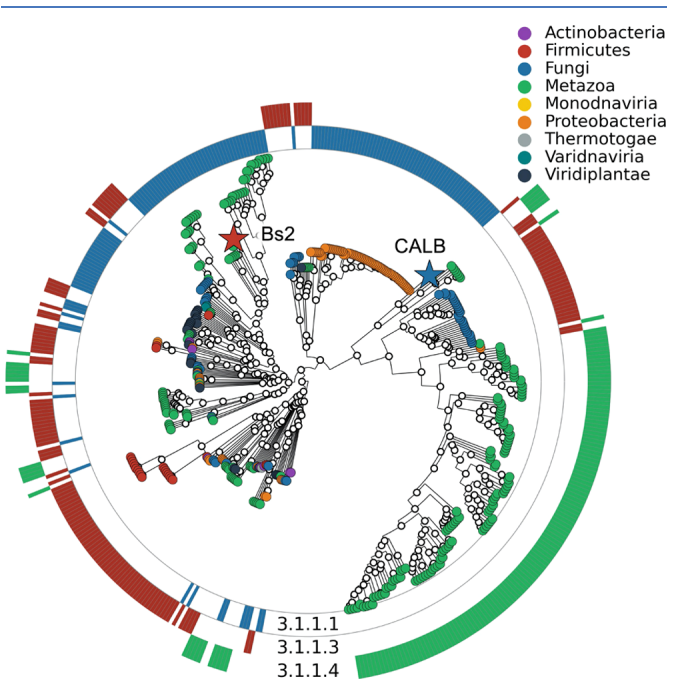

Figure 3. Phylogenetic tree of sample enzymes belonging to EC 3.1.1. External circles codified by each EC number studied. Enzymes were also classified by phylum. The red and blue stars correspond to Bs2 and CALB, respectively.

concluded that Bs2 and CALB are distantly related proteins converged in a function. These EC domains were taken since a reasonable number of crystallographic structures were available in the PDB database, ${ }^{47}$ making it possible to be used for CNN training.

Reaction Mechanism of Bs2 as an Amidase. The hydrolytic reaction of $\mathrm{N}$-(4-nitrophenyl)-butyramide catalyzed by Bs2 was investigated using QM/MM methodologies, assuming the mechanism previously suggested for serine hydrolases. ${ }^{26,48}$ Free energy surfaces (FES) of every step of the reaction were computed in terms of potentials of mean force (PMFs). The resulting FESs reveal that, as expected, the reaction takes place in four steps involving acylation of the substrate, release of the leaving group (4-nitroaniline), hydrolysis of the acyl enzyme complex, and culminating in the regeneration of the active site by final product formation (Figures $1 \mathrm{c}$ and 4).

The free energy profile derived from the FESs shows that the rate-limiting step for the formation of the first product, 4nitroaniline in I2, corresponds to the first step in which Ser198 activated by His 399 attacks $\mathrm{C} 1$ of the substate that covalently binds to the enzyme (see Figure 5a). The computed overall energy barrier for this step was $19.2 \mathrm{kcal} \cdot \mathrm{mol}^{-1}$. The rate-limiting step of the deacylation corresponds to the final step during which the butyric acid product is formed and the enzyme is regenerated $\left(20.0 \mathrm{kcal} \cdot \mathrm{mol}^{-1}\right)$.

The predictions derived from the $\mathrm{QM} / \mathrm{MM}$ computational protocol were assessed experimentally by measuring the release of 4-nitroaniline that according to the results of our calculations can sufficiently serve as a gauge for the catalytic efficiency of Bs2. As observed in Table 1 , the experimentally rate constant $k_{\text {cat }}$ determined by a UV-vis spectrophotometric assay was found to be $0.0185 \pm 0.0007 \mathrm{~s}^{-1}$, which is 2 orders of magnitude higher than previously reported. ${ }^{24,25}$ A possible explanation for this difference could be associated with the purity of the starting material or the difference in the linker used between the enzyme and hexahistidine tag. Additionally, previous studies employed low protein concentrations $\left(66 \mathrm{ng} \cdot \mathrm{mL}^{-1}\right)^{25}$ which may be prone to error during kinetic measurement. Despite these discrepancies, the rate constant obtained in our studies is in excellent agreement with the computationally assessed enzyme activity. In the frame of the transition-state theory, ${ }^{49-51}$ the measured kinetic rate constant corresponds to an energy barrier of 19.5 $\mathrm{kcal} \cdot \mathrm{mol}^{-1}$ at $294 \mathrm{~K}$, which is very close to the computationally predicted value of $19.2 \mathrm{kcal} \cdot \mathrm{mol}^{-1}$ obtained at $300 \mathrm{~K}$.

Although the mechanism of the studied reaction is rather predictable and follows the classical order of chemical transformations, it is of interest to identify some features of Bs2 that allow catalysis of the same secondary reaction as in CALB. Therefore, for the rest of this work, a comparative analysis of catalytic efficiency in these two promiscuous enzymes will be presented.

Promiscuity of Bs2 vs CALB. After analyzing the free energy profile of the reaction pathway determined for both enzymes (Figure 5), it is noticeable that the kinetics of the acylation and deacylation steps are significantly different despite the rate-limiting step of the full process corresponds to the last step in both enzymes. Comparison of the full chemical reaction shows how the overall chemical steps in Bs2 is a slightly endergonic process, while the full reaction in CALB is slightly exergonic. At this point, we must keep in mind that the reported free energy profiles correspond to total energies of the chemical system plus the full solvated proteins for the chemical steps. Both proteins can behave slightly different along the full catalytic process, until all products are released and the enzyme recovered for the next catalytic cycle. Regarding the deacylation step, the energies of TS3 and TS4 relative to the reactant complex (RC) are much higher in the Bs2 reaction in comparison to the same steps in CALB. Nevertheless, the overstabilization of intermediate 2 in CALB makes the overall free energy barrier, determined by the energy of TS4 relative to I2, much higher than in the Bs2 cycle (24.2 vs $20.0 \mathrm{kcal} \cdot \mathrm{mol}^{-1}$, respectively). Nevertheless, keeping in mind that the kinetic experiments are based on the determination of 4-nitroaniline released in intermediate 2, a deeper analysis will be based on just the acylation process where, as mentioned above, a very good agreement was obtained between the experimental rate constants and the predicted activation free energies (see Table $1)$.

The computed free energy barrier of the second step, with respect to the intermediate 1 (I1), is the same in both enzymatic systems suggesting that the stabilization of this intermediate 1 dictates the final shape of the energetic profile of the acylation process (see Figure 5). A more stable I1 in Bs2 is reflected in an overall lower free energy barrier computed for the second step with respect to RC. Interestingly, the different behavior displayed by Bs2 and CALB with respect to the acylation step can be compared with recent $\mathrm{QM} / \mathrm{MM}$ studies carried out in our laboratory on other proteases such as the cruzain ${ }^{52}$ or SARS CoV-2 $\mathrm{M}^{\text {pro53,54 }}$ cysteine proteases, or the $20 \mathrm{~S}$ subunit of proteasome, ${ }^{55}$ where the protonation of the amine leaving group 

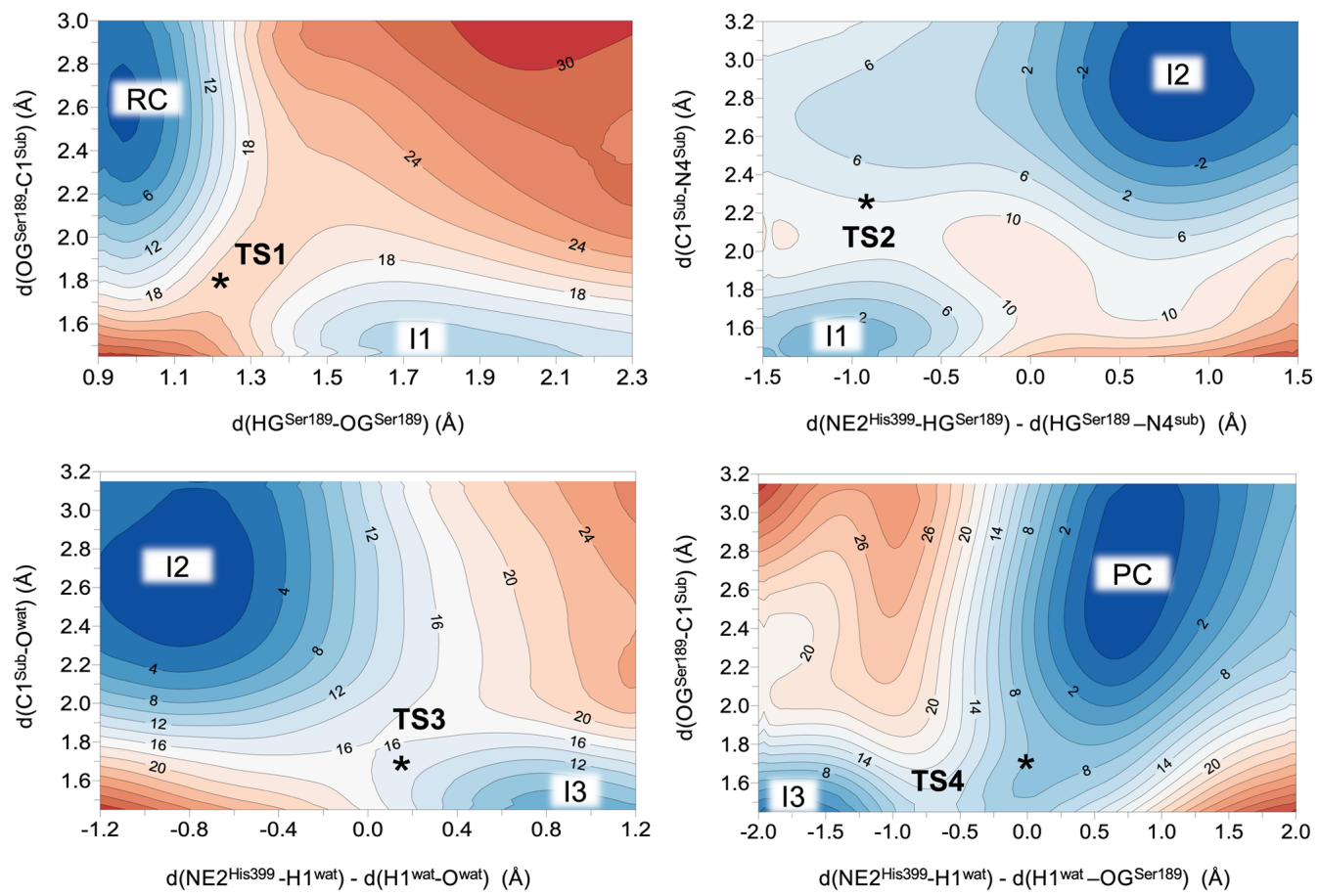

Figure 4. M06-2X:AM1/OPLS-AA free energy surfaces of the hydrolysis of N-(4-nitrophenyl)-butyramide catalyzed by wild-type Bs2. Distances are given in angstrom and isoenergetic lines are in $\mathrm{kcal} \cdot \mathrm{mol}^{-1}$. The black stars indicate the position of single transition-state (TS) structures fully optimized at M06-2X/OPLS-AA level of theory.

appears to be crucial. In the present study, the difference of 5.7 $\mathrm{kcal} \cdot \mathrm{mol}^{-1}$ in the stabilization of I1 between Bs 2 and CALB can be explained based on electrostatic properties (see the Supporting Information). We observed a much higher positive electrostatic potential on the carbonyl oxygen (O2) of the substrate in Bs2 than in the corresponding atom within CALB during the transition from RC to I2. This, together with the variations of the charge distribution on the substrate from $\mathrm{RC}$ to I1 (see a full list of computed CHelpG charges of the key atoms of the system at M06-2X/MM level in the Supporting Information), can be at the origin of the relative energies of I2: the negatively charged $\mathrm{O} 2$ in $\mathrm{I} 1$ is more stabilized in $\mathrm{Bs} 2$ than in CALB. Nevertheless, because a higher electrostatic potential is also measured on the NE2 atom of the catalytic histidine residue (His399 in Bs2 and His224 in CALB) from RC to I2, CALB would stabilize the positive charge that appears in the imidazole ring in I1 more effectively than Bs2 (280.3 and - 132.7 $\mathrm{kJ} \cdot \mathrm{mol}^{-1} \cdot \mathrm{e}^{-1}$ in Bs2 and CALB, respectively). Considering the final relative energies of $\mathrm{I} 2$ in both systems, it can be assumed that the effect on the carbonyl group of the substrate appears to be more relevant than the effect on histidine, which is in agreement with previous computational studies on the substrate promiscuity of CALB. $^{26}$

Interestingly, subtle differences can be detected between the reaction pathways of Bs2 and CALB in the second step of the studied process. The proton transfer and $\mathrm{C}-\mathrm{N}$ bond breaking occur in a concerted but asynchronous manner in both enzymes but the timing of the two events is completely different (Figure 6). In $\mathrm{Bs} 2$, the breaking of the $\mathrm{C}-\mathrm{N}$ bond precedes the proton transfer from the catalytic His to the nitrogen of 4-nitroaniline. An inverse order of events was found in the reaction catalyzed by CALB where the transfer of the proton occurs before the breaking of the $\mathrm{C}-\mathrm{N}$ bond. This geometrical analysis is in agreement with the analysis of the evolution of charges. Thus, from the CHelpG charges computed at M06-2X/MM level in TS2 (see the Supporting Information for the full list of charges), a significant difference is observed in the nitrogen $\mathrm{N} 4$ atom of the substrate with -0.91 and -0.49 au in Bs2 and CALB, respectively. This confirms that, while in $\mathrm{Bs} 2$ the breaking of the $\mathrm{C}-\mathrm{N}$ clearly precedes the proton transfer and there is less negative charge built on the carbonyl oxygen $\mathrm{O} 2(-0.842$ and -1.086 au in Bs2 and CALB, respectively), the more advanced proton transfer in CALB results in a more advanced double bond formation between $\mathrm{O} 2$ and $\mathrm{C} 1$ (see Figure 2 for numbering of the atoms). As suggested by the minimum energy paths traced in Figure 6, once crossing the TS2, the rest of the process involving the breaking of the $\mathrm{C} 1-\mathrm{N} 4$ bond is barrierless in CALB, while in Bs2 the energy barrier is mostly associated with the breaking of the amide bond.

The difference in the mechanism of the second step can be further revealed by analysis of the electrostatic forces generated by the protein on the scissile $\mathrm{C} 1-\mathrm{N} 4$ bond (see Figure 7). The calculation of the electric field generated on the $\mathrm{C}$ and $\mathrm{N}$ atoms in I1 and TS2, combined with the charges computed on these atoms, shows stronger attractive forces between these two atoms in Bs2 than in CALB. Nevertheless, similar energy barriers computed from I1 are in agreement with the similar evolution of resulting electrostatic forces from I1 to TS2. Interestingly, 

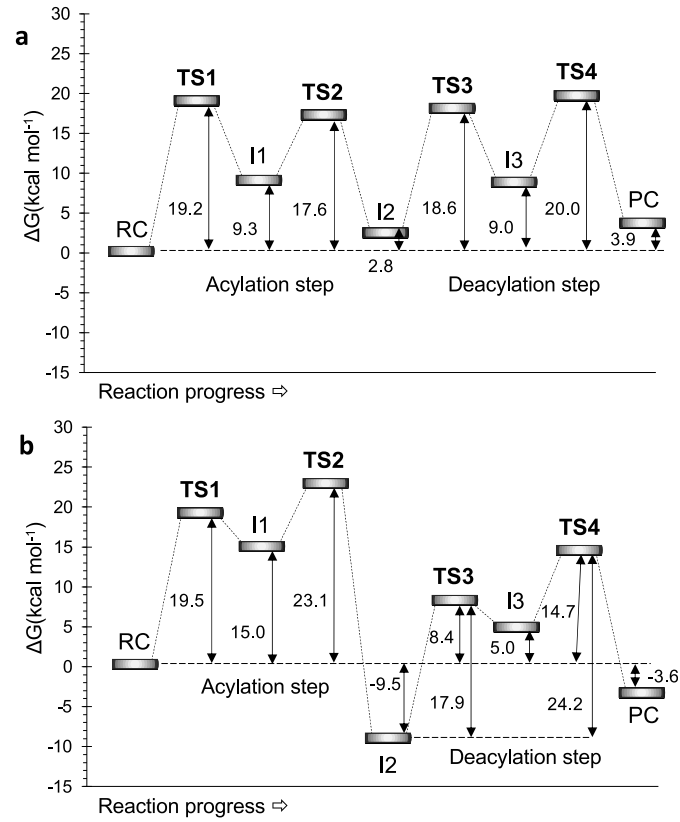

Figure 5. Free energy profiles of the hydrolysis of $N$-(4-nitrophenyl)butyramide catalyzed by (a) Bs2 and (b) CALB (from ref 26) computed at M06-2X/OPLS-AA level.

slightly weaker attractive electrostatic forces are detected between the two atoms of the scissile bond in TS2 than in I1, in both systems.

Three-Dimensional Convolutional Neural Network (3D CNN). We trained a 3D CNN to test, first, whether our machine learning protocol would be able to reproduce the classification of the two enzymes into their EC number based on the surroundings of its active site. One of the most successful architectures in the Deep Learning paradigm is the ResNet ${ }^{42}$ for its very fast convergency and high accuracy in classification tasks. Consequently, a 3D ResNet50 to classify the proteins by EC number was used. We achieved an overall accuracy of $99.8 \%$, implying that the network was capable to correctly classify the proteins into its functional class based on the surrounding of the active site.

Similar to the experimental protocols, we performed an alanine scanning experiment whereby residues key to its correct classification by the CNN protocol (and hence to enzyme functionality) were identified through site-directed alanine replacement (see the Supporting Information for details). We reasoned that if the misclassification rate increases when a mutation is done to a specific residue, it might be important among the family. Performing consecutive iterations and accumulating the relevant mutations allows the identification of residues that the network has learned to be common in the family. According to the results, the network was able to classify Bs2 and CALB with its correct EC number with an accuracy of 98.1 and $99.6 \%$, respectively. Nevertheless, it must be noted that the small initial sample of structures in each family makes this approach poorly diverse, but it can be successfully used to elucidate the common features of each family. Moreover, relevant findings from the alanine scan indicate crucial residues (see the Supporting Information for an illustration of their location) in the structure of Bs2 and CALB that define their classification in their corresponding families (Figure 8). A score of 1 corresponds to a perfectly classified protein, while a score of -1 refers to a perfectly misclassified class.

Our CNN method suggests that Glu188 is crucial to Bs2, and a detailed analysis confirms that this residue is involved in a hydrogen bonding network in the vicinity of the active site, including Ser 215 and the catalytic residues Ser 189 and Glu310 (see Figure 8c). Hence, Glu188 may play a role in the structural stabilization of the active site. Moreover, keeping in mind that this is a titratable residue, its protonation state, which can be modulated by the environment, may be decisive in the binding of charged substrates and the resulting electrostatics in the active site.

Other crucial networks in Bs2 involve aromatic and hydrophobic residues. The $\mathrm{CH}-\pi$ interaction between Met358 and Phe271 resulted in a water bridge interaction with a superficial His322. Accordingly, Met358 helps stabilizing the overall protein structure. Phe 398 and Phe315 also form a sandwich along with Tyr 312 through complex $\pi-\pi$ interactions. These residues play a role in the stabilization of the substrate forming a $\pi-\pi$ stacking network, as highlighted by Bornscheuer and co-workers ${ }^{24}$ and further confirmed by our simulations. Leu362 forms a hydrophobic pocket along with Phe363 and Leu273 where the acyl chain of the substrate is placed. This hydrophobic pocket is essential for the acceptance of acyl chain substrates. Finally, Trp102 might be involved in the stabilization of Tyr118 with a hydrogen bond established with the nitro group of the substrate.

In CALB, the mutagenesis has a considerable impact (Figure $8 b)$. This can be explained either due to the higher diversity in the trained dataset which enables identification of more crucial features or, simply, the distinctive features of this enzyme classification. Trp104 forms a hydrophobic pocket which permits the correct placement of the substrate in the active site, similar to the role played by Phe398 and Phe315 in Bs2. Based on our findings, this residue appears to be imperative because mutation for this residue resulted in misclassification. From the localized structures at M06-2X/MM level, Ile189, Val190, and Thr138 form a hydrophobic pocket and interact with the acyl chain of the substrate (Figure $8 \mathrm{~d}$ ). Moreover, the lateral chain of Thr138 established a strong hydrogen bond with the backbone of Ile189, assisting the formation of a rigid pocket that has a similar role to the one formed by Leu362, Leu273, and

Table 1. Experimental and Theoretical Kinetic Values for the Hydrolysis of $\mathrm{N}$-(4-Nitrophenyl)-butyramide Catalyzed by Bs2 and CALB

$\begin{array}{lccccc}\text { enzyme } & k_{\text {cat }}\left(10^{-3} \mathrm{~s}^{-1}\right) & K_{\mathrm{M}}(\mathrm{mM}) & k_{\text {cat }} / K_{\mathrm{M}}\left(\mathrm{s}^{-1} \cdot \mathrm{M}^{-1}\right) & \Delta G^{\text {exp }}\left(\mathrm{kcal} \cdot \mathrm{mol}^{-1}\right) \text { at } 294 \mathrm{~K} & \Delta G^{\text {theor }}\left(\mathrm{kcal} \cdot \mathrm{mol}^{-1}\right) \text { at } 300 \mathrm{~K} \\ \text { Bs2 } & 18.54 \pm 0.66 & 0.182 \pm 0.043 & 108 \pm 27 & 19.5 & 19.2 \\ \text { CALB }^{a} & 0.29 \pm 0.06 & 4.2 \pm 1.3 & 0.069 \pm 0.026 & 22.7 & 23.1\end{array}$

${ }^{a}$ Results from our previous studies. ${ }^{26}$ 

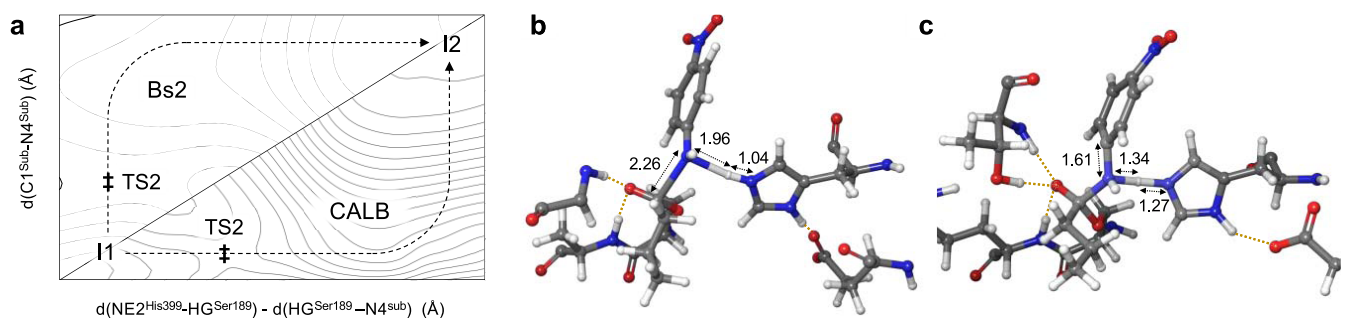

Figure 6. Schematic representation of the different reaction pathways of step 2 between Bs 2 and CALB, with their respective transition-state structures. (a) Path followed by both enzymes on the FES of step 2. Double daggers represent the localized TS2 at M06-2X/MM level. (b) Localized TS2 at M062X/MM level in Bs2. (c) Localized TS2 at M06-2X/MM level in CALB.
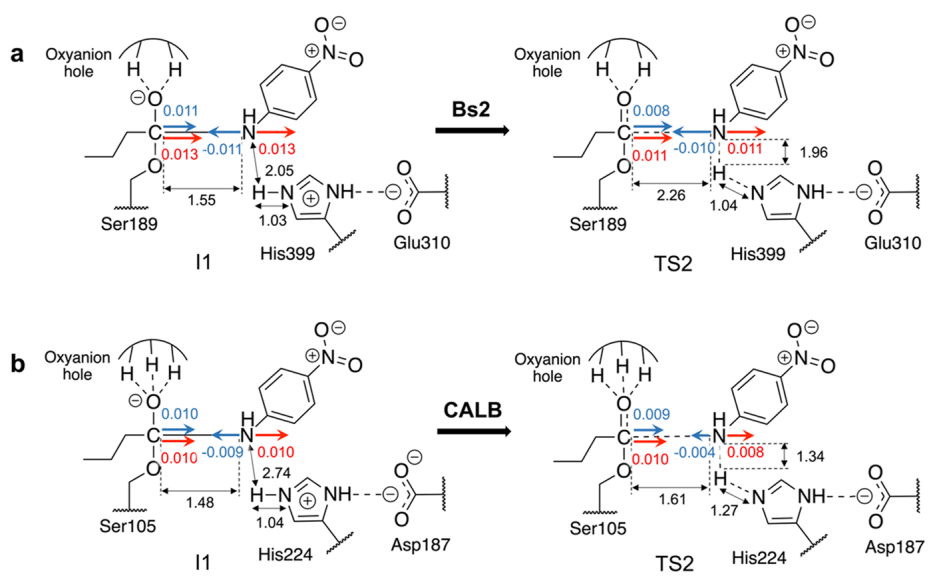

Figure 7. Schematic representation of the electrostatic features of the active site in (a) Bs2 and (b) CALB, in I1 and TS2 of the acylation step. Projections of the electric field created by the proteins in the $\mathrm{C}-\mathrm{N}$ peptide bond direction are shown as red arrows in $\mathrm{C} 1$ and $\mathrm{N} 4$ atoms, blue arrows are the resulting electrostatic forces (given in au $\times 10^{3}$ ) projected on the direction of the vector defined from $\mathrm{C} 1$ to $\mathrm{N} 4$, and black arrows represent the distance between atoms in angstrom.

Phe363 in Bs2. Our previous studies ${ }^{26}$ revealed that Asp134, located in proximity to the substrate, is protonated at $\mathrm{pH} 7$ and it is involved in a complex hydrogen bonding network including Gln 157 and the residues used in forming the oxyanion hole, Gln106, and Thr40. These interactions likely assist the formation of the oxyanion hole that is essential for the activity.

Another noteworthy residue that assists the structural stabilization of the active site is Gln193, which establishes a hydrogen bond between the catalytic Asp187 through a water bridge. This type of interaction has also been seen in Bs2 (the network of Glu188, Ser215, and Glu310). Polar residues such as Ser184 and Ser227 have been identified as their mutations resulting in misclassification of CALB after three iterations of the alanine scan. These serine residues are positioned behind the catalytic His224 and seem to be involved in the maintenance of the tertiary structure in forming hydrogen bond interactions with the backbone of residues Asp223 and Ile222. Finally, another residue identified by CNN as important is Thr40. This amino acid is part of the oxyanion hole and establishes a hydrogen bond with the carbonyl oxygen of the substrate via its lateral chain. The other two hydrogen bonds of the oxyanion hole are created by the backbone. The fact that the backbone is not represented in the CNN limits the identification of the oxyanion hole in both Bs2 and CALB. Even though limitations exist, our findings could guide further research and contribute to expanding the knowledge of the structural conformation of both enzymes.

Interestingly, some of the Bs2 residues that our $\mathrm{CNN}$ reveals as relevant for the structure have already been previously selected on directed evolution and mutagenesis studies. For instance, Bornscheuer and co-workers found that Glu118, ${ }^{25}$ Phe314, and Phe $315^{56}$ have a very important role in the kinetic performance of Bs2. In a directed evolution study by Arnold and collaborators, ${ }^{57}$ despite many of the tested mutations related to the surface, the mutation of Met358 was found to improve the kinetic activity in organic solvents. Other studies have reported that Trp104 is essential and forms a hydrophobic pocket in CALB, ${ }^{58-60}$ while residues such as Ile189, Val190, and Thr138 have also been proven to be crucial in the kinetic performance of CALB. ${ }^{60-62}$

\section{CONCLUSIONS}

The present study has focused on understanding and explaining how two structurally different enzymes, Bs 2 and CALB, have evolved converged specificity to catalyze the same amidase reaction. The combined experimental and computational approach in analyzing the reaction mechanism of the hydrolysis of $\mathrm{N}$-(4-nitrophenyl)-butyramide catalyzed by Bs2 has allowed 

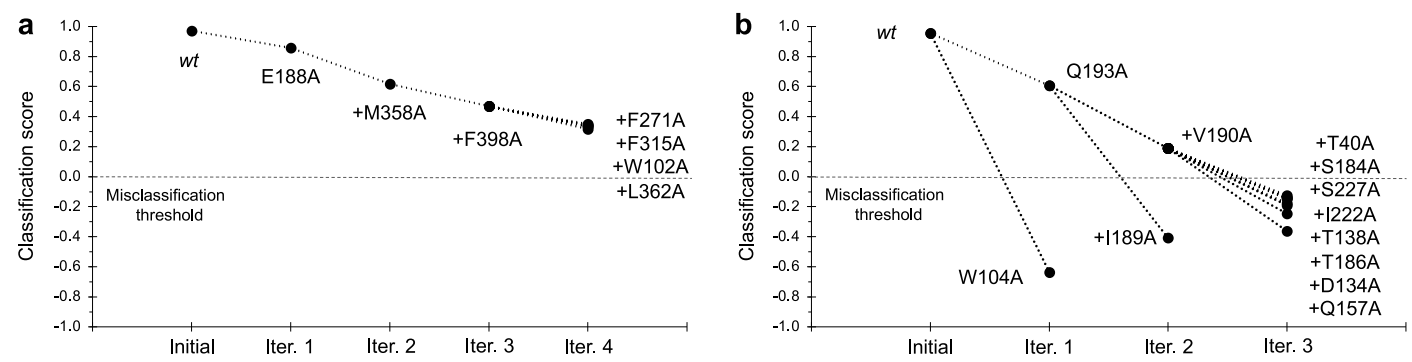

C

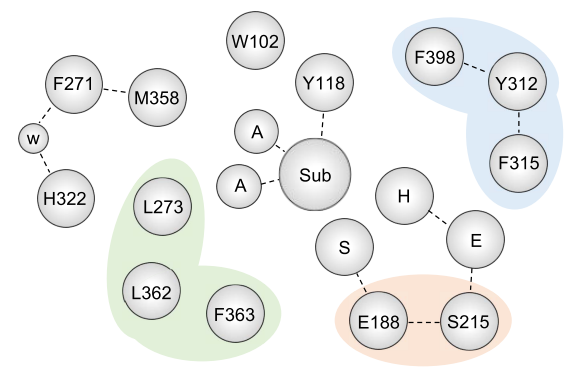

d

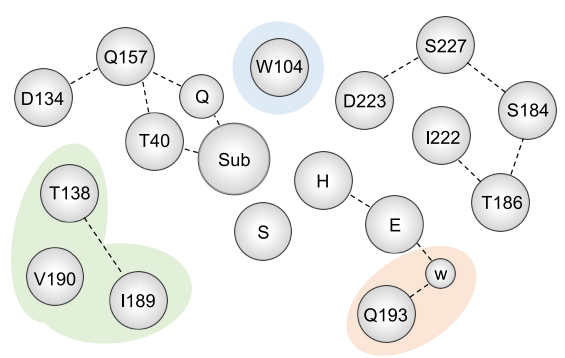

Figure 8. In silico CNN-mediated alanine scan in the vicinity of the active sites of Bs2 and CALB. (a) Four iterations of mutagenesis in Bs2. (b) Three iterations of mutagenesis in CALB. Previous mutations were accumulated in each iteration. (c) Schematic representation of the key residues in the vicinity of the active site in Bs2. (d) Schematic representation of the key residues in CALB. The green area corresponds to the hydrophobic acylacceptor pocket, blue highlights the hydrophobic cap and the ring stacking area, and the orange area corresponds to the residues involved in the stabilization of the catalytic site.

deep comparative analysis with our previous study on the same reaction catalyzed by CALB. ${ }^{26}$ The first conclusion that derives from our results is the excellent agreement between our predicted free energy barriers and the experimentally determined rate constants for the acylation step of the process. Subsequently, we confirm that Bs2 and CALB catalyze the full reaction in a similar fashion, with the same rate-limiting step corresponding to the last step of the deacylation process. However, significant mechanistic differences were identified in the acylation stage. The QM/MM free energy landscape of Bs2 illustrated that the rate-limiting step for the formation of the first product, 4-nitroaniline, corresponds to the first step in which Ser 198 activated by His 399 attacks C1 of the substrate, forming an enzyme covalent intermediate. In contrast, in the case of CALB, the kinetics of the acylation stage are determined by the barrier of the proton transfer and the substrate $\mathrm{C}-\mathrm{N}$ bond breaking occurring in the second step. Moreover, despite this second step is concerted in both enzymes, the timing of the events taking place are completely different: in Bs2, the breaking of the $\mathrm{C}-\mathrm{N}$ bond precedes the proton transfer from the catalytic His to the nitrogen of the 4-nitroaniline, while the inverse order is found in the reaction catalyzed by CALB. This can be reasoned based on protein electrostatics; the charges on the $\mathrm{C}$ and $\mathrm{N}$ atoms of the scissile bond provoke a slight difference in the electrostatic forces on these two atoms. Moreover, the overall barrier difference can be explained by a more stable I1 in Bs2 due to a stabilization of the negative charge accumulated in the carbonyl oxygen atom by the electrostatic potential created by the protein. The evolutive analysis of the protein geometries using deep learning approaches such as convolutional neural networks (CNN) has proved to be useful in predicting the classification of both enzymes and confirming their significant structural differences. Three-dimensional CNN has been used to analyze the vicinity of the active sites in Bs2 and CALB, revealing residues that are decisive in their $3 \mathrm{D}$ structures. According to the $3 \mathrm{D} C N N$ results, $\mathrm{Bs} 2$ would be a more robust protein scaffold to perform mutagenesis studies to improve the, in this case, amidase activity without dramatically perturbing the structure of the protein. On the contrary, mutations on CALB appear to have significant effects on the 3D structure of the protein. This 3D CNN analysis can be considered a practical illustration of the use of machine learning protocols that, together with the comparative analysis of the reactivity and electrostatic effects, can be decisive to unravel the origin of the promiscuity of enzymes, which in turn can be the bedrock of new strategies in protein engineering.

\section{ASSOCIATED CONTENT}

\section{SI Supporting Information}

The Supporting Information is available free of charge at https://pubs.acs.org/doi/10.1021/acscatal.1c02150.

Sequence alignments of crystal structures; details of computational and experimental methods; time dependence of RMSD of backbone atoms in the equilibration MD simulations and $B$ factors; key interatomic distances and CHelpG charges of structures optimized at M06-2X/ $\mathrm{MM}$ level; electrostatic potential and electric field created by the proteins Bs 2 and CALB on key atoms in the different states along the reaction; representation of the key residues identified by the alanine scan using the CNN on both Bs2 and CALB; Cartesian coordinates of transition-state structures localized at M06-2X/MM and 
imaginary frequencies; map of plasmid with wild-type Bs2 gene in pET-28a vector used for recombinant protein expression; LC chromatograms; mass spectra; and Michaelis-Menten plots (PDF)

$x y z$ coordinates of TS1 and TS2 of Bs2 (XYZ) $x y z$ coordinates of TS3 and TS4 of Bs2 (XYZ)

\section{AUTHOR INFORMATION}

\section{Corresponding Authors}

Louis Luk - Cardiff Catalysis Institute, School of Chemistry, Cardiff University, Cardiff CF10 3AT, United Kingdom; ○ orcid.org/0000-0002-7864-6261; Email: lukly@ cardiff.ac.uk

Katarzyna Swiderek - Institute of Advanced Materials (INAM), Universitat Jaume I, 12071 Castellón, Spain; ○ orcid.org/0000-0002-7528-1551; Email: swiderek@ uji.es

Vicent Moliner - Institute of Advanced Materials (INAM), Universitat Jaume I, 12071 Castellón, Spain; $\odot$ orcid.org/ 0000-0002-3665-3391; Email: moliner@uji.es

\section{Authors}

Miquel À. Galmés - Institute of Advanced Materials (INAM), Universitat Jaume I, 12071 Castellón, Spain

Alexander R. Nödling - Cardiff Catalysis Institute, School of Chemistry, Cardiff University, Cardiff CF10 3AT, United

Kingdom; ๑ orcid.org/0000-0002-3483-6266

Complete contact information is available at:

https://pubs.acs.org/10.1021/acscatal.1c02150

\section{Notes}

The authors declare no competing financial interest.

\section{ACKNOWLEDGMENTS}

The authors thank the Spanish Ministerio de Ciencia e Innovación (grants PGC2018-094852-B-C21 and PID2019107098RJ-I00), Generalitat Valenciana (grants AICO/2019/ 195 and SEJI/2020/007), Universitat Jaume I (grants UJIB2017-31 and UJI-A2019-04), the Leverhulme Trust (grant RPG-2017-195), and the UK's Wellcome Trust (grant 202056/ Z/16/Z). M.A.G. thanks Universitat Jaume I for FPI-UJI grant (PREDOC/2017/23). The authors acknowledge computational resources from the Servei d'Informàtica of Universitat Jaume I and T.R.I.N.I.T.Y cluster funded by Generalitat Valenciana and Universitat Jaume I.

\section{REFERENCES}

(1) Aharoni, A.; Gaidukov, L.; Khersonsky, O.; Gould, S. M.; Roodveldt, C.; Tawfik, D. S. The "evolvability" of Promiscuous Protein Functions. Nat. Genet. 2005, 37, 73-76.

(2) Humble, M. S.; Berglund, P. Biocatalytic Promiscuity. Eur. J. Org. Chem. 2011, 2011, 3391-3401.

(3) Voordeckers, K.; Brown, C. A.; Vanneste, K.; van der Zande, E.; Voet, A.; Maere, S.; Verstrepen, K. J. Reconstruction of Ancestral Metabolic Enzymes Reveals Molecular Mechanisms Underlying Evolutionary Innovation through Gene Duplication. PLoS Biol. 2012, 10, No. e1001446

(4) Copley, S. D. Shining a Light on Enzyme Promiscuity. Curr. Opin. Struct. Biol. 2017, 47, 167-175.

(5) Yang, G.; Miton, C. M.; Tokuriki, N. A Mechanistic View of Enzyme Evolution. Protein Sci. 2020, 29, 1724-1747.

(6) Glasner, M. E.; Gerlt, J. A.; Babbitt, P. C. Evolution of Enzyme Superfamilies. Curr. Opin. Chem. Biol. 2006, 10, 492-497.
(7) Ulusu, N. N. Evolution of Enzyme Kinetic Mechanisms. J. Mol. Evol. 2015, 80, 251-257.

(8) Petrović, D.; Risso, V. A.; Kamerlin, S. C. L.; Sanchez-Ruiz, J. M. Conformational Dynamics and Enzyme Evolution. J. R. Soc. Interface 2018, 15, No. 20180330.

(9) Kaltenbach, M.; Tokuriki, N. Dynamics and Constraints of Enzyme Evolution. J. Exp. Zool., Part B 2014, 322, 468-487.

(10) Mitchell, J. B. Enzyme Function and Its Evolution. Curr. Opin. Struct. Biol. 2017, 47, 151-156.

(11) Gherardini, P. F.; Wass, M. N.; Helmer-Citterich, M.; Sternberg, M. J. E. Convergent Evolution of Enzyme Active Sites Is Not a Rare Phenomenon. J. Mol. Biol. 2007, 372, 817-845.

(12) Drenth, J.; Hol, W. G.; Jansonius, J. N.; Koekoek, R. A Comparison of the Three-Dimensional Structures of Subtilisin BPN' and Subtilisin Novo. Cold Spring Harbor Symp. Quant. Biol. 1972, 36, 107-116.

(13) Kraut, J. Serine Proteases: Structure and Mechanism of Catalysis. Annu. Rev. Biochem. 1977, 46, 331-358.

(14) Zhang, M.; Van Etten, R. L.; Stauffacher, C. V. Crystal Structure of Bovine Heart Phosphotyrosyl Phosphatase at 2.2-A Resolution. Biochemistry 1994, 33, 11097-11105.

(15) Penning, T. M.; Bennett, M. J.; Smith-Hoog, S.; Schlegel, B. P.; Jez, J. M.; Lewis, M. Structure and Function of 3 Alpha-Hydroxysteroid Dehydrogenase. Steroids 1997, 62, 101-111.

(16) Hegyi, H.; Gerstein, M. The Relationship between Protein Structure and Function: A Comprehensive Survey with Application to the Yeast Genome. J. Mol. Biol. 1999, 288, 147-164.

(17) Doolittle, R. F. Convergent Evolution: The Need to Be Explicit. Trends Biochem. Sci. 1994, 19, 15-18.

(18) LeCun, Y.; Bengio, Y.; Hinton, G. Deep Learning. Nature 2015, $521,436-444$.

(19) Jiménez, J.; Skalič, M.; Martínez-Rosell, G.; De Fabritiis, G. KDEEP: Protein-Ligand Absolute Binding Affinity Prediction via 3DConvolutional Neural Networks. J. Chem. Inf. Model 2018, 58, 287296.

(20) Alley, E. C.; Khimulya, G.; Biswas, S.; AlQuraishi, M.; Church, G. M. Unified Rational Protein Engineering with Sequence-Based Deep Representation Learning. Nat. Methods 2019, 16, 1315-1322.

(21) Cao, H.; Wang, J.; He, L.; Qi, Y.; Zhang, J. Z. DeepDDG: Predicting the Stability Change of Protein Point Mutations Using Neural Networks. J. Chem. Inf. Model 2019, 59, 1508-1514.

(22) Senior, A. W.; Evans, R.; Jumper, J.; Kirkpatrick, J.; Sifre, L.; Green, T.; Qin, C.; Zídek, A.; Nelson, A. W. R.; Bridgland, A.; Penedones, H.; Petersen, S.; Simonyan, K.; Crossan, S.; Kohli, P.; Jones, D. T.; Silver, D.; Kavukcuoglu, K.; Hassabis, D. Improved Protein Structure Prediction Using Potentials from Deep Learning. Nature 2020, 577, 706-710.

(23) Chang, A.; Jeske, L.; Ulbrich, S.; Hofmann, J.; Koblitz, J.; Schomburg, I.; Neumann-Schaal, M.; Jahn, D.; Schomburg, D. BRENDA, the ELIXIR Core Data Resource in 2021: New Developments and Updates. Nucleic Acids Res. 2021, 49, D498-D508.

(24) Hackenschmidt, S.; Moldenhauer, E. J.; Behrens, G. A.; Gand, M.; Pavlidis, I. V.; Bornscheuer, U. T. Enhancement of Promiscuous Amidase Activity of a Bacillus subtilis Esterase by Formation of a $\pi-\pi$ Network. Chem CatChem 2014, 6, 1015-1020.

(25) Kourist, R.; Bartsch, S.; Fransson, L.; Hult, K.; Bornscheuer, U. T. Understanding Promiscuous Amidase Activity of an Esterase from Bacillus subtilis. ChemBioChem 2008, 9, 67-69.

(26) Galmés, M. A.; García-Junceda, E.; Świderek, K.; Moliner, V. Exploring the Origin of Amidase Substrate Promiscuity in CALB by a Computational Approach. ACS Catal. 2020, 10, 1938-1946.

(27) Ribitsch, D.; Heumann, S.; Trotscha, E.; Herrero Acero, E.; Greimel, K.; Leber, R.; Birner-Gruenberger, R.; Deller, S.; Eiteljoerg, I.; Remler, P.; Weber, T.; Siegert, P.; Maurer, K. H.; Donelli, I.; Freddi, G.; Schwab, H.; Guebitz, G. M. Hydrolysis of Polyethyleneterephthalate by P-Nitrobenzylesterase from Bacillus subtilis. Biotechnol. Prog. 2011, 27, 951-960.

(28) Carniel, A.; Valoni, É.; Nicomedes, J.; Gomes, A. d. C.; de Castro, A. M. Lipase from Candida antarctica (CALB) and Cutinase from 
Humicola Insolens Act Synergistically for PET Hydrolysis to Terephthalic Acid. Process Biochem. 2017, 59, 84-90.

(29) Osuna, S. The Challenge of Predicting Distal Active Site Mutations in Computational Enzyme Design. Wiley Interdiscip. Rev.: Comput. Mol. Sci. 2021, 11, No. e1502.

(30) Consortium, T. U. UniProt: A Worldwide Hub of Protein Knowledge. Nucleic Acids Res. 2019, 47, D506-D515.

(31) Spiller, B.; Gershenson, A.; Arnold, F. H.; Stevens, R. C. A Structural View of Evolutionary Divergence. Proc. Natl. Acad. Sci. U.S.A. 1999, 96, 12305-12310.

(32) Sali, A.; Blundell, T. L. Comparative Protein Modelling by Satisfaction of Spatial Restraints. J. Mol. Biol. 1993, 234, 779-815.

(33) Olsson, M. H. M.; Søndergaard, C. R.; Rostkowski, M.; Jensen, J. H. PROPKA3: Consistent Treatment of Internal and Surface Residues in Empirical p Kapredictions. J. Chem. Theory Comput. 2011, 7, 525537.

(34) Søndergaard, C. R.; Olsson, M. H. M.; Rostkowski, M.; Jensen, J. H. Improved Treatment of Ligands and Coupling Effects in Empirical Calculation and Rationalization of PKa Values. J. Chem. Theory Comput. 2011, 7, 2284-2295.

(35) Jorgensen, W. L.; Chandrasekhar, J.; Madura, J. D.; Impey, R. W.; Klein, M. L. Comparison of Simple Potential Functions for Simulating Liquid Water. J. Chem. Phys. 1983, 79, 926-935.

(36) Duan, Y.; Wu, C.; Chowdhury, S.; Lee, M. C..; Xiong, G.; Zhang, W.; Yang, R.; Cieplak, P.; Luo, R.; Lee, T.; Caldwell, J.; Wang, J.; Kollman, P. A Point-Charge Force Field for Molecular Mechanics Simulations of Proteins Based on Condensed-Phase. J. Comput. Chem. 2003, 24, 1999-2012.

(37) Phillips, J. C.; Braun, R.; Wang, W.; Gumbart, J.; Tajkhorshid, E.; Villa, E.; Chipot, C.; Skeel, R. D.; Kalé, L.; Schulten, K. Scalable Molecular Dynamics with NAMD. J. Comput. Chem. 2005, 26, 17811802 .

(38) Dewar, M. J. S.; Zoebisch, E. G.; Healy, E. F.; Stewart, J. J. P. Development and Use of Quantum Mechanical Molecular Models. 76. AM1: A New General Purpose Quantum Mechanical Molecular Model. J. Am. Chem. Soc. 1985, 107, 3902-3909.

(39) Zhao, Y.; Truhlar, D. G. The M06 Suite of Density Functionals for Main Group Thermochemistry, Thermochemical Kinetics, Noncovalent Interactions, Excited States, and Transition Elements: Two New Functionals and Systematic Testing of Four M06-Class Functionals and 12 Other Function. Theor. Chem. Acc. 2008, 120, 215-241.

(40) Jorgensen, W. L.; Maxwell, D. S.; Tirado-Rives, J. Development and Testing of the OPLS All-Atom Force Field on Conformational Energetics and Properties of Organic Liquids. J. Am. Chem. Soc. 1996, $118,11225-11236$

(41) Field, M. J.; Albe, M.; Bret, C.; Proust-De Martin, F.; Thomas, A. The Dynamo Library for Molecular Simulations Using Hybrid Quantum Mechanical and Molecular Mechanical Potentials. J. Comput. Chem. 2000, 21, 1088-1100.

(42) He, K.; Zhang, X.; Ren, S.; Sun, J. In Deep Residual Learning for Image Recognition, 2016 IEEE Conference on Computer Vision and Pattern Recognition (CVPR), 2016; pp 770-778.

(43) Chollet, F. Keras; GitHub, 2015.

(44) Abadi, M.; Agarwal, A.; Barham, P.; Brevdo, E.; Chen, Z.; Citro, C.; Corrado, G. S.; Davis, A.; Dean, J.; Devin, M.; Ghemawat, S.; Goodfellow, I.; Harp, A.; Irving, G.; Isard, M.; Jia, Y.; Jozefowicz, R.; Kaiser, L.; Kudlur, M.; Levenberg, J.; Mane, D.; Monga, R.; Moore, S.; Murray, D.; Olah, C.; Schuster, M.; Shlens, J.; Steiner, B.; Sutskever, I.; Talwar, K.; Tucker, P.; Vanhoucke, V.; Vasudevan, V.; Viegas, F.; Vinyals, O.; Warden, P.; Wattenberg, M.; Wicke, M.; Yu, Y.; Zheng, X. TensorFlow: A System for Large-Scale Machine Learning. In 12th USENIX Symposium on Operating Systems Design and Implementation (OSDI 16); 2016; pp 265-283.

(45) Dawson, N. L.; Lewis, T. E.; Das, S.; Lees, J. G.; Lee, D.; Ashford, P.; Orengo, C. A.; Sillitoe, I. CATH: An Expanded Resource to Predict Protein Function through Structure and Sequence. Nucleic Acids Res. 2017, 45, D289-D295.

(46) UniProt Consortium. UniProt: The Universal Protein Knowledgebase in 2021. Nucleic Acids Res. 2021, 49, D480-D489.
(47) Berman, H. M.; Westbrook, J.; Feng, Z.; Gilliland, G.; Bhat, T. N.; Weissig, H.; Shindyalov, I. N.; Bourne, P. E. The Protein Data Bank. Nucleic Acids Res. 2000, 28, 235-242.

(48) Bordes, I.; Recatalá, J.; Świderek, K.; Moliner, V. Is Promiscuous CALB a Good Scaffold for Designing New Epoxidases? Molecules 2015 , 20, 17789-17806.

(49) Glasstone, S.; Laidler, K.; Eyring, H. The Theory of Rate Processes: The Kinetics of Chemical Reactions, Viscosity, Diffusion and Electrochemical Phenomena; McGraw-Hill: New York, 1941.

(50) Keck, J. C. Variational Theory of Reaction Rates. Adv. Chem. Phys. 1967, 85-121.

(51) Truhlar, D. G.; Garrett, B. C.; Klippenstein, S. J. Current Status of Transition-State Theory. J. Phys. Chem. A 1996, 100, 12771-12800.

(52) Arafet, K.; Ferrer, S.; Moliner, V. Computational Study of the Catalytic Mechanism of the Cruzain Cysteine Protease. ACS Catal. 2017, 7, 1207-1215.

(53) Swiderek, K.; Moliner, V. Revealing the Molecular Mechanisms of Proteolysis of SARS-CoV-2 Mpro by QM/MM Computational Methods. Chem. Sci. 2020, 11, 10626-10630.

(54) Arafet, K.; Serrano-Aparicio, N.; Lodola, A.; Mulholland, A. J.; González, F. V.; Świderek, K.; Moliner, V. Mechanism of Inhibition of SARS-CoV-2 Mpro by N3 Peptidyl Michael Acceptor Explained by QM/MM Simulations and Design of New Derivatives with Tunable Chemical Reactivity. Chem. Sci. 2021, 12, 1433-1444.

(55) Serrano-Aparicio, N.; Moliner, V.; Swiderek, K. Nature of Irreversible Inhibition of Human 20S Proteasome by Salinosporamide A. The Critical Role of Lys-Asp Dyad Revealed from Electrostatic Effects Analysis. ACS Catal. 2021, 11, 3575-3589.

(56) Hackenschmidt, S.; Moldenhauer, E. J.; Behrens, G. A.; Gand, M.; Pavlidis, I. V.; Bornscheuer, U. T. Enhancement of Promiscuous Amidase Activity of a Bacillus subtilis Esterase by Formation of a $\pi-\pi$ Network. Chem CatChem 2014, 6, 1015-1020.

(57) Moore, J. C.; Arnold, F. H. Directed Evolution of a ParaNitrobenzyl Esterase for Aqueous-Organic Solvents. Nat. Biotechnol. 1996, 14, 458-467.

(58) Suplatov, D. A.; Besenmatter, W.; Švedas, V. K.; Svendsen, A. Bioinformatic Analysis of Alpha/Beta-Hydrolase Fold Enzymes Reveals Subfamily-Specific Positions Responsible for Discrimination of Amidase and Lipase Activities. Protein Eng., Des. Sel. 2012, 25, 689697.

(59) Xu, J.; Cen, Y.; Singh, W.; Fan, J.; Wu, L.; Lin, X.; Zhou, J.; Huang, M.; Reetz, M. T.; Wu, Q. Stereodivergent Protein Engineering of a Lipase to Access All Possible Stereoisomers of Chiral Esters with Two Stereocenters. J. Am. Chem. Soc. 2019, 141, 7934-7945.

(60) Santos, A. G.; da Rocha, G. O.; de Andrade, J. B. Dissecting the Evolvability Landscape of the CalB Active Site toward Aromatic Substrates. Sci. Rep. 2019, 9, No. 1.

(61) Montanier, C. Y.; Chabot, N.; Emond, S.; Guieysse, D.; RemaudSiméon, M.; Peruch, F.; André, I. Engineering of Candida antarctica Lipase B for Poly( $\varepsilon$-Caprolactone) Synthesis. Eur. Polym. J. 2017, 95, 809-819.

(62) Shen, J. W.; Qi, J. M.; Zhang, X. J.; Liu, Z. Q.; Zheng, Y. G. Significantly Increased Catalytic Activity of: Candida antarctica Lipase $\mathrm{B}$ for the Resolution of Cis - $( \pm)$-Dimethyl 1-Acetylpiperidine-2,3Dicarboxylate. Catal. Sci. Technol. 2018, 8, 4718-4725. 


\subsubsection{Redesign of Bs2 based on electrostatic features of GALB (Article 4)}

As stated in the Introduction chapter, enzyme reactivity can be rationalized in terms of electrostatic preorganization of the enzyme. ${ }^{15-20}$ Thus, the electrostatic potential and electric field exerted on key atoms along a specified reaction coordinate determine the reaction efficiency and mechanism. Following this idea, we proposed a rational-based approach focused on the optimization of the electrostatic preorganization of the active site.

From previous studies, we saw that Bs2 and CALB are mechanistically similar, ${ }^{15,20}$ but structurally different although they share common features in the vicinity of the active site. ${ }^{20}$ Moreover, we determined that Bs2 might be a more robust scaffold for future mutagenesis studies. Therefore, we decided to combine the best electrostatic features of Bs2 and CALB to propose a catalytically improved Bs2 variant.

The rate-determining step in Bs2 is the first one, where the Ser189 attacks the carbonyl carbon forming a tetrahedral intermediate, I1. In this step the transfer of the proton from Ser 189 to His399 is crucial. Thus, focusing on facilitating the transfer of the proton in this step could be a good strategy to obtain a more efficient Bs2 variant in terms of $k_{\text {cat. }}$. We focused on performing mutations on Bs2, inspired by the information derived from CALB, to stabilize the positive charge accumulated in His399 in this step, by generating a negative electrostatic potential on the $\mathrm{N} \varepsilon$ of His399.

As already explained, one of the objectives of the present thesis is to propose a computer-based method to design new enzymes. In particular, the bedrock of our proposed method is the comparison between two enzymes capable of catalyzing the same reaction to deduce the best of each one. When dealing with two very different enzymes, comparing the environment generated by the surrounding of the active site onto the 


\subsection{A computer-assisted rational redesign}

substrate is a challenging task. A possible solution to tackle this limitation could be to perform a structural alignment using RQs by aligning the common parts within the active site and therefore allowing the comparison of structurally non-related proteins, with a mechanistically similar TS. The structural alignment of the QM/MM MD trajectories of TS1 of both Bs2 and CALB was done as explained in section 3.5.2. Then, the electrostatic potential per residue was computed on the $\mathrm{N} \varepsilon$ of His399, identifying a series of favorable and unfavorable residues from the electrostatic perspective. After the careful analysis of the electrostatic features (Figure 4.12), an F398D Bs2 variant was proposed.

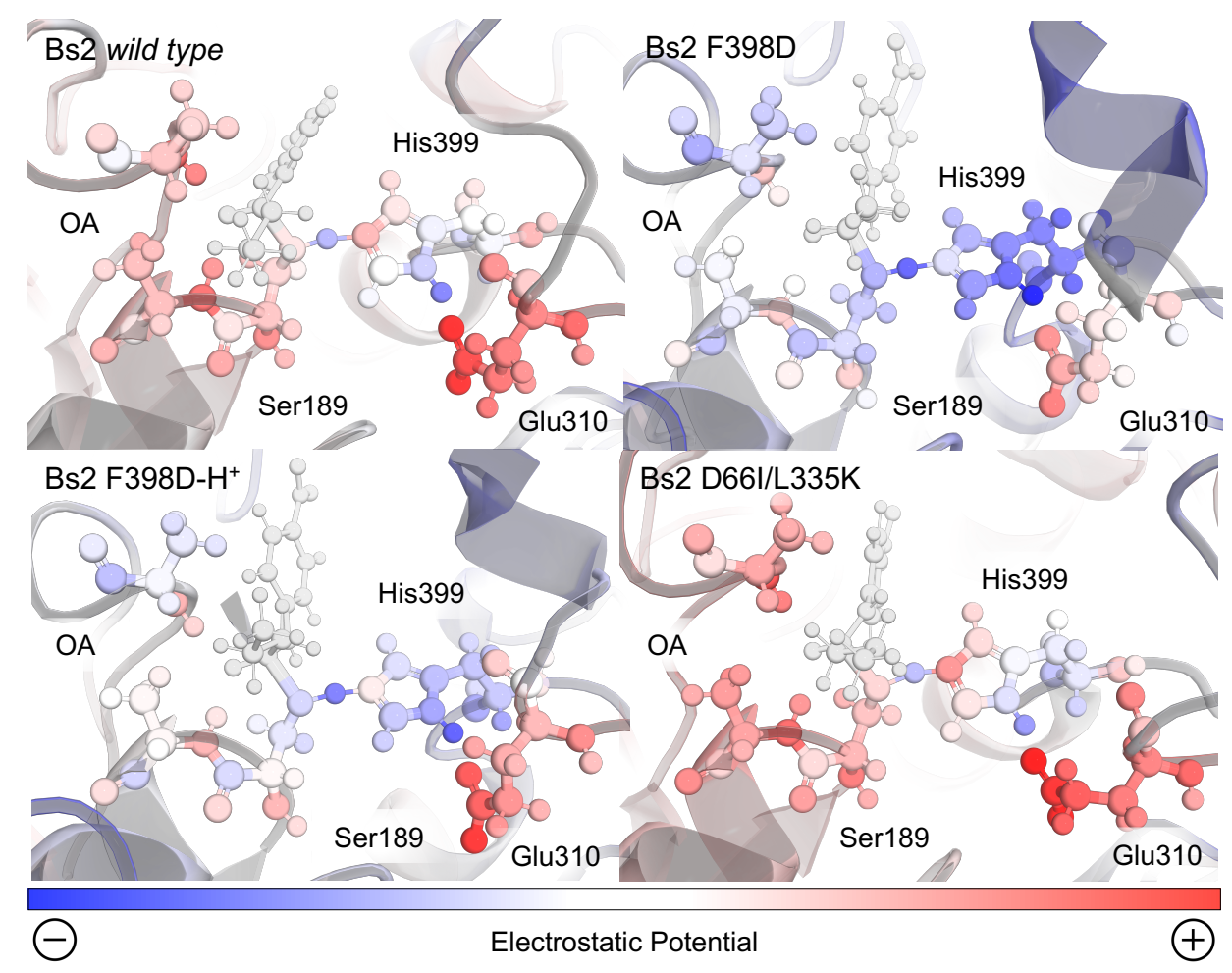

Figure 4.12 General representation of the active site in the TS2 of the four variants of Bs2. The colors of each of the atoms are a representation of the electrostatic potential generated by the protein on each of these atoms. The blue represents a more negative electrostatic potential while the red indicates a more positive value. The catalytic triad and the oxyanions hole $(\mathrm{OA})$ are also presented. The substrate is represented in light grey. All the values are qualitative. 
From the QM/MM free energy calculations, we predicted an impressive improvement in terms of the reduction of the activation free energy for this variant, which supports our idea that the stabilization of the positive charge generated on I1 due to the transfer of the proton leads to a decrease of the barrier of the first step of the reaction (Figure 4.13). We also proved that generating a positive electrostatic potential on this very same atom, by the D66I/L335K Bs2 variant, leads to an increase of the energy barrier of this first step (Figure 4.13), confirming a linear correlation between activation free energies and electrostatic potential.

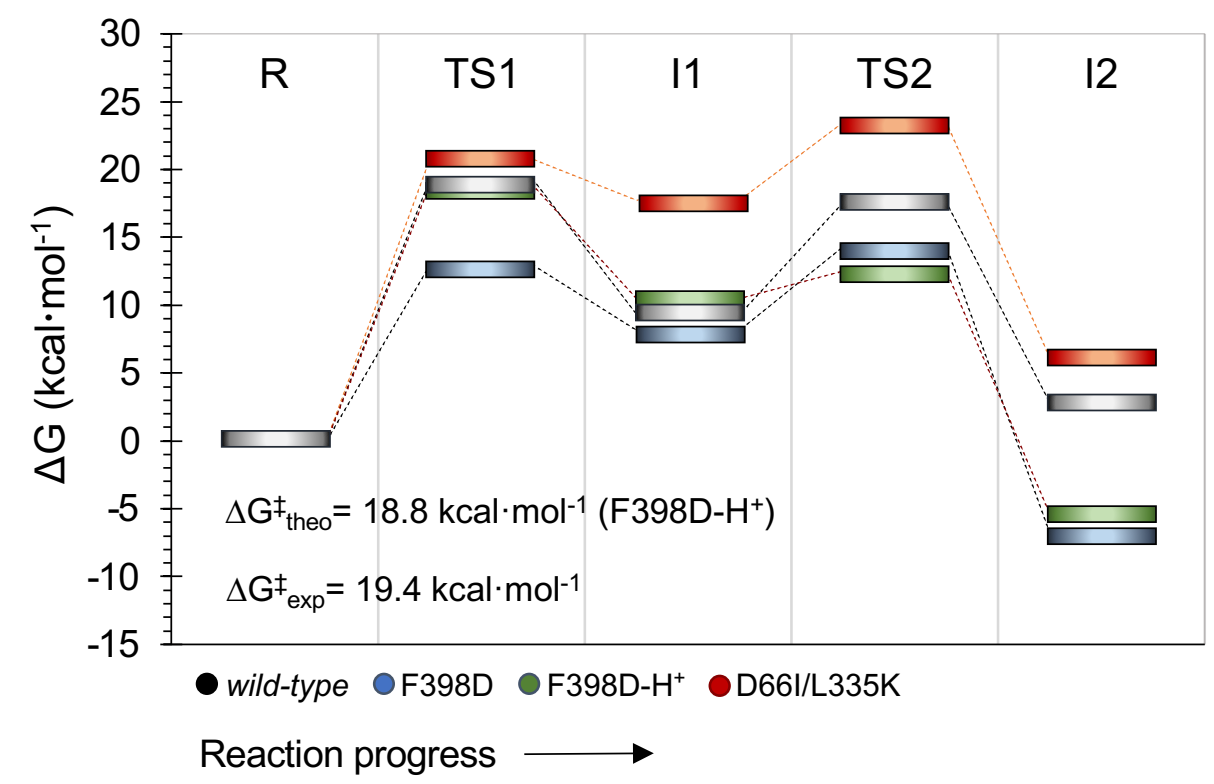

Figure 4.13. Free energy profile obtained at M06-2X/OPLS-AA level for the wild-type Bs2 and the three studied variants. Only the acylation step (from R to I2) is showed.

However, even though the preliminary estimation of the $\mathrm{pK}_{\mathrm{a}}$ of F398D predicted a deprotonated D398, a deep analysis from long MD simulations revealed that a protonated $\mathrm{F} 398 \mathrm{D}-\mathrm{H}^{+}$would be more likely and it should be explored. In this case, the electrostatic effect is diluted due to the protonated D398, hence predicting a less dramatic decrease of the barrier 


\subsection{A computer-assisted rational redesign}

of the first step. This prediction on F398D- $\mathrm{H}^{+}$Bs2 variant was experimentally confirmed by our collaborators from the group of Dr. Louis Luk of Cardiff University, whose kinetic rate constants were in very good agreement with the computational results.

For future studies not only the single mutation of F398D will be considered, but the use of other non-titratable residues including artificial amino acids can be a useful strategy. Despite the individual electrostatic contribution of the individual new residues was small, several mutations could act in a concerted manner. Alternatively, the F398D-H $\mathrm{H}^{+}$Bs2 variant can also be subjected to further analysis to provide information about how this F398 can remain deprotonated. 


\section{Computational design of an amidase by combining the best electrostatic features of two promiscuous hydrolases.}

Miquel À. Galmés, ${ }^{1}$ Alexander $\mathrm{R}$ Nödling, ${ }^{2}$ Kaining He, ${ }^{2}$ Louis Luk, ${ }^{2, *}$ Katarzyna Świderek, ${ }^{1, *}$ Vicent Moliner ${ }^{1, *}$

1. BioComp Group, Institute of Advanced Materials (INAM), Universitat Jaume I, 12071 Castellón, Spain.

2. School of Chemistry, Cardiff University, Main Building, Park Pl, Cardiff CF10 3AT (United Kingdom)

corresponding authors:

L. Luk: Phone number: +44 292251 0161; email: lukly@cardiff.ac.uk

K. Świderek: Phone number: +34964728070; email: swiderek@uji.es

V. Moliner: Phone number: +34964728084; email: moliner@uji.es

Keywords: QM/MM molecular dynamics, promiscuous enzymes, enzyme design, electrostatic effects 


\section{ABSTRACT}

While there has been emerging interest in designing new enzymes to solve practical challenges, the use of computer-based approaches to redesign catalytically active proteins remains largely unexplored. Here, a rational QM/MM MD strategy based on the combination of the best electrostatic properties of enzymes with activity on a common reaction is presented. The computational protocol has been applied to the re-design of the protein scaffold of an existing promiscuous esterase from Bacillus subtilis Bs2 to enhance its secondary amidase activity. After alignment of Bs2 with a non-homologous amidase Candida antarctica lipase B (CALB) within rotation quaternions, a relevant spatial aspartate residue of the latter was transferred to the former as means to favor the electrostatics of the transition state formation where a clear separation of charge takes place. Although the calculations for the Bs2-F398D variant predicted an unprecedented $10^{4}$-fold increased catalytic activity over that of the wild-type enzyme, deeper computational insights reveal a significant shift in the $p \mathrm{~K}_{\mathrm{a}}$ of the inserted aspartate, resulting in a modest catalytic effect. This prediction was experimentally confirmed as a 1.3 fold increase in activity which can be further improved with mutations. Despite the intrinsic limitations, the simplicity of the presented approach offers an alternative route based on computational techniques for enzyme engineer.

\section{Table of contents}

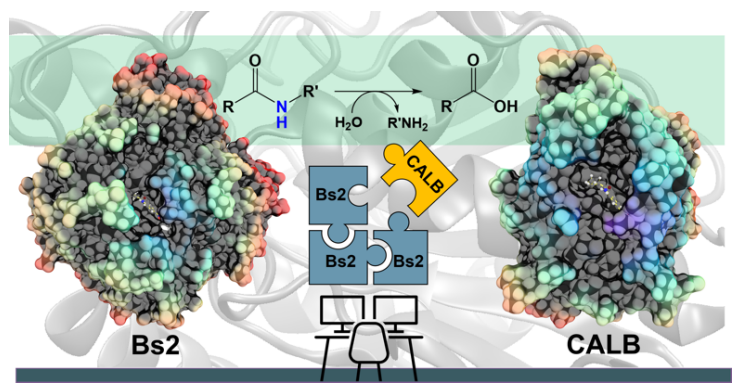




\section{INTRODUCTION}

The application of enzymes for desired chemical transformations has been demonstrated by the report of novel and functional designed structures. ${ }^{1-5}$ Recent advances in molecular biology and screening technologies have enabled the creation of enzymes via directed evolution. By mimicking the process of natural evolution, iterative cycles of (semi-)random mutations facilitate improvement of proteins in the laboratory through screening and selection, hence the identification of active variants. ${ }^{6-12}$ Minimal structural information is needed for this strategy and regions distant from the active site that can influence the reaction rate can also be identified. Nevertheless, directed evolution is limited by the fact that, even when the most efficient high-throughput system is used, only a fraction can be sampled within a set timeframe. ${ }^{13}$ Furthermore, development of an efficient screening system for a tailored reaction remains challenging. Recent machine-learning (ML) methods have been proposed to expedite evolution and expand the number of properties that can be optimized. ${ }^{14,15}$ However, in order to create enzymes with novel reactivities by means of ML methods, protein engineers will have to use proteins with sequences not assigned to the designated reaction or assigned with properties other than those of specific interest. Sequence-function data from engineering experiments must be collected to catalogue the natural diversity of proteins in order to convert ML into a useful tool. ${ }^{15}$

An alternative approach whose potentials have not been fully revealed is rational design, a technique that modifies selected residues at specific positions of an already existing protein scaffold through analysis of existing mechanistic and structural data. ${ }^{16}$ In order to reveal structures of the protein along the full catalytic process under physiological conditions, including metastable transition state (TS) structures, computer simulations are essential. Among all the different computer-assisted design strategies, two philosophies can be identified: the redesign of the active 
site of an existing substate-promiscuous enzyme and the de novo design that constructs an enzyme "from scratch". The use of promiscuous enzymes results to be a very promising starting point for the design of new highly efficient biocatalyst. ${ }^{17,18}$ However, the knowledge about the particular molecular mechanisms that permits enzymes to catalyze more than one chemical reaction is still under debate. ${ }^{19-22}$

Because both the enzyme redesign and the de novo design approach require knowledge of the TS of the reaction to be catalyzed, quantum mechanical (QM) calculations offer crucial complementary information that accelerates the development of novel designed reactions. Moreover, multiscale methods are the only tool that can offer a detailed atomistic picture of the reactions in the active site of the enzyme, which can be dramatically different from that in the gas phase or in solution. In multiscale methods, electrons of the reacting fragments are explicitly described by QM methods and the large and complex interacting environment (the fully solvated protein) is described by molecular mechanics (MM) force fields. The mechanism of a reaction in the active site of an enzyme can be determined within these hybrid QM/MM methods through the extensive exploration of the Free Energy Surface (FES). This allows the determination of the ratelimiting step in a multi-step process and, within the framework of Transition State Theory (TST), ${ }^{23}$ to predict rate constants directly comparable with experiments.

One of the challenges that remains non-trivial to optimize secondary activity of reactivitypromisucous enzyme which can be illustrated in the study of Bacillus subtilis esterase Bs2. While Bs2 is recognized as a serine hydrolase whose primary reaction is the hydrolysis of esters, it can also catalyze the hydrolysis of the amide bond of $N$-(4-nitrophenyl)-butyramide as a secondary reaction (Figure 1). ${ }^{22,24}$ Previously directed evolution experiments by Arnold and co-workers resulted in a 7-mutation variant with 100-fold enhancement of the esterase activity (using para- 
nitrobenzyl butyrate as substrate). ${ }^{25}$ Bornscheuer and co-workers used a combination of directed evolution and rational design based on docking and classical energy minimization to get a 3-fold increase of the amidase activity of Bs2 after two single mutations. ${ }^{22}$ In a larger context, despite the successes of different computer-assisted designs of new enzymes, it has been argued that the high activities of the best artificial enzymes have been largely due to directed evolution and the contribution of computation was comparatively modest. ${ }^{26}$

a:

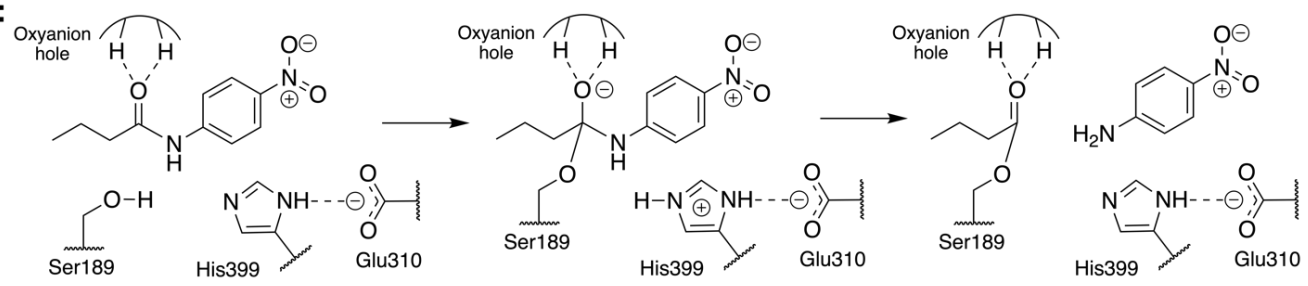
Reactants Complex Intermediate 1 Intermediate 2

b:

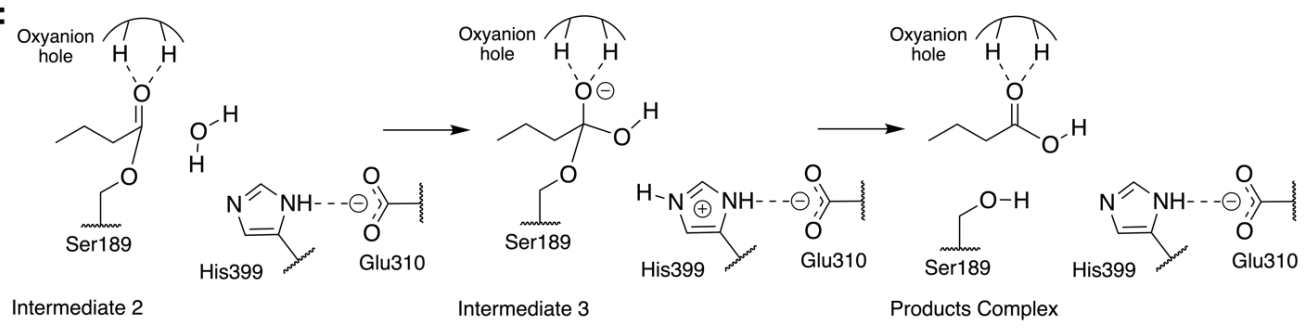

Figure 1. Schematic representation of the reaction mechanism of the hydrolysis of the $\mathrm{N}$-(4nitrophenyl)-butyramide catalyzed by Bs2. (a) Acylation step: the nucleophilic addition of Ser189 to the carbonyl followed by the breaking of C-N bond is triggered by His399-assisted proton shuffling, and the leaving group, in this case, is 4-nitroaniline. (b) Hydrolysis step: the nucleophilic addition of a water molecule followed by the resolution of the acyl-enzyme complex is triggered by His399-assisted protein shuffling, yielding butyric acid as product.

We envisaged that mutations that optimize the preorganization of the protein environment will result in a variant that exhibits improved activity for a desired reaction. ${ }^{27}$ Based on our recent 
QM/MM studies of different enzymatic reactions, we have quantified and shown how the reactivity of different proteins can be rationalized from their electrostatic properties, ${ }^{28-33}$ as the pioneering works proposed by Warshel and co-workers. ${ }^{34-36}$ The computed changes of the electrostatic potential or the electric field exerted by the studied proteins on key atoms of the substrates reflect that there is a small reorganization of these entities when evolving from the reactant state (RS) to the TS at the lowest energy cost. ${ }^{28-32}$ The electrostatic effects within the active site of the enzyme, therefore, appear to be critical for the electronic reorganization of the reactants during chemical transformations. These studies support the idea that electrostatic properties of enzymes are the origin of their catalytic features ${ }^{37}$ and their detailed understanding could be useful in future computer-assisted protein design methods.

To engineer enzymes with optimal electrostatic preorganization, comparative analysis between unrelated natural enzymes that catalyze the same chemical reactions can be a promising strategy. In previous studies we have shown that Candida antarctica lipase B (CALB) also displays amidase with activity similar to that of Bs2, though being non-homologous with each other. ${ }^{32,33} \mathrm{QM} / \mathrm{MM}$ studies of the amidase reaction catalyzed by the wild-type Bs2 and CALB enzymes were previously conducted. ${ }^{32,33} \mathrm{We}$ expect that favorable features of each enzyme could be isolated and combined to design a new enzyme with improved catalytic activity for the secondary amidase reaction. In the present paper, based on our knowledge derived from previous comparative studies, and by applying the concept of electrostatic pre-organisation, ${ }^{28-32,37-40}$ a variant with improved activity for the designated amidase reaction was generated. After overlapping the structures of both proteins in one of the located TSs, through the use of a rotation quaternion around selected atoms of the substrate, a catalytically improved Bs2 variant was delineated. In particular, residues of Bs2 with an unfavorable electrostatic effect on catalysis were substituted by those placed in an 
equivalent spatial position in CALB with a favorable effect, as explained in detailed below. QM/MM FES of the full catalytic reaction in the proposed variant, combined with the experimental characterizations, will be used to propose a general computer-based strategy that can be potentially used to design new enzymes.

\section{MATERIALS AND METHODS}

Computational methods. Primary sequence of wild-type $p$-nitrobenzyl (PNB) esterase sequence from Bacillus subtilis (with ID P37967) was taken from UniProt. ${ }^{41}$ Due to the lack of crystallized structure for this specific variant, the framework for the required model, a natural variant of the protein (strain 168) was prepared based on a PNB esterase from a different organism (Bs2; PDB ID: 1QE3). ${ }^{25}$ Details can be found in previous publications ${ }^{33}$ and the Supporting Information. Based on the analysis of the previously computed FES of the amidase reaction catalyzed by wildtype $\mathrm{CALB}^{32}$ and $\mathrm{Bs}^{33}$ with the $N$-(4-nitrophenyl)-butyramide as substrate, the structural alignment of the two proteins was subsequently done within the TS1 structures localized at QM/MM level in both systems. The QM sub-set of atoms was described with the M06-2X hybrid functional ${ }^{42}$ and the solvent water molecules and the protein treated with the TIP3 $\mathrm{P}^{43}$ and the OPLS-AA ${ }^{44}$ force field, respectively, as implemented in fDynamo library. ${ }^{45}$ Then, a short $20 \mathrm{ps}$ QM/MM MD was done freezing the geometries of the quantum partition. The alignment of the structures from the trajectory was done using a rotation quaternion ${ }^{46}$ around the positions of atoms of the amide bond and the two neighboring carbon atoms of the substrate (Supplementary Fig. S1). When both proteins were aligned, structures were compared based on the averaged position of the centers of mass of every residue of the protein. Residues of Bs2 and CALB were paired based on the proximity of their centers of mass, i.e., the pair of a residue from Bs2 is the residue from CALB 
whose center of mass lies the nearest to the tested residue (Supplementary Fig. S2). Finally, the electrostatic potential generated on $\mathrm{N} \varepsilon$ of the catalytic histidine residues of Bs 2 and CALB by the residues whose center of mass lies within $15 \AA$ of the center of mass of the substrate was computed. The proposed variants, F398D, F398D with protonated aspartate (F398D-H $\mathrm{H}^{+}$from now on), and D66I/L335K were prepared from the structure of the wild-type Bs2. Then, counterions were placed in the most electrostatically favorable positions in order to neutralize the three systems. Subsequently, the systems containing the protein, counterions, and the substrate were solvated by placing them in $100 \times 80 \times 80 \AA^{3}$ pre-equilibrated boxes of TIP3 $\mathrm{P}^{43}$ water molecules. After initial energy minimizations, the systems were heated to $303 \mathrm{~K}$ with $0.1 \mathrm{~K}$ temperature increment and equilibrated during short (100ps) NPT MD simulations, followed by non-accelerated classical NVT MD simulations with a time step of $1 \mathrm{fs}$. The substrate was described with the same force field parameters as determined in our previous works. ${ }^{32,33}$ During the $100 \mathrm{~ns}$ MD simulations all atoms were free to move with periodic boundary conditions and cut-offs for nonbonding interactions were applied using a smooth switching function between 14.5 and $16 \AA$. Timedependent evolution of the root mean square deviations (RMSD) and B-factors for all three variants of Bs2 confirm that they were equilibrated and did not suffer dramatic structural modifications (Supplementary Fig. S3).

After setting up the model for F398D, F398D-H+ ${ }^{+}$and D66I/L335K, the reaction was studied using a QM/MM approach from the equilibrated structures. The semiempirical $\mathrm{AM} 1{ }^{47}$ method and the M06- $2 \mathrm{X}^{42}$ density functional were used to describe the QM sub-set of atoms, corresponding to the active site (Supplementary Fig. S4). The OPLS-AA and TIP3P classical force fields were used to treat the protein and the solvent water molecules, respectively, as implemented in fDynamo library. After PESs were computed, exploring the appropriate distinguished reaction coordinates, FESs for 
each of the chemical step were calculated in terms of PMFs using umbrella sampling (US) technique $^{48,49}$ (Supplementary Fig. S5-S7). Structures corresponding to the stationary points were finally optimized at M06-2X/MM level and IRC paths were traced down to the precedent and posterior energy minima structures.

Experimental methods: A short summary of the experimental procedures is provided while a more detailed description of the experimental procedures is reported in the Supporting Information. The synthesis of $N$-(4-nitrophenyl)-butyramide was performed according to a known procedure. ${ }^{32}$ The production of wild-type Bs2 and Bs2 F398D was based on a previously reported procedure. $^{22}$

For the 96 well-plate kinetic assay, stock solutions of the respective Bs2 variant (in $50 \mathrm{mM} \mathrm{NaP}$, $\mathrm{pH}$ 7.0) and $\mathrm{N}$-(4-nitrophenyl)-butyramide (in DMSO) were prepared. The protein stock solution was kept on ice until use and was freshly prepared before each usage. DMSO and substrate stock solution were added to wells of a 96 transparent well-plate to a total of $15 \mu \mathrm{L}$. Buffer $(50 \mathrm{mM}$ $\left.\mathrm{NaP}_{\mathrm{i}}, \mathrm{pH} 7.0\right)$ was added to a total volume of $135 \mu \mathrm{L}(150 \mu \mathrm{L}$ in case of controls to monitor substrate stability). The plate was transferred into a plate reader, double orbitally shaken for $5 \mathrm{~s}$ and the absorption at $\lambda_{\mathrm{Ex}}=405 \mathrm{~nm}$ measured to check correct substrate distribution. Then $15 \mu \mathrm{L}$ of protein stock solution were added to each well except the enzyme free controls within 5 min. Final assay conditions were $150 \mu \mathrm{L}$ volume, 10\% DMSO, N-(4-nitrophenyl)-butyramide (10, 50, $100,250500,1000,2000,3000 \mu \mathrm{M}), 20 \mu \mathrm{g} / \mathrm{mL}$ protein. The plate was sealed with an airtight and UV-Vis transparent self-adhesive plastic cover sheet. After sealing, the plate was placed into the plate reader and the assay was monitored for $14 \mathrm{~h}$. 


\section{RESULTS AND DISCUSSION}

Structural and electrostatic comparison of Bs2 vs CALB. Previously studies of the amidase mechanism catalyzed by $\mathrm{CALB}^{32}$ and $\mathrm{Bs}^{33}$ (Figure 1) showed a clear separation of charges in this first step, where a negative charge is accumulated in the carbonyl oxygen atom of the substrate and a positive charge is generated in the $\mathrm{N} \varepsilon$ atom of His399 (Figure 1). The stabilization of the negative charge is achieved through interactions with the oxyanion hole that is present in both enzymes through the generation of a microenvironment with positive electrostatic potential. ${ }^{28,32}$ Nevertheless, additional attempts to increase the positive electrostatic potential should not result in a significant catalytic effect. ${ }^{32}$ Consequently, in the present study we focused on identifying residues in Bs2 and CALB contributing to generate a negative electrostatic potential on the $\mathrm{N} \varepsilon$ atom of the catalytic histidine residue (His399 or His224 in Bs2 and CALB, respectively), which assists in the positive charge transfer from Ser189/Ser105 that takes place in the first step.

In order to compare TS1 of two separate protein environments that have significantly low sequence identity, residues located in equivalent spatial positions in both proteins need to be identified (Supplementary Fig. S1). The structural alignment of the proteins was done by using a rotation quaternion ${ }^{46}$ around the positions of the atoms of the amide bond of the substrate, and the two adjacent carbon atoms. In particular, the matching was done by pairing residues of Bs2 and CALB whose averaged centers of masses overlap (or are in close proximity) to each other. The first conclusion derived from the results was the good overlapping between the active site triad SerHis-Glu in Bs2 and the Ser-His-Asp counterpart in CALB (Supplementary Fig. S2), implying that both enzymes are preorganized for breaking the $\mathrm{C}-\mathrm{N}$ bond of the substrate.

The electrostatic potential on the $\mathrm{N} \varepsilon$ atom of the active site histidine was computed per residue in Bs2 and CALB. We aimed to seek residues that stabilize the formation of TS1 in CALB which are 
not found in Bs2 (Figure 2 and Supplementary Fig. S8). The residues that generate a negative potential on $\mathrm{N} \varepsilon$ can be considered as favorable for catalysis whilst those adding a positive potential detrimental for the enzyme activity. The difference between Bs2 and CALB revealed "hot spot" candidates with a positive value for mutagenesis for enhancing the amidase activity in Bs2, whereas residues with negative values proposed to decrease activity.
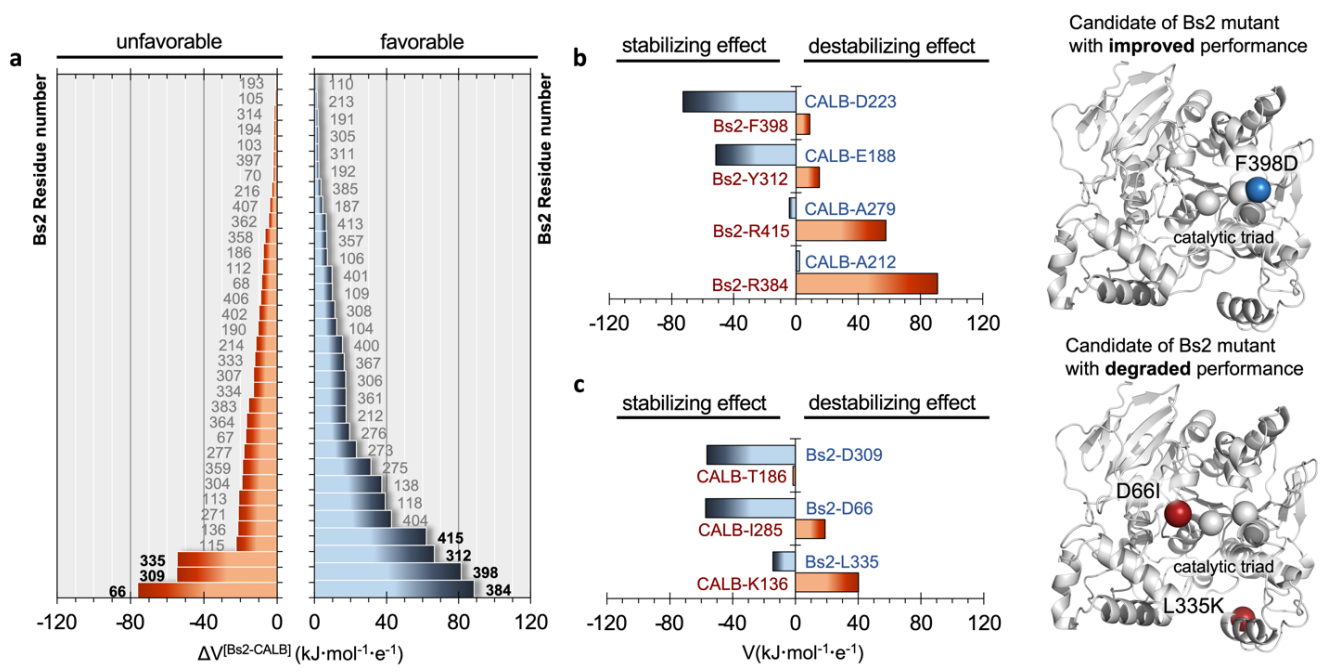

Figure 2. Comparison of electrostatic potential generated by Bs2 and CALB. (a) Difference in the electrostatic potential $(\Delta \mathrm{V})$ generated on the $\mathrm{N} \varepsilon$ atom of the catalytic histidine in the TS1 structure by each residue of Bs2 and CALB, calculated as $\mathrm{V}_{\mathrm{i}}(\mathrm{Bs} 2)-\mathrm{V}_{\mathrm{j}}(\mathrm{CALB})$ where $\mathrm{i}$ and $\mathrm{j}$ are the corresponding paired residues of each enzyme. Only residue numbers of Bs2 are labelled indicating the possible candidates for mutations. The residues are ordered by their estimated role in improving or degrading enzymatic activity: positions with $\Delta \mathrm{V}>0$ have a favorable effect on activity upon mutation (right panel), while those with $\Delta \mathrm{V}<0$ provide the unfavorable effect (left panel). (b) Electrostatic potential generated by selected residues with destabilizing effects in Bs2 (in red) and the corresponding residues from CALB in the same position that have a stabilizing effect (in blue). The proposed mutations to improve Bs2 catalytic activity are shown on the right side of the panel as blue balls. (c) Selected residues with stabilizing effects in Bs2 (blue labels) and the corresponding residues from CALB in the same position that have a destabilizing effect 
(red labels). The proposed mutations to degrade Bs2 catalytic activity are shown on the right side of the panel as red balls.

\section{Bs2 Redesign.}

As summarized in Figure 2b, four residues that generate an unfavorable potential on the catalytic His399 in Bs2 were identified (F398, Y312, R415, and R384) by comparison with those located in an equivalent spatial position in CALB with stabilizing or not-unfavorable effects (D223, E188, A279, and A212). Subsequently, four separate amino acid replacements in Bs2, including F398D, Y312E, R415A, and R384A, were investigated. After the analysis of the surroundings of R384 and R415 in Bs2, these positions were excluded because they form a complex hydrogen-bonding network with the close residues of the amino acids, and the mutation to Ala could be reflected in a large impact in the local structure due to the change in the size and polarity losing all the key hydrogen bond interactions (Supplementary Fig. S9). As determined by the calculations with the empirical program PropKa v.3.0.3, ${ }^{50,51}$ substitution of Y312 with glutamate causes a $\mathrm{p} K_{\mathrm{a}}$ shift, suggesting that the carboxylic group is protonated in the variant, and hence the predicted increase in the negative electrostatic potential becomes diluted. By elimination, we propose to create just the F398D Bs2 variant. Nevertheless, although the predicted $\mathrm{p} K_{\mathrm{a}}$ indicates a deprotonated Asp when inserted in position 398 of $\mathrm{Bs} 2$, the possibility of a protonated $\mathrm{F} 398 \mathrm{D}-\mathrm{H}^{+}$variant was also investigated as a result of structural analysis of long MD simulations on this variant (Supplementary Fig. S10). Indeed, estimation of the pKa of the aspartate introduced in position 398 of Bs2 with PropKa using the equilibrated structure of the protonated F398D- $\mathrm{H}^{+}$variant confirms a shift in its pKa (from 5.1, in the F398D structure to 7.9 when using the F398D-H ${ }^{+}$ structure). 


\section{In silico amidase activity test of the F398D and F398D-H ${ }^{+}$Bs2 variants.}

Molecular dynamics (MD) simulations of the Bs2 F398D and F398D- $\mathrm{H}^{+}$variants indicated that the overall protein architecture remains largely unchanged but the protonation of Asp398 appears as a plausible possibility (Supplementary Fig. S10). Its catalysis was examined by generating the free energy landscape of the acylation step of the amidase reaction (Supplementary Fig. S5 and Fig. S6). The barrier of the first step of the reaction decreased $4.2 \mathrm{kcal} \cdot \mathrm{mol}^{-1}$ in the $\mathrm{F} 398 \mathrm{D}$ variant when compared to the same step catalyzed by the wild-type Bs2 (Figure 3). As anticipated, the F398D exerts a stabilization effect for the formation first intermediate INT1, decreasing the energy of TS2 relative to the reactants by $3.2 \mathrm{kcal} \cdot \mathrm{mol}^{-1}$. In the other words, we predicted a significant reduction in the energy barrier of the rate determining step for the acylation step, from 19.2 $\mathrm{kcal} \cdot \mathrm{mol}^{-1}$ (TS1 in the wild-type) to $14.3 \mathrm{kcal} \cdot \mathrm{mol}^{-1}$ (TS2 in the F398D), which would correspond to an increase of reaction rate by $>3000$-fold by applying TST at $303 \mathrm{~K}$. Nevertheless, in the case of the protonated $\mathrm{F} 398 \mathrm{D}-\mathrm{H}^{+}$variant this impact is less dramatic generating a lower negative potential on the catalytic His399. In this case the overall barrier of the first step of the reaction is similar to the wild-type Bs2, with just a small decrease from $19.2 \mathrm{kcal} \cdot \mathrm{mol}^{-1}$ in the wild-type Bs2 to $18.8 \mathrm{kcal} \cdot \mathrm{mol}^{-1}$ in the $\mathrm{F} 398 \mathrm{D}-\mathrm{H}^{+}$variant. This difference in the energy barrier indicates a negligible effect.

In order to verify the robustness of our computational strategy, an additional in silico proof test was carried out by designing a Bs2 variant with decreased activity. Based on the comparative analysis of the electrostatic properties of Bs2 and CALB, two possible mutations were identified to decrease the activity of the enzyme including D66I and L335K (see Figure 2c). Replacement of D309 with threonine was discarded due to the loss of hydrogen bond interactions. For the D66I/L335K mutation, there is a meaningful increase in the free energy barrier for the C-N bond 
cleavage, up to $23.5 \mathrm{kcal} \cdot \mathrm{mol}^{-1}$ (Figure 3a and Supplementary Fig. S7), which aligns with our predictions based on the importance of protein electrostatic. In all, a clear correlation between the free energy barriers and the electrostatic potential generated on the catalytic His399 was observed. The lower the electrostatic potential on the $\mathrm{N} \varepsilon$ results in a lower energy barrier (Figure 3b).
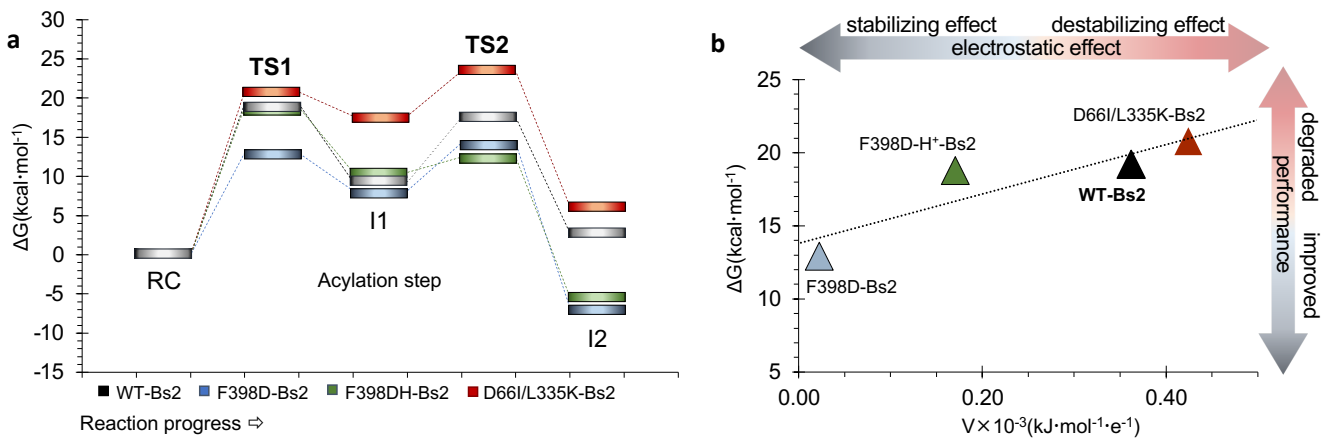

Figure 3. (a), QM/MM Free energy profiles of the acylation step catalyzed by wild-type Bs2 (in grey), by the improved F398D Bs2 variant (in blue), the protonated F398D-H $\mathrm{H}^{+}$Bs2 variant (in green), and the degraded D66I/L335K Bs2 variant (in red). Data from wild-type Bs2 was taken from ref 33. (b), Correlation between the activation free energy of acylation step and the electrostatic potential in the $\mathrm{N} \varepsilon$ atom of His.

\section{Kinetic measurements of the amidase activity of the F398D Bs2 variant.}

The predictions derived from the $\mathrm{QM} / \mathrm{MM}$ computational protocol were assessed by experimentally comparing the catalytic activities of the wild-type Bs2 $2^{33}$ and F398D variant. The experimental turnover constants $\left(k_{\text {cat }}\right)$ measured via UV-Vis spectrophotometric assay are higher than those previously reported by two orders of magnitude (Figure 4a). ${ }^{22,24}$ This could be caused by a potentially different arrangement of the hexahistidine tag that could result from a discrepancy 
in the assay setup. Attempts to use the original assays were found to be unreliable with nonreproducible kinetic constants generated, and hence the present 96-well plate assay methodology that was proven to be successful in our previous work on $\mathrm{CALB}^{32}$ was applied. The $k_{\text {cat }}$ constant for the F398D variant is 1.3-fold higher than that for the wild-type. The free energy barrier derived from this experimentally measured rate constan is in agreement with the computationally assessed enzyme activity for the protonated $\mathrm{F} 398 \mathrm{D}-\mathrm{H}^{+} \mathrm{Bs} 2$ variant $\left(\Delta \mathrm{G}^{\star}\right.$ exp $=19.4 \mathrm{kcal} \cdot \mathrm{mol}^{-1}$ and $\Delta \mathrm{G}^{\ddagger}$ theo $=$ $18.8 \mathrm{kcal} \cdot \mathrm{mol}^{-1}$, respectively). On the other hand, the $K_{\mathrm{M}}$ constant for the mutant was noticeably higher resulting in a decrease in the overall catalytic efficiency $k_{\text {cat }} / K_{\mathrm{M}}$. As the binding step was not explored in the simulation studies, this effect was not predicted. The increase of the $K_{\mathrm{M}}$ in the mutant variant could be explained by the loss of key hydrogen bonds that R415 and Y118 establish with the nitro group of the substrate (Supplementary Figure S10a and S10b). The mutation of F398 to Asp causes a conformational change in the vicinity of the active site forcing R415 and Y118 to lose the contacts with the substrate (Supplementary Figure S11a and S11b). This observation is supported by the analysis of electrostatic interactions during the MD simulation. Interactions of the substrate with mainly G105, Y118, and R415, could have a role in the stabilization of the Michaelis complex, and those interactions are more favorable in the wild-type than in the F398D$\mathrm{H}^{+}$Bs2 variant (Supplementary Figure S12). 
a<smiles>CCCC(=O)Nc1ccc([N+](=O)[O-])cc1</smiles>

b

\begin{tabular}{c|c|c|c|c} 
Enzyme & $\boldsymbol{v}_{\max }\left(\mathbf{1 0}^{-\mathbf{3}} \boldsymbol{\mu} \mathbf{M ~ s}^{-1}\right)$ & $\boldsymbol{K}_{\text {cat }}\left(\mathbf{1 0}^{-\mathbf{3}} \mathbf{s}^{-1}\right)$ & $\boldsymbol{K}_{\mathrm{M}}\left(\boldsymbol{\mu \mathrm { M } ^ { - 1 } )}\right.$ & $\boldsymbol{K}_{\mathrm{cat}} / \boldsymbol{K}_{\mathrm{M}}\left(\mathbf{s}^{-\mathbf{1}} \mathbf{M}^{-1}\right)$ \\
\hline Bs2 wild-type & $6.7 \pm 0.2$ & $18.5 \pm 0.7$ & $180 \pm 40$ & $110 \pm 30$ \\
Bs2 F398D & $8.4 \pm 0.4$ & $23.1 \pm 1.0$ & $500 \pm 50$ & $46 \pm 3$
\end{tabular}

C

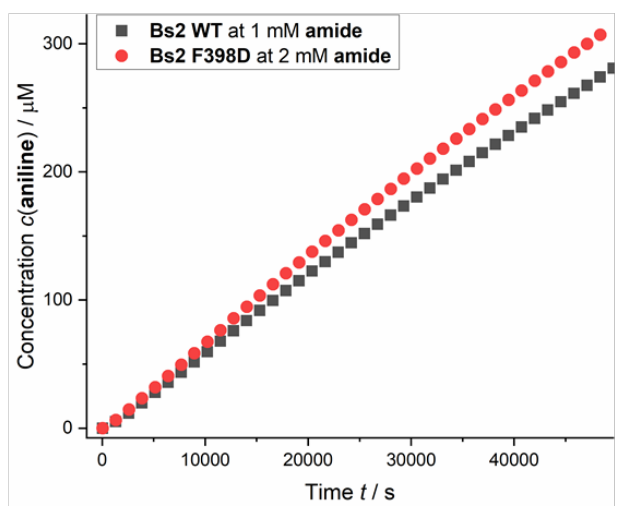

d

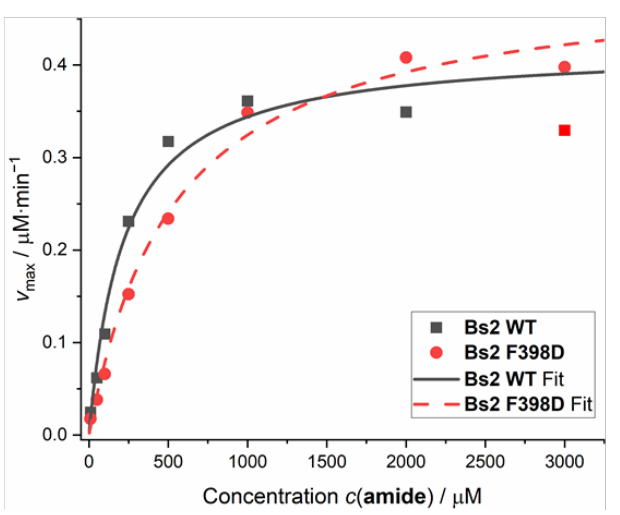

Figure 4. (a) Model assay reaction and conditions to spectrophotometrically determine the catalytic activities. (b) Table summarizing the kinetic parameters of wild-type Bs2 and F398D Bs2 variant for the reaction shown in a. (c) Comparison of 4-nitroaniline formation over time between wild-type Bs2 and F398D Bs2 variant. (d) Comparison of Michaelis-Menten plots of wild-type Bs2 and F398D Bs2 variant. 


\section{CONCLUSIONS}

We have illustrated a computational strategy to redesign enzymes with enhanced catalytic activity. An in-depth analysis on the electrostatic properties of the chemical transformations under the effect of the protein along the reaction, explored by QM/MM MD simulations, illustrated that the ratelimiting transition states can be further stabilized by modifying the electrostatic field on key fragments of the chemical system through site-directed mutagenesis. In particular, comparative analysis between Bs2 and CALB has facilitated the identification of key residues with a different impact for the amidase reaction; residues of the former with an unfavorable effect on catalysis are substituted by those at equivalent spatial positions in CALB that exert a favorable effect. Our in silico method predicts an, a priori, increase of 4 orders of magnitude on the measurable rate constant after a single mutation of Phe398 to aspartate which is $\sim 10 \AA$ away from the active site. In addition, the same strategy was employed to prepare variants of Bs2 with lower catalytic activities, as a control test of the method. The resulting FESs for the acylation step of the amidase reaction catalyzed by the different variants show a linear correlation between the activation free energy of the acylation step and the electrostatic potential in the $\mathrm{N} \varepsilon$ atom of His. Though estimation of the $\mathrm{p} K_{\mathrm{a}}$ of Asp398 in Bs2 suggests to be deprotonated, an in deep structural analysis of the F398D Bs2 variant during long MD simulations suggest that the newly inserted aspartate residue could be in fact protonated, rendering a different but stable protein conformational structure. QM/MM FESs with this F398D-H $\mathrm{H}^{+}$variant predicts a lower electrostatic effect which results in a less dramatic decrease of the energy barrier of the reaction, as confirmed by the experimental kinetic measurement of the variant where Phe398 was converted to aspartate.

While a variant with much better activity has not been obtained, there was excellent agreement between the predicted activation free energies and the experimentally determined rate constants. 
For future work, several non-titratable residues including artificial amino acids can be introduced together, instead of only converting Phe398 to aspartate which resulted in an unwanted shift in pKa. While their individual contribution is less considerable, according to our analysis (see Figure 2a), these non-titratable residues when introduced together can act concertedly to drastically modify the protein electrostatics. Alternatively, this F398D first-generation variant can be subjected to additional investigations, including QM/MM and structural analyses, which provides guidance on how additional modifications can enhance the new aspartate residue to remain deprotonated. Improvement on the $K_{\mathrm{M}}$ values should also be made as means to improve the catalytic efficiency of the redesigned enzyme. Supported by the good agreement between theoretical and experimental results, as well as the linear correlation between the electrostatic properties and the activation energy barriers, we hope that upon optimization the presented computer-guided rational design will become a valuable component in the toolkit of enzyme redesign and engineering. 


\section{Acknowledgements}

This work was supported by the Spanish Ministerio de Ciencia, Innovación y Universidades (ref. PGC2018-094852-B-C21 and PID2019-107098RJ-I00), the Generalitat Valenciana (ref. AICO/2019/195 and SEJI/2020/007), Universitat Jaume I (UJI-A2019-04 and UJI-B2020-03), Cardiff University through the start-up fund provided by the Cardiff School of Chemistry. MAG thanks Universitat Jaume I for FPI-UJI grant (PREDOC/2017/23). Authors acknowledge computational resources from the Servei d'Informàtica of Universitat Jaume I.

\section{Supporting Information}

The Supporting Information is available free of charge at https://pubs.acs.org/doi/

Overlapped centers of masses of paired residues from Bs2 and CALB; Structural alignment of Bs2 and CALB; Analysis of MD simulations; Schematic representation of the QM region; M062X:AM1/OPLS-AA free energy surfaces; electrostatic potential generated by Bs2 and CALB per residue; Structural analysis of the wild-type and mutants of Bs2; key interatomic distances and charges; computational and experimental details; Cartesian coordinates of M06-2X/MM TSs optimized structures and imaginary frequencies; LC chromatograms and mass spectrum; Michaelis-Menten plots.

\section{Author contributions}

M.A.G. carried out all the calculations. K.S. and V.M. supervised the computational work and designed the project. L.Y.P.L and A.R.N. designed the kinetic measurements. K.H. performed cloning, mutagenesis and initial synthesis of the wild-type under supervision of A.R.N. A.R.N. 
performed the synthesis of the mutants and all the kinetic experiments. All authors contributed to analyzing the results and writing the manuscript.

\section{Competing interests}

The authors declare no competing interests.

\section{Additional information}

Supplementary information is available for this paper at https://doi....

\section{ORCID Information}

Miquel À. Galmés: 0000-0002-9000-9396

Alexander R Nödling: 0000-0002-3483-6266

Louis Luk: 0000-0002-7864-6261

Katarzyna Świderek: 0000-0002-7528-1551

Vicent Moliner: 0000-0002-3665-3391 


\section{REFERENCES}

(1) Chen, K.; Arnold, F. H. Tuning the Activity of an Enzyme for Unusual Environments: Sequential Random Mutagenesis of Subtilisin E for Catalysis in Dimethylformamide. Proc. Natl. Acad. Sci. U. S. A. 1993, 90, 5618-5622.

(2) Arnold, F. H. Combinatorial and Computational Challenges for Biocatalyst Design. Nature 2001, 409, 253-257.

(3) Blomberg, R.; Kries, H.; Pinkas, D. M.; Mittl, P. R. E.; Grütter, M. G.; Privett, H. K.; Mayo, S. L.; Hilvert, D. Precision Is Essential for Efficient Catalysis in an Evolved Kemp Eliminase. Nature 2013, 503, 418-421.

(4) Huang, P.-S.; Boyken, S. E.; Baker, D. The Coming of Age of de Novo Protein Design. Nature 2016, 537, 320-327.

(5) Zeymer, C.; Hilvert, D. Directed Evolution of Protein Catalysts. Annu. Rev. Biochem. 2018, $87,131-157$.

(6) Stemmer, W. P. C. Searching Sequence Space. Bio/Technology 1995, 13, 549-553.

(7) Kuchner, O.; Arnold, F. H. Directed Evolution of Enzyme Catalysts. Trends Biotechnol. 1997, 15, 523-530.

(8) Bornscheuer, U. T. Directed Evolution of Enzymes. Angew. Chemie Int. Ed. 1998, 37, 3105-3108.

(9) MacBeath, G.; Kast, P.; Hilvert, D. Redesigning Enzyme Topology by Directed Evolution. Science (80-. ). 1998, 279, 1958-1961.

(10) Yoshikuni, Y.; Ferrin, T. E.; Keasling, J. D. Designed Divergent Evolution of Enzyme Function. Nature 2006, 440, 1078-1082.

(11) Arnold, F. H. Directed Evolution: Bringing New Chemistry to Life. Angew. Chemie Int. Ed. 2018, 57, 4143-4148.

(12) Otten, R.; Pádua, R. A. P.; Bunzel, H. A.; Nguyen, V.; Pitsawong, W.; Patterson, M.; Sui, S.; Perry, S. L.; Cohen, A. E.; Hilvert, D.; Kern, D. How Directed Evolution Reshapes the Energy Landscape in an Enzyme to Boost Catalysis. Science (80-. ). 2020, 370, 1442 LP 1446.

(13) Bunzel, H. A.; Garrabou, X.; Pott, M.; Hilvert, D. Speeding up Enzyme Discovery and Engineering with Ultrahigh-Throughput Methods. Curr. Opin. Struct. Biol. 2018, 48, 149156. 
(14) Fox, R. J.; Davis, S. C.; Mundorff, E. C.; Newman, L. M.; Gavrilovic, V.; Ma, S. K.; Chung, L. M.; Ching, C.; Tam, S.; Muley, S.; Grate, J.; Gruber, J.; Whitman, J. C.; Sheldon, R. A.; Huisman, G. W. Improving Catalytic Function by ProSAR-Driven Enzyme Evolution. Nat. Biotechnol. 2007, 25, 338-344.

(15) Yang, K. K.; Wu, Z.; Arnold, F. H. Machine-Learning-Guided Directed Evolution for Protein Engineering. Nat. Methods 2019, 16, 687-694.

(16) Świderek, K.; Tuñón, I.; Moliner, V. Predicting Enzymatic Reactivity: From Theory to Design. Wiley Interdiscip. Rev. Comput. Mol. Sci. 2014, 4, 407-421.

(17) Nobeli, I.; Favia, A. D.; Thornton, J. M. Protein Promiscuity and Its Implications for Biotechnology. Nat. Biotechnol. 2009, 27, 157-167.

(18) Leveson-Gower, R. B.; Mayer, C.; Roelfes, G. The Importance of Catalytic Promiscuity for Enzyme Design and Evolution. Nat. Rev. Chem. 2019, 3, 687-705.

(19) Deslongchamps, P. Stereoelectronic Control in the Cleavage of Tetrahedral Intermediates in the Hydrolysis of Esters and Amides. Tetrahedron 1975, 31, 2463-2490.

(20) Busto, E.; Gotor-Fernández, V.; Gotor, V. Hydrolases: Catalytically Promiscuous Enzymes for Non-Conventional Reactions in Organic Synthesis. Chem. Soc. Rev. 2010, 39, 45044523 .

(21) Syrén, P. O.; Hendil-Forssell, P.; Aumailley, L.; Besenmatter, W.; Gounine, F.; Svendsen, A.; Martinelle, M.; Hult, K. Esterases with an Introduced Amidase-Like Hydrogen Bond in the Transition State Have Increased Amidase Specificity. ChemBioChem 2012, 13, 645648.

(22) Hackenschmidt, S.; Moldenhauer, E. J.; Behrens, G. A.; Gand, M.; Pavlidis, I. V.; Bornscheuer, U. T. Enhancement of Promiscuous Amidase Activity of a Bacillus Subtilis Esterase by Formation of a $\pi-\pi$ Network. ChemCatChem 2014, 6, 1015-1020.

(23) Truhlar, D. G.; Garrett, B. C.; Klippenstein, S. J. Current Status of Transition-State Theory. J. Phys. Chem. 1996, 100, 12771-12800.

(24) Kourist, R.; Bartsch, S.; Fransson, L.; Hult, K.; Bornscheuer, U. T. Understanding Promiscuous Amidase Activity of an Esterase from Bacillus Subtilis. ChemBioChem 2008, 9, 67-69.

(25) Spiller, B.; Gershenson, A.; Arnold, F. H.; Stevens, R. C. A Structural View of Evolutionary Divergence. Proc. Natl. Acad. Sci. 1999, 96, 12305-12310. 
(26) Jindal, G.; Slanska, K.; Kolev, V.; Damborsky, J.; Prokop, Z.; Warshel, A. Exploring the Challenges of Computational Enzyme Design by Rebuilding the Active Site of a Dehalogenase. Proc. Natl. Acad. Sci. 2019, 116, 389 LP - 394.

(27) Frushicheva, M. P.; Cao, J.; Warshel, A. Challenges and Advances in Validating Enzyme Design Proposals: The Case of Kemp Eliminase Catalysis. Biochemistry 2011, 50, 38493858 .

(28) Świderek, K.; Martí, S.; Moliner, V. Theoretical Study of Primary Reaction of Pseudozyma Antarctica Lipase B as the Starting Point to Understand Its Promiscuity. ACS Catal. 2014, 4, 426-434.

(29) Świderek, K.; Tuñón, I.; Moliner, V.; Bertran, J. Protein Flexibility and Preorganization in the Design of Enzymes. The Kemp Elimination Catalyzed by HG3.17. ACS Catal. 2015, 5, $2587-2595$.

(30) Krzemińska, A.; Moliner, V.; Świderek, K. Dynamic and Electrostatic Effects on the Reaction Catalyzed by HIV-1 Protease. J. Am. Chem. Soc. 2016, 138, 16283-16298.

(31) Świderek, K.; Tunón, I.; Williams, I. H.; Moliner, V. Insights on the Origin of Catalysis on Glycine N-Methyltransferase from Computational Modeling. J. Am. Chem. Soc. 2018, 140, $4327-4334$.

(32) Galmés, M. A.; García-Junceda, E.; Świderek, K.; Moliner, V. Exploring the Origin of Amidase Substrate Promiscuity in CALB by a Computational Approach. ACS Catal. 2020, 10, 1938-1946.

(33) Galmés, M. À.; Nödling, A. R.; Luk, L.; Świderek, K.; Moliner, V. Combined Theoretical and Experimental Study to Unravel the Differences in Promiscuous Amidase Activity of Two Nonhomologous Enzymes. ACS Catal. 2021, 8635-8644.

(34) Warshel, A. Energetics of Enzyme Catalysis. Proc. Natl. Acad. Sci. U. S. A. 1978, 75.

(35) Warshel, A. Electrostatic Origin of the Catalytic Power of Enzymes and the Role of Preorganized Active Sites. Journal of Biological Chemistry. 1998.

(36) Olsson, M. H. M.; Parson, W. W.; Warshel, A. Dynamical Contributions to Enzyme Catalysis: Critical Tests of a Popular Hypothesis. Chemical Reviews. 2006.

(37) Warshel, A.; Sharma, P. K.; Kato, M.; Xiang, Y.; Liu, H.; Olsson, M. H. M. Electrostatic Basis for Enzyme Catalysis. Chem. Rev. 2006, 106, 3210-3235.

(38) García-Meseguer, R.; Martí, S.; Ruiz-Pernía, J. J.; Moliner, V.; Tunón, I. Studying the Role 
of Protein Dynamics in an SN2 Enzyme Reaction Using Free-Energy Surfaces and Solvent Coordinates. Nat. Chem. 2013, 5, 566-571.

(39) Kholodar, S. A.; Ghosh, A. K.; Swiderek, K.; Moliner, V.; Kohen, A.; Świderek, K.; Moliner, V.; Kohen, A. Parallel Reaction Pathways and Noncovalent Intermediates in Thymidylate Synthase Revealed by Experimental and Computational Tools. Proc. Natl. Acad. Sci. U. S. A. 2018, 115, 10311-10314.

(40) De Raffele, D.; Martí, S.; Moliner, V. QM/MM Theoretical Studies of a de Novo RetroAldolase Design. ACS Catal. 2019, 9, 2482-2492.

(41) Consortium, T. U. UniProt: A Worldwide Hub of Protein Knowledge. Nucleic Acids Res. 2018, 47, D506-D515.

(42) Zhao, Y.; Truhlar, D. G. The M06 Suite of Density Functionals for Main Group Thermochemistry, Thermochemical Kinetics, Noncovalent Interactions, Excited States, and Transition Elements: Two New Functionals and Systematic Testing of Four M06-Class Functionals and 12 Other Function. Theor. Chem. Acc. 2008, 120, 215-241.

(43) Jorgensen, W. L.; Chandrasekhar, J.; Madura, J. D.; Impey, R. W.; Klein, M. L. Comparison of Simple Potential Functions for Simulating Liquid Water. J. Chem. Phys. 1983, 79, 926935.

(44) Jorgensen, W. L.; Maxwell, D. S.; Tirado-Rives, J. Development and Testing of the OPLS All-Atom Force Field on Conformational Energetics and Properties of Organic Liquids. $J$. Am. Chem. Soc. 1996, 118, 11225-11236.

(45) Field, M. J.; Albe, M.; Bret, C.; Proust-De Martin, F.; Thomas, A. The Dynamo Library for Molecular Simulations Using Hybrid Quantum Mechanical and Molecular Mechanical Potentials. J. Comput. Chem. 2000, 21, 1088-1100.

(46) Kneller, G. R. Superposition of Molecular Structures Using Quaternions. Mol. Simul. 1991, 7, 113-119.

(47) Dewar, M. J. S.; Zoebisch, E. G.; Healy, E. F.; Stewart, J. J. P. Development and Use of Quantum Mechanical Molecular Models. 76. AM1: A New General Purpose Quantum Mechanical Molecular Model. J. Am. Chem. Soc. 1985, 107, 3902-3909.

(48) Roux, B. The Calculation of the Potential of Mean Force Using Computer-Simulation. Comput. Phys. Commun. 1995, 91, 275-282.

(49) Torrie, G. M.; Valleau, J. P. Non-Physical Sampling Distibutions in Monte-Carlo Free- 
Energy Estimation - Umbrella Sampling. J. Comput. Phys. 1977, 23, 187-199.

(50) Olsson, M. H. M.; SØndergaard, C. R.; Rostkowski, M.; Jensen, J. H. PROPKA3: Consistent Treatment of Internal and Surface Residues in Empirical p Kapredictions. $J$. Chem. Theory Comput. 2011, 7, 525-537.

(51) Søndergaard, C. R.; Olsson, M. H. M.; Rostkowski, M.; Jensen, J. H. Improved Treatment of Ligands and Coupling Effects in Empirical Calculation and Rationalization of PKa Values. J Chem Theory Comput 2011, 7, 2284-2295. 



\subsection{References}

(1) Busto, E.; Gotor-Fernández, V.; Gotor, V. Hydrolases: Catalytically Promiscuous Enzymes for Non-Conventional Reactions in Organic Synthesis. Chem. Soc. Rev. 2010, 39, 4504-4523.

(2) Gotor-Fernández, V.; Brieva, R.; Gotor, V. Lipases: Useful Biocatalysts for the Preparation of Pharmaceuticals. J. Mol. Catal. B Enzym. 2006, 40, 111-120.

(3) Wikmark, Y.; Humble, M. S.; Bäckvall, J. E. Combinatorial Library Based Engineering of Candida Antarctica Lipase a for Enantioselective Transacylation of Sec-Alcohols in Organic Solvent. Angew. Chemie - Int. Ed. 2015, 54, 42844288.

(4) Humble, M. S.; Carlqvist, P.; Branneby, C.; Allnér, O.; Frise, A.; Hult, K.; Berglund, P.; Brinck, T. Direct Epoxidation in Candida Antarctica Lipase B Studied by Experiment and Theory. ChemBioChem 2008, 9, 2443-2451.

(5) Chen, X. Y.; Chen, G. J.; Wang, J. L.; Wu, Q.; Lin, X. F. Lipase/AcetamideCatalyzed Carbon-Carbon Bond Formations: A Mechanistic View. Adv. Synth. Catal. 2013, 355, 864-868.

(6) Sarmah, N.; Revathi, D.; Sheelu, G.; Rani, K. Y.; Sridhar, S.; Mehtab, V.; Sumana, C. Recent Advances on Sources and Industrial Applications of Lipases. Biotechnol. Prog. 2018, 34, 5-28.

(7) Torres-Gavilán, A.; Castillo, E.; López-Munguía, A. The Amidase Activity of Candida Antarctica Lipase B Is Dependent on Specific Structural Features of the Substrates. J. Mol. Catal. B Enzym. 2006, 41, 136-140.

(8) Buchholz, K. A Breakthrough in Enzyme Technology to Fight Penicillin Resistance-Industrial Application of Penicillin Amidase. Appl. Microbiol. Biotechnol. 2016, 100, 3825-3839.

(9) Sheldon, R. A.; Brady, D. Broadening the Scope of Biocatalysis in Sustainable Organic Synthesis. ChemSusChem 2019, 12, 2859-2881.

(10) Prileschajew, N. Oxydation Ungesättigter Verbindungen Mittels Organischer Superoxyde. Berichte der Dtsch. Chem. Gesellschaft 1909, 42, 4811-4815.

(11) Kourist, R.; Bartsch, S.; Fransson, L.; Hult, K.; Bornscheuer, U. T. Understanding Promiscuous Amidase Activity of an Esterase from Bacillus Subtilis. ChemBioChem 2008, 9, 67-69.

(12) Carniel, A.; Valoni, É.; Nicomedes, J.; Gomes, A. da C.; Castro, A. M. de. Lipase from Candida Antarctica (CALB) and Cutinase from Humicola Insolens Act Synergistically for PET Hydrolysis to Terephthalic Acid. Process Biochem. 2017, 59, 84-90.

(13) Ribitsch, D.; Heumann, S.; Trotscha, E.; Herrero Acero, E.; Greimel, K.; Leber, 


\subsection{References}

R.; Birner-Gruenberger, R.; Deller, S.; Eiteljoerg, I.; Remler, P.; et al. Hydrolysis of Polyethyleneterephthalate by P-Nitrobenzylesterase from Bacillus Subtilis. Biotechnol. Prog. 2011, 27, 951-960.

(14) Hackenschmidt, S.; Moldenhauer, E. J.; Behrens, G. A.; Gand, M.; Pavlidis, I. V.; Bornscheuer, U. T. Enhancement of Promiscuous Amidase Activity of a Bacillus Subtilis Esterase by Formation of a $\pi-\pi$ Network. ChemCatChem 2014, 6, 1015-1020.

(15) Galmés, M.; García-Junceda, E.; Świderek, K.; Moliner, V. Exploring the Origin of Amidase Substrate Promiscuity in CALB by a Computational Approach. ACS Catal. 2020, 10, 1938-1946.

(16) Świderek, K.; Martí, S.; Moliner, V. Theoretical Study of Primary Reaction of Pseudozyma Antarctica Lipase B as the Starting Point to Understand Its Promiscuity. ACS Catal. 2014, 4, 426-434.

(17) Krzemińska, A.; Moliner, V.; Świderek, K. Dynamic and Electrostatic Effects on the Reaction Catalyzed by HIV-1 Protease. J. Am. Chem. Soc. 2016, 138, 1628316298.

(18) Świderek, K.; Tuñón, I.; Williams, I. H.; Moliner, V. Insights on the Origin of Catalysis on Glycine N-Methyltransferase from Computational Modeling. J. Am. Chem. Soc. 2018, 140, 4327-4334.

(19) Świderek, K.; Tunón, I.; Moliner, V.; Bertran, J. Protein Flexibility and Preorganization in the Design of Enzymes. The Kemp Elimination Catalyzed by HG3.17. ACS Catal. 2015, 5, 2587-2595.

(20) Galmés, M.; Nödling, A. R.; Luk, L.; Świderek, K.; Moliner, V. Combined Theoretical and Experimental Study to Unravel the Differences in Promiscuous Amidase Activity of Two Nonhomologous Enzymes. ACS Catal. 2021, 11, 86358644. 
Chapter 5

Conclusions and future perspectives 
- 176 - 
In the present thesis, the promiscuous activity of two $\mathrm{SHs}$ was studied. Firstly, the hydrolysis of amides as a secondary reaction was evaluated in CALB, by experimental and computational approaches. We showed that CALB is dependent on specific electrostatic features of the substrate, thus introducing a nitro group in the para position of a benzyl-acyl substrate is needed for the hydrolysis of the amide bond. This nitro group promotes the displacement of the electrons towards the ring, which at the same time facilitates the nucleophilic attack of the catalytic Ser 105 decreasing the free energy barrier of the first step of the reaction. This behavior was confirmed by experimental enzyme kinetics studies, which was in very good agreement with the computational studies. Based on the exploration of the free energy landscape with the use of US methods, we determined that the hydrolysis of the $\mathcal{N}$-(4-nitrophenyl)-butyramide goes through a 4-step mechanism. The formation of the acyl-enzyme complex and the release of the leaving group occurred in the first and second steps, while the hydrolysis of the acyl chain and the regeneration of the active site took place in the third and last steps. The deep analysis of the QM/MM MD results revealed the role of the enzyme in generating an electric field that not only polarizes the substrates but stabilizes the charge distribution in the rate-determining TS. Interestingly, these results revealed that the mutations oriented to reduce the overall activation energy barrier should be focused on generating the proper electrostatic effects to facilitate the transfer of the proton from His224 to the nitrogen of the substrate in the second step of the reaction, where a dramatic charge rearrangement was detected.

To expand the knowledge of the molecular mechanisms that guide the promiscuity in CALB, the epoxidation of short alkenes was studied. We showed how the direct epoxidation of a short 4-carbon alkene, 2-butenal, with hydrogen peroxide takes place in two steps. The first step involves the attack of one oxygen of the hydrogen peroxide to one carbon of the double bond, together with the transfer of the proton from the oxygen to the catalytic His224. The second step involves the closing of the epoxide and 
the formation of a water molecule. Due to the presence of asymmetric carbon in the substrate, two enantiomeric products could be obtained. The analysis of the FES suggested that CALB should favor the enantiomeric behavior towards the (S)-enantiomer. However, from the population analysis of long MD simulations and the free energies of binding derived from alchemical FEP methods, a preferred (R)- enantioselectivity was detected. Thus, the lack of experimental enantioselectivity might be explained due to the compensation of both effects. Additionally, we showed that the production of epoxides from the alternative chemical synthesis using peroxy-acids is less efficient than the direct enzymatic approach, proposing CALB as a green environmentally friendly alternative to produce epoxides.

We pursued the opportunity to use all the knowledge on the promiscuous mechanism of CALB to propose a computational-assisted protocol towards the improvement of a related serine hydrolase. Consequently, we studied the promiscuous amidase activity of Bs2, trying to give insights about the similarities and differences between both enzymes. We focused on how two structurally different enzymes catalyze the same secondary amidase reaction on $\mathcal{N}$-(4-nitrophenyl)-butyramide. We confirmed that Bs2 hydrolyzed this substrate in a similar mechanistic manner as it is done by CALB. The experimental kinetic studies carried out by our collaborators confirmed, with very good agreement, the computationally predicted energy barriers derived from the QM/MM calculations. The fact that the second step between Bs2 and CALB was mechanistically different could be explained based on protein electrostatics. The forces exerted on the G-N of the substrate are slightly different in Bs2 when comparing with CALB. These forces promote an early breaking of the amide bond in Bs2 followed by the proton transfer, whereas in CALB the proton transfer occurs in the first place. Moreover, the overstabilization of the first intermediate in Bs2 with respect to GALB could be explained by the stabilization of the negative charge accumulated on the carbonyl oxygen of the substrate. To understand which are the significant structural differences in the vicinity of 
the active site, a map of structural determinants was built using a 3D-GNN. According to our results, some similarities can be found within the key residues of the active site pocket, and it can be derived that Bs2 would be a more robust scaffold for mutagenesis studies aiming to improve the amidase activity.

Considering the findings of these studies, our proposed protocol for the rational design of new enzymes is based on combining electrostatic features of, in this case, CALB and Bs2. From the deep comparative analysis of the vicinity of the active site of structurally aligned enzymes, we proposed a Bs2 variant, F398D, that should increase its catalytic activity. The structural comparison of TS structures related to the first step of the reaction identified a series of key residues with favorable and unfavorable impacts on the amidase reaction in terms of electrostatic properties. QM/MM studies based on improved and degraded Bs2 variants showed a strong linear correlation between the free energy barriers and the electrostatic potential generated by the enzyme on the catalytic His399. The F398D Bs2 variant predicted a significant decrease in the activation of free energy barrier but, though the estimation of the $\mathrm{pK}_{\mathrm{a}}$ of the F398D Bs2 variant predicted a deprotonated D398, a detailed analysis of long MD simulations revealed that the effect of the mutation will lead to a stable protonated $\mathrm{F} 398 \mathrm{D}-\mathrm{H}^{+}$variant. In this case, the electrostatic effect was lower, hence a less dramatic decrease in the free energy barrier was obtained. The experimental kinetic studies confirmed a protonated F398D- $\mathrm{H}^{+}$Bs2 variant with very good agreement in the catalytic rate constants.

For future perspectives, several strategies could be followed towards the improvement of the catalytic efficiency of Bs2 and other serine hydrolases based on this proposed computational-assisted protein engineering method. Since introducing a negatively charged residue in the protein is a risky strategy, a second-generation of Bs2 mutants could be based on the improvement of electrostatic features using different approaches such as 
Chapter 5. Conclusions and future perspectives

the use of artificial amino acids or the accumulation of several mutations with less impact but acting in a concerted manner. Alternatively, the F398D-H ${ }^{+}$Bs2 variant could also be subjected to further analysis focused on providing more information about how this Phe398 can remain deprotonated. The final conclusion of this work is the proposal of a computational protocol that could be used to guide a rational-based method that can be part of the molecular biology toolbox for the obtention of improved biocatalysts 


\section{Appendix. List of abbreviations}

AM1 Austin Model 1

Bs2 para-nitrobenzyl esterase from Bacillus subtilis

CALB Candida antarctica Lipase B

CEH Carboxylic Ester Hydrolases

CNN Convolutional Neural Network

DFT Density Functional Theory

DMSODimethyl Sulfoxide

EC Enzyme Commission

EVB Empirical Valence Bond

FEP Free Energy Perturbation

FES Free Energy Surface

FF $\quad$ Force Field

GAFF General Amber Force Field

GD Gradient Descent

HF Hartree-Fock

HPLC High-Performance Liquid Chromatography

I Intermediate

IAD Innovation-Amplification-Divergence

IRC Intrinsic Reaction Coordinate

KIE Kinetic Isotope Effects

MD Molecular Dynamics

MEP Minimum Energy Path

ML Machine Learning

MM Molecular Mechanics

MNDO Modified Neglect of Diatomic Overlap 
Appendix. List of abbreviations

MO Molecular Orbital

NDDO Neglect of Differential Diatomic Overlap

NMR Nuclear Magnetic Resonance

OPLS-AA Optimized potentials for liquid simulations

P Products

PDB Protein Data Bank

PES Potential Energy Surface

PMF Potential of Mean Force

QM/MM Quantum Mechanics / Molecular Mechanics

R Reactants

ReLU Rectified Linear Unit function

ResNet Residual Network

RQ Rotation Quaternion

SE Semiempirical

SH Serine Hydrolase

TLC Thin-Layer Chromatography

TS Transition State

TST Transition State Theory

US Umbrella Sampling

WHAM Weighted Histogram Analysis Method 


\section{Annex A}

Supporting Information of Article 1: "Exploring the Origin of Amidase Substrate Promiscuity in CALB by a Computational Approach" 



\title{
Supporting Information
}

\section{Exploring the Origin of Amidase Substrate Promiscuity in CALB by a Computational Approach.}

\author{
Miquel À. Galmés, ${ }^{1}$ Eduardo García-Junceda, ${ }^{2, *}$ Katarzyna Świderek, ${ }^{1, *}$ Vicent Moliner ${ }^{1, *}$ \\ 1 Departament de Química Física i Analítica, Universitat Jaume I, 12071 Castellón, Spain. \\ 2 Departamento de Química Orgánica Biológica, Instituto de Química Orgánica General, CSIC \\ Juan de la Cierva 3, 28006 Madrid, Spain.
}

* corresponding authors:

Eduardo García-Junceda, e-mail: eduardo.junceda@csic.es

Katarzyna Świderek,

e-mail: swiderek@uji.es

Vicent Moliner,

e-mail:moliner@uji.es

KEYWORDS: Computational chemistry, Enzyme catalysis, Enzyme promiscuity, QM/MM, Molecular Dynamics, Free Energy Surfaces 


\section{TABLE OF CONTENTS}

- Computational methods S3

- Computational details of the molecular models set up S3

- Computational details of the QM/MM simulations

S8

○ Potential Energy Surfaces $\quad$ S8

○ Spline corrections $\quad$ S9

- Free Energy Surfaces

S9

○ Kinetic Isotope Effects $\quad$ S9

- Experimental Methods $\quad$ S11

○ Synthesis of benzylbutyramide (1)

S11

○ Synthesis of N-(4-nitrophenyl)-butyramide (2) S11

- Enzymatic assays of the hydrolysis of $\mathbf{1} \quad \mathrm{S} 12$

○ Enzymatic assays of the hydrolysis of $2 \quad S 12$

- Results

S14

- NMR Spectra of the substrates $\quad$ S14

$\begin{array}{lr}\text { - Classic MD simulations } & \text { S17 }\end{array}$

- QM/MM localized structures

S18

- Determined Kinetic Isotope Effects

S22

○ Kinetics of hydrolysis of $\mathbf{2}$ 
- References 


\section{COMPUTATIONAL METHODS}

\section{Computational details of the molecular models set up}

Initial geometry of the protein was obtained from the PDB structure of Candida antarctica Lipase B (PDB ID: 1TCA). ${ }^{1}$ The protonation state of titratable residues were determined at $\mathrm{pH}$ equivalent to 7 using the semiempirical program PropKa ver. 3.0.3.2,3 The calculations determined that Asp134 must be protonated and all histidines as neutral with hydrogen atom added in $\mathrm{N} \delta$ position. Moreover, three disulphide bridges between residues Cys22 and Cys64, Cys216 and Cys258, and Cys293 and Cys311 were identified. Hydrogen atoms were added to the structure and the system was solvated by placing it in a $100 \times 80 \times 80 \AA^{3}$ pre-equilibrated box of water molecules. Any water with an oxygen atom lying in a radius of $2.8 \AA$ from a heavy atom of the protein was deleted. The geometry of the system was optimized using a series of optimization algorithms. ${ }^{4}$ A final equilibration was done to fully relax the entire system. In order to study the amidase activity of CALB, the system was set up by building the corresponding substrates, benzylbutyramide (1) and $\mathrm{N}$-(4-nitrophenyl)-butyramide (2), into the active site pocket of a pre-equilibrated CALB ${ }^{4}$ In order to equilibrate the total system a classical MD simulation was done to the Michaelis complex of each system. Substrates were parametrized with Antechamber ${ }^{5}$ at AM1 level. Parameters for 1 and 2 are showed in Tables S1 and S2, respectively. Finally 5 ns of classical MD using the NVT ensemble were run to equilibrate the system with AMBER force field, ${ }^{6}$ as implemented in NAMD software. ${ }^{7}$ In order to avoid the diffusion of the substrate, the distance between $\mathrm{OG}_{\text {Ser105 }}$ and $\mathrm{C}_{\text {Subs }}$ was constraint applying a force constant of $2000 \mathrm{~kJ} \cdot \mathrm{mol}^{-1} \cdot \AA^{-2}$. 
Table S1. Atom types, charges and parameters obtained for $\mathbf{1}$.

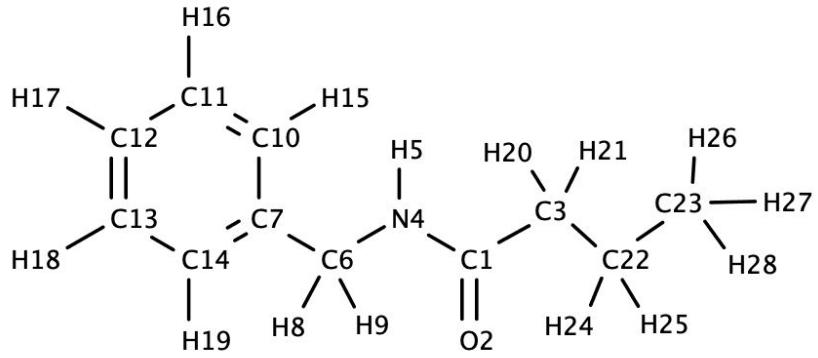

$\begin{array}{llllll}\text { Atom name } & \text { Atom type } & \text { Charge } & \text { Atom name } & \text { Atom type } & \text { Charge } \\ \mathrm{C} 23 & \mathrm{c} 3 & -0.0941 & \mathrm{C} 6 & \mathrm{c} 3 & 0.1233 \\ \mathrm{H} 26 & \mathrm{hc} & 0.0374 & \mathrm{H} 8 & \mathrm{~h} 1 & 0.0712 \\ \mathrm{H} 27 & \mathrm{hc} & 0.0374 & \mathrm{H} 9 & \mathrm{~h} 1 & 0.0712 \\ \mathrm{H} 28 & \mathrm{hc} & 0.0374 & \mathrm{C} 7 & \mathrm{ca} & -0.1213 \\ \mathrm{C} 22 & \mathrm{c} 3 & -0.0784 & \mathrm{C} 10 & \mathrm{ca} & -0.1175 \\ \mathrm{H} 24 & \mathrm{hc} & 0.0447 & \mathrm{H} 15 & \text { ha } & 0.1355 \\ \mathrm{H} 25 & \mathrm{hc} & 0.0447 & \mathrm{C} 11 & \text { ca } & -0.1315 \\ \mathrm{C} 3 & \mathrm{c} 3 & -0.1564 & \mathrm{H} 16 & \text { ha } & 0.1330 \\ \mathrm{H} 20 & \mathrm{hc} & 0.0727 & \mathrm{C} 12 & \text { ca } & -0.1240 \\ \mathrm{H} 21 & \mathrm{hc} & 0.0727 & \mathrm{H} 17 & \text { ha } & 0.1320 \\ \mathrm{C} 1 & \mathrm{c} & 0.6511 & \mathrm{C} 13 & \text { ca } & -0.1315 \\ \mathrm{O} 2 & \mathrm{o} & -0.6061 & \mathrm{H} 18 & \text { ha } & 0.1330 \\ \mathrm{~N} 4 & \mathrm{n} & -0.5569 & \mathrm{C} 14 & \text { ca } & -0.1175 \\ \mathrm{H} 5 & \mathrm{hn} & 0.3015 & \mathrm{H} 19 & \text { ha } & 0.1355\end{array}$

Parameters:

$\begin{array}{lll}\text { MASS } & & \\ \mathrm{c} 3 & 12.01 & 0.878 \\ \mathrm{hc} & 1.008 & 0.135 \\ \mathrm{c} & 12.01 & 0.616 \\ \mathrm{o} & 16.00 & 0.434 \\ \mathrm{n} & 14.01 & 0.530 \\ \mathrm{hn} & 1.008 & 0.161 \\ \mathrm{~h} 1 & 1.008 & 0.135 \\ \mathrm{ca} & 12.01 & 0.360 \\ \mathrm{ha} & 1.008 & 0.135\end{array}$

\section{BOND}

c3-hc

c3-c3

c3-c

$\mathrm{c}-\mathrm{o}$

$\mathrm{c}-\mathrm{n}$

$\mathrm{n}-\mathrm{hn}$

$\mathrm{n}-\mathrm{c} 3$

c3-h1

c3-ca

ca-ca

ca-ha

$\begin{array}{ll}330.60 & 1.097 \\ 300.90 & 1.538 \\ 313.00 & 1.524 \\ 637.70 & 1.218 \\ 427.60 & 1.379 \\ 403.20 & 1.013 \\ 328.70 & 1.462 \\ 330.60 & 1.097 \\ 321.00 & 1.516 \\ 461.10 & 1.398 \\ 345.80 & 1.086\end{array}$




$\begin{array}{lll}\text { ANGLE } & & \\ \text { c3-c3-hc } & 46.340 & 109.800 \\ \text { c3-c3-c3 } & 62.860 & 111.510 \\ \text { hc-c3-hc } & 39.400 & 107.580 \\ \text { c3-c3-c } & 63.270 & 111.040 \\ \text { c3-c-o } & 67.400 & 123.200 \\ \text { c3-c -n } & 66.790 & 115.180 \\ \text { hc-c3-c } & 46.930 & 108.770 \\ \text { c -n -hn } & 48.330 & 117.550 \\ \text { c -n -c3 } & 63.390 & 120.690 \\ \text { o-c -n } & 74.220 & 123.050 \\ \mathrm{n}-\mathrm{c} 3-\mathrm{h} 1 & 49.840 & 108.880 \\ \mathrm{n}-\mathrm{c} 3-\mathrm{ca} & 66.210 & 112.380 \\ \mathrm{hn}-\mathrm{n}-\mathrm{c} 3 & 45.800 & 117.680 \\ \mathrm{c} 3-\mathrm{ca}-\mathrm{ca} & 63.530 & 120.770 \\ \mathrm{~h} 1-\mathrm{c} 3-\mathrm{h} 1 & 39.240 & 108.460 \\ \mathrm{~h} 1-\mathrm{c} 3-\mathrm{ca} & 46.990 & 109.560 \\ \text { ca-ca-ha } & 48.180 & 119.880 \\ \text { ca-ca-ca } & 66.620 & 120.020\end{array}$

\section{DIHEDRALS}

$\begin{array}{lrrrr}\text { c3-c3-c3-hc } & 1 & 0.160 & 0.000 & 3.000 \\ \text { c3-c3-c3-c } & 1 & 0.156 & 0.000 & 3.000 \\ \text { hc-c3-c3-hc } & 1 & 0.150 & 0.000 & 3.000 \\ \text { c3-c3-c -o } & 1 & 0.000 & 180.000 & 2.000 \\ \text { c3-c3-c -n } & 1 & 0.100 & 0.000 & -4.000 \\ \text { c3-c3-c-n } & 1 & 0.070 & 0.000 & 2.000 \\ \text { hc-c3-c3-c } & 1 & 0.156 & 0.000 & 3.000 \\ \text { c3-c -n -hn } & 1 & 2.500 & 180.000 & 2.000 \\ \text { c3-c -n -c3 } & 1 & 0.000 & 0.000 & -2.000 \\ \text { c3-c -n -c3 } & 1 & 1.500 & 180.000 & 1.000 \\ \text { hc-c3-c -o } & 1 & 0.800 & 0.000 & -1.000 \\ \text { hc-c3-c -o } & 1 & 0.000 & 0.000 & -2.000 \\ \text { hc-c3-c-o } & 1 & 0.080 & 180.000 & 3.000 \\ \text { hc-c3-c -n } & 1 & 0.000 & 180.000 & 2.000 \\ \text { c -n -c3-h1 } & 1 & 0.000 & 0.000 & 2.000 \\ \text { c -n -c3-ca } & 1 & 0.000 & 0.000 & 2.000 \\ \text { o -c -n -hn } & 1 & 2.500 & 180.000 & -2.000 \\ \text { o -c -n -hn } & 1 & 2.000 & 0.000 & 1.000 \\ \text { o -c -n -c3 } & 1 & 2.500 & 180.000 & 2.000 \\ \text { n-c3-ca-ca } & 1 & 0.000 & 0.000 & 2.000 \\ \text { hn-n -c3-h1 } & 1 & 0.000 & 0.000 & 2.000 \\ \text { hn-n -c3-ca } & 1 & 0.000 & 0.000 & 2.000 \\ \text { c3-ca-ca-ha } & 1 & 3.625 & 180.000 & 2.000 \\ \text { c3-ca-ca-ca } & 1 & 3.625 & 180.000 & 2.000 \\ \text { h1-c3-ca-ca } & 1 & 0.000 & 0.000 & 2.000 \\ \text { ca-ca-ca-ha } & 1 & 3.625 & 180.000 & 2.000 \\ \text { ca-ca-ca-ca } & 1 & 3.625 & 180.000 & 2.000 \\ \text { ha-ca-ca-ha } & 1 & 3.625 & 180.000 & 2.000\end{array}$

\section{IMPROPER}

\section{c3-n -c -o}

c -c3-n-hn

c3-ca-ca-ca$$
10.5
$$$$
1.1
$$$$
1.1
$$$$
180.0 \quad 2.0
$$

ca-ca-ca-ha

$\begin{array}{lll}1.1 & 180.0 & 2.0\end{array}$

$\begin{array}{lll}\text { NONBON } & & \\ \mathrm{c} 3 & 1.9080 & 0.1094 \\ \mathrm{hc} & 1.4870 & 0.0157 \\ \mathrm{c} & 1.9080 & 0.0860 \\ \mathrm{o} & 1.6612 & 0.2100 \\ \mathrm{n} & 1.8240 & 0.1700 \\ \mathrm{hn} & 0.6000 & 0.0157 \\ \mathrm{~h} 1 & 1.3870 & 0.0157 \\ \mathrm{ca} & 1.9080 & 0.0860 \\ \mathrm{ha} & 1.4590 & 0.0150\end{array}$


Table S2. Atom types, charges and parameters obtained for 2.

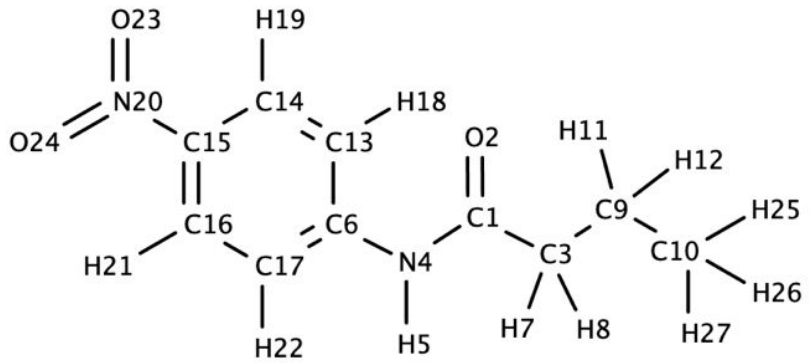

$\begin{array}{llllll}\text { Atom name } & \text { Atom type } & \text { Charge } & \text { Atom name } & \text { Atom type } & \text { Charge } \\ \text { C10 } & \text { c3 } & -0.0981 & \text { C6 } & \text { ca } & 0.1226 \\ \text { H25 } & \text { hc } & 0.0370 & \text { C13 } & \text { ca } & -0.1920 \\ \text { H26 } & \text { hc } & 0.0370 & \text { H18 } & \text { ha } & 0.1650 \\ \text { H27 } & \text { hc } & 0.0370 & \text { C14 } & \text { ca } & -0.0360 \\ \text { C9 } & \text { c3 } & -0.0864 & \text { H19 } & \text { ha } & 0.1715 \\ \text { H11 } & \text { hc } & 0.0552 & \mathrm{C} 15 & \text { ca } & -0.2172 \\ \text { H12 } & \text { hc } & 0.0552 & \text { N20 } & \text { no } & 0.3192 \\ \text { C3 } & \text { c3 } & -0.1584 & \text { O23 } & \text { o } & -0.2145 \\ \text { H7 } & \text { hc } & 0.0812 & \text { O24 } & \text { o } & -0.2145 \\ \text { H8 } & \text { hc } & 0.0812 & \mathrm{C} 16 & \text { ca } & -0.0360 \\ \text { C1 } & \text { c } & 0.6681 & \mathrm{H} 21 & \text { ha } & 0.1715 \\ \text { O2 } & \text { o } & -0.5731 & \mathrm{C} 17 & \text { ca } & -0.1920 \\ \text { N4 } & \mathrm{n} & -0.4731 & \mathrm{H} 22 & \text { ha } & 0.1650 \\ \text { H5 } & \text { hn } & 0.3235 & & & \end{array}$

Parameters:

$\begin{array}{lll}\text { MASS } & & \\ \mathrm{c} 3 & 12.01 & 0.878 \\ \mathrm{hc} & 1.008 & 0.135 \\ \mathrm{c} & 12.01 & 0.616 \\ \mathrm{o} & 16.00 & 0.434 \\ \mathrm{n} & 14.01 & 0.530 \\ \mathrm{hn} & 1.008 & 0.161 \\ \mathrm{ca} & 12.01 & 0.360 \\ \mathrm{ha} & 1.008 & 0.135 \\ \text { no } & 14.01 & 0.530\end{array}$

$\begin{array}{lll}\text { BOND } & & \\ \text { c3-hc } & 330.60 & 1.097 \\ \text { c3-c3 } & 300.90 & 1.538 \\ \text { c3-c } & 313.00 & 1.524 \\ \text { c -o } & 637.70 & 1.218 \\ \text { c -n } & 427.60 & 1.379 \\ \text { n -hn } & 403.20 & 1.013 \\ \text { n -ca } & 384.20 & 1.412 \\ \text { ca-ca } & 461.10 & 1.398 \\ \text { ca-ha } & 345.80 & 1.086 \\ \text { ca-no } & 321.70 & 1.469 \\ \text { no-o } & 741.80 & 1.226\end{array}$




$\begin{array}{lll}\text { ANGLE } & & \\ \text { c3-c3-hc } & 46.340 & 109.800 \\ \text { c3-c3-c3 } & 62.860 & 111.510 \\ \text { hc-c3-hc } & 39.400 & 107.580 \\ \text { c3-c3-c } & 63.270 & 111.040 \\ \text { c3-c -o } & 67.400 & 123.200 \\ \text { c3-c -n } & 66.790 & 115.180 \\ \text { hc-c3-c } & 46.930 & 108.770 \\ \text { c -n -hn } & 48.330 & 117.550 \\ \text { c -n -ca } & 63.820 & 123.710 \\ \text { o -c -n } & 74.220 & 123.050 \\ \text { n -ca-ca } & 67.870 & 120.190 \\ \text { hn-n -ca } & 47.630 & 116.000 \\ \text { ca-ca-ha } & 48.180 & 119.880 \\ \text { ca-ca-ca } & 66.620 & 120.020 \\ \text { ca-ca-no } & 66.770 & 119.010 \\ \text { ca-no-o } & 68.700 & 117.760 \\ \text { o -no-o } & 76.730 & 125.080\end{array}$

\section{DIHEDRALS}

$\begin{array}{llrrr}\text { c3-c3-c3-hc } & 1 & 0.160 & 0.000 & 3.000 \\ \text { c3-c3-c3-c } & 1 & 0.156 & 0.000 & 3.000 \\ \text { hc-c3-c3-hc } & 1 & 0.150 & 0.000 & 3.000 \\ \text { c3-c3-c-o } & 1 & 0.000 & 180.000 & 2.000 \\ \text { c3-c3-c -n } & 1 & 0.100 & 0.000 & -4.000 \\ \text { c3-c3-c -n } & 1 & 0.070 & 0.000 & 2.000 \\ \text { hc-c3-c3-c } & 1 & 0.156 & 0.000 & 3.000 \\ \text { c3-c -n -hn } & 1 & 2.500 & 180.000 & 2.000 \\ \text { c3-c -n -ca } & 1 & 2.500 & 180.000 & 2.000 \\ \text { hc-c3-c -o } & 1 & 0.800 & 0.000 & -1.000 \\ \text { hc-c3-c-o } & 1 & 0.000 & 0.000 & -2.000 \\ \text { hc-c3-c -o } & 1 & 0.080 & 180.000 & 3.000 \\ \text { hc-c3-c -n } & 1 & 0.000 & 180.000 & 2.000 \\ \text { c -n -ca-ca } & 1 & 0.450 & 180.000 & 2.000 \\ \text { o-c -n -hn } & 1 & 2.500 & 180.000 & -2.000 \\ \text { o-c -n -hn } & 1 & 2.000 & 0.000 & 1.000 \\ \text { o-c -n -ca } & 1 & 2.500 & 180.000 & 2.000 \\ \text { n-ca-ca-ha } & 1 & 3.625 & 180.000 & 2.000 \\ \text { n -ca-ca-ca } & 1 & 3.625 & 180.000 & 2.000 \\ \text { hn-n -ca-ca } & 1 & 0.450 & 180.000 & 2.000 \\ \text { ca-ca-ca-ha } & 1 & 3.625 & 180.000 & 2.000 \\ \text { ca-ca-ca-ca } & 1 & 3.625 & 180.000 & 2.000 \\ \text { ca-ca-ca-no } & 1 & 3.625 & 180.000 & 2.000 \\ \text { ha-ca-ca-ha } & 1 & 3.625 & 180.000 & 2.000 \\ \text { ca-ca-no-o } & 1 & 0.600 & 180.000 & 2.000 \\ \text { ha-ca-ca-no } & 1 & 3.625 & 180.000 & 2.000\end{array}$

\section{IMPROPER}

$\begin{array}{lllll}\text { c3-n -c -o } & 10.5 & 180.0 & 2.0 & \text { General improper torsional angle (2 general atom types) } \\ \text { c -ca-n -hn } & 1.1 & 180.0 & 2.0 & \text { General improper torsional angle (2 general atom types) } \\ \text { ca-ca-ca-n } & 1.1 & 180.0 & 2.0 & \text { Using default value } \\ \text { ca-ca-ca-ha } & 1.1 & 180.0 & 2.0 & \text { General improper torsional angle (2 general atom types) } \\ \text { ca-ca-ca-no } & 1.1 & 180.0 & 2.0 & \text { Using default value } \\ \text { ca-o -no-o } & 1.1 & 180.0 & 2.0 & \text { Using default value }\end{array}$

\section{NONBON}

$\begin{array}{lcc}\text { c3 } & 1.9080 & 0.1094 \\ \text { hc } & 1.4870 & 0.0157 \\ \mathrm{c} & 1.9080 & 0.0860 \\ \mathrm{o} & 1.6612 & 0.2100 \\ \mathrm{n} & 1.8240 & 0.1700 \\ \mathrm{hn} & 0.600 & 0.0157 \\ \mathrm{ca} & 1.9080 & 0.0860 \\ \text { ha } & 1.4590 & 0.0150 \\ \text { no } & 1.8240 & 0.1700\end{array}$




\section{Computational details of the QM/MM simulations}

In this work, an additive hybrid QM/MM scheme was employed for the construction of the total Hamiltonian, $\hat{\mathrm{H}}_{\mathrm{eff}}$, where the total energy is obtained from the sum of each contribution to the energy.

$\hat{\mathrm{H}}_{\mathrm{eff}}=\hat{\mathrm{H}}_{\mathrm{QM}}+\hat{\mathrm{H}}_{\mathrm{QM} / \mathrm{MM}}^{\mathrm{elec}}+\hat{\mathrm{H}}_{\mathrm{QM} / \mathrm{MM}}^{\mathrm{VdW}}+\hat{\mathrm{H}}_{\mathrm{MM}}$

Here, $\hat{\mathrm{H}}_{\mathrm{QM}}$ describes the atoms in the QM part, $\hat{\mathrm{H}}_{\mathrm{QM} / \mathrm{MM}}$ defines the interaction between the $\mathrm{QM}$ and $\mathrm{MM}$ region and $\hat{\mathrm{H}}_{\mathrm{MM}}$ describes the rest of the MM part.

\section{Potential Energy Surfaces}

Exploration of the Potential Energy Surfaces (PES) was carried out by choosing the appropriate combination of internal coordinates $\left(\xi_{i}\right)$ in every single step of the reaction. A harmonic constraint was used to maintain the proper interatomic distances along the reaction coordinate, and a series of conjugate gradient optimizations and L-BFGS-B optimization algorithms were applied to obtain the final potential energy of the minimized constrained geometry. The QM sub-set of atoms were described by the Austin Model $1(\mathrm{AM} 1)^{8}$ semiempirical Hamiltonian.

After the exploration of stationary points on the PES, the structures corresponding to reactants and products states (RS and PS, respectively), intermediates (Is) and transition state (TSs) were localized applying the Baker's algorithm. ${ }^{9}$ Minimum energy path was traced down to reactants and products following the Intrinsic Reaction Coordinate ${ }^{10}$ (IRC) method from every localized TS structure. 


\section{Spline corrections}

A correction term is interpolated to any value along the reaction coordinates in the FES. A continuous energy function is used to obtain the corrected PMFs:

$E=E_{L L / M M}+S\left[\Delta E_{L L}^{H L}\left(\xi_{1}, \xi_{2}\right)\right]$

where $S$ is the two-dimensional spline function and $\Delta E_{L L}^{H L}$ is the difference between the energies obtained at low-level (LL) and high-level (HL) of theory of the QM part. The AM1 semiempirical Hamiltonian was used as LL method, while a density functional theory (DFT)-based method was selected for the HL energy calculation. In particular, HL energy calculations were performed by means of the hybrid M06-2X ${ }^{11}$ functional using the standard $6-31+\mathrm{G}(\mathrm{d}, \mathrm{p})$ basis set. These calculations were carried out using the Gaussian09 program. ${ }^{12}$

\section{Free Energy Surfaces}

FESs were obtained, in terms of $2 \mathrm{D}-\mathrm{PMF},{ }^{13}$ for every step of the reaction using the Umbrella Sampling approach ${ }^{13,14}$ combined with the Weighted Histogram Analysis Method (WHAM). ${ }^{15}$ Series of MD simulations were performed adding a constraint along the selected reaction coordinates with an umbrella force constant of $2500 \mathrm{~kJ} \cdot \mathrm{mol}^{-1} \cdot \AA^{-2}$. In every window QM/MM MD simulations were performed with a total of 5 ps of equilibration and 20 ps of production at $303 \mathrm{~K}$ using the Langevin-Verlet algorithm ${ }^{16}$ with a time step of $1 \mathrm{fs}$. Structures obtained in previously computed PESs were used as starting points for the MD simulations in every window.

\section{Kinetic Isotope Effects}

Averaged KIEs were calculated from isotopic substitution of the key atoms from localized RS and TS structures. From the definition of the free energy of a state and using the Transition State 
Theory, the ratio between the rate constants corresponding to the heavy and the light atoms can be computed by means of equation S3.

$K I E=\frac{\left(\frac{Q_{T S}}{Q_{R S}}\right)_{L}}{\left(\frac{Q_{T S}}{Q_{R S}}\right)_{H}} e^{(-1 /(R T))\left(\Delta Z P E_{L}-\Delta Z P E_{H}\right)}$

Here, $Q$ refers to the total partition function which was computed as the product of the translational, rotational and vibrational partition functions of isotopologs in RS and TS structures, and $\triangle Z P E$ refers to difference of the zero point energy between RS and TS in the light (L) and heavy (H) isotopologs. ${ }^{17,18}$ KIEs were computed at M06-2X/MM and AM1/MM level of theory. In the case of AM1/MM KIEs, ten structures representing each of the TS and the RS were extracted from a MD simulation constraining the key distances to maintain their characteristic geometries. These structures were localized to have 10 representative structures of each state. Those were used to compute the averaged KIEs. The Hessian was computed for the QM subset of atoms. For M062X/MM KIEs, one RS structure and one TS structure for each step of the reaction were localized at this high level of theory. 


\section{EXPERIMENTAL METHODS}

\section{Synthesis of benzylbutyramide (1)}

The synthesis of the substrate benzylbutyramide was carried out following an enzymatic approach. Commercial immobilized CALB (Novozyme 435) was used to synthetize 1, from benzylamine and butyric acid. Equimolar solution of $50 \mathrm{mM}$ of butyric acid and benzylamine in 2-methyl-2butanol, was incubated during 120 hours in sealed vessels with argon atmosphere and molecular sieves to minimize the available soluble water that is formed in the reaction. The reaction was followed by Thin-Layer Chromatography (TLC), with a mobile phase of hexane:ethyl acetate (2:3), until complete conversion. The product was purified by Silica column with hexane:ethyl acetate (1:5) as mobile phase. Solvent was finally evaporated in a rotatory evaporator, obtaining pure product, which was characterized by mass spectrometry $(\mathrm{m} / \mathrm{z}=178.2)$ and NMR (Figure $\mathrm{S} 1)$. The overall yield obtained was of $48 \%$.

\section{Synthesis of N-(4-nitrophenyl)-butyramide (2)}

Butyryl chloride dissolved in $5 \mathrm{~mL}$ anhydrous dichloromethane (4.32 mmols) was added to $10 \mathrm{~mL}$ of a solution of 4-nitroaniline $(3.60 \mathrm{mmols})$ with pyridine $(7.20 \mathrm{mmols})$ in anhydrous dichloromethane to act as a base to catalyze the reaction. The molar ration of butyryl chloride:4nitroaniline:pyridine was set to 1.2:1:2. Reaction was performed in sealed vessels in argon atmosphere at room temperature over-night. Once reaction was completed, further washes with $10 \% \mathrm{HCl}$ were performed. 2 was purified by Silica column with hexane:ethyl acetate $(4: 1)$ as mobile phase. Solvent was evaporated in a rotatory evaporator obtaining yellow crystals. The product was characterized by NMR (Figure S2) and the overall yield was $74 \%$. 


\section{Enzymatic assays of the hydrolysis of 1.}

In order to evaluate the amidase reaction of CALB, experimental kinetics parameters of hydrolysis of $\mathbf{1}$ were obtained. Commercial free form of wild type CALB was purchased from Sigma-Aldrich. Kinetics assays were performed in $20 \mathrm{mM}$ phosphate buffer at $\mathrm{pH} 7$, with $10 \%$ of organic solvent, acetonitrile in this case. The addition of organic solvent is due to the poor solubility of $\mathbf{1}$ in aqueous solution. Different organic solvents were discarded due to interferences with the signal during HPLC acquisitions. Real concentration of enzyme in the commercial powder was determined by Bradford. Final concentration of enzyme in the assay was adjusted to $105 \mu \mathrm{g} \cdot \mathrm{mL}^{-1}$ and concentrations of 1 were set to $0 \mathrm{mM}, 0.2 \mathrm{mM}, 0.5 \mathrm{mM}, 1 \mathrm{mM}, 5 \mathrm{mM}$ in a final volume of 500 $\mu \mathrm{L}$. Reactions were done at $37^{\circ} \mathrm{C}$ and $100 \mathrm{rpm}$. Aliquots of $50 \mu \mathrm{L}$ were taken at $0 \mathrm{~h}, 3 \mathrm{~h}, 5 \mathrm{~h}, 7 \mathrm{~h}$, $24 \mathrm{~h}$ and $48 \mathrm{~h}$, and were frozen at $-20^{\circ} \mathrm{C}$ to stop the progression of the reaction.

Formation of the benzylamine was monitored by HPLC (JASCO UK Limited). A diluted sample of each aliquot was injected into a C18 column and a mixture of water:acetonitrile 50:50 with 0.5 $\%$ of trifluoroacetic acid was used as a mobile phase with a $1 \mathrm{~mL} \cdot \mathrm{min}^{-1}$ flow. Elution of products (benzylamine) was followed by absorbance at $206 \mathrm{~nm}$ (retention time of benzylamine $=3.1 \mathrm{~min}$; retention time of benzylbutyramide $=4.5 \mathrm{~min})$.

\section{Enzymatic assays of the hydrolysis of 2}

To assay the amidase activity on $\mathbf{2}$ commercial wild type CALB was used (Sigma-Aldrich). The poor solubility of the substrate makes impossible to perform reactions in pure aqueous solutions so addition of organic solvent is required. Reactions were done in $20 \mathrm{mM}$ phosphate buffer at $\mathrm{pH}$ 7, with $10 \%$ of DMSO. Concentrations of 2 were set to $0 \mathrm{mM}, 0.5 \mathrm{mM}, 1.0 \mathrm{mM}, 1.5 \mathrm{mM}, 2.0$ $\mathrm{mM}, 2.5 \mathrm{mM}$ and $3 \mathrm{mM}$ and final concentration of protein was set to $35 \mu \mathrm{g} \cdot \mathrm{mL}^{-1}$. Reactions were 
carried out in a final volume of $150 \mu \mathrm{L}$ in microtiter plates at $37^{\circ} \mathrm{C}$ and $100 \mathrm{rpm}$. Reactions were done in triplicates. The progression of the reaction was monitored during 24 hours by the increment of absorbance at $405 \mathrm{~nm}$ using a spectrophotometer (Spectra max Plus 384 from Molecular Devices). The use of this spectrophotometric technique permits the use of DMSO instead of acetonitrile in the case of $\mathbf{2}$. The use of spectrophotometric approaches is not possible in the case of $\mathbf{1}$, because of the very high similar peaks of absorbance of substrates and products (maximum absorbance peak of 1, substrate: $250 \mathrm{~nm}$; and benzylamine, product: $245 \mathrm{~nm}$ ). 


\section{RESULTS}

\section{NMR Spectra of the substrates}

${ }^{1} \mathrm{H}$ NMR spectra were done to characterize the substrates. Spectra for $\mathbf{1}$ is depicted in Figure S1 and for $\mathbf{2}$ in Figure S2.

Benzylbutyramide: ${ }^{1} \mathrm{H}$ NMR (300 MHz, Chloroform- $d$ ) $\delta 7.47-7.17(\mathrm{~m}, 3 \mathrm{H}), 5.76-5.67$ (m, $1 \mathrm{H}), 4.44(\mathrm{~d}, J=5.7 \mathrm{~Hz}, 1 \mathrm{H}), 2.32-2.00(\mathrm{~m}, 6 \mathrm{H}), 1.85-1.54(\mathrm{~m}, 2 \mathrm{H}), 1.14-0.82(\mathrm{~m}, 2 \mathrm{H})$.

$N$-(4-nitrophenyl)-butyramide: ${ }^{1} \mathrm{H}$ NMR (300 MHz, Chloroform-d) $\delta 8.27$ - $8.16(\mathrm{~m}, 2 \mathrm{H}), 7.76$ $7.65(\mathrm{~m}, 2 \mathrm{H}), 7.37(\mathrm{~s}, 2 \mathrm{H}), 2.40(\mathrm{dd}, J=7.8,7.1 \mathrm{~Hz}, 2 \mathrm{H}), 1.88-1.69(\mathrm{~m}, 2 \mathrm{H}), 1.03(\mathrm{t}, J=7.4 \mathrm{~Hz}$, $3 \mathrm{H})$. 


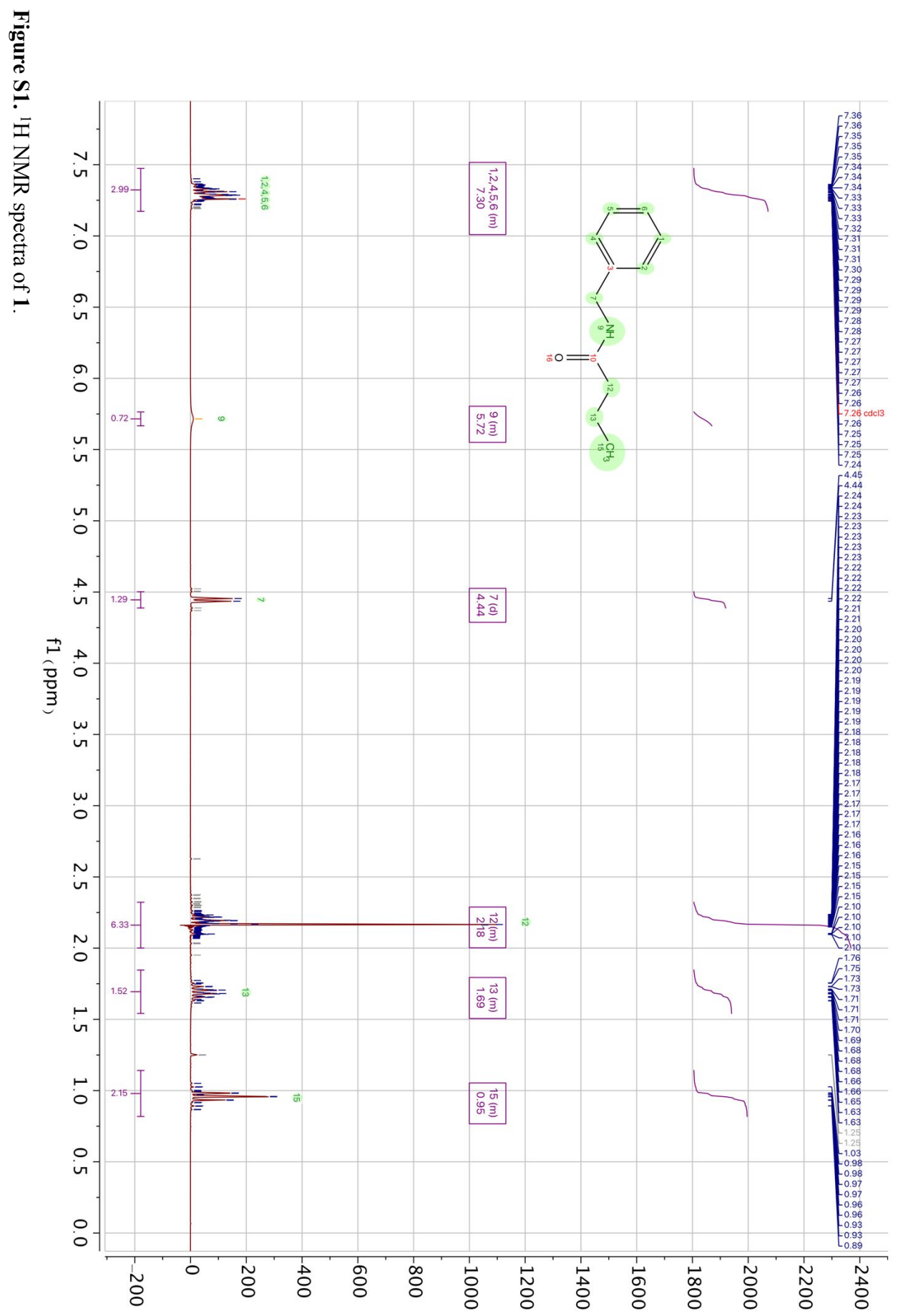

ลุ 


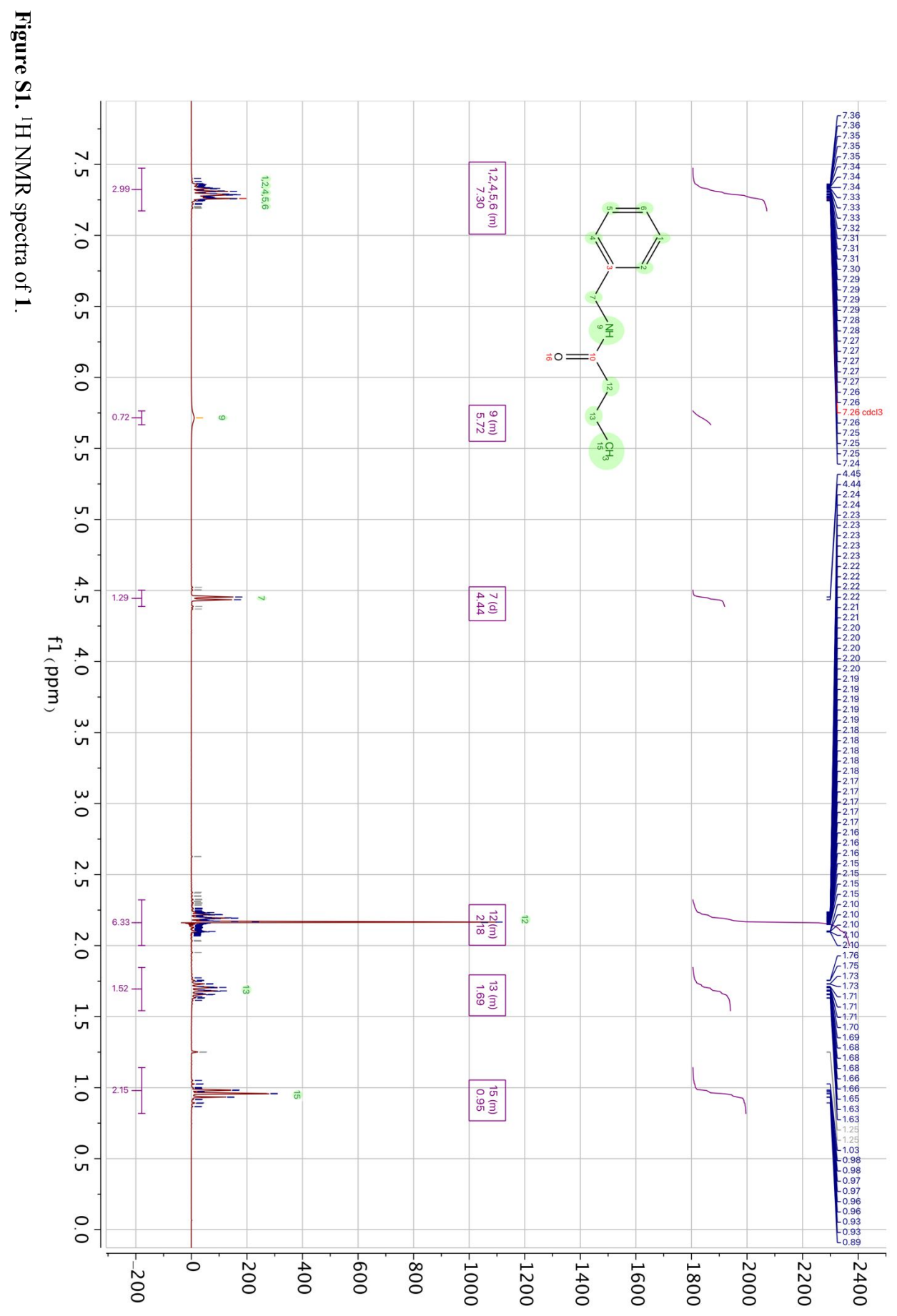

ڤ્ 


\section{Classic MD simulations}

MD simulations were done in order to equilibrate the system containing $\mathbf{1}$ and $\mathbf{2}$ as substrates. The time dependent evolution of the RMSD for both systems is depicted in Figures S3 and S4.

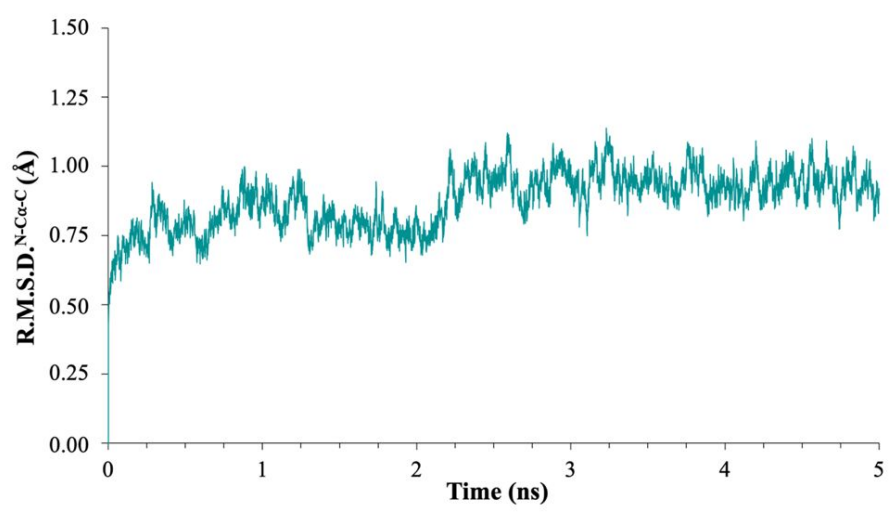

Figure S3. Time-dependent evolution of the RMSD of the atoms belonging to the protein backbone during the $5 \mathrm{~ns}$ MD trajectory of the 1-CALB system.

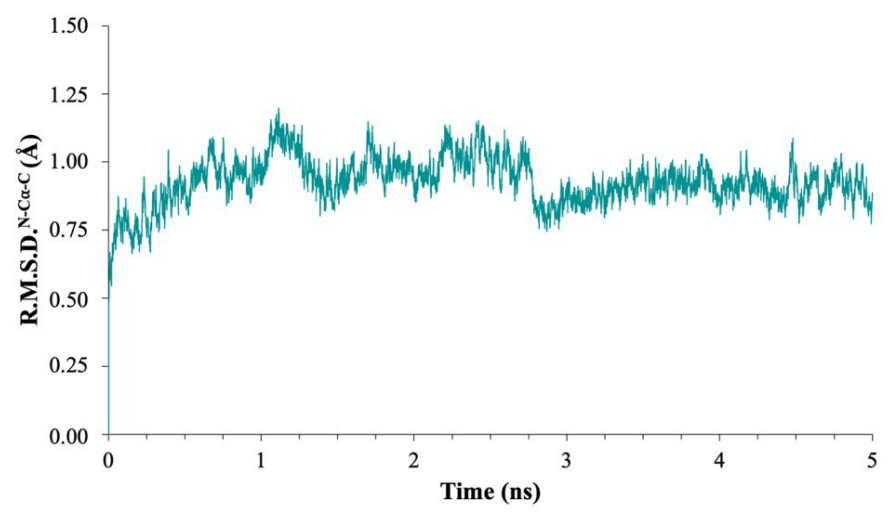

Figure S4. Time-dependent evolution of the RMSD of the atoms belonging to the protein backbone during the $5 \mathrm{~ns}$ MD trajectory of the 2-CALB system. 


\section{QM/MM localized structures}

Transition state structures localized at M06-2X level of theory are depicted in Figure S5. Averaged key distances of the structures localized at AM1/MM and M06-2X/MM are showed in Table S3 and Table S4 respectively. Averaged charges of key atoms were calculated from a QM/MM MD simulation at M06-2X/MM level and are shown in Table S5. Charges and polarization of the atoms belonging to the amide group of $\mathbf{2}$ produced by the residues belonging to the active site can be found in table S6.
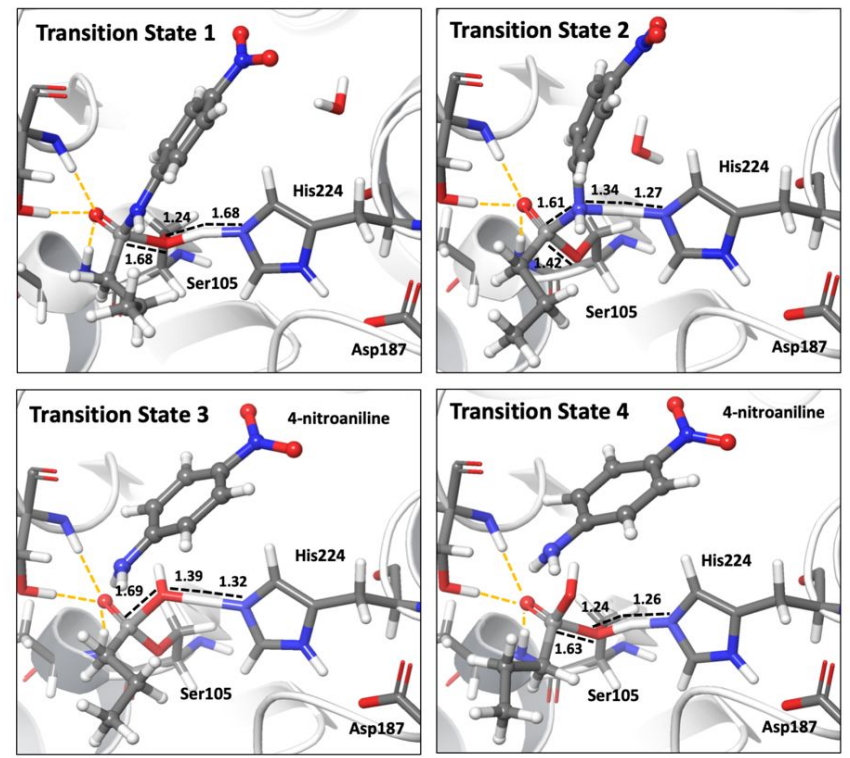

Figure S5 M06-2X/MM TS structures appearing along the hydrolysis of 2 catalyzed by CALB. Key protein residues are showed in liquorice, and substrate and products are showed in ball and sticks representation. Distances are in $\AA$. 
Table S3 Averaged key distances obtained from the structures located at AM1/MM level for the hydrolysis of $\mathbf{2}$ and used to compute averaged KIEs listed in Table S7.

\begin{tabular}{|c|c|c|c|c|c|}
\hline & RS & TS1 & TS2 & TS3 & TS4 \\
\hline $\mathrm{OG}_{\text {Ser } 105}-\mathrm{Cl}_{\text {subs }}$ & $2.51 \pm 0.06$ & $1.605 \pm 0.012$ & $1.4424 \pm 0.0019$ & $1.4199 \pm 0.0014$ & $1.538 \pm 0.006$ \\
\hline $\mathrm{OG}_{\text {Ser } 105}-\mathrm{HG}_{\text {Ser } 105}$ & $0.9631 \pm 0.0005$ & $1.299 \pm 0.016$ & $2.28 \pm 0.05$ & & \\
\hline $\mathrm{NE} 2_{\mathrm{His2} 24}-\mathrm{HG}_{\mathrm{Ser} 105}$ & $2.37 \pm 0.07$ & $1.245 \pm 0015$ & $1.253 \pm 0.006$ & - & - \\
\hline $\mathrm{C} 1_{\text {subs }}-\mathrm{N} 4_{\text {subs }}$ & $1.3823 \pm 0.0023$ & $1.460 \pm 0.005$ & $1.556 \pm 0.005$ & - & - \\
\hline $\mathrm{HG}_{\text {Ser } 105}-\mathrm{N} 4_{\text {subs }}$ & $2.78 \pm 0.05$ & $2.68 \pm 0.03$ & $1.393 \pm 0.013$ & - & - \\
\hline $\mathrm{O}_{\text {wat }}-\mathrm{C} 1_{\text {subs }}$ & - & - & - & $1.51 \pm 0.24$ & $1.4 \pm 0.3$ \\
\hline $\mathrm{O}_{\text {wat }}-\mathrm{H} 1_{\text {wat }}$ & - & - & - & $1.273 \pm 0.011$ & $2.26 \pm 0.03$ \\
\hline $\mathrm{H} 1_{\text {wat }}-\mathrm{NE} 2_{\mathrm{His} 224}$ & - & - & - & $1.256 \pm 0.012$ & $1.252 \pm 0.009$ \\
\hline $\mathrm{H} 1_{\text {wat }}-\mathrm{OG}_{\text {Ser } 105}$ & & & & $2.289 \pm 0.024$ & $1.292 \pm 0.007$ \\
\hline $\mathrm{Cl}_{\text {Ser } 105}-\mathrm{O} 2_{\text {subs }}$ & $1.2589 \pm 0.0020$ & $1.2928 \pm 0.0011$ & $1.3053 \pm 0.0011$ & $1.2980 \pm 0.0014$ & $1.2928 \pm 0.0009$ \\
\hline $\mathrm{O} 2_{\text {subs }}-\mathrm{H}_{\text {Thr } 40}$ & $1.94 \pm 0.08$ & $1.79 \pm 0.05$ & $1.78 \pm 0.06$ & $1.72 \pm 0.03$ & $1.82 \pm 0.04$ \\
\hline $\mathrm{O} 2_{\text {subs }}-\mathrm{HO}_{\mathrm{Thr} 40}$ & $1.92 \pm 0.04$ & $1.90 \pm 0.03$ & $1.86 \pm 0.04$ & $1.843 \pm 0.018$ & $1.82 \pm 0.04$ \\
\hline $\mathrm{O} 2_{\text {subs }}-\mathrm{H}_{\mathrm{Gln} 106}$ & $2.17 \pm 0.18$ & $1.90 \pm 0.04$ & $2.03 \pm 0.07$ & $1.91 \pm 0.03$ & $1.94 \pm 0.04$ \\
\hline
\end{tabular}


Table S4 Key inter-atomic distances of the different states appearing along the hydrolysis of $\mathbf{2}$ catalyzed by CALB. Structures optimized at M06-2X/MM level. All distances are given in $\AA$.

\begin{tabular}{|c|c|c|c|c|c|c|c|c|c|}
\hline & RS & TS1 & INT1 & TS2 & INT2 & TS3 & INT3 & TS4 & PS \\
\hline $\mathrm{OG}_{\mathrm{Ser} 105}-\mathrm{C} 1_{\text {subs }}$ & 2.32 & 1.68 & 1.51 & 1.42 & 1.32 & 1.39 & 1.43 & 1.63 & 2.20 \\
\hline $\mathrm{OG}_{\text {Ser } 105}-\mathrm{HG}_{\text {Ser } 105}$ & 1.00 & 1.24 & 1.81 & 2.50 & 2.53 & - & - & - & - \\
\hline $\mathrm{NE} 2_{\mathrm{His} 224}-\mathrm{HG}_{\mathrm{Ser} 105}$ & 1.65 & 1.26 & 1.04 & 1.27 & 2.91 & - & - & - & - \\
\hline $\mathrm{C} 1_{\text {subs }}-\mathrm{N} 4_{\text {subs }}$ & 1.36 & 1.44 & 1.48 & 1.61 & 2.84 & - & - & - & - \\
\hline $\mathrm{HG}_{\mathrm{Ser} 105}-\mathrm{N} 4_{\text {subs }}$ & 2.76 & 2.66 & 2.74 & 1.34 & 1.02 & - & - & - & - \\
\hline $\mathrm{O}_{\text {wat }}-\mathrm{C} 1_{\text {subs }}$ & - & - & - & - & 4.33 & 1.69 & 1.49 & 1.40 & 1.33 \\
\hline $\mathrm{O}_{\text {wat }}-\mathrm{H} 1_{\text {wat }}$ & - & - & - & - & 0.97 & 1.17 & 1.60 & 2.40 & 2.55 \\
\hline $\mathrm{H} 1_{\text {wat }}-\mathrm{NE} 2_{\text {His } 224}$ & - & - & - & - & 3.77 & 1.32 & 1.05 & 1.26 & 1.64 \\
\hline $\mathrm{H} 1_{\text {wat }}-\mathrm{OG}_{\text {Ser } 105}$ & - & - & - & - & 4.78 & 2.61 & 2.78 & 1.24 & 1.00 \\
\hline $\mathrm{HD} 1_{\text {His } 224}-\mathrm{OD} 2_{\mathrm{Asp} 187}$ & 2.02 & 2.08 & 2.04 & 2.19 & 2.15 & 2.18 & 2.14 & 2.03 & 2.00 \\
\hline $\mathrm{C} 1_{\text {subs }}-\mathrm{O} 2_{\text {subs }}$ & 1.26 & 1.29 & 1.31 & 1.30 & 1.23 & 1.30 & 1.32 & 1.29 & 1.24 \\
\hline $\mathrm{O} 2_{\text {subs }}-\mathrm{H}_{\text {Thr } 40}$ & 1.77 & 1.77 & 1.68 & 1.82 & 1.86 & 1.95 & 1.88 & 1.97 & 1.85 \\
\hline $\mathrm{O} 2_{\text {subs }}-\mathrm{HO}_{\text {Thr } 40}$ & 1.85 & 1.87 & 1.65 & 1.81 & 1.66 & 1.97 & 1.77 & 1.88 & 1.66 \\
\hline $\mathrm{O} 2_{\text {subs }}-\mathrm{H}_{\mathrm{Gln} 106}$ & 2.14 & 1.95 & 1.78 & 2.05 & 1.85 & 1.93 & 1.79 & 1.89 & 1.91 \\
\hline
\end{tabular}


Table S5. Charges of the key atoms of the 2 computed with the CHelpG method at M06-2X/MM level as an average of 20 ps MD simulations on the states involved in the full chemical reaction, from reactants state (RS) to Products state (PS). N4 only participates in the first two steps of the reaction. Charges are in a.u.

\begin{tabular}{cccc}
\hline & $\mathrm{C} 1$ & $\mathrm{O} 2$ & $\mathrm{~N} 4$ \\
\hline RS & $0.85 \pm 0.03$ & $-0.884 \pm 0.012$ & $-0.77 \pm 0.03$ \\
TS1 & $0.92 \pm 0.04$ & $-1.009 \pm 0.020$ & $-0.888 \pm 0.023$ \\
INT1 & $1.04 \pm 0.03$ & $-1.104 \pm 0.017$ & $-0.89 \pm 0.03$ \\
TS2 & $0.85 \pm 0.04$ & $-1.086 \pm 0.015$ & $-0.49 \pm 0.03$ \\
INT2 & $0.82 \pm 0.03$ & $-0.806 \pm 0.013$ & $-0.78 \pm 0.04$ \\
TS3 & $0.74 \pm 0.04$ & $-0.965 \pm 0.022$ & - \\
INT3 & $0.73 \pm 0.03$ & $-1.039 \pm 0.015$ & - \\
TS4 & $0.96 \pm 0.04$ & $-1.030 \pm 0.021$ & - \\
PS & $0.76 \pm 0.03$ & $-0.848 \pm 0.013$ & - \\
\hline
\end{tabular}

Table S6. Charges of the key atoms of 2 computed with the CHelpG method at M06-2X/MM level in gas phase and including, as point charges, the atoms of the residues belonging to the oxyanion hole formed by residues Thr40 and Gln106 (Oxyanion), the residues Ser105, His224, and Asp187 (Catalytic Triad), residues Thr40, Gln106, Ser105, His224, and Asp187 (Oxyanion hole + Catalytic Triad), and the full protein. Charges are in a.u.

\begin{tabular}{|c|c|c|c|c|c|}
\hline Atom & Gas Phase & Oxyanion Hole & Catalytic Triad & $\begin{array}{c}\text { Oxyanion Hole } \\
+ \\
\text { Catalytic Triad }\end{array}$ & Full Protein \\
\hline $\mathrm{C} 1$ & 0.78 & 0.87 & 0.98 & 1.10 & 1.11 \\
\hline $\mathrm{O} 2$ & -0.56 & -0.79 & -0.71 & -0.91 & -0.94 \\
\hline N4 & -0.77 & -0.72 & -0.95 & -0.98 & -0.97 \\
\hline
\end{tabular}




\section{Determined Kinetic Isotope Effects}

Averaged KIE at AM1/MM level are showed in table S7. As explained above, 10 structures of RS and each TS were localized at AM1/MM and averaged KIE were computed. Slightly differences on key distances and KIE exist between M06-2X/MM and AM1/MM which may be due to differences in the computed charges of the methods.

Table S7 Averaged KIEs computed at AM1/MM level for the reaction of hydrolysis of 2.

\begin{tabular}{ccccc}
\hline & TS1 & TS2 & TS3 & TS4 \\
\hline${ }^{14}[1-\mathrm{C}]$ & $1.043 \pm 0.005$ & $1.016 \pm 0.005$ & $0.999 \pm 0.004$ & $1.007 \pm 0.004$ \\
${ }^{15}[4-\mathrm{N}]$ & $1.0008 \pm 0.0021$ & $1.0006 \pm 0.0021$ & - & - \\
${ }^{18}[2-\mathrm{O}]$ & $0.994 \pm 0.004$ & $0.992 \pm 0.004$ & $0.981 \pm 0.005$ & $0.987 \pm 0.003$ \\
\hline
\end{tabular}

It is interesting to stress that the KIEs values obtained at M06-2X/MM level (see Table 1 of the text) are in good agreement to those average values calculated at AM1/MM level (see Table S6) in the case of ${ }^{14} \mathrm{C}$ and ${ }^{15} \mathrm{~N}$ substitutions. On the contrary, the ${ }^{18} \mathrm{O}-\mathrm{KIE}$ values at AM1/MM level (see Table S6) are slightly inverse, from 0.6 to $1.9 \%$. This can be rationalized based on the analysis of the inter-atomic interactions established between $\mathrm{O} 2$ and the residues of the oxyanion hole (Thr40 and Gln106). In all TS structures localized at AM1/MM, O2 atom forms stronger H-bond interaction than in the reactants state, due to the accumulation of negative charge of this atom along the reaction. This result explains the inverse ${ }^{18} \mathrm{O}-\mathrm{KIEs}$ obtained at $\mathrm{AM} 1 / \mathrm{MM}$ level. This effect is not so dramatic at M06-2X/MM level, where the variations of the distances between $\mathrm{O} 2$ atom and the residues of the oxyanion hole from reactants state to the different TSs, are not so meaningful (see Table S4). 


\section{Kinetics of hydrolysis of 2}

Hydrolysis of 2 by CALB was evaluated at increasing concentrations of substrate as explained above. A Michaelis-Menten curve was fitted on the data (Figure S6), and kinetics constants were measured.

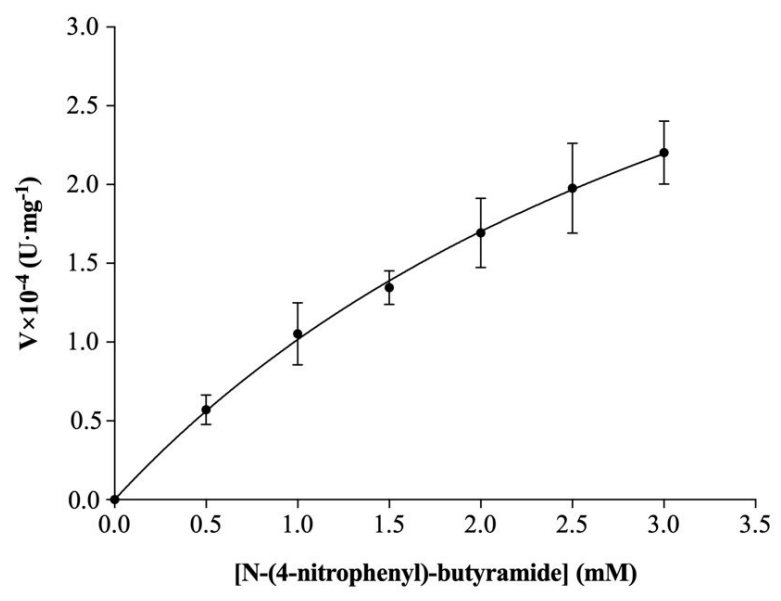

Figure S6. Kinetics of the hydrolysis of 2 by CALB. Velocities were calculated at increasing concentrations of substrate. 


\section{REFERENCES}

(1) Uppenberg, J.; Morgens, H.; Shamkant, P.; Alwyn, J. T. The Sequence, Crystal Structure Determination and Refinement of Two Crystal Forms of Lipase B from Candida Antarctica. Structure 1994, 2, 293-308.

(2) Olsson, M. H. M.; SØndergaard, C. R.; Rostkowski, M.; Jensen, J. H. PROPKA3: Consistent Treatment of Internal and Surface Residues in Empirical p Kapredictions. $J$. Chem. Theory Comput. 2011, 7, 525-537.

(3) Søndergaard, C. R.; Olsson, M. H. M.; Rostkowski, M.; Jensen, J. H. Improved Treatment of Ligands and Coupling Effects in Empirical Calculation and Rationalization of $\mathrm{PKa}$ Values. J Chem Theory Comput 2011, 7, 2284-2295.

(4) Świderek, K.; Martí, S.; Moliner, V. Theoretical Study of Primary Reaction of Pseudozyma Antarctica Lipase B as the Starting Point to Understand Its Promiscuity. ACS Catal. 2014, $4,426-434$.

(5) Wang, J.; Wang, W.; Kollman, P. A.; Case, D. A. Automatic Atom Type and Bond Type Perception in Molecular Mechanical Calculations. J. Mol. Graph. Model. 2006, 25, 247260.

(6) Zhang, W. E. I.; Yang, R.; Cieplak, P.; Luo, R. A. Y.; Lee, T.; Caldwell, J.; Wang, J.; Kollman, P. A Point-Charge Force Field for Molecular Mechanics Simulations of Proteins Based on Condensed-Phase. J. Comput. Chem. 2003, 24, 1999-2012.

(7) Phillips, J. C.; Braun, R.; Wang, W. E. I.; Gumbart, J.; Tajkhorshid, E.; Villa, E.; Chipot, C.; Skeel, R. D.; Poincare, H. Scalable Molecular Dynamics with NAMD. J. Comput. Chem. 2005, 26, 1781-1802.

(8) Dewar, M. J. S.; Zoebisch, E. G.; Healy, E. F.; Stewart, J. J. P. Development and Use of Quantum Mechanical Molecular Models. 76. AM1: A New General Purpose Quantum Mechanical Molecular Model. J. Am. Chem. Soc. 1985, 107, 3902-3909.

(9) Baker, J. An Algorithm for the Location of Transition States. J. Comput. Chem. 1986, 7, 
$385-395$.

(10) Fukui, K. The Path of Chemical Reactions - The IRC Approach. Acc. Chem. Res. 1981, 14, $363-368$.

(11) Zhao, Y.; Truhlar, D. G. The M06 Suite of Density Functionals for Main Group Thermochemistry, Thermochemical Kinetics, Noncovalent Interactions, Excited States, and Transition Elements: Two New Functionals and Systematic Testing of Four M06-Class Functionals and 12 Other Function. Theor. Chem. Acc. 2008, 120, 215-241.

(12) Frisch, M. J.; Trucks, G. W.; Schlegel, H. B.; Scuseria, G. E.; Robb, M. A.; Cheeseman, J. R.; Scalmani, G.; Barone, V.; Mennucci, B.; Petersson, G. A.; Nakatsuji, H.; Caricato, M.; Li, X.; Hratchian, H. P.; Izmaylov, A. F.; Bloino, J.; Zheng, G.; Sonnenberg, J. L.; Hada, M.; Ehara, M.; Toyota, K.; Fukuda, R.; Hasegawa, J.; Ishida, M.; Nakajima, T.; Honda, Y.; Kitao, O.; Nakai, H.; Vreven, T.; Montgomery, J. A., Jr.; Peralta, J. E.; Ogliaro, F.; Bearpark, M.; Heyd, J. J.; Brothers, E.; Kudin, K. N.; Staroverov, V. N.; Kobayashi, R.; Normand, J.; Raghavachari, K.; Rendell, A.; Burant, J. C.; Iyengar, S. S.; Tomasi, J.; Cossi, M.; Rega, N.; Millam, J. M.; Klene, M.; Knox, J. E.; Cross, J. B.; Bakken, V.; Adamo, C.; Jaramillo, J.; Gomperts, R.; Stratmann, R. E.; Yazyev, O.; Austin, A. J.; Cammi, R.; Pomelli, C.; Ochterski, J. W.; Martin, R. L.; Morokuma, K.; Zakrzewski, V. G.; Voth, G. A.; Salvador, P.; Dannenberg, J. J.; Dapprich, S.; Daniels, A. D.; Farkas, O.; Foresman, J. B.; Ortiz, J. V.; Cioslowski, J.; Fox, D. J. Gaussian 09, Revision E.01. Gaussian, Inc.: Wallingford, CT 2009.

(13) Roux, B. The Calculation of the Potential of Mean Force Using Computer-Simulation. Comput. Phys. Commun. 1995, 91, 275-282.

(14) Torrie, G. M.; Valleau, J. P. Non-Physical Sampling Distibutions in Monte-Carlo FreeEnergy Estimation - Umbrella Sampling. J. Comput. Phys. 1977, 23, 187-199.

(15) Kumar, S.; Rosenberg, J. M.; Bouzida, D.; Swendsen, R. H.; Kollman, P. A. The Weighted Histogram Analysis Method for Free-energy Calculations on Biomolecules. I. The Method. J. Comput. Chem. 1992, 13, 1011-1021.

(16) Verlet, L. Computer "Experiments" on Classical Fluids. I. Thermodynamical Properties of 
Lennard-Jones Molecules. Phys. Rev. 1967, 159, 98-103.

(17) Martí, S.; Moliner, V.; Tuñón, I.; Williams, I. H. QM / MM Calculations of Kinetic Isotope Effects in the Chorismate Mutase Active Site. Org. Biomol. Chem. 2003, 1, 483-487.

(18) Martí, S.; Moliner, V.; Tuñón, I. Improving the QM/MM Description of Chemical Processes: A Dual Level Strategy to Explore the Potential Energy Surface in Very Large Systems. J. Chem. Theory Comput. 2005, 1, 1008-1016. 



\section{Annex B}

Supporting Information of Article 2:

${ }^{66}$ Computational Studies Suggest

Promiscuous Candida antarctica

Lipase B as an Environmentally

Friendly Alternative for the

Production of Epoxides" 



\title{
Supporting Information
}

\section{Computational Studies Suggest Promiscuous}

\author{
Candida antarctica Lipase B as an
}

\section{Environmentally Friendly Alternative for the}

\author{
Production of Epoxides
}

\author{
Miquel A. Galmés, Katarzyna Świderek, * Vicent Moliner*
}

Institute of Advanced Materials (INAM), Universitat Jaume I, 12071 Castellón, Spain

Corresponding authors:

swiderek@uji.es

moliner@uji.es 
Table S1. Atom types, charges and parameters obtained for 2-butenal.

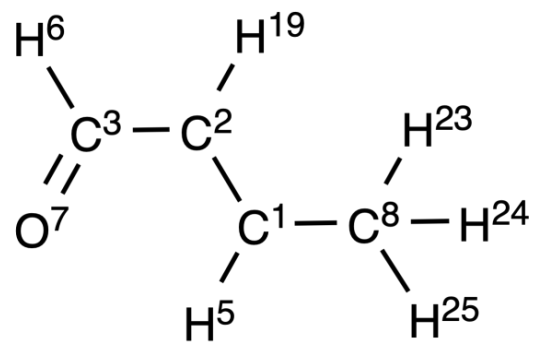

$\begin{array}{lll}\text { Atom name } & \text { Atom type } & \text { Charge } \\ \text { C1 } & \text { c2 } & -0.0922 \\ \text { C2 } & \text { ce } & -0.2792 \\ \text { C3 } & \text { c } & 0.5601 \\ \text { H5 } & \text { ha } & 0.1490 \\ \text { H6 } & \text { h4 } & -0.0098 \\ \text { O7 } & \text { o } & -0.5261 \\ \text { C8 } & \text { c3 } & -0.0809 \\ \text { H19 } & \text { ha } & 0.1220 \\ \text { H23 } & \text { hc } & 0.0524 \\ \text { H24 } & \text { hc } & 0.0524 \\ \text { H25 } & \text { hc } & 0.0524\end{array}$

\section{Parameters:}

\section{MASS}

o

16.000

0.434

BOND

$\begin{array}{lll}\text { c } & 12.010 & 0.616\end{array}$

$\begin{array}{lll}\text { h4 } & 1.008 & 0.135\end{array}$

$\begin{array}{lll}\text { ce } & 12.010 & 0.360\end{array}$

$\begin{array}{lll}\text { ha } & 1.008 & 0.135\end{array}$

$\begin{array}{lll}\text { c2 } & 12.010 & 0.360\end{array}$

$\begin{array}{lll}\text { c3 } & 12.010 & 0.878\end{array}$

hc

1.008

0.135

$\begin{array}{lll}\text { c -o } & 637.700 & 1.218 \\ \text { c -4 } & 310.700 & 1.112 \\ \text { c -ce } & 354.500 & 1.482 \\ \text { ce-ha } & 342.500 & 1.088 \\ \text { c2-ce } & 547.300 & 1.346 \\ \text { c2-ha } & 343.100 & 1.088 \\ \text { c2-c3 } & 326.800 & 1.510 \\ \text { c3-hc } & 330.600 & 1.097\end{array}$

\section{ANGLE}

h4-c -o

$$
54.200
$$

120.700

NONBON

ce-c -o

68.800

123.200

o

1.6612

0.2100

c -ce-ha

46.500

116.460

1.9080

0.0860

c -ce-c2

65.500

120.420

1.4090

0.0150

ce-c -h4

46.700

114.890

1.9080

0.0860

ce-c2-ha

49.500

120.450

1.4590

0.0150

c3-c2-ce

64.000

123.150

1.9080

0.0860

c2

1.9080

0.1094 


$\begin{array}{llllll}\text { c2-ce-ha } & 49.600 & 119.940 & \text { hc } & 1.4870 & 0.0157 \\ \text { c2-c3-hc } & 47.000 & 110.360 & & & \\ \text { c3-c2-ha } & 45.900 & 115.680 & & & \\ \text { hc-c3-hc } & 39.400 & 107.580 & & & \end{array}$

\section{DIHE}

$\begin{array}{lllll}\text { o -c -ce-ha } & 4 & 8.700 & 180.000 & 2.000 \\ \text { o -c -ce-c2 } & 4 & 8.700 & 180.000 & 2.000 \\ \text { ha-c2-ce-c } & 4 & 26.600 & 180.000 & 2.000 \\ \text { c3-c2-ce-c } & 4 & 26.600 & 180.000 & 2.000 \\ \text { h4-c -ce-ha } & 4 & 8.700 & 180.000 & 2.000 \\ \text { h4-c -ce-c2 } & 4 & 8.700 & 180.000 & 2.000 \\ \text { ce-c2-c3-hc } & 6 & 0.000 & 0.000 & 2.000 \\ \text { ha-c2-ce-ha } & 4 & 26.600 & 180.000 & 2.000 \\ \text { c3-c2-ce-ha } & 4 & 26.600 & 180.000 & 2.000 \\ \text { ha-c2-c3-hc } & 6 & 0.000 & 0.000 & 2.000\end{array}$

\section{IMPROPER}

$\begin{array}{llll}\text { ce-h4-c -o } & 10.5 & 180.0 & 2.0 \\ \text { c -c2-ce-ha } & 1.1 & 180.0 & 2.0 \\ \text { c3-ce-c2-ha } & 1.1 & 180.0 & 2.0\end{array}$

Table S2. Atom types, charges and parameters obtained for hydrogen peroxide.

$$
\mathrm{H}^{4}-\mathrm{O}^{20}
$$

$\begin{array}{lll}\text { Atom name } & \text { Atom type } & \text { Charge } \\ \text { H4 } & \text { ho } & 0.412 \\ \text { O20 } & \text { oh } & -0.412 \\ \text { O21 } & \text { oh } & -0.412 \\ \text { H22 } & \text { ho } & 0.412\end{array}$

\section{Parameters:}

\section{MASS}

$\begin{array}{lll}\text { oh } & 16.000 & 0.465 \\ \text { ho } & 1.008 & 0.135\end{array}$

\section{BOND}




$\begin{array}{lll}\text { ho-oh } & 371.400 & 0.973 \\ \text { oh-oh } & 340.500 & 1.469\end{array}$

ANGLE

$\begin{array}{lllll}\text { ho-oh-oh } & 49.300 & 98.720 & & \\ \begin{array}{l}\text { DIHE } \\ \text { ho-oh-oh-ho }\end{array} & 1 & 1.600 & 0.000 & 2.000 \\ \text { NONBON } & & & & \\ \text { oh } & 1.7210 & 0.2104 & & \\ \text { ho } & 0.0000 & 0.0000 & & \end{array}$

Table S3. Atom types, charges and parameters obtained for perbutyric acid.

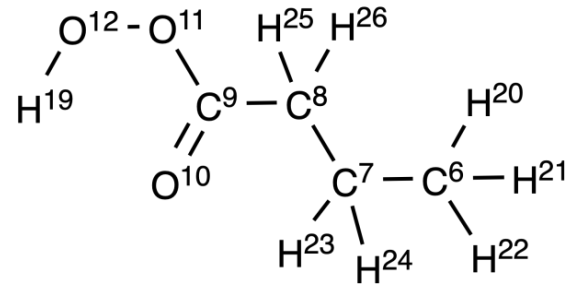

$\begin{array}{lll}\text { Atom name } & \text { Atom type } & \text { Charge } \\ \text { C6 } & \text { ct } & -0.0951 \\ \text { C7 } & \text { ct } & -0.0794 \\ \text { C8 } & \text { ct } & -0.1304 \\ \text { C9 } & \text { c } & 0.5981 \\ \text { O10 } & \text { o } & -0.514 \\ \text { O11 } & \text { os } & -0.2281 \\ \text { O12 } & \text { oh } & -0.368 \\ \text { H19 } & \text { ho } & 0.413 \\ \text { H20 } & \text { hc } & 0.039367 \\ \text { H21 } & \text { hc } & 0.039367 \\ \text { H22 } & \text { hc } & 0.039367 \\ \text { H23 } & \text { hc } & 0.0577 \\ \text { H24 } & \text { hc } & 0.0577 \\ \text { H25 } & \text { hc } & 0.0852 \\ \text { H26 } & \text { hc } & 0.0852\end{array}$




\section{Parameters:}

MASS

$\begin{array}{llllll}\text { ct } & 12.010 & 0.878 & \text { ct-hc } & 330.600 & 1.097 \\ \text { hc } & 1.008 & 0.135 & \text { ct-ct } & 300.900 & 1.538 \\ \text { c } & 12.010 & 0.616 & \text { c-ct } & 313.000 & 1.524 \\ \text { o } & 16.000 & 0.434 & \text { c-o } & 637.700 & 1.218 \\ \text { os } & 16.000 & 0.465 & \text { c-os } & 390.800 & 1.358 \\ \text { oh } & 16.000 & 0.465 & \text { oh-os } & 354.900 & 1.456 \\ \text { ho } & 1.008 & 0.135 & \text { ho-oh } & 371.400 & 0.973\end{array}$

\section{ANGLE}

ct-ct-he

$$
46.300
$$

109.800

ct-ct-ct

62.900

111.510

ct

hc-ct-he

39.400

107.580

c-ct-ct

63.300

111.040

ct-c-o

67.400

123.200

68.900

110.720

ct-c-os

46.900

108.770

c-os-oh

66.200

110.500

o-c-os

75.300

123.250

ho-oh-os

49.500

99.680

\section{DIHE}

$\begin{array}{lll}0.160 & 0.000 & 3.000 \\ 1.400 & 0.000 & 3.000 \\ 0.150 & 0.000 & 3.000 \\ 0.000 & 180.000 & 2.000 \\ 0.000 & 180.000 & 2.000 \\ 1.400 & 0.000 & 3.000 \\ 5.400 & 180.000 & 2.000 \\ 0.800 & 0.000 & -1.000 \\ 0.000 & 0.000 & -2.000 \\ 0.080 & 180.000 & 3.000 \\ 0.000 & 180.000 & 2.000 \\ 1.600 & 0.000 & 2.000 \\ 5.400 & 180.000 & 2.000\end{array}$

\section{IMPROPER}

ct-o-c-os

1.1

180.0

2.0 


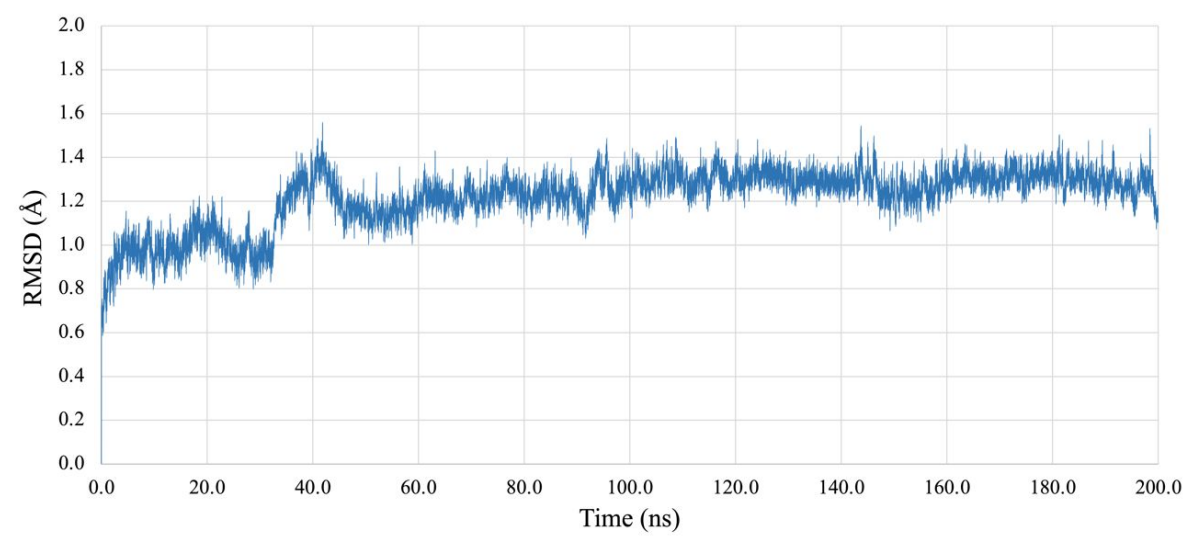

Figure S1. Root mean square deviation (RMSD) of the position of $\mathrm{Ca}, \mathrm{C}$, and $\mathrm{N}$ atoms of the backbone along $200 \mathrm{~ns}$ of MD simulation.

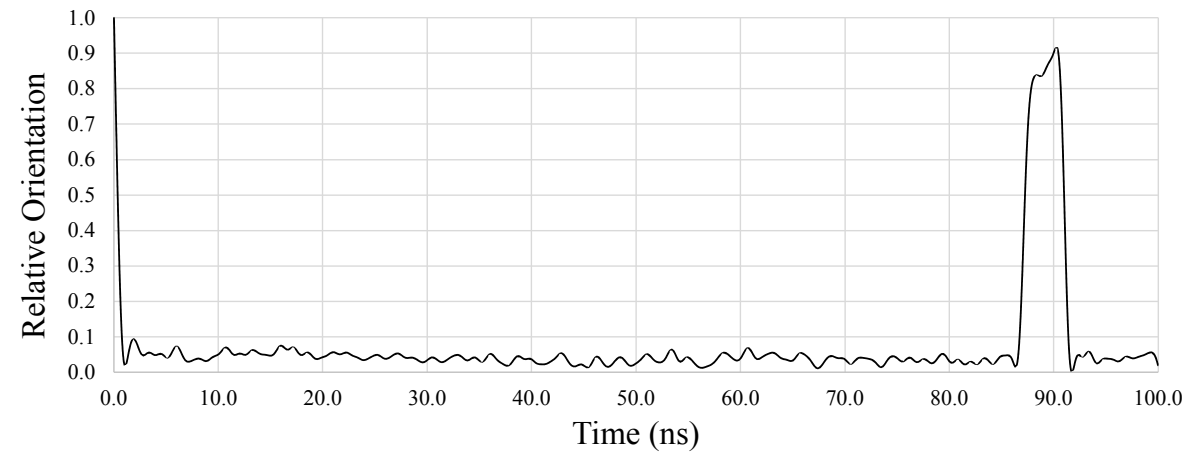

Figure S2. Relative orientation of the substrate 2-butenal along 100 ns of classical MD starting from the geometry of S-enantiomer. Baseline 0.0 corresponds to (R)-enantiomer and 1.0 is the corresponding (S)-geometry. 

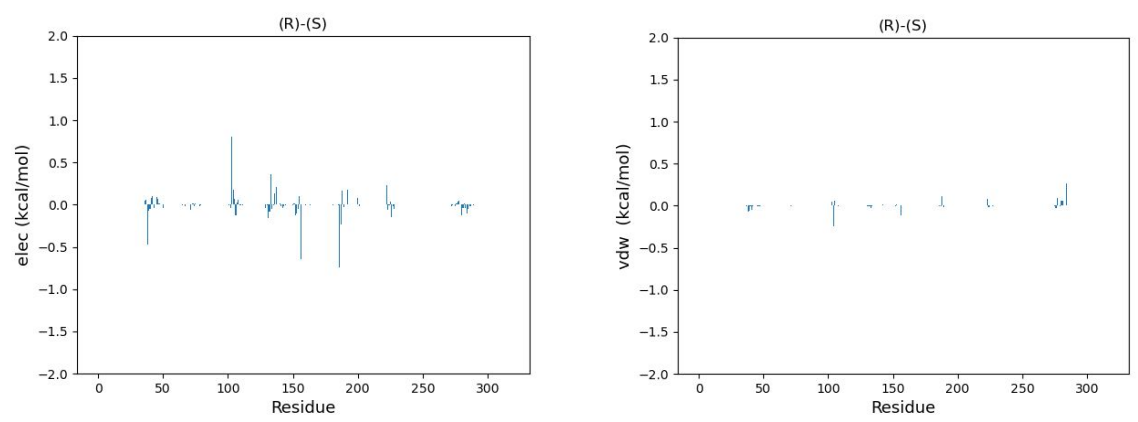

Figure S3. Interaction energies between the substrate (2-butenal) and the protein, by residue, computed along the 200 ns MD simulation displayed as the difference between the (R)- and (S)- enantiomers. Left panel corresponds to the electrostatic interactions while the right panel shows the van der Waals interactions. 

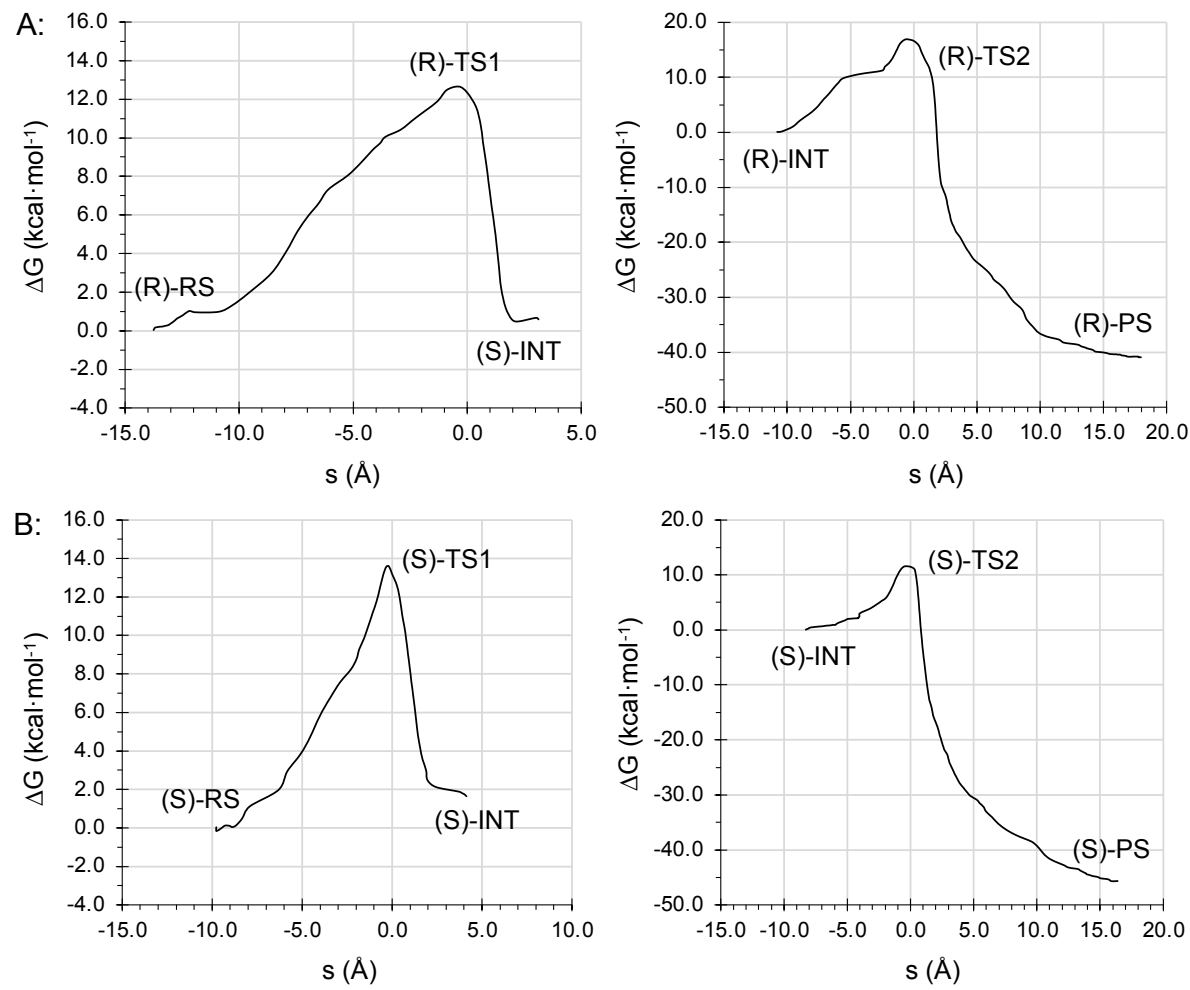

Figure S4. Free energy profile along the two-step mechanism of the direct epoxidation of 2-butenal obtained with free energy perturbation method at B3LYP/MM level of theory. Top A panel corresponds to the R enantiomer and $\mathbf{B}$ shows the evolution of $\mathrm{S}$ enantiomer. Energies are given in $\mathrm{kcal} \cdot \mathrm{mol}^{-1}$ and distances are represented as $s$ coordinate in $\AA$. 
Table S4. FEP activation energies for direct epoxidation computed at B3LYP/MM level and errors computed as first order expansion. The energies are in $\mathrm{kcal} \cdot \mathrm{mol}^{-1}$.

\begin{tabular}{lcc}
\hline & R-enantiomer & S-enantiomer \\
\hline$\Delta \mathrm{F}_{\mathrm{RC} \rightarrow \mathrm{TS} 1}$ & $12.40 \pm 0.15$ & $16.44 \pm 0.14$ \\
$\Delta \mathrm{F}_{\mathrm{INT} \rightarrow \mathrm{TS} 2}$ & $13.09 \pm 0.12$ & $11.52 \pm 0.10$ \\
\hline
\end{tabular}
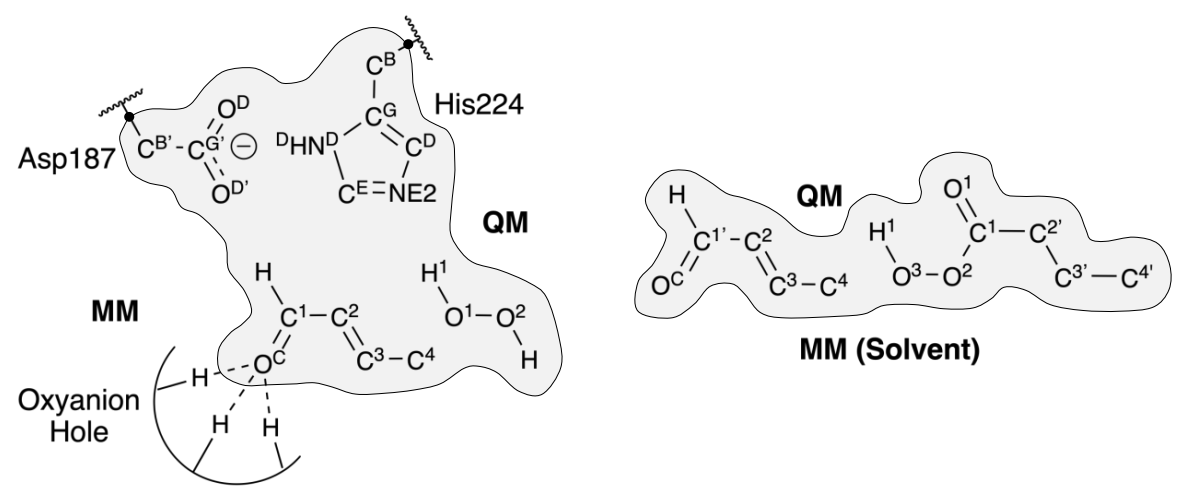

Figure S5. Schematic representation of QM partition. A: CALB active site and its QM sub-set represented as a shaded region. Black dots represent link atoms introduced between $\mathrm{QM}$ and $\mathrm{MM}$ region. B: QM region for the epoxidation reaction using peroxyacids in chloroform. The solvent was treated as MM. 
Table S5. Key inter-atomic distances of the different states appearing along the direct epoxidation of 2-butenal wit hydrogen peroxide by CALB. Structures optimized at B3LYP/MM level. All distances are given in $\AA$.

\begin{tabular}{lcccccccccccc}
\hline & \multicolumn{1}{c}{ Enantiomer R } & \multicolumn{8}{c}{ Enantiomer S } \\
& RS & TS1 & INT & INT & TS2 & PS & RS & TS1 & INT & INT & TS2 & PS \\
\hline $\mathrm{NE} 2-\mathrm{H}^{1}$ & 1.60 & 1.25 & 1.02 & 1.02 & 1.03 & 1.78 & 1.60 & 1.19 & 1.03 & 1.03 & 1.06 & 1.78 \\
$\mathrm{H}^{1}-\mathrm{O}^{1}$ & 1.03 & 1.22 & 1.86 & 1.84 & 1.97 & 3.02 & 1.02 & 1.31 & 1.88 & 1.87 & 2.14 & 3.24 \\
$\mathrm{NE2}-\mathrm{O}^{2}$ & 3.44 & 3.08 & 2.94 & 2.96 & 2.61 & 2.77 & 3.19 & 2.96 & 3.20 & 3.16 & 2.53 & 2.78 \\
$\mathrm{H}^{1}-\mathrm{O}^{2}$ & 2.02 & 2.06 & 2.21 & 2.23 & 2.10 & 1.00 & 1.94 & 2.14 & 2.64 & 2.59 & 1.63 & 1.00 \\
$\mathrm{O}^{1}-\mathrm{O}^{2}$ & 1.45 & 1.44 & 1.46 & 1.46 & 1.88 & 3.29 & 1.46 & 1.46 & 1.48 & 1.49 & 1.91 & 3.34 \\
$\mathrm{O}^{1}-\mathrm{C}^{3}$ & 3.00 & 2.06 & 1.50 & 1.50 & 1.41 & 1.42 & 3.29 & 2.07 & 1.50 & 1.50 & 1.40 & 1.43 \\
$\mathrm{O}^{1}-\mathrm{C}^{2}$ & 3.28 & 2.77 & 2.43 & 2.43 & 1.85 & 1.44 & 3.44 & 2.71 & 2.36 & 2.36 & 1.89 & 1.43 \\
$\mathrm{C}^{2}-\mathrm{C}^{3}$ & 1.35 & 1.39 & 1.47 & 1.47 & 1.48 & 1.49 & 1.35 & 1.39 & 1.48 & 1.48 & 1.49 & 1.49 \\
$\mathrm{O}^{\mathrm{c}}-\mathrm{HO}^{\mathrm{T} 40}$ & 1.82 & 1.79 & 1.74 & 1.73 & 1.73 & 1.74 & 1.78 & 1.81 & 1.77 & 1.76 & 1.76 & 1.81 \\
$\mathrm{O}^{\mathrm{c}}-\mathrm{HN}^{\mathrm{T} 40}$ & 1.71 & 1.77 & 1.73 & 1.74 & 1.67 & 1.84 & 1.87 & 1.90 & 1.88 & 1.91 & 1.94 & 1.75 \\
$\mathrm{O}^{\mathrm{c}}-\mathrm{H}^{\mathrm{Q} 106}$ & 2.23 & 2.04 & 2.05 & 2.14 & 2.25 & 2.29 & 2.23 & 2.01 & 2.04 & 2.04 & 2.02 & 2.35 \\
$\mathrm{H}^{\mathrm{D}}-\mathrm{O}^{\mathrm{D}}$ & 1.77 & 1.78 & 1.68 & 1.68 & 1.86 & 1.75 & 1.88 & 1.84 & 1.71 & 1.69 & 1.82 & 2.04 \\
$\mathrm{H}^{\mathrm{D}}-\mathrm{N}^{\mathrm{D}}$ & 1.04 & 1.04 & 1.06 & 1.06 & 1.04 & 1.04 & 1.03 & 1.04 & 1.06 & 1.06 & 1.04 & 1.03 \\
\hline
\end{tabular}


Table S6. CHelpG charges (in a.u.) of the heavy atoms of the amide substrate computed at $\mathrm{B} 3 \mathrm{LYP} / \mathrm{MM}$ level on the states involved in the full chemical reaction, from reactants state (RS) to Products state (PS) of R-enantiomer.

\begin{tabular}{|c|c|c|c|c|c|c|}
\hline & \multicolumn{6}{|c|}{ R-enantiomer } \\
\hline & RS & TS1 & INT & INT & TS2 & PS \\
\hline $\mathrm{C}^{1}$ & 0.087 & 0.133 & 0.313 & 0.308 & 0.362 & 0.272 \\
\hline $\mathrm{C}^{2}$ & -0.362 & -0.467 & -0.543 & -0.539 & -0.453 & -0.362 \\
\hline $\mathrm{C}^{3}$ & 0.577 & 0.491 & 0.415 & 0.425 & 0.538 & 0.793 \\
\hline $\mathrm{C}^{4}$ & -0.018 & 0.060 & -0.105 & -0.104 & -0.032 & -0.202 \\
\hline $\mathrm{O}^{\mathrm{C}}$ & -0.766 & -0.816 & -0.927 & -0.934 & -0.803 & -0.769 \\
\hline $\mathrm{O}^{1}$ & -0.294 & -0.278 & -0.194 & -0.185 & -0.180 & -0.200 \\
\hline $\mathrm{O}^{2}$ & -0.486 & -0.498 & -0.486 & -0.484 & -0.794 & -0.812 \\
\hline $\mathrm{C}^{\mathrm{B}}$ & -0.280 & -0.286 & -0.253 & -0.302 & -0.330 & -0.282 \\
\hline $\mathrm{C}^{\mathrm{G}}$ & 0.196 & 0.231 & 0.288 & 0.304 & 0.239 & 0.194 \\
\hline $\mathrm{N}^{\mathrm{D}}$ & -0.380 & -0.341 & -0.328 & -0.349 & -0.364 & -0.419 \\
\hline $\mathrm{C}^{\mathrm{E}}$ & 0.226 & 0.244 & 0.101 & 0.114 & 0.191 & 0.228 \\
\hline NE2 & -0.476 & -0.402 & -0.064 & -0.063 & -0.182 & -0.471 \\
\hline $\mathrm{C}^{\mathrm{D}}$ & -0.043 & -0.116 & -0.243 & -0.259 & -0.117 & -0.044 \\
\hline $\mathrm{C}^{\mathrm{B}^{\prime}}$ & -0.425 & -0.420 & -0.419 & -0.413 & -0.441 & -0.433 \\
\hline $\mathrm{C}^{\mathrm{G}^{\prime}}$ & 1.115 & 1.113 & 1.097 & 1.084 & 1.103 & 1.133 \\
\hline $\mathrm{O}^{\mathrm{D}}$ & -0.929 & -0.938 & -0.925 & -0.931 & -0.936 & -0.946 \\
\hline $\mathrm{O}^{\mathrm{D}^{\prime}}$ & -0.985 & -0.976 & -0.973 & -0.976 & -0.986 & -1.002 \\
\hline
\end{tabular}


Table S7. CHelpG charges (in a.u.) of the heavy atoms of the amide substrate computed at $\mathrm{B} 3 \mathrm{LYP} / \mathrm{MM}$ level on the states involved in the full chemical reaction, from reactants state (RS) to Products state (PS) of S-enantiomer.

\begin{tabular}{lcccccc}
\hline & & & \multicolumn{2}{c}{ S-enantiomer } & \\
& RS & TS1 & INT & INT & TS2 & PS \\
\hline $\mathrm{C}^{1}$ & 0.141 & 0.220 & 0.236 & 0.201 & 0.290 & 0.091 \\
$\mathrm{C}^{2}$ & -0.463 & -0.526 & -0.569 & -0.541 & -0.518 & -0.176 \\
$\mathrm{C}^{3}$ & 0.723 & 0.555 & 0.499 & 0.481 & 0.586 & 0.751 \\
$\mathrm{C}^{4}$ & 0.033 & -0.076 & 0.022 & 0.008 & -0.004 & -0.102 \\
$\mathrm{O}^{\mathrm{C}}$ & -0.796 & -0.818 & -0.922 & -0.922 & -0.832 & -0.778 \\
$\mathrm{O}^{1}$ & -0.384 & -0.140 & -0.149 & -0.142 & -0.187 & -0.268 \\
$\mathrm{O}^{2}$ & -0.371 & -0.476 & -0.447 & -0.436 & -0.692 & -0.847 \\
$\mathrm{C}^{\mathrm{B}}$ & -0.209 & -0.230 & -0.261 & -0.264 & -0.182 & -0.215 \\
$\mathrm{C}^{\mathrm{G}}$ & 0.180 & 0.262 & 0.301 & 0.304 & 0.201 & 0.129 \\
$\mathrm{~N}^{\mathrm{D}}$ & -0.273 & -0.317 & -0.359 & -0.375 & -0.250 & -0.198 \\
$\mathrm{C}^{\mathrm{E}}$ & 0.039 & -0.015 & 0.024 & 0.037 & -0.084 & -0.031 \\
$\mathrm{NE}^{2}$ & -0.314 & 0.026 & 0.073 & 0.080 & 0.091 & -0.261 \\
$\mathrm{C}^{\mathrm{D}}$ & -0.106 & -0.255 & -0.266 & -0.273 & -0.198 & -0.120 \\
$\mathrm{C}^{\mathrm{B}}$ & -0.372 & -0.375 & -0.389 & -0.404 & -0.396 & -0.411 \\
$\mathrm{C}^{\mathrm{G}^{\prime}}$ & 1.115 & 1.099 & 1.087 & 1.092 & 1.100 & 1.121 \\
$\mathrm{O}^{\mathrm{D}}$ & -0.964 & -0.953 & -0.939 & -0.941 & -0.956 & -0.972 \\
$\mathrm{O}^{\mathrm{D}}$ & -0.980 & -0.976 & -0.975 & -0.986 & -0.982 & -0.974 \\
\hline & & & & & &
\end{tabular}




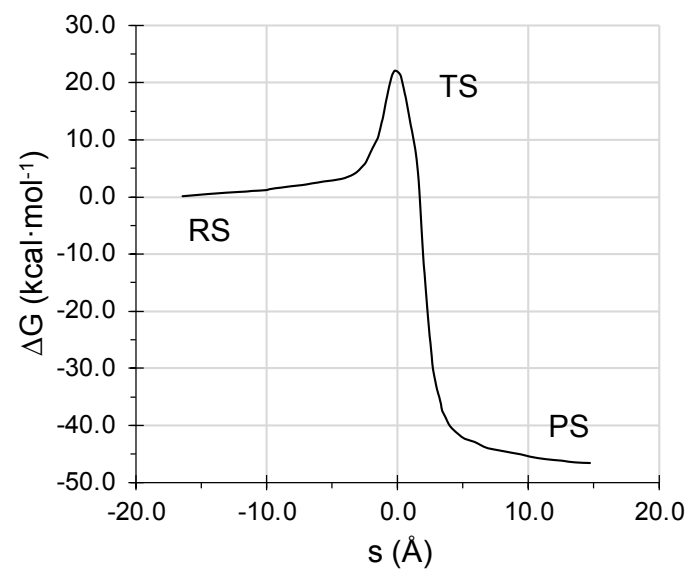

Figure S6 Free energy profile along the epoxidation of 2-butenal by peroxyacids in chloroform obtained with free energy perturbation method at B3LYP/MM level of theory. Energies are gives in $\mathrm{kcal} \cdot \mathrm{mol}^{-1}$ and distances are represented as $s$ coordinate in $\AA$.

Table S8. FEP activation energies for the Prilezhaev reaction in chlorofom computed at B3LYP/MM level and errors computed as first order expansion. The energies are in $\mathrm{kcal} \cdot \mathrm{mol}^{-1}$.

Prilezhaev reaction in Chlorofom

$\Delta \mathrm{F}_{\mathrm{RC} \rightarrow \mathrm{TS}} \quad 21.73 \pm 0.30$


Table S9. Key inter-atomic distances (in $\AA$ ) of the different states appearing along the epoxidation of 2-butenal by perbutyric acid in solution. Structures optimized at B3LYP/MM level.

\begin{tabular}{lccc}
\hline & \multicolumn{3}{c}{ Chloroform } \\
& RS & TS & PS \\
\hline $\mathrm{C}^{2}-\mathrm{C}^{3}$ & 1.34 & 1.39 & 1.49 \\
$\mathrm{C}^{2}-\mathrm{O}^{3}$ & 4.08 & 2.26 & 1.45 \\
$\mathrm{C}^{3}-\mathrm{O}^{3}$ & 4.20 & 1.84 & 1.44 \\
$\mathrm{O}^{3}-\mathrm{H}^{1}$ & 0.99 & 1.01 & 2.82 \\
$\mathrm{O}^{2}-\mathrm{O}^{3}$ & 1.44 & 1.81 & 3.75 \\
$\mathrm{O}^{1}-\mathrm{H}^{1}$ & 1.88 & 1.70 & 0.97 \\
$\mathrm{C}^{1}-\mathrm{O}^{1}$ & 1.22 & 1.24 & 1.35 \\
$\mathrm{C}^{1}-\mathrm{O}^{2}$ & 1.36 & 1.31 & 1.22 \\
\hline
\end{tabular}


Table S10. ChelpG atomic charges (in a.u.) of the different structures appearing along the epoxidation of 2-butenal by perbutyric acid in solution. Structures optimized at B3LYP/MM level.

\begin{tabular}{cccc}
\hline & \multicolumn{3}{c}{ Chloroform } \\
& RS & TS & PS \\
\hline $\mathrm{C}^{1}$ & 0.600 & 0.523 & 0.599 \\
$\mathrm{C}^{2}$ & -0.332 & -0.282 & -0.083 \\
$\mathrm{C}^{3}$ & -0.019 & 0.180 & 0.083 \\
$\mathrm{C}^{4}$ & -0.034 & -0.190 & -0.092 \\
$\mathrm{O}^{\mathrm{C}}$ & -0.549 & -0.494 & -0.532 \\
$\mathrm{C}^{1}$ & 0.751 & 0.773 & 0.810 \\
$\mathrm{C}^{2}$ & -0.361 & -0.232 & -0.273 \\
$\mathrm{C}^{3}$ & 0.309 & 0.310 & 0.261 \\
$\mathrm{C}^{4}$ & -0.153 & -0.169 & -0.140 \\
$\mathrm{O}^{1}$ & -0.577 & -0.624 & -0.639 \\
$\mathrm{O}^{2}$ & -0.223 & -0.449 & -0.605 \\
$\mathrm{O}^{3}$ & -0.391 & -0.301 & -0.352 \\
\hline
\end{tabular}





\section{Annex G}

Supporting Information of Article 3:

${ }^{66}$ Combined Theoretical and

Experimental Study to Unravel the

Differences in Promiscuous

Amidase Activity of Two

Nonhomologous Enzymes" 



\section{SUPPLEMENTARY INFORMATION}

\section{A Combined Theoretical and Experimental Study to Unravel}

the Differences in Promiscuous Amidase Activity of Two

\section{Nonhomologous Enzymes}

Miquel À. Galmés, ${ }^{1}$ Alexander R Nödling, ${ }^{2}$ Louis Luk, ${ }^{2, *}$ Katarzyna Świderek, ${ }^{1, *}$ Vicent Moliner ${ }^{1, *}$

1. Institute of Advanced Materials (INAM), 12071 Castellón, Spain.

2. School of Chemistry, Cardiff University, Main Building, Park Pl, Cardiff CF10 3AT, UK.

corresponding authors:

L. Luk:1ukly@cardiff.ac.uk; K. Świderek: swiderek@uji.es; V. Moliner: moliner@uji.es 


\section{RESULTS AND ANALYSIS}

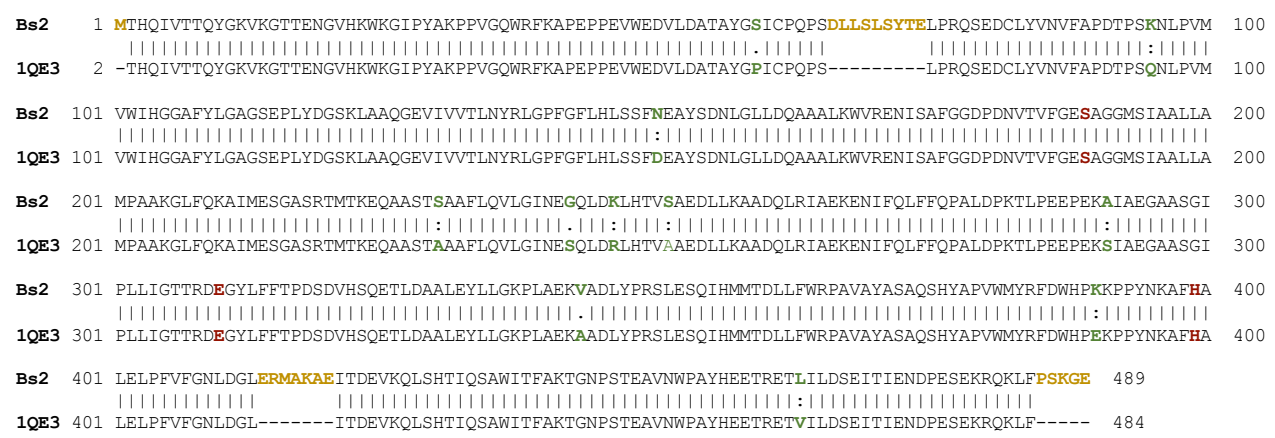

Figure S1. Sequence alignment between Bs2 available crystal structure (PDB ID: 1QE3, NRRL B8079 strain) and sequence of Bs2 from strain 168. In red the residues of catalytic triad are highlighted. In yellow missing fragments of protein, and in green the differences in sequence between both models are indicated.
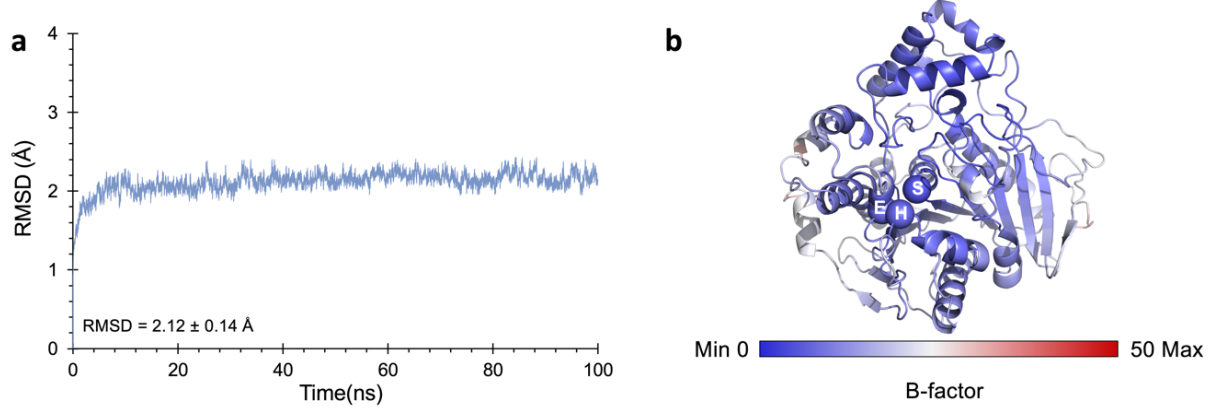

Figure S2. Analysis of $100 \mathrm{~ns}$ molecular dynamic simulations. a: Root mean square deviation (RMSD) of the position of $\mathrm{C} \alpha, \mathrm{C}$, and $\mathrm{N}$ atoms of the backbone along 100ns of MD simulation. b: Representation of the computed B-factor for Bs2. The catalytic triad is highlighted as spheres. 


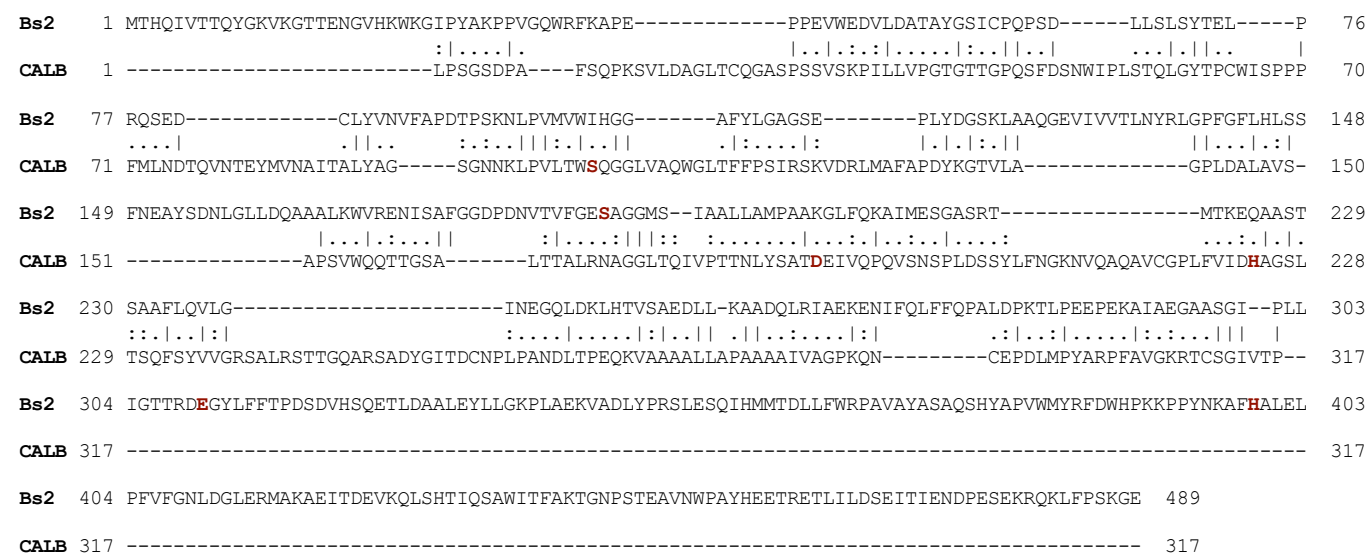

Figure S3. Sequence alignment between CALB and Bs2. The alignment was done using the Needleman-Wunsch ${ }^{1}$ algorithm implemented in EMBOSS-6.6.0 $0^{2}$ package (scoring matrix: BLOSUM62; ${ }^{3}$ gap penalty: 10 ; gap extension penalty: 0.5$)$. Residues of catalytic triad are highlighted in red. 
Table S1. Key inter-atomic distances of the different states appearing along the hydrolysis of $N$-(4-nitrophenyl)-butyramide catalyzed by wild-type Bs2. Structures optimized at M062X/MM level. All distances are given in $\AA$.

\begin{tabular}{|c|c|c|c|c|c|c|c|c|c|c|c|c|}
\hline & $\mathrm{RC}$ & TS1 & INT1 & INT1 & TS2 & INT2 & INT2 & TS3 & INT3 & INT3 & TS4 & $\mathrm{PC}$ \\
\hline $\mathrm{OG}_{\text {Ser } 189}-\mathrm{C} 1_{\text {subs }}$ & 2.32 & 1.80 & 1.49 & 1.48 & 1.35 & 1.33 & 1.33 & 1.40 & 1.46 & 1.44 & 1.71 & 2.35 \\
\hline $\mathrm{OG}_{\text {Ser } 189}-\mathrm{HG}_{\text {Ser } 189}$ & 1.00 & 1.22 & 2.08 & 2.44 & 2.71 & 2.58 & - & - & - & - & - & - \\
\hline NE2 $2_{\text {His } 399}-\mathrm{HG}_{\text {Ser1 } 189}$ & 1.69 & 1.32 & 1.03 & 1.03 & 1.04 & 1.76 & - & - & - & - & - & - \\
\hline $\mathrm{C} 1_{\text {subs }}-\mathrm{N} 4_{\text {subs }}$ & 1.37 & 1.44 & 1.53 & 1.55 & 2.26 & 2.62 & - & - & - & - & - & - \\
\hline $\mathrm{HG}_{\text {Ser189 }}-\mathrm{N} 4_{\text {subs }}$ & 2.76 & 2.62 & 2.25 & 2.05 & 1.96 & 1.05 & - & - & - & - & - & - \\
\hline $\mathrm{O}_{\text {wat }}-\mathrm{C} 1_{\text {subs }}$ & - & - & - & - & - & - & 2.53 & 1.69 & 1.49 & 1.48 & 1.41 & 1.34 \\
\hline $\mathrm{O}_{\text {wat }}-\mathrm{H} 1_{\text {wat }}$ & - & - & - & - & - & - & 0.98 & 1.19 & 1.74 & 1.98 & 2.44 & 2.69 \\
\hline $\mathrm{H} 1_{\text {wat }}-\mathrm{NE} 2_{\text {His } 399}$ & - & - & - & - & - & - & 1.91 & 1.34 & 1.05 & 1.03 & 1.30 & 1.74 \\
\hline $\mathrm{H} 1_{\text {wat }}-\mathrm{OG}_{\text {Ser } 189}$ & - & - & - & - & - & - & 2.88 & 2.52 & 2.60 & 2.76 & 1.31 & 1.00 \\
\hline $\begin{array}{l}\mathrm{HD}_{\text {His399- }} \\
\mathrm{OE} 1_{\text {Glu310 }}\end{array}$ & 1.64 & 1.60 & 1.50 & 1.55 & 1.55 & 1.68 & 1.77 & 1.72 & 1.62 & 1.69 & 1.54 & 1.75 \\
\hline $\begin{array}{l}\mathrm{HD} 1_{\text {His } 399}- \\
\mathrm{ND} 1_{\text {His } 399}\end{array}$ & 1.04 & 1.04 & 1.07 & 1.07 & 1.07 & 1.03 & 1.03 & 1.04 & 1.05 & 1.04 & 1.06 & 1.03 \\
\hline $\mathrm{C} 1_{\text {subs }}-\mathrm{O} 2_{\text {subs }}$ & 1.24 & 1.27 & 1.31 & 1.31 & 1.24 & 1.23 & 1.23 & 1.27 & 1.31 & 1.31 & 1.27 & 1.23 \\
\hline $\mathrm{O} 2_{\text {subs }}-\mathrm{H}_{\text {Ala190 }}$ & 1.93 & 1.85 & 1.79 & 1.87 & 1.81 & 1.79 & 1.77 & 1.93 & 1.87 & 1.76 & 1.72 & 1.96 \\
\hline $\mathrm{O} 2_{\text {subs }}-\mathrm{H}_{\text {Ala107 }}$ & 1.80 & 1.87 & 1.86 & 1.89 & 1.94 & 1.93 & 1.98 & 1.93 & 1.90 & 1.74 & 1.90 & 1.72 \\
\hline
\end{tabular}


Table S2. CHelpG charges (in a.u.) of the key atoms of the amide substrate computed at M062X/MM level on the states involved in the full chemical reaction catalyzed by the wild-type Bs2 and CALB (data from ref 11) from reactants complex (RC) to products complex (PC).

\section{Bs2}

\begin{tabular}{ccccccc}
\hline & $\mathrm{C}_{\text {subs }}$ & $\mathrm{N}_{\text {subs }}$ & $\mathrm{O}_{\text {subs }}$ & $\mathrm{NE}_{\text {His399 }}$ & $\mathrm{OG}_{\text {Ser189 }}$ & $\mathrm{O}_{\text {wat }}$ \\
\hline RC & 0.794 & -0.865 & -0.846 & -0.268 & -0.554 & \\
TS1 & 0.790 & -0.850 & -0.947 & -0.103 & -0.513 & - \\
INT1 & 0.823 & -0.826 & -1.051 & -0.142 & -0.572 & - \\
INT1 & 0.790 & -0.822 & -1.040 & -0.156 & -0.542 & - \\
TS2 & 0.768 & -0.906 & -0.842 & -0.187 & -0.482 & - \\
INT2 & 0.696 & -0.927 & -0.793 & -0.396 & -0.423 & - \\
INT2 & 0.901 & - & -0.810 & -0.327 & -0.340 & -0.829 \\
TS3 & 0.909 & - & -0.965 & -0.183 & -0.455 & -0.687 \\
INT3 & 0.942 & - & -1.064 & -0.118 & -0.502 & -0.737 \\
INT3 & 0.818 & - & -1.061 & -0.213 & -0.494 & -0.935 \\
TS4 & 1.013 & - & -0.987 & -0.142 & -0.536 & -0.825 \\
PC & 0.747 & - & -0.805 & -0.256 & -0.536 & -0.673
\end{tabular}

\section{CALB}

\begin{tabular}{cccc}
\hline & $\mathrm{C} 1$ & $\mathrm{O} 2$ & $\mathrm{~N} 4$ \\
\hline RS & $0.85 \pm 0.03$ & $-0.884 \pm 0.012$ & $-0.77 \pm 0.03$ \\
TS1 & $0.92 \pm 0.04$ & $-1.009 \pm 0.020$ & $-0.888 \pm 0.023$ \\
INT1 & $1.04 \pm 0.03$ & $-1.104 \pm 0.017$ & $-0.89 \pm 0.03$ \\
TS2 & $0.85 \pm 0.04$ & $-1.086 \pm 0.015$ & $-0.49 \pm 0.03$ \\
INT2 & $0.82 \pm 0.03$ & $-0.806 \pm 0.013$ & $-0.78 \pm 0.04$ \\
TS3 & $0.74 \pm 0.04$ & $-0.965 \pm 0.022$ & - \\
INT3 & $0.73 \pm 0.03$ & $-1.039 \pm 0.015$ & - \\
TS4 & $0.96 \pm 0.04$ & $-1.030 \pm 0.021$ & - \\
PS & $0.76 \pm 0.03$ & $-0.848 \pm 0.013$ & - \\
\hline
\end{tabular}


Table S3. Electrostatic potential (in $\mathrm{kJ} \cdot \mathrm{mol}^{-1} \cdot \mathrm{e}^{-1}$ ) generated by the enzyme on the key atoms of the reaction along the states appearing in the acylation step in Bs2 and CALB.

\begin{tabular}{ccccccccccc}
\hline & & \multicolumn{3}{c}{ Bs2 } & & & \multicolumn{5}{c}{ CALB } \\
& C1 & N4 & O2 & OG & NE2 & C1 & N4 & O2 & OG & NE2 \\
\hline RC & 386.8 & 287.8 & 525.2 & 380.5 & 285.4 & -10.9 & -85.2 & 150.1 & -68.3 & -134.5 \\
TS1 & 387.4 & 289.1 & 528.1 & 373.9 & 288.9 & -3.4 & -76.4 & 169.0 & -58.3 & -131.0 \\
I1 & 393.8 & 290.5 & 536.9 & 385.5 & 280.3 & -0.3 & -73.5 & 178.3 & -52.7 & -132.7 \\
TS2 & 384.9 & 249.5 & 509.4 & 369.3 & 246.7 & -13.5 & -89.6 & 150.6 & -47.0 & -131.5 \\
I2 & 410.9 & 250.7 & 519.8 & 395.4 & 271.4 & -11.2 & -118.6 & 142.5 & -55.8 & -142.6 \\
\hline
\end{tabular}

Table S4. Projection of the electric field created by the proteins Bs2 and CALB in the C-N peptide bond direction, computed in $\mathrm{C} 1$ and $\mathrm{N} 4$ atoms, and the resulting electrostatic forces on the direction of the vector defined from $\mathrm{C} 1$ to $\mathrm{N} 4$ atoms. All values are in a.u.

\begin{tabular}{cccccccccc}
\hline & \multicolumn{4}{c}{ Bs2 } & \multicolumn{3}{c}{ CALB } \\
& \multicolumn{2}{c}{ Electric Field } & \multicolumn{2}{c}{ Force } & \multicolumn{2}{c}{ Electric Field } & \multicolumn{2}{c}{ Force } \\
& $\mathrm{C} 1$ & $\mathrm{~N} 4$ & $\mathrm{C} 1$ & $\mathrm{~N} 4$ & $\mathrm{C} 1$ & $\mathrm{~N} 4$ & $\mathrm{C} 1$ & $\mathrm{~N} 4$ \\
\hline $\mathrm{RC}$ & 0.0138 & 0.0135 & 0.0110 & -0.0117 & 0.0119 & 0.0095 & 0.0101 & -0.0073 \\
TS1 & 0.0129 & 0.0132 & 0.0102 & -0.0112 & 0.0102 & 0.0096 & 0.0094 & -0.0085 \\
INT1 & 0.0134 & 0.0129 & 0.0110 & -0.0107 & 0.0097 & 0.0096 & 0.0101 & -0.0085 \\
TS2 & 0.0110 & 0.0110 & 0.0084 & -0.0100 & 0.0104 & 0.0076 & 0.0089 & -0.0037 \\
INT2 & 0.0128 & 0.0102 & 0.0089 & -0.0095 & 0.007 & 0.0062 & 0.0057 & -0.0048 \\
\hline
\end{tabular}



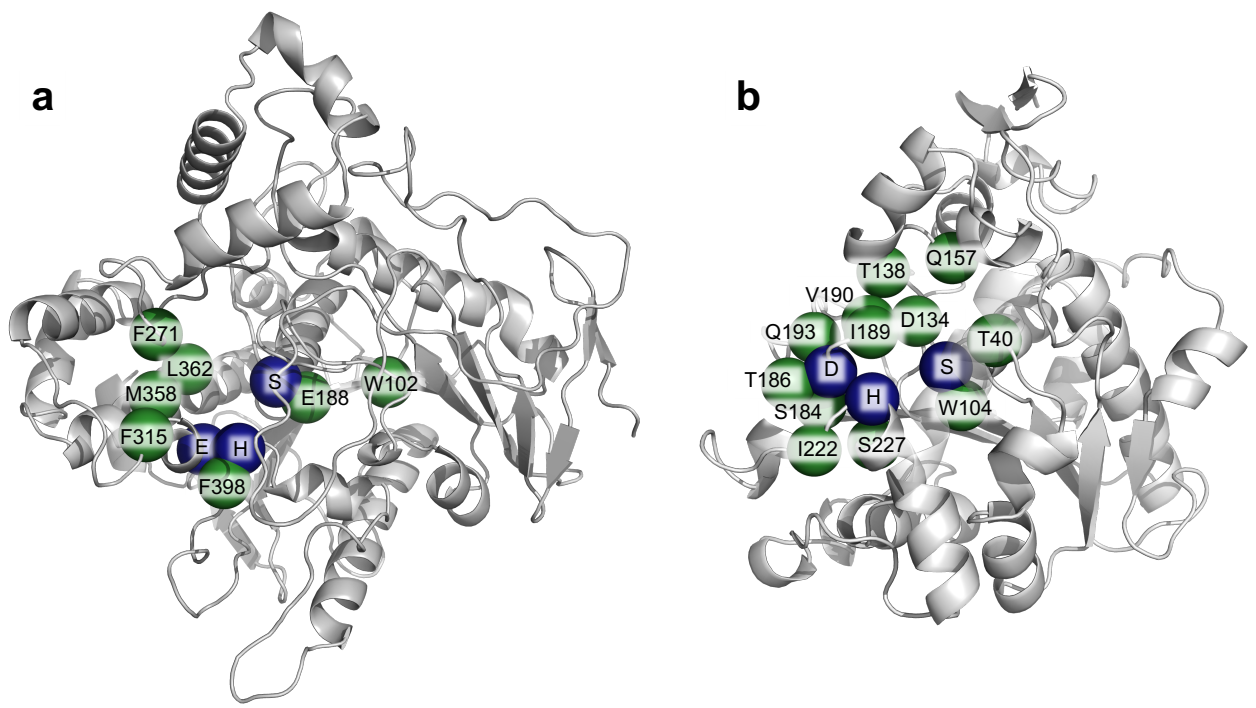

Figure S4. Representation of the key residues identified by the alanine scan using the CNN on both Bs2 (a) and CALB (b). Spheres correspond to the CA of each residue. In blue is marked the catalytic triad while in green the key residues are highlighted. 


\section{COMPUTATIONAL METHODS}

Models set up. Wild type $p$-nitrobenzyl (PNB) esterase sequence from Bacillus subtilis (with ID P37967) that corresponds to the protein used in the experimental measurements ${ }^{4}$ was initially taken from UniProt ${ }^{5}$ accessible resource of protein sequence and functional information. Due to the lack of crystallized structure for this specific variant, the framework for the required model, a natural variant of the protein (strain 168), was prepared based on PNB esterase from the same organism (Bs2; PDB ID: 1QE3). ${ }^{6}$ Both enzymes share $97.6 \%$ of sequence identity. Thus, the X-ray structure was used as a template to build the Bs2 model by adding the missing residues and introducing required mutations. All changes were done using Modeller. ${ }^{7}$ The differences in sequence together with the regions of missing residues are presented in Figure S1.

$N$-(4-nitrophenyl)-butyramide substrate was placed inside the active site pocket covalently bound to catalytic Ser189 in the form of intermediate 1, in order to avoid possible substrate dissociation to the solvent during MD simulations. The protonation state of titratable residues was determined at $\mathrm{pH} 7$ by estimating $\mathrm{pKa}$ shifts generated by the local environment on titratable groups using the empirical program PropKa ver. 3.0.3. ${ }^{8,9}$ The values of $\mathrm{pKa}$ obtained for Glu188 (8.72), Glu214 (7.52), and Glu402 (10.32) ensured their protonated state. Additionally, all histidine residues present in the enzyme were found to be neutral, with pKa values varying between 4.12 and 6.19. After a detailed inspection of surrounding of each histidine residue, it was concluded that all should be protonated in $\mathrm{N} \delta$ position. Thus, once the hydrogen atoms were added to the structure, the 20 counterions ions were placed in the most electrostatically favorable positions in order to neutralize the system. Subsequently, the system was solvated by placing it in a $100 \times 80 \times 80 \AA^{3}$ pre-equilibrated box of TIP3 $\mathrm{P}^{10}$ water molecules. Any water with an oxygen atom lying in a radius of $2.8 \AA$ from a heavy atom of the 
protein was deleted. In order to equilibrate the total system, classical MD simulations were done. For $N$-(4-nitrophenyl)-butyramide the same force field parameters were used as determined in our previous work. ${ }^{11}$ Such prepared model was optimized, then the system was heated to $303 \mathrm{~K}$ with $0.1 \mathrm{~K}$ temperature increment and equilibrated during short (100 ps) NPT MD simulations, the proper $100 \mathrm{~ns}$ of non-accelerated classical MD simulations were done using the NVT ensemble with AMBER force field, ${ }^{12}$ as implemented in NAMD software. ${ }^{13}$ The temperature during the MD simulation was controlled using the Langevin thermostat. ${ }^{14}$ In order to improve the time of simulations, cut-offs for nonbonding interactions were applied using a smooth switching function between 14.5 and $16 \AA$. During MD simulations all atoms were free to move. Periodic boundary conditions were used. Time-dependent evolution of the root mean square deviations (RMDS) together with B-factor are depicted in Figure S2. Thus, one structure from the MD simulation was chosen for further study applying QM/MM approach. The starting structures were selected by its proximity to the average values of RMSD.

QM/MM simulations. In present work, the standard additive hybrid QM/MM scheme was used to construct the total Hamiltonian, $\widehat{\mathrm{H}}_{\mathrm{QM} / \mathrm{MM}}$, where the total energy $E_{Q M / M M}$ is obtained as the sum of specific contributions, as presented in equation 1:

$$
E_{Q M / M M}=\left\langle\Psi\left|\widehat{H_{O}}\right| \Psi\right\rangle+\left(\sum\left\langle\Psi\left|\frac{q_{M M}}{r_{e, M M}}\right| \Psi\right\rangle+\sum \sum \frac{z_{Q M} q_{M M}}{r_{Q M, M M}}\right)+E_{Q M / M M}^{v d W}+E_{M M}
$$

where $E_{M M}$ is the energy of the MM subsystem term, $E_{Q M-M M}^{v d W}$ the van der Waals interaction energy between the QM and MM subsystems and $E_{Q M-M M}^{\text {elect }}$ includes both the Coulombic interaction of the QM nuclei $\left(Z_{Q M}\right)$ and the electrostatic interaction of the polarized electronic wave function $\left(q_{M M}\right)$ with the charges of the protein $\left(q_{M M}\right)$. The region described by quantum 
mechanics includes the side chains of the catalytic Ser189, His399 and Glu310 residues as well as the full substrate, and one water molecule, as shown in Figure 2 of the main text. Three-link atoms ${ }^{15}$ were inserted where the QM/MM boundary intersected covalent bonds: these were placed between the Ca-Cb for Ser189, His299 and Glu310. Finally, in QM part the 55 and 42 atoms were defined including link atoms, for acylation and deacylation process, respectively. The lower number of atoms included in the QM region for the deacylation step is a consequence of the fact that the first product of the studied reaction is leaving the active site in the step preceding this deacylation process. The rest of the protein, counterions and solvent molecules (in total 73077 atoms) were represented by classical OPLS-AA force field ${ }^{16}$ and TIP3P force fields, respectively, as implemented in fDynamo library. ${ }^{17}$ The Austin Model 1 (AM1) $)^{18}$ semiempirical Hamiltonian and the Minnesota Functional M06-2X, ${ }^{19}$ with the standard 6$31+\mathrm{G}(\mathrm{d}, \mathrm{p})$ basis set, were used to treat the QM sub-set of atoms, as implemented in Mopac ${ }^{20}$ and Gaussian $09,{ }^{21}$ respectively. The atom positions of all residues presented beyond $25 \AA$ from the substrate were frozen and the same cut-offs as in MD simulations were applied for the nonbonding interactions.

Potential Energy Surfaces. Potential Energy Surfaces (PES) were explored by choosing and scanning the appropriate combination of internal coordinates $\left(\xi_{i}\right)$ assuming their dominant role in the shape of the reaction coordinate. Thus, a combination of different distances was controlled during exploration of all four chemical steps being part of the complete reaction path. In the first step of the reaction, the PES was generated by controlling the distance between nitrogen, NE2 atom of His399 and hydrogen, HG atoms of Ser189, together with the distance between oxygen, OG atom of Ser189 and carbonyl carbon, C1 atom of a substrate, directing acylation process. In the second step antisymmetric combination of nitrogen, NE2 atom and hydrogen $\mathrm{HG}$, attached to His399 and this hydrogen atom and its acceptor, nitrogen, N4 atom of the substrate, together with carbon-nitrogen (C1-N4) bond of a substrate were controlled. 
PES of the third step that starts the process of deacylation was generated by scanning the antisymmetric combination of distance between oxygen, $\mathrm{O}^{\text {wat }}$ atom of the water molecule and hydrogen, $\mathrm{H}^{\text {wat }}$ of the same molecule and the same hydrogen atom and nitrogen, NE2 atom of His399, together with a distance corresponding to the formation of the covalent bond between oxygen, $\mathrm{O}^{\text {wat }}$ from water and carbon, $\mathrm{C} 1$ atom of the substrate. The final, fourth step of reaction is explored controlling the antisymmetric combination of distance between nitrogen, NE2 atom of His399 and hydrogen, $\mathrm{H}^{\text {wat }}$ atom and the same hydrogen atom and oxygen, OG atom of the Ser189, together with elongation distance between oxygen, OG atom of Ser 189 and carbon, $\mathrm{C} 1$ atom of the substrate.

In order to explore all PESs, the harmonic constraint of $5000 \mathrm{~kJ} \cdot \mathrm{mol}^{-1} \cdot \AA^{-2}$ was used to maintain the proper interatomic distances along the reaction coordinate, and a series of conjugate gradient optimizations and L-BFGS-B optimization algorithms ${ }^{22}$ were applied to obtain the final potential energy of the minimized constrained geometry. The QM sub-set of atoms were described by the Austin Model 1 (AM1) semiempirical Hamiltonian. The distances evolution was controlled by applying small size change of $0.1 \AA$ when the distance between two heavy atoms was explored, or $0.05 \AA$ when the transfer of light hydrogen atom was involved.

A micro-macro iteration optimization algorithm ${ }^{23,24}$ together with the Baker's algorithm ${ }^{25,26}$ was used to localize, optimize, and characterize the transition states (TS) and structures using a Hessian matrix containing all the coordinates of the QM subsystem, whereas the gradient norm of the remaining movable atoms was maintained less than $0.25 \mathrm{kcal} \mathrm{mol}^{-1} \AA^{-1}$. The Intrinsic Reaction Coordinate ${ }^{10}$ (IRC) was traced down from located TSs to the connecting valleys in mass-weighted Cartesian coordinates. And the same micro-macro iteration optimization algorithm was used to optimized reactant complex (RC), intermediates (Is) and product complex (PC). The existence of the saddle-points, as well as those located in minima, was confirmed by frequency calculation. Thus, for TS structures, only one imaginary value of 
frequency was registered, while for structures from located in the minimum of the PESs no imaginary values were found.

Free Energy Surfaces. FESs were obtained, in terms of two-dimensional potential mean force $(2 \mathrm{D}-\mathrm{PMF}),{ }^{27}$ for every step of the reaction using the Umbrella Sampling approach ${ }^{27,28}$ combined with the Weighted Histogram Analysis Method (WHAM) ${ }^{29}$ The procedure for the PMF calculation is straightforward and requires a series of molecular dynamics simulations in which the distinguished reaction coordinate variable, $\xi$, is constrained around particular values. The values of the variables sampled during the simulations are then pieced together to construct a distribution function from which the PMF is obtained as a function of the distinguished reaction coordinate $(\mathrm{W}(\xi))$. The PMF is related to the normalized probability of finding the system at a particular value of the chosen coordinate by eq 2 :

$$
W(\xi)=C-k T \ln \int \rho\left(r^{N}\right) \delta\left(\xi\left(r^{N}\right)-\xi\right) d r^{N-1}
$$

The activation free energy can be then expressed as:

$$
\Delta G^{\ddagger}(\xi)=W\left(\xi^{\ddagger}\right)-\left[W\left(\xi^{R}\right)+G_{\xi}\left(\xi^{R}\right)\right]
$$

where the superscripts indicate the value of the reaction coordinate at the reactants $(\mathrm{R})$, and at the TS $(\dagger)$, and $G_{\xi}\left(\xi^{R}\right)$ is the free energy associated with setting the reaction coordinate to a specific value at the reactant state. Normally this last term makes a small contribution, and the activation free energy is directly estimated from the PMF change between the maximum of the profile and the reactant's minimum:

$$
\Delta G^{\ddagger}(\xi) \approx W\left(\xi^{\ddagger}\right)-W\left(\xi^{R}\right)=\Delta W^{\ddagger}(\xi)
$$

The selection of the reaction coordinate is usually trivial when the mechanism can be driven by a single internal coordinate or a simple combination (as the antisymmetric combination of two interatomic distances). However, this is not the case for all possible steps of the reaction subject of study in this paper where many coordinates are participating. Instead, we were 
compelled to obtain a much more computationally demanding 2D-PMF using two coordinates: $\xi_{1}$ and $\xi_{2}$. The $2 \mathrm{D}-\mathrm{PMF}$ is related to the probability of finding the system at particular values of these two coordinates:

$$
W(\xi)=C^{\prime}-k T \ln \int \rho\left(r^{N}\right) \delta\left(\xi_{1}\left(r^{N}\right)-\xi_{1}\right) \delta\left(\xi_{2}\left(r^{N}\right)-\xi_{2}\right) d r^{N-2}
$$

To estimate the activation free energy from this quantity, we recovered one-dimensional PMF changes tracing a maximum probability reaction path on the 2D-PMF surface and integrating over the perpendicular coordinate.

Thus, a series of MD simulations were performed adding a constraint for the selected reaction coordinates with an umbrella force constant of $2500 \mathrm{~kJ} \cdot \mathrm{mol}^{-1} \cdot \AA^{-2}$. In every window, QM/MM MD simulations were performed with a total of $5 \mathrm{ps}$ of equilibration and $20 \mathrm{ps}$ of production at $303 \mathrm{~K}$ using the Langevin-Verlet algorithm ${ }^{30}$ with a time step of $1 \mathrm{fs}$. Structures obtained in previously computed PESs were used as starting points for the MD simulations in every window. The total number of calculations for each surface is summarized in Table S5.

Table S5. The total amount of calculations required to produce each AM1/MM 2D-PMF surface corrected at M06-2X:AM1/MM level of theory.

\begin{tabular}{cccccc}
\hline & Step 1 & Step 2 & Step 3 & Step 4 & TOTAL \\
\hline Geometry optimization (AM1/MM) & 403 & 1659 & 976 & 854 & 3892 \\
QM/MM MD simulation (AM1/MM) & 403 & 1659 & 976 & 854 & 3892 \\
Single Point Energy calculations (M06-2X/MM) & 403 & 1659 & 976 & 854 & 3892 \\
\hline TOTAL & 1209 & 4977 & 2928 & 2562 & $\mathbf{1 1 6 7 6}$ \\
\hline
\end{tabular}

Spline corrections. In order to improve lower quality results associated with the low-level semiempirical calculations, high-level corrections were applied using Density Functional Theory (DFT). As already described in the literature, ${ }^{31,32}$ a correction term $S\left[\Delta E_{L L}^{H L}\left(\xi_{1}, \xi_{2}\right)\right]$ is interpolated to any value along reaction coordinates in the FES. A continuous energy function is used to obtain the corrected PMFs: 


$$
E=E_{L L / M M}+S\left[\Delta E_{L L}^{H L}\left(\xi_{1}, \xi_{2}\right)\right]
$$

where $S$ is the two-dimensional spline function and $\Delta E_{L L}^{H L}$ is the difference between the energies obtained at low-level (LL) and high-level (HL) of the theory of the QM part. The AM1 semiempirical Hamiltonian was used as LL method, while the DFT method was selected for the HL energy calculation. In particular, HL energy calculations were performed by means of the hybrid M06-2X functional using the standard $6-31+\mathrm{G}(\mathrm{d}, \mathrm{p})$ basis set. These calculations were carried out using the Gaussian09 program.

Phylogeny. The phylogenetic tree was build using a random sample of 150 sequences of proteins belonging to EC 3.1.1.1, EC 3.1.1.3 and EC 3.1.1.4. Fasta sequences were taken from Uniprot $^{33}$ database. Multiple sequence alignment was done using Clustal Omega ${ }^{34}$. Then the tree was constructed using BioPython ${ }^{35}$ library. Distances were calculated using BLOSUM62 3 substitution matrix and the tree was constructed with the UPGMA ${ }^{36}$ clustering method. The final tree was plotted using the $\mathrm{GraPhl}_{\mathrm{An}}^{37}$ package.

3D Convolutional Neural Network. Crystallographic data of enzymes belonging to EC 3.1.1.1, EC 3.1.1.3 and EC 3.1.1.4 was taken from $\mathrm{PDB}^{38}$ database. These proteins were selected based on its classification in Uniprot ${ }^{33}$ database. In order to avoid overrepresentation of some structures, redundancy was cleaned based on sequence alignment between all the set of proteins. All proteins that shared more than $85 \%$ of sequence identity were eliminated. The alignment was done using the Needleman-Wunch ${ }^{1}$ algorithm. A final list of the codes used to build the dataset is showed in Table S6. The catalytic site of each protein was then labeled based on the curated information available in Uniprot. Then, a cube of $20 \times 20 \times 20 \AA^{3}$ was centered on the geometrical center of the catalytic site. Placing the cube in the catalytic site permits the analysis of only the near vicinity of the active site. The cube was then divided into voxels of $1 \AA$ to ensure that only one atom lay inside each voxel. Subsequently, all atoms that 
lay inside the cube were selected and codified into a 13 vector. Each position of the vector represented a specific characteristic of the codified residue or atom, i.e., atom type $(\mathrm{C}, \mathrm{N}, \mathrm{O}$ or S), atom belonging to the backbone, methyl group, negative or positive charge, polar or nonpolar, hydrogen bond donor or acceptor, or aromatic residue. If the characteristic was present in the atom the corresponding position of the vector was set to 1 , and a 0 otherwise. A final cube of dimension $20 \times 20 \times 20 \AA^{3}$ and 13 channels was obtained for each protein of the initial dataset. In order to mimic the electron density in a real protein, a series of Gaussian filters were applied in each channel. These filters were calibrated to resemble de Van der Waals radius of each atom type. This process was done using Python language and NumPy ${ }^{39}$ and $\mathrm{SciPy}^{40}$ libraries.

Table S6. PDB IDs of the proteins used for the CNN training.

\begin{tabular}{|c|c|}
\hline EC number & PDB ID \\
\hline EC 3.1.1.1 & $\begin{array}{l}\text { 2O7V, 5FV4, 1K4Y, 1AUR, 3P2M, 5THM, 3KVN, 1TQH, 4JGG, 2R11, } \\
4 \mathrm{CCY}\end{array}$ \\
\hline EC 3.1.1.3 & $\begin{array}{l}\text { 5CUR, 3RAR, 1THG, 1GZ7, 1THG, 1LLF, 3GUU, 1K8Q, 1HLG, 1HPL, } \\
\text { 1N8S, 1ETH, 1GPL, 2PVS, 1BU8, 4FKB, 5LIP, 2ES4, 1EX9, 6QPR, } \\
\text { 1LGY, } 6 \text { XS3 }\end{array}$ \\
\hline EC 3.1.1.4 & $\begin{array}{l}\text { 1QD6, 4BP2, 5IZR, 1TC8, 1OZ6, 1POB, 1GP7, 1M8T, 1P7O, 4RFP, 1PP2, } \\
\text { 1IJL, 1VAP, 1BUN, 1OO1, 6AL3, 5TFV, 2NOT, 5VET, 3G8G, 4HG9, } \\
\text { 1PO8, 1VIP, 1RGB, 2GHN, }\end{array}$ \\
\hline
\end{tabular}

In order to up-sample the dataset a series of random rotations were applied to the cubes in $\mathrm{x}, \mathrm{y}$ and $\mathrm{z}$ axis. Due to the different number of proteins in each of the EC classes, the dataset was balanced by upsampling each class independently to obtain an equal number of samples per class in the dataset. The network was trained with a ratio train:test:validation of 0.7:0.15:0.15. In this case, we are not talking about a specific number of samples in each dataset because the 
cubes and the random rotations were done on the fly while the network was trained so each mini batch was in this sense different. For that reason, we trained until convergency of the loss. It must be said that our aim is not to obtain a general classifier, which would require a much bigger and diverse protein set, but a tool that permits the analysis of Bs2 and CALB. For that reason, structures of $\mathrm{Bs} 2$ and $\mathrm{CALB}$ were not used in the $\mathrm{CNN}$ training.

A ResNet $50^{41}$ architecture was chosen to build the CNN. In this case a set of 3D convolutional layers and 3D max pooling layers were applied maintaining the original architecture of the network. For the training of the $\mathrm{CNN}$, the Adam ${ }^{42}$ optimizer was used and the categorical crossentropy was chosen a loss function. A mini batch of 16 was set for each iteration, and an exponential decay learning rate was scheduled (starting at $10^{-4}$ ). The $\mathrm{CNN}$ was build using Keras v.2.4.3 ${ }^{43}$ and Tensorflow v.2.4.1. ${ }^{44}$

In order to validate the $\mathrm{CNN}$ for our particular task, 1000 samples applying random rotations of Bs2 and CALB were predicted, and the ratio of correct classifications was used as measure of the performance of the $\mathrm{CNN}$.

Finally, an alanine scan was done in order to figure out which are the structural determinants in both Bs2 and CALB. For that, all the residues that had an atom inside the cube were mutated to alanine consecutively, obtaining a library of mutants. The mutations were introduced using Modeller. ${ }^{7}$ Then, 1000 predictions were done in the same way as explained before. With this strategy we were able to highlight which are the residues that the CNN has learned to be crucial for its correct classification. A classification score was computed by the difference between the classification ratio of the actual class and the ratio of the most probable of the remaining classes (equation 7).

$$
S=\frac{c_{i 1}}{t_{i 1}}-\frac{c_{\max (i 2, i 3)}}{t_{\max (i 2, i 3)}}
$$


In the above equation $s$ stands for the score, $c$ is the number of correctly classified instances, $t$ is the total number of instances, $i 1$ stands for the actual class and $i 2$ and $i 3$ are the rest of the classes. In this sense a score of 1 corresponds to a perfectly classified mutant and a score of -1 is the one completely misclassified. Finally, after each iteration the mutation that dropped the score the most was accumulated for the next iteration. In this way we performed up to 5 iterations. 


\section{STRUCTURES COORDINATES}

Table S7. X, Y and Z coordinates of QM atoms for Transition State Structures optimized at

M06-2X/MM level for acylation step of the reaction of hydrolysis taking place in the active

site of Bs2.

\begin{tabular}{|c|c|c|c|c|c|c|c|}
\hline \multicolumn{8}{|c|}{ Acylation step } \\
\hline \multicolumn{4}{|c|}{ Transition State $1\left(v_{\mathrm{i}}=-907.500 \mathrm{~cm}^{-1}\right)$} & \multicolumn{4}{|c|}{ Transition State $2\left(v_{\mathrm{i}}=-103.200 \mathrm{~cm}^{-1}\right)$} \\
\hline Atoms & $\mathrm{X}$ & $y$ & $\mathrm{Z}$ & Atoms & $\mathrm{x}$ & $y$ & Z \\
\hline $\mathrm{C}$ & 45.471 & 44.021 & 52.769 & $\mathrm{C}$ & 45.424 & 43.836 & 52.633 \\
\hline $\mathrm{H}$ & 46.369 & 44.586 & 53.017 & $\mathrm{H}$ & 46.267 & 44.347 & 53.096 \\
\hline $\mathrm{H}$ & 45.012 & 44.525 & 51.912 & $\mathrm{H}$ & 45.095 & 44.428 & 51.780 \\
\hline $\mathrm{O}$ & 44.583 & 44.140 & 53.888 & $\mathrm{O}$ & 44.392 & 43.777 & 53.624 \\
\hline $\mathrm{H}$ & 45.068 & 44.609 & 54.901 & $\mathrm{H}$ & 44.783 & 45.514 & 55.661 \\
\hline $\mathrm{C}$ & 48.614 & 41.110 & 59.894 & $\mathrm{C}$ & 48.545 & 41.092 & 59.950 \\
\hline $\mathrm{H}$ & 48.447 & 40.206 & 60.490 & $\mathrm{H}$ & 48.411 & 40.169 & 60.524 \\
\hline $\mathrm{H}$ & 47.796 & 41.151 & 59.168 & $\mathrm{H}$ & 47.710 & 41.143 & 59.244 \\
\hline $\mathrm{C}$ & 49.925 & 40.969 & 59.125 & $\mathrm{C}$ & 49.832 & 40.982 & 59.140 \\
\hline $\mathrm{H}$ & 50.803 & 41.082 & 59.768 & $\mathrm{H}$ & 50.727 & 41.148 & 59.747 \\
\hline $\mathrm{H}$ & 49.968 & 39.959 & 58.706 & $\mathrm{H}$ & 49.912 & 39.968 & 58.737 \\
\hline $\mathrm{C}$ & 50.101 & 41.910 & 57.939 & $\mathrm{C}$ & 49.963 & 41.891 & 57.932 \\
\hline $\mathrm{O}$ & 49.231 & 42.789 & 57.701 & $\mathrm{O}$ & 49.151 & 42.831 & 57.715 \\
\hline $\mathrm{O}$ & 51.104 & 41.728 & 57.191 & $\mathrm{O}$ & 50.920 & 41.605 & 57.151 \\
\hline $\mathrm{C}$ & 47.478 & 45.820 & 59.213 & $\mathrm{C}$ & 47.505 & 45.848 & 59.446 \\
\hline $\mathrm{H}$ & 47.161 & 45.209 & 60.063 & $\mathrm{H}$ & 47.237 & 45.187 & 60.271 \\
\hline $\mathrm{H}$ & 47.113 & 46.835 & 59.398 & $\mathrm{H}$ & 47.168 & 46.856 & 59.701 \\
\hline $\mathrm{C}$ & 46.789 & 45.274 & 57.965 & $\mathrm{C}$ & 46.761 & 45.428 & 58.203 \\
\hline $\mathrm{N}$ & 47.002 & 43.974 & 57.581 & $\mathrm{~N}$ & 47.007 & 44.249 & 57.528 \\
\hline $\mathrm{H}$ & 47.748 & 43.340 & 57.945 & $\mathrm{H}$ & 47.784 & 43.552 & 57.749 \\
\hline $\mathrm{C}$ & 46.346 & 43.741 & 56.448 & $\mathrm{C}$ & 46.236 & 44.191 & 56.450 \\
\hline $\mathrm{H}$ & 46.373 & 42.798 & 55.924 & $\mathrm{H}$ & 46.221 & 43.390 & 55.728 \\
\hline $\mathrm{N}$ & 45.670 & 44.812 & 56.056 & $\mathrm{~N}$ & 45.482 & 45.289 & 56.398 \\
\hline $\mathrm{C}$ & 45.939 & 45.792 & 57.006 & $\mathrm{C}$ & 45.797 & 46.084 & 57.482 \\
\hline $\mathrm{H}$ & 45.520 & 46.785 & 56.933 & $\mathrm{H}$ & 45.339 & 47.048 & 57.642 \\
\hline $\mathrm{C}$ & 42.965 & 44.864 & 53.581 & $\mathrm{C}$ & 43.165 & 44.284 & 53.353 \\
\hline $\mathrm{C}$ & 42.155 & 43.939 & 54.483 & $\mathrm{C}$ & 42.176 & 43.756 & 54.351 \\
\hline $\mathrm{H}$ & 42.620 & 43.909 & 55.475 & $\mathrm{H}$ & 42.625 & 43.770 & 55.348 \\
\hline $\mathrm{H}$ & 41.175 & 44.422 & 54.612 & $\mathrm{H}$ & 41.296 & 44.403 & 54.364 \\
\hline $\mathrm{C}$ & 41.971 & 42.540 & 53.924 & $\mathrm{C}$ & 41.829 & 42.320 & 53.930 \\
\hline $\mathrm{C}$ & 41.008 & 41.711 & 54.765 & $\mathrm{C}$ & 40.984 & 41.592 & 54.962 \\
\hline $\mathrm{H}$ & 40.026 & 42.197 & 54.820 & $\mathrm{H}$ & 40.051 & 42.131 & 55.157 \\
\hline $\mathrm{H}$ & 40.866 & 40.716 & 54.333 & $\mathrm{H}$ & 40.732 & 40.588 & 54.608 \\
\hline $\mathrm{H}$ & 41.371 & 41.594 & 55.791 & $\mathrm{H}$ & 41.518 & 41.496 & 55.912 \\
\hline $\mathrm{H}$ & 41.601 & 42.614 & 52.894 & $\mathrm{H}$ & 41.310 & 42.342 & 52.963 \\
\hline $\mathrm{H}$ & 42.947 & 42.048 & 53.863 & $\mathrm{H}$ & 42.755 & 41.760 & 53.759 \\
\hline $\mathrm{O}$ & 42.813 & 44.812 & 52.321 & $\mathrm{O}$ & 42.850 & 44.625 & 52.203 \\
\hline $\mathrm{N}$ & 43.052 & 46.149 & 54.233 & $\mathrm{~N}$ & 43.369 & 46.220 & 54.495 \\
\hline $\mathrm{H}$ & 42.399 & 46.277 & 55.018 & $\mathrm{H}$ & 42.617 & 46.411 & 55.179 \\
\hline $\mathrm{C}$ & 43.757 & 47.256 & 53.858 & $\mathrm{C}$ & 43.906 & 47.351 & 54.061 \\
\hline $\mathrm{C}$ & 44.609 & 47.292 & 52.731 & $\mathrm{C}$ & 44.810 & 47.361 & 52.934 \\
\hline $\mathrm{C}$ & 45.345 & 48.423 & 52.439 & $\mathrm{C}$ & 45.486 & 48.487 & 52.557 \\
\hline $\mathrm{C}$ & 45.255 & 49.543 & 53.273 & $\mathrm{C}$ & 45.330 & 49.683 & 53.298 \\
\hline $\mathrm{C}$ & 44.403 & 49.549 & 54.387 & $\mathrm{C}$ & 44.398 & 49.738 & 54.365 \\
\hline $\mathrm{C}$ & 43.656 & 48.423 & 54.662 & $\mathrm{C}$ & 43.673 & 48.629 & 54.698 \\
\hline $\mathrm{H}$ & 43.001 & 48.407 & 55.530 & $\mathrm{H}$ & 42.961 & 48.664 & 55.518 \\
\hline $\mathrm{H}$ & 44.332 & 50.431 & 55.020 & $\mathrm{H}$ & 44.265 & 50.667 & 54.912 \\
\hline $\mathrm{N}$ & 46.066 & 50.696 & 53.001 & $\mathrm{~N}$ & 46.135 & 50.785 & 53.025 \\
\hline $\mathrm{O}$ & 46.685 & 50.767 & 51.945 & $\mathrm{O}$ & 46.878 & 50.803 & 52.033 \\
\hline $\mathrm{O}$ & 46.130 & 51.588 & 53.856 & $\mathrm{O}$ & 46.117 & 51.751 & 53.824 \\
\hline $\mathrm{H}$ & 45.996 & 48.423 & 51.571 & $\mathrm{H}$ & 46.156 & 48.476 & 51.705 \\
\hline $\mathrm{H}$ & 44.677 & 46.432 & 52.084 & $\mathrm{H}$ & 44.943 & 46.439 & 52.383 \\
\hline $\mathrm{H}$ & 48.478 & 45.821 & 59.198 & $\mathrm{H}$ & 48.503 & 45.848 & 59.373 \\
\hline $\mathrm{H}$ & 48.571 & 41.899 & 60.507 & $\mathrm{H}$ & 48.511 & 41.867 & 60.580 \\
\hline $\mathrm{H}$ & 45.730 & 43.098 & 52.486 & $\mathrm{H}$ & 45.696 & 42.917 & 52.346 \\
\hline
\end{tabular}


Table S8. X, Y and Z coordinates of QM atoms for Transition State Structures optimized at M06-2X/MM level for deacylation step of the reaction of hydrolysis taking place in the active site of Bs2.

\begin{tabular}{|c|c|c|c|c|c|c|c|}
\hline \multicolumn{8}{|c|}{ Deacylation step } \\
\hline \multicolumn{4}{|c|}{ Transition State $3\left(v_{i}=-646.598 \mathrm{~cm}^{-1}\right)$} & \multicolumn{4}{|c|}{ Transition State $4\left(v_{i}=-1212.657 \mathrm{~cm}^{-1}\right)$} \\
\hline Atoms & $\mathrm{X}$ & $y$ & $\mathrm{Z}$ & Atoms & $\mathrm{X}$ & $y$ & $Z$ \\
\hline $\mathrm{C}$ & 5.472 & 0.119 & -1.252 & $\mathrm{C}$ & 52.634 & 45.258 & 40.947 \\
\hline $\mathrm{H}$ & 5.977 & -0.698 & -1.770 & $\mathrm{H}$ & 52.915 & 44.309 & 40.494 \\
\hline $\mathrm{H}$ & 4.637 & 0.453 & -1.873 & $\mathrm{H}$ & 51.846 & 45.686 & 40.321 \\
\hline $\mathrm{O}$ & 6.414 & 1.177 & -1.069 & $\mathrm{O}$ & 53.801 & 46.107 & 40.837 \\
\hline $\mathrm{H}$ & 8.058 & 1.197 & -2.973 & $\mathrm{H}$ & 54.845 & 45.575 & 40.245 \\
\hline $\mathrm{C}$ & 12.640 & -2.987 & 1.405 & $\mathrm{C}$ & 59.844 & 42.390 & 43.238 \\
\hline $\mathrm{H}$ & 13.245 & -2.843 & 2.307 & $\mathrm{H}$ & 60.339 & 42.777 & 44.134 \\
\hline $\mathrm{H}$ & 11.921 & -2.163 & 1.376 & $\mathrm{H}$ & 59.049 & 43.115 & 43.029 \\
\hline $\mathrm{C}$ & 11.854 & -4.286 & 1.521 & $\mathrm{C}$ & 59.193 & 41.050 & 43.577 \\
\hline $\mathrm{H}$ & 12.480 & -5.172 & 1.379 & $\mathrm{H}$ & 59.893 & 40.210 & 43.544 \\
\hline $\mathrm{H}$ & 11.438 & -4.352 & 2.532 & $\mathrm{H}$ & 58.821 & 41.106 & 44.603 \\
\hline $\mathrm{C}$ & 10.657 & -4.418 & 0.586 & $\mathrm{C}$ & 57.987 & 40.662 & 42.733 \\
\hline $\mathrm{O}$ & 10.403 & -3.520 & -0.259 & $\mathrm{O}$ & 57.718 & 41.297 & 41.677 \\
\hline $\mathrm{O}$ & 9.924 & -5.437 & 0.766 & $\mathrm{O}$ & 57.251 & 39.731 & 43.165 \\
\hline $\mathrm{C}$ & 12.010 & -1.891 & -3.329 & $\mathrm{C}$ & 59.049 & 43.151 & 38.627 \\
\hline $\mathrm{H}$ & 12.841 & -1.595 & -2.684 & $\mathrm{H}$ & 59.930 & 43.580 & 39.110 \\
\hline $\mathrm{H}$ & 12.264 & -1.576 & -4.346 & $\mathrm{H}$ & 59.076 & 43.455 & 37.577 \\
\hline $\mathrm{C}$ & 10.780 & -1.107 & -2.917 & $\mathrm{C}$ & 57.805 & 43.803 & 39.284 \\
\hline $\mathrm{N}$ & 10.169 & -1.247 & -1.692 & $\mathrm{~N}$ & 57.510 & 43.559 & 40.605 \\
\hline $\mathrm{H}$ & 10.413 & -1.957 & -0.978 & $\mathrm{H}$ & 57.873 & 42.744 & 41.175 \\
\hline $\mathrm{C}$ & 9.129 & -0.404 & -1.632 & $\mathrm{C}$ & 56.418 & 44.238 & 40.951 \\
\hline $\mathrm{H}$ & 8.469 & -0.306 & -0.783 & $\mathrm{H}$ & 55.982 & 44.223 & 41.936 \\
\hline $\mathrm{N}$ & 9.025 & 0.296 & -2.754 & $\mathrm{~N}$ & 55.950 & 44.959 & 39.940 \\
\hline $\mathrm{C}$ & 10.052 & -0.139 & -3.571 & $\mathrm{C}$ & 56.821 & 44.692 & 38.876 \\
\hline $\mathrm{H}$ & 10.203 & 0.263 & -4.562 & $\mathrm{H}$ & 56.686 & 45.134 & 37.900 \\
\hline $\mathrm{C}$ & 6.260 & 2.364 & -1.801 & $\mathrm{C}$ & 53.511 & 47.603 & 40.072 \\
\hline $\mathrm{C}$ & 7.156 & 3.395 & -1.126 & $\mathrm{C}$ & 54.524 & 48.488 & 40.797 \\
\hline $\mathrm{H}$ & 8.170 & 2.988 & -1.043 & $\mathrm{H}$ & 55.488 & 47.968 & 40.848 \\
\hline $\mathrm{H}$ & 7.207 & 4.281 & -1.770 & $\mathrm{H}$ & 54.683 & 49.362 & 40.149 \\
\hline $\mathrm{C}$ & 6.629 & 3.758 & 0.259 & $\mathrm{C}$ & 54.091 & 48.962 & 42.176 \\
\hline $\mathrm{C}$ & 7.575 & 4.691 & 0.997 & $\mathrm{C}$ & 55.120 & 49.914 & 42.783 \\
\hline $\mathrm{H}$ & 7.727 & 5.620 & 0.433 & $\mathrm{H}$ & 54.790 & 50.291 & 43.755 \\
\hline $\mathrm{H}$ & 7.179 & 4.958 & 1.982 & $\mathrm{H}$ & 56.088 & 49.420 & 42.925 \\
\hline $\mathrm{H}$ & 8.557 & 4.229 & 1.135 & $\mathrm{H}$ & 55.289 & 50.777 & 42.127 \\
\hline $\mathrm{H}$ & 5.637 & 4.217 & 0.166 & $\mathrm{H}$ & 53.117 & 49.456 & 42.099 \\
\hline $\mathrm{H}$ & 6.488 & 2.842 & 0.841 & $\mathrm{H}$ & 53.942 & 48.098 & 42.834 \\
\hline $\mathrm{O}$ & 5.082 & 2.706 & -2.148 & $\mathrm{O}$ & 52.273 & 47.883 & 40.158 \\
\hline $\mathrm{O}$ & 7.168 & 1.959 & -3.165 & $\mathrm{O}$ & 53.947 & 47.299 & 38.769 \\
\hline $\mathrm{H}$ & 7.495 & 2.779 & -3.577 & $\mathrm{H}$ & 54.749 & 47.829 & 38.530 \\
\hline $\mathrm{H}$ & 11.956 & -2.889 & -3.305 & $\mathrm{H}$ & 59.137 & 42.157 & 38.680 \\
\hline $\mathrm{H}$ & 13.245 & -2.944 & 0.609 & $\mathrm{H}$ & 60.508 & 42.395 & 42.490 \\
\hline $\mathrm{H}$ & 5.153 & -0.191 & -0.356 & $\mathrm{H}$ & 52.261 & 45.073 & 41.856 \\
\hline
\end{tabular}




\section{EXPERIMENTAL METHODS}

General information. Deionized water was obtained by an Elga PURELAB Option system (15 M $\Omega \cdot \mathrm{cm})$. Analytical Thin Layer Chromatography (TLC) was carried out with silica gel 60 F254 aluminum sheets from Merck. Detection was carried out using UV light $(\lambda=254 \mathrm{~nm}$ and $366 \mathrm{~nm}$ ), followed by immersion in permanganate or cerium ammonium molybdate staining solution with subsequent development via careful heating with a heat gun. Flash column chromatography was performed using silica gel (pore size $60 \AA, 0.040-0.063 \mathrm{~mm}$ ). $\mathrm{N}$-(4nitrophenyl)-butyramide was synthesized using a known procedure. ${ }^{11}$ All other solvents and reagents were obtained from commercial sources and used as received.

Plasmid miniprep-kit and gel extraction-kit were purchased from Qiagen. DNA oligos were purchased from Sigma-Aldrich. The gene encoding for wild-type Bs $2^{4}$ with a C-terminal Histag (see below) was purchased as a double-stranded fragment form Thermo Fisher Scientifcic GeneArt. Gibson Assembly was performed using New England Biolabs NEBuilder ${ }^{\circledR}$ HiFi DNA Assembly master mix. Restriction enzymes and required reagents were obtained from Thermo Fisher Scientific. Takara PrimeSTAR Max was employed for site-directed mutagenesis. All kits and enzymes were used exactly following the maufacturers' protocols. DNA sequencing of constructed plasmids and mutants was obtained from Eurofins Genomics using T7 promoter and terminator primers.

A VWR 3510 benchtop $\mathrm{pH}$ Meter connected to a Jenway micro $\mathrm{pH}$ electrode or a $V W R$ Universal $\mathrm{pH}$ electrode were used for the $\mathrm{pH}$ adjustment of buffers and reaction mixtures employing either $1.0 \mathrm{M}$ or $0.1 \mathrm{M}$ sodium hydroxide solution or hydrochloric acid.

Size exclusion chromatography was performed using a GE Healthcare ÄKTA Purifier workstation or a Bio-Rad NGC Medium-Pressure Liquid Chromatography System. 
Protein concentrations were determined using a Thermo Scientific NanoDrop One spectrophotometer measuring the absorption at $280 \mathrm{~nm}$ using the

Protein liquid chromatography-mass spectrometry (LC-MS) was performed on a Waters Acquity H-Class UPLC system combined with a Waters Synapt G2-Si quadrupole time of flight mass spectrometer. A Waters Acquity UPLC Protein C4 BEH column $300 \AA, 1.7 \mu \mathrm{m}(2.1 \times$ $100 \mathrm{~mm}$ ) held at $60{ }^{\circ} \mathrm{C}$ was applied. A flow rate of $0.2 \mathrm{~mL} / \mathrm{min}$ and the gradient of eluents A and B highlighted below were employed.

\begin{tabular}{c|c|c} 
Time / $\min$ & $\mathbf{A}\left(\mathbf{H}_{\mathbf{2}} \mathbf{O}, \mathbf{0 . 1} \% \mathbf{C H O} \mathbf{H}_{\mathbf{H}}\right) / \%$ & $\begin{array}{c}\mathbf{B}\left(\mathbf{A C N}, \mathbf{0 . 1} \% \mathbf{C H O} \mathbf{H}_{\mathbf{H}}\right) / \\
\text { \% }\end{array}$ \\
\hline 0 & 95 & 5 \\
3 & 95 & 5 \\
50 & 35 & 65 \\
52 & 3 & 97 \\
54 & 3 & 97 \\
56 & 95 & 5 \\
60 & 95 & 5
\end{tabular}

The data was collected in positive electrospray ionization mode and analyzed using Waters MassLynx 4.1. Deconvoluted mass spectra were generate using the maximum entropy 1 (MaxEnt 1) software.

Cloning, expression and purification of recombinant proteins. The gene encoding for the wild-type Bs2 with a C-terminal linker and His-tag (GSSHHHHHHSSG), and 27 and 20 bases either side complimentary to the vector was purchased as a double-stranded fragment. After a NcoI and BamHI restriction enzyme digested pET28a vector the wild-type Bs2 gene was cloned in-between by Gibson assembly. The incorporation of the wild-type Bs2 gene was confirmed by DNA sequencing. 
The plasmid containing the gene for wild-type Bs2 was transformed into $\mathrm{Ca}^{2+}$ chemically competent BL21 (DE3) cells and grown on LB agar plates supplemented with kanamycin (50 $\mu \mathrm{g} / \mathrm{mL}$ ) at $37^{\circ} \mathrm{C}$ overnight. One colony from the plate was picked to inoculate a $10 \mathrm{~mL} \mathrm{LB}$ starter culture containing kanamycin $(50 \mu \mathrm{g} / \mathrm{mL})$ and grown at $37^{\circ} \mathrm{C}$ and $180 \mathrm{rpm}$ overnight. The starter culture was diluted into $1 \mathrm{~L}$ of fresh LB media containing kanamycin $(50 \mu \mathrm{g} / \mathrm{mL})$. The cells were grown at $37{ }^{\circ} \mathrm{C}$ and $200 \mathrm{rpm}$, until they reached an OD600 of 0.8 , and IPTG was added to reach a final concentration of $1.0 \mathrm{mM}$. The cells were then incubated overnight at $20{ }^{\circ} \mathrm{C}$. The cultures were harvested by centrifugation $\left(4,000 \mathrm{rpm}, 4{ }^{\circ} \mathrm{C}, 30 \mathrm{~min}\right)$ and the dry pellet was stored at $-20^{\circ} \mathrm{C}$.

The pellet was subjected to a freeze-thaw cycle, resuspended in $25 \mathrm{~mL}$ of lysis buffer 1 (50 $\mathrm{mM} \mathrm{NaP}, 300 \mathrm{mM} \mathrm{NaCl}, \mathrm{pH} 7.5$ ) and lysed by sonication (7 min, $5 \mathrm{~s}$ on, $10 \mathrm{~s}$ off). The insoluble fraction was removed by centrifugation at $18000 \mathrm{rpm}$ for $25 \mathrm{~min}$ at $4{ }^{\circ} \mathrm{C}$. The supernatant was mixed with $3 \mathrm{~mL}$ of Ni-NTA affinity resin for His-tag affinity purification which was equilibrated with the lysis buffer. After incubation at $4{ }^{\circ} \mathrm{C}$ for $0.5 \mathrm{~h}$ the resin was washed twice with 1.5 volumes of resin wash buffer $1(50 \mathrm{mM} \mathrm{NaP}, 300 \mathrm{mM} \mathrm{NaCl}, 10 \mathrm{mM}$ imidazole, $\mathrm{pH}$ 7.5). The protein was eluted with elution buffer $(5 \times$ resin volume, $50 \mathrm{mM} \mathrm{NaP}$, $300 \mathrm{mM} \mathrm{NaCl}, 250 \mathrm{mM}$ imidazole, $\mathrm{pH}$ 7.5). Samples of the wash and elution fractions were collected and run on SDS-PAGE gel $(12 \% \mathrm{w} / \mathrm{v})$. The elution fractions containing the respective Bs2 variant were pooled and concentrated to $5 \mathrm{~mL}$ using Amicon ultra centrifugation with a $10 \mathrm{kDa}$ cut-off. The concentrated protein solution was applied to size exclusion chromatography (Generon ProSEC 26/60 3-70 HR column, $50 \mathrm{mM} \mathrm{NaP}$, pH 7.0). Fractions containing protein (analysis by following $280 \mathrm{~nm}$ UV trace) were collected and the samples loaded on SDS-PAGE to check the purity of the protein $(12 \% \mathrm{w} / \mathrm{v})$. Fractions containing Bs2 were pooled, transferred to a centrifugal concentrator with a $10 \mathrm{kDa}$ cut-off, and were concentrated to $15-20 \mathrm{mg} / \mathrm{mL}$ as determined by nanodrop measurement at $280 \mathrm{~nm}$, with $\varepsilon_{280}=$ 
$80330 \mathrm{M}^{-1} \mathrm{~cm}^{-1}$ calculated using https://web.expasy.org/protparam/. The protein solutions were stored at $4{ }^{\circ} \mathrm{C}$ until further usage within a week. It should be noted that the solutions showed no significant loss in activity over 4 weeks (longer stability has not been monitored) and only minimal protein precipitation was observed.

\section{Nucleotide sequence.}

ATGACCCACCAGATTGTTACCACACAGTATGGTAAAGTGAAAGGCACCACCGAAAATGGTGT TCATAAATGGAAAGGTATCCCGTATGCAAAACCGCCTGTTGGTCAGTGGCGTTTTAAAGCAC CGGAACCGCCTGAAGTTTGGGAAGATGTTCTGGATGCAACCGCATATGGTAGCATTTGTCCG CAGCCGAGCGATCTGCTGAGCCTGAGCTATACCGAACTGCCTCGTCAGAGCGAAGATTGTCT GTATGTTAATGTTTTTGCACCGGATACGCCGAGCAAAAATCTGCCGGTTATGGTTTGGATTC ATGGTGGTGCATTTTATCTTGGTGCAGGTAGCGAACCGCTGTATGATGGTAGCAAACTGGCA GCACAGGGTGAAGTTATTGTTGTTACCCTGAATTATCGTCTGGGTCCGTTTGGTTTTCTGCA TCTGAGCAGCTTTAATGAAGCCTATAGCGATAATCTGGGTCTGCTGGATCAGGCAGCAGCAC TGAAATGGGTTCGTGAAAACATTAGCGCATTTGGTGGTGATCCGGATAATGTTACCGTTTTT GGTGAAAGTGCCGGTGGTATGAGCATTGCAGCACTGCTGGCCATGCCTGCAGCAAAAGGTCT GTTTCAGAAAGCAATTATGGAAAGCGGTGCAAGCCGTACCATGACCAAAGAACAGGCAGCAA GTACCAGCGCAGCATTTCTGCAGGTTCTGGGTATTAATGAAGGTCAGCTGGATAAACTGCAT ACCGTTAGCGCAGAAGATTTACTGAAAGCAGCAGATCAGCTGCGTATTGCAGAAAAAGAAAA CATCTTTCAGCTGTTTTTTCAGCCTGCACTGGATCCGAAAACACTGCCGGAAGAACCGGAAA AAGCAATTGCAGAAGGTGCAGCAAGCGGTATTCCGCTGCTGATTGGTACAACCCGTGATGAA GGTTACCTGTTTTTTACTCCGGATAGTGATGTTCATAGCCAAGAAACCCTGGATGCAGCCCT GGAATATCTGCTGGGTAAACCGCTGGCCGAAAAAGTTGCAGATCTGTATCCGCGTAGCCTGG AAAGCCAGATTCATATGATGACGGATCTGCTGTTTTGGCGTCCGGCAGTTGCATATGCCAGC GCACAGAGCCATTATGCACCGGTTTGGATGTATCGTTTTGATTGGCATCCGAAAAAACCTCC GTATAACAAAGCATTTCATGCACTGGAACTGCCGTTTGTTTTTGGTAATCTGGATGGTCTGG AACGTATGGCAAAAGCAGAAATTACCGATGAAGTGAAACAACTGAGCCATACCATTCAGAGC GCATGGATTACCTTTGCAAAAACCGGTAATCCGAGCACCGAAGCAGTTAATTGGCCTGCATA TCATGAAGAAACCCGTGAAACCCTGATTCTGGATAGCGAAATTACCATTGAAAATGATCCGG AAAGCGAGAAACGTCAGAAACTGTTTCCGAGCAAAGGTGAAGGTAGCAGCCATCACCATCAT CATCATAGCAGTGGTTAA

\section{Protein sequence.}

MTHQ IVTTQYGKVKGTTENGVHKWKGI PYAKP PVGQWRFKAPEPPEVWEDVLDATAYGS ICP QPSDLLSLSYTELPRQSEDCLYVNVFAPDTPSKNLPVMVWI HGGAFYLGAGSEPLYDGSKLA AQGEVIVVTLNYRLGPFGFLHLSSFNEAYSDNLGLLDQAAALKWVRENISAFGGDPDNVTVF GESAGGMS IAALLAMPAAKGLFQKAIMESGASRTMTKEQAASTSAAFLQVLGINEGQLDKLH TVSAEDLLKAADQLRIAEKENI FQLFFQPALDPKTLPEEPEKAIAEGAAS GI PLL IGTTRDE GYLFFTPDSDVHSQETLDAALEYLLGKPLAEKVADLYPRSLESQI HMMTDLLFWRPAVAYAS AQSHYAPVWMYRF DWHPKKPPYNKAFHALELPFVFGNLDGLERMAKAEITDEVKQLSHTIQS AWITEAKTGNPSTEAVNWPAYHEETRETLILDSEITIENDPESEKRQKLFPSKGEGSSHHHH HHSSG*

Protein Mass. $\quad$ wild-type Bs2 $(-\mathrm{Met})=55140.21 \mathrm{Da}$ 
96 well-plate kinetic assay. Stock solutions of the wild-type $\mathrm{Bs} 2$ (in $50 \mathrm{mM} \mathrm{NaP}, \mathrm{pH} 7.0$ ) and $N$-(4-nitrophenyl)-butyramide (in DMSO) were prepared. The protein stock solution was kept on ice until use and was freshly prepared before each usage. DMSO and substrate stock solution were added to wells of a 96 transparent well-plate to a total of $15 \mu \mathrm{L}$. Buffer $(50 \mathrm{mM} \mathrm{NaP}$, $\mathrm{pH}$ 7.0) was added to a total volume of $135 \mu \mathrm{L}(150 \mu \mathrm{L}$ in case of controls to monitor substrate stability). The plate was transferred into a platereader, double orbitally shaken for $5 \mathrm{~s}$ and the absorption at $\lambda_{\mathrm{Ex}}=405 \mathrm{~nm}$ measured to check correct substrate distribution. Then $15 \mu \mathrm{L}$ of protein stock solution were added to each well except the enzyme free controls within 5 min. Final assay conditions were $150 \mu \mathrm{L}$ volume, $10 \%$ DMSO, $N$-(4-nitrophenyl)-butyramide (10, $50,100,250500,1000,2000,3000 \mu \mathrm{M}), 20 \mu \mathrm{g} / \mathrm{mL}$ protein. The plate was sealed with an airtight and UV-Vis transparent self-adhesive plastic cover sheet. After sealing, the plate was placed into the plate reader and the assay was monitored using the following program:

Temperature: $\quad 21.0 \pm 1.0^{\circ} \mathrm{C}$

Number of repeats: $\quad 42$

Delay between repeats: $1200 \mathrm{~s}$

Shaking duration: $\quad 5.0 \mathrm{~s}$

Shaking diameter: $\quad 0.70 \mathrm{~mm}$

Shaking type: Double orbit

Delay duration: $\quad 5.0 \mathrm{~s}$

Wavelength $\lambda_{\text {Ex }}$ : $\quad 405 \mathrm{~nm}$

Kinetic assay analysis. In order to convert the absorption reading obtained from the kinetic assay into concentrations for the determination of kinetic data a calibration curve was generated using $10,50,100,250500,1000,2000,3000 \mu \mathrm{M}$ of 4-nitroaniline in $150 \mu \mathrm{L}$ buffer $(50 \mathrm{mM}$ $\left.\mathrm{NaP}_{\mathrm{i}}, \mathrm{pH} 7.0,10 \% \mathrm{DMSO}\right)$. Raw absorption data was converted into product concentrations and data analysis with Origin 2020 was performed to obtained kinetic data assuming Michaelis-Menten kinetics. The maximum velocities $v_{\max }$ were obtained from the linear range of the product concentration vs. time plots and used to calculate $k_{\text {cat }}$ and $K_{\mathrm{M}}$ for wild-type Bs2. 


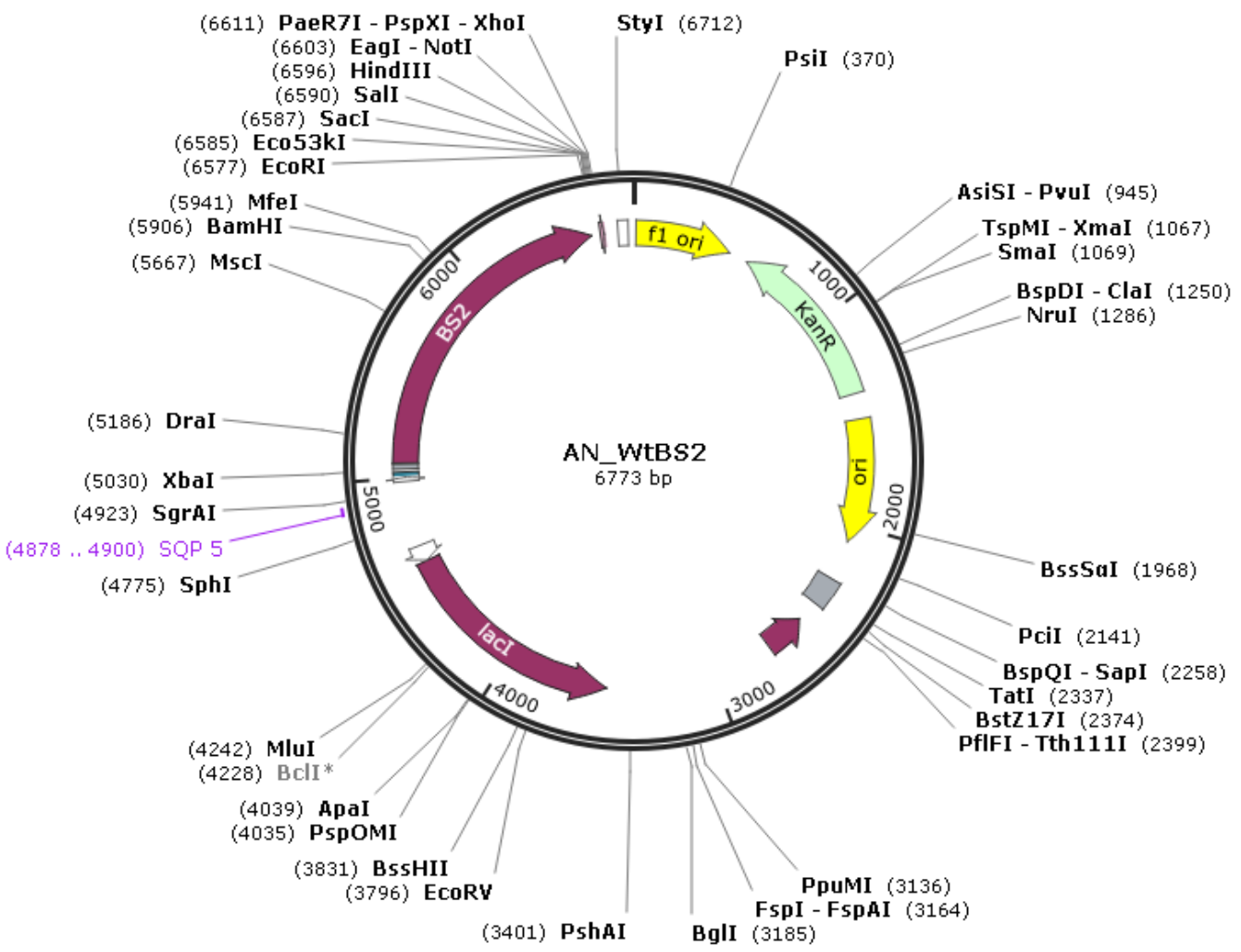

Figure S5. Plasmid map of plasmid with wild-type Bs2 gene in pET-28a vector used for recombinant protein expression. 


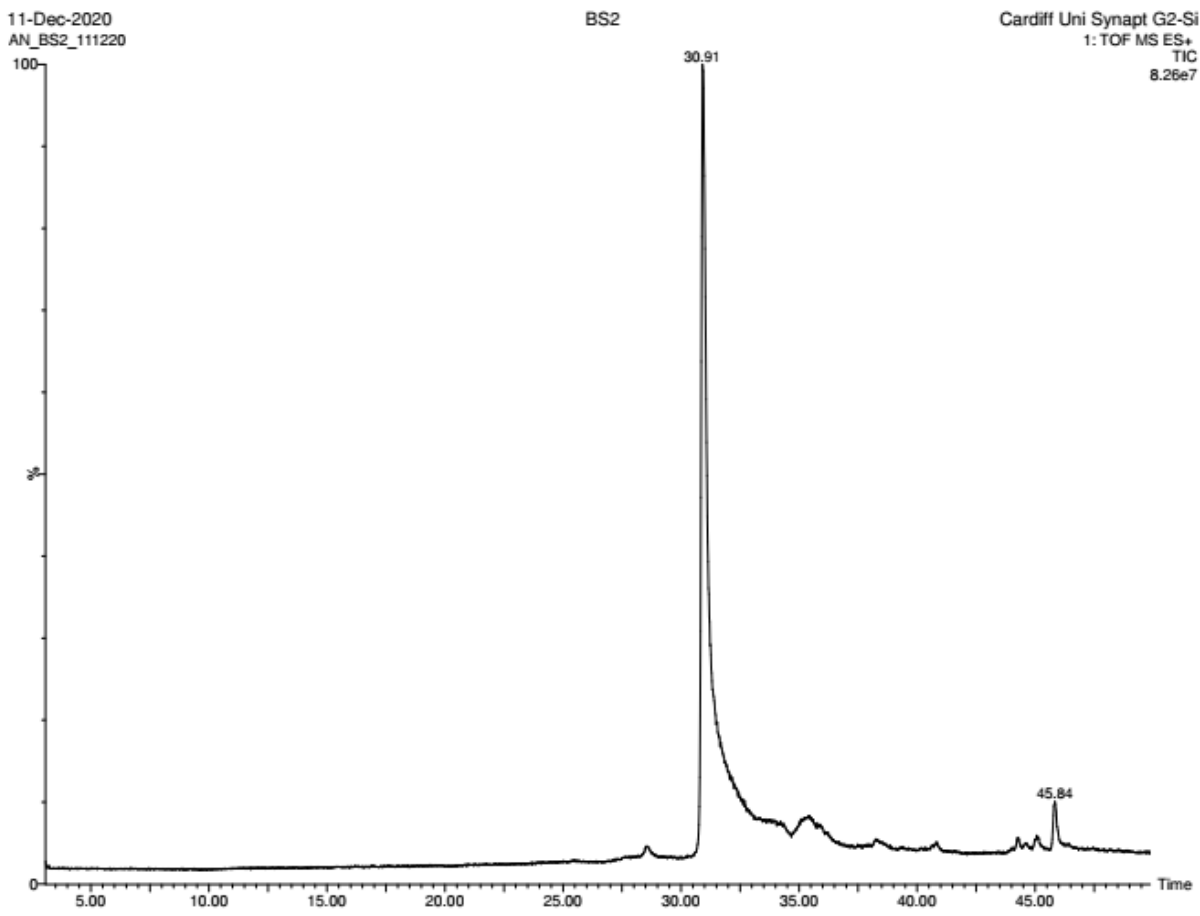

Figure S6. LC chromatogram of purified wild-type Bs2. 


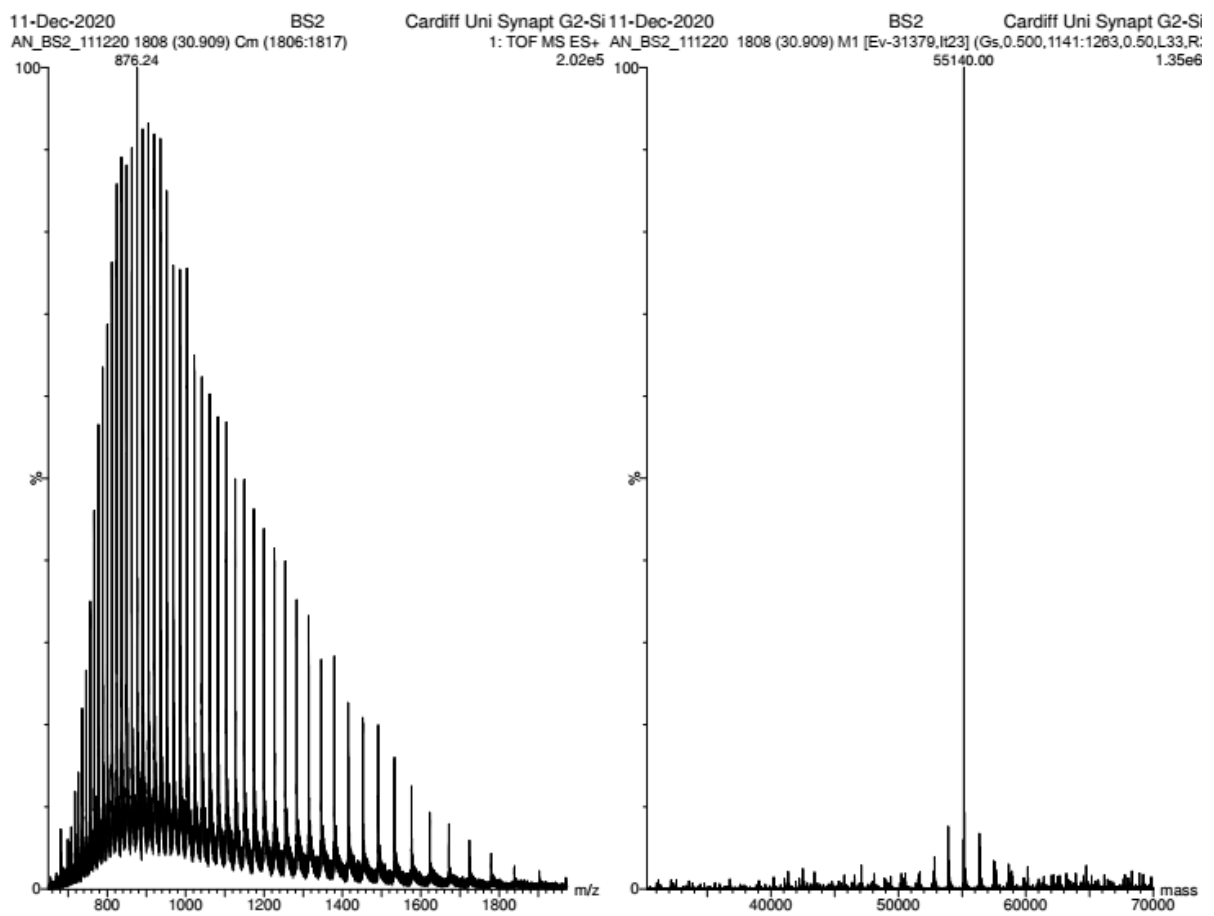

Figure S7. Raw and deconvoluted mass spectrum of purified wild-type Bs2. 


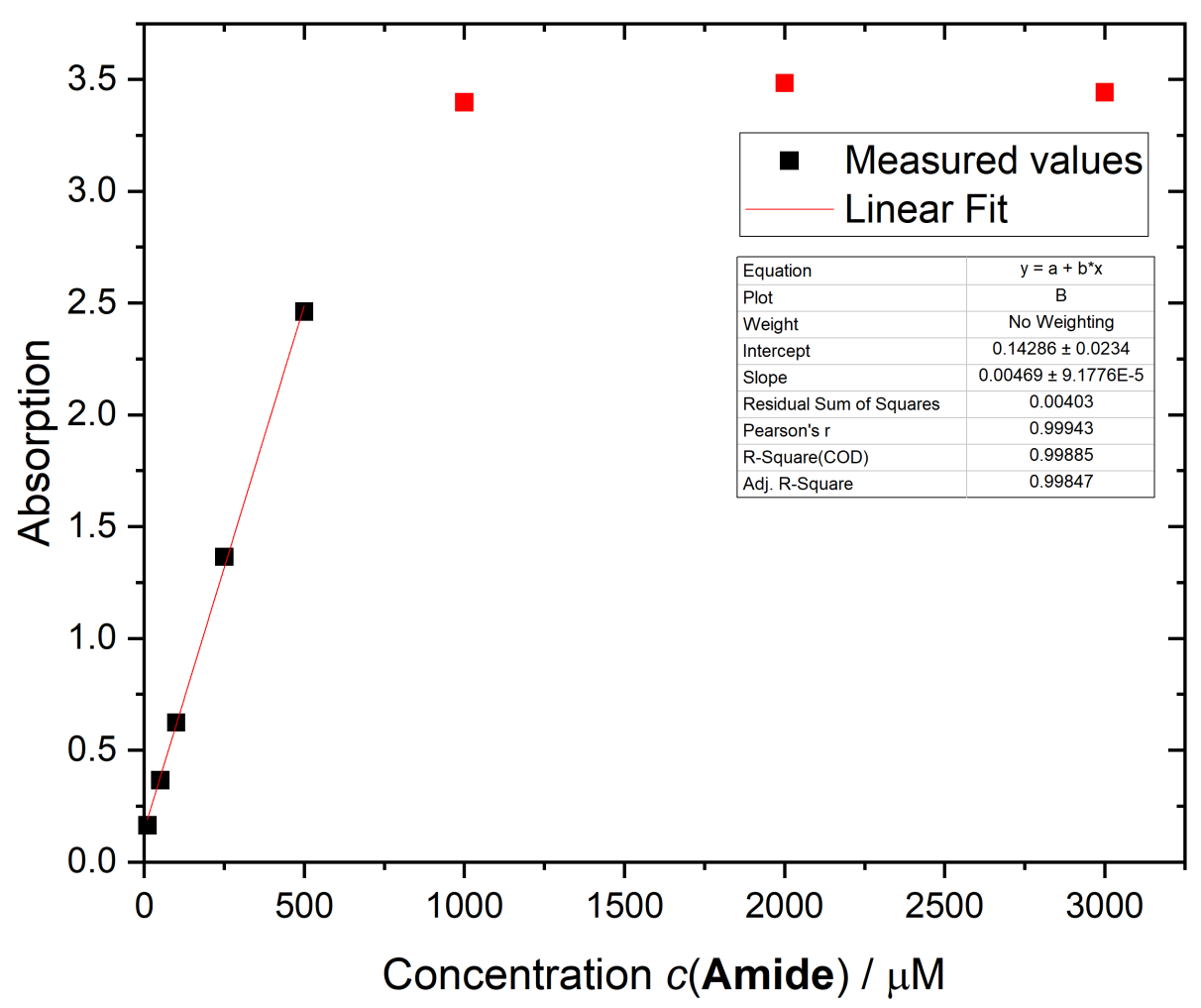

Figure S8. Calibration curve for the 4-nitroaniline concentration in the assay mixture. 


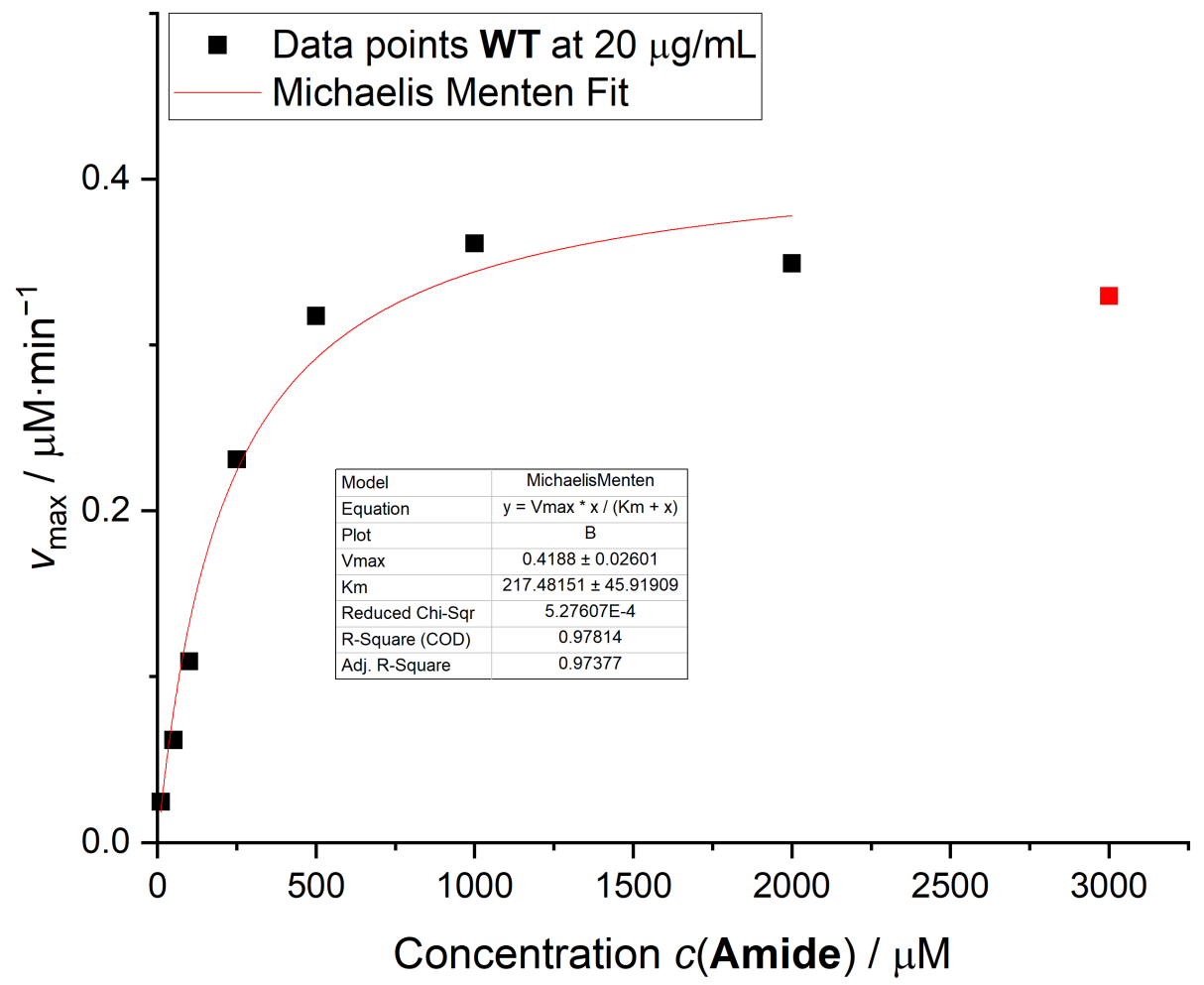

Figure S9. Michaelis-Menten plot for wild-type Bs2, run 1. 


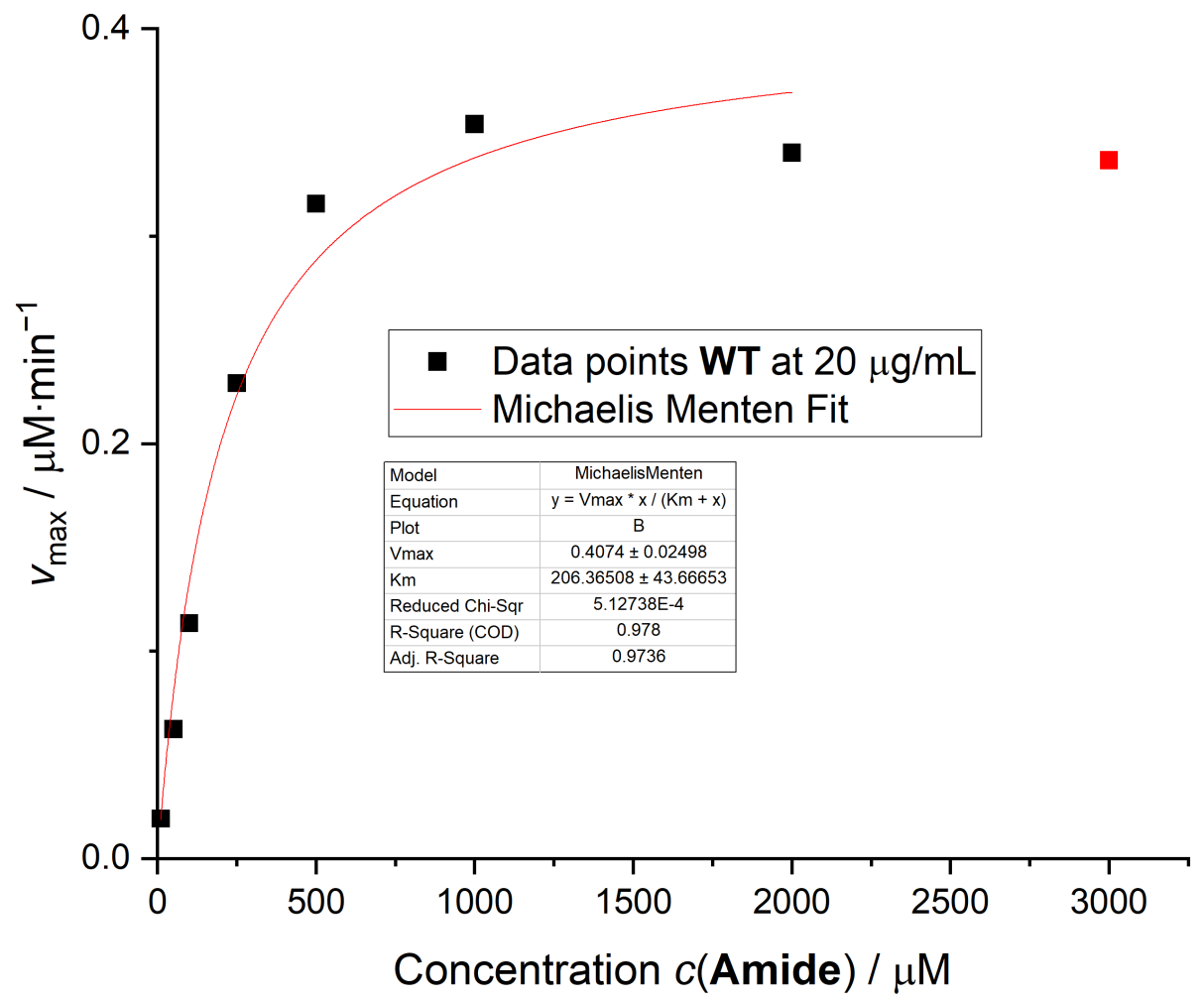

Figure S10. Michaelis-Menten plot for wild-type Bs2, run 2. 


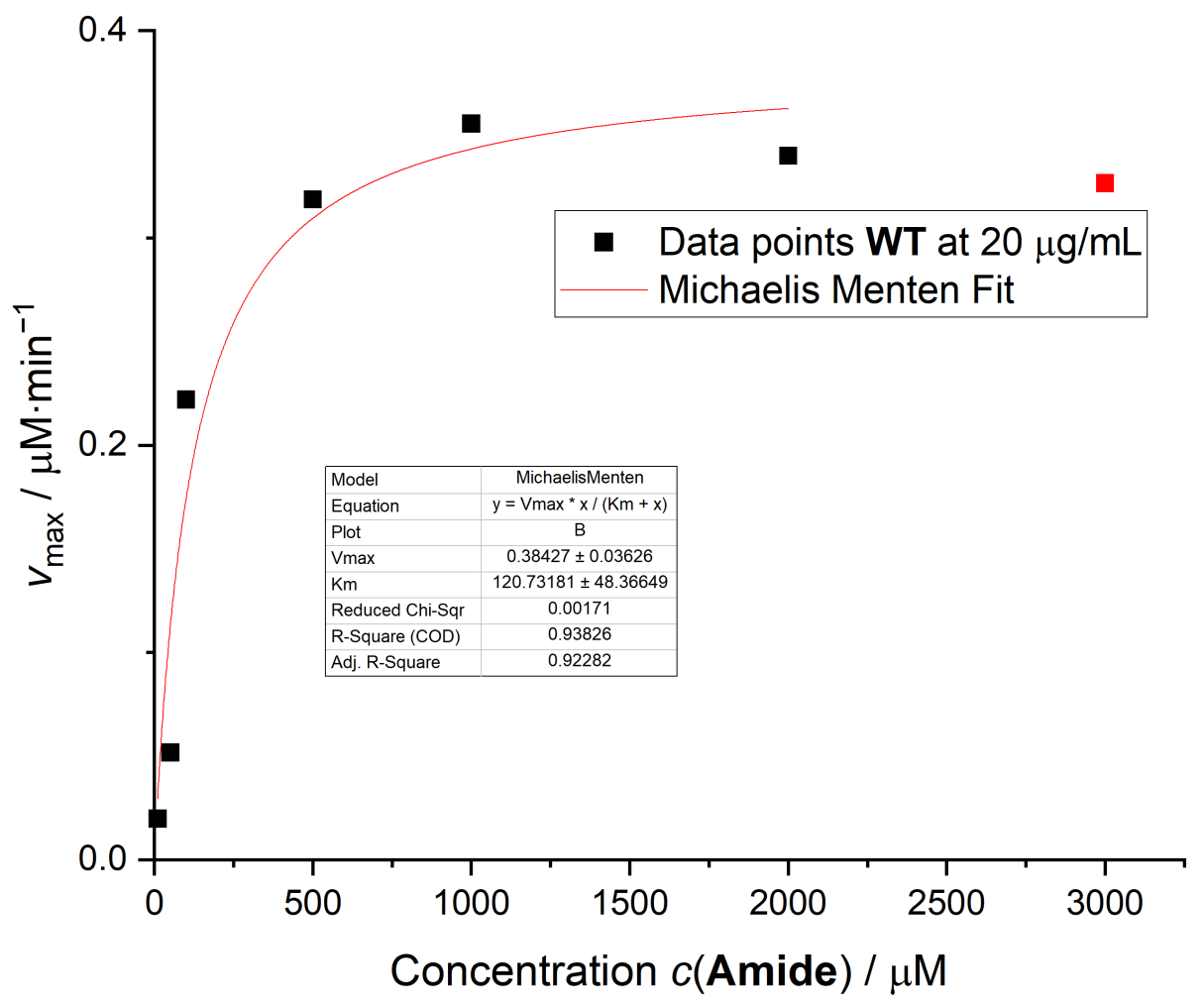

Figure S11. Michaelis-Menten plot for wild-type Bs2, run 3. 


\section{REFERENCES}

(1) Needleman, S. B.; Wunsch, C. D. A General Method Applicable to the Search for Similarities in the Amino Acid Sequence of Two Proteins. J. Mol. Biol. 1970, 48, 443453.

(2) Rice, P.; Longden, I.; Bleasby, A. EMBOSS: The European Molecular Biology Open Software Suite. Trends Genet. 2000, 16, 276-277.

(3) Henikoff, S.; Henikoff, J. G. Amino Acid Substitution Matrices from Protein Blocks. Proc. Natl. Acad. Sci. U. S. A. 1992, 89, 10915-10919.

(4) Hackenschmidt, S.; Moldenhauer, E. J.; Behrens, G. A.; Gand, M.; Pavlidis, I. V.; Bornscheuer, U. T. Enhancement of Promiscuous Amidase Activity of a Bacillus Subtilis Esterase by Formation of a $\pi-\pi$ Network. ChemCatChem 2014, 6, 1015-1020.

(5) Consortium, T. U. UniProt: A Worldwide Hub of Protein Knowledge. Nucleic Acids Res. 2018, 47, D506-D515.

(6) Spiller, B.; Gershenson, A.; Arnold, F. H.; Stevens, R. C. A Structural View of Evolutionary Divergence. Proc. Natl. Acad. Sci. U. S. A. 1999, 96, 12305-12310.

(7) Sali, A.; Blundell, T. L. Comparative Protein Modelling by Satisfaction of Spatial Restraints. J. Mol. Biol. 1993, 234, 779-815.

(8) Olsson, M. H. M.; SØndergaard, C. R.; Rostkowski, M.; Jensen, J. H. PROPKA3: Consistent Treatment of Internal and Surface Residues in Empirical p Kapredictions. $J$. Chem. Theory Comput. 2011, 7, 525-537.

(9) Søndergaard, C. R.; Olsson, M. H. M.; Rostkowski, M.; Jensen, J. H. Improved Treatment of Ligands and Coupling Effects in Empirical Calculation and Rationalization of PKa Values. J Chem Theory Comput 2011, 7, 2284-2295.

(10) Jorgensen, W. L.; Chandrasekhar, J.; Madura, J. D.; Impey, R. W.; Klein, M. L. Comparison of Simple Potential Functions for Simulating Liquid Water. J. Chem. Phys. 1983, 79, 926-935.

(11) Galmés, M. A.; García-Junceda, E.; Świderek, K.; Moliner, V. Exploring the Origin of Amidase Substrate Promiscuity in CALB by a Computational Approach. ACS Catal. 2020, 10, 1938-1946.

(12) Zhang, W. E. I.; Yang, R.; Cieplak, P.; Luo, R. A. Y.; Lee, T.; Caldwell, J.; Wang, J.; 
Kollman, P. A Point-Charge Force Field for Molecular Mechanics Simulations of Proteins Based on Condensed-Phase. J. Comput. Chem. 2003, 24, 1999-2012.

(13) Phillips, J. C.; Braun, R.; Wang, W. E. I.; Gumbart, J.; Tajkhorshid, E.; Villa, E.; Chipot, C.; Skeel, R. D.; Poincare, H. Scalable Molecular Dynamics with NAMD. J. Comput. Chem. 2005, 26, 1781-1802.

(14) Grest, G. S.; Kremer, K. Molecular Dynamics Simulation for Polymers in the Presence of a Heat Bath. Phys. Rev. A 1986, 33, 3628-3631.

(15) Field, M. J.; Bash, P. A.; Karplus, M. A Combined Quantum Mechanical and Molecular Mechanical Potential for Molecular Dynamics Simulations. J. Comput. Chem. 1990, 11, 700-733.

(16) Jorgensen, W. L.; Maxwell, D. S.; Tirado-Rives, J. Development and Testing of the OPLS All-Atom Force Field on Conformational Energetics and Properties of Organic Liquids. J. Am. Chem. Soc. 1996, 118, 11225-11236.

(17) Field, M. J.; Albe, M.; Bret, C.; Proust-De Martin, F.; Thomas, A. The Dynamo Library for Molecular Simulations Using Hybrid Quantum Mechanical and Molecular Mechanical Potentials. J. Comput. Chem. 2000, 21, 1088-1100.

(18) Dewar, M. J. S.; Zoebisch, E. G.; Healy, E. F.; Stewart, J. J. P. Development and Use of Quantum Mechanical Molecular Models. 76. AM1: A New General Purpose Quantum Mechanical Molecular Model. J. Am. Chem. Soc. 1985, 107, 3902-3909.

(19) Zhao, Y.; Truhlar, D. G. The M06 Suite of Density Functionals for Main Group Thermochemistry, Thermochemical Kinetics, Noncovalent Interactions, Excited States, and Transition Elements: Two New Functionals and Systematic Testing of Four M06-Class Functionals and 12 Other Function. Theor. Chem. Acc. 2008, 120, 215241.

(20) Stewart, J. J. P. Quantum Chemistry Program Exchange 455. 1996, 6.

(21) Frisch, M. J.; Trucks, G. W.; Schlegel, H. B.; Scuseria, G. E.; Robb, M. A.; Cheeseman, J. R.; Scalmani, G.; Barone, V.; Mennucci, B.; Petersson, G. A.; Nakatsuji, H.; Caricato, M.; Li, X.; Hratchian, H. P.; Izmaylov, A. F.; Bloino, J.; Zheng, G.; Sonnenberg, J. L.; Hada, M.; Ehara, M.; Toyota, K.; Fukuda, R.; Hasegawa, J.; Ishida, M.; Nakajima, T.; Honda, Y.; Kitao, O.; Nakai, H.; Vreven, T.; Montgomery, J. A., Jr.; Peralta, J. E.; Ogliaro, F.; Bearpark, M.; Heyd, J. J.; Brothers, 
E.; Kudin, K. N.; Staroverov, V. N.; Kobayashi, R.; Normand, J.; Raghavachari, K.; Rendell, A.; Burant, J. C.; Iyengar, S. S.; Tomasi, J.; Cossi, M.; Rega, N.; Millam, J. M.; Klene, M.; Knox, J. E.; Cross, J. B.; Bakken, V.; Adamo, C.; Jaramillo, J.; Gomperts, R.; Stratmann, R. E.; Yazyev, O.; Austin, A. J.; Cammi, R.; Pomelli, C.; Ochterski, J. W.; Martin, R. L.; Morokuma, K.; Zakrzewski, V. G.; Voth, G. A.; Salvador, P.; Dannenberg, J. J.; Dapprich, S.; Daniels, A. D.; Farkas, O.; Foresman, J. B.; Ortiz, J. V.; Cioslowski, J.; Fox, D. J. Gaussian 09, Revision E.01. Gaussian, Inc.: Wallingford, CT 2009.

(22) Byrd, R. H.; Lu, P.; Nocedal, J.; Zhu, C. A Limited Memory Algorithm for Bound Constrained Optimization. SIAM J. Sci. Comput. 1995, 16, 1190-1208.

(23) J. Turner, A.; Moliner, V.; H. Williams, I. Transition-State Structural Refinement with GRACE and CHARMM: Flexible QM/MM Modelling for Lactate Dehydrogenase. Phys. Chem. Chem. Phys. 1999, 1, 1323-1331.

(24) Martí, S.; Moliner, V.; Tuñón, I. Improving the QM/MM Description of Chemical Processes: A Dual Level Strategy to Explore the Potential Energy Surface in Very Large Systems. J. Chem. Theory Comput. 2005, 1, 1008-1016.

(25) Baker, J.; Kessi, A.; Delley, B. The Generation and Use of Delocalized Internal Coordinates in Geometry Optimization. J. Chem. Phys. 1996, 105, 192-212.

(26) Baker, J. Constrained Optimization in Delocalized Internal Coordinates. J. Comput. Chem. 1997, 18, 1079-1095.

(27) Roux, B. The Calculation of the Potential of Mean Force Using Computer-Simulation. Comput. Phys. Commun. 1995, 91, 275-282.

(28) Torrie, G. M.; Valleau, J. P. Non-Physical Sampling Distibutions in Monte-Carlo FreeEnergy Estimation - Umbrella Sampling. J. Comput. Phys. 1977, 23, 187-199.

(29) Kumar, S.; Rosenberg, J. M.; Bouzida, D.; Swendsen, R. H.; Kollman, P. A. The Weighted Histogram Analysis Method for Free-energy Calculations on Biomolecules. I. The Method. J. Comput. Chem. 1992, 13, 1011-1021.

(30) Verlet, L. Computer "Experiments" on Classical Fluids. I. Thermodynamical Properties of Lennard-Jones Molecules. Phys. Rev. 1967, 159, 98-103.

(31) Ruiz-Pernía, J. J.; Silla, E.; Tuñón, I.; Martí, S.; Moliner, V. Hybrid QM/MM Potentials of Mean Force with Interpolated Corrections. J. Phys. Chem. B 2004, 108, 
$8427-8433$.

(32) Ruiz-Pernía, J. J.; Silla, E.; Tuñón, I.; Martí, S. Hybrid Quantum Mechanics/Molecular Mechanics Simulations with Two-Dimensional Interpolated Corrections: Application to Enzymatic Processes. J. Phys. Chem. B 2006, 110, 17663-17670.

(33) UniProt: The Universal Protein Knowledgebase in 2021. Nucleic Acids Res. 2021, 49, D480-D489.

(34) Sievers, F.; Wilm, A.; Dineen, D.; Gibson, T. J.; Karplus, K.; Li, W.; Lopez, R.; McWilliam, H.; Remmert, M.; Soeding, J.; Thompson, J. D.; Higgins, D. G. Fast, Scalable Generation of High-Quality Protein Multiple Sequence Alignments Using Clustal Omega. Mol. Syst. Biol. 2011, 7.

(35) Cock, P. J. A.; Antao, T.; Chang, J. T.; Chapman, B. A.; Cox, C. J.; Dalke, A.; Friedberg, I.; Hamelryck, T.; Kauff, F.; Wilczynski, B.; de Hoon, M. J. L. Biopython: Freely Available Python Tools for Computational Molecular Biology and Bioinformatics. BIOINFORMATICS 2009, 25, 1422-1423.

(36) Sokal, R. R.; Michener, C. D.; Kansas., U. of. A Statistical Method for Evaluating Systematic Relationships; [University of Kansas]: [Lawrence, Kan.], 1958.

(37) Asnicar, F.; Weingart, G.; Tickle, T. L.; Huttenhower, C.; Segata, N. Compact Graphical Representation of Phylogenetic Data and Metadata with GraPhlAn. PeerJ 2015, 3 .

(38) Berman, H. M.; Westbrook, J.; Feng, Z.; Gilliland, G.; Bhat, T. N.; Weissig, H.; Shindyalov, I. N.; Bourne, P. E. The Protein Data Bank. Nucleic Acids Res. 2000, 28, $235-242$.

(39) Harris, C. R.; Millman, K. J.; van der Walt, S. J.; Gommers, R.; Virtanen, P.; Cournapeau, D.; Wieser, E.; Taylor, J.; Berg, S.; Smith, N. J.; Kern, R.; Picus, M.; Hoyer, S.; van Kerkwijk, M. H.; Brett, M.; Haldane, A.; del Río, J.; Wiebe, M.; Peterson, P.; Gérard-Marchant, P.; Sheppard, K.; Reddy, T.; Weckesser, W.; Abbasi, H.; Gohlke, C.; Oliphant, T.E. Array Programming with $\{$ NumPy $\}$. Nature 2020, 585, $357-362$.

(40) Virtanen, P.; Gommers, R.; Oliphant, T. E.; Haberland, M.; Reddy, T.; Cournapeau, D.; Burovski, E.; Peterson, P.; Weckesser, W.; Bright, J.; van der Walt, S. J.; Brett, M.; Wilson, J.; Millman, K. J.; Mayorov, N.; Nelson, A. R. J.; Jones, E.; Kern, R.; Larson, 
E.; Carey, C.J.; Polat, I.; Feng, Y.; Moore, E.W.; VanderPlas, J.; Laxalde, D.; Perktold, J.; Cimrman, R.; Henriksen, I.; Quintero, E.A.; Harris, C.R.; Archibald, A.M.; Ribeiro, A.H.; Pedregosa, F.; van Mulbregt, P.; SciPy 1.0 Contributors. \{SciPy\} 1.0:

Fundamental Algorithms for Scientific Computing in Python. Nat. Methods 2020, 17, 261-272.

(41) He, K.; Zhang, X.; Ren, S.; Sun, J. Deep Residual Learning for Image Recognition. In 2016 IEEE Conference on Computer Vision and Pattern Recognition (CVPR); 2016; pp 770-778.

(42) Kingma, D. P.; Ba, J. Adam: A Method for Stochastic Optimization. CoRR 2015, $a b s / 1412.6$.

(43) Chollet, F.; others. Keras. GitHub 2015.

(44) Abadi, M.; Agarwal, A.; Barham, P.; Brevdo, E.; Chen, Z.; Citro, C.; Corrado, G. S.; Davis, A.; Dean, J.; Devin, M.; Ghemawat, S.; Goodfellow, I.; Harp, A.; Irving, G.; Isard, M.; Jia, Y.; Jozefowicz, R.; Kaiser, L.; Kudlur, M.; Levenberg, J.; Mane, D.; Monga, R.; Moore, S.; Murray, D.; Olah, C.; Schuster, M.; Shlens, J.; Steiner, B.; Sutskever, I.; Talwar, K.; Tucker, P.; Vanhoucke, V.; Vasudevan, V.; Viegas, F.; Vinyals, O.; Warden, P.; Wattenberg, M.; Wicke, M.; Yu, Y.; Zheng, X. TensorFlow: Large-Scale Machine Learning on Heterogeneous Distributed Systems. 2016. 


\section{Annex D}

Supporting Information of Article 4: "Computational design of an amidase by combining the best electrostatic features of two promiscuous hydrolases" 



\section{SUPPLEMENTARY INFORMATION}

\section{Computational design of an amidase by combining the best electrostatic features of two promiscuous hydrolases.}

Miquel À. Galmés, ${ }^{1}$ Alexander R Nödling, ${ }^{2}$ Kaining He, ${ }^{2}$ Louis Luk, ${ }^{2, *}$ Katarzyna Świderek, ${ }^{1, *}$ Vicent Moliner ${ }^{1, *}$

1. BioComp Group, Institute of Advanced Materials (INAM), Universitat Jaume I, 12071 Castellón, Spain.

2. School of Chemistry, Cardiff University, Main Building, Park Pl, Cardiff CF10 3AT (United Kingdom)

corresponding authors:

L. Luk: lukly@cardiff.ac.uk;

K. Świderek: swiderek@uji.es;

V. Moliner: moliner@uji.es 


\section{RESULTS AND ANALYSIS}
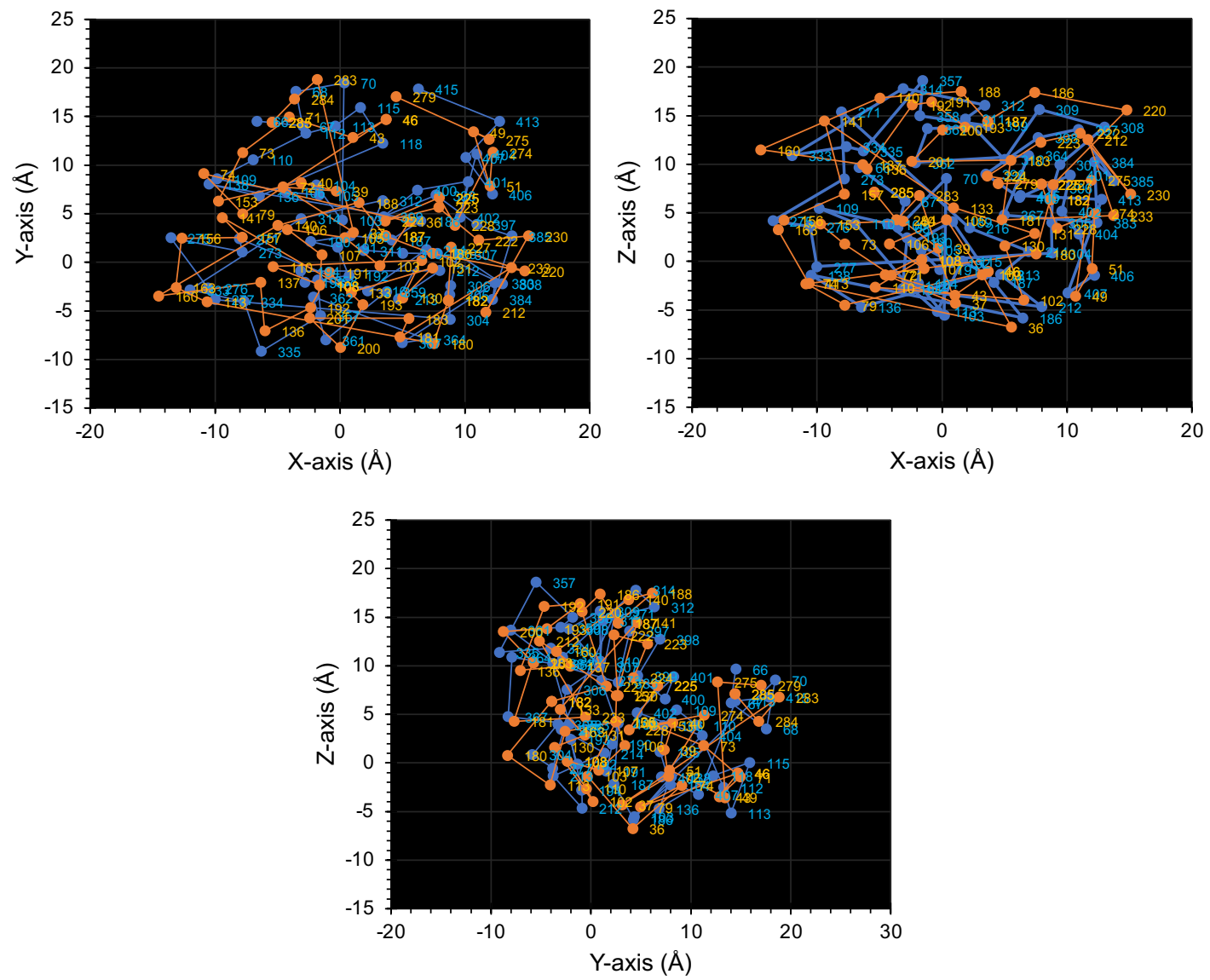

Figure S1. Overlapped centers of masses of paired residues from Bs2 (in blue) and CALB (in orange) in 3-dimensional space. 


\begin{tabular}{|c|c|c|c|c|c|c|c|c|c|c|c|c|c|c|c|}
\hline \multirow[t]{4}{*}{ Bs 2} & 66 & 67 & 68 & 70 & 103 & 104 & 105 & 106 & 109 & 110 & 112 & 113 & 115 & 118 & 136 \\
\hline & ASP & LEU & LEU & LEU & ILE & HIS & GLY & GLY & TYR & LEU & ALA & GLY & GLU & TYR & TYR \\
\hline & - & $\mid$ & | & $\mid$ & 1 & + & | | & - & - & | | & : & - & - & - & - \\
\hline & ILE & ILE & ALA & ALA & VAL & MET & GLY & THR & SER & LEU & PHE & THR & GLN & GLN & ASN \\
\hline CALB & 285 & 285 & 284 & 283 & 37 & 72 & 39 & 40 & 153 & 73 & 71 & 43 & 46 & 46 & 79 \\
\hline \multirow[t]{4}{*}{ Bs 2} & 138 & 186 & 187 & 189 & 190 & 191 & 192 & 193 & 194 & 212 & 213 & 214 & 216 & 271 & 273 \\
\hline & LEU & PHE & GLY & SER & ALA & GLY & GLY & MET & SER & ILE & MET & GLU & GLY & PHE & LEU \\
\hline & - & : & • & || & - & || & || & I & • & I & 1 & - & • & : & • \\
\hline & ASN & LEU & THR & SER & GLN & GLY & GLY & GLY & VAL & LEU & ALA & PHE & PRO & ALA & GLN \\
\hline CALB & 74 & 36 & 103 & 105 & 106 & 107 & 108 & 108 & 110 & 102 & 130 & 131 & 133 & 141 & 157 \\
\hline \multirow[t]{4}{*}{ Bs 2} & 275 & 276 & 277 & 304 & 305 & 306 & 307 & 308 & 309 & 310 & 311 & 312 & 314 & 333 & 334 \\
\hline & PHE & GLN & PRO & ILE & GLY & THR & THR & ARG & ASP & GLU & GLY & TYR & PHE & TYR & LEU \\
\hline & • & • & o & • & | & • & 1 & + & - & | & - & - & : & : & \\
\hline & GLN & LEU & TRP & THR & LEU & LEU & SER & PHE & THR & ASP & ASP & GLU & LEU & GLY & GLY \\
\hline CALB & 156 & 163 & 113 & 180 & 182 & 182 & 227 & 220 & 186 & 187 & 187 & 188 & 140 & 160 & 137 \\
\hline \multirow[t]{4}{*}{ Bs 2} & 335 & 357 & 358 & 359 & 361 & 362 & 364 & 367 & 383 & 384 & 385 & 397 & 398 & 399 & 400 \\
\hline & LEU & MET & MET & THR & LEU & LEU & TRP & $A L A$ & TYR & ARG & PHE & ALA & PHE & HIS & ALA \\
\hline & + & - & • & $\mid$ & - & • & 1 & - & - & + & . & | & - & | | & || \\
\hline & LYS & PRO & GLN & GLN & ASP & SER & TYR & ASN & SER & ALA & SER & ILE & ASP & HIS & ALA \\
\hline CALB & 136 & 192 & 191 & 193 & 200 & 201 & 183 & 181 & 233 & 212 & 230 & 222 & 223 & 224 & 225 \\
\hline \multirow[t]{4}{*}{ Bs 2} & 401 & 402 & 404 & 406 & 407 & 413 & 415 & & & & & & & & \\
\hline & LEU & GLU & PRO & VAL & PHE & LEU & ARG & & & & & & & & \\
\hline & 1 & - & • & • & - & 1 & + & & & & & & & & \\
\hline & $A L A$ & LEU & ALA & ASN & ASP & ALA & ALA & & & & & & & & \\
\hline CALB & 225 & 228 & 274 & 51 & 49 & 275 & 279 & & & & & & & & \\
\hline
\end{tabular}

Figure S2. Structural alignment of Bs2 and CALB. In red the paired residues of catalytic triad are highlighted. Double "| |" is used to mark equal residues; single "|" refers to a change to a residue of the same family (polar, non-polar, positively charged, negatively charged and aromatic); colon ":” is marking the change between non-polar to aromatic; simple dot "." refers to the change between polar and nonpolar residues; "o" is used for the changes among polar and aromatic residues; "+" refers to the change in a positively charged residue; and "_" refers to a change in a negatively charged residue. 

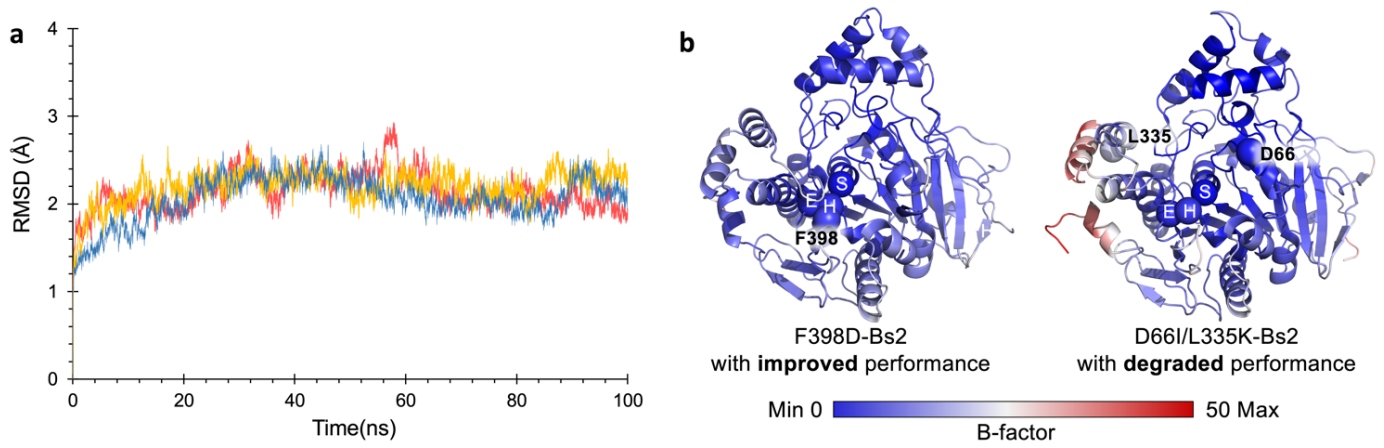

Figure S3. Analysis of 100 ns molecular dynamic simulations. a, Root mean square deviation (RMSD) of the position of $\mathrm{C} \alpha, \mathrm{C}$, and $\mathrm{N}$ atoms of the backbone along $100 \mathrm{~ns}$ of MD simulations computed for the F398D Bs2 variant (blue), F398D-H ${ }^{+}$Bs2 variant (yellow) and D66I/L335K Bs2 variant (red) along MD simulations. b, B-factor per atom computed as atomic oscillations around the equilibrium positions recorded during MD simulations. The red regions correspond to the flexible parts of the protein, the blue areas are static. 


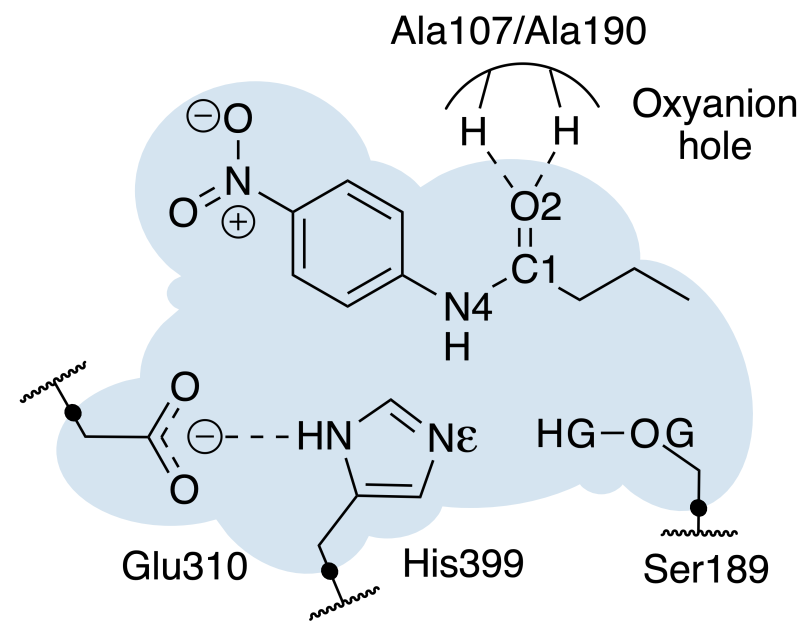

Figure S4. Schematic representation of the QM sub-set region (shadowed region). Black dots represent link atoms between QM and MM region. The H-bond interactions between $N$-(4-nitrophenyl)-butyramide substrate and the oxyanion hole, are shown as dashed lines. 

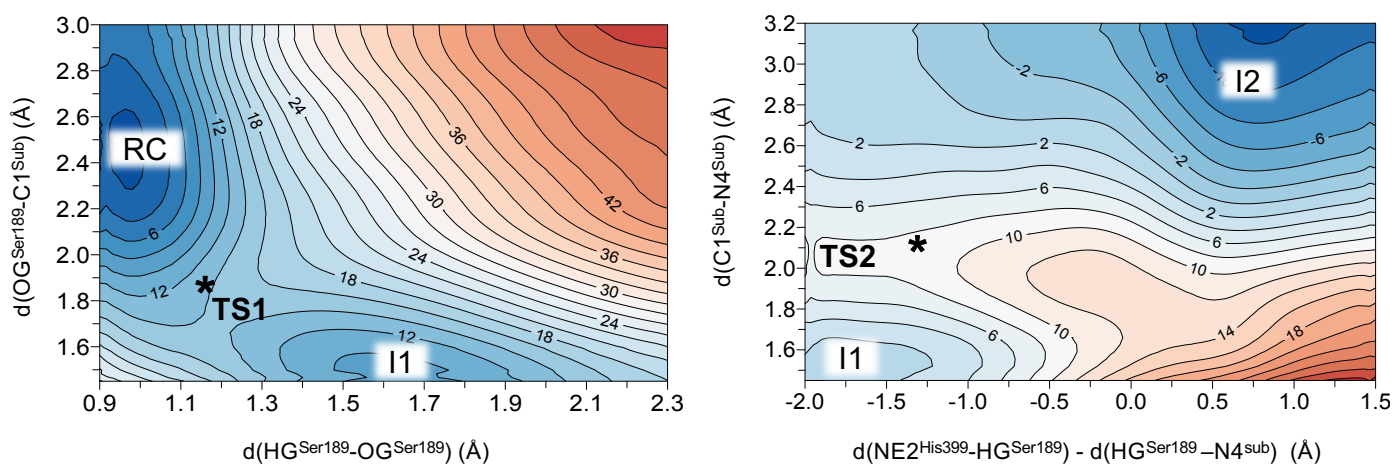

Figure S5. M06-2X:AM1/OPLS-AA Free Energy Surfaces of F398D Bs2 variant. Distances are given in $\AA$ and isoenergetic lines are in $\mathrm{kcal} \cdot \mathrm{mol}^{-1}$. The black stars indicate the position of single TS structures localized at potential energy surface computed at M06-2X/OPLS-AA level of theory.
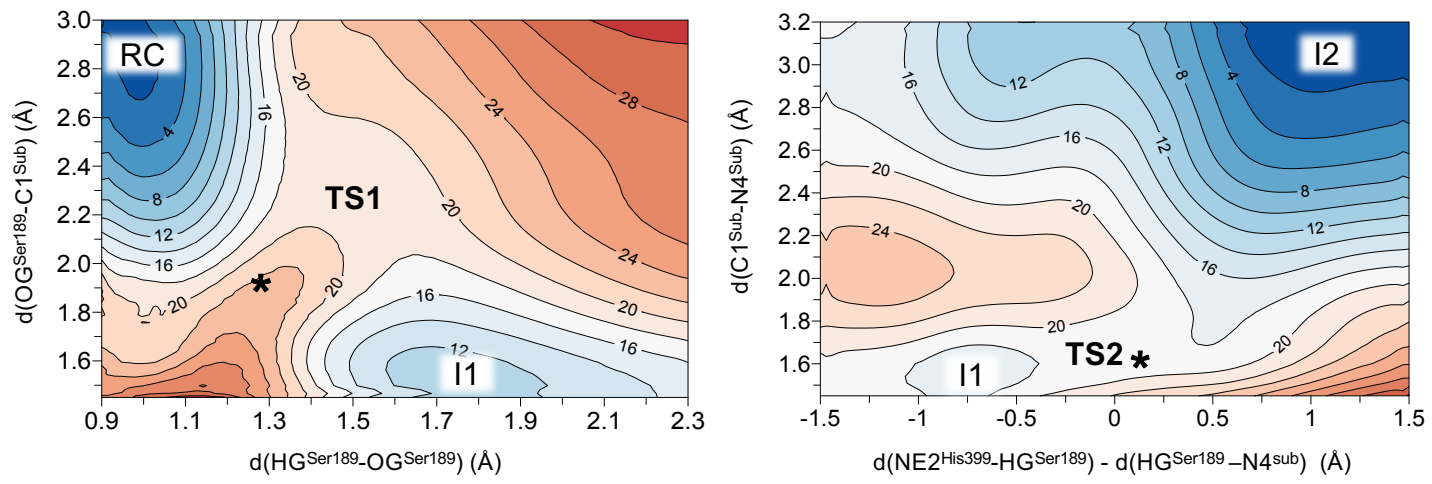

Figure S6. M06-2X:AM1/OPLS-AA Free Energy Surfaces of F398D-H ${ }^{+}$Bs2 variant. Distances are given in $\AA$ and isoenergetic lines are in $\mathrm{kcal} \cdot \mathrm{mol}^{-1}$. The black stars indicate the position of single TS structures localized at potential energy surface computed at M06-2X/OPLS-AA level of theory. 

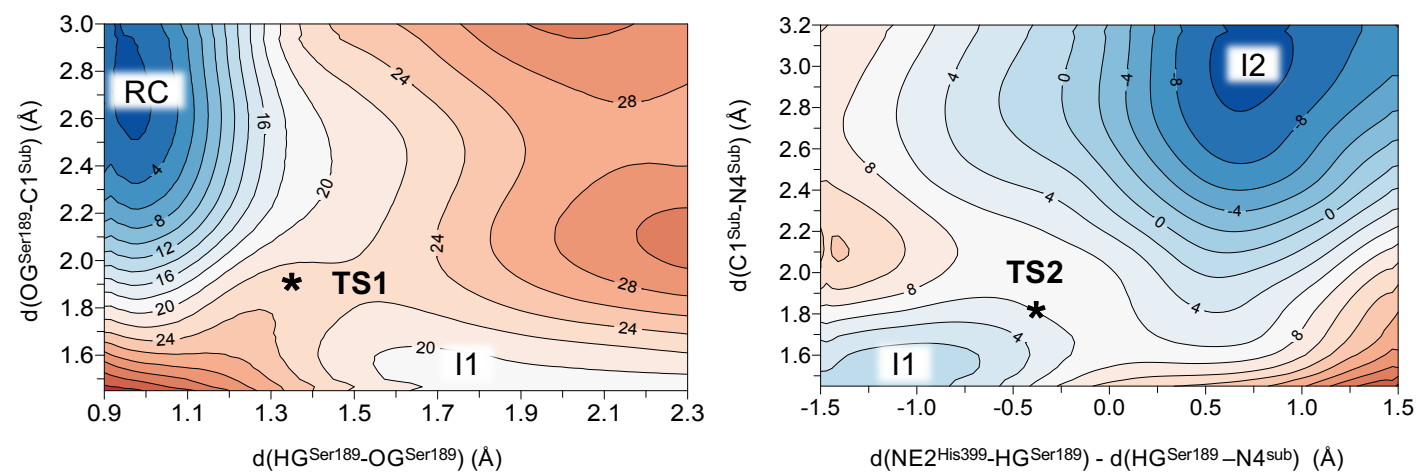

Figure S7. M06-2X:AM1/OPLS-AA Free Energy Surfaces of D66I/L335K Bs2 variant. Distances are given in $\AA$ and isoenergetic lines are in $\mathrm{kcal} \cdot \mathrm{mol}^{-1}$. The black stars indicate the position of single TS structures localized at potential energy surface computed at M06-2X/OPLS-AA level of theory. 


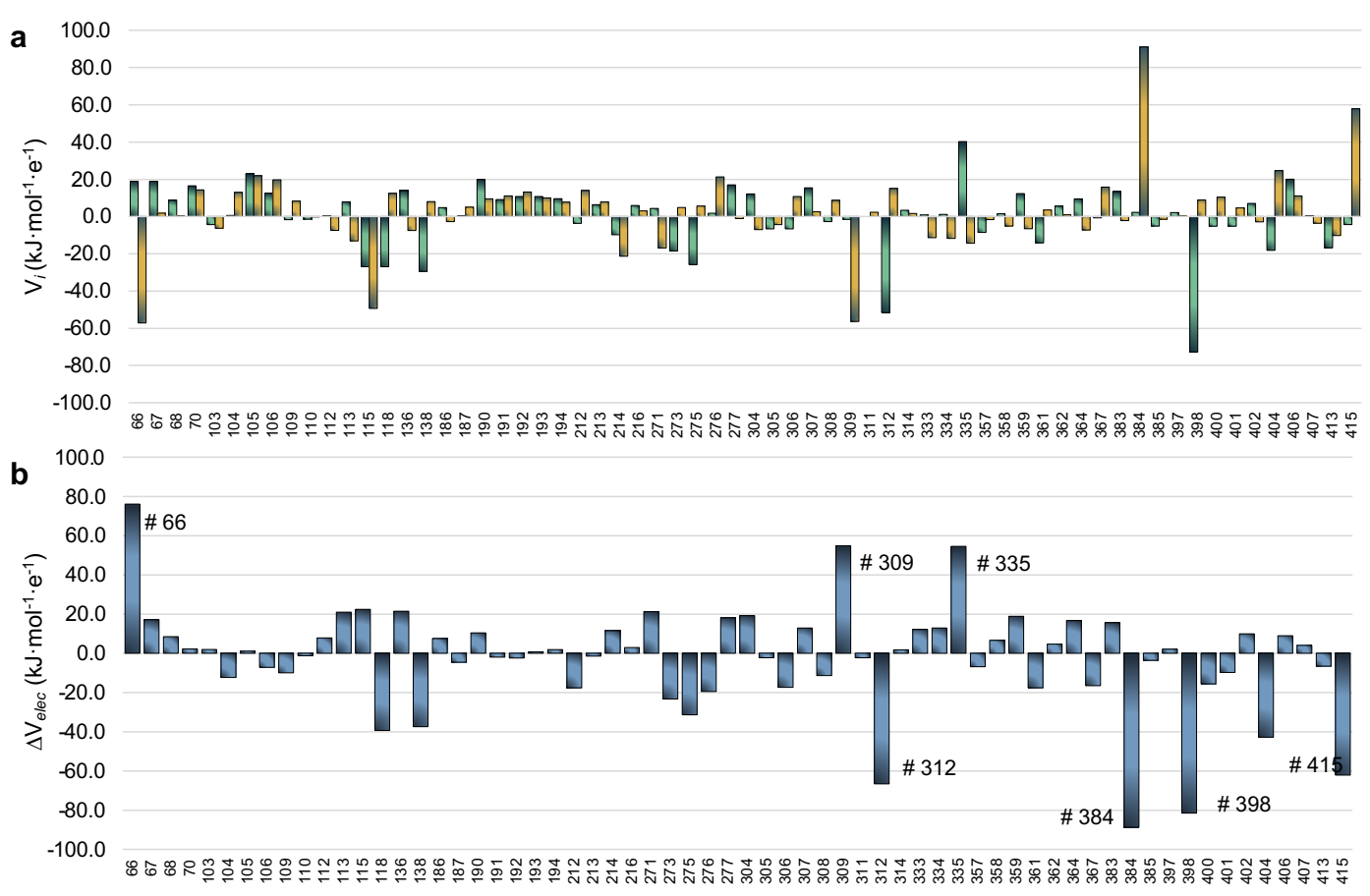

Figure S8. The electrostatic potential generated by Bs2 and CALB per residue. a, Electrostatic potential generated per residue on the $\mathrm{N} \varepsilon$ atom of the active site histidine (His399/His224) in the TS1 structure optimized in Bs2 (yellow bars) and in CALB (green bars). b, the difference in the electrostatic potential per residue between Bs2 and CALB. $\Delta \mathrm{V}_{\text {elec }}$ values were calculated as $\mathrm{V}_{\mathrm{i}}^{\mathrm{Bs} 2}-\mathrm{V}_{\mathrm{i}}^{\mathrm{CALB}}$ were $i$ is the corresponding residue in the aligned pairs. Only the residues within $15 \AA$ from the center of mass of the substrate were taken into consideration. 

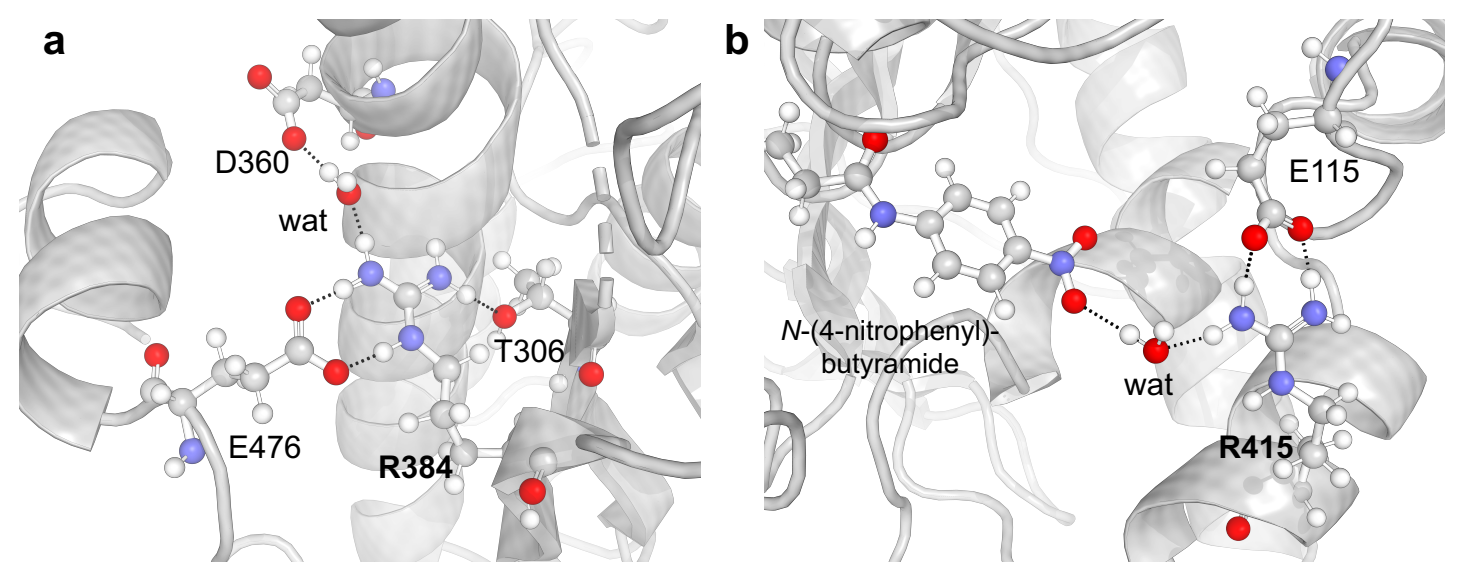

Figure S9. Detailed view of the surrounding of R384 and R415 in Bs2. a, Hydrogen bonding network of R384 with the lateral chains of E476, D360 and T306. b, Hydrogen bond interactions of R415 with E115 and the substrate $N$-(4-nitrophenyl)-butyramide. 


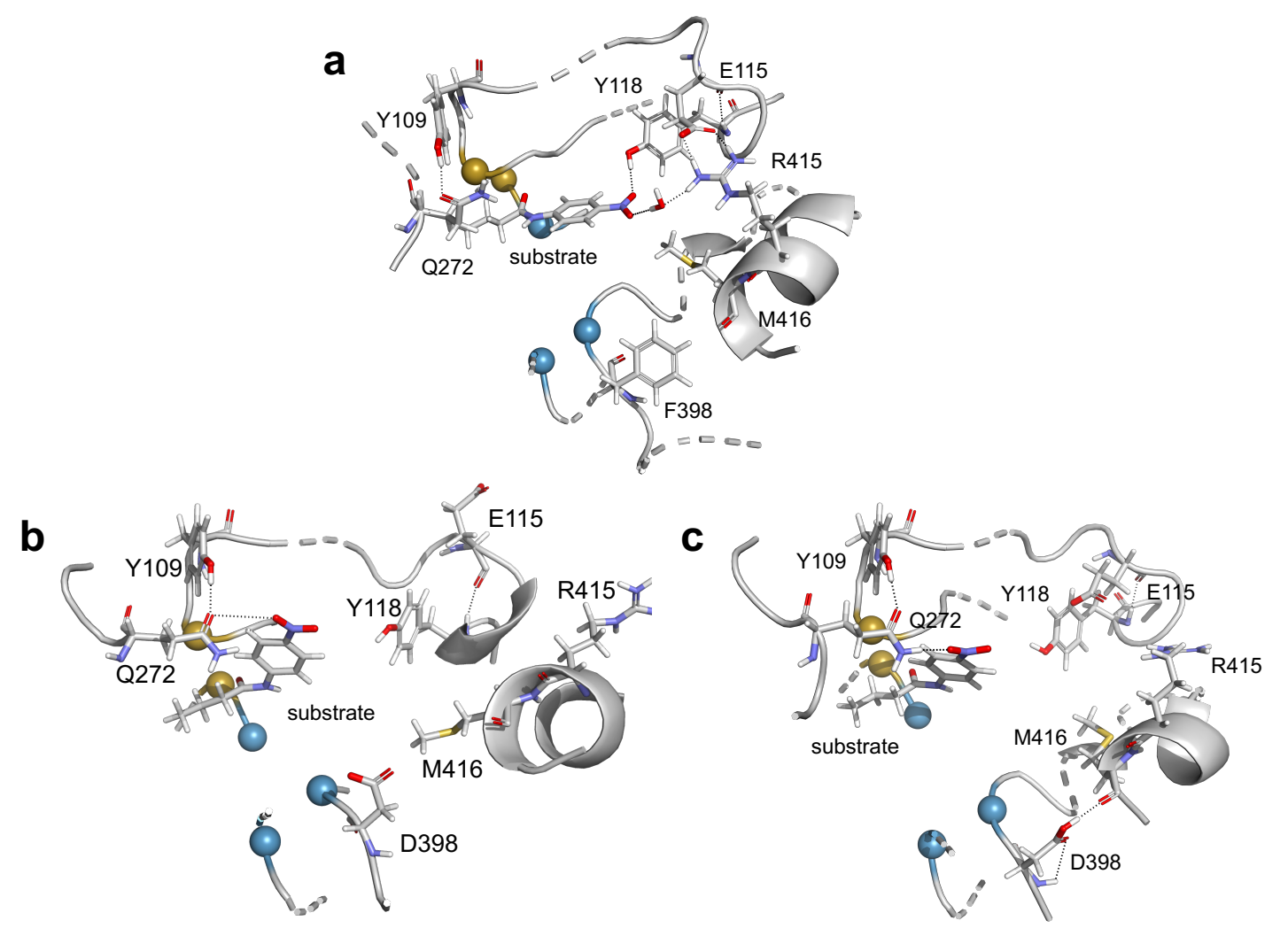

Figure S10. Detailed image of the arrangement of the substrate contacts in wild-type Bs2 and F398D-H ${ }^{+}$Bs2. a, interactions of Y118 and R415 with the nitro group of $N$-(4-nitrophenyl)-butyramide in wild-type Bs2. b, interactions of Y109 and Q272 with the nitro group of $N$-(4-nitrophenyl)-butyramide in F398D Bs2. c, interactions of Y109 and Q272 with the nitro group of $N$-(4-nitrophenyl)-butyramide in F398D-H $\mathrm{H}^{+}$Bs2.The mutation F398D-H $\mathrm{H}^{+}$leads to the formation of a hydrogen bond with the backbone of M416, promoting the rearrangement of the interactions with the substrate. Blue spheres represent the catalytic triad while yellow spheres are the residues involved in the oxyanion hole. 

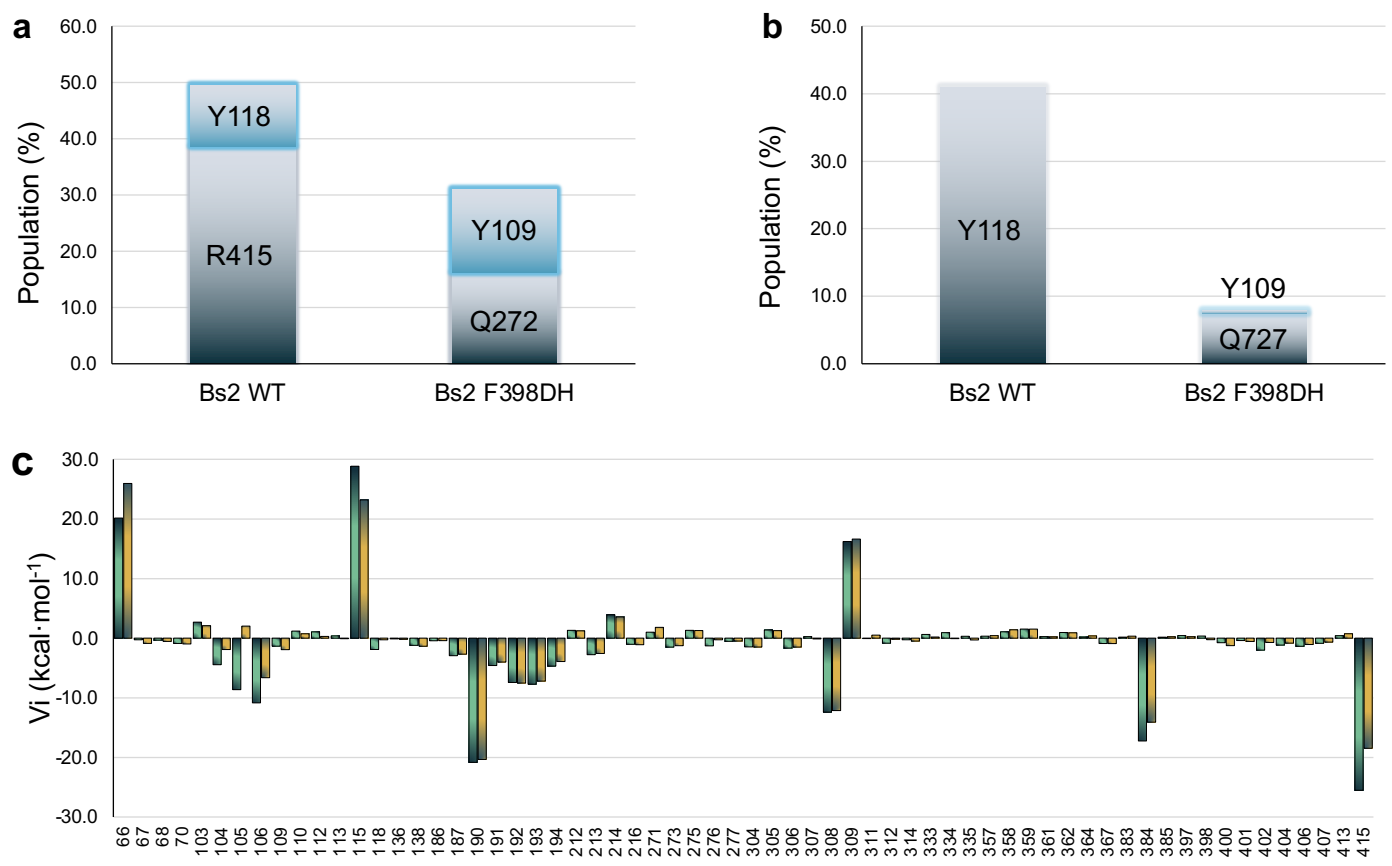

Figure S11. Analysis of contacts and interactions of wild-type Bs2 and F398D-H $\mathrm{H}^{+}$Bs2 along the molecular dynamics simulation. a, water bridge contacts of key residues with the nitro group of the substrate. $\mathbf{b}$, hydrogen bond contacts of key residues with the nitro group of the substrate. c, electrostatic interactions of the residues within $15 \AA$ with the substrate along the molecular dynamics simulation. Green bars represent the interactions in the wild-type Bs2 while yellow ones correspond to the F398D- $\mathrm{H}^{+}$Bs2 variant. 

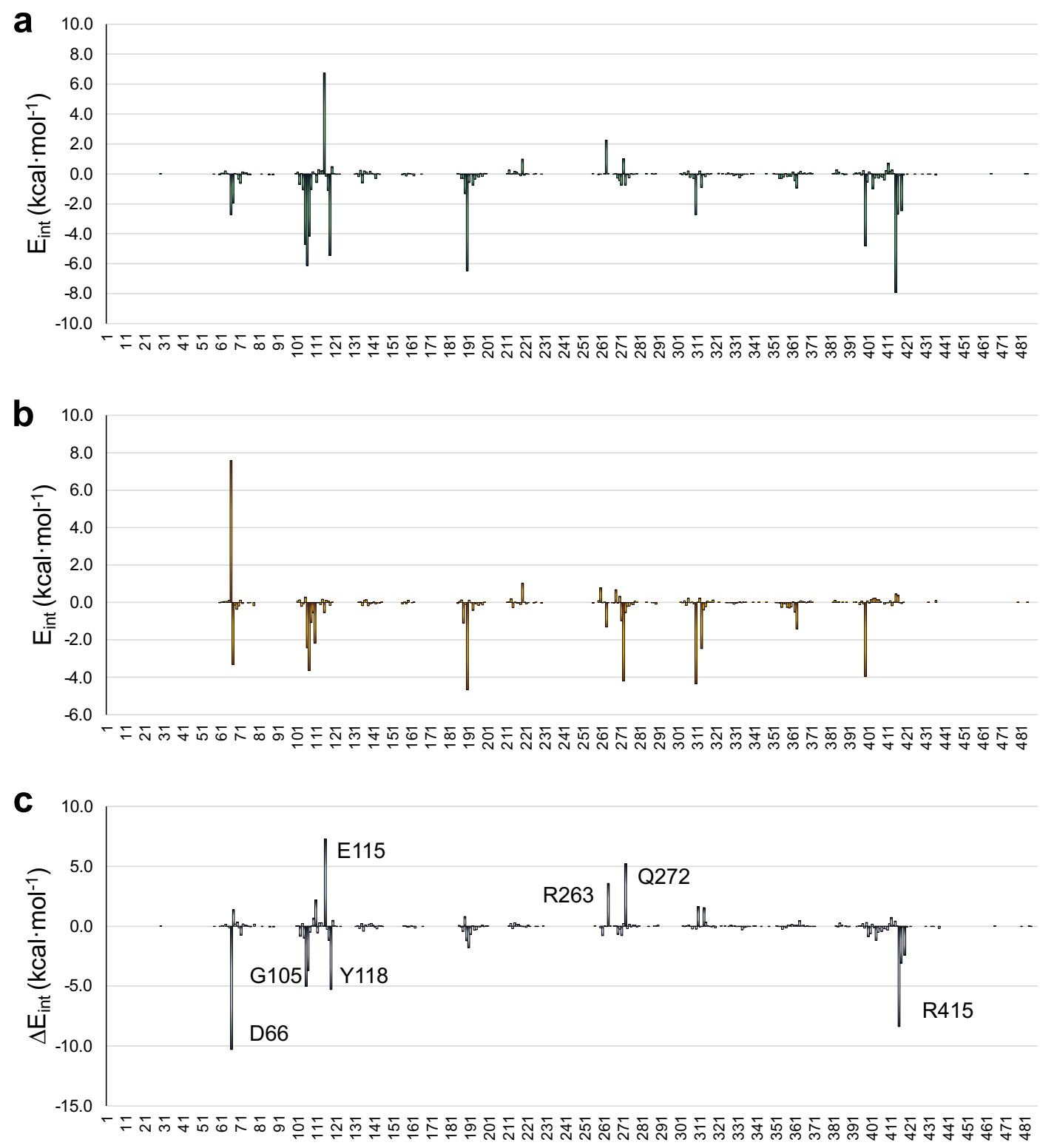

Figure S12. Interaction energies of the protein by residue with the substrate. a, interactions in wild type Bs2; b, interactions in the F398D-H $\mathrm{H}^{+} \mathrm{Bs} 2$ variant; $\mathbf{c}$, difference of interactions energies computed as $\mathrm{E}_{\text {int }}{ }^{\text {wild-type }}-\mathrm{E}_{\text {int }}{ }^{\mathrm{F} 398 \mathrm{D}-\mathrm{H}+}$. 
Table S1. Key inter-atomic distances of the different states appearing along the acylation step of $N$-(4nitrophenyl)-butyramide catalyzed by F398D Bs2 variant. Structures optimized at M06-2X/MM level. All distances are given in $\AA$.

\begin{tabular}{lcccccc}
\hline & $\mathrm{RC}$ & $\mathrm{TS} 1$ & $\mathrm{INT1}$ & $\mathrm{INT1}$ & $\mathrm{TS} 2$ & INT2 \\
\hline $\mathrm{OG}_{\text {Ser189 }}-\mathrm{C}_{\text {subs }}$ & 2.26 & 1.87 & 1.50 & 1.48 & 1.38 & 1.32 \\
$\mathrm{OG}_{\text {Ser189 }}-\mathrm{HG}_{\text {Ser189 }}$ & 1.03 & 1.16 & 1.75 & 1.74 & 1.89 & 2.48 \\
$\mathrm{~N} \varepsilon_{\text {His399 }}-\mathrm{HG}_{\text {Ser189 }}$ & 1.52 & 1.34 & 1.04 & 1.04 & 1.02 & 1.81 \\
$\mathrm{C}_{\text {subs }}-\mathrm{N} 4_{\text {subs }}$ & 1.43 & 1.48 & 1.56 & 1.56 & 2.12 & 3.07 \\
$\mathrm{HG}_{\text {Ser189 }}-\mathrm{N} 4_{\text {subs }}$ & 2.72 & 2.54 & 2.45 & 2.48 & 2.33 & 1.05 \\
$\mathrm{HD}_{\text {His399 }}-\mathrm{OE} 1_{\text {Glu310 }}$ & 1.68 & 1.61 & 1.44 & 1.43 & 1.42 & 1.74 \\
$\mathrm{HD}_{\text {His399 }}-\mathrm{ND} 1_{\text {His399 }}$ & 1.05 & 1.05 & 1.10 & 1.11 & 1.11 & 1.04 \\
$\mathrm{C}_{\text {subs }}-\mathrm{O} 2_{\text {subs }}$ & 1.22 & 1.24 & 1.29 & 1.29 & 1.23 & 1.23 \\
$\mathrm{O} 2_{\text {subs }}-\mathrm{H}_{\text {Ala190 }}$ & 1.80 & 1.83 & 1.71 & 1.71 & 1.66 & 1.75 \\
$\mathrm{O} 2_{\text {subs }}-\mathrm{H}_{\text {Ala107 }}$ & 1.69 & 1.72 & 1.72 & 1.69 & 1.75 & 1.77 \\
\hline
\end{tabular}

Table S2. ESP charges (in a.u.) of the key atoms of the amide substrate computed at M06-2X/MM level on the states involved in the acylation step catalyzed by the F398D Bs2 variant from reactants state (RS) to Intermediate 2 (INT2).

\begin{tabular}{lcccccc}
\hline & RC & TS1 & INT1 & INT1 & TS2 & INT2 \\
\hline $\mathrm{C}_{\text {subs }}$ & 0.568 & 0.813 & 0.816 & 0.817 & 0.731 & 0.721 \\
$\mathrm{~N}_{\text {subs }}$ & -0.592 & -0.690 & -0.628 & -0.739 & -0.648 & -0.779 \\
$\mathrm{O}_{\text {subs }}$ & -0.694 & -0.828 & -0.983 & -0.744 & -0.795 & -0.748 \\
$\mathrm{~N} \varepsilon_{\text {His399 }}$ & -0.070 & -0.057 & -0.076 & -0.075 & -0.028 & -0.352 \\
$\mathrm{OG}_{\text {Ser189 }}$ & -0.485 & -0.479 & -0.480 & -0.444 & -0.401 & -0.399 \\
\hline
\end{tabular}


Table S3. Key inter-atomic distances of the different states appearing along the acylation step of $N$-(4nitrophenyl)-butyramide catalyzed by F398D-H $\mathrm{H}^{+} \mathrm{Bs} 2$ variant. Structures optimized at M06-2X/MM level. All distances are given in $\AA$.

\begin{tabular}{lcccccc}
\hline & $\mathrm{RC}$ & $\mathrm{TS} 1$ & $\mathrm{INT1}$ & INT1 & TS2 & INT2 \\
\hline $\mathrm{OG}_{\text {Ser189 }}-\mathrm{C}_{\text {subs }}$ & 2.37 & 1.92 & 1.50 & 1.48 & 1.45 & \\
$\mathrm{OG}_{\text {Ser189 }}-\mathrm{HG}_{\text {Ser189 }}$ & 1.01 & 1.29 & 2.52 & 2.95 & 2.67 & \\
$\mathrm{~N} \varepsilon_{\text {His399 }}-\mathrm{HG}_{\text {Ser189 }}$ & 1.73 & 1.28 & 1.03 & 1.03 & 1.39 & \\
$\mathrm{C}_{\text {subs }}-\mathrm{N} 4_{\text {subs }}$ & 1.42 & 1.47 & 1.54 & 1.54 & 1.62 & \\
$\mathrm{HG}_{\text {Ser189 }}-\mathrm{N} 4_{\text {subs }}$ & 2.72 & 2.59 & 2.04 & 2.06 & 1.26 & \\
$\mathrm{HD}_{\text {His399 }}-\mathrm{OE} 1_{\text {Glu310 }}$ & 1.73 & 1.28 & 1.03 & 1.03 & 1.39 & \\
$\mathrm{HD}_{\text {His399 }}-\mathrm{ND} 1_{\text {His399 }}$ & 1.01 & 1.29 & 2.52 & 2.95 & 2.67 & \\
$\mathrm{C} 1_{\text {subs }}-\mathrm{O} 2_{\text {subs }}$ & 1.68 & 1.60 & 1.59 & 1.57 & 1.70 & \\
$\mathrm{O} 2_{\text {subs }}-\mathrm{H}_{\text {Ala190 }}$ & 1.03 & 1.05 & 1.06 & 1.06 & 1.04 & \\
$\mathrm{O}_{\text {subs }}-\mathrm{H}_{\text {Ala107 }}$ & 1.22 & 1.24 & 1.30 & 1.30 & 1.29 & \\
\hline
\end{tabular}

Table S4. ESP charges (in a.u.) of the key atoms of the amide substrate computed at M06-2X/MM level on the states involved in the acylation step catalyzed by the $\mathrm{F} 398 \mathrm{D}-\mathrm{H}^{+} \mathrm{Bs} 2$ variant from reactants state (RS) to Intermediate 2 (INT2).

\begin{tabular}{lcccccc}
\hline & RC & TS1 & INT1 & INT1 & TS2 & INT2 \\
\hline $\mathrm{C} 1_{\text {subs }}$ & 0.755 & 0.959 & 0.930 & 0.853 & 0.745 & \\
$\mathrm{~N} 4_{\text {subs }}$ & -0.776 & -0.836 & -0.797 & -0.715 & -0.395 & \\
$\mathrm{O} 2_{\text {subs }}$ & -0.692 & -0.840 & -0.988 & -0.978 & -0.950 & \\
$\mathrm{~N} \varepsilon_{\text {His399 }}$ & -0.229 & -0.135 & -0.092 & -0.092 & -0.198 & \\
$\mathrm{OG}_{\text {Ser189 }}$ & -0.573 & -0.655 & -0.561 & -0.540 & -0.510 & \\
\hline
\end{tabular}


Table S5. Key inter-atomic distances of the different states appearing along the acylation step of $N$-(4nitrophenyl)-butyramide catalyzed by D66I/L335K Bs2 variant. Structures optimized at M06-2X/MM level. All distances are given in $\AA$.

\begin{tabular}{lcccccc}
\hline & $\mathrm{RC}$ & $\mathrm{TS} 1$ & $\mathrm{INT1}$ & $\mathrm{INT1}$ & $\mathrm{TS} 2$ & INT2 \\
\hline $\mathrm{OG}_{\text {Ser189 }}-\mathrm{C}_{\text {subs }}$ & 2.44 & 1.91 & 1.49 & 1.48 & 1.41 & 1.33 \\
$\mathrm{OG}_{\text {Ser189 }}-\mathrm{HG}_{\text {Ser189 }}$ & 1.00 & 1.35 & 2.26 & 2.48 & 2.46 & 2.65 \\
$\mathrm{~N} \varepsilon_{\text {His399 }}-\mathrm{HG}_{\text {Ser189 }}$ & 1.67 & 1.19 & 1.04 & 1.05 & 1.50 & 1.77 \\
$\mathrm{C}_{\text {subs }}-\mathrm{N} 4_{\text {subs }}$ & 1.35 & 1.41 & 1.53 & 1.55 & 1.82 & 2.70 \\
$\mathrm{HG}_{\text {Ser189 }}-\mathrm{N} 4_{\text {subs }}$ & 2.68 & 2.62 & 1.89 & 1.76 & 1.12 & 1.04 \\
$\mathrm{HD}_{\text {His399 }}-\mathrm{OE} 1_{\text {Glu310 }}$ & 1.00 & 1.35 & 2.26 & 2.48 & 2.46 & 2.65 \\
$\mathrm{HD}_{\text {His399 }}-\mathrm{ND} 1_{\text {His399 }}$ & 1.68 & 1.59 & 1.56 & 1.57 & 1.68 & 1.70 \\
$\mathrm{C} 1_{\text {subs }}-\mathrm{O} 2_{\text {subs }}$ & 1.04 & 1.05 & 1.06 & 1.06 & 1.04 & 1.03 \\
$\mathrm{O}_{\text {subs }}-\mathrm{H}_{\text {Ala190 }}$ & 1.25 & 1.27 & 1.31 & 1.31 & 1.27 & 1.23 \\
$\mathrm{O}_{\text {subs }}-\mathrm{H}_{\text {Ala107 }}$ & 2.14 & 1.89 & 1.84 & 1.89 & 1.99 & 1.85 \\
\hline
\end{tabular}

Table S6. ESP charges (in a.u.) of the key atoms of the amide substrate computed at M06-2X/MM level on the states involved in the acylation step reaction catalyzed by the D66I/L335K Bs2 variant from reactants state (RS) to Intermediate 2 (INT2).

\begin{tabular}{lcccccc}
\hline & $\mathrm{RC}$ & $\mathrm{TS} 1$ & $\mathrm{INT1}$ & $\mathrm{INT1}$ & $\mathrm{TS} 2$ & $\mathrm{INT} 2$ \\
\hline $\mathrm{C} 1_{\text {subs }}$ & 0.847 & 0.970 & 0.837 & 0.938 & 0.946 & 0.928 \\
$\mathrm{~N} 4_{\text {subs }}$ & -0.858 & -0.963 & -0.757 & -0.845 & -0.680 & -1.048 \\
$\mathrm{O} 2_{\text {subs }}$ & -0.905 & -0.985 & -1.084 & -1.112 & -1.059 & -0.903 \\
$\mathrm{~N} \varepsilon_{\text {His399 }}$ & -0.122 & -0.095 & -0.173 & -0.211 & -0.305 & -0.371 \\
$\mathrm{OG}_{\text {Ser189 }}$ & -0.512 & -0.662 & -0.545 & -0.615 & -0.565 & -0.491 \\
\hline
\end{tabular}




\section{COMPUTATIONAL METHODS}

Models set up. Wild type $p$-nitrobenzyl (PNB) esterase sequence from Bacillus subtilis (with ID P37967) that corresponds to the protein used in the experimental measurements ${ }^{1}$ was initially taken from UniProt ${ }^{2}$ accessible resource of protein sequence and functional information. Due to the lack of crystallized structure for this specific variant, the framework for the required model, a natural variant of the protein (strain 168), was prepared based on PNB esterase from the same organism (Bs2; PDB ID: 1QE3). ${ }^{3}$ Both enzymes share $97.6 \%$ of sequence identity. Thus, the X-ray structure was used as a template to build the Bs2 model by adding the missing residues and introducing required mutations. All changes were done using Modeller. ${ }^{4}$

Based on the structure of the wild type which was studied in previous works ${ }^{5}$, three variants were prepared, F398D, protonated F398D- $\mathrm{H}^{+}$and $\mathrm{D} 66 \mathrm{I} / \mathrm{L} 335 \mathrm{~K}$. The protonation state of titratable residues was determined at $\mathrm{pH} 7$ by estimating $\mathrm{pKa}$ shifts generated by the local environment on titratable groups using the empirical program PropKa ver. 3.0.3. ${ }^{6,7}$ The values of pKa obtained for Glu188 (8.72), Glu214 (7.52), and Glu402 (10.32) ensured their protonated state. Additionally, all histidines residues present in the enzyme were found to be neutral, with pKa values varying between 4.12 and 6.19. After a detailed inspection of surrounding of each histidine residue, it was concluded that all should be protonated in $\mathrm{N} \delta$ position. $N$-(4-nitrophenyl)-butyramide substrate was placed inside the active site pocket covalently bound to catalytic Ser189 in the form of intermediate 1, in order to avoid possible substrate dissociation to the solvent during MD simulations. Thus, once the hydrogen atoms were added to the structure, the counterions ions were placed in the most electrostatically favorable positions to neutralize the system. Depending on the variant of the enzyme the 21,20 and18 sodium ions were added, for F398D, F398D-H ${ }^{+}$ and D66I/L335K, variant of Bs2, respectively. Subsequently, the system was solvated by placing it in a $100 \times 80 \times 80 \AA^{3}$ pre-equilibrated box of TIP $3 \mathrm{P}^{8}$ water molecules. Any water with an oxygen atom lying 
in a radius of $2.8 \AA$ from a heavy atom of the protein was deleted. In order to equilibrate the total system, classical MD simulations were done. For $N$-(4-nitrophenyl)-butyramide the same force field parameters were used as determined in our previous work. ${ }^{9}$ Such prepared model was optimized, then the system was heated to $303 \mathrm{~K}$ with $0.1 \mathrm{~K}$ temperature increment and equilibrated during short (100 ps) NPT MD simulations, the proper 50 ns of non-accelerated classical MD simulations were done using the NVT ensemble with AMBER force field, ${ }^{10}$ as implemented in NAMD software. ${ }^{11}$ The temperature during the MD simulation was controlled using the Langevin thermostat. ${ }^{12}$ In order to improve the time of simulations, cut-offs for nonbonding interactions were applied using a smooth switching function between 14.5 and $16 \AA$. During MD simulations all atoms were free to move. Periodic boundary conditions were used. Time-dependent evolution of the root mean square deviations (RMSD) for all three variants and Bfactors for F398D and D66I/L335K Bs2 variants are depicted in Figure S3. All the analysis of the MD, including RMSD, B-factors and contacts was done using AmberTools. ${ }^{13}$ Based on the RMSD obtained from MD simulations it was concluded that all three models of Bs2 variants that will be studied by QM/MM methods (F398D, F398D-H $\mathrm{H}^{+}$and D66I/L335K) do not suffer any dramatic changes during MD simulations, and their values oscillate within the standard deviation, not higher than $0.25 \AA$. Thus, the one structure from each of the MD simulations was chosen for further study applying QM/MM approach. The starting structures were selected by its proximity to the average values of RMSD.

QM/MM simulations. In present work, the standard additive hybrid QM/MM scheme was used to construct the total Hamiltonian, $\widehat{\mathrm{H}}_{\mathrm{QM} / \mathrm{MM}}$, where the total energy $E_{Q M / M M}$ is obtained as the sum of specific contributions, as presented in equation 1 :

$$
E_{Q M / M M}=\left\langle\Psi\left|\widehat{H_{o}}\right| \Psi\right\rangle+\left(\sum\left\langle\Psi\left|\frac{q_{M M}}{r_{e, M M}}\right| \Psi\right\rangle+\sum \sum \frac{z_{Q M} q_{M M}}{r_{Q M, M M}}\right)+E_{Q M / M M}^{v d W}+E_{M M}
$$


where $E_{M M}$ is the energy of the MM subsystem term, $E_{Q M-M M}^{v d W}$ the van der Waals interaction energy between the QM and MM subsystems and $E_{Q M-M M}^{e l e c t}$ includes both the Coulombic interaction of the QM nuclei $\left(Z_{Q M}\right)$ and the electrostatic interaction of the polarized electronic wave function $\left(q_{M M}\right)$ with the charges of the protein $\left(q_{M M}\right)$. The region described by quantum mechanics includes the side chains of the catalytic Ser189, His399 and Glu310 residues as well as the full substrate, and one water molecule, as shown in Figure S4. Three-link atoms ${ }^{14}$ were inserted where the QM/MM boundary intersected covalent bonds: these were placed between the Ca-Cb for Ser189, His299 and Glu310.

Finally, in QM part the 55 were defined including link atoms. The rest of the protein, counterions and solvent molecules (in total 73070 atoms in F398D and F398D- $\mathrm{H}^{+}$, and 73085 atoms in D66I/L335K variants) were represented by classical OPLS-AA force field ${ }^{15}$ and TIP3P force fields, respectively, as implemented in fDynamo library. ${ }^{16}$ The Austin Model 1 (AM1) ${ }^{17}$ semiempirical Hamiltonian and the Minnesota Functional M06-2X, ${ }^{18}$ with the standard 6-31+G(d,p) basis set, were used to treat the QM subset of atoms, as implemented in Mopac ${ }^{19}$ and Gaussian $09,{ }^{20}$ respectively. The atom positions of all residues presented beyond $25 \AA$ from the substrate were frozen and the same cut-offs as in MD simulations were applied for the nonbonding interactions.

Potential Energy Surfaces. Potential Energy Surfaces (PES) were explored by choosing and scanning the appropriate combination of internal coordinates $\left(\xi_{i}\right)$ assuming their dominant role in the shape of the reaction coordinate. Thus, a combination of different distances was controlled during exploration of all four chemical steps being part of the complete reaction path. In the first step of the reaction, the PES was generated by controlling the distance between nitrogen, $\mathrm{N} \varepsilon$ atom of His399 and hydrogen, $\mathrm{HG}$ atoms of Ser189, together with the distance between oxygen, OG atom of Ser189 and carbonyl carbon, C1 atom of a substrate, directing acylation process. In the second step antisymmetric combination of nitrogen, $\mathrm{N} \varepsilon$ 
atom and hydrogen $\mathrm{HG}$, attached to His399 and this hydrogen atom and its acceptor, nitrogen, N4 atom of the substrate, together with carbon-nitrogen (C1-N4) bond of a substrate were controlled.

In order to explore all PESs, the harmonic constraint of $5000 \mathrm{~kJ} \cdot \mathrm{mol}^{-1} \cdot \AA^{-2}$ was used to maintain the proper interatomic distances along the reaction coordinate, and a series of conjugate gradient optimizations and L-BFGS-B optimization algorithms ${ }^{21}$ were applied to obtain the final potential energy of the minimized constrained geometry. The QM sub-set of atoms were described by the Austin Model 1 (AM1) semiempirical Hamiltonian. The distances evolution was controlled by applying small size change of 0.1 $\AA$ when the distance between two heavy atoms was explored, or $0.05 \AA$ when the transfer of light hydrogen atom was involved.

A micro-macro iteration optimization algorithm ${ }^{22,23}$ together with the Baker's algorithm ${ }^{24,25}$ was used to localize, optimize, and characterize the transition states (TS) and structures using a Hessian matrix containing all the coordinates of the QM subsystem, whereas the gradient norm of the remaining movable atoms was maintained less than $0.25 \mathrm{kcal} \mathrm{mol}^{-1} \AA^{-1}$. the Intrinsic Reaction Coordinate ${ }^{10}$ (IRC) were traced down from located TSs to the connecting valleys in mass-weighted Cartesian coordinates. And the same micro-macro iteration optimization algorithm was used to optimized reactant complex (RC) and intermediates (Is). The existence of the saddle-points, as well as those located in minima, was confirmed by frequency calculation. Thus, for TS structures, only one imaginary value of frequency was registered, while for structures from located in the minimum of the PESs no imaginary values were found.

Free Energy Surfaces. FESs were obtained, in terms of two-dimensional potential mean force (2DPMF) ${ }^{26}$ for every step of the reaction using the Umbrella Sampling approach ${ }^{26,27}$ combined with the Weighted Histogram Analysis Method (WHAM). ${ }^{28}$ The procedure for the PMF calculation is straightforward and requires a series of molecular dynamics simulations in which the distinguished reaction coordinate variable, $\xi$, is constrained around particular values. The values of the variables 
sampled during the simulations are then pieced together to construct a distribution function from which the PMF is obtained as a function of the distinguished reaction coordinate $(\mathrm{W}(\xi))$. The PMF is related to the normalized probability of finding the system at a particular value of the chosen coordinate by eq 2 :

$$
W(\xi)=C-k T \ln \int \rho\left(r^{N}\right) \delta\left(\xi\left(r^{N}\right)-\xi\right) d r^{N-1}
$$

The activation free energy can be then expressed as:

$$
\Delta G^{\ddagger}(\xi)=W\left(\xi^{\ddagger}\right)-\left[W\left(\xi^{R}\right)+G_{\xi}\left(\xi^{R}\right)\right]
$$

where the superscripts indicate the value of the reaction coordinate at the reactants $(\mathrm{R})$, and at the TS ( $\$)$, and $G_{\xi}\left(\xi^{R}\right)$ is the free energy associated with setting the reaction coordinate to a specific value at the reactant state. Normally this last term makes a small contribution, and the activation free energy is directly estimated from the PMF change between the maximum of the profile and the reactant's minimum:

$$
\Delta G^{\ddagger}(\xi) \approx W\left(\xi^{\ddagger}\right)-W\left(\xi^{R}\right)=\Delta W^{\ddagger}(\xi)
$$

The selection of the reaction coordinate is usually trivial when the mechanism can be driven by a single internal coordinate or a simple combination (as the antisymmetric combination of two interatomic distances). However, this is not the case for all possible steps of the reaction subject of study in this paper where many coordinates are participating. Instead, we were compelled to obtain a much more computationally demanding $2 \mathrm{D}-\mathrm{PMF}$ using two coordinates: $\xi_{1}$ and $\xi_{2}$. The $2 \mathrm{D}-\mathrm{PMF}$ is related to the probability of finding the system at particular values of these two coordinates:

$$
W(\xi)=C^{\prime}-k T \ln \int \rho\left(r^{N}\right) \delta\left(\xi_{1}\left(r^{N}\right)-\xi_{1}\right) \delta\left(\xi_{2}\left(r^{N}\right)-\xi_{2}\right) d r^{N-2}
$$

To estimate the activation free energy from this quantity, we recovered one-dimensional PMF changes tracing a maximum probability reaction path on the 2D-PMF surface and integrating over the perpendicular coordinate. 
Thus, a series of MD simulations were performed adding a constraint for the selected reaction coordinates with an umbrella force constant of $2500 \mathrm{~kJ} \cdot \mathrm{mol}^{-1} \cdot \AA^{-2}$. In every window, QM/MM MD simulations were performed with a total of 5 ps of equilibration and 20 ps of production at $303 \mathrm{~K}$ using the Langevin-Verlet algorithm ${ }^{29}$ with a time step of $1 \mathrm{fs}$. Structures obtained in previously computed PESs were used as starting points for the MD simulations in every window.

Spline corrections. In order to improve lower quality results associated with the lower-level semiempirical calculations, high-level corrections were applied using Density Functional Theory (DFT). As already described in the literature, ${ }^{30,31}$ a correction term $S\left[\Delta E_{L L}^{H L}\left(\xi_{1}, \xi_{2}\right)\right]$ is interpolated to any value along reaction coordinates in the FES. A continuous energy function is used to obtain the corrected PMFs:

$$
E=E_{L L / M M}+S\left[\Delta E_{L L}^{H L}\left(\xi_{1}, \xi_{2}\right)\right]
$$

where $S$ is the two-dimensional spline function and $\Delta E_{L L}^{H L}$ is the difference between the energies obtained at low-level (LL) and high-level (HL) of the theory of the QM part. The AM1 semiempirical Hamiltonian was used as LL method, while the DFT method was selected for the HL energy calculation. In particular, HL energy calculations were performed by means of the hybrid M06-2X functional using the standard 6$31+\mathrm{G}(\mathrm{d}, \mathrm{p})$ basis set. These calculations were carried out using the Gaussian09 program.

Structural alignment of CALB and Bs2. To compare the environment generated by the enzyme surrounding the substrate a structural alignment was done between Bs2 and CALB. For this purpose, TS1 structures localized at M06-2X/MM level in both systems were used. Due to the poor percentage of sequence identity between enzymes a structural alignment based in sequence similarity was unachievable. For that reason, an alternative procedure was used involving the geometrical overlapping of the positions of atoms of the amide bond and the neighboring carbon atoms (Figure S12). Structures of protein were 
forced to align accordingly, that was achieved using a rotation quaternion around those selected atoms highlighted in Figure S13.

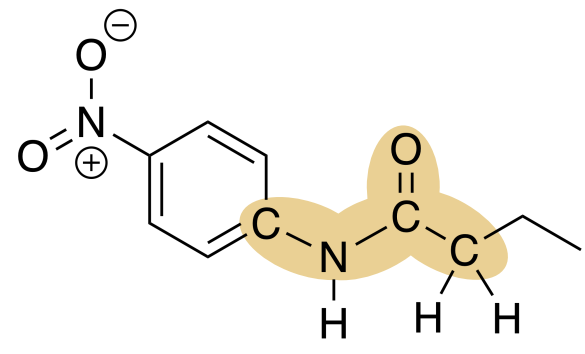

Figure S13. Structure of the substrate with highlighted atoms (in yellow) used as observed and reference points for geometrical overlapping.

The superimposition was done using quaternions implemented in QMCube. ${ }^{32}$ This vector formulation for molecule rotations is useful in large vectorized systems. It basically assumes that the optimal superposition can be found by finding the normalized eigenvector of the smallest eigenvalue of the quaternion $4 \times 4$ matrix, where the components of this eigenvector represent the optimal orientation of target structure towards the reference. Once the parameters of the quaternion are found, the molecule can be rotated applying the optimal rotation quaternion-derived matrix to the target molecule. ${ }^{33}$

When both proteins were aligned, structures were compared based on the position of the centers of mass of every residue inside each of protein. Residues of Bs2 and CALB were paired based on the proximity of their centers of mass, i.e. the pair of a residue from Bs 2 is the residue from CALB which center of mass lies the nearest to the tested residue. In order to reduce the error of alignment, the centers of mass were averaged along a $20 \mathrm{ps}$ constrained $\mathrm{QM} / \mathrm{MM} \mathrm{MD}$ on TS1 of both proteins. The pairing alignment is depicted in Figure S2. 
Electrostatic potential per residue. The electrostatic potential generated on $\mathrm{N} \varepsilon$ of the catalytic His399 was computed. Only the residues which center of mass lies within $15 \AA$ of the center of mass of the substrate were considered. Electrostatic potential per residue $\left(V_{\text {res }}\right)$ was computed based on equation 6 ,

$$
V_{\text {res }}=\sum_{i=1}^{n} \frac{q_{i}}{4 \pi \varepsilon \cdot r_{i}}
$$

where $\mathrm{n}$ corresponds to the number of atoms in the residue, $q_{i}$ is the charge of the atom taken from the OPLS-AA force field, and $r_{i}$ is the distance between atom $i$ and $\mathrm{N} \varepsilon$ of His399. Cut-offs of 14.5 and $16 \AA$ were applied. The electrostatic potential per residue was averaged along a 20 ps constrained QM/MM MD simulation on TS1. The electrostatic potential per residue in CALB and Bs2 is depicted in Figure S3. 


\section{STRUCTURES COORDINATES}

Table S7. X, Y and Z coordinates of QM atoms for Transition State Structures optimized at M06-2X/MM level for the acylation step of the F398D Bs2 variant.

\begin{tabular}{|c|c|c|c|c|c|c|c|}
\hline \multicolumn{8}{|c|}{ Acylation step } \\
\hline \multicolumn{4}{|c|}{ Transition State $1\left(v_{\mathrm{i}}=-383.062 \mathrm{~cm}^{-1}\right)$} & \multicolumn{4}{|c|}{ Transition State $2\left(v_{i}=-166.294 \mathrm{~cm}^{-1}\right)$} \\
\hline Atoms & $\mathrm{x}$ & $\mathrm{y}$ & $\mathrm{Z}$ & Atoms & $\mathrm{X}$ & $\mathrm{y}$ & $\mathrm{Z}$ \\
\hline $\mathrm{C}$ & 48.592 & 45.418 & 50.575 & $\mathrm{C}$ & 48.433 & 45.542 & 50.535 \\
\hline $\mathrm{H}$ & 49.662 & 45.396 & 50.318 & $\mathrm{H}$ & 49.517 & 45.623 & 50.396 \\
\hline $\mathrm{H}$ & 48.060 & 45.841 & 49.707 & $\mathrm{H}$ & 47.929 & 45.954 & 49.657 \\
\hline $\mathrm{O}$ & 48.430 & 46.285 & 51.690 & $\mathrm{O}$ & 48.104 & 46.295 & 51.705 \\
\hline $\mathrm{H}$ & 49.456 & 46.719 & 52.019 & $\mathrm{H}$ & 49.687 & 47.213 & 52.180 \\
\hline $\mathrm{C}$ & 54.375 & 45.243 & 56.520 & $\mathrm{C}$ & 54.371 & 45.232 & 56.502 \\
\hline $\mathrm{H}$ & 54.281 & 44.964 & 57.574 & $\mathrm{H}$ & 54.283 & 44.940 & 57.553 \\
\hline $\mathrm{H}$ & 53.356 & 45.475 & 56.187 & $\mathrm{H}$ & 53.350 & 45.469 & 56.179 \\
\hline $\mathrm{C}$ & 54.876 & 44.032 & 55.739 & $\mathrm{C}$ & 54.865 & 44.030 & 55.705 \\
\hline $\mathrm{H}$ & 55.960 & 43.916 & 55.821 & $\mathrm{H}$ & 55.950 & 43.913 & 55.773 \\
\hline $\mathrm{H}$ & 54.442 & 43.122 & 56.164 & $\mathrm{H}$ & 54.438 & 43.115 & 56.124 \\
\hline $\mathrm{C}$ & 54.562 & 43.998 & 54.246 & $\mathrm{C}$ & 54.535 & 44.001 & 54.220 \\
\hline $\mathrm{O}$ & 54.238 & 45.057 & 53.643 & $\mathrm{O}$ & 54.152 & 45.056 & 53.616 \\
\hline $\mathrm{O}$ & 54.662 & 42.873 & 53.688 & $\mathrm{O}$ & 54.673 & 42.901 & 53.639 \\
\hline $\mathrm{C}$ & 53.919 & 48.895 & 52.974 & $\mathrm{C}$ & 53.944 & 48.884 & 52.996 \\
\hline $\mathrm{H}$ & 54.073 & 49.215 & 54.008 & $\mathrm{H}$ & 54.099 & 49.184 & 54.035 \\
\hline $\mathrm{H}$ & 53.838 & 49.791 & 52.354 & $\mathrm{H}$ & 53.864 & 49.787 & 52.389 \\
\hline $\mathrm{C}$ & 52.649 & 48.081 & 52.878 & $\mathrm{C}$ & 52.676 & 48.087 & 52.890 \\
\hline $\mathrm{N}$ & 52.511 & 46.906 & 53.576 & $\mathrm{~N}$ & 52.506 & 46.873 & 53.529 \\
\hline $\mathrm{H}$ & 53.292 & 46.321 & 53.968 & $\mathrm{H}$ & 53.302 & 46.173 & 53.854 \\
\hline $\mathrm{C}$ & 51.329 & 46.361 & 53.281 & $\mathrm{C}$ & 51.293 & 46.409 & 53.273 \\
\hline $\mathrm{H}$ & 50.988 & 45.415 & 53.680 & $\mathrm{H}$ & 50.893 & 45.471 & 53.629 \\
\hline $\mathrm{N}$ & 50.655 & 47.132 & 52.437 & $\mathrm{~N}$ & 50.659 & 47.284 & 52.489 \\
\hline $\mathrm{C}$ & 51.477 & 48.215 & 52.168 & $\mathrm{C}$ & 51.509 & 48.334 & 52.220 \\
\hline $\mathrm{H}$ & 51.160 & 49.018 & 51.519 & $\mathrm{H}$ & 51.191 & 49.160 & 51.603 \\
\hline $\mathrm{C}$ & 47.315 & 47.773 & 51.521 & $\mathrm{C}$ & 47.165 & 47.299 & 51.626 \\
\hline $\mathrm{C}$ & 47.281 & 48.052 & 53.016 & $\mathrm{C}$ & 47.003 & 47.910 & 52.999 \\
\hline $\mathrm{H}$ & 48.311 & 48.085 & 53.390 & $\mathrm{H}$ & 47.924 & 48.418 & 53.301 \\
\hline $\mathrm{H}$ & 46.875 & 49.060 & 53.161 & $\mathrm{H}$ & 46.204 & 48.652 & 52.934 \\
\hline $\mathrm{C}$ & 46.433 & 47.077 & 53.824 & $\mathrm{C}$ & 46.616 & 46.838 & 54.026 \\
\hline $\mathrm{C}$ & 46.363 & 47.508 & 55.290 & $\mathrm{C}$ & 46.320 & 47.455 & 55.390 \\
\hline $\mathrm{H}$ & 45.902 & 48.495 & 55.406 & $\mathrm{H}$ & 45.464 & 48.135 & 55.353 \\
\hline $\mathrm{H}$ & 45.775 & 46.805 & 55.886 & $\mathrm{H}$ & 46.092 & 46.683 & 56.130 \\
\hline $\mathrm{H}$ & 47.365 & 47.560 & 55.730 & $\mathrm{H}$ & 47.179 & 48.024 & 55.757 \\
\hline $\mathrm{H}$ & 45.426 & 47.022 & 53.395 & $\mathrm{H}$ & 45.745 & 46.275 & 53.668 \\
\hline $\mathrm{H}$ & 46.864 & 46.075 & 53.734 & $\mathrm{H}$ & 47.437 & 46.118 & 54.110 \\
\hline $\mathrm{O}$ & 46.322 & 47.400 & 50.873 & $\mathrm{O}$ & 46.259 & 47.255 & 50.790 \\
\hline $\mathrm{N}$ & 48.296 & 48.615 & 50.794 & $\mathrm{~N}$ & 48.552 & 48.624 & 50.721 \\
\hline $\mathrm{H}$ & 48.815 & 48.115 & 50.082 & $\mathrm{H}$ & 49.171 & 48.183 & 50.038 \\
\hline $\mathrm{C}$ & 48.279 & 49.969 & 50.657 & $\mathrm{C}$ & 48.486 & 49.927 & 50.517 \\
\hline $\mathrm{C}$ & 49.254 & 50.573 & 49.814 & $\mathrm{C}$ & 49.334 & 50.623 & 49.581 \\
\hline $\mathrm{C}$ & 49.393 & 51.940 & 49.752 & $\mathrm{C}$ & 49.410 & 51.987 & 49.577 \\
\hline $\mathrm{C}$ & 48.546 & 52.757 & 50.514 & $\mathrm{C}$ & 48.595 & 52.751 & 50.445 \\
\hline $\mathrm{C}$ & 47.505 & 52.203 & 51.263 & $\mathrm{C}$ & 47.624 & 52.109 & 51.245 \\
\hline $\mathrm{C}$ & 47.356 & 50.830 & 51.310 & $\mathrm{C}$ & 47.558 & 50.743 & 51.263 \\
\hline $\mathrm{H}$ & 46.521 & 50.419 & 51.861 & $\mathrm{H}$ & 46.816 & 50.249 & 51.877 \\
\hline $\mathrm{H}$ & 46.827 & 52.851 & 51.813 & $\mathrm{H}$ & 46.952 & 52.708 & 51.852 \\
\hline $\mathrm{N}$ & 48.800 & 54.168 & 50.593 & $\mathrm{~N}$ & 48.838 & 54.121 & 50.608 \\
\hline $\mathrm{O}$ & 49.705 & 54.646 & 49.898 & $\mathrm{O}$ & 49.736 & 54.662 & 49.925 \\
\hline $\mathrm{O}$ & 48.127 & 54.843 & 51.358 & $\mathrm{O}$ & 48.185 & 54.760 & 51.439 \\
\hline $\mathrm{H}$ & 50.170 & 52.382 & 49.140 & $\mathrm{H}$ & 50.101 & 52.495 & 48.916 \\
\hline $\mathrm{H}$ & 49.926 & 49.945 & 49.236 & $\mathrm{H}$ & 49.969 & 50.042 & 48.915 \\
\hline $\mathrm{H}$ & 54.745 & 48.390 & 52.724 & $\mathrm{H}$ & 54.763 & 48.375 & 52.732 \\
\hline $\mathrm{H}$ & 54.945 & 46.063 & 56.473 & $\mathrm{H}$ & 54.943 & 46.051 & 56.460 \\
\hline $\mathrm{H}$ & 48.295 & 44.474 & 50.721 & $\mathrm{H}$ & 48.201 & 44.582 & 50.688 \\
\hline
\end{tabular}


Table S8. X, Y and Z coordinates of QM atoms for Transition State Structures optimized at M06-2X/MM level for the acylation step of the F398D-H $\mathrm{H}^{+}$Bs2 variant.

\begin{tabular}{|c|c|c|c|c|c|c|c|}
\hline \multicolumn{8}{|c|}{ Acylation step } \\
\hline \multicolumn{4}{|c|}{ Transition State $1\left(v_{\mathrm{i}}=-1051.441 \mathrm{~cm}^{-1}\right)$} & \multicolumn{4}{|c|}{ Transition State $2\left(v_{i}=-1007.015 \mathrm{~cm}^{-1}\right)$} \\
\hline Atoms & $\mathrm{X}$ & $\mathrm{y}$ & $\mathrm{Z}$ & Atoms & $\mathrm{X}$ & $\mathrm{y}$ & $\mathrm{Z}$ \\
\hline $\mathrm{C}$ & 44.224 & 43.709 & 52.695 & $\mathrm{C}$ & 44.120 & 43.553 & 52.600 \\
\hline $\mathrm{H}$ & 45.016 & 44.388 & 53.034 & $\mathrm{H}$ & 44.818 & 44.208 & 53.136 \\
\hline $\mathrm{H}$ & 43.741 & 44.205 & 51.837 & $\mathrm{H}$ & 43.702 & 44.112 & 51.754 \\
\hline $\mathrm{O}$ & 43.277 & 43.587 & 53.748 & $\mathrm{O}$ & 43.094 & 43.153 & 53.494 \\
\hline $\mathrm{H}$ & 43.563 & 44.229 & 54.828 & $\mathrm{H}$ & 42.608 & 45.205 & 55.131 \\
\hline $\mathrm{C}$ & 46.591 & 41.656 & 59.922 & $\mathrm{C}$ & 46.585 & 41.711 & 59.882 \\
\hline $\mathrm{H}$ & 46.396 & 40.633 & 60.262 & $\mathrm{H}$ & 46.441 & 40.677 & 60.215 \\
\hline $\mathrm{H}$ & 45.929 & 41.798 & 59.060 & $\mathrm{H}$ & 45.906 & 41.841 & 59.032 \\
\hline $\mathrm{C}$ & 48.059 & 41.728 & 59.453 & $\mathrm{C}$ & 48.037 & 41.859 & 59.389 \\
\hline $\mathrm{H}$ & 48.748 & 41.867 & 60.293 & $\mathrm{H}$ & 48.727 & 42.068 & 60.213 \\
\hline $\mathrm{H}$ & 48.318 & 40.769 & 58.993 & $\mathrm{H}$ & 48.354 & 40.906 & 58.954 \\
\hline $\mathrm{C}$ & 48.442 & 42.787 & 58.415 & $\mathrm{C}$ & 48.341 & 42.899 & 58.303 \\
\hline $\mathrm{O}$ & 47.692 & 43.766 & 58.202 & $\mathrm{O}$ & 47.502 & 43.785 & 58.014 \\
\hline $\mathrm{O}$ & 49.528 & 42.592 & 57.771 & $\mathrm{O}$ & 49.460 & 42.761 & 57.701 \\
\hline $\mathrm{C}$ & 44.854 & 46.163 & 59.331 & $\mathrm{C}$ & 44.963 & 46.184 & 59.563 \\
\hline $\mathrm{H}$ & 44.434 & 45.531 & 60.117 & $\mathrm{H}$ & 44.626 & 45.458 & 60.307 \\
\hline $\mathrm{H}$ & 44.290 & 47.099 & 59.343 & $\mathrm{H}$ & 44.419 & 47.111 & 59.762 \\
\hline $\mathrm{C}$ & 44.606 & 45.457 & 57.988 & $\mathrm{C}$ & 44.516 & 45.693 & 58.195 \\
\hline $\mathrm{N}$ & 45.247 & 44.271 & 57.714 & $\mathrm{~N}$ & 44.977 & 44.533 & 57.615 \\
\hline $\mathrm{H}$ & 46.099 & 43.911 & 58.201 & $\mathrm{H}$ & 45.818 & 44.014 & 57.927 \\
\hline $\mathrm{C}$ & 44.870 & 43.824 & 56.519 & $\mathrm{C}$ & 44.316 & 44.326 & 56.468 \\
\hline $\mathrm{H}$ & 45.242 & 42.917 & 56.068 & $\mathrm{H}$ & 44.483 & 43.488 & 55.806 \\
\hline $\mathrm{N}$ & 43.978 & 44.634 & 55.967 & $\mathrm{~N}$ & 43.425 & 45.285 & 56.257 \\
\hline $\mathrm{C}$ & 43.806 & 45.678 & 56.879 & $\mathrm{C}$ & 43.558 & 46.161 & 57.325 \\
\hline $\mathrm{H}$ & 43.150 & 46.508 & 56.666 & $\mathrm{H}$ & 42.972 & 47.066 & 57.399 \\
\hline $\mathrm{C}$ & 41.489 & 44.046 & 53.220 & $\mathrm{C}$ & 41.788 & 43.751 & 53.327 \\
\hline $\mathrm{C}$ & 40.814 & 43.087 & 54.194 & $\mathrm{C}$ & 40.915 & 42.908 & 54.281 \\
\hline $\mathrm{H}$ & 41.436 & 42.997 & 55.089 & $\mathrm{H}$ & 41.515 & 42.644 & 55.158 \\
\hline $\mathrm{H}$ & 39.869 & 43.531 & 54.527 & $\mathrm{H}$ & 40.060 & 43.482 & 54.648 \\
\hline $\mathrm{C}$ & 40.543 & 41.728 & 53.554 & $\mathrm{C}$ & 40.413 & 41.647 & 53.579 \\
\hline $\mathrm{C}$ & 39.713 & 40.809 & 54.446 & $\mathrm{C}$ & 39.484 & 40.810 & 54.455 \\
\hline $\mathrm{H}$ & 40.217 & 40.615 & 55.398 & $\mathrm{H}$ & 39.988 & 40.493 & 55.374 \\
\hline $\mathrm{H}$ & 38.736 & 41.254 & 54.669 & $\mathrm{H}$ & 38.593 & 41.379 & 54.743 \\
\hline $\mathrm{H}$ & 39.538 & 39.845 & 53.960 & $\mathrm{H}$ & 39.151 & 39.909 & 53.932 \\
\hline $\mathrm{H}$ & 40.023 & 41.886 & 52.602 & $\mathrm{H}$ & 39.894 & 41.947 & 52.662 \\
\hline $\mathrm{H}$ & 41.498 & 41.258 & 53.294 & $\mathrm{H}$ & 41.271 & 41.048 & 53.255 \\
\hline $\mathrm{O}$ & 41.418 & 43.853 & 51.993 & $\mathrm{O}$ & 41.390 & 43.874 & 52.105 \\
\hline $\mathrm{N}$ & 41.587 & 45.436 & 53.676 & $\mathrm{~N}$ & 42.021 & 45.203 & 54.016 \\
\hline $\mathrm{H}$ & 42.500 & 45.899 & 53.528 & $\mathrm{H}$ & 42.773 & 45.693 & 53.489 \\
\hline $\mathrm{C}$ & 40.541 & 46.277 & 53.893 & $\mathrm{C}$ & 40.916 & 46.117 & 54.079 \\
\hline $\mathrm{C}$ & 40.840 & 47.631 & 54.214 & $\mathrm{C}$ & 41.188 & 47.419 & 54.527 \\
\hline $\mathrm{C}$ & 39.857 & 48.508 & 54.599 & $\mathrm{C}$ & 40.159 & 48.306 & 54.800 \\
\hline $\mathrm{C}$ & 38.528 & 48.062 & 54.679 & $\mathrm{C}$ & 38.847 & 47.873 & 54.614 \\
\hline $\mathrm{C}$ & 38.182 & 46.772 & 54.272 & $\mathrm{C}$ & 38.550 & 46.630 & 54.067 \\
\hline $\mathrm{C}$ & 39.170 & 45.902 & 53.843 & $\mathrm{C}$ & 39.593 & 45.760 & 53.775 \\
\hline $\mathrm{H}$ & 38.878 & 44.927 & 53.474 & $\mathrm{H}$ & 39.379 & 44.807 & 53.311 \\
\hline $\mathrm{H}$ & 37.141 & 46.463 & 54.285 & $\mathrm{H}$ & 37.519 & 46.344 & 53.890 \\
\hline $\mathrm{N}$ & 37.546 & 48.930 & 55.255 & $\mathrm{~N}$ & 37.756 & 48.713 & 55.090 \\
\hline $\mathrm{O}$ & 36.488 & 48.457 & 55.666 & $\mathrm{O}$ & 36.640 & 48.222 & 55.173 \\
\hline $\mathrm{O}$ & 37.800 & 50.126 & 55.349 & $\mathrm{O}$ & 38.006 & 49.863 & 55.420 \\
\hline $\mathrm{H}$ & 40.102 & 49.525 & 54.881 & $\mathrm{H}$ & 40.362 & 49.298 & 55.185 \\
\hline $\mathrm{H}$ & 41.878 & 47.947 & 54.184 & $\mathrm{H}$ & 42.225 & 47.713 & 54.674 \\
\hline $\mathrm{H}$ & 45.807 & 46.345 & 59.577 & $\mathrm{H}$ & 45.936 & 46.344 & 59.731 \\
\hline $\mathrm{H}$ & 46.321 & 42.276 & 60.659 & $\mathrm{H}$ & 46.306 & 42.313 & 60.631 \\
\hline $\mathrm{H}$ & 44.673 & 42.872 & 52.383 & $\mathrm{H}$ & 44.625 & 42.750 & 52.283 \\
\hline
\end{tabular}


Table S9. X, Y and Z coordinates of QM atoms for Transition State Structures optimized at M06-2X/MM level for the acylation step of the reaction of the D66I/L335K Bs2 variant.

\begin{tabular}{|c|c|c|c|c|c|c|c|}
\hline \multicolumn{8}{|c|}{ Acylation step } \\
\hline \multicolumn{4}{|c|}{ Transition State $1\left(v_{\mathrm{i}}=-672.467 \mathrm{~cm}^{-1}\right)$} & \multicolumn{4}{|c|}{ Transition State $2\left(v_{\mathrm{i}}=-164.117 \mathrm{~cm}^{-1}\right)$} \\
\hline Atoms & $\mathrm{x}$ & $\mathrm{y}$ & $\mathrm{Z}$ & Atoms & $\mathrm{X}$ & $\mathrm{y}$ & $\mathrm{Z}$ \\
\hline $\mathrm{C}$ & 49.182 & 44.719 & 50.456 & $\mathrm{C}$ & 48.981 & 44.552 & 50.594 \\
\hline $\mathrm{H}$ & 50.226 & 44.892 & 50.135 & $\mathrm{H}$ & 50.054 & 44.765 & 50.484 \\
\hline $\mathrm{H}$ & 48.556 & 45.251 & 49.722 & $\mathrm{H}$ & 48.465 & 45.130 & 49.825 \\
\hline $\mathrm{O}$ & 48.999 & 45.298 & 51.728 & $\mathrm{O}$ & 48.584 & 44.996 & 51.886 \\
\hline $\mathrm{H}$ & 50.048 & 45.931 & 52.289 & $\mathrm{H}$ & 49.691 & 47.074 & 52.602 \\
\hline $\mathrm{C}$ & 56.620 & 42.266 & 55.271 & $\mathrm{C}$ & 56.638 & 42.244 & 55.295 \\
\hline $\mathrm{H}$ & 57.285 & 41.778 & 54.562 & $\mathrm{H}$ & 57.297 & 41.782 & 54.563 \\
\hline $\mathrm{H}$ & 56.600 & 41.615 & 56.154 & $\mathrm{H}$ & 56.604 & 41.553 & 56.145 \\
\hline $\mathrm{C}$ & 55.225 & 42.300 & 54.624 & $\mathrm{C}$ & 55.240 & 42.304 & 54.642 \\
\hline $\mathrm{H}$ & 54.794 & 41.296 & 54.679 & $\mathrm{H}$ & 54.810 & 41.297 & 54.674 \\
\hline $\mathrm{H}$ & 54.528 & 42.972 & 55.132 & $\mathrm{H}$ & 54.545 & 42.966 & 55.164 \\
\hline $\mathrm{C}$ & 55.293 & 42.651 & 53.140 & $\mathrm{C}$ & 55.262 & 42.681 & 53.161 \\
\hline $\mathrm{O}$ & 54.604 & 43.623 & 52.716 & $\mathrm{O}$ & 54.605 & 43.684 & 52.783 \\
\hline $\mathrm{O}$ & 55.996 & 41.942 & 52.379 & $\mathrm{O}$ & 55.892 & 41.934 & 52.354 \\
\hline $\mathrm{C}$ & 54.411 & 47.214 & 54.198 & $\mathrm{C}$ & 54.512 & 47.275 & 54.324 \\
\hline $\mathrm{H}$ & 54.512 & 46.974 & 55.260 & $\mathrm{H}$ & 54.611 & 46.983 & 55.372 \\
\hline $\mathrm{H}$ & 54.380 & 48.303 & 54.117 & $\mathrm{H}$ & 54.554 & 48.366 & 54.281 \\
\hline $\mathrm{C}$ & 53.089 & 46.644 & 53.702 & $\mathrm{C}$ & 53.167 & 46.834 & 53.809 \\
\hline $\mathrm{N}$ & 52.913 & 45.278 & 53.650 & $\mathrm{~N}$ & 52.856 & 45.515 & 53.571 \\
\hline $\mathrm{H}$ & 53.678 & 44.558 & 53.609 & $\mathrm{H}$ & 53.531 & 44.727 & 53.543 \\
\hline $\mathrm{C}$ & 51.718 & 45.010 & 53.127 & $\mathrm{C}$ & 51.613 & 45.460 & 53.061 \\
\hline $\mathrm{H}$ & 51.330 & 44.018 & 52.946 & $\mathrm{H}$ & 51.115 & 44.541 & 52.785 \\
\hline $\mathrm{N}$ & 51.074 & 46.134 & 52.856 & $\mathrm{~N}$ & 51.091 & 46.668 & 52.970 \\
\hline $\mathrm{C}$ & 51.914 & 47.179 & 53.216 & $\mathrm{C}$ & 52.052 & 47.539 & 53.434 \\
\hline $\mathrm{H}$ & 51.617 & 48.212 & 53.117 & $\mathrm{H}$ & 51.883 & 48.605 & 53.468 \\
\hline $\mathrm{C}$ & 47.515 & 46.489 & 51.918 & $\mathrm{C}$ & 47.638 & 46.020 & 52.072 \\
\hline $\mathrm{C}$ & 46.965 & 45.800 & 53.156 & $\mathrm{C}$ & 47.055 & 45.819 & 53.461 \\
\hline $\mathrm{H}$ & 45.996 & 46.286 & 53.351 & $\mathrm{H}$ & 46.428 & 46.693 & 53.680 \\
\hline $\mathrm{H}$ & 46.748 & 44.770 & 52.858 & $\mathrm{H}$ & 46.378 & 44.963 & 53.333 \\
\hline $\mathrm{C}$ & 47.795 & 45.816 & 54.434 & $\mathrm{C}$ & 48.022 & 45.535 & 54.601 \\
\hline $\mathrm{C}$ & 47.070 & 45.048 & 55.537 & $\mathrm{C}$ & 47.256 & 45.026 & 55.818 \\
\hline $\mathrm{H}$ & 46.894 & 44.009 & 55.238 & $\mathrm{H}$ & 46.720 & 44.103 & 55.571 \\
\hline $\mathrm{H}$ & 47.634 & 45.048 & 56.471 & $\mathrm{H}$ & 47.915 & 44.824 & 56.664 \\
\hline $\mathrm{H}$ & 46.091 & 45.496 & 55.743 & $\mathrm{H}$ & 46.511 & 45.760 & 56.144 \\
\hline $\mathrm{H}$ & 48.770 & 45.363 & 54.233 & $\mathrm{H}$ & 48.745 & 44.784 & 54.267 \\
\hline $\mathrm{H}$ & 47.985 & 46.839 & 54.782 & $\mathrm{H}$ & 48.594 & 46.434 & 54.859 \\
\hline $\mathrm{O}$ & 46.869 & 46.370 & 50.835 & $\mathrm{O}$ & 46.810 & 46.281 & 51.143 \\
\hline $\mathrm{N}$ & 48.131 & 47.722 & 52.211 & $\mathrm{~N}$ & 48.676 & 47.490 & 52.366 \\
\hline $\mathrm{H}$ & 48.244 & 47.977 & 53.188 & $\mathrm{H}$ & 48.376 & 47.936 & 53.240 \\
\hline $\mathrm{C}$ & 48.510 & 48.685 & 51.283 & $\mathrm{C}$ & 48.815 & 48.485 & 51.367 \\
\hline $\mathrm{C}$ & 48.365 & 50.045 & 51.611 & $\mathrm{C}$ & 48.327 & 49.783 & 51.575 \\
\hline $\mathrm{C}$ & 48.767 & 51.032 & 50.724 & $\mathrm{C}$ & 48.601 & 50.797 & 50.665 \\
\hline $\mathrm{C}$ & 49.334 & 50.656 & 49.511 & $\mathrm{C}$ & 49.351 & 50.490 & 49.534 \\
\hline $\mathrm{C}$ & 49.509 & 49.315 & 49.167 & $\mathrm{C}$ & 49.827 & 49.204 & 49.285 \\
\hline $\mathrm{C}$ & 49.084 & 48.331 & 50.046 & $\mathrm{C}$ & 49.549 & 48.201 & 50.204 \\
\hline $\mathrm{H}$ & 49.240 & 47.283 & 49.820 & $\mathrm{H}$ & 49.967 & 47.208 & 50.069 \\
\hline $\mathrm{H}$ & 50.028 & 49.068 & 48.246 & $\mathrm{H}$ & 50.463 & 49.027 & 48.423 \\
\hline $\mathrm{N}$ & 49.793 & 51.678 & 48.584 & $\mathrm{~N}$ & 49.721 & 51.557 & 48.606 \\
\hline $\mathrm{O}$ & 49.847 & 52.845 & 48.981 & $\mathrm{O}$ & 49.603 & 52.720 & 48.989 \\
\hline $\mathrm{O}$ & 50.099 & 51.346 & 47.455 & $\mathrm{O}$ & 50.129 & 51.248 & 47.504 \\
\hline $\mathrm{H}$ & 48.648 & 52.081 & 50.971 & $\mathrm{H}$ & 48.275 & 51.815 & 50.846 \\
\hline $\mathrm{H}$ & 47.939 & 50.332 & 52.568 & $\mathrm{H}$ & 47.795 & 50.023 & 52.491 \\
\hline $\mathrm{H}$ & 55.217 & 46.873 & 53.715 & $\mathrm{H}$ & 55.287 & 46.905 & 53.812 \\
\hline $\mathrm{H}$ & 57.020 & 43.146 & 55.525 & $\mathrm{H}$ & 57.052 & 43.101 & 55.601 \\
\hline $\mathrm{H}$ & 49.012 & 43.738 & 50.360 & $\mathrm{H}$ & 48.846 & 43.571 & 50.455 \\
\hline
\end{tabular}




\section{EXPERIMENTAL METHODS}

General information. Deionized water was obtained by an Elga PURELAB Option system $(15 \mathrm{M} \Omega \cdot \mathrm{cm})$. Analytical Thin Layer Chromatography (TLC) was carried out with silica gel 60 F254 aluminum sheets from Merck. Detection was carried out using UV light ( $\lambda=254 \mathrm{~nm}$ and $366 \mathrm{~nm})$, followed by immersion in permanganate or cerium ammonium molybdate staining solution with subsequent development via careful heating with a heat gun. Flash column chromatography was performed using silica gel (pore size $60 \AA, 0.040-0.063 \mathrm{~mm}) . N$-(4-nitrophenyl)-butyramide was synthesized using a known procedure. ${ }^{9}$ All other solvents and reagents were obtained from commercial sources and used as received.

Plasmid miniprep-kit and gel extraction-kit were purchased from Qiagen. DNA oligos were purchased from Sigma-Aldrich. The gene encoding for wild-type Bs $2^{1}$ with a C-terminal His-tag (see below) was purchased as a double-stranded fragment form Thermo Fisher Scientifcic GeneArt. Gibson Assembly was performed using New England Biolabs NEBuilder ${ }^{\circledR} H i F i$ DNA Assembly master mix. Restriction enzymes and required reagents were obtained from Thermo Fisher Scientific. Takara PrimeSTAR Max was employed for site-directed mutagenesis. All kits and enzymes were used exactly following the manufacturers' protocols. DNA sequencing of constructed plasmids and mutants was obtained from Eurofins Genomics using T7 promoter and terminator primers.

A VWR 3510 benchtop $\mathrm{pH}$ Meter connected to a Jenway micro $\mathrm{pH}$ electrode or a $V W R$ Universal $\mathrm{pH}$ electrode were used for the $\mathrm{pH}$ adjustment of buffers and reaction mixtures employing either $1.0 \mathrm{M}$ or 0.1 M sodium hydroxide solution or hydrochloric acid.

Size exclusion chromatography was performed using a GE Healthcare ÄKTA Purifier workstation or a Bio-Rad NGC Medium-Pressure Liquid Chromatography System. 
Protein concentrations were determined using a Thermo Scientific NanoDrop One spectrophotometer measuring the absorption at $280 \mathrm{~nm}$ using the

Protein liquid chromatography-mass spectrometry (LC-MS) was performed on a Waters Acquity H-Class UPLC system combined with a Waters Synapt G2-Si quadrupole time of flight mass spectrometer. A Waters Acquity UPLC Protein C4 BEH column $300 \AA$, $1.7 \mu \mathrm{m}(2.1 \times 100 \mathrm{~mm})$ held at $60{ }^{\circ} \mathrm{C}$ was applied. A flow rate of $0.2 \mathrm{~mL} / \mathrm{min}$ and the gradient of eluents A and B highlighted below were employed.

\begin{tabular}{c|c|c} 
Time / $\mathbf{m i n}$ & $\mathbf{A}\left(\mathrm{H}_{\mathbf{2}} \mathbf{O}, \mathbf{0 . 1} \% \mathbf{C H O} \mathbf{C H}_{2} \mathbf{H}\right) / \%$ & $\mathbf{B}\left(\mathbf{A C N}, \mathbf{0 . 1} \% \mathbf{C H O} \mathbf{C H}_{2} \mathbf{H}\right) / \%$ \\
\hline 0 & 95 & 5 \\
50 & 95 & 5 \\
52 & 35 & 65 \\
54 & 3 & 97 \\
56 & 3 & 97 \\
60 & 95 & 5 \\
\end{tabular}

The data was collected in positive electrospray ionization mode and analyzed using Waters MassLynx 4.1. Deconvoluted mass spectra were generate using the maximum entropy 1 (MaxEnt 1) software.

Cloning, expression and purification of recombinant proteins. The gene encoding for the wild-type Bs2 with a C-terminal linker and His-tag (GSSHHHHHHSSG), and 27 and 20 bases either side complimentary to the vector was purchased as a double-stranded fragment. After a NcoI and BamHI restriction enzyme digested pET28a vector the wild-type Bs2 gene was cloned in-between by Gibson assembly. The incorporation of the wild-type Bs2 gene was confirmed by DNA sequencing. Finally, Bs2 F398D was prepared by site-directed mutagenesis. 
The plasmid containing the gene for Bs2 F398D was transformed into $\mathrm{Ca}^{2+}$ chemically competent BL21 (DE3) cells and grown on LB agar plates supplemented with kanamycin $(50 \mu \mathrm{g} / \mathrm{mL})$ at $37{ }^{\circ} \mathrm{C}$ overnight. One colony from the plate was picked to inoculate a $10 \mathrm{~mL} \mathrm{LB}$ starter culture containing kanamycin (50 $\mu \mathrm{g} / \mathrm{mL}$ ) and grown at $37^{\circ} \mathrm{C}$ and $180 \mathrm{rpm}$ overnight. The starter culture was diluted into $1 \mathrm{~L}$ of fresh LB media containing kanamycin $(50 \mu \mathrm{g} / \mathrm{mL})$. The cells were grown at $37^{\circ} \mathrm{C}$ and $200 \mathrm{rpm}$, until they reached an OD600 of 0.8 , and IPTG was added to reach a final concentration of $1.0 \mathrm{mM}$. The cells were then incubated overnight at $20{ }^{\circ} \mathrm{C}$. The cultures were harvested by centrifugation $\left(4,000 \mathrm{rpm}, 4{ }^{\circ} \mathrm{C}, 30 \mathrm{~min}\right)$ and the dry pellet was stored at $-20^{\circ} \mathrm{C}$.

The pellet was subjected to a freeze-thaw cycle, resuspended in $25 \mathrm{~mL}$ of lysis buffer $1(50 \mathrm{mM} \mathrm{NaP}$, $300 \mathrm{mM} \mathrm{NaCl}, \mathrm{pH} \mathrm{7.5)}$ and lysed by sonication (7 min, $5 \mathrm{~s}$ on, $10 \mathrm{~s}$ off). The insoluble fraction was removed by centrifugation at $18000 \mathrm{rpm}$ for $25 \mathrm{~min}$ at $4{ }^{\circ} \mathrm{C}$. The supernatant was mixed with $3 \mathrm{~mL}$ of $\mathrm{Ni}$ NTA affinity resin for His-tag affinity purification which was equilibrated with the lysis buffer. After incubation at $4{ }^{\circ} \mathrm{C}$ for $0.5 \mathrm{~h}$ the resin was washed twice with 1.5 volumes of resin wash buffer $1(50 \mathrm{mM}$ $\mathrm{NaP}_{\mathrm{i}}, 300 \mathrm{mM} \mathrm{NaCl}, 10 \mathrm{mM}$ imidazole, $\mathrm{pH}$ 7.5). The protein was eluted with elution buffer (5× resin volume, $50 \mathrm{mM} \mathrm{NaP}$, $300 \mathrm{mM} \mathrm{NaCl}, 250 \mathrm{mM}$ imidazole, $\mathrm{pH}$ 7.5). Samples of the wash and elution fractions were collected and run on SDS-PAGE gel $(12 \% \mathrm{w} / \mathrm{v})$. The elution fractions containing the respective Bs2 variant were pooled and concentrated to $5 \mathrm{~mL}$ using Amicon ultra centrifugation with a $10 \mathrm{kDa}$ cutoff. The concentrated protein solution was applied to size exclusion chromatography (Generon ProSEC 26/60 3-70 HR column, $50 \mathrm{mM} \mathrm{NaP}$, $\mathrm{pH}$ 7.0). Fractions containing protein (analysis by following $280 \mathrm{~nm}$ UV trace) were collected and the samples loaded on SDS-PAGE to check the purity of the protein $(12 \% \mathrm{w} / \mathrm{v})$. Fractions containing the respective Bs2 variant were pooled, transferred to a centrifugal concentrator with a $10 \mathrm{kDa}$ cutoff, and were concentrated to $15-20 \mathrm{mg} / \mathrm{mL}$ as determined by nanodrop measurement at $280 \mathrm{~nm}$, with $\varepsilon_{280}=80330 \mathrm{M}^{-1} \mathrm{~cm}^{-1}$ calculated using https://web.expasy.org/protparam. 
The protein solutions were stored at $4{ }^{\circ} \mathrm{C}$ until further usage within a week. It should be noted that the solutions showed no significant loss in activity over 4 weeks (longer stability has not been monitored) and only minimal protein precipitation was observed.

Site-directed mutagenesis. The F398D mutation was introduced by site-directed mutagenesis PCR using Takara PrimeSTAR Max polymerase and the accompanying buffers, dNTPs and primers mentioned below. A $50 \mu \mathrm{L}$ PCR reaction was prepared according to the instructions, the reaction mixture was distributed equally $(12.5 \mu \mathrm{L})$ over 4 PCR tubes, and four different annealing temperatures in $2{ }^{\circ} \mathrm{C}$ increments were used. The PCR products were analyzed by agarose gel, correct size bands extracted, and clones of each plasmid sent for DNA sequencing. Primers used are given in the following with the site of mutation marked in bold red.

Forward primer: ACCTCCGTATAACAAAGCAGATCATGCACTGG

Reverse primer: CTTTGTTATACGGAGGTTTTTTCGGATGCC

\section{Nucleotide sequence.}

ATGACCCACCAGATTGTTACCACACAGTATGGTAAAGTGAAAGGCACCACCGAAAATGGTGTTCATAAA TGGAAAGGTATCCCGTATGCAAAACCGCCTGTTGGTCAGTGGCGTTTTAAAGCACCGGAACCGCCTGAA GTTTGGGAAGATGTTCTGGATGCAACCGCATATGGTAGCATTTGTCCGCAGCCGAGCGATCTGCTGAGC CTGAGCTATACCGAACTGCCTCGTCAGAGCGAAGATTGTCTGTATGTTAATGTTTTTGCACCGGATACG CCGAGCAAAAATCTGCCGGTTATGGTTTGGATTCATGGTGGTGCATTTTATCTTGGTGCAGGTAGCGAA CCGCTGTATGATGGTAGCAAACTGGCAGCACAGGGTGAAGTTATTGTTGTTACCCTGAATTATCGTCTG GGTCCGTTTGGTTTTCTGCATCTGAGCAGCTTTAATGAAGCCTATAGCGATAATCTGGGTCTGCTGGAT CAGGCAGCAGCACTGAAATGGGTTCGTGAAAACATTAGCGCATTTGGTGGTGATCCGGATAATGTTACC GTTTTTGGTGAAAGTGCCGGTGGTATGAGCATTGCAGCACTGCTGGCCATGCCTGCAGCAAAAGGTCTG TTTCAGAAAGCAATTATGGAAAGCGGTGCAAGCCGTACCATGACCAAAGAACAGGCAGCAAGTACCAGC GCAGCATTTCTGCAGGTTCTGGGTATTAATGAAGGTCAGCTGGATAAACTGCATACCGTTAGCGCAGAA GATTTACTGAAAGCAGCAGATCAGCTGCGTATTGCAGAAAAAGAAAACATCTTTCAGCTGTTTTTTCAG CCTGCACTGGATCCGAAAACACTGCCGGAAGAACCGGAAAAAGCAATTGCAGAAGGTGCAGCAAGCGGT ATTCCGCTGCTGATTGGTACAACCCGTGATGAAGGTTACCTGTTTTTTACTCCGGATAGTGATGTTCAT AGCCAAGAAACCCTGGATGCAGCCCTGGAATATCTGCTGGGTAAACCGCTGGCCGAAAAAGTTGCAGAT CTGTATCCGCGTAGCCTGGAAAGCCAGATTCATATGATGACGGATCTGCTGTTTTGGCGTCCGGCAGTT GCATATGCCAGCGCACAGAGCCATTATGCACCGGTTTGGATGTATCGTTTTGATTGGCATCCGAAAAAA 
CCTCCGTATAACAAAGCATTTCATGCACTGGAACTGCCGTTTGTTTTTGGTAATCTGGATGGTCTGGAA CGTATGGCAAAAGCAGAAATTACCGATGAAGTGAAACAACTGAGCCATACCATTCAGAGCGCATGGATT ACCTTTGCAAAAACCGGTAATCCGAGCACCGAAGCAGTTAATTGGCCTGCATATCATGAAGAAACCCGT GAAACCCTGATTCTGGATAGCGAAATTACCATTGAAAATGATCCGGAAAGCGAGAAACGTCAGAAACTG TTTCCGAGCAAAgGTGAAgGTAGCAGCCATCACCATCATCATCATAGCAGTGGTTAA

\section{Protein sequence.}

MTHQIVTTQYGKVKGTTENGVHKWKGI PYAKP PVGQWRFKAPEPPEVWEDVLDATAYGS ICPQPS DLLS LSYTELPRQSEDCLYVNVFAP DTP SKNLPVMVWI HGGAFYLGAGSEP LYDGSKLAAQGEVIVVTLNYRL GPFGFLHLS SFNEAYS DNLGLLDQAAALKWVREN ISAFGGDP DNVTVFGESAGGMS IAALLAMPAAKGL FQKAIMESGASRTMTKEQAASTSAAFLQVLGINEGQLDKLHTVSAEDLLKAADQLRIAEKENIFQLFFQ PALDPKTLPEEPEKAIAEGAASGI PLLIGTTRDEGYLFFTPDSDVHSQETLDAALEYLLGKPLAEKVAD LYPRSLESQ I HMMT DLLFWRPAVAYASAQSHYAPVWMYRF DWHPKKP PYNKAFHALELPFVFGNLDGLE RMAKAEITDEVKQLSHTIQSAWITFAKTGNPSTEAVNWPAYHEETRETLILDSEITIENDPESEKRQKL FPSKGEGSSHHHHHHSSG*

Protein Mass. $\quad$ wild-type Bs2 $(-\mathrm{Met})=55140.21 \mathrm{Da}$ Bs2 F398D (-Met) $=55108.12 \mathrm{Da}$

96 well-plate kinetic assay. Stock solutions of the $\mathrm{Bs} 2$ variant (in $50 \mathrm{mM} \mathrm{NaP}, \mathrm{pH} \mathrm{7.0)}$ ) and $N$-(4nitrophenyl)-butyramide (in DMSO) were prepared. The protein stock solution was kept on ice until use and was freshly prepared before each usage. DMSO and and substrate stock solution were added to wells of a 96 transparent well-plate to a total of $15 \mu \mathrm{L}$. Buffer $(50 \mathrm{mM} \mathrm{NaP}$, $\mathrm{pH} 7.0)$ was added to a total volume of $135 \mu \mathrm{L}(150 \mu \mathrm{L}$ in case of controls to monitor substrate stability). The plate was transferred into a platereader, double orbitally shaken for $5 \mathrm{~s}$ and the absorption at $\lambda_{\mathrm{Ex}}=405 \mathrm{~nm}$ measured to check correct substrate distribution. Then $15 \mu \mathrm{L}$ of protein stock solution were added to each well except the enzyme free controls within $5 \mathrm{~min}$. Final assay conditions were $150 \mu \mathrm{L}$ volume, 10\% DMSO, $N$-(4-nitrophenyl)butyramide $(10,50,100,250500,1000,2000,3000 \mu \mathrm{M}), 20 \mu \mathrm{g} / \mathrm{mL}$ protein. The plate was sealed with an airtight and UV-Vis transparent self-adhesive plastic cover sheet. After sealing, the plate was placed into the plate reader and the assay was monitored using the following program:

Temperature: $21.0 \pm 1.0^{\circ} \mathrm{C}$

Number of repeats: 
Delay between repeats: $\quad 1200 \mathrm{~s}$

Shaking duration: $\quad 5.0 \mathrm{~s}$

Shaking diameter: $\quad 0.70 \mathrm{~mm}$

Shaking type: Double orbit

Delay duration: $\quad 5.0 \mathrm{~s}$

Wavelength $\lambda_{\mathrm{Ex}}$ : $\quad 405 \mathrm{~nm}$

Kinetic assay analysis. In order to convert the absorption reading obtained from the kinetic assay înto concentrations for the determination of kinetic data a calibration curve was generated using 10, 50, 100, $250500,1000,2000,3000 \mu \mathrm{M}$ of 4-nitroaniline in $150 \mu \mathrm{L}$ buffer (50 mM NaPi, pH 7.0, 10\% DMSO). Raw absorption data was converted into product concentrations and data analysis with Origin 2020 was performed to obtained kinetic data assuming Michaelis-Menten kinetics. The maximum velocities $v_{\max }$ were obtained from the linear range of the product concentration vs. time plots and used to calculate $k_{\text {cat }}$ and $K_{\mathrm{M}}$ for Bs2 F398D. 


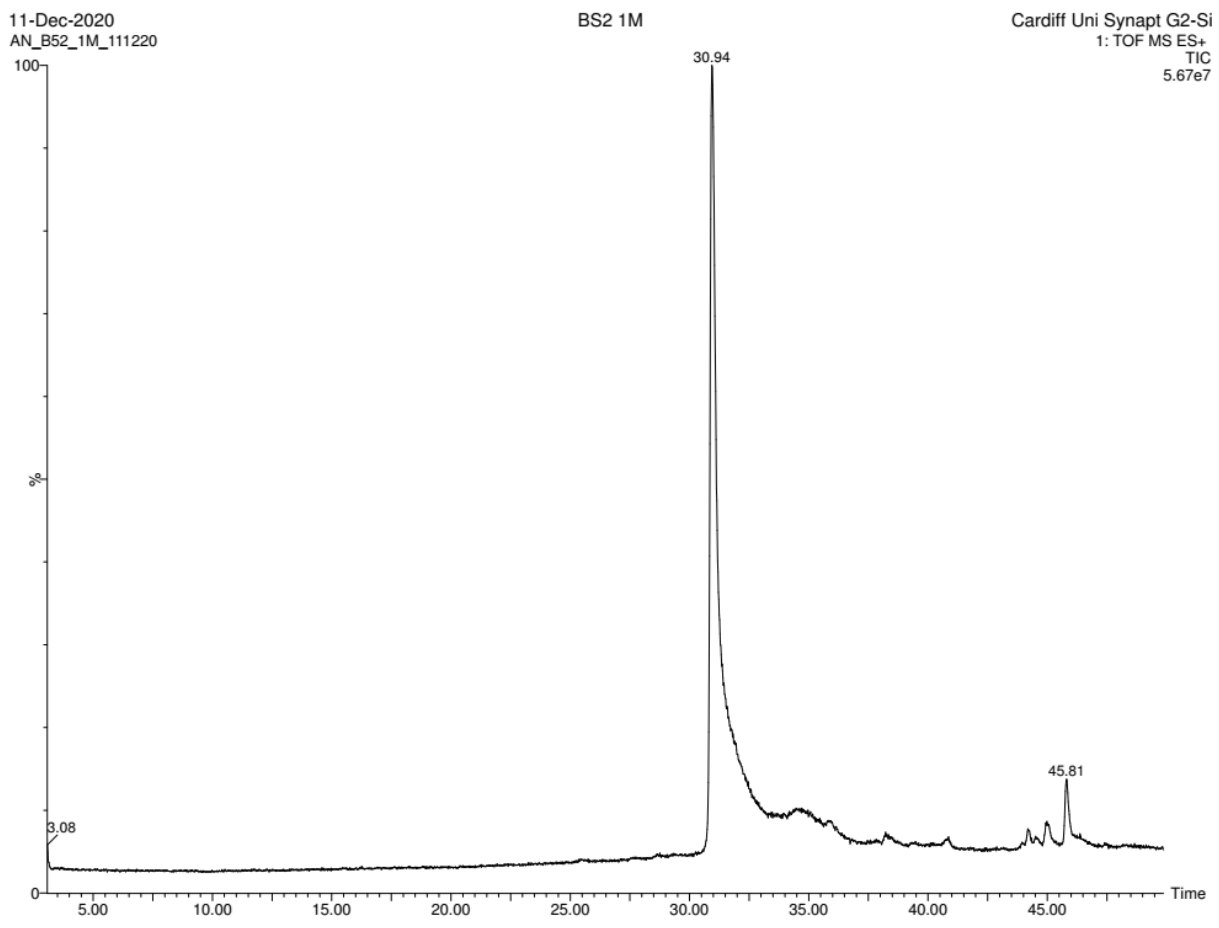

Figure S14. LC chromatogram of purified Bs2 F398D. 

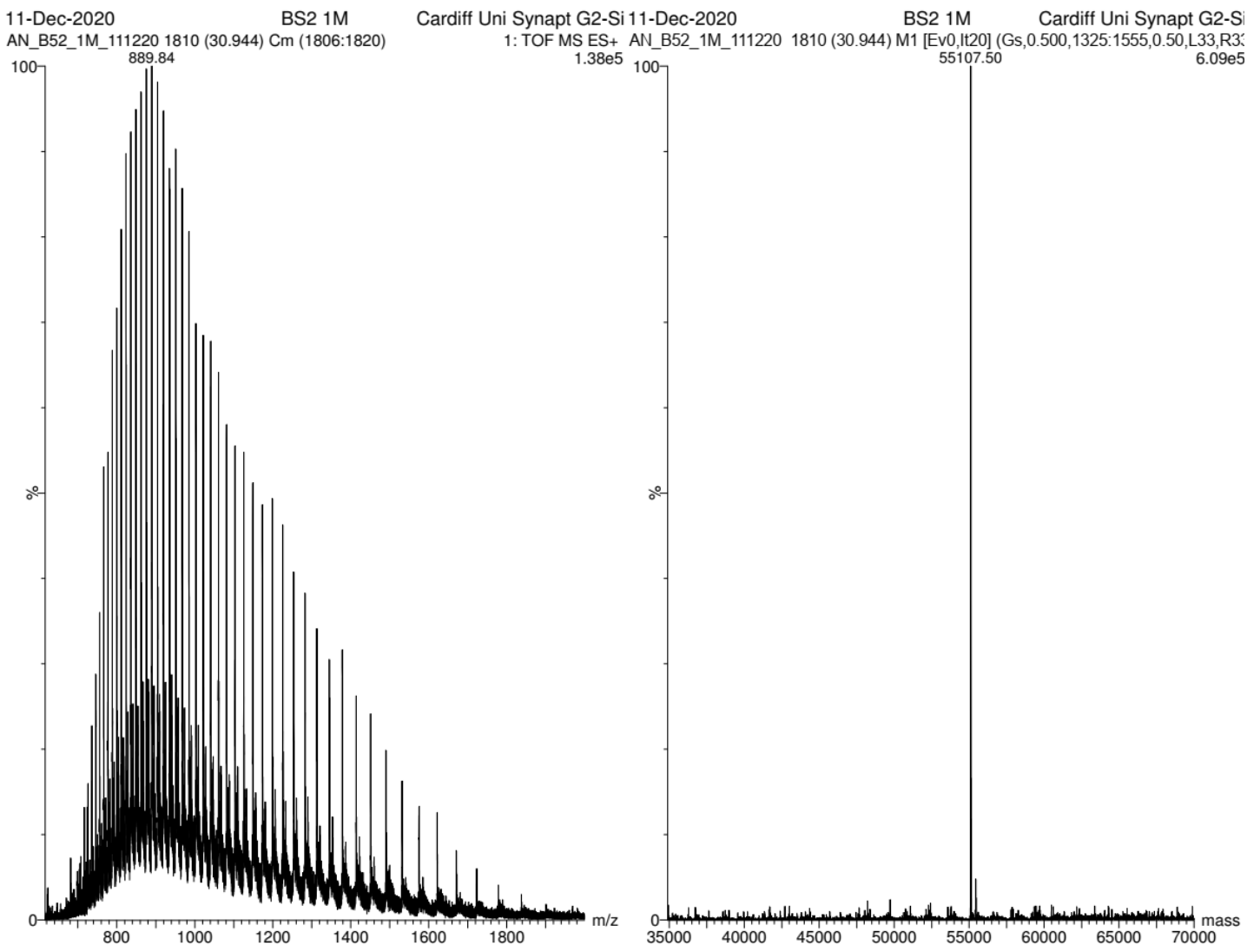

Figure S15. Raw and deconvoluted mass spectrum of purified Bs2 F398D. 


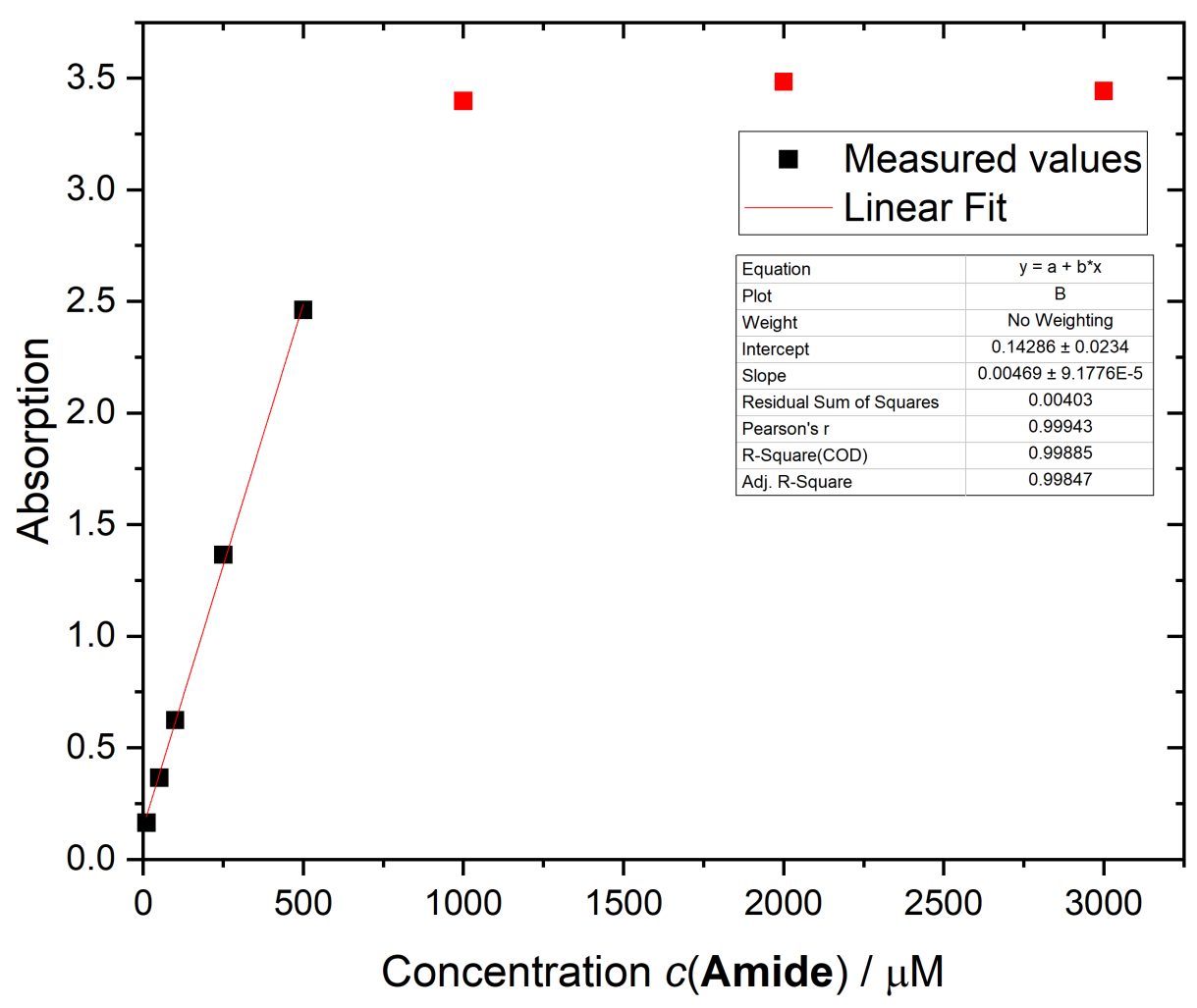

Figure S16. Calibration curve for the 4-nitroaniline concentration in the assay mixture. 


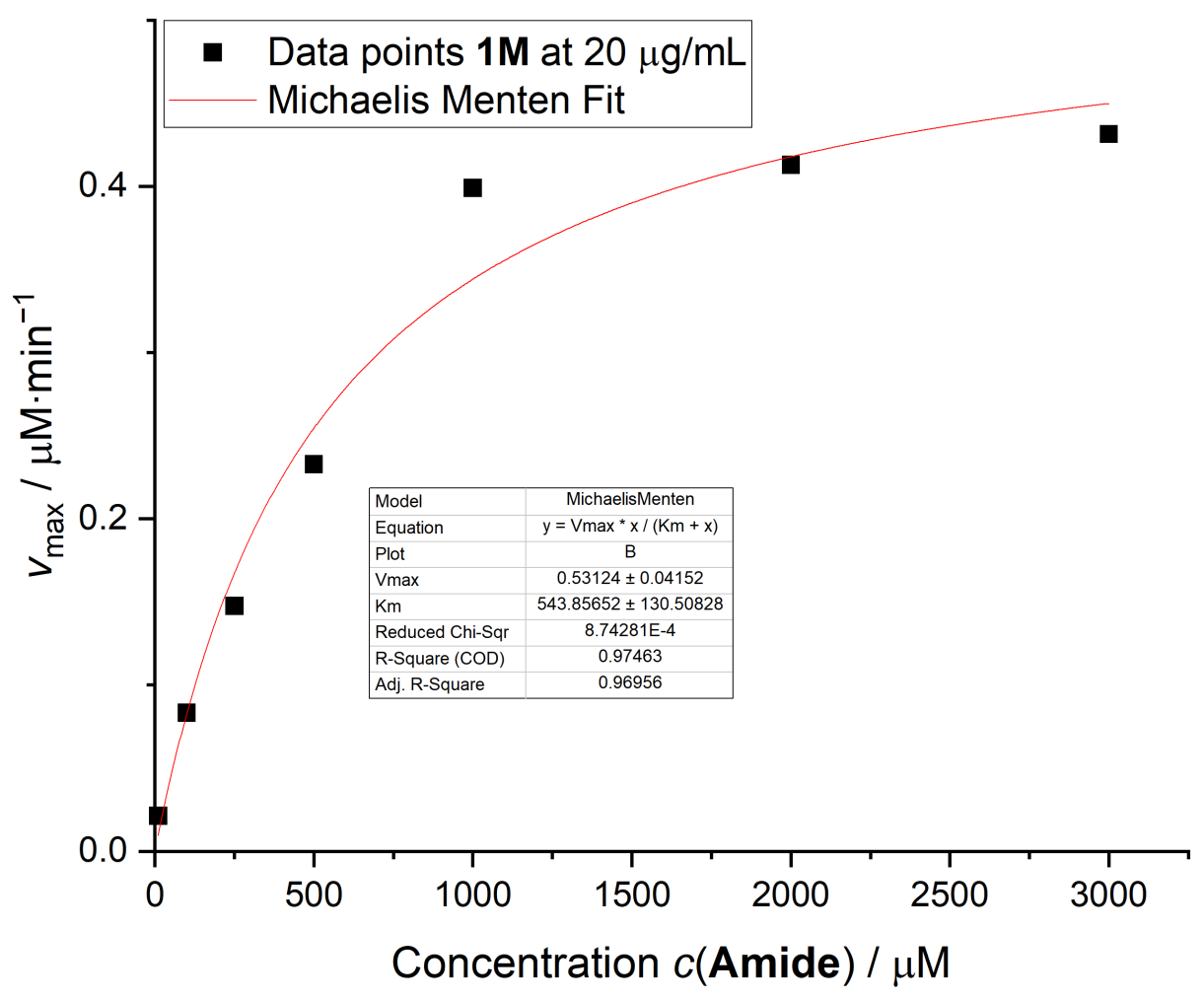

Figure S17. Michaelis-Menten plot for Bs2 F398D, run 1. 


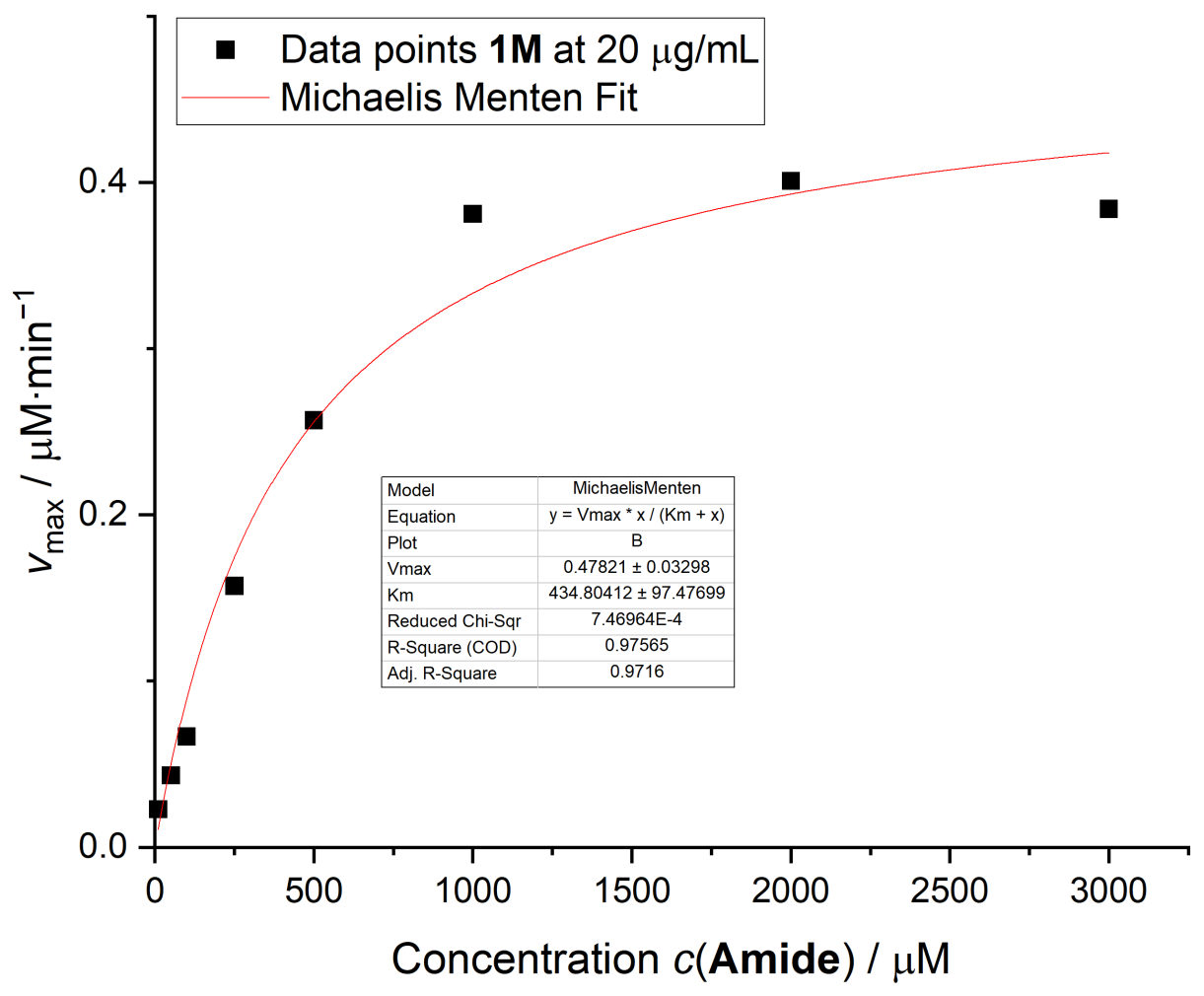

Figure S18. Michaelis-Menten plot for Bs2 F398D, run 2. 


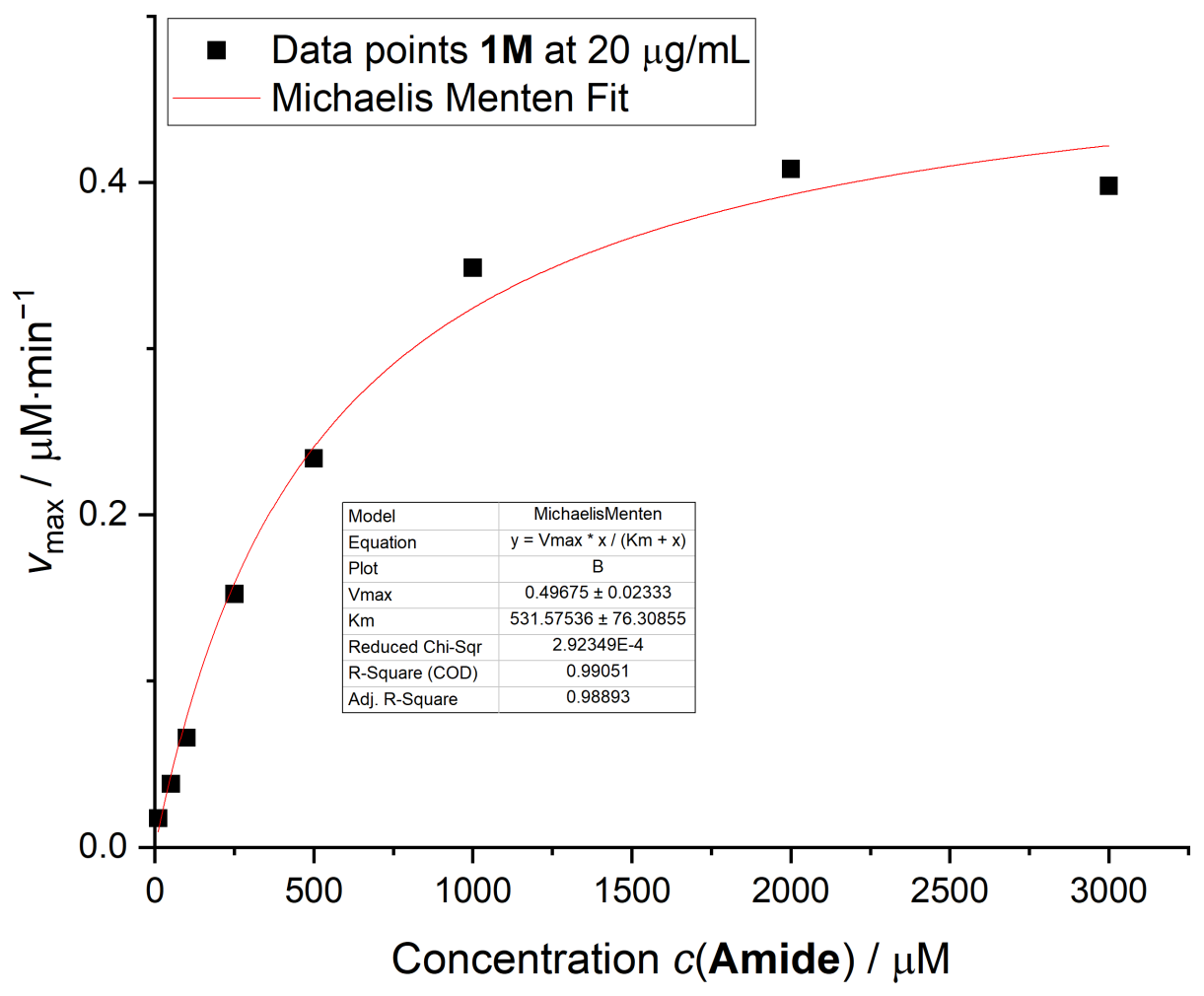

Figure S19. Michaelis-Menten plot for Bs2 F398D, run 3. 


\section{REFERENCES}

(1) Hackenschmidt, S.; Moldenhauer, E. J.; Behrens, G. A.; Gand, M.; Pavlidis, I. V.; Bornscheuer, U. T. Enhancement of Promiscuous Amidase Activity of a Bacillus Subtilis Esterase by Formation of a $\pi-\pi$ Network. ChemCatChem 2014, 6, 1015-1020.

(2) Consortium, T. U. UniProt: A Worldwide Hub of Protein Knowledge. Nucleic Acids Res. 2018, 47, D506-D515.

(3) Spiller, B.; Gershenson, A.; Arnold, F. H.; Stevens, R. C. A Structural View of Evolutionary Divergence. Proc. Natl. Acad. Sci. 1999, 96, 12305-12310.

(4) Sali, A.; Blundell, T. L. Comparative Protein Modelling by Satisfaction of Spatial Restraints. J. Mol. Biol. 1993, 234, 779-815.

(5) Galmés, M. À.; Nödling, A. R.; Luk, L.; Świderek, K.; Moliner, V. Combined Theoretical and Experimental Study to Unravel the Differences in Promiscuous Amidase Activity of Two Nonhomologous Enzymes. ACS Catal. 2021, 8635-8644.

(6) Olsson, M. H. M.; SØndergaard, C. R.; Rostkowski, M.; Jensen, J. H. PROPKA3: Consistent Treatment of Internal and Surface Residues in Empirical p Kapredictions. J. Chem. Theory Comput. 2011, 7, 525-537.

(7) Søndergaard, C. R.; Olsson, M. H. M.; Rostkowski, M.; Jensen, J. H. Improved Treatment of Ligands and Coupling Effects in Empirical Calculation and Rationalization of PKa Values. J Chem Theory Comput 2011, 7, 2284-2295.

(8) Jorgensen, W. L.; Chandrasekhar, J.; Madura, J. D.; Impey, R. W.; Klein, M. L. Comparison of Simple Potential Functions for Simulating Liquid Water. J. Chem. Phys. 1983, 79, 926-935.

(9) Galmés, M. A.; García-Junceda, E.; Świderek, K.; Moliner, V. Exploring the Origin of Amidase Substrate Promiscuity in CALB by a Computational Approach. ACS Catal. 2020, 10, 1938-1946.

(10) Duan, Y.; Wu, C.; Chowdhury, S.; Lee, M. C.; Xiong, G.; Zhang, W. E. I.; Yang, R.; Cieplak, P.; Luo, R. A. Y.; Lee, T.; Caldwell, J.; Wang, J.; Kollman, P. A Point-Charge Force Field for Molecular Mechanics Simulations of Proteins Based on Condensed-Phase. J. Comput. Chem. 2003, 24, 1999-2012.

(11) Phillips, J. C.; Braun, R.; Wang, W. E. I.; Gumbart, J.; Tajkhorshid, E.; Villa, E.; Chipot, C.; Skeel, R. D.; Poincare, H. Scalable Molecular Dynamics with NAMD. J. Comput. Chem. 2005, 26, 1781- 
1802.

(12) Grest, G. S.; Kremer, K. Molecular Dynamics Simulation for Polymers in the Presence of a Heat Bath. Phys. Rev. A 1986, 33, 3628-3631.

(13) Case, D. A.; Aktulga, H. M.; Belfon, K.; Ben-Shalom, I. Y.; Brozell, S. R.; Cerutti, D. S.; Cheatham, T. E. I. I. I.; Cruzeiro, V. W. D.; Darden, T. A.; Duke, R. E.; Giambasu, G.; Gilson, M. K.; Gohlke, H.; Goetz, A. W.; Harris, R.; Izadi, S.; Izmailov, S. A.; Jin, C.; Kasavajhala, K.; et al. Amber 2021. University of California: San Francisco 2021.

(14) Field, M. J.; Bash, P. A.; Karplus, M. A Combined Quantum Mechanical and Molecular Mechanical Potential for Molecular Dynamics Simulations. J. Comput. Chem. 1990, 11, 700-733.

(15) Jorgensen, W. L.; Maxwell, D. S.; Tirado-Rives, J. Development and Testing of the OPLS AllAtom Force Field on Conformational Energetics and Properties of Organic Liquids. J. Am. Chem. Soc. 1996, 118, 11225-11236.

(16) Field, M. J.; Albe, M.; Bret, C.; Proust-De Martin, F.; Thomas, A. The Dynamo Library for Molecular Simulations Using Hybrid Quantum Mechanical and Molecular Mechanical Potentials. J. Comput. Chem. 2000, 21, 1088-1100.

(17) Dewar, M. J. S.; Zoebisch, E. G.; Healy, E. F.; Stewart, J. J. P. Development and Use of Quantum Mechanical Molecular Models. 76. AM1: A New General Purpose Quantum Mechanical Molecular Model. J. Am. Chem. Soc. 1985, 107, 3902-3909.

(18) Zhao, Y.; Truhlar, D. G. The M06 Suite of Density Functionals for Main Group Thermochemistry, Thermochemical Kinetics, Noncovalent Interactions, Excited States, and Transition Elements: Two New Functionals and Systematic Testing of Four M06-Class Functionals and 12 Other Function. Theor. Chem. Acc. 2008, 120, 215-241.

(19) Stewart, J. J. P. Quantum Chemistry Program Exchange 455. 1996, 6.

(20) Frisch, M. J.; Trucks, G. W.; Schlegel, H. B.; Scuseria, G. E.; Robb, M. A.; Cheeseman, J. R.; Scalmani, G.; Barone, V.; Petersson, G. A.; Nakatsuji, H.; Li, X.; Caricato, M.; Marenich, A. V; Bloino, J.; Janesko, B. G.; Gomperts, R.; Mennucci, B.; Hratchian, H. P.; Ortiz, J. V; et al. Gaussian 09, Revision E.01. Gaussian, Inc.: Wallingford, CT 2009.

(21) Byrd, R. H.; Lu, P.; Nocedal, J.; Zhu, C. A Limited Memory Algorithm for Bound Constrained Optimization. SIAM J. Sci. Comput. 1995, 16, 1190-1208. 
(22) J. Turner, A.; Moliner, V.; H. Williams, I. Transition-State Structural Refinement with GRACE and CHARMM: Flexible QM/MM Modelling for Lactate Dehydrogenase. Phys. Chem. Chem. Phys. 1999, 1, 1323-1331.

(23) Martí, S.; Moliner, V.; Tuñón, I. Improving the QM/MM Description of Chemical Processes: A Dual Level Strategy to Explore the Potential Energy Surface in Very Large Systems. J. Chem. Theory Comput. 2005, 1, 1008-1016.

(24) Baker, J.; Kessi, A.; Delley, B. The Generation and Use of Delocalized Internal Coordinates in Geometry Optimization. J. Chem. Phys. 1996, 105, 192-212.

(25) Baker, J. Constrained Optimization in Delocalized Internal Coordinates. J. Comput. Chem. 1997, 18, 1079-1095.

(26) Roux, B. The Calculation of the Potential of Mean Force Using Computer-Simulation. Comput. Phys. Commun. 1995, 91, 275-282.

(27) Torrie, G. M.; Valleau, J. P. Non-Physical Sampling Distibutions in Monte-Carlo Free-Energy Estimation - Umbrella Sampling. J. Comput. Phys. 1977, 23, 187-199.

(28) Kumar, S.; Rosenberg, J. M.; Bouzida, D.; Swendsen, R. H.; Kollman, P. A. The Weighted Histogram Analysis Method for Free-energy Calculations on Biomolecules. I. The Method. J. Comput. Chem. 1992, 13, 1011-1021.

(29) Verlet, L. Computer "Experiments" on Classical Fluids. I. Thermodynamical Properties of Lennard-Jones Molecules. Phys. Rev. 1967, 159, 98-103.

(30) Ruiz-Pernía, J. J.; Silla, E.; Tuñón, I.; Martí, S.; Moliner, V. Hybrid QM/MM Potentials of Mean Force with Interpolated Corrections. J. Phys. Chem. B 2004, 108, 8427-8433.

(31) Ruiz-Pernía, J. J.; Silla, E.; Tuñón, I.; Martí, S. Hybrid Quantum Mechanics/Molecular Mechanics Simulations with Two-Dimensional Interpolated Corrections: Application to Enzymatic Processes. J. Phys. Chem. B 2006, 110, 17663-17670.

(32) Martí, S. QMCube (QM3): An All-Purpose Suite for Multi Scale QM/MM Calculations.

(33) Kneller, G. R. Superposition of Molecular Structures Using Quaternions. Mol. Simul. 1991, 7, 113119. 



\section{Annex D}

Co-author's consent to use the Articles presented in this thesis 

I, Vicent Moliner, hereby authorise Miquel Àngel Galmés Ordinas to include the publications listed below in his doctoral thesis. In addition, I waive the right to use those articles as part of any other doctoral thesis.

List of articles:

(1) Galmés, M. A.; García-Junceda, E.; Świderek, K.; Moliner, V. Exploring the Origin of Amidase Substrate Promiscuity in CALB by a Computational Approach. ACS Catal. 2020, 10, 1938-1946.

(2) Galmés, M. À.; Świderek, K.; Moliner, V. Computational Studies Suggest Promiscuous Candida Antarctica Lipase B as an Environmentally Friendly Alternative for the Production of Epoxides. J. Chem. Inf. Model. 2021.

(3) Galmés, M. À.; Nödling, A. R.; Luk, L.; Świderek, K.; Moliner, V. Combined Theoretical and Experimental Study to Unravel the Differences in Promiscuous Amidase Activity of Two Nonhomologous Enzymes. ACS Catal. 2021, 8635-8644.

(4) Galmés, M. À.; Nödling, A. R.; He, K.; Luk, L.; Świderek, K.; Moliner, V. Computational Design of an Amidase by Combining the Best Electrostatic Features of Two Promiscuous Hydrolases. 2021. Manuscript submitted for publication.

Signed,

$\begin{array}{ll}\text { VICENTE| } & \text { Firmado digitalmente } \\ \text { por VICENTE|MOLINER| } \\ \text { MOLINER| } & \text { IBANEZZ } \\ \text { IBAÑEZ } & \text { Fecha: 2021.10.06 } \\ \text { IBN:52:49+02'00' }\end{array}$

In accordance with article 28 of the Regulations on doctoral studies of the Universitat Jaume I in Castelló, regulated by RD 99/2011, at the Universitat Jaume I (Approved by the Governing Council at its meeting no. 8/2020 held on 2 October 2020):

"(...)

4. In the case of joint publications, all the co-authors must explicitly state their approval that the doctoral student presented the work as part of her/his thesis and the express waiver of presenting this same work as part of another doctoral thesis. This authorisation must be attached as documentation when the evaluation of the thesis begins." 

I, Katarzyna Świderek, hereby authorise Miquel Àngel Galmés Ordinas to include the publications listed below in his doctoral thesis. In addition, I waive the right to use those articles as part of any other doctoral thesis.

List of articles:

(1) Galmés, M. A.; García-Junceda, E.; Świderek, K.; Moliner, V. Exploring the Origin of Amidase Substrate Promiscuity in CALB by a Computational Approach. ACS Catal. 2020, 10, 1938-1946.

(2) Galmés, M. À.; Świderek, K.; Moliner, V. Computational Studies Suggest Promiscuous Candida Antarctica Lipase B as an Environmentally Friendly Alternative for the Production of Epoxides. J. Chem. Inf. Model. 2021.

(3) Galmés, M. À.; Nödling, A. R.; Luk, L.; Świderek, K.; Moliner, V. Combined Theoretical and Experimental Study to Unravel the Differences in Promiscuous Amidase Activity of Two Nonhomologous Enzymes. ACS Catal. 2021, 8635-8644.

(4) Galmés, M. À.; Nödling, A. R.; He, K.; Luk, L.; Świderek, K.; Moliner, V. Computational Design of an Amidase by Combining the Best Electrostatic Features of Two Promiscuous Hydrolases. 2021. Manuscript submitted for publication.

Signed,

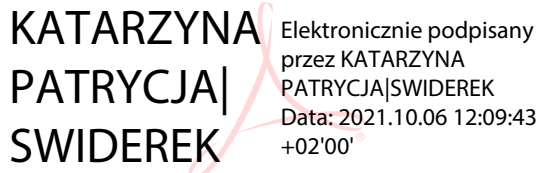

In accordance with article 28 of the Regulations on doctoral studies of the Universitat Jaume I in Castelló, regulated by RD 99/2011, at the Universitat Jaume I (Approved by the Governing Council at its meeting no. 8/2020 held on 2 October 2020):

"(...)

4. In the case of joint publications, all the co-authors must explicitly state their approval that the doctoral student presented the work as part of her/his thesis and the express waiver of presenting this same work as part of another doctoral thesis. This authorisation must be attached as documentation when the evaluation of the thesis begins." 



\section{I UNIVERSITAT \\ JAUME I}

Escola de Doctorat $\cdot$ ED

I, Eduardo García-Junceda, hereby authorise Miquel Àngel Galmés Ordinas to include the publications listed below in his doctoral thesis. In addition, I waive the right to use those articles as part of any other doctoral thesis.

List of articles:

(1) Galmés, M. A.; García-Junceda, E.; Świderek, K.; Moliner, V. Exploring the Origin of Amidase Substrate Promiscuity in CALB by a Computational Approach. ACS Catal. 2020, 10, 1938-1946.

Madrid, 06 de octubre de 2021

Signed,

Eduardo García-Junceda

$\begin{array}{ll}\text { GARCIA-JUNCEDA } & \begin{array}{l}\text { Firmado digitalmente por } \\ \text { GARCIA-JUNCEDA }\end{array} \\ \text { REDONDO } & \text { REDONDO EDUARDO - DNI } \\ \text { EDUARDO - DNI } & \text { 05384079D } \\ \text { 05384079D } & \begin{array}{l}\text { Fecha: 2021.10.06 12:18:26 } \\ +02^{\prime} 00^{\prime}\end{array}\end{array}$

In accordance with article 28 of the Regulations on doctoral studies of the Universitat Jaume I in Castelló, regulated by RD 99/2011, at the Universitat Jaume I (Approved by the Governing Council at its meeting no. 8/2020 held on 2 October 2020):

"(...)

4. In the case of joint publications, all the co-authors must explicitly state their approval that the doctoral student presented the work as part of her/his thesis and the express waiver of presenting this same work as part of another doctoral thesis. This authorisation must be attached as documentation when the evaluation of the thesis begins." 



\section{U UNVEBSTAT \\ JAUME I}

Escola de Doctorat $\cdot$ ED

Cardiff, $23^{\text {rd }}$ October, 2021

I, Louis Luk, hereby authorise Miquel Àngel Galmés Ordinas to include the publications listed below in his doctoral thesis. In addition, I waive the right to use those articles as part of any other doctoral thesis.

List of articles:

(1) Galmés, M. À.; Nödling, A. R.; Luk, L.; Świderek, K.; Moliner, V. Combined Theoretical and Experimental Study to Unravel the Differences in Promiscuous Amidase Activity of Two Nonhomologous Enzymes. ACS Catal. 2021, 8635-8644.

(2) Galmés, M. À.; Nödling, A. R.; He, K.; Luk, L.; Świderek, K.; Moliner, V. Computational Design of an Amidase by Combining the Best Electrostatic Features of Two Promiscuous Hydrolases. 2021. Manuscript submitted for publication.

Signed,

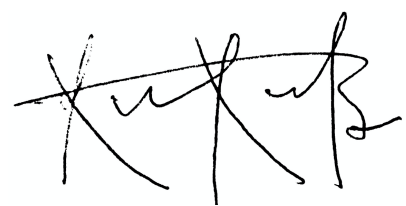

In accordance with article 28 of the Regulations on doctoral studies of the Universitat Jaume I in Castelló, regulated by RD 99/2011, at the Universitat Jaume I (Approved by the Governing Council at its meeting no. 8/2020 held on 2 October 2020):

"(...)

4. In the case of joint publications, all the co-authors must explicitly state their approval that the doctoral student presented the work as part of her/his thesis and the express waiver of presenting this same work as part of another doctoral thesis. This authorisation must be attached as documentation when the evaluation of the thesis begins." 

I, Alexander R Nödling, hereby authorise Miquel Àngel Galmés Ordinas to include the publications listed below in his doctoral thesis. In addition, I waive the right to use those articles as part of any other doctoral thesis.

List of articles:

(1) Galmés, M. À.; Nödling, A. R.; Luk, L.; Świderek, K.; Moliner, V. Combined Theoretical and Experimental Study to Unravel the Differences in Promiscuous Amidase Activity of Two Nonhomologous Enzymes. ACS Catal. 2021, 8635-8644.

(2) Galmés, M. À.; Nödling, A. R.; He, K.; Luk, L.; Świderek, K.; Moliner, V. Computational Design of an Amidase by Combining the Best Electrostatic Features of Two Promiscuous Hydrolases. 2021. Manuscript submitted for publication.

Signed,

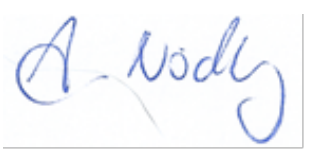

In accordance with article 28 of the Regulations on doctoral studies of the Universitat Jaume I in Castelló, regulated by RD 99/2011, at the Universitat Jaume I (Approved by the Governing Council at its meeting no. 8/2020 held on 2 October 2020):

"(...)

4. In the case of joint publications, all the co-authors must explicitly state their approval that the doctoral student presented the work as part of her/his thesis and the express waiver of presenting this same work as part of another doctoral thesis. This authorisation must be attached as documentation when the evaluation of the thesis begins." 

I, Kaining He, hereby authorise Miquel Àngel Galmés Ordinas to include the publications listed below in his doctoral thesis. In addition, I waive the right to use those articles as part of any other doctoral thesis.

List of articles:

(1) Galmés, M. À.; Nödling, A. R.; He, K.; Luk, L.; Świderek, K.; Moliner, V. Computational Design of an Amidase by Combining the Best Electrostatic Features of Two Promiscuous Hydrolases. 2021. Manuscript submitted for publication.

signed, Kaiming He

In accordance with article 28 of the Regulations on doctoral studies of the Universitat Jaume I in Castello, regulated by RD 99/2011, at the Universitat Jaume I (Approved by the Governing Council at its meeting no. 8/2020 held on 2 October 2020):

"(...)

4. In the case of joint publications, all the co-authors must explicitly state their approval that the doctoral student presented the work as part of her/his thesis and the express waiver of presenting this same work as part of another doctoral thesis. This authorisation must be attached as documentation when the evaluation of the thesis begins." 


